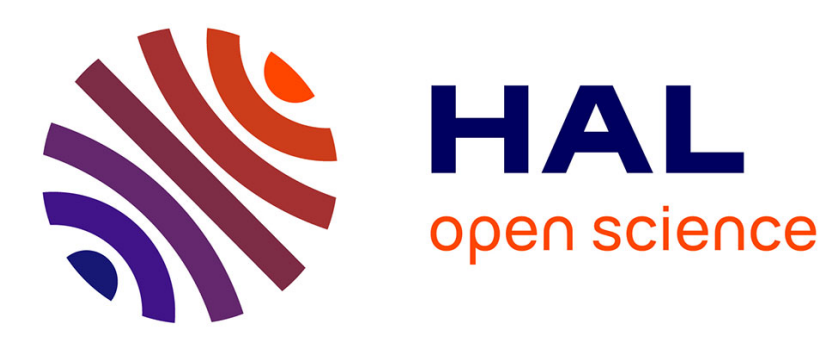

\title{
Equations aux dérivées partielles
}

Thierry Gallouët, Raphaele Herbin

\section{To cite this version:}

Thierry Gallouët, Raphaele Herbin. Equations aux dérivées partielles: Cours de Master 2 de mathématiques Université Aix Marseille. Master. France. 2015. cel-01196782v3

\section{HAL Id: cel-01196782 https://hal.science/cel-01196782v3}

Submitted on 14 Oct 2021 (v3), last revised 23 Jun 2022 (v4)

HAL is a multi-disciplinary open access archive for the deposit and dissemination of scientific research documents, whether they are published or not. The documents may come from teaching and research institutions in France or abroad, or from public or private research centers.
L'archive ouverte pluridisciplinaire HAL, est destinée au dépôt et à la diffusion de documents scientifiques de niveau recherche, publiés ou non, émanant des établissements d'enseignement et de recherche français ou étrangers, des laboratoires publics ou privés. 


\section{Université Aix Marseille}

\section{Master 2 de mathématiques}

\section{Equations aux dérivées partielles}

Thierry Gallouët, Raphaèle Herbin

8 octobre 2021 


\section{Table des matières}

1 Espaces de Sobolev

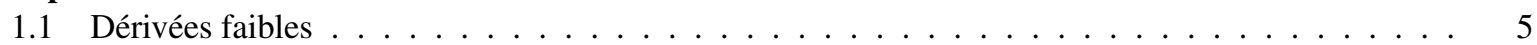

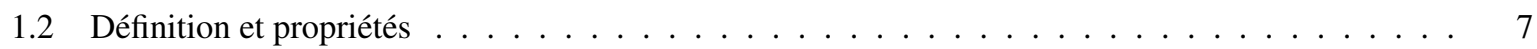

1.3 Rappels d'analyse fonctionnelle $\ldots \ldots \ldots \ldots \ldots \ldots \ldots \ldots$

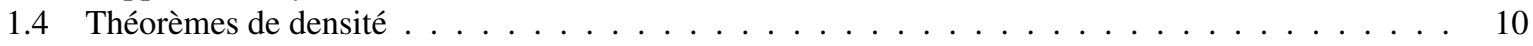

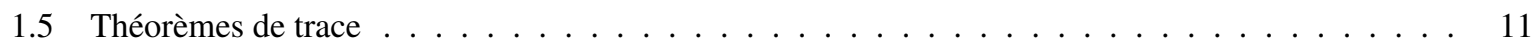

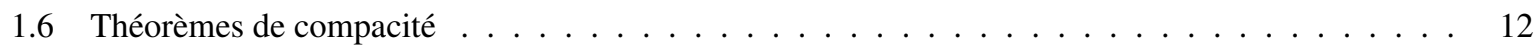

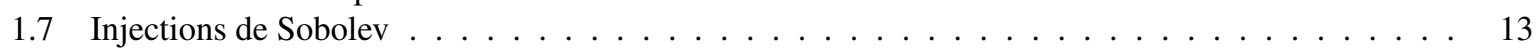

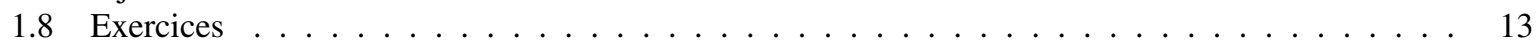

\begin{tabular}{|lll}
2 & Problèmes elliptiques linéaires & 32
\end{tabular}

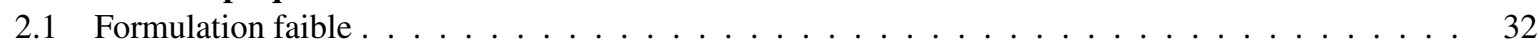

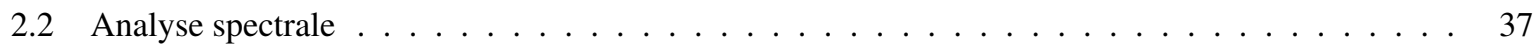

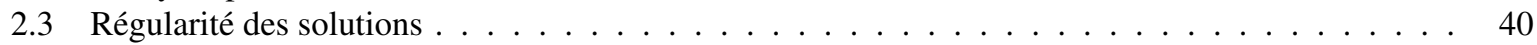

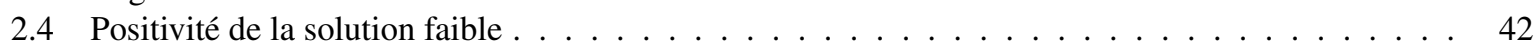

2.5 Condition de Dirichlet non homogène $\ldots \ldots \ldots \ldots \ldots \ldots \ldots \ldots \ldots$

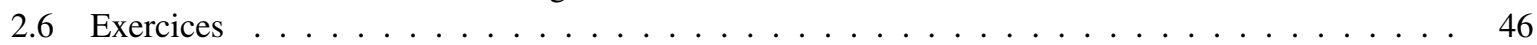

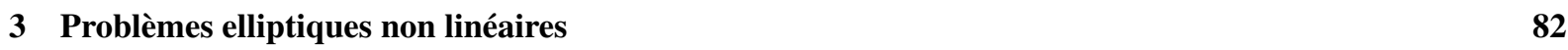

3.1 Méthodes de compacité . . . . . . . . . . . . . . . . . . . . . . . . . 82

3.1 .1 Degré topologique et théorème de Schauder . . . . . . . . . . . . . . . . . . . . 82

3.1 .2 Existence par le théorème de Schauder $\ldots \ldots \ldots \ldots \ldots$. . . . . . . . . . . 85

3.1 .3 Existence par degré topologique $\ldots \ldots \ldots \ldots \ldots$. . . . . . . . . . . . . . 86

3.2 Méthodes de monotonie. . . . . . . . . . . . . . . . . . . . . . . . . . . . 95

3.2 .1 Introduction . . . . . . . . . . . . . . . . . . . . . . . . . . . . 95

3.2 .2 Opérateurs de Leray-Lions $\ldots \ldots \ldots \ldots$. . . . . . . . . . . . . . . . . 96

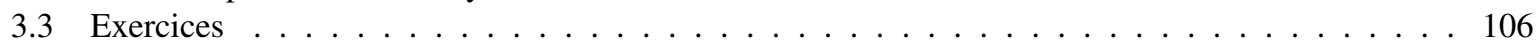

\begin{tabular}{|lll}
4 & Problèmes paraboliques & 124
\end{tabular}

4.1 Aperçu des méthodes . . . . . . . . . . . . . . . . . . . . . . . . . . . . . . . . . . . 124

4.2 Intégration à valeur vectorielle $\ldots \ldots \ldots \ldots$. . . . . . . . . . . . . . . . . . . . . 133

4.3 Étude de l'équation de la chaleur . . . . . . . . . . . . . . . . . . . . . . . . . . 140

4.4 Problèmes paraboliques non linéaires $\ldots \ldots \ldots \ldots \ldots$

4.4 .1 Premier exemple : diffusion non linéaire . . . . . . . . . . . . . . . . . . . . 154

4.4 .2 Deuxième exemple : diffusion convection non linéaire . . . . . . . . . . . . . . . . 155

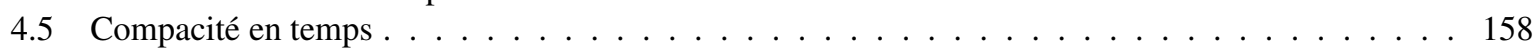


4.6 Exercices . . . . . . . . . . . . . . . . . . . . . . . . . . 171

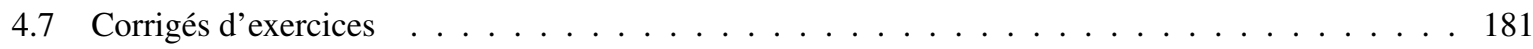

\begin{tabular}{|lll}
5 & Problèmes hyperboliques & 196
\end{tabular}

5.1 Le cas unidimensionnel . . . . . . . . . . . . . . . . . . . . . . . . . . . . . . . 196

$5.1 .1 \quad$ Solutions classiques et courbes caractéristiques . . . . . . . . . . . . . . . . . . 197

5.1 .2 Solutions faibles $\ldots \ldots \ldots \ldots \ldots$

5.1 .3 Solution entropique. . . . . . . . . . . . . . . . . . . . . . . . . 203

5.1 .4 Conditions limites $\ldots \ldots \ldots$

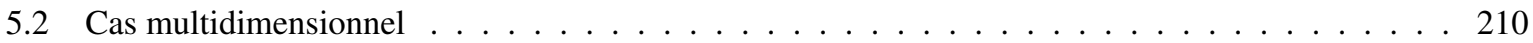

5.2 .1 Cas sans condition limite . . . . . . . . . . . . . . . . . . . . . 211

$5.2 .2 \quad$ Cas des conditions aux limites . . . . . . . . . . . . . . . . . . . . . . . 217

5.3 Systèmes hyperboliques . . . . . . . . . . . . . . . . . . . . . . . . . . . . 219

5.3 .1 Définitions . . . . . . . . . . . . . . . . . . . . . . . . . . . . . . . . . . . . . 219

5.3 .2 Solutions faibles, solutions entropiques . . . . . . . . . . . . . . . . . . . . 220

5.3 .3 Résolution du problème de Riemann . . . . . . . . . . . . . . . . . . . . . . . . . 223

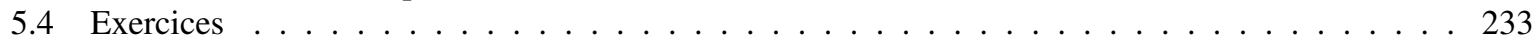

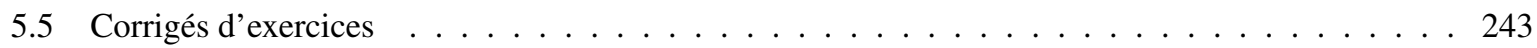




\section{Introduction}

Ce cours décrit quelques outils pour l'étude mathématique des équations aux dérivées partielles (EDP).

Une équation différentielle aux dérivées partielles est une équation mathématique dont l'inconnue est une fonction de plusieurs variables qui fait intervenir les dérivées partielles de cette fonction par rapport à ces variables.

Les équations aux dérivées partielles interviennent dans de nombreux modèles de physique, d'ingénierie ou de biologie, par exemple tels que la propagation de la chaleur ou du son, l'écoulement des fluides, l'électrodynamique, les épidémies. Elles peuvent être linéaires, c'est le cas de l'équation de la chaleur par exemple, ou non linéaires, comme les équations de Barré de Saint-Venant qui décrivent le mouvement d'un fluide de faible épaisseur.

Les outils que nous développons dans ce livre ont comme objectif d'obtenir des résultats d'existence (et souvent d'unicité) pour quelques exemples de problèmes d'EDP de nature diverse (elliptique, parabolique ou hyperbolique). Plusieurs exemples de problèmes non linéaires sont étudiés, avec des outils récents permettant de montrer l'existence et éventuellement l'unicité de la solution.

Le contenu de ce livre est issu de plusieurs cours de master 2ème année que les auteurs ont enseigné à l'université d'Aix-Marseille, à l'ENS Lyon et à l'université de Savoie; nous tenons à remercier nos étudiants pour leur intérêt et leurs remarques. Même si nous rappelons la plupart des notions avancées que nous utilisons, il va de soi qu'une lecture profitable de ce livre nécessite de bonnes connaissances d'analyse réelle, de théorie d'intégration de Lebesgue et d'analyse fonctionnelle.

L'ouvrage comporte de très nombreux exercices et problèmes, la plupart sont résolus. Certains de ces problèmes ont pour objet la démonstration de résultats relativement récents.

Nous rappelons au chapitre 1 la définition des espaces fonctionnels de Sobolev nécessaires pour écrire les équations considérées, à partir des notions de dérivée faible et dérivée par transposition. Quelques résultats d'analyse fonctionnelle sont ensuite énoncés, ainsi que des théorèmes classiques de densité, de trace et de compacité, et les fameuses injections de Sobolev.

Le chapitre 2 est consacré aux opérateurs elliptiques linéaires, dont un exemple type est l'equation de la chaleur en régime stationnaire, qui fait intervenir les dérivées partielles d'ordre 2 de la fonction inconnue (la température) par rapport aux variables d'espace. On étudie leur formulation faible ou variationnelle, ainsi que la régularité de leurs solutions. Les exercices de ce chapitre permettent d'approfondir des résultats d'analyse spectrale, d'introduire les espaces de Sobolev à poids, ou encore de prouver l'existence des solutions au problème de Stokes ou aux équations de Schrödinger.

Les opérateurs elliptiques non linéaires sont étudiés au chapitre 3. On distingue deux types de méthodes pour montrer l'existence des solutions : les méthodes de compacité, soit par point fixe, soit par degré topologique, et les méthodes de monotonie. On donne des exemples d'application de ces méthodes sur plusieurs problèmes non linéaires, dans le texte de cours et dans les exercices : en particulier par point fixe de Schauder pour un opérateur de diffusion semi-linéaire, par degré topologique pour un opérateur de diffusion convection réaction non linéaire, et par monotonie pour des opérateurs de type Leray-Lions.

Le chapitre 4 est consacré aux équations de type parabolique, dont un exemple type est l'équation de la chaleur en régime transitoire, qui fait intervenir la dérivée partielle d'ordre 1 de la fonction inconnue par rapport au temps et les 
dérivées partielles d'ordre 2 par rapport aux variables d'espace. On commence par un aperçu des méthodes utilisées pour leur étude théorique. Elles nécessitent l'intégration à valeur dans un espace de Banach, dont les principes sont rappelés au second paragraphe, ainsi que l'extension des notions de dérivée faible et par transposition dans ces espaces. L'existence, unicité et régularité des solutions de l'équation de la chaleur est ensuite abordée. On donne également la démonstration de l'équivalence de deux formulations faibles classiquement utilisées en analyse et analyse numérique. Les méthodes de point fixe, de degré topologique et de monotonie sont appliquées à plusieurs exemples d'équations paraboliques non linéaires dans le texte du cours (diffusion, convection-diffusion-réaction, opérateurs de Leray-Lions) et dans les exercices (Stefan). Les résultats de compacité de type Aubin-Simon sont généralisés à des suites d'espace, pour permet leur utilisation dans le cadre de l'étude de convergence de schémas numériques.

Enfin, le dernier chapitre s'intéresse aux lois de conservation hyperboliques (équations et systèmes), qui font intervenir les dérivées d'ordre 1 de la fonction inconnue. Les solutions de ces équations ont un comportement très différent de celles des équations paraboliques, car, même en partant de données initiales régulières, les solutions peuvent devenir discontinues et donc ne pas avoir de sens classique. On étudie d'abord le cas scalaire, avec un problème de Cauchy unidimensionnel, c.à.d. d'une équation hyperbolique faisant intervenir une fonction inconnue d'une variable d'espace et de la variable temps, avec une condition initiale et posée sur tout $\mathbb{R}$. On introduit la notion de solution entropique, qui permet de déterminer une solution unique. Le cas des conditions limites est succinctement exposé et repris plus en détails dans le cadre multidimensionnel. On étudie ensuite le cas des systèmes hyperboliques dans le cas unidimensionnel avec la variable spatiale dans $\mathbb{R}$ tout entier. Le cas des systèmes hyperboliques avec condition aux limites et le cas multidimensionnel ne sont pas abordés sans ce cours, la théorie mathématique des systèmes hyperboliques étant d'ailleurs encore très incomplète. Les systèmes hyperboliques interviennent souvent en mécanique des fluides, et leur résolution numérique fait intervenir le problème de Riemann (problème de Cauchy avec donnée constante à gauche et à droite). Nous donnons une étude approfondie du problème de Riemann, avec, en particulier en exercice, la solution complète du problème de Riemann pour les équations de Barré de St-Venant.

Nous donnons en fin de ce livre une bibliographie des articles phares relatifs aux résultats exposés, ainsi que de quelques ouvrages de référence. Il va de soi que cette bibliographie est loin d'être exhaustive. 


\section{Chapitre 1}

\section{Espaces de Sobolev}

Les espaces de Sobolev ${ }^{1}$ sont des espaces fonctionnels, c'est-à-dire des espaces dont les élements sont des fonctions, et ces fonctions sont telles que leurs puissances et les puissances de leurs dérivées (au sens de la transposition, ou au sens faible, que nous allons préciser) sont intégrables au sens de Lebesgue ${ }^{2}$. Tout comme les espaces de Lebesgue, ces espaces sont des espaces de Banach ${ }^{34}$. Le fait que les espaces de Sobolev sont complets est très important pour démontrer l'existence de solutions à de nombreuses équations aux dérivées partielles.

Les résultats concernant les espaces de Sobolev ainsi que les rappels d'analyse fonctionnelle et de théorie de l'intégration ne sont que pour la plupart énoncés dans ce chapitre. On pourra trouver les démonstrations et approfondir le sujet dans les ouvrages [1],[8] et [15] par exemple.

\subsection{Dérivées faibles}

La notion de dérivée faible apparaît déjà dans un article célebre de Jean Leray ${ }^{5}$ paru en 1934, sur les équations de ${ }^{6}$-Stokes 7 ; dans cet article, elle apparaît sous le nom de "quasi-dérivée"([20] page 205). Cette notion est fondamentale pour l'étude de l'existence de solution pour beaucoup d'équations aux dérivées partielles.

Dans toute la suite, $\Omega$ désigne un ouvert de $\mathbb{R}^{N}, N \geq 1$, et on note $C_{c}^{\infty}(\Omega)$ l'ensemble des fonctions de classe $C^{\infty}$ et à support compact sur $\Omega$ c'est-à-dire

$$
C_{c}^{\infty}(\Omega)=\left\{u \in C^{\infty}(\Omega) ; \exists K \subset \Omega, K \text { compact } ; u=0 \text { sur } K^{c}\right\} .
$$

De plus, l'ouvert $\Omega$ sera toujours muni de la tribu de Borel (ou tribu borélienne), notée $\mathcal{B}(\Omega)$, et de la mesure de Lebesgue, notée $\lambda$ si $N=1$ et $\lambda_{N}$ si $N>1$. Les intégrales seront toujours par rapport à cette mesure de Lebesgue, sauf indication contraire.

Le lemme suivant est absolument fondamental, car il permet de confondre une fonction localement intégrable $f \in L_{\text {loc }}^{1}(\Omega)$ avec l'application linéaire $T_{f}: C_{c}^{\infty}(\Omega) \rightarrow \mathbb{R}$ définie par $\varphi \in C_{c}^{\infty}(\Omega) \mapsto T_{f}(\varphi)=\int_{\Omega} f(x) \varphi(x) \mathrm{d} x$.

1. Sergueï Lvovitch Sobolev, (1908-1989) est un mathématicien et physicien russe.

2. Henri-Léon Lebesgue (1875-1941), mathématicien français, reconnu pour sa théorie d'intégration

3. Un espace de Banach est un espace vectoriel normé complet.

4. Stefan Banach (1892-1945), mathématicien polonais, fondateur de l'analyse fonctionnelle moderne.

5. Jean Leray, 1906 -1998, est un mathématicien français; il a travaillé sur les équations aux dérivées partielles et sur la topologie algébrique.

6. Claude Louis Marie Henri Navier (1785-1836), ingénieur, mathématicien et économiste français du XIXe siècle, qui établit les équations décrivant le mouvement des fluides.

7. George Gabriel Stokes (1819-1903) physicien et mathématicien irlandais, professeur à l'Université de Cambridge, Angleterre. 
On rappelle que $f \in L_{\text {loc }}^{1}(\Omega)$ si, pour tout sous-ensemble compact $K$ de $\Omega$, la restriction $\left.f\right|_{K}$ de $f$ à $K$ est un élément de $L^{1}(K)$.

Lemme 1.1 (Egalité presque partout dans $L^{\mathbf{1}}$ ) Soit $\Omega$ un ouvert de $\mathbb{R}^{N}, N \geq 1$, et soient $f$ et $g \in L_{\text {loc }}^{1}(\Omega)$. Alors :

$$
\left[\forall \varphi \in C_{c}^{\infty}(\Omega), \int_{\Omega} f(x) \varphi(x) \mathrm{d} x=\int_{\Omega} g(x) \varphi(x) \mathrm{d} x\right] \Longleftrightarrow[f=g \quad p . p . .]
$$

Démonstration : La démonstration de ce lemme utilise la régularisation d'une fonction intégrable par la convolution avec une suite de noyaux régularisants : voir [15, Exercice 8.7 page 480].

On note $\mathcal{D}(\Omega)$ l'espace $C_{c}^{\infty}(\Omega)$ et $\mathcal{D}^{\star}(\Omega)$ l'ensemble des formes linéaires sur $\mathcal{D}(\Omega)$ : on dit que $\mathcal{D}^{\star}(\Omega)$ est le dual algébrique ${ }^{8}$ de $\mathcal{D}(\Omega)$. Si $T \in \mathcal{D}^{\star}(\Omega)$ et $\varphi \in \mathcal{D}(\Omega)$, le réel $T(\varphi)$ s'appelle l'“action de $T$ sur $\varphi$ " et sera noté $\langle T, \varphi\rangle_{\mathcal{D}^{\star}(\Omega), \mathcal{D}(\Omega)}$ ou $\langle T, \varphi\rangle_{\mathcal{D}^{\star}(\Omega), \mathcal{D}(\Omega)}$. Le lemme 1.1 nous permet de définir la dérivée par transposition d'une fonction $L_{\mathrm{loc}}^{1}$ de la manière suivante :

Définition 1.2 (Dérivée par transposition) Soit $\Omega$ un ouvert de $\mathbb{R}^{N}, N \geq 1$; soit $\mathcal{D}(\Omega)=C_{c}^{\infty}(\Omega)$ et $\mathcal{D}^{\star}(\Omega)$ son dual algébrique, c'est-à-dire l'ensemble des formes linéaires sur $\mathcal{D}(\Omega)$;

- Soit $f \in L_{\mathrm{loc}}^{1}(\Omega)$, on appelle dérivée par transposition de f par rapport à sa i-ème variable la forme linéaire $D_{i} f \operatorname{sur} C_{c}^{\infty}(\Omega)$ définie par :

$$
\left\langle D_{i} f, \varphi\right\rangle_{\mathcal{D}^{\star}(\Omega), \mathcal{D}(\Omega)}=-\int_{\Omega} f \partial_{i} \varphi \mathrm{d} x .
$$

où $\partial_{i} \varphi$ désigne la dérivée partielle classique de $\varphi$ par rapport à sa $i$-ème variable. Donc $D_{i} f$ est un élément de $\mathcal{D}^{\star}(\Omega)$ Noter que si $f \in C^{1}(\Omega)$, alors $D_{i} f$ n'est autre que $\partial_{i} f$ car on confond $\partial_{i} f$ et $T_{\partial_{i} f}$ (qui est l'élément de $\mathcal{D}^{\star}(\Omega)$ induit par $\left.\partial_{i} f\right)$. Il s'agit donc bien d'une généralisation de la notion de dérivée.

Si la forme linéaire $D_{i} f$ peut étre confondue avec une fonction localement intégrable au sens du lemme 1.1 . on dit que $f$ admet une dérivée faible.

- Soit $T \in \mathcal{D}^{\star}(\Omega)$; on définit la dérivée par transposition $D_{i} T$ de $T$ par :

$$
\left\langle D_{i} T, \varphi\right\rangle_{\mathcal{D}^{\star}(\Omega), \mathcal{D}(\Omega)}=-\left\langle T, \partial_{i} \varphi\right\rangle_{\mathcal{D}^{\star}(\Omega), \mathcal{D}(\Omega)}, \forall \varphi \in C_{c}^{\infty}(\Omega) .
$$

La notion de dérivée au sens des distributions est un peu plus délicate à définir car elle demande la définition d'une topologie sur $C_{c}^{\infty}(\Omega)$. Nous n'en aurons pas besoin dans le cadre de ce cours. Cependant lorsque $C_{c}^{\infty}(\Omega)$ est muni de cette topologie, les deux définitions coïncident.

Voici un exemple de dérivée par transposition. La fonction de Heaviside 9 définie par

$$
H(x)=\left\{\begin{array}{l}
1 \text { if } x \geq 0, \\
0 \text { if } x<0,
\end{array}\right.
$$

est localement intégrable. Elle admet donc une dérivée par transposition. Pour calculer cette dérivée, notée $D H$, on remarque, pour $\varphi \in C_{c}^{\infty}(\mathbb{R})$, on a :

8. Dans le cadre de la théorie des distributions, l'espace $\mathcal{D}(\Omega)$ est munie d'une topologie (assez compliquée, et dont on n'a pas besoin ici) ; l'espace dual topologique de $\mathcal{D}(\Omega)$ est le sous-espace de $\mathcal{D}^{\star}(\Omega)$ constitué des formes linéaires continues pour cette topologie, on le note $\mathcal{D}^{\prime}(\Omega)$. Ici et dans toute la suite de ce cours, nous n'utiliserons pas les distributions, et donc nous ne munissons pas $\mathcal{D}(\Omega)$ d'une topologie.

9. Oliver Heaviside (1850 - 1925) physicien britannique autodidacte. 


$$
-\int_{\mathbb{R}} H(x) \varphi^{\prime}(x) \mathrm{d} x=-\int_{0}^{+\infty} \varphi^{\prime}(x) \mathrm{d} x=\varphi(0),
$$

et donc $D H$ est la forme linéaire qui à $\varphi$ associe sa valeur en 0 , qu'on appelle aussi "mesure de Dirac en 0" : $D H=\delta_{0}$. Par contre, cette dérivée n'est pas une dérivée faible, car $\delta_{0}$ ne peut pas étre assimilée à une fonction de $L_{\text {loc }}^{1}$, au sens où il n'existe pas de fonction $g \in L_{\text {loc }}^{1}(\mathbb{R})$ telle que $\delta_{0}(\varphi)=\int_{\mathbb{R}} g \varphi \mathrm{d} x$ pour tout $\varphi \in C_{c}^{\infty}(\mathbb{R})($ voir exercice 1.1).

La définition 1.2 permet de définir des dérivées par transposition d'une fonction $L_{\text {loc }}^{1}$ (ou d'un élément de $\mathcal{D}^{\star}(\Omega)$ ) à tous les ordres. Par l'identification d'une fonction $L_{\text {loc }}^{1}$ avec l'élément de $\mathcal{D}^{\star}(\Omega)$ qu'elle représente, on peut aussi définir la notion de dérivée faible à tous les ordres. Plus précisément, pour $\alpha=\left(\alpha_{1}, \ldots \alpha_{N}\right) \in \mathbb{N}^{N}$, et $u \in L_{\text {loc }}^{1}\left(\mathbb{R}^{N}\right)$, on définit la dérivée faible $D_{1}^{\alpha_{1}} \ldots D_{N}^{\alpha_{N}} u \in L_{\text {loc }}^{1}\left(\mathbb{R}^{N}\right)$, si elle existe, par

$$
\int_{\mathbb{R}^{N}} D_{1}^{\alpha_{1}} \ldots D_{N}^{\alpha_{N}} u(x) \varphi(x) \mathrm{d} x=(-1)^{|\alpha|} \int_{\mathbb{R}^{N}} u(x) \partial_{1}^{\alpha_{1}} \ldots \partial_{N}^{\alpha_{N}} \varphi(x) \mathrm{d} x, \forall \varphi \in C_{c}^{\infty}\left(\mathbb{R}^{N}\right),
$$

où $|\alpha|=\alpha_{1}+\ldots+\alpha_{N}$ et $\partial_{i}^{\alpha_{i}} \varphi$ désigne la dérivée partielle (classique) d'ordre $\alpha_{i}$ par rapport à la $i$-ème variable.

Remarque $1.3\left(\right.$ Convergence dans $\left.\mathcal{D}^{\star}\right)$ Soit $\Omega$ un ouvert de $\mathbb{R}^{N}(N \geq 1),\left(T_{n}\right)_{n \in \mathbb{N}}$ une suite déléments de $\mathcal{D}^{\star}(\Omega)$ et $T \in \mathcal{D}^{\star}(\Omega)$. On dit que $T_{n} \rightarrow T$ dans $\mathcal{D}^{\star}(\Omega)$, quand $n \rightarrow+\infty$, si

$$
\left\langle T_{n}, \phi\right\rangle_{\mathcal{D}^{\star}(\Omega), \mathcal{D}(\Omega)} \rightarrow\langle T, \phi\rangle_{\mathcal{D}^{\star}(\Omega), \mathcal{D}(\Omega)} \text { pour tout } \phi \in C_{c}^{\infty}(\Omega) .
$$

Il s'agit donc de la convergence simple dans l'ensemble des applications de $C_{c}^{\infty}(\Omega)$ dans $\mathbb{R}$.

Lorsque l'on s'intéresse aux distributions, on ajoute une structure topologique à l'espace $C_{c}^{\infty}(\Omega)$ (non décrite dans ce livre) et, au lieu de travailler avec $\mathcal{D}^{\star}(\Omega)$, on travaille avec l'espace strictement plus petit des applications linéaires continues de $C_{c}^{\infty}(\Omega)$ dans $\mathbb{R}$, espace qu'on note $\mathcal{D}^{\prime}(\Omega)$. Toutefois, même lorsque l'on travaille avec l'espace $\mathcal{D}^{\prime}(\Omega)$, la notion de convergence est toujours donnée par 1.2 .

\subsection{Définition et propriétés}

Définition 1.4 (Espaces de Sobolev) Soit $\Omega$ un ouvert de $\mathbb{R}^{N}, N \geq 1$.

1. L'espace $H^{1}(\Omega)$ est défini par :

$$
H^{1}(\Omega)=\left\{u \in L^{2}(\Omega) \text { t.q. } D_{i} u \in L^{2}(\Omega), \forall i=1, \ldots, N\right\} .
$$

Dans cette définition, lorsqu'on écrit $D_{i} u \in L^{2}(\Omega)$, on sous-entend

$$
\exists g \in L^{2}(\Omega) ;\left\langle D_{i} f, \varphi\right\rangle_{\mathcal{D}^{\star}(\Omega), \mathcal{D}(\Omega)}=-\int_{\Omega} g \varphi \mathrm{d} x, \forall \varphi \in C_{c}^{\infty}(\Omega) .
$$

2. L'espace $H^{m}(\Omega)$ est défini pour $m \in \mathbb{N}$ par :

$$
H^{m}(\Omega)=\left\{u \in L^{2}(\Omega) ; D^{\alpha} u \in L^{2}(\Omega) \forall \alpha \in \mathbb{N}^{N} ;|\alpha| \leq m\right\} .
$$

3. L'espace $W^{m, p}(\Omega)$ est défini pour $1 \leqslant p \leqslant \infty$ et $m \in \mathbb{N}$, par

$$
W^{m, p}(\Omega)=\left\{u \in L^{p}(\Omega) ; D^{\alpha} u \in L^{p}(\Omega), \forall \alpha \in \mathbb{N}^{N} ;|\alpha| \leq m .\right\}
$$

4. Noter que pour $m=0$, l'espace $W^{m, p}(\Omega)$ est l'espace de Lebesgue $L^{p}(\Omega)$. 
On note $(\cdot \mid \cdot)_{L^{2}}$ le produit scalaire dans $L^{2}(\Omega)$, i.e.

$$
(u \mid v)_{L^{2}}=\int_{\Omega} u v \mathrm{~d} x
$$

et $\|\cdot\|_{L^{p}}$ la norme dans $L^{p}(\Omega)$., i.e.

$$
\|u\|_{L^{p}}=\left(\left(\int_{\Omega}|u|^{p} \mathrm{~d} x\right)^{\frac{1}{p}} .\right.
$$

Proposition 1.5 (Structure d'espace vectoriel) Les espaces $H^{m}(\Omega)$ sont des espaces de Hilbert $\left.\right|^{10}{ }^{11}$ lorqu'on les munit du produit scalaire

$$
(u \mid v)_{H^{m}}=\sum_{|\alpha| \leq m}\left(D^{\alpha} u \mid D^{\alpha} v\right)_{L^{2}}
$$

Noter que $W^{m, 2}(\Omega)=H^{m}(\Omega)$.

Une norme naturelle sur $W^{m, p}(\Omega)$ est définie par :

$$
\|u\|_{W^{m, p}}=\left\{\begin{array}{l}
\left(\sum_{0 \leqslant|\alpha| \leqslant m}\left\|D^{\alpha} u\right\|_{L^{p}}^{p}\right)^{1 \mid p}, \quad \text { si } 1 \leqslant p<+\infty ; \\
\max _{0 \leqslant|\alpha| \leqslant m}\left\|D^{\alpha} u\right\|_{L^{\infty}}, \quad \text { si } p=+\infty .
\end{array}\right.
$$

Muni de cette norme $W^{m, p}(\Omega)$ est un espace de Banach. On peut montrer que la norme :

$$
\|u\|_{m, p}= \begin{cases}\sum_{0 \leqslant|\alpha| \leqslant m}\left\|D^{\alpha} u\right\|_{L^{p}}, & 1 \leqslant p<+\infty ; \\ \sum_{0 \leqslant|\alpha| \leqslant m}\left\|D^{\alpha} u\right\|_{L^{\infty}}, & p=+\infty .\end{cases}
$$

est une norme équivalente à la norme définie par (1.3) : ceci est une conséquence de l'équivalence entre les normes dans $\mathbb{R}^{q}$ où $q=\operatorname{card}\left(\left\{\alpha \in \mathbb{N}^{N}|\alpha| \leq m\right\}\right)$. Les deux normes sont notées indifféremment $\|\cdot\|_{m, p}$ ou $\|\cdot\|_{W^{m, p},}$. L'intérêt principal de la norme (1.3) est que dans le cas $p=2$ elle confère à $H^{m}$ une structure hilbertienne, ce qui n'est pas le cas avec la norme définie par (1.4)

Remarque 1.6 (Espaces de Sobolev et continuité) En une dimension d'espace $(N=1)$, avec $a, b \in \mathbb{R}, a<b$, $1 \leq p \leq+\infty$, tout élément de $W^{1, p}(] a, b[)$ (qui est donc une classe de fonctions) peut étre assimilé à une fonction continue, au sens où il existe un représentant de la classe qui est continu (ce représentant continu est unique, voir à ce propos l'exercice 1.3 . Ceci tient au fait qu'en dimension 1, toute (classe de) fonction(s) de $W^{1, p}(] a, b[)$ peut s'écrire comme l'intégrale de sa dérivée.

$$
u \in W^{1, p}(] a, b[) \Longleftrightarrow\left\{\exists \tilde{u} \in C([a, b]) \text { et } v \in L^{p}(] a, b[) ; u=\tilde{u} \text { p.p. et } \tilde{u}(x)=\tilde{u}(a)+\int_{a}^{x} v(s) d s\right\} .
$$

En dimension strictement supérieure à 1 , ceci est faux. En particulier $H^{1}(\Omega) \not \subset C(\bar{\Omega})$, comme le prouve l'exemple suivant : soit $\Omega=\left\{x=\left(x_{1}, x_{2}\right)^{t} \in \mathbb{R}^{2},\left|x_{i}\right|<\frac{1}{2}, i=1,2\right\}$, et $u$ la fonction définie sur $\Omega$ par $u(x)=$ $(-\ln (|x|))^{\gamma}$, avec $\left.\gamma \in\right] 0,1 / 2\left[\right.$. Alors $u \in H^{1}(\Omega)$ mais $u \notin L^{\infty}(\Omega)$ (voir exercice 1.5), et donc en particulier, $u \notin C(\bar{\Omega})$.

10. David Hilbert (1862-1943), mathématicien allemand, connu pour ses travaux dans de nombreuseuses branches des mathématiques. Il énonça en 190023 problèmes, dont certains ne sont toujours pas résolus.

11. On rappelle qu'un espace Hilbert est un espace vectoriel normé complet dont la norme est induite par un produit scalaire. 
Proposition 1.7 (Séparabilité) Soit $\Omega$ un ouvert de $\mathbb{R}^{N}(N \geq 1), m \in \mathbb{N}$ et $1 \leq p<+\infty$; l'espace $W^{m, p}(\Omega)$ is espace séparable ( c'est-à-dire un espace vectoriel normé qui contient une partie dénombrable dense).

La preuve de cette proposition fait l'objet de l'exercice 1.9, où l'on montre aussi par un contre-exemple que le résultat de séparabilité n'est pas vrai pour $p=+\infty$.

La notion de séparabilité est importante, car elle permet d'approcher aussi près que l'on veut n'importe quel élément de l'espace par un élément d'une famille dénombrable : dans le cadre d'un espace de Hilbert, on peut montrer que cette propriété est équivalente à l'existence d'une base hilbertienne (voir par exemple]15, Proposition 6.62]).

Nous rappelons maintenant la notion importante d'espace réflexif.

Définition 1.8 (Espace réflexif) Soit $E$ un espace vectoriel normé réel. On note $E^{\prime}$ son dual topologique, c'està-dire l'ensemble des formes linéaires continues de $E$ dans $\mathbb{R}$ muni de sa norme naturelle ( $E^{\prime}$ est un espace de Banach). Pour tout $x \in E$, on définit l'application $J_{x}$ de $E^{\prime}$ dans $\mathbb{R}$ par $J_{x}(T)=T(x)$ pour tout $T \in E^{\prime}$. On a

$$
\left|J_{x}(T)\right|=|T(x)| \leq\|T\|_{E^{\prime}}\|x\|_{E}
$$

et donc $J_{x}$ est une forme linéaire continue sur $E^{\prime}$, ce qu'on note $J_{x} \in E^{\prime \prime}$ où $E^{\prime \prime}$ est le bidual de $E$, c'est-à-dire le dual topologique de $E^{\prime}$. On peut montrer par le théorème de Hahn ${ }^{12}$-Banach qu'on rappelle dans le paragraphe suivant, que $\left\|J_{x}\right\|_{E^{\prime \prime}}=\|x\|_{E}$.

L'application $J$, définie de $E$ dans $E^{\prime \prime}$ par $J(x)=J_{x}$ pour tout $x \in E$, est donc une isométrie linéaire de $E$ sur son image, notée $\operatorname{Im}(J)$, et on a évidemment $\operatorname{Im}(J) \subset E^{\prime \prime}$.

On dit que E est un espace réflexif si $\operatorname{Im}(J)=E^{\prime \prime}$, ce qui revient à dire que $J$ est surjective.

Notons que tout espace réflexif E est forcément complet puisque le dual d'un espace vectoriel normé quelconque est toujours complet.

Proposition 1.9 (Réflexivité) Soit $\Omega$ un ouvert de $\mathbb{R}^{N}, N \geq 1$, et $m \in \mathbb{N}$. Pour tout $\left.p \in\right] 1,+\infty[$, l'espace $W^{m, p}(\Omega)$ est un espace réflexif.

La preuve de ce résultat fait l'objet de l'exercice 1.10 .

\subsection{Rappels d'analyse fonctionnelle}

Commençons par un théorème fondamental (voir par exemple [8]) :

Théorème 1.10 (Hahn-Banach) Soit $E$ un espace vectoriel sur $\mathbb{R}$ et $p$ une fonction convexe définie de $E$ dans $\mathbb{R}$. Soit $F$ un sous-espace vectoriel de $E$, et $T$ une forme linéaire sur $F$ qui vérifie $T(x) \leq p(x)$ pour tout $x \in F$. Il existe alors un une forme linéaire de $E$ dans $\mathbb{R}$, égale à $T$ sur $F$, qui prolonge $T$ sur l'espace $E$ tout entier, et qui vérifie encore la condition : $T(x) \leq p(x)$ pour tout $x \in E$.

Le corollaire suivant est essentiel :

Corollaire 1.11 (Prolongement d'une application linéaire) Soit E un espace normé, $F$ un sous-espace de E et $T$ une forme linéaire continue sur $F$. On peut alors prolonger $T$ en une application continue définie sur $E$, de même norme que $T$.

Un résultat bien connu sur les espaces de dimension finie est donné dans le théorème suivant :

12. Hans Hahn (1879-1934) mathématicien et philosophe autrichien, connu pour ses contributions en analyse fonctionnelle, topologie, théorie des ensembles et analyse réelle 
Théorème 1.12 (CNS sur la dimension) Un espace de Banach E est de dimension finie si et seulement si sa boule unité fermée est compacte.

Les notions de convergence faible et faible- $\star$ seront fondamentales pour la suite.

Définition 1.13 (Convergence faible et faible- $\star$ ) Soit $E$ un espace de Banach.

1. Convergence faible Soient $\left(u_{n}\right)_{n \in \mathbb{N}} \subset E$ et $u \in E$. On dit que $u_{n} \rightarrow u$ faiblement dans $E$ lorsque $n \rightarrow \infty$ si $T\left(u_{n}\right) \rightarrow T(u)$ pour tout $T \in E^{\prime}$.

2. Convergence faible $-\star$ Soient $\left(T_{n}\right)_{n \in \mathbb{N}} \subset E^{\prime}$ et $u \in E^{\prime}$. On dit que $T_{n} \rightarrow T$ dans $E^{\prime}$ faible- $\star$ si $T_{n}(x) \rightarrow$ $T(x)$ pour tout $x \in E$.

Théorème 1.14 (Compacité faible- $\star$ des bornés du dual d'un espace séparable) Soit $E$ un espace de Banach séparable, et soit $\left(T_{n}\right)_{n \in \mathbb{N}}$ une suite bornée de $E^{\prime}$ ( c'est-à-dire telle qu'il existe $C \in \mathbb{R}_{+}$tel que $\left(\left\|T_{n}\right\|_{E^{\prime}} \leq C\right.$ pour tout $n \in \mathbb{N})$. Alors il existe une sous-suite, encore notée $\left(T_{n}\right)_{n \in \mathbb{N}}$, et $T \in E^{\prime}$ telle que $T_{n} \rightarrow T$ dans $E^{\prime}$ faible-ネ.

Une application importante de ce théorème est la suivante : si $\Omega$ est un ouvert de $\mathbb{R}^{N}$ et $\left(u_{n}\right)_{n \in \mathbb{N}}$ est une suite bornée de $L^{\infty}(\Omega)$, alors il existe une sous suite encore notée $\left(u_{n}\right)_{n \in \mathbb{N}}$ et $u \in L^{\infty}(\Omega)$ tels que $\int_{\Omega} u_{n} \varphi \mathrm{d} x \rightarrow$ $\int_{\Omega} u \varphi \mathrm{d} x$ pour tout $\varphi \in L^{1}(\Omega)$. Ceci découle du fait qu'il existe une isométrie naturelle entre $L^{\infty}(\Omega)$ et le dual de $L^{1}(\Omega)$ et que $L^{1}(\Omega)$ est séparable.

Théorème 1.15 (Compacité faible des bornés d'un espace réflexif) Soit $E$ un espace de Banach réflexif, et soit $\left(u_{n}\right)_{n \in \mathbb{N}}$ une suite bornée de $E$ ( c'est-à-dire telle qu'il existe $C \in \mathbb{R}_{+}$t.q. $\left(\left\|u_{n}\right\|_{E} \leq C\right.$ pour tout $n \in \mathbb{N}$ ). Alors il existe une sous-suite, encore notée $\left(u_{n}\right)_{n \in \mathbb{N}}$, et $u \in E$ telle que $u_{n} \rightarrow u$ dans $E$ faiblement.

Noter qu'un espace de Hilbert est toujours un espace de Banach réflexif.

\subsection{Théorèmes de densité}

Définition 1.16 (Frontière lipschitzienne) Un ouvert borné $\Omega$ de $\mathbb{R}^{N}$ est dit à frontière lipschitzienne s'il existe $n \in \mathbb{N}$ et des ouverts $\left(\Omega_{0}, \Omega_{1}, \ldots, \Omega_{n}\right)$ de $\mathbb{R}^{N}$ ainsi que des applications $\left(\phi_{0}, \phi_{1}, \ldots, \phi_{n}\right)$ telles que :

1. $\bar{\Omega} \subset \bigcup_{i=0}^{n} \Omega_{i}$ et $\Omega_{0} \subset \Omega$.

2. $\phi_{0}: \Omega_{0} \rightarrow B_{1, N}=\left\{x \in \mathbb{R}^{N}\right.$ t.q. $\left.\|x\|<1\right\}$ est bijective et $\phi_{0}$ et $\phi_{0}^{-1}$ sont lipschitziennes,

3. Pour tout $i \geq 1, \phi_{i}: \Omega_{i} \rightarrow B_{1, N}$ est bijective et $\phi_{i}$ et $\phi_{i}^{-1}$ sont lipschitziennes, et $\phi_{i}\left(\Omega_{i} \cap \Omega\right)=B_{1, N} \cap \mathbb{R}_{+}^{N}$ et $\phi_{i}\left(\Omega_{i} \cap \partial \Omega\right)=B_{1, N} \cap\left\{(0, y), y \in \mathbb{R}^{N_{-}}\right\}$( où $\mathbb{R}_{+}^{N}=\left\{(x, y) \in \mathbb{R}^{N} ; x \in \mathbb{R}, x>0\right.$ et $\left.y \in \mathbb{R}^{N-1}\right\}$.)

Remarque 1.17 (Frontière fortement lipschitzienne) Un ouvert borné $\Omega$ de $\mathbb{R}^{N}$ est dit à frontière fortement lipschitzienne si le bord de $\Omega$ est localement le graphe d'une fonction lipschitzienne et que $\Omega$ est (localement) d'un seul côté de ce graphe. Un ouvert (borné) à frontière fortement lipschitzienne est un ouvert à frontière lipschitzienne mais la réciproque est fausse comme le montre l'exercice [1.13, voir aussi [12].

Théorème 1.18 (Densité et prolongement) Soit $\Omega$ est un ouvert borné à frontière lipschitzienne et $1 \leq p \leq+\infty$, alors :

1. Si $p<+\infty$, l'ensemble $C^{\infty}(\bar{\Omega})$ des restrictions à $\Omega$ des fonctions $C_{c}^{\infty}\left(\mathbb{R}^{N}\right)$ est dense dans $W^{1, p}(\Omega)$.

2. Il existe une application linéaire continue $P: W^{1, p}(\Omega) \rightarrow W^{1, p}\left(\mathbb{R}^{N}\right)$ telle que

$$
\forall u \in W^{1, p}(\Omega), P(u)=u \text { p.p. dans } \Omega .
$$


Des résultats analogues sont vrais avec $W^{m, p}(\Omega)(m>1)$ au lieu de $W^{1, p}(\Omega)$ mais demandent plus de régularité $\operatorname{sur} \Omega$ (voir [1]). La démonstration se fait par troncature et régularisation; un cas particulier fait l'objet de l'exercice 1.18

On peut montrer aussi que $C_{c}^{\infty}\left(\mathbb{R}^{N}\right)$ est dense dans $W^{m, p}\left(\mathbb{R}^{N}\right)$ si $N \geq 1, m \in N$ et $1 \leq p<+\infty$. Mais ceci est faux si on remplace $\mathbb{R}^{N}$ par $\Omega$ avec $\Omega$ ouvert borné et $m>0$. Par exemple, si $\Omega$ est un ouvert borné, l'espace $C_{c}^{\infty}(\Omega)$ n'est pas dense dans $H^{1}(\Omega)$. Son adhérence est un sous espace strict de $H^{1}(\Omega)$, qu'on note $H_{0}^{1}(\Omega)$.

Définition 1.19 (L'espace $\boldsymbol{H}_{\mathbf{0}}^{\mathbf{1}}(\boldsymbol{\Omega})$ ) Soit $\Omega$ un ouvert de $\mathbb{R}^{N}, N \geq 1$.

1. On appelle $H_{0}^{1}(\Omega)$ l'adhérence de $C_{c}^{\infty}(\Omega)$ dans $H^{1}(\Omega)$, ce qu'on note aussi : $H_{0}^{1}(\Omega)=\overline{C_{c}^{\infty}(\Omega)} H^{1}(\Omega)$.

2. Pour $m>0$ et $1 \leq p<+\infty$, on définit le sous espace $W_{0}^{m, p}(\Omega)$ de $W^{m, p}(\Omega)$ comme l'adhérence de $C_{c}^{\infty}(\Omega)$ dans $W^{m, p}(\Omega)$ :

$$
W_{0}^{m, p}(\Omega)={\overline{C_{c}^{\infty}(\Omega)}}^{W^{m, p}(\Omega)} .
$$

Comme cela a été dit précédemment, si $\Omega=\mathbb{R}^{N}$ on a $H_{0}^{1}(\Omega)=H^{1}(\Omega)$ alors que l'inclusion est stricte si $\Omega$ est un ouvert borné.

Remarque 1.20 (Espace $C^{k}(\bar{\Omega})$ ) Soit $\Omega$ un ouvert de $\mathbb{R}^{N}, N \geq 1$, et $k \in \mathbb{N}^{\star} \cup\{+\infty\}$. Soit $\varphi$ une fonction de $\Omega$ dans $\mathbb{R}$. On dit que $\varphi \in C^{k}(\bar{\Omega})$ s'il existe une fonction $\psi$ de $\mathbb{R}^{N}$ dans $\mathbb{R}$, de classe $C^{k}$ telle que $\psi=\varphi$ dans $\Omega$. Si $\Omega$ est borné, il est bien sûr possible de demander que la fonction $\psi$ soit à support compact dans $\mathbb{R}^{N}$, comme cela a été fait dans le théorème 1.18 Il est intéressant de noter qu'il est possible de prendre la même définition pour $k=0$. En effet, si $\varphi$ est continue de $\bar{\Omega}$ dans $\mathbb{R}$, il existe alors $\psi$ continue de $\mathbb{R}^{N}$ dans $\mathbb{R}$ telle que $\psi=\varphi$ dans $\bar{\Omega}$, voir l'exercice 1.14

\subsection{Théorèmes de trace}

Théorème 1.21 (Trace, demi-espace) Soit $\Omega=\mathbb{R}_{+}^{N}=\left\{(x, y) \in \mathbb{R}^{N} ; x \in \mathbb{R}, x>0\right.$ et $\left.y \in \mathbb{R}^{N-1}\right\}$. Pour tout p tel que $1 \leq p<+\infty$, il existe une unique application linéaire continue $\gamma$ de $W^{1, p}(\Omega)$ dans $L^{p}\left(\mathbb{R}^{N-1}\right)$ telle que $\gamma u=u(0, \cdot)$ p.p sur $\mathbb{R}^{N-1}$ (au sens de la mesure de Lebesgue $N-1$ dimensionnelle) si $u \in C_{c}^{\infty}\left(\overline{\mathbb{R}_{+}^{N}}\right)$.

Remarque 1.22 (Lien avec la trace classique.) On suppose que $\Omega=\mathbb{R}_{+}^{N}$. Alors :

1. Si $u \in H^{1}(\Omega) \cap C(\bar{\Omega})$, on a alors $\gamma u=u$ p.p sur $\partial \Omega$ (au sens de la mesure de Lebesgue $N-1$ dimensionnelle).

2. $\operatorname{Ker} \gamma=W_{0}^{1, p}\left(\mathbb{R}_{+}^{N}\right)$.

Voir à ce propos l'exercice 1.17 .

Théorème 1.23 (Trace, ouvert borné) Soit $\Omega$ un ouvert borné à frontière lipschitzienne et $1 \leq p<+\infty$. Alors, il existe une unique application $\gamma$ (linéaire continue) définie de $W^{1, p}(\Omega)$ dans $L^{p}(\partial \Omega)$ et tel que

$$
\gamma u=u \text { p.p. sur } \partial \Omega \text { si } u \in W^{1, p}(\Omega) \cap C(\bar{\Omega}) \text {. }
$$

Ici encore, p.p. est à prendre au sens de la mesure de Lebesgue $N-1$ dimensionnelle sur $\partial \Omega$.

De plus $\operatorname{Ker} \gamma=W_{0}^{1, p}(\Omega)$.

Remarquons que si $p>N$, on peut montrer (voir théorème 1.28 que $W^{1, p}(\Omega) \subset C(\bar{\Omega})$ et $\gamma u$ est alors la valeur de $u$ au bord au sens classique.

Le théorème suivant généralise la propriété d'intégration par parties des fonctions régulières. 


\section{Théorème 1.24 (Intégration par parties)}

- Si $\Omega=\mathbb{R}_{+}^{N}\left(=\left\{x=\left(x_{1}, \ldots, x_{N}\right)^{t} \in \mathbb{R}^{N} ; x_{1}>0\right\}\right)$, alors

$$
\left\{\begin{array}{l}
\text { Si } 2 \leq i \leq N, \int_{\Omega} u D_{i} v \mathrm{~d} x=-\int_{\Omega} D_{i} u v \mathrm{~d} x, \forall(u, v) \in\left(H^{1}(\Omega)\right)^{2}, \\
\text { Si } i=1, \int_{\Omega} u D_{1} v \mathrm{~d} x=-\int_{\Omega} D_{1} u v \mathrm{~d} x+\int_{\partial \Omega} \gamma u(y) \gamma v(y) \mathrm{d} \gamma(y), \forall(u, v) \in\left(H^{1}(\Omega)\right)^{2},
\end{array}\right.
$$

- si $\Omega$ est un ouvert borné à frontière lipschitzienne, alors, pour tout $i=1, \ldots, N$,

$$
\int_{\Omega} u D_{i} v \mathrm{~d} x=-\int_{\Omega} D_{i} u v \mathrm{~d} x+\int_{\partial \Omega} \gamma u(y) \gamma v(y) n_{i}(y) \mathrm{d} \gamma(y), \forall(u, v) \in\left(H^{1}(\Omega)\right)^{2},
$$

où $\gamma u$ désigne la trace de u sur la frontière $\partial \Omega$ et $\mathrm{d} \gamma(y)$ désigne l'intégration par rapport à la mesure adéquate sur $\partial \Omega$ ( c'est-à-dire la mesure de Hausdorff $\left.{ }^{13}\right]$ sur $\partial \Omega$ qu'on peut voir comme une mesure de Lebesque $(N-1)$ dimensionnelle), et $\boldsymbol{n}=\left(n_{1}, \ldots, n_{N}\right)^{t}$ est la normale à $\partial \Omega$ extérieure à $\Omega$.

\subsection{Théorèmes de compacité}

Le théorème de compacité de Rellich ${ }^{14}$ et ses généralisations sont une conséquence du théorème de compacité de Kolmogorov ${ }^{15}$ (voir [15, chapitre 8]).

Théorème 1.25 (Rellich) Soit $\Omega$ un ouvert borné de $\mathbb{R}^{N}(N \geq 1)$ et $1 \leq p<+\infty$. Toute partie bornée de $W_{0}^{1, p}(\Omega)$ est relativement compacte dans $L^{p}(\Omega)$. Ceci revient à dire que de toute suite bornée de $W_{0}^{1, p}(\Omega)$, on peut extraire une sous-suite qui converge dans $L^{p}(\Omega)$.

Le théorème précédent reste vrai avec $W^{1, p}(\Omega)$ à condition de supposer la frontière lipschitzienne.

Théorème 1.26 (Compacité des bornés de $W^{1, p}(\Omega)$ dans $L^{p}(\Omega)$ ) Soit $\Omega$ un ouvert borné de $\mathbb{R}^{N}(N \geq 1)$, à frontière lipschitzienne, et $1 \leq p<+\infty$. Toute partie bornée de $W^{1, p}(\Omega)$ est relativement compacte dans $L^{p}(\Omega)$. Ceci revient à dire que de toute suite bornée de $W^{1, p}(\Omega)$, on peut extraire une sous-suite qui converge dans $L^{p}(\Omega)$.

Nous aurons aussi besoin d'une version du théorème 1.25 dans les espaces duaux de $L^{p}$ et $W_{0}^{1, p}$. Comme, pour $p<+\infty$, le dual de $L^{p}$ est identifié à l'espace $L^{q}$ avec $q=p /(p-1)$, et que le dual de $W_{0}^{1, p}$ est noté $W^{-1, q}$, on obtient le théorème 1.27 .

Théorème 1.27 (Compacité des bornés de $L^{q}(\Omega)$ dans $W^{-1, q}(\Omega)$ ) Soit $\Omega$ un ouvert borné de $\mathbb{R}^{N}(N \geq 1)$ et $1<q<+\infty$. Toute partie bornée de $L^{q}(\Omega)$ est relativement compacte dans $W^{-1, q}(\Omega)$.

En particulier, pour $q=2$, l'espace $W^{-1,2}(\Omega)$ est aussi noté $H^{-1}(\Omega)$. Toute partie bornée de $L^{2}(\Omega)$ est donc relativement compacte dans $H^{-1}(\Omega)$.

Démonstration Ce résultat est une conséquence du théorème de Rellich 1.25 , voir l'exercice 1.23 .

13. Felix Hausdorff, mathématicien allemand (1868,1942), également écrivain et philosophe, connu pour ses travaux en topologie et théorie des ensembles. Il se suicida en famille en 1942 pour éviter la déportation en camp de concentration.

14. Franz Rellich (1906-1955) mathématician autrichien-allemand, spécialiste de physique mathématique et d'équations aux dérivées partielles.

15. Andrey Nikolaevich Kolmogorov (1903-1987) mathématician soviétique connu pour ses contributions en probabilités, topologie, logique, turbulence, mécanique, théorie de l'information et complexité. 


\subsection{Injections de Sobolev}

Le théorème suivant concerne les injections de Sobolev; ces injections établissent le fait qu'une fonction dont une certaine puissance d'elle-même et de sa dérivée est intégrable ( c'est-à-dire $u \in W^{1, p}$ ) est en fait dans un "meilleur" espace (en terme d'intégration ou de régularité). On distingue trois cas différents, selon que la puissance est inférieure strictement, égale, ou supérieure strictement à la dimension de l'espace $N$.

Théorème 1.28 (Injections de Sobolev) Soit $\Omega$ un ouvert de $\mathbb{R}^{N}, N \geq 1$ qui est soit borné à frontière lipschitzienne, soit égal à $\mathbb{R}^{N}$.

1. Si $1 \leq p<N$, alors $W^{1, p}(\Omega) \subset L^{p^{\star}}(\Omega)$, avec $p^{\star}=\frac{N p}{N-p}$, et l'injection est continue, c'est-à-dire qu'il existe $C \in \mathbb{R}_{+}$(ne dépendant que de $p, N$ et $\Omega$ ) tel que

$$
\forall u \in W^{1, p}(\Omega),\|u\|_{L^{p^{\star}}} \leq C\|u\|_{W^{1, p}} \text { ce qu'on note } W^{1, p}(\Omega) \hookrightarrow L^{p^{\star}}(\Omega) ;
$$

on a en particulier

$$
W^{1,1}(\Omega) \hookrightarrow L^{\frac{N}{N-1}}(\Omega) .
$$

Pour $N=1$, le cas $p=N$ est autorisé. On a donc aussi

$$
W^{1,1}(\Omega) \hookrightarrow L^{\infty}(\Omega) \text { si } N=1 .
$$

2. Si $p>N$, alors

$$
W^{1, p}(\Omega) \subset C^{0,1-\frac{N}{p}}(\Omega)
$$

où , pour $\alpha>0, C^{0, \alpha}(\Omega)$ est l'ensemble des fonctions höldériennes d'exposant $\alpha$ défini par

$$
C^{0, \alpha}(\Omega)=\left\{u \in C(\Omega, \mathbb{R})|\exists k \in \mathbb{R} ;| u(x)-u(y) \mid \leq k\|x-y\|^{\alpha}, \forall(x, y) \in \Omega^{2}\right\} .
$$

La démonstration de ce résultat fait l'objet de l'exercice 1.15

3. Dans le cas où $\Omega$ est borné à frontière lipschitzienne, $W^{1, N}(\Omega) \hookrightarrow L^{q}(\Omega)$ pour tout q tel que $1 \leq q<+\infty$ (et le cas $q=\infty$ est autorisé si $N=1$ ). Ce résultat est faux dans le cas où $\Omega=\mathbb{R}^{N}$, voir un contre exemple à l'exercice 1.5

Si $\Omega$ est un ouvert borné sans hypothèse de régularité sur la frontière, les trois assertions précédentes restent vraies si l'on remplace l'espace $W^{1, p}(\Omega)$ par l'espace $W_{0}^{1, p}(\Omega)$.

Remarque 1.29 (Compacité de l'injection de $\boldsymbol{W}_{0}^{1, p}(\Omega)$ dans $L^{q}(\Omega)$ ) Soit $\Omega$ un ouvert borné de $\mathbb{R}^{N}$. Une conséquence simple du théorème d'injection de Sobolev (théorème 1.28) et du théorème de compacité de Rellich (théorème 1.25 est que l'application $u \mapsto u$ est compacte de $W_{0}^{1, p}(\Omega)$ dans $L^{q}(\Omega)$ si $1 \leq p \leq N$ et $q<p^{\star}=\frac{p N}{N-p}$.

Si $p>N$, une conséquence simple de théorème d'injection de Sobolev (théorème1.28) et du théorème (classique) d'Ascoli est que l'application $u \mapsto u$ est compacte de $W_{0}^{1, p}(\Omega)$ dans $C(\bar{\Omega})$.

\subsection{Exercices}

Exercice 1.1 (Exemple de dérivée) $\quad$ Soient $N \geq 1, \Omega=\left\{x=\left(x_{1}, \ldots, x_{N}\right)^{t} \in \mathbb{R}^{N},\left|x_{i}\right|<1, i=1, \ldots, N\right\}$ et $u: \mathbb{R}^{N} \rightarrow \mathbb{R}$ définie par $u(x)=1$ si $x \in \Omega$ et $u(x)=0$ si $x \notin \Omega$.

1. Pour $i=\{1, \ldots, N\}$ et $\varphi \in C_{c}^{\infty}\left(\mathbb{R}^{N}\right)$, montrer que $\int_{\mathbb{R}^{N}} u(x) \partial_{i} \varphi(x) \mathrm{d} x$ ne dépend que des valeurs prises par $\varphi$ sur le bord de $\Omega$. 
2. Montrer que $u \notin W^{1,1}\left(\mathbb{R}^{N}\right)$.

Corrigé-

1. On prend, par exemple, $i=1$ (les autres valeurs de $i$ se traitent de manière similaire). Pour tout $\varphi \in C_{c}^{\infty}\left(\mathbb{R}^{N}\right)$ on a

$$
\int_{\mathbb{R}^{N}} u(x) \frac{\partial \varphi}{\partial x_{1}}(x) \mathrm{d} x=\int_{]-1,1\left[\left[^{N}\right.\right.} \frac{\partial \varphi}{\partial x_{1}}(x) \mathrm{d} x=\int_{]-1,1\left[^{N-1}\right.}\left(\int_{-1}^{1} \frac{\partial \varphi}{\partial x_{1}}\left(x_{1}, y\right) \mathrm{d} x_{1}\right) \mathrm{d} y
$$

et donc

$$
\int_{\mathbb{R}^{N}} u(x) \frac{\partial \varphi}{\partial x_{1}}(x) \mathrm{d} x=\int_{]_{-1,1}\left[{ }^{N-1}\right.} \varphi(1, y) \mathrm{d} y-\int_{]_{-1,1}\left[{ }^{N-1}\right.} \varphi(-1, y) \mathrm{d} y .
$$

Ceci montre bien que $\int_{\mathbb{R}^{N}} u(x) \frac{\partial \varphi}{\partial x_{1}}(x) \mathrm{d} x$ ne dépend que des valeurs prises par $\varphi$ sur le bord de $\Omega$.

2. On raisonne par l'absurde. On suppose que $u \in W^{1,1}\left(\mathbb{R}^{N}\right)$. Il existe alors (en particulier) $g \in L^{1}\left(\mathbb{R}^{N}\right)$ t.q.

$$
\int_{\mathbb{R}^{N}} u(x) \frac{\partial \varphi}{\partial x_{1}}(x) \mathrm{d} x=\int_{\mathbb{R}^{N}} g(x) \varphi(x) \mathrm{d} x \text { pour tout } \varphi \in C_{c}^{\infty}\left(\mathbb{R}^{N}\right) .
$$

Pour $n \in \mathbb{N}^{\star}$, on pose $\left.A_{n}=\right] 1-\frac{1}{n}, 1+\frac{1}{n}[\times]-1,1\left[^{N-1}\right.$.

On choisit une fonction $\varphi \in C_{c}^{\infty}\left(\mathbb{R}^{N}\right)$ t.q. $\varphi(x) \geq 0$ pour tout $x \in \mathbb{R}^{N}, \varphi(x)=0$ si $x \notin A_{1}$ et $\varphi(x)=1$ si $x=(1, y)$ avec $y \in]-\frac{1}{2}, \frac{1}{2}\left[{ }^{N-1}\right.$ (une telle fonction $\varphi$ existe). Pour $n \in \mathbb{N}^{\star}$, on définit alors $\varphi_{n}$ par $\left.\varphi_{n}\left(1+x_{1}, y\right)\right)=$ $\varphi\left(1+n x_{1}, y\right)$ pour tout $x_{1} \in \mathbb{R}$ et $y \in \mathbb{R}^{N-1}$ (de sorte que $\varphi_{n}=0$ hors de $A_{n}$ ).

Pour $n \in \mathbb{N}^{\star}$, on a bien $\varphi_{n} \in C_{c}^{\infty}\left(\mathbb{R}^{N}\right)$ et le choix de $\varphi_{n}$ donne

et

$$
\int_{\mathbb{R}^{N}} u(x) \frac{\partial \varphi_{n}}{\partial x_{1}}(x) \mathrm{d} x=\int_{]_{-1,1\left[^{N-1}\right.}} \varphi_{n}(1, y) \mathrm{d} y-\int_{]_{-1,1\left[^{N-1}\right.}} \varphi_{n}(-1, y) \mathrm{d} y \geq 1
$$

$$
\left|\int_{\mathbb{R}^{N}} g(x) \varphi_{n}(x) \mathrm{d} x\right| \leq \int_{A_{n}}|g(x)| \mathrm{d} x .
$$

On a donc $\int_{A_{n}}|g(x)| \mathrm{d} x \geq 1$ pour tout $n \in \mathbb{N}^{\star}$, ce qui est impossible car la mesure de Lebesgue ( $N$-dimensionnelle) de $A_{n}$ tend vers 0 quand $n \rightarrow+\infty$.

Exercice 1.2 (Une fonction à dérivée nulle est constante p.p.) Soit $u \in L_{\text {loc }}^{1}(] 0,1[)$ telle que $D u=0$. Montrer que

$$
\exists a \in \mathbb{R} ; u=a \text { p.p. }
$$

Corrigé - On se donne $\varphi_{0} \in C_{c}^{\infty}(] 0,1[)$ t.q. $\int_{0}^{1} \varphi_{0}(x) \mathrm{d} x=1$.

Pour $\psi \in C_{c}^{\infty}(] 0,1[)$, on définit la fonction $\varphi$ par

$$
\left.\varphi(x)=\int_{0}^{x} \psi(t) \mathrm{d} t-\left(\int_{0}^{1} \psi(t) d t\right) \int_{0}^{x} \varphi_{0}(t) d t . \text { pour } x \in\right] 0,1[.
$$

Avec ce choix de $\varphi$ on a $\varphi \in C_{c}^{\infty}(] 0,1[)$ et donc, comme $D u=0$,

$$
0=\langle D u, \varphi\rangle_{\mathcal{D}^{\star}, C_{c}^{\infty}}=-\int_{0}^{1} u(x) \varphi^{\prime}(x) \mathrm{d} x .
$$

Comme $\varphi^{\prime}=\psi-\left(\int_{0}^{1} \psi(t) d t\right) \varphi_{0}$, on a donc

$$
\int_{0}^{1} u(x) \psi(x) \mathrm{d} x-\left(\int_{0}^{1} \psi(t) d t\right)\left(\int_{0}^{1} u(x) \varphi_{0}(x) \mathrm{d} x\right)=0 .
$$

On pose $a=\int_{0}^{1} u(x) \varphi_{0}(x) \mathrm{d} x$, on a ainsi

$$
\int_{0}^{1} u(x) \psi(x) \mathrm{d} x=\int_{0}^{1} a \psi(x) \mathrm{d} x \text { pour tout } \psi \in C_{c}^{\infty}(] 0,1[) .
$$


Le lemme 1.1 donne alors $u=$ a p.p.

Une autre méthode consiste à considérer d'abord le cas $u \in L^{1}(] 0,1[)$ et procéder, par exemple, par densité. La fonction $u$ peut étre approchée par convolution par des noyaux régularisants $\rho_{n} q u$ 'on prend à support dans $]-\frac{1}{n}, \frac{1}{n}[$. En prolongeant $u$ par 0 en dehors de $[0,1]$, on pose $u_{n}=u \star \rho_{n}$. On a alors $u_{n}^{\prime}=u \star \rho_{n}^{\prime}$. On montre alors que $u_{n}^{\prime}(x)=$ $-<D u, \rho_{n}(x-\cdot)>$ pour tout $\left.x \in\right] \frac{1}{n}, 1-\frac{1}{n}\left[\right.$, et on conclut que $u_{n}^{\prime}(x)=0$ pour tout $\left.x \in\right] \frac{1}{n}, 1-\frac{1}{n}[$.

On termine le raisonnement en utilisant le fait que $u_{n} 1_{] \frac{1}{n}, 1-\frac{1}{n}[}$ tend vers $u$ dans $L^{1}$.

Dans le cas $u \in L_{\mathrm{loc}}^{1}(] 0,1[)$ on considére d'abord la fonction $u_{\varepsilon}=u 1_{[\varepsilon, 1-\varepsilon]}$ avec $\varepsilon>0$.

L'intérêt de cette deuxième méthode est qu'elle se généralise au cas multidimensionnel (voir l'exercice 1.4.

Exercice 1.3 (Espace de Sobolev en 1d) Soit $1 \leq p \leq \infty$ et $u \in W^{1, p}(] 0,1[)$.

1. Soit $u \in W^{1, p}(] 0,1[)$.

(a) Montrer qu'il existe $C \in \mathbb{R}$ t.q. $u(x)=C+\int_{0}^{x} D u(t) d t$, pour presque tout $\left.x \in\right] 0,1[$. En déduire que $u \in C([0,1], \mathbb{R})$ (au sens où il existe $v \in C([0,1], \mathbb{R})$ t.q. $u=v$ p.p. sur $] 0,1[$; en identifiant $u$ et $v$, on peut donc dire que $\left.W^{1, p}(] 0,1[) \subset C([0,1], \mathbb{R})\right)$.

(b) Montrer que $\|u\|_{\infty} \leq\|u\|_{W^{1, p}(] 0,1[)}$.

(c) Si $p>1$, Montrer que $u$ est une fonction höldérienne d'exposant $1-(1 / p)$.

2. Soit $u \in C([0,1], \mathbb{R})$. On suppose qu'il existe $w \in L^{p}(] 0,1[)$ t.q. $u(x)=u(0)+\int_{0}^{x} w(t) d t$, pour tout $\left.x \in\right] 0,1[$. Montrer que $u \in W^{1, p}(] 0,1[)$ et $D u=w$.

Corrigé-

1.(a) Pour $x \in[0,1]$, on pose $F(x)=\int_{0}^{x} D u(t) d t$. Comme $D u \in L^{1}(] 0,1[)$, on a $F \in C([0,1], \mathbb{R})$. On peut aussi montrer que $F$ est dérivable p.p. et que $F^{\prime}=D$ u p.p. mais cela est inutile ici. On s'intéresse plutôt à la dérivée par transposition de $F$, c'est-à-dire à $D F$ et on va montrer que $D F=D u$.

Soit $\varphi \in C_{c}^{\infty}(] 0,1[)$, on $a$

$$
\langle D F, \varphi\rangle_{\mathcal{D}^{\star}, C_{c}^{\infty}}=-\int_{0}^{1} F(x) \varphi^{\prime}(x) \mathrm{d} x=-\int_{0}^{1}\left(\int_{0}^{1} 1_{10, x[}[(t) D u(t) \mathrm{d} t) \varphi^{\prime}(x) \mathrm{d} x .\right.
$$

En remarquant que $1_{0, x[}(t)=1_{t, 1}(x)$ pour tout $\left.t, x \in\right] 0,1[$ et en utilisant le théorème de Fubini, on a donc

$$
\langle D F, \varphi\rangle_{\mathcal{D}^{\star}, C_{c}^{\infty}}=-\int_{0}^{1}\left(\int_{0}^{1} 1_{] t, 1[}(x) \varphi^{\prime}(t) \mathrm{d} x\right) D u(t) \mathrm{d} t=\int_{0}^{1} \varphi(t) D u(t) \mathrm{d} t,
$$

ce qui prouve que $D F=D u$.

On a donc $D(u-F)=0$ et l'exercice 1.2 donne alors l'existence de $C \in \mathbb{R}$ t.q. $u-F=C$ p.p. c'est-à-dire

$$
\left.u(x)=C+\int_{0}^{x} D u(t) d t \text { pour presque tout } x \in\right] 0,1[.
$$

(b) On choisit maintenant pour u (qui est une classe de fonctions) son représentant continu; on a alors pour tout $x \in[0,1]$

$$
u(x)=u(0)+\int_{0}^{x} D u(t) d t .
$$

On a alors aussi pour tout $x, y \in[0,1], u(x)=u(y)+\int_{y}^{x} D u(t) d t$, et on en déduit que

$$
|u(x)| \leq|u(y)|+\int_{0}^{1}|D u(t)| \mathrm{d} t .
$$

En intégrant cette inégalité sur $[0,1]$ (par rapport à y), on obtient pour tout $x \in[0,1]$

$$
|u(x)| \leq\|u\|_{L^{1}}+\|D u\|_{L^{1}}=\|u\|_{W^{1,1}},
$$

et donc, en prenant le max sur $x$ et en utilisant l'inégalité de Hölder,

$$
\|u\|_{L^{\infty}} \leq\|u\|_{L^{1}}+\|D u\|_{L^{1}} \leq\|u\|_{L^{p}}+\|D u\|_{L^{p}}=\|u\|_{W^{1, p}} .
$$


(c) On choisit toujours pour $u$ son représentant continu. Soient $x, y \in[0,1], y>x$, on a

$$
u(y)-u(x)=\int_{x}^{y} D u(t) d t
$$

et donc, en utilisant l'inégalité de Hölder,

$$
|u(y)-u(x)| \leq\left(\int_{x}^{y}|D u(t)|^{p} d t\right)^{\frac{1}{p}}|y-x|^{1-\frac{1}{p}} \leq\|u\|_{W^{1, p}}|y-x|^{1-\frac{1}{p}} .
$$

2. Il est clair que $u \in L^{p}(] 0,1[)$. Pour montrer que $u \in W^{1, p}(] 0,1[)$ il suffit de montrer que Du $=w$ c'est-à-dire que $\langle D u, \varphi\rangle_{\mathcal{D}^{\star}, C_{c}^{\infty}}=\int_{0}^{1} w(t) \varphi(t) d t$ pour tout $\varphi \in C_{c}^{\infty}(] 0,1[)$.

Soit $\varphi \in C_{c}^{\infty}(] 0,1[)$, on a

$$
\langle D u, \varphi\rangle_{\mathcal{D}^{\star}, C_{c}^{\infty}}=-\int_{0}^{1} u(t) \varphi^{\prime}(t) d t=-\int_{0}^{1}\left(\int_{0}^{t} w(x) \mathrm{d} x\right) \varphi^{\prime}(t) d t=-\int_{0}^{1}\left(\int_{0}^{1} 1_{0, t[}(x) w(x) \mathrm{d} x\right) \varphi^{\prime}(t) d t .
$$

En utilisant une nouvelle fois le théorème de Fubini et le fait que $1_{] 0, t[}(x)=1_{] x, 1[}(t)$ (pour tout $\left.x, t \in\right] 0,1[$ ), on obtient

$\langle D u, \varphi\rangle_{\mathcal{D}^{\star}, C_{c}^{\infty}}=-\int_{0}^{1}\left(\int_{0}^{1} 1_{] x, 1[}(t) \varphi^{\prime}(t) d t\right) w(x) \mathrm{d} x=-\int_{0}^{1}\left(\int_{x}^{1} \varphi^{\prime}(t) d t\right) w(x) \mathrm{d} x=\int_{0}^{1} \varphi(x) w(x) \mathrm{d} x$, ce qui donne bien $D u=w$.

Exercice 1.4 (Une fonction à gradient nul est constante p.p.) $]$ Soient $N \geq 1, B=\left\{x \in \mathbb{R}^{N},|x|<1\right\}$ et $u \in L_{\text {loc }}^{1}(B)$.

1. On suppose que $D_{i} u=0$ pour tout $i \in\{1, \ldots, N\}$. Montrer qu'il existe $a \in \mathbb{R}$ t.q. $u=a$ p.p.. ( $u$ est donc la fonction constante égale à $a$.) [On pourra, par exemple, raisonner ainsi :

Soit $\varepsilon \in] 0,1 / 2\left[\right.$ et $\left(\rho_{n}\right)_{n \in \mathbb{N}^{\star}}$ une suite de noyaux régularisants, c'est-à-dire :

$$
\begin{aligned}
& \rho \in C_{c}^{\infty}\left(\mathbb{R}^{N}, \mathbb{R}\right), \int_{\mathbb{R}^{N}} \rho \mathrm{d} x=1, \rho \geq 0, \rho(x)=0 \text { si }|x| \geq 1, \\
& \text { et, pour } n \in \mathbb{N}^{\star}, x \in \mathbb{R}^{N}, \rho_{n}(x)=n^{N} \rho(n x) .
\end{aligned}
$$

On pose $u_{\varepsilon}(x)=u$ si $|x| \leq 1-\varepsilon$ et $u_{\varepsilon}=0$ sinon. Puis, on pose $u_{\varepsilon, n}=u_{\varepsilon} \star \rho_{n}$.

Montrer que $u_{\varepsilon, n} \in C_{c}^{\infty}\left(\mathbb{R}^{N}, \mathbb{R}\right)$ et que, si $1 / n<\varepsilon, u_{\varepsilon, n}$ est constante sur la boule de centre 0 et de rayon $1-2 \varepsilon$. Puis, conclure....]

2. On suppose que $D_{i} u$ est une fonction continue, pour tout $i \in\{1, \ldots, N\}$. Montrer que $u \in C^{1}(B, \mathbb{R})$ (au sens "il existe $v \in C^{1}(B, \mathbb{R})$ t.q. $u=v$ p.p."). [On pourra, par exemple, reprendre l'indication de la 1ère question et raisonner ainsi : Montrer que pour tout $x, y \in \mathbb{R}^{N}$ on a

$$
u_{\varepsilon, n}(y)-u_{\varepsilon, n}(x)=\int_{0}^{1} \nabla u_{\varepsilon, n}(t y+(1-t) x) \cdot(y-x) \mathrm{d} t
$$

et que pour $z$ dans la boule de centre 0 et rayon $1-2 \varepsilon$ et $i \in\{1, \ldots, N\}$ on a

$$
\left(\partial u_{\varepsilon, n} / \partial x_{i}\right)(z)=\int_{B} D_{i} u(\bar{z}) \rho_{n}(z-\bar{z}) d \bar{z}
$$

En déduire que pour presque tout $x, y \in B$, on a, avec $D u=\left\{D_{1} u, \ldots, D_{N} u\right\}^{t}$,

$$
u(y)-u(x)=\int_{0}^{1} D u(t y+(1-t) x) \cdot(y-x) \mathrm{d} t .
$$

Montrer alors que $u$ est continue et que la formule précédente est vraie pour tout $x, y \in B$. Conclure enfin que $u \in C^{1}(B, \mathbb{R})$.] 
3. On reprend ici la 1ère question en remplaçant $B$ par un ouvert quelconque de $\mathbb{R}^{N}$. Montrer que $u$ est constante sur chaque composante connexe de $B$. (Comme d'habitude, $u$ constante signifie qu'il existe $a \in \mathbb{R}$ t.q. $u=a$ p.p..)

Corrigé -

1. On a $u_{\varepsilon} \in L^{1}\left(\mathbb{R}^{N}\right)$. Pour tout $n \in \mathbb{N}^{\star}$, la fonction $u_{\varepsilon, n}$ est donc bien définie sur tout $\mathbb{R}^{N}$. Le fait que $u_{\varepsilon, n}$ soit de classe $C^{\infty}$ est classique et les dérivées de $u_{\varepsilon, n}$ sont égales à la convolution de $u_{\varepsilon}$ avec les dérivées de $\rho_{n}$. Il est facile aussi de voir que $u_{\varepsilon, n}$ est une fonction à support compact car $u_{\varepsilon}$ et $\rho_{n}$ sontdes fonctions à support compact.

On note $B_{r}$ la boule de centre 0 et de rayon $r$. On montre maintenant que pour tout $i$ la fonction $\frac{\partial u_{\varepsilon, n}}{\partial x_{i}}$ est nulle sur $B_{1-2 \varepsilon}$ si $1 / n<\varepsilon$.

Soit $i \in\{1, \ldots, N\}$ et $x \in \mathbb{R}^{N}$, on a

$$
\frac{\partial u_{\varepsilon, n}}{\partial x_{i}}(x)=\left(u_{\varepsilon} \star \frac{\partial \rho_{n}}{\partial x_{i}}\right)(x)=\int_{\mathbb{R}^{N}} u_{\varepsilon}(y) \frac{\partial \rho_{n}}{\partial x_{i}}(x-y) d y .
$$

Si $1 / n<\varepsilon$ et $x \in B_{1-2 \varepsilon}$, la fonction $\rho_{n}(x-\cdot)$ appartient à $C_{c}^{\infty}(B)$ et est nulle hors de $B_{1-\varepsilon}$. On remarque aussi que

$$
\frac{\partial \rho_{n}(x-\cdot)}{\partial x_{i}}=-\frac{\partial \rho_{n}}{\partial x_{i}}(x-\cdot) \text {. }
$$

(La notation $\partial / \partial x_{i}$ désigne la dérivée par rapport à la i-ème variable, à ne pas confondre avec la i-ème composante de $x$ dans la formule précédente....) On obtient ainsi

$$
\frac{\partial u_{\varepsilon, n}}{\partial x_{i}}(x)=\int_{B} u(y) \frac{\partial \rho_{n}}{\partial x_{i}}(x-y) d y=\left\langle D_{i} u, \rho_{n}(x-\cdot)\right\rangle_{\mathcal{D}^{\star}(B), C_{c}^{\infty}(B)}=0 .
$$

On a ainsi montré que pour $1 / n<\varepsilon$, la fonction $\frac{\partial u_{\varepsilon, n}}{\partial x_{i}}$ est, pour tout $i$, nulle sur $B_{1-2 \varepsilon}$. On en déduit que la fonction $u_{\varepsilon, n}$ est constante sur $B_{1-2 \varepsilon}$. En effet, il suffit de remarquer que pour tout $x \in B_{1-2 \varepsilon}$ on a

$$
u_{\varepsilon, n}(x)-u_{\varepsilon, n}(0)=\int_{0}^{1} \nabla u_{\varepsilon, n}(t x) \cdot x \mathrm{~d} t=0 .
$$

Comme $u_{\varepsilon} \in L^{1}\left(\mathbb{R}^{N}\right)$, la suite $\left(u_{\varepsilon, n}\right)_{n \in \mathbb{N}}$ converge dans $L^{1}\left(\mathbb{R}^{N}\right)$ vers $u_{\varepsilon}$. En considérant les restrictions de ces fonctions à la boule $B_{1-2 \varepsilon}$ (sur la laquelle $u_{\varepsilon}=u$ ), la suite $\left(u_{\varepsilon, n}\right)_{n \in \mathbb{N}}$ converge dans $L^{1}\left(B_{1-2 \varepsilon}\right)$ vers $u$. Comme $u_{\varepsilon, n}$ est une fonction constante sur $B_{1-2 \varepsilon}$ (pour $1 / n<\varepsilon$ ) sa limite (dans $L^{1}$ ) est donc aussi une fonction constante. Ceci montre que la fonction u est constante sur $B_{1-2 \varepsilon}$, c'est-à-dire qu'il existe $a_{\varepsilon} \in \mathbb{R}$ t.q. u $=a_{\varepsilon}$ p.p. sur $B_{1-2 \varepsilon}$. Comme $\varepsilon>0$ est arbitraire, on en déduit que $a_{\varepsilon}$ ne dépend pas de $\varepsilon$ et que u est constante sur $B$.

2. Soit $\varepsilon>0$. La fonction $u_{\varepsilon, n}$ est de classe $C^{\infty}$. On a donc bien, pour tout $x, y \in \mathbb{R}^{N}$,

$$
u_{\varepsilon, n}(y)-u_{\varepsilon, n}(x)=\int_{0}^{1} \nabla u_{\varepsilon, n}(t y+(1-t) x) \cdot(y-x) \mathrm{d} t .
$$

Dans cette formule $\nabla u_{\varepsilon, n}$ désigne la fonction vectorielle définie par les dérivées classiques de $u_{\varepsilon, n}$.

Pour $z \in \mathbb{R}^{N}$ et $i \in\{1, \ldots, N\}$ on a

$$
\frac{\partial u_{\varepsilon, n}}{\partial x_{i}}(z)=\int_{\mathbb{R}^{N}} u_{\varepsilon}(\bar{z}) \frac{\partial \rho_{n}}{\partial x_{i}}(z-\bar{z}) d \bar{z}
$$

Si $z \in B_{1-2 \varepsilon}$ et $1 / n<\varepsilon$, la fonction $\rho_{n}(z-\cdot)$ appartient à $C_{c}^{\infty}(B)$ et est nulle hors de $B_{1-\varepsilon}$ (et sur $B_{1-\varepsilon}$ on a $u_{\varepsilon}=u$ ). On en déduit

$$
\frac{\partial u_{\varepsilon, n}}{\partial x_{i}}(z)=\left\langle D_{i} u, \rho_{n}(z-\cdot)\right\rangle_{\mathcal{D}^{\star}(B), C_{c}^{\infty}(B)}=\int_{B} D_{i} u(\bar{z}) \rho_{n}(z-\bar{z}) d \bar{z} .
$$

Comme $D_{i}(u)$ est uniformément continue sur $B_{1-\varepsilon}$, on déduit de la formule précédente que $\partial u_{\varepsilon, n} / \partial x_{i}$ converge vers $D_{i} u$ uniformément sur $B_{1-2 \varepsilon}$. On a donc, pour tout $x, y \in B_{1-2 \varepsilon}$

$$
\lim _{n \rightarrow+\infty} \int_{0}^{1} \nabla u_{\varepsilon, n}(t y+(1-t) x) \cdot(y-x) \mathrm{d} t .=\int_{0}^{1} D u(t y+(1-t) x) \cdot(y-x) \mathrm{d} t .
$$


La suite $\left(u_{\varepsilon, n}\right)_{n \in \mathbb{N}^{*}}$ converge dans $L^{1}\left(\mathbb{R}^{N}\right)$ vers $u_{\varepsilon}$. Après extraction éventuelle d'une sous suite, on peut donc supposer que cette suite converge p.p. vers $u_{\varepsilon}$ et donc p.p. vers $u$ sur la boule $B_{1-\varepsilon}$. En passant à la limite quand $n \rightarrow+\infty$ dans l'égalité 1.6 , on obtient pour presque tout $x$, y dans $B_{1-2 \varepsilon}$

$$
u(y)-u(x)=\int_{0}^{1} D u(t y+(1-t) x) \cdot(y-x) \mathrm{d} t .
$$

Comme $\varepsilon>0$ est arbitraire, la formule 1.7 est valable pour presque tout $x, y \in B$.

Pour conclure, on fixe un point $x \in B$ pour lequel 1.7 est vraie pour presque tout $y \in B$ et on pose

$$
v(y)=u(x)+\int_{0}^{1} D u(t y+(1-t) x) \cdot(y-x) \mathrm{d} t \text { pour tout } y \in B .
$$

La fonction $v$ est de classe $C^{1}$ (car Du est une fonction continue et donc $v$ est dérivable sur tout $B$ et $\nabla v=D u$ ). Comme $u=v$ p.p., ceci termine la question.

3. On note $\Omega$ l'ouvert remplaçant B. Le raisonnement précédent montre que sur toute boule incluse dans $\Omega$, u est p.p. égale à une constante. Pour que l'égalité soit vraie sur toute la boule (et non seulement p.p.), il suffit de définir v sur $\Omega$ par

$$
v(x)=\lim _{h \rightarrow 0, h>0} \frac{1}{\lambda_{d}\left(B_{x, h}\right)} \int_{B(x, h)} u(y) d y \text { pour tout } x \in \Omega,
$$

où $B(x, h)$ désigne la boule de centre $x$ et de rayon $h$ et $\lambda_{d}(B(x, h))$ la mesure de Lebesgue d-dimensionelle de cette boule. On a alors $u=v$ p.p. ( $v$ est donc un représentant de la classe $u$ ) et sur toute boule incluse dans $\Omega$, $v$ est égale à une constante.

La fonction $v$ est donc localement constante. On en déduit que $v$ est constante sur chaque composante connexe de $\Omega$. En effet, soit $x \in \Omega$ et $U$ la composante connexe de $\Omega$ contenant $x$. On pose $a=v(x)$. L'ensemble $\{y \in U ; v(y)=a\}$ est un ouvert non vide de $U$ et l'ensemble $\{y \in U ; v(y) \neq a\}$ est aussi un ouvert de $U$ disjoint du précédent. Par connexité de $U$ ce dernier ensemble est donc vide, ce qui prouve que $v=$ a sur tout $U$.

\section{Exercice 1.5 (Une fonction $\boldsymbol{H}^{\mathbf{1}}$ n'est pas forcément continue, $\boldsymbol{d}>\mathbf{1}$ )}

Soit $\left.\Omega=\left\{x=\left(x_{1}, x_{2}\right)^{t} \in \mathbb{R}^{2},\left|x_{i}\right|<\frac{1}{2}, i=1,2\right\}, \gamma \in\right] 0,1 / 2[$.

Soit $u: \Omega \rightarrow \mathbb{R}$ définie par $u(x)=(-\ln (|x|))^{\gamma}$. Montrer que $u \in H^{1}(\Omega)$. En déduire que $H^{1}(\Omega) \not \subset C(\bar{\Omega})$.

Corrigé - La fonction u est de classe $C^{\infty}$ sur $\bar{\Omega} \backslash\{0\}$ (en remarquant que $|x| \leq \sqrt{2} / 2<1$ pour tout $x \in \bar{\Omega}$ ). Les dérivées classiques de u sont pour $x=\left(x_{1}, x_{2}\right)^{t} \neq 0$

$$
\frac{\partial u}{\partial x_{i}}(x)=-\gamma(-\ln (|x|))^{\gamma-1} \frac{x_{i}}{|x|^{2}}, i=1,2 .
$$

Il est facile de voir que $u \in L^{2}(\Omega)$ (et même $u \in L^{p}(\Omega)$ pour tout $1 \leq p<+\infty$ ). Comme $\gamma<1 / 2$, on peut aussi montrer que les dérivées classiques de u sont dans $L^{2}(\Omega)$. Il suffit pour cela de remarquer que, pour a $>0$,

$$
\int_{0}^{a} \frac{1}{r|\ln (r)|^{2(1-\gamma)}} d r<+\infty
$$

Pour montrer que $u \in H^{1}(\Omega)$, il suffit donc de montrer que les dérivées par transposition de u sont représentées par les dérivées classiques, c'est-à-dire que pour tout $\varphi \in C_{c}^{\infty}(\Omega)$ et pour $i=1,2$, on a

$$
\int_{\Omega} u(x) \frac{\partial \varphi}{\partial x_{i}}(x) \mathrm{d} x=-\int_{\Omega} \frac{\partial u}{\partial x_{i}}(x) \varphi(x) \mathrm{d} x .
$$

On montre maintenant 1.8 pour $i=1$ (bien sûr, $i=2$ se traite de manière semblable). Soit $\varphi \in C_{c}^{\infty}(\Omega)$ et $0<\varepsilon<1 / 2$. On pose $L_{\varepsilon}=[-\varepsilon, \varepsilon] \times[-1 / 2,1 / 2]$. En intégrant par parties, on a

$$
\int_{\Omega \backslash L_{\varepsilon}} u(x) \frac{\partial \varphi}{\partial x_{1}}(x) \mathrm{d} x=-\int_{\Omega \backslash L_{\varepsilon}} \frac{\partial u}{\partial x_{1}}(x) \varphi(x) \mathrm{d} x-\int_{-1 / 2}^{1 / 2} u\left(\varepsilon, x_{2}\right)\left(\varphi\left(\varepsilon, x_{2}\right)-\varphi\left(-\varepsilon, x_{2}\right)\right) \mathrm{d} x_{2} .
$$

(On a utilisé ici le fait que $u\left(\varepsilon, x_{2}\right)=u\left(-\varepsilon, x_{2}\right)$.)

Par convergence dominée, on a

$$
\lim _{\varepsilon \rightarrow 0} \int_{\Omega \backslash L_{\varepsilon}} u(x) \frac{\partial \varphi}{\partial x_{1}}(x) \mathrm{d} x=\int_{\Omega} u(x) \frac{\partial \varphi}{\partial x_{1}}(x) \mathrm{d} x \text { et } \lim _{\varepsilon \rightarrow 0} \int_{\Omega \backslash L_{\varepsilon}} \frac{\partial u}{\partial x_{1}}(x) \varphi(x) \mathrm{d} x=\int_{\Omega} \frac{\partial u}{\partial x_{1}}(x) \varphi(x) \mathrm{d} x
$$


Il reste à montrer que le deuxième terme du membre de droite de 1.9] tend vers 0 . Ceci se fait en remarquant que la fonction $\varphi$ est régulière, il existe donc $C$ ne dépendant que de $\varphi$ t.q.

$$
\left|\int_{-1 / 2}^{1 / 2} u\left(\varepsilon, x_{2}\right)\left(\varphi\left(\varepsilon, x_{2}\right)-\varphi\left(-\varepsilon, x_{2}\right)\right) \mathrm{d} x_{2}\right| \leq|\ln (\varepsilon)|^{\gamma} C \varepsilon
$$

On en déduit bien que

$$
\lim _{\varepsilon \rightarrow 0} \int_{-1 / 2}^{1 / 2} u\left(\varepsilon, x_{2}\right)\left(\varphi\left(\varepsilon, x_{2}\right)-\varphi\left(-\varepsilon, x_{2}\right)\right) \mathrm{d} x_{2}=0
$$

ce qui termine la démonstration de 1.8 pour $i=1$. Finalement, on a bien ainsi montré que les dérivées par transposition de u sont représentées par les dérivées classiques et que $u \in H^{1}(\Omega)$.

Exercice 1.6 (Laplacien d'un élément de $\boldsymbol{H}_{\mathbf{0}}^{\mathbf{1}}(\boldsymbol{\Omega})$ )

Soit $\Omega$ un ouvert borné de $\mathbb{R}^{N}(N \geq 1)$ et $u \in H_{0}^{1}(\Omega)$.

1. Montrer que, pour tout $\varphi \in C_{c}^{\infty}(\Omega)$, on a

$$
\langle\Delta u, \varphi\rangle_{\mathcal{D}^{\star}(\Omega), C_{c}^{\infty}(\Omega)}=\int u(x) \Delta \varphi(x) \mathrm{d} x=-\int_{\Omega} \nabla u(x) \cdot \nabla \varphi(x) \mathrm{d} x .
$$

2. On rappelle que $H_{0}^{1}(\Omega)$ est un s.e.v. fermé de $H^{1}(\Omega)$. Muni de la norme de $H^{1}(\Omega)$, l'espace $H_{0}^{1}(\Omega)$ est donc un espace de Hilbert. On note $H^{-1}(\Omega)$ le dual (topologique) de $H_{0}^{1}(\Omega)$. Déduire de la question précédente que $\Delta u \in H^{-1}(\Omega)$ ( c'est-à-dire que l'élément de $\mathcal{D}^{\star}(\Omega)$, noté $\Delta u$, se prolonge de manière unique en un élément de $H^{-1}(\Omega)$, encore notée $\Delta u$ ) et que

$$
\|\Delta u\|_{H^{-1}(\Omega)} \leq\||\nabla u|\|_{L^{2}(\Omega)}
$$

NB. En fait, on montrera au chapitre 2 que sur $H_{0}^{1}(\Omega)$ la norme $H^{1}(\Omega)$ est équivalente à la norme notée $\|\cdot\|_{H_{0}^{1}(\Omega)}$ définie par $\|u\|_{H_{0}^{1}(\Omega)}=\||\nabla u|\|_{L^{2}(\Omega)}$. Avec ce choix de norme sur $H_{0}^{1}(\Omega)$ on obtient $\|\Delta u\|_{H^{-1}(\Omega)}=\|u\|_{H_{0}^{1}(\Omega)}$.

Corrigé -

1. Par définition de la dérivée par transposition, on a

$$
\langle\Delta u, \varphi\rangle_{\mathcal{D}^{\star}(\Omega), C_{c}^{\infty}(\Omega)}=\sum_{i=1}^{N}\left\langle D_{i} D_{i} u, \varphi\right\rangle_{\mathcal{D}^{\star}(\Omega), C_{c}^{\infty}(\Omega)}=\sum_{i=1}^{N} \int_{\Omega} u(x) \partial_{i}^{2} \varphi(x) \mathrm{d} x .
$$

Puis, comme $u \in H_{0}^{1}(\Omega)$, la forme linéaire $\left(\right.$ sur $\left.C_{c}^{\infty}(\Omega)\right) D_{i}$ u est représentée par un élément de $L^{2}(\Omega)$ encore noté $D_{i} u$ et on a, pour tout $i \in\{1, \ldots, N\}$,

$$
\int_{\Omega} u(x) \partial_{i}^{2} \varphi(x) \mathrm{d} x=-\left\langle D_{i} u, \partial_{i} \varphi\right\rangle_{\mathcal{D}^{\star}(\Omega), C_{c}^{\infty}(\Omega)}=-\int_{\Omega} D_{i} u(x) \partial_{i} \varphi(x) \mathrm{d} x .
$$

Comme $\nabla$ u est l'élément de $L^{2}(\Omega)^{N}$ dont les composantes sont les $D_{i} u$, on obtient bien

$$
\langle\Delta u, \varphi\rangle_{\mathcal{D}^{*}(\Omega), C_{c}^{\infty}(\Omega)}=-\int_{\Omega} \nabla u(x) \cdot \nabla \varphi(x) \mathrm{d} x .
$$

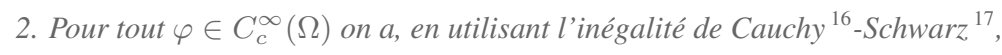

$$
\left|\langle\Delta u, \varphi\rangle_{\mathcal{D}^{*}(\Omega), C_{c}^{\infty}(\Omega)}\right| \leq \int_{\Omega}|\nabla u(x)||\nabla \varphi(x)| \mathrm{d} x \leq\||\nabla u|\|_{L^{2}(\Omega)}\||\nabla \varphi|\|_{L^{2}(\Omega)} \leq\|u\|_{H^{1}(\Omega)}\|\varphi\|_{H^{1}(\Omega)} .
$$

16. Augustin Louis, baron Cauchy, (1789-1857) est un mathématicien français à qui l'on doit en particulier de nombreux résultats en analyse. Il a malheureusement négligé, en tant que membre de l'Académie des sciences, les travaux d'Evariste Galois et Niels Abel, redécouverts plus tard, et qui ont profondément marqué les mathématiques du 20ème siècle.

17. Hermann Schwarz (1843-1921) est un mathématicien allemand, connu pour ses travaux en analyse complexe. (À ne pas confondre avec Laurent Schwartz, mathématicien français du 20ème siècle). 
Ceci montre que l'application $\varphi \mapsto\langle\Delta u, \varphi\rangle_{\mathcal{D}^{\star}(\Omega), C_{c}^{\infty}(\Omega)}$ est linéaire continue de $C_{c}^{\infty}(\Omega)$, muni de la norme $H^{1}(\Omega)$, dans $\mathbb{R}$. Comme $\mathcal{D}^{\star}(\Omega)$ est dense dans $H_{0}^{1}(\Omega)$ cette application se prolonge donc, par densité, de manière unique en une application linéaire continue de $H_{0}^{1}(\Omega)$ dans $\mathbb{R}$ ( c'est-à-dire en un élément de $H^{-1}(\Omega)$ ). Cet élément de $H^{-1}(\Omega)$ est encore noté $\Delta u$ et le prolongement par densité donne, pour tout $v \in H_{0}^{1}(\Omega)$,

$$
\langle\Delta u, v\rangle_{H^{-1}(\Omega), H_{0}^{1}(\Omega)}=-\int_{\Omega} \nabla u(x) \cdot \nabla v(x) \mathrm{d} x .
$$

On a donc, pour tout $v \in H_{0}^{1}(\Omega)$,

$$
\left|\langle\Delta u, v\rangle_{H^{-1}(\Omega), H_{0}^{1}(\Omega)}\right| \leq \int_{\Omega}|\nabla u(x)||\nabla v(x)| \mathrm{d} x \leq\|u\|_{H^{1}(\Omega)}\|v\|_{H^{1}(\Omega)} .
$$

L'espace $H_{0}^{1}(\Omega)$ est muni de la norme $H^{1}(\Omega)$, ce qui donne

on déduit alors de 1.10 que

$$
\|\Delta u\|_{H^{-1}(\Omega)}=\sup _{v \in H_{0}^{1}(\Omega), v \neq 0} \frac{\langle\Delta u, v\rangle_{H^{-1}(\Omega), H_{0}^{1}(\Omega)}}{\|v\|_{H^{1}(\Omega)}} .
$$

$$
\|\Delta u\|_{H^{-1}(\Omega)} \leq\|u\|_{H^{1}(\Omega)} .
$$

Exercice 1.7 (Petits pièges) Pour $x \in \mathbb{R}^{2} \backslash\{0\}$, on pose $G(x)=\ln (|x|)$.

1. Montrer que $G \in C^{\infty}\left(\mathbb{R}^{2} \backslash\{0\}\right)$ et $\Delta G=0$ (au sens classique) dans $\mathbb{R}^{2} \backslash\{0\}$. En déduire que $\Delta G=0$ dans $\mathcal{D}^{\star}\left(\mathbb{R}^{2}\right) \backslash\{0\}$. Que vaut $\Delta G$ dans $\mathcal{D}^{\star}\left(\mathbb{R}^{2}\right)$ ?

2. Montrer que $G \in L_{l o c}^{p}\left(\mathbb{R}^{2}\right)$ pour tout $p<+\infty$ et $\nabla G \in L_{l o c}^{p}\left(\mathbb{R}^{2}\right)$ pour $p<2$.

3. On prend dans cette question $\Omega=] 0,1[2$. Montrer que

$$
u \in L^{2}(\Omega), \Delta u \in H^{-1}(\Omega) \nRightarrow u \in H^{1}(\Omega) .
$$

Montrer que

$$
v \in\left(H^{-1}(\Omega)\right)^{2}, \operatorname{div}(v)=0 \text { dans } \mathcal{D}^{\star}(\Omega) \text { et } \operatorname{rot}(\mathrm{v})=0 \text { dans } \mathcal{D}^{\star}(\Omega) \nRightarrow \mathrm{v} \in\left(\mathrm{L}^{2}(\Omega)\right)^{2} .
$$

4. (Singularité éliminable) Soit $\Omega$ un ouvert de $\mathbb{R}^{2}$ contenant 0 . On suppose ici que $u \in H^{1}(\Omega)$ et que $\Delta u=0$ dans $\mathcal{D}^{\star}(\Omega \backslash\{0\})$. Montrer que $\Delta u=0$ dans $\mathcal{D}^{\star}(\Omega)$.

Exercice 1.8 (Trois applications de Hahn-Banach) Soit $E$ un espace de Banach réel.

1. Soit $x \in E, x \neq 0$. Montrer qu'il existe $T \in E^{\prime}$ t.q. $T(x)=\|x\|_{E}$ et $\|T\|_{E^{\prime}}=1$.

2. Soient $F$ un s.e.v de $E$ et $x \in E$. Montrer que $x \notin \bar{F}$ si et seulement si il existe $T \in E^{\prime}$ t.q. $T(x) \neq 0$ et $T(y)=0$ pour tout $y \in F$.

3. Pour $x \in E$, on définit $J_{x}$ de $E^{\prime}$ dans $\mathbb{R}$ par $J_{x}(T)=T(x)$ pour tout $T \in E^{\prime}$. Montrer que $J_{x} \in E^{\prime \prime}$ pour tout $x \in E$ et que l'application $J: x \mapsto J_{x}$ est une isométrie de $E$ sur $J(E) \subset E$ ". (Définition : On dit que $E$ est réflexif si $J(E)=E^{\prime \prime}$.)

Indication -

1. Définir T sur la droite engendrée par $x$ et prolonger T par Hahn Banach.

2. Sens "si" : Sens facile.

Sens "seulement si" : Construire l'application linéaire $T$ sur $\mathbb{R} x \oplus F$ par $T(x)=1$ et $T(y)=0$ pour tout $y \in F$; montrer que T est continue et conclure par Hahn Banach. La continuité de T est le point le plus technique : on peut par exemple remarquer que si $x \notin \bar{F}$, alors il existe $\varepsilon>0$ tel que $B(0, \varepsilon) \cap F=\emptyset$, et montrer ensuite que $T(z) \leq \frac{1}{\varepsilon}\|z\|$ pour tout $z \in G=\mathbb{R} x \oplus F$, ce qui montre la continuité de $T$ sur $G$. 
3. La linéarité et la continuité de $J$ sont faciles. Il reste à montrer le caractére isométrique. Soit $x \in$ E. Il est facile de voir que $|J(x)| \leq\|x\|_{E}$. Pour montrer l'égalité, considérer l'application $T$ de la première question.

Exercice 1.9 (Séparabilité de $\boldsymbol{L}^{p}$ ) On désigne par $L^{p}$ l'espace $L_{\mathbb{R}}^{p}(\mathbb{R}, \mathcal{B}(\mathbb{R}), \lambda)$.

1. Soit $1 \leq p<\infty$. Montrer que $L^{p}$ est séparable.

2. Montrer que $L^{\infty}(\mathbb{R})$ n'est pas séparable.

Indication -

1. On pourra onstruire une famille dénombrable dense de $C_{c}(\mathbb{R})$ en considérant pour $n \in \mathbb{N}$, l'ensemble $A_{n}$ des fonctions qui sont nulles sur $[-n, n]^{c}$ et qui sont constantes par morceaux et à valeur rationnelles sur tous les intervalles de la forme $\left[\frac{i}{n}, \frac{i+1}{n}\left[, i \in \mathbb{Z}\right.\right.$. Vérifier que les ensembles $A_{n}$ sont dénombrables et montrer ensuite que $A=\cup_{n} \in \mathbb{N} A_{n}$ est dense dans $L^{p}$.

2. Soit $B$ l'ensemble des fonctions constantes sur les intervalles $[i, i+1[, i \in \mathbb{Z}$, et qui ne prenent que les valeurs 0 ou 1. Vérifier que $B$ est une partie non dénombrable de $L^{\infty}$ et que si A est une partie dense de $L^{\infty}(\mathbb{R})$ il existe une injection de A dans $B$.

Exercice 1.10 (Réflexivité de $L^{p}$ si $1<p<\infty$ )

Soient $(X, T, m)$ un espace mesuré $\sigma$-fini et $1<p<\infty$, montrer que $L^{p}(X, T, m)$ est un espace de Banach réflexif.

\section{Exercice 1.11 (Séparabilité et réflexivité d'un s.e.v. fermé)}

Soient $E$ un espace de Banach (réel) et $F$ un s.e.v. fermé de $E$. Montrer que :

1. $E$ séparable $\Rightarrow F$ séparable.

2. $E$ réflexif $\Rightarrow F$ réflexif.

Exercice 1.12 (Fonctions lipschitziennes) Soit $\Omega$ un ouvert borné de $\mathbb{R}^{N}$ à frontière lipschitzienne.

1. Soit $u: \Omega \rightarrow \mathbb{R}$ une fonction lipschitzienne. Montrer que $u \in W^{1, \infty}(\Omega)$.

2. Soit $u \in W^{1, \infty}(\Omega)$. Montrer que $u$ est lipschitzienne (au sens : il existe $v: \Omega \rightarrow \mathbb{R}$ lipschitzienne t.q. $u=v$ p.p.).

Exercice 1.13 (Exemple d'ouvert lipschitzien non fortement lipschitzien) On appelle ouvert lipschitzien un ouvert à frontière lipschitzienne (définition 1.16 et ouvert fortement lipschitzien un ouvert à frontière fortement lipschitzienne (remarque 1.17).

Pour construire un exemple d'ouvert lipschitzien non fortement lipschitzien, l'idée est de construire un ouvert qui ne vérifie pas la propriété du segment.

Définition 1.30 (Propriété du segment) On dit qu'un ouvert $\Omega$ de $\mathbb{R}^{N}$ satisfait la propriété du segment si que pour tout $z \in \partial \Omega$, il existe $d \in \mathbb{R}^{N}, d \neq 0$ et $t \in \mathbb{R}_{+}^{\star}$ t.q. $\left.\left.\{z+s d, s \in] 0, t\right]\right\} \subset \Omega$.

Un ouvert fortement lipschitzien de $R^{N}$ vérifie la propriété du segment : il suffit pour s'en convaincre de considérer (sans restriction de généralité) que la frontière est localement alignée avec l'axe $x_{1}$ en coordonnées cartésiennes et de choisir $d=(0, \ldots, 0,1)$ Pour construire un ouvert qui ne vérifie pas la propriété du segment, l'idée utilisée ici (due, semble-t-il à Zerner, voir [17]) est de prendre pour ouvert une "route" allant vers le point $(0,0)$ avec une infinité de virages, sans changer le rayon de courbure des virages (ce qui donne que l'ouvert est lipschitzien) mais 
(bien sûr) en faisant en sorte que la largeur des virages tende vers 0 quand on se rapproche de $(0,0)$. A cause des virages, l'ouvert ne vérifie pas la propriété du segment et donc n'est pas fortement lipschitzien.

Construction de l'ouvert $\Omega$ - Soit $\bar{\varphi}$ une fonction continue de $[0,1]$ dans $\mathbb{R}$, nulle en 0 et 1 . On suppose que

$$
\bar{\varphi}\left(\frac{1}{4}\right) \geq 1
$$

La fonction $\bar{\varphi}$ peut être, par exemple, une fonction "chapeau" ou une fonction de classe $C^{\infty}$ à support compact dans $] 0,1\left[\right.$. On pose $a_{n}=1 / 2^{n}$ pour $n \in \mathbb{N}$ et et on définit $\varphi$ de $[0,1]$ dans $\mathbb{R}$ en posant

$$
\left.\left.\varphi(x)=a_{n-1} \bar{\varphi}\left(\frac{x-a_{n}}{a_{n-1}}\right) \text { si } x \in\right] a_{n}, a_{n-1}\right] \text { et } n \geq 1 .
$$

La fonction $\varphi$ est donc lipschitzienne de $[0,1]$ dans $\mathbb{R}$ (en ajoutant $\varphi(0)=0$ ). On remarque aussi que $\varphi\left(a_{n}\right)=0$. On pose alors $\Omega=\left\{(x, y) \in \mathbb{R}^{2} ; x \in\right] 0,1[, \varphi(x)-x<y<\varphi(x)\}$.

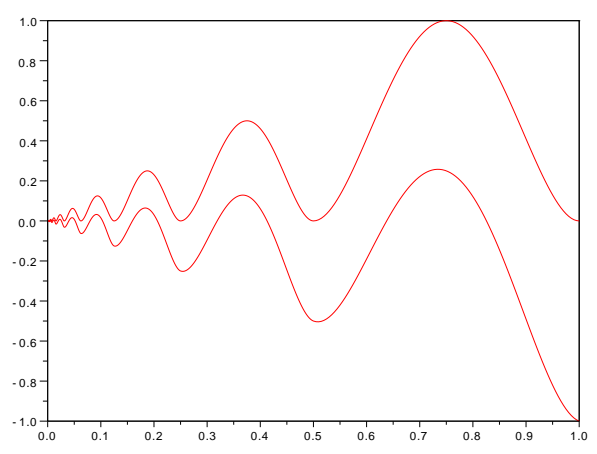

FIGURE 1.1 - L'ouvert $\Omega$

1. L'ouvert $\Omega$ n'est pas fortement lipschitzien - Montrer que la propriété du segment n'est pas vérifiée pour $\Omega$ et $z=0$ et en déduire que $\Omega$ n'est pas fortement lipschitzien.

2. L'ouvert $\Omega$ est faiblement lipschitzien - On pose $T=\{(x, y), x \in] 0,1[,-x<y<x\}$. On définit une bijection $\psi$ de $\Omega$ dans le triangle $T$ en posant

$$
\psi(x, y)=(x, x+2(y-\varphi(x))) .
$$

Montrer que $\psi$ est lipschitzienne ainsi que son inverse et en déduire que $\Omega$ est un ouvert lipschitzien.

Corrigé -

1. Soit $d=\left(d_{1}, d_{2}\right) \in \mathbb{R}^{2}, d \neq 0$ et $t \in \mathbb{R}_{+}^{\star}$. On va montrer que le segment $\left.\left.\{s d, s \in] 0, t\right]\right\}$ rencontre le complémentaire de $\Omega$. On pose $S=\{s d, s \in] 0, t]\}$. Comme $\Omega \subset \mathbb{R}_{+}^{\star} \times \mathbb{R}$, on a, bien sûr, $S \subset \Omega^{c}$ si $d_{1} \leq 0$. On suppose donc $d_{1}>0$ et (comme t est arbitraire) il suffit de considérer le cas $d_{1}=1$.

Si $d_{2} \geq 0$, on a $a_{n} d \in S$ pour $n$ t.q. $a_{n} \leq$ t et $a_{n} d \notin \Omega$ car $a_{n} d_{2} \geq \varphi\left(a_{n}\right)=0$. Donc, S rencontre $\Omega^{c}$. 
Si $d_{2}<0$, on a $\left(a_{n}+\frac{a_{n}}{2}\right) d \in S$ pour n t.q. $a_{n}+\frac{a_{n}}{2} \leq t$. Mais en utilisant 1.11, on a

$$
\varphi\left(a_{n}+\frac{a_{n}}{2}\right)=a_{n-1} \bar{\varphi}\left(\frac{1}{4}\right) \geq a_{n-1} \geq a_{n}+\frac{a_{n}}{2} .
$$

On a donc $\varphi\left(a_{n}+\frac{a_{n}}{2}\right)-\left(a_{n}+\frac{a_{n}}{2}\right) \geq 0$ et, comme $\left(a_{n}+\frac{a_{n}}{2}\right) d_{2}<0$, ceci montre que $\left(a_{n}+\frac{a_{n}}{2}\right) d \notin \Omega$. On a ainsi montré que $S$ rencontre $\Omega^{c}$.

Finalement, on a bien montré que $\Omega$ n'est pas fortement lipschitzien.

2. La fonction $\psi$ est lipschitzienne (car $\varphi$ l'est) et son inverse aussi car son inverse est l'application $\bar{\psi}$ définie par

$$
\bar{\psi}(x, y)=\left(x, \varphi(x)+\frac{1}{2}(y-\varphi(x))\right) .
$$

Comme le triangle $T$ est fortement lipschitzien, on en déduit (assez facilement) que $\Omega$ est lipschitzien.

Exercice 1.14 (Prolongement d'une fonction continue) Soient $\Omega$ un ouvert de $\mathbb{R}^{N}, N \geq 1$, et $f$ une fonction continue de $\bar{\Omega}$ dans $\mathbb{R}$. Le but de l'exercice est de montrer qu'il existe $g$ continue de $\mathbb{R}^{N}$ dans $\mathbb{R}$ telle que $g=f$ dans $\bar{\Omega}$.

Si $x \in \bar{\Omega}$, on pose $g(x)=f(x)$.

Si $x \notin \bar{\Omega}$, on pose $d_{x}=\inf \{|x-y|, y \in \Omega\}$ (on a donc $d_{x}>0$ ), $B_{x}=\left\{z \in \mathbb{R}^{N},|x-z|<2 d_{x}\right\}$ et

$$
g(x)=\frac{\int_{\Omega \cap B_{x}} f(z) d z}{\int_{\Omega \cap B_{x}} d z} .
$$

Montrer que $g$ est bien définie et est continue de $\mathbb{R}^{N}$ dans $\mathbb{R}$.

\section{Exercice 1.15 (Inégalités de Sobolev pour $p>N$ )}

L'objet de cet exercice est de démontrer l'injection de Sobolev pour $p>N$.

Si $x \in \mathbb{R}^{N}(N \geq 1)$, on note $x=\left(x_{1}, \bar{x}\right)$, avec $x_{1} \in \mathbb{R}$ et $\bar{x} \in \mathbb{R}^{N-1}$. On note $H=\{(t,(1-|t|) a), t \in]-1,1[$, $\left.a \in B_{N-1}\right\}$, où $B_{N-1}=\left\{x \in \mathbb{R}^{N-1},|x|<1\right\}$. (On rappelle que $|\cdot|$ désigne toujours la norme euclidienne.) Soit $N<p<\infty$.

1. Soit $u \in C^{1}\left(\mathbb{R}^{N}, \mathbb{R}\right)$. Montrer qu'il existe $C_{1} \in \mathbb{R}$, ne dépendant que de $N$ et $p$, t.q.

$$
|u(1,0)-u(-1,0)| \leq C_{1}\|(|\nabla u|)\|_{L^{p}(H)} .
$$

[On pourra commencer par écrire $u(1,0)-u(0, a)$ comme une intégrale utilisant convenablement $\nabla u(t,(1-t) a)$ pour $t \in] 0,1$, et intégrer pour $a \in B_{N-1}$ pour comparer $\mathrm{u}(1,0)$ et sa moyenne sur $B_{N-1}$. On pourra se limiter au cas $N=2$, pour éviter des complications inutiles.]

2. Soit $u \in C_{c}^{1}\left(\mathbb{R}^{N}, \mathbb{R}\right)$. Montrer qu'il existe $C_{2} \in \mathbb{R}$, ne dépendant que de $N$ et $p$, t.q.

$$
|u(x)-u(y)| \leq C_{2}\|(|\nabla u|)\|_{L^{p}\left(\mathbb{R}^{N}\right)}|x-y|^{1-\frac{N}{p}} .
$$

[Après, éventuellement, une rotation et une translation, on peut supposer que $x=(b, 0)$ et $y=(-b, 0)$. Se ramener alors à 1.12.]

Pour $\alpha \in] 0,1]$ et $K$ sous ensemble fermé de $\mathbb{R}^{N}$, on note

$$
C^{0, \alpha}(K)=\left\{u \in C(K, \mathbb{R}),\|u\|_{L^{\infty}(K)}<\infty \text { et } \sup _{x, y \in K, x \neq y} \frac{|u(x)-u(y)|}{|x-y|^{\alpha}}<\infty\right\},
$$


et, si $u \in C^{0, \alpha}(K)$,

$$
\|u\|_{0, \alpha}=\|u\|_{L^{\infty}(K)}+\sup _{x, y \in K, x \neq y} \frac{|u(x)-u(y)|}{|x-y|^{\alpha}} .
$$

Noter que $C^{0, \alpha}(K)$, muni de cette norme, est un espace de Banach.

3. Soit $u \in C_{c}^{1}\left(\mathbb{R}^{N}, \mathbb{R}\right)$. Montrer qu'il existe $C_{3} \in \mathbb{R}$, ne dépendant que de $N$ et $p$, t.q.

$$
\|u\|_{L^{\infty}\left(\mathbb{R}^{N}\right)} \leq C_{3}\|u\|_{W^{1, p}\left(\mathbb{R}^{N}\right)} .
$$

[Cette question est plus délicate... Il faut utiliser 1.13) et le fait que $u \in L^{p}\left(\mathbb{R}^{N}\right)$.]

4. (Injection de Sobolev dans $\mathbb{R}^{N}$.) Montrer que $W^{1, p}\left(\mathbb{R}^{N}\right) \subset C^{0, \alpha}\left(\mathbb{R}^{N}\right)$, avec $\alpha=1-\frac{N}{p}$, et qu'il existe $C_{4} \in \mathbb{R}$, ne dépendant que de $N$ et $p$, t.q.

$$
\|u\|_{C^{0, \alpha}\left(\mathbb{R}^{N}\right)} \leq C_{4}\|u\|_{W^{1, p}\left(\mathbb{R}^{N}\right)} .
$$

5. (Injection de Sobolev dans $\Omega$.) Soient $\Omega$ un ouvert borné de $\mathbb{R}^{N}(N \geq 1)$, à frontière lipschitzienne.

Montrer que $W^{1, p}(\Omega) \subset C^{0, \alpha}(\bar{\Omega})$, avec $\alpha=1-\frac{N}{p}$, et qu'il existe $C_{5} \in \mathbb{R}$, ne dépendant que de $\Omega, N$ et $p$, t.q.

$$
\|u\|_{C^{0, \alpha}(\bar{\Omega})} \leq C_{5}\|u\|_{W^{1, p}(\Omega)} .
$$

Exercice 1.16 (Inégalités de Sobolev pour $p \leq N$ ) L'objet de cet exercice est de démontrer l'injection de Sobolev pour $1 \leq p \leq N$.

La démonstration proposée ici est due à L. Nirenberg ${ }^{18}$. Elle consiste à faire d'abord le cas $p=1$, puis à en déduire le cas $1<p<N$. Historiquement, la cas $1<p<N$ à été démontré avant le cas $p=1$ (et le cas $p=1$ est longtemps resté un problème ouvert).

1. Soit $u \in C_{c}^{1}\left(\mathbb{R}^{N}\right)$.

(a) On suppose ici $N=1$. Montrer que $\|u\|_{\infty} \leq\left\|u^{\prime}\right\|_{1}$.

(b) Par récurrence sur $N$, montrer que $\|u\|_{N /(N-1)} \leq\left\|\frac{\partial u}{\partial x_{1}}\right\|_{1}^{1 / N} \ldots\left\|\frac{\partial u}{\partial x_{N}}\right\|_{1}^{1 / N}$.

(c) Montrer que $\|u\|_{N /(N-1)} \leq\||\nabla u|\|_{1}$.

(d) Soit $1 \leq p<N$. Montrer qu'il existe $C_{N, p}$ ne dépendant que $N$ et $p$ t.q. $\|u\|_{p^{\star}} \leq C_{N, p}\||\nabla u|\|_{p}$, avec $p^{\star}=(N p) /(N-p)$.

2. Soit $1 \leq p<N$. Montrer que $\|u\|_{p^{\star}} \leq C_{N, p}\||\nabla u|\|_{p}$, pour tout $u \in W^{1, p}\left(\mathbb{R}^{N}\right)\left(C_{N, p}\right.$ et $p^{\star}$ sont donnés à la question précédente). En déduire que l'injection de $W^{1, p}\left(\mathbb{R}^{N}\right)$ dans $L^{q}\left(\mathbb{R}^{N}\right)$ est continue pour tout $q \in\left[p, p^{\star}\right]$.

3. Soit $p=N$. Montrer que l'injection de $W^{1, N}\left(\mathbb{R}^{N}\right)$ dans $L^{q}\left(\mathbb{R}^{N}\right)$ est continue pour tout $q \in[N, \infty[$ (Pour $N=1$, le cas $q=\infty$ est autorisé).

4. On suppose maintenant que $\Omega$ est un ouvert borné à frontière lipschitzienne. Pour $1 \leq p<N$, Montrer que l'injection de $W^{1, p}(\Omega)$ dans $L^{q}(\Omega)$ est continue pour tout $q \in\left[p, p^{\star}\right]\left(p^{\star}=(N p) /(N-p)\right)$. Montrer que l'injection de $W^{1, N}(\Omega)$ dans $L^{q}(\Omega)$ est continue pour tout $q \in[N, \infty[$ (Pour $N=1$, le cas $q=\infty$ est autorisé).

Corrigé -

18. Louis Nirenberg (1925-2020), mathématicien canadien, spécialiste de l'analyse des équations aux dérivées partielles 
1.(a) Comme $u \in C_{c}^{1}(\mathbb{R})$ on a, pour $x \in \mathbb{R}, u(x)=\int_{-\infty}^{x} u^{\prime}(t) d t$ et donc

On en déduit bien $\|u\|_{\infty} \leq\left\|u^{\prime}\right\|_{1}$.

$$
|u(x)| \leq \int_{-\infty}^{x}\left|u^{\prime}(t)\right| \mathrm{d} t \leq\left\|u^{\prime}\right\|_{1}
$$

(b) La question précédente permet d'initialiser la récurrence, on a pour tout toute fonction u appartenant à $C_{c}^{1}(\mathbb{R})$, $\|u\|_{\infty} \leq\left\|u^{\prime}\right\|_{1}$.

Soit maintenant $N \geq 1$. On suppose que pour toute fonction u appartenant à $C_{c}^{1}\left(\mathbb{R}^{N}\right)$, on a

$$
\|u\|_{N /(N-1)} \leq\left\|\frac{\partial u}{\partial x_{1}}\right\|_{1}^{1 / N} \cdots\left\|\frac{\partial u}{\partial x_{N}}\right\|_{1}^{1 / N} .
$$

Soit $u \in C_{c}^{1}\left(\mathbb{R}^{N+1}\right)$. Pour $x \in \mathbb{R}^{N+1}$ on note $x=\left(x_{1}, y\right)^{t}$ avec $x_{1} \in \mathbb{R}$ et $y \in \mathbb{R}^{N}$. Pour $x_{1} \in \mathbb{R}$, l'inégalité de Hölder donne

$$
\begin{aligned}
& \int_{\mathbb{R}^{N}}\left|u\left(x_{1}, y\right)\right|^{\frac{N+1}{N}} \mathrm{~d} y=\int_{\mathbb{R}^{N}}\left|u\left(x_{1}, y\right)\right|\left|u\left(x_{1}, y\right)\right|^{\frac{1}{N}} d y \\
& \leq\left(\int_{\mathbb{R}^{N}}\left|u\left(x_{1}, y\right)\right|^{\frac{N}{N-1}} d y\right)^{\frac{N-1}{N}}\left(\int_{\mathbb{R}^{N}}\left|u\left(x_{1}, y\right)\right| \mathrm{d} y\right)^{\frac{1}{N}} .
\end{aligned}
$$

On applique l'hypothèse de récurrence à la fonction $y \mapsto u\left(x_{1}, y\right)$ (qui est bien dans $C_{c}^{1}\left(\mathbb{R}^{N}\right)$ ), on obtient

$$
\left\|u\left(x_{1}, \cdot\right)\right\|_{L^{\frac{N}{N-1}}\left(\mathbb{R}^{N}\right)} \leq\left\|\frac{\partial u}{\partial x_{2}}\left(x_{1}, \cdot\right)\right\|_{L^{1}\left(\mathbb{R}^{N}\right)}^{1 / N} \cdots\left\|\frac{\partial u}{\partial x_{N+1}}\left(x_{1}, \cdot\right)\right\|_{L^{1}\left(\mathbb{R}^{N}\right)}^{1 / N} .
$$

D'autre part, en appliquant le cas $N=1$ (démontré à la question (a)) à la fonction $z \mapsto u_{(} z, y$ ) (qui est bien dans $C_{c}^{1}(\mathbb{R})$ ), on a pour tout $y \in \mathbb{R}^{N}$

$$
\left|u\left(x_{1}, y\right)\right| \leq\left\|\frac{\partial u}{\partial x_{1}}(\cdot, y)\right\|_{L^{1}(\mathbb{R})} .
$$

et donc, en intégrant par rapport à y,

En reportant ces majorations dans 1.17 on obtient pour tout $x_{1} \in \mathbb{R}$

$$
\int_{\mathbb{R}^{N}}\left|u\left(x_{1}, y\right)\right| \mathrm{d} y \leq\left\|\frac{\partial u}{\partial x_{1}}\right\|_{L^{1}\left(\mathbb{R}^{N+1}\right)}
$$

$$
\int_{\mathbb{R}^{N}}\left|u\left(x_{1}, y\right)\right|^{\frac{N+1}{N}} \mathrm{~d} y \leq\left\|\frac{\partial u}{\partial x_{2}}\left(x_{1}, \cdot\right)\right\|_{L^{1}\left(\mathbb{R}^{N}\right)}^{1 / N} \cdots\left\|\frac{\partial u}{\partial x_{N+1}}\left(x_{1}, \cdot\right)\right\|_{L^{1}\left(\mathbb{R}^{N}\right)}^{1 / N}\left\|\frac{\partial u}{\partial x_{1}}\left(x_{1}, \cdot\right)\right\|_{L^{1}\left(\mathbb{R}^{N+1}\right)}^{\frac{1}{N}} .
$$

En intégrant cette inégalité par rapport à $x_{1}$ et en utilisant une nouvelle fois l'inégalité de Hölder (avec le produit deN fonctions dans $L^{N}$, on obtient bien l'inégalité désirée, c'est-à-dire

ou encore

$$
\|u\|_{\frac{N+1}{N}}^{\frac{N}{N+1}} \leq\left\|\frac{\partial u}{\partial x_{1}}\right\|_{1}^{1 / N} \ldots\left\|\frac{\partial u}{\partial x_{N+1}}\right\|_{1}^{1 / N}
$$

$$
\|u\|_{\frac{N+1}{N}} \leq\left\|\frac{\partial u}{\partial x_{1}}\right\|_{1}^{\frac{1}{N+1}} \cdots\left\|\frac{\partial u}{\partial x_{N+1}}\right\|_{1}^{\frac{1}{N+1}} .
$$

Ce qui termine la récurrence.

(c) La moyenne géométrique de $N$ nombres positifs est plus petite que la moyenne arithmétique de ces mêmes nombres. (Ceci peut se démontrer en utilisant, par exemple, la convexité de la fonction exponentielle.)

On en déduit que

$$
\|u\|_{N /(N-1)} \leq\left\|\frac{\partial u}{\partial x_{1}}\right\|_{1}^{1 / N} \ldots\left\|\frac{\partial u}{\partial x_{N}}\right\|_{1}^{1 / N} \leq \frac{1}{N} \sum_{i=1}^{N}\left\|\frac{\partial u}{\partial x_{i}}\right\|_{1} .
$$

Comme $\left\|\frac{\partial u}{\partial x_{i}}\right\|_{1} \leq\||\nabla u|\|_{1}$ pour tout $i$, on a bien

$$
\|u\|_{N /(N-1)} \leq\||\nabla u|\|_{1} .
$$

(d) Pour $p=1$, on a vu que $C_{N, p}=1$ convient. On suppose maintenant $1<p<N$.

On pose $\alpha=\frac{p(N-1)}{N-p}$ (de sorte que $\left.\alpha \frac{N}{N-1}=p^{\star}\right)$ et $v=|u|^{\alpha-1} u$. 
Comme $\alpha>1$ et $u \in C_{c}^{1}\left(\mathbb{R}^{N}\right)$, on a aussi $v \in C_{c}^{1}\left(\mathbb{R}^{N}\right)$. On peut donc appliquer le résultat de la question (c) $\grave{a}$ la fonction $v$. On obtient

$$
\left(\int_{\mathbb{R}^{N}}|u(x)|^{p^{\star}}\right)^{\frac{N-1}{N}}=\left(\int_{\mathbb{R}^{N}}|u(x)|^{\alpha \frac{N}{N-1}}\right)^{\frac{N-1}{N}} \leq\||\nabla v|\|_{1} .
$$

Comme $|\nabla v|=\alpha|u|^{\alpha-1}|\nabla u|$, l'inégalité de Hölder (avec $p$ et $q=p /(p-1)$ ) donne

$$
\||\nabla v|\|_{1}=\alpha\left|\left\||u|^{\alpha-1}|\nabla u|\right\|_{1} \leq \alpha\left\|\left.|| u\right|^{\alpha-1}\right\|_{q}\||\nabla u|\|_{p} .\right.
$$

Comme $(\alpha-1) q=(\alpha-1) p /(p-1)=p^{\star}$, on a donc

$$
\|u\|_{p^{\star}}^{\frac{p^{\star}(N-1)}{N}}=\left(\int_{\mathbb{R}^{N}}|u(x)|^{p^{\star}}\right)^{\frac{N-1}{N}} \leq \alpha\|u\|_{p^{\star}}^{\frac{p^{\star}(p-1)}{p}}\||\nabla u|\|_{p} .
$$

Ce qui donne, avec $C_{N, p}=\alpha=\frac{p(N-1)}{N-p}$,

$$
\|u\|_{p^{\star}} \leq C_{N, p}\||\nabla u|\|_{p} .
$$

2. Soit $u \in W^{1, p}\left(\mathbb{R}^{N}\right)$, il existe une suite $\left(u_{n}\right)_{n \in \mathbb{N}}$ de fonctions appartenant à $C_{c}^{1}\left(\mathbb{R}^{N}\right)$ t.q. $u_{n} \rightarrow$ u dans $W^{1, p}\left(\mathbb{R}^{N}\right)$ quand $n \rightarrow+\infty$. Par la question précédente, cette suite est de Cauchy dans $L^{p^{\star}}$. Par unicité de la limite (par exemple dans $L_{\mathrm{loc}}^{1}\left(\mathbb{R}^{N}\right)$ ) cette limite est nécessairement égale à $u$. On peut alors passer à la limite quand $n \rightarrow+\infty$ dans l'inégalité $\left\|u_{n}\right\|_{p^{\star}} \leq C_{N, p}\left\|\left|\nabla u_{n}\right|\right\|_{p}$ et on obtient ainsi

$$
\|u\|_{p^{\star}} \leq C_{N, p}\||\nabla u|\|_{p} \text { pour tout } u \in W^{1, p}\left(\mathbb{R}^{N}\right) .
$$

Ceci donne l'injection continue de $W^{1, p}\left(\mathbb{R}^{N}\right)$ dans $L^{p^{\star}}\left(\mathbb{R}^{N}\right)$.

L'injection continue de $W^{1, p}\left(\mathbb{R}^{N}\right)$ dans $L^{p}\left(\mathbb{R}^{N}\right)$ est immédiate car $\|u\|_{p} \leq\|u\|_{W^{1, p}}$ pour tout $u \in W^{1, p}\left(\mathbb{R}^{N}\right)$.

Soit maintenant $q \in] p, p^{\star}\left[\right.$. Pour montrer que $W^{1, p}\left(\mathbb{R}^{N}\right)$ s'injecte continûment dans $L^{q}\left(\mathbb{R}^{N}\right)$ il suffit d'utiliser l'inégalité classique suivante (qui se démontre avec l'inégalité de Hölder) avec $p<q<r=p^{\star}$.

$$
\|u\|_{q} \leq\|u\|_{p}^{\theta}\|u\|_{r}^{1-\theta}
$$

avec $\left.\theta=\frac{p(r-q)}{q(r-p)} \in\right] 0,1[$.

3. Le cas $N=1$ est facile. La question 2 donne l'injection continue de $W^{1,1}(\mathbb{R})$ dans $L^{\infty}(\mathbb{R})$. Comme $W^{1,1}(\mathbb{R})$ s'injecte aussi continûment dans $L^{1}(\mathbb{R})$, on obtient aussi une injection continu de $W^{1,1}(\mathbb{R})$ dans $L^{q}(\mathbb{R})$ pour tout $q \in] 1,+\infty[$ (en utilisant 1.18 avec $r=+\infty, p=1$ et $\theta=1 / q$ ).

On suppose maintenant $N>1$. On a bien une injection continue de $W^{1, N}(\mathbb{R})$ dans $L^{N}(\mathbb{R})$. Le seul cas à considérer est donc $N<q<+\infty$. Plusieurs démonstration sont possibles. Une première démonstration consiste à utiliser pour $|u|^{\alpha}$, avec $u \in C_{c}^{1}\left(\mathbb{R}^{N}\right)$, l'inégalité démontrée à la question $1(c)$, puis à utiliser l'inégalité de Hölder (pour faire apparître $\left.|\nabla u|^{N}\right)$ et l'inégalité 1.18 . Enfin, on conclut avec la densité de $C_{c}^{1}\left(\mathbb{R}^{N}\right)$ dans $W^{1, N}\left(\mathbb{R}^{N}\right)$. On donne ci dessous une démonstration probablement plus longue mais qui utilise de manière intéressante le caractère homogène de la norme. de la norme.

Soit $N<q<+\infty$. Il existe alors $p \in] 1, N\left[\right.$ t.q. $p^{\star}=N p /(N-p)=q$. On va utiliser la question 1 avec cette valeur de $p$.

On définit $\varphi$ de $\mathbb{R}$ dans $\mathbb{R}$, de classe $C^{1}$, par :

$$
\begin{aligned}
& \varphi(s)=0 \text { si }|s| \leq 1, \\
& \varphi(s)=\frac{1}{2}(|s|-1)^{2} \text { si } 1<|s| \leq 2, \\
& \varphi(s)=|s|-\frac{3}{2} \text { si } 2<|s| .
\end{aligned}
$$

On a $|\varphi(u)| \leq 1$ et $\left|\varphi^{\prime}(s)\right| \leq 1$ pour tout $s \in \mathbb{R}$.

Soit $u \in C_{c}^{1}\left(\mathbb{R}^{N}\right)$ t.q $\|u\|_{W^{1, N}}=1$. On a $\varphi(u) \in C_{c}^{1}\left(\mathbb{R}^{N}\right)$. Par question 1 (et la définition $\varphi$ ) on obtient

$$
\|\varphi(u)\|_{q}^{p} \leq C_{N, p}\||\nabla \varphi(u)|\|_{p} \leq C_{N, p} \int_{\{|u| \geq 1\}}|\nabla u(x)|^{p} \mathrm{~d} x \leq C_{N, p}\left(\int_{\mathbb{R}^{N}}|\nabla u(x)|^{N} \mathrm{~d} x\right)^{\frac{p}{N}} \lambda_{d}\left(\{|u| \geq 1\}^{1-\frac{p}{N}} .\right.
$$

On a $\lambda_{d}\left(\{|u| \geq 1\} \leq \int_{\mathbb{R}^{N}}|u(x)|^{N} \mathrm{~d} x \leq 1\right.$ et $\int_{\mathbb{R}^{N}}|\nabla u(x)|^{N} \mathrm{~d} x \leq 1$. On en déduit

$$
\|\varphi(u)\|_{q}^{p} \leq C_{N, p}
$$




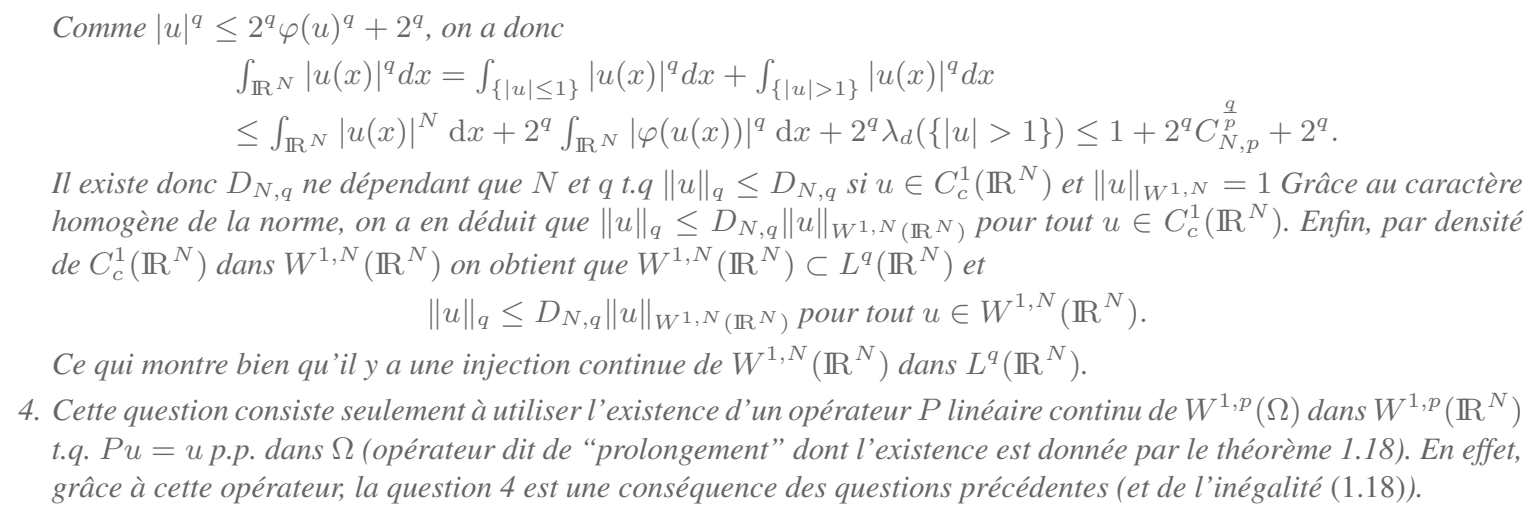

\section{Exercice 1.17 (Noyau de l'opérateur trace)}

Soient $\Omega=\mathbb{R}_{+}^{N}, 1 \leq p<\infty$ et $\gamma: W^{1, p}(\Omega) \rightarrow L^{p}(\partial \Omega)$ l'opérateur trace défini au théorème 1.21

1. Montrer que $\operatorname{Ker} \gamma=W_{0}^{1, p}(\Omega)$.

2. Soit $u \in W^{1, p}(\Omega) \cap C(\bar{\Omega})$. Montrer que $\gamma u=u$ p.p. (pour la mesure de lebesgue $N-1$-dimensionnelle sur $\partial \Omega)$.

\section{Exercice 1.18 (Prolongement $\boldsymbol{H}^{\mathbf{2}}$ )}

Soient $N \geq 1, \Omega=\mathbb{R}_{+}^{N}$ et $p \in[1, \infty[$.

1. Montrer que $C^{\infty}(\bar{\Omega})$ est dense dans $W^{2, p}(\Omega)$ [On pourra s'inspirer de la démonstration de la densité de $C^{\infty}(\bar{\Omega})$ dans $\left.W^{1, p}(\Omega)\right]$.

2. Montrer qu'il existe un opérateur $P$ linéaire continu de $W^{2, p}(\Omega)$ dans $W^{2, p}\left(\mathbb{R}^{N}\right)$ tel que $P u=u$ p.p. dans $\Omega$, pour tout $u \in W^{2, p}(\Omega)$ [On pourra chercher $P$ sous la forme $P u\left(x_{1}, y\right)=\alpha u\left(-x_{1}, y\right)+\beta u\left(-2 x_{1}, y\right)$, pour $x_{1} \in \mathbb{R}_{-}$et $\left.y \in \mathbb{R}^{N-1}\right]$.

3. On prend maintenant $p=\infty$. A-t-on $C^{\infty}(\bar{\Omega})$ est dense dans $W^{2, \infty}(\Omega)$ ? Existe-t-il un opérateur $P$ linéaire continu de $W^{2, \infty}(\Omega)$ dans $W^{2, \infty}\left(\mathbb{R}^{N}\right)$ tel que $P u=u$ p.p. dans $\Omega$, pour tout $u \in W^{2, p}(\Omega)$ ? (justifier vos réponses...).

Exercice 1.19 (Convergence faible et opérateur continu) Soit $E$ et $F$ deux espaces de Banach et $T$ une application linéaire continue de $E$ dans $F$. Soit $\left(u_{n}\right)_{n \in \mathbb{N}}$ une suite d'éléments de $E$ et $u$ dans $E$. On suppose que $u_{n} \rightarrow u$ faiblement dans $E$ quand $n \rightarrow+\infty$. Montrer que $T\left(u_{n}\right) \rightarrow T(u)$ faiblement dans $F$. On donne maintenant deux applications de ce résultat.

1. Soit $E$ et $F$ deux espaces de Banach. On suppose que $E$ s'injecte continûment dans $F$, c'est-à-dire que $E \subset F$ et que l'application $u \mapsto u$ est continue de $E$ dans $F$. Soit $\left(u_{n}\right)_{n \in \mathbb{N}}$ une suite d'éléments de $E$ et $u$ dans $E$. On suppose que $u_{n} \rightarrow u$ faiblement dans $E$ quand $n \rightarrow+\infty$. Montrer que $u_{n} \rightarrow u$ faiblement dans $F$.

2. Soit $\Omega$ est un ouvert borné de $\mathbb{R}^{N}, N \geq 1,\left(u_{n}\right)_{n \in \mathbb{N}}$ une suite d'éléments de $H_{0}^{1}(\Omega)$ et $u$ dans $H_{0}^{1}(\Omega)$. On suppose que $u_{n} \rightarrow u$ faiblement dans $H_{0}^{1}(\Omega)$ quand $n \rightarrow+\infty$. Montrer que $D_{i} u_{n} \rightarrow D_{i} u$ faiblement dans $L^{2}(\Omega)$ quand $n \rightarrow+\infty$.

Exercice 1.20 (Fonction non continue appartenant à $H^{1}\left(\mathbb{R}^{2}\right) \cap L^{\infty}\left(\mathbb{R}^{2}\right)$ ) Dans cet exercice, on construit $v$ t.q. $v \in H^{1}\left(\mathbb{R}^{2}\right) \cap L^{\infty}\left(\mathbb{R}^{2}\right)$ et $v \notin C\left(\mathbb{R}^{2}, \mathbb{R}\right)$ ( c'est-à-dire qu'il n'existe pas $w \in C\left(\mathbb{R}^{2}, \mathbb{R}\right)$ t.q. $v=w$ p.p.). Pour cela, on reprend la fonction de l'exercice 1.5 .

Soit $\gamma \in] 0,1 / 2\left[\right.$ et $u$ définie par $u(x)=(-\ln (|x|))^{\gamma}$ si $|x|<1$ et $u(x)=0$ si $|x| \geq 1$.

Pour chaque $n \in \mathbb{N}^{\star}$, on définit $u_{n}$ en posant $u_{n}(x)=u(x)-n$ si $n \leq u(x)<n+1$ et $u_{n}(x)=0$ sinon. 
1. Montrer que $u_{n} \in H^{1}\left(\mathbb{R}^{2}\right)$ pour tout $n \in \mathbb{N}^{\star}$ et que

$$
\sum_{n=1}^{\infty}\left\|\left|\nabla u_{n}\right|\right\|_{L^{2}\left(\mathbb{R}^{2}\right)}^{2}<+\infty
$$

[Utiliser l'exercice 1.5]

2. Montrer que $u_{n}$ prend ses valeurs entre 0 et 1 et que le support de $u_{n}$ est une boule dont le diamètre tend vers 0 quand $n \rightarrow+\infty$.

Pour $n \in \mathbb{N}^{\star}$, on pose $x_{n}=(1 / n, 0) \in \mathbb{R}^{2}$ et on choisit $m_{n}$ t.q. le support de $u_{n}$ soit une boule de diamètre plus petit que $(1 / 2)(1 / n-1 /(n+1))$ et t.q. la suite $\left(m_{n}\right)_{n \in \mathbb{N}^{\star}}$ soit strictement croissante. Puis on pose, pour $x \in \mathbb{R}^{2}, v_{n}(x)=u_{m_{n}}\left(x-x_{n}\right)$.

3. Montrer que toutes les fonctions $v_{n}$ ont des supports disjoints.

4. On pose $v=\sum_{n \in \mathbb{N}^{\star}} v_{n}$. Montrer que la fonction $v$ appartient à $H^{1}\left(\mathbb{R}^{2}\right) \cap L^{\infty}\left(\mathbb{R}^{2}\right)$. Montrer que $v$ est continue sur $\mathbb{R}^{2} \backslash\{0\}$ mais n'est pas continue en 0 .

Exercice 1.21 (Sur l'injection de $\boldsymbol{W}^{1,1}$ dans $\boldsymbol{L}^{1^{\star}}$ ) Soit $\Omega$ un ouvert borné connexe de $\mathbb{R}^{N}(N \geq 1)$ à frontière lipschitzienne. Soit $\omega$ une partie borélienne de $\Omega$ de mesure de Lebesgue strictement positive, c'est-àdire $\lambda_{N}(\omega)>0$ en désignant par $\lambda_{N}$ la mesure de Lebesgue sur les boréliens de $\mathbb{R}^{N}$.

On définit l'ensemble $W_{\omega}$ par

$$
W_{\omega}=\left\{u \in W^{1,1}(\Omega) \text { tel que } u=0 \text { p.p. dans } \omega\right\} .
$$

le but de cet exercice est de montrer, par deux méthodes différentes, qu'il existe $C$, dépendant seulement de $\Omega$ et $\omega$, tel que

$$
\|u\|_{L^{p}(\Omega)} \leq C\||\nabla u|\|_{L^{1}(\Omega)} \text { pour tout } u \in W_{\omega} \text { et pour tout } 1 \leq p \leq \frac{N}{N-1} .
$$

\section{I- Première méthode (méthode directe)}

1. On suppose qu'il existe une suite $\left(u_{n}\right)_{n \in \mathbb{N}^{\star}}$ d'éléments de $W_{\omega}$ t.q. $\left\|u_{n}\right\|_{L^{1}(\Omega)}=1$ pour tout $n \in \mathbb{N}^{\star}$ et

$$
\left\|u_{n}\right\|_{L^{1}(\Omega)} \geq n\left\|\left|\nabla u_{n}\right|\right\|_{L^{1}(\Omega)} \text { pour tout } n \in \mathbb{N}^{\star} .
$$

En utilisant un théorème de compacité du cours (chapitre 1), montrer qu'on peut supposer, après extraction d'une sous suite, que $u_{n} \rightarrow u$ dans $L^{1}(\Omega)$ quand $n \rightarrow+\infty$. Montrer alors que $u=0$ p.p. et que $\|u\|_{L^{1}(\Omega)}=1$ (ce qui est impossible...).

2. Déduire de la question précédente qu'il existe $C_{1}$, dépendant seulement de $\Omega$ et $\omega$, tel que

$$
\|u\|_{L^{1}(\Omega)} \leq C_{1}\||\nabla u|\|_{L^{1}(\Omega)} \text { pour tout } u \in W_{\omega} .
$$

3. On rappelle (théorème 1.28 qu'il existe $C_{2}$, dépendant seulement de $\Omega$, tel que, en posant $1^{\star}=N /(N-1)$, $\|u\|_{L^{1^{\star}}(\Omega)} \leq C_{2}\|u\|_{W^{1,1}(\Omega)}$ pour tout $u \in W^{1,1}(\Omega)$. Avec la question précédente, en déduire qu'il existe $C$, dépendant seulement de $\Omega$ et $\omega$, vérifiant $[1.19$.

\section{II- Deuxième méthode (en passant par la moyenne de $u$ )}

1. Soit $H=\left\{u \in W^{1,1}(\Omega)\right.$ t.q. $\left.\int_{\Omega} u(x) \mathrm{d} x=0\right\}$. Montrer qu'il existe $C_{3}$ ne dépendant que de $\Omega$ t.q. $\|u\|_{L^{1}(\Omega)} \leq$ $C_{3}\||\nabla u|\|_{L^{1}(\Omega)}$ pour tout $u \in H$.

En utilisant le rappel de la question 3 de la première partie, en déduire qu'il existe $C_{4}$ ne dépendant que de $\Omega$ t.q.

$$
\|u-m\|_{L^{1^{\star}}(\Omega)} \leq C_{4}\||\nabla u|\|_{L^{1}(\Omega)} \text { pour tout } u \in W^{1,1}(\Omega),
$$

avec $m \lambda_{N}(\Omega)=\int_{\Omega} u(x) \mathrm{d} x$. [On pourra remarquer que $u-m \in H$.] 
2. Soit $u \in W_{\omega}$ et $m$ tel que $m \lambda_{N}(\Omega)=\int_{\Omega} u(x) \mathrm{d} x$. Montrer que

$$
|m| \leq \frac{C_{4}}{\lambda_{N}(\omega)^{1 / 1^{\star}}}\||\nabla u|\|_{L^{1}(\Omega)}
$$

et en déduire que

$$
\|u\|_{L^{1^{\star}}(\Omega)} \leq C_{4}\left(1+\left(\frac{\lambda_{N}(\Omega)}{\lambda_{N}(\omega)}\right)^{1 / 1^{\star}}\right)\||\nabla u|\|_{L^{1}(\Omega)} .
$$

\section{Exercice 1.22 (Partition de l'unité)}

Soit $K$ un compact de $\mathbb{R}^{N}(N \geq 1)$ et $\Omega_{1}, \ldots, \Omega_{n}, n \in \mathbb{N}^{\star}$, une famille finie d'ouverts tels que $K \subset \cup_{i=1}^{n} \Omega_{i}$. On va montrer ici qu'il existe des fonctions $\varphi_{1}, \ldots, \varphi_{n}$ telles que

(p1) Pour tout $i \in\{1, \ldots, n\}, \varphi_{i} \in C_{c}^{\infty}\left(\mathbb{R}^{N}, \mathbb{R}\right), \bar{S}_{i} \subset \Omega_{i}$ avec $S_{i}=\left\{x \in \mathbb{R}^{N}, \varphi_{i}(x) \neq 0\right\}$,

(p2) $\sum_{i=1}^{n} \varphi_{i}=1$ sur $K$.

Pour $\varepsilon>0$ et $i \in\{1, \ldots, n\}$, on pose $\Omega_{i, \varepsilon}=\left\{x \in \Omega_{i}, d\left(x, \Omega_{i}^{c}\right)>\varepsilon\right\}$ où $d\left(x, \Omega_{i}^{c}\right)=\inf \left\{|x-y|, y \notin \Omega_{i}\right\}$.

1. Montrer qu'il existe $\varepsilon>0$ tel que $K \subset \cup_{i=1}^{n} \Omega_{i, \varepsilon}$.

Soit maintenant $\varepsilon$ donné par la question 1

2. Montrer qu'il existe $n$ fonctions $f_{1}, \ldots, f_{n}$ telles que, pour tout $i, f_{i}=0$ on $\Omega_{i, \varepsilon}^{c}$ et $\sum_{i=1}^{n} f_{i}=1$ on $\cup_{i=1}^{n} \Omega_{i, \varepsilon}$.

3. Par une méthode de regularisation, montrer l'existence de $n$ functions $\varphi_{1}, \ldots, \varphi_{n}$ satisfaisant (p1) et (p2).

Corrigé -

1. Pour $i \in\{1, \ldots, n\}$, on a $\Omega_{i}=\cup_{\varepsilon>0} \Omega_{i, \varepsilon}$ et donc $K \subset \cup_{i=1}^{n} \cup_{\varepsilon>0} \Omega_{i, \varepsilon}$. Comme $K$ est compact et $\Omega_{i, \varepsilon}$ est ouvert (pour tout $i$ ete), il existe $\varepsilon_{1}, \ldots, \varepsilon_{n}$ tels que $\varepsilon_{i}>0$ pour tout $i$ et $K \subset \cup_{i=1}^{n} \Omega_{i, \varepsilon_{i}}$. En prenant $\varepsilon=\min \left\{\varepsilon_{1}, \ldots, \varepsilon_{n}\right\}$, on a $K \subset \cup_{i=1}^{n} \Omega_{i, \varepsilon}$.

2. Pour tout $i \in\{1, \ldots, n\}$, on pose

$$
\begin{aligned}
& f_{i}(x)=1 \text { if } x \in\left(\Omega_{i, \varepsilon} \backslash \cup_{j<i} \Omega_{j, \varepsilon}\right), \\
& f_{i}(x)=0 \text { if } x \notin\left(\Omega_{i, \varepsilon} \backslash \cup_{j<i} \Omega_{j, \varepsilon}\right) .
\end{aligned}
$$

Avec cette définition, on obtient $f_{i}=0$ on $\Omega_{i, \varepsilon}^{c}$. De plus, si $x \in \cup_{i=1}^{n} \Omega_{i, \varepsilon}$. et $i=\min \left\{j ; x \in \Omega_{j, \varepsilon}\right.$, on a $f_{i}(x)=1$ et $f_{j}(x)=0$ if $j \neq i$. Donc $\sum_{i=1}^{n} f_{i}(x)=1$.

3. Comme K est compact et $\left(\cup_{i=1}^{n} \Omega_{i, \varepsilon}\right)^{c}$ est fermé, on a d $\left(K,\left(\cup_{i=1}^{n} \Omega_{i, \varepsilon}\right)^{c}\right)=\delta>0$.

On prend $\eta$ tel que $0<\eta<\min \{\delta, \varepsilon\}$. Soit $\rho \in C_{c}^{\infty}\left(\mathbb{R}^{n}, \mathbb{R}\right)$ tel que $\rho(x)=0$ si $|x| \geq \eta$ et tel que $\int_{\mathbb{R}^{n}} \rho(x) \mathrm{d} x=1$.

Pour $i \in\{1, \ldots, n\}$ on prend $\varphi_{i}=f_{i} \star \rho$. On a $\varphi_{i} \in \mathcal{C}_{c}^{\infty}\left(\mathbb{R}^{n}, \mathbb{R}\right)$ et comme $f_{i}=0$ sur $\Omega_{i, \varepsilon}^{c}$ et $\eta<\varepsilon$, la fonction $\varphi_{i}$ satisfait (p1).

Comme $\sum_{i=1}^{n} \varphi_{i}=\left(\sum_{i=1}^{n} f_{i}\right) \star \rho$ et $\eta<\delta$, les fonctions $\varphi_{i}$ satisfont $(p 2)$.

\section{Exercice 1.23 (Opérateur transposé, continuité et compacité)}

Soient $E, F$ deux espaces de Banach (réels) et $T \in \mathcal{L}(E, F)$. Pour $g \in F^{\prime}$ on définit $T^{t} g \in E^{\prime} \operatorname{par}\left\langle T^{t} g, u\right\rangle_{E^{\prime}, E}=$ $\langle g, T u\rangle_{F^{\prime}, F}$.

1. Vérifier que $T^{t} g$ est bien un élément de $E^{\prime}$ pour tout $g \in F^{\prime}$ et que $T^{t} \in \mathcal{L}\left(F^{\prime}, E^{\prime}\right)$.

Corrigé - Soit $g \in F^{\prime}$. L'application $u \mapsto\langle g, T u\rangle_{F^{\prime}, F}$ est linéaire et

$$
\left|\langle g, T u\rangle_{F^{\prime}, F}\right| \leq\|T\|_{\mathcal{L}(E, F)}\|g\|_{F^{\prime}}\|u\|_{E} .
$$

Ceci prouve que $T^{t} g$ est un élément de $E^{\prime}$ et que $\left\|T^{t} g\right\|_{E^{\prime}} \leq\|T\|_{\mathcal{L}(E, F)}\|g\|_{F^{\prime}}$. On a donc aussi $T^{t} \in \mathcal{L}\left(F^{\prime}, E^{\prime}\right)$ et $\left\|T^{t}\right\|_{\mathcal{L}\left(F^{\prime}, E^{\prime}\right)} \leq\|T\|_{\mathcal{L}(E, F)}$. 
2. Montrer que $\left\|T^{t}\right\|_{\mathcal{L}\left(F^{\prime}, E^{\prime}\right)}=\|T\|_{\mathcal{L}(E, F)}$.

Corrigé - Soit $u \in E$. Une conséquence du théorème de Hahn-Banach (voir exercice 1.8 question 1) donne l'existence de $g \in F^{\prime}$ tel que $\|g\|_{F^{\prime}}=1$ et $\|T u\|_{F}=\langle g, T u\rangle_{F^{\prime}, F}$. En effet, en posant $v=T u \in F$ et en supposant $v \quad 0$ (le cas $v=0$ est trivial), on choisit $\widetilde{g}: \mathbb{R} v \rightarrow \mathbb{R}$ linéaire et telle que $\widetilde{g}(v)=\|v\|$. On a donc $\|\widetilde{g}\|=1$. Par le théorème de Hahn-Banach, on peut prolonger $\widetilde{g}$ en $g \in F^{\prime}$ avec $\|g\|_{F^{\prime}}=1$ et $\langle g, v\rangle_{F^{\prime}, F}=\|v\|_{F}$, ou encore $\|T u\|_{F}=\langle g, T u\rangle_{F^{\prime}, F}$.

On a donc $\|T u\|_{F} \leq\left\|T^{t}\right\|_{\mathcal{L}\left(F^{\prime}, E^{\prime}\right)}\|u\|_{E}$. Ceci prouve que $\|T\|_{\mathcal{L}(E, F)} \leq\left\|T^{t}\right\|_{\mathcal{L}\left(F^{\prime}, E^{\prime}\right)}$ et donc finalement $\|T\|_{\mathcal{L}(E, F)}=$ $\left\|T^{t}\right\|_{\mathcal{L}\left(F^{\prime}, E^{\prime}\right)}$.

On suppose maintenant que $T$ est un opérateur compact, c'est-à-dire que de toute suite bornée de $E$ on peut extraire une sous suite dont l'image par $T$ converge dans $F$.

On note $B_{E}=\left\{u \in E,\|u\|_{E} \leq 1\right\}$.

3. Montrer que $T\left(B_{E}\right)$ est précompacte, c.à.d. que pour tout $\varepsilon>0$, il existe $I \subset B_{E}$ tel que card $(I)<+\infty$ et

$$
T\left(B_{E}\right)=\left\{T(u), u \in B_{E}\right\} \subset \cup_{u \in I} B_{F}(T u, \varepsilon),
$$

où $B_{F}(T u, \varepsilon)=\left\{v \in F,\|v-T u\|_{F}<\varepsilon\right\}$.

Corrigé - Par hypothèse, la boule $T\left(B_{E}\right)$ est relativement compacte donc précompacte. En effet, soit $\varepsilon>0$, supposons par l'absurde qu'il n'existe pas de recouvrement fini de $T\left(B_{E}\right)$ par des boules de la forme $B_{F}(T u, \varepsilon)$. Soit $u_{0} \in B_{E}$, on a donc $T\left(B_{E}\right) \not \subset B_{F}\left(T u_{0}, \varepsilon\right)$ Puis, par récurrence, on suppose $u_{0}, \ldots, u_{n}$ choisis dans $B_{E}$. Comme $T\left(B_{E}\right) \not \subset \cup_{i=0}^{n} B_{F}\left(T u_{i}, \varepsilon\right)$, on choisit $u_{n+1} \in B_{E}$ tel que $T\left(u_{n+1}\right) \notin \cup_{i=0}^{n} B_{F}\left(T u_{i}, \varepsilon\right)$. On construit ainsi une suite $\left(u_{n}\right)_{n \in \mathbb{N}}$ de $B_{E}$ telle que la suite $\left(T\left(u_{n}\right)\right)_{n \in \mathbb{N}}$ n'admet aucune sous suite convergente $\left(\right.$ car $\left\|T\left(u_{n}\right)-T\left(u_{m}\right)\right\|_{F} \geq \varepsilon$ si $n \neq m)$, ce qui est en contradiction avec l'hypothèse de compacité de $T$.

Pour $p \in \mathbb{N}^{\star}$, on choisit $I_{p}$ conformément à la question 3 avec $\varepsilon=1 / p$ et on pose $I=\cup_{p \in \mathbb{N}^{\star}} I_{p}$ (de sorte que $I$ est dénombrable). Soit $\left(g_{n}\right)_{n \in \mathbb{N}}$ une suite bornée de $F^{\prime}$.

4. Montrer qu'il existe une sous suite de la suite $\left(g_{n}\right)_{n \in \mathbb{N}}$ telle que pour cette sous suite, encore notée $\left(g_{n}\right)_{n \in \mathbb{N}}$, la suite $\left(\left\langle T^{t} g_{n}, u\right\rangle_{E^{\prime}, E}\right)_{n \in \mathbb{N}}$ converge pour tout $u \in I$. [Utiliser le procédé diagonal.]

Corrigé - Pour tout $u \in I$, la suite $\left(\left\langle T^{t} g_{n}, u\right\rangle_{E^{\prime}, E}\right)_{n \in \mathbb{N}}$ est bornée dans $\mathbb{R}$. Elle admet donc une sous suite convergente. Comme I est dénombrable, le procédé diagonal, décrit par exemple dans la preuve de la proposition 8.19 de [15], permet d'extraire une sous suite telle que la suite $\left(\left\langle T^{t} g_{n}, u\right\rangle_{E^{\prime}, E}\right)_{n \in \mathbb{N}}$ est convergente pour tout $u \in I$. Dans la suite on note $f_{u}$ cette limite.

Noter que, pour cette question, il suffit que $I_{p}$ soit fini ou dénombrable.

Pour les deux questions suivantes on considère cette sous suite.

5. Montrer que la suite $\left(\left\langle T^{t} g_{n}, u\right\rangle_{E^{\prime}, E}\right)_{n \in \mathbb{N}}$ converge pour tout $u \in E$.

Corrigé - Soit $u \in B_{E}$. On remarque alors que la suite $\left(\left\langle T^{t} g_{n}, u\right\rangle_{E^{\prime}, E}\right)_{n \in \mathbb{N}}$ est de Cauchy. En effet, Soit $\varepsilon>0$. On choisit $p \in \mathbb{N}^{\star}$ tel que $1 / p \leq \varepsilon$. Il existe $v \in I_{p}$ tel que $\|T v-T u\|_{F} \leq \varepsilon$. On a alors, pour tout $n, m \in \mathbb{N}$, avec $C=\sup _{n}\left\|g_{n}\right\|_{F^{\prime}}$,

$$
\begin{aligned}
\left|\left\langle T^{t} g_{n}, u\right\rangle_{E^{\prime}, E}-\left\langle T^{t} g_{m}, u\right\rangle_{E^{\prime}, E}\right| & \leq\left|\left\langle T^{t} g_{n}, v\right\rangle_{E^{\prime}, E}-\left\langle T^{t} g_{m}, v\right\rangle_{E^{\prime}, E}\right| \\
& +\left|\left\langle g_{n}, T v-T u\right\rangle_{F^{\prime}, F}\right|+\left|\left\langle g_{m}, T v-T u\right\rangle_{F^{\prime}, F}\right| \\
& \leq\left|\left\langle T^{t} g_{n}, v\right\rangle_{E^{\prime}, E}-\left\langle T^{t} g_{m}, v\right\rangle_{E^{\prime}, E}\right|+2 C \varepsilon .
\end{aligned}
$$

Puis, comme $v \in I_{p} \subset I$, il existe $n_{0}$ tel que $\left|\left\langle T^{t} g_{n}, v\right\rangle_{E^{\prime}, E}-\left\langle T^{t} g_{m}, v\right\rangle_{E^{\prime}, E}\right| \leq \varepsilon$ pour $n, m \geq n_{0}$. On a donc, pour $n, m \geq n_{0}$,

$$
\left|\left\langle T^{t} g_{n}, u\right\rangle_{E^{\prime}, E}-\left\langle T^{t} g_{m}, u\right\rangle_{E^{\prime}, E}\right| \leq(2 C+1) \varepsilon
$$


Ceci montre bien que la suite $\left(\left\langle T^{t} g_{n}, u\right\rangle_{E^{\prime}, E}\right)_{n \in \mathbb{N}}$ est de Cauchy.

Si $u \in E, u \neq 0$, on se ramène au cas précédent en divisant u par sa norme. On obtient bien ainsi la convergence de la suite $\left(\left\langle T^{t} g_{n}, u\right\rangle_{E^{\prime}, E}\right)_{n \in \mathbb{N}}$ pour tout $u \in E$ et nous notons encore $f_{u}$ cette limite.

L'application $u \mapsto f_{u}$ est trivialement linéaire (de $E$ dans $\left.\mathbb{R}\right)$ car limite d'applications linéraires. Mais elle est aussi continue car $\left|f_{u}\right| \leq C\|T\|_{\mathcal{L}(E, F)}\|u\|_{E}$. Il existe donc $f \in E^{\prime}$ tel que $f_{u}=\langle f, u\rangle_{E^{\prime}, E}$ pour tout $u \in E$.

Cette question montre que $T^{t} g_{n} \rightarrow f \star$-faiblement dans $E^{\prime}$ quand $n \rightarrow+\infty$.

6. Montrer qu'il existe $f \in E^{\prime}$ tel que $T^{t} g_{n} \rightarrow f$ dans $E^{\prime}$.

Corrigé - C'est pour cette question que l'on va utiliser que $I_{p}$ est fini. On reprend la méthode de la question précédente.

Soit $\varepsilon>0$. On choisit $p \in \mathbb{N}^{\star}$ tel que $1 / p \leq \varepsilon$.

Soit $u \in B_{E}$. Il existe $v \in I_{p}$ tel que $\|T v-T u\|_{F} \leq \varepsilon$. L'inégalité 1.21 donne alors

$$
\left|\left\langle T^{t} g_{n}, u\right\rangle_{E^{\prime}, E}-\left\langle T^{t} g_{m}, u\right\rangle_{E^{\prime}, E}\right| \leq(2 C+1) \varepsilon,
$$

pour $n, m \geq n_{0}$. Mais, comme $I_{p}$ est fini, $n_{0}$ peut être choisit inépendamment de $v$ (et donc de $u$ ). On obtient ainsi, quand $m \rightarrow+\infty$ dans 1.22 , pour tout $n \geq n_{0}$ et tout $u \in B_{E}$,

$$
\left|\left\langle T^{t} g_{n}, u\right\rangle_{E^{\prime}, E}-\langle f, u\rangle_{E^{\prime}, E}\right| \leq(2 C+1) \varepsilon,
$$

et donc, pour tout $n \geq n_{0},\left\|T^{t} g_{n}-f\right\|_{E^{\prime}} \leq(2 C+1) \varepsilon$.

On a bien montré que $T^{t} g_{n} \rightarrow f$ dans $E^{\prime}$.

\section{Déduire des questions précédentes que $T^{t}$ est un opérateur compact.}

Corrigé - On a montré que de toute suite bornée de $F^{\prime}$ on peut extraire une sous suite dont l'image par $T^{t}$ converge dans $E^{\prime}$. Ceci montre bien que $T^{t}$ est un opérateur compact.

8. Démontrer le résultat de compacité des bornés de $L^{q}(\Omega)$ dans $W^{-1, q}(\Omega)$ énoncé dans le théorème 1.27

Corrigé - Soit $\Omega$ un ouvert borné de $\mathbb{R}^{N}(N \geq 1)$ et $1<q<+\infty$. Le théorème 1.25 montre que l'application $T: u \mapsto u$ de $W_{0}^{1, p}(\Omega)$ dans $L^{p}(\Omega)$ est compacte. L'opérateur $T^{t}$ de $L^{p}(\Omega)^{\prime}$ dans $W_{0}^{1, p}(\Omega)^{\prime}$ est donc compact. L'espace $L^{p}(\Omega)^{\prime}$ est identifié à $L^{q}(\Omega)$ avec $q=p /(p-1)$ et $W_{0}^{1, p}(\Omega)^{\prime}$ est noté $W^{-1, q}(\Omega)$.

Pour $u \in\left(L^{p}\right)^{\prime}(\Omega)$ et $v \in W_{0}^{1, p}(\Omega)$, comme $L^{p}(\Omega)^{\prime}$ est identifié à $L^{q}(\Omega)$,

$$
\left\langle T^{t} u, v\right\rangle_{W^{-1, q}, W_{0}^{1, p}}=\langle u, T v\rangle_{\left(L^{p}\right)^{\prime}, L^{p}}=\int_{\Omega} u(x) v(x) \mathrm{d} x,
$$

c'est-à-dire que $T^{t} u$ est identifié à $u$. L'application $u \mapsto u$ est donc compacte de $L^{q}(\Omega)$ dans $W^{-1, q}(\Omega)$. 


\section{Chapitre 2}

\section{Problèmes elliptiques linéaires}

\subsection{Formulation faible}

Soit $\Omega$ un ouvert borné de $\mathbb{R}^{N}(N \geq 1)$ de frontière $\partial \Omega=\bar{\Omega} \backslash \Omega$. Soient $a_{i, j} \in L^{\infty}(\Omega)$, pour $i, j=1, \ldots, N$. On suppose que les fonctions $a_{i, j}$ vérifient l'hypothèse d'ellipticité uniforme, c'est-à-dire :

$$
\exists \alpha>0 ; \forall \xi=\left(\xi_{1}, \ldots, \xi_{N}\right) \in \mathbb{R}^{N}, \sum_{i, j=1}^{N} a_{i, j} \xi_{i} \xi_{j} \geq \alpha|\xi|^{2} \text { p.p. dans } \Omega .
$$

On se donne $f \in L^{2}(\Omega)$ et $g: \partial \Omega \rightarrow \mathbb{R}$, et on cherche une solution au problème :

$$
\begin{gathered}
-\sum_{i=1}^{N} \sum_{j=1}^{N} \partial_{i}\left(a_{i, j}(x) \partial_{j} u\right)(x)=f(x), x \in \Omega, \\
u(x)=g(x), x \in \partial \Omega,
\end{gathered}
$$

où $\partial_{i} u$ désigne la dérivée partielle de $u$ par rapport à sa $i$-ème variable.

Exemple 2.1 (Le Laplacien) Si on prend $a_{i, j}=\delta_{i, j}$ ( c'est-à-dire 1 si $i=j$, 0 si $i \neq j$ ), alors le problème (2.2) devient

$$
\begin{gathered}
-\Delta u=f \text { sur } \Omega, \\
u=g \text { sur } \partial \Omega .
\end{gathered}
$$

Définition 2.2 (Solution classique) On suppose que $a_{i, j} \in C^{1}(\bar{\Omega})$ pour tout $i, j=1, \ldots, N$. On suppose que $f \in C(\bar{\Omega})$ et $g \in C(\partial \Omega)$. On appelle alors solution classique de $[2.2]$ une fonction $u \in C^{2}(\bar{\Omega})$ vérifiant $[2.2$.

On rappelle que pour tout $k \in \mathbb{N} \cup\{+\infty\}, C^{k}(\bar{\Omega})$ désigne l'ensemble des restrictions à $\Omega$ des fonctions appartenant à $C^{k}\left(\mathbb{R}^{N}\right)$.

Il n'existe pas forcément de solution classique à (2.2). Mais il existe des solutions en un sens plus faible que l'on va définir ci-après. Pour comprendre leur nature, considérons d'abord le cas $g=0$, avec $a_{i, j} \in C^{1}(\bar{\Omega})$ et $f \in C(\bar{\Omega})$, et supposons qu'il existe une solution classique $u \in C^{2}(\bar{\Omega})$. Par définition, celle ci vérifie :

$$
-\sum_{i=1}^{N} \sum_{j=1}^{N} \partial_{i}\left(a_{i, j}(x) \partial_{j} u\right)(x)=f(x), \forall x \in \Omega .
$$


Soit $\varphi \in C_{c}^{\infty}(\Omega)$; multiplions l'équation précédente par $\varphi(x)$ et intégrons sur $\Omega$ :

$$
-\int_{\Omega}\left(\sum_{i=1}^{N} \sum_{j=1}^{N} \partial_{i}\left(a_{i, j}(x) \partial_{j} u\right)(x)\right) \varphi(x) \mathrm{d} x=\int_{\Omega} f(x) \varphi(x) \mathrm{d} x, \forall \varphi \in C_{c}^{\infty}(\Omega) .
$$

Une intégration par parties donne alors :

$$
\int_{\Omega}\left(\sum_{i=1}^{N} \sum_{j=1}^{N} a_{i, j}(x) \partial_{j} u(x) \partial_{i} \varphi(x)\right) \mathrm{d} x=\int_{\Omega} f(x) \varphi(x) \mathrm{d} x, \forall \varphi \in C_{c}^{\infty}(\Omega) .
$$

Comme $u \in C^{2}(\bar{\Omega})$, on a $\partial_{j} u \in C^{1}(\bar{\Omega}) \subset C(\bar{\Omega}) \subset L^{2}(\Omega)$, et $D_{j} u=\partial_{j} u$ p.p (come cela a été vu au Chapitre 1 . De plus $u \in C(\bar{\Omega}) \subset L^{2}(\Omega)$ et donc $u \in H^{1}(\Omega)$. Enfin, comme $u=0$ sur $\partial \Omega$, on a finalement $u \in H_{0}^{1}(\Omega)$ (voir l'exercice 1.17).

Soit $v \in H_{0}^{1}(\Omega)$, par densité de $C_{c}^{\infty}(\Omega)$ dans $H_{0}^{1}(\Omega)$, il existe une suite $\left(\varphi_{n}\right)_{n \in \mathbb{N}} \subset C_{c}^{\infty}(\Omega)$ telle que $\varphi_{n} \rightarrow v$ dans $H^{1}(\Omega)$, c'est-à-dire $\varphi_{n} \rightarrow v$ dans $L^{2}(\Omega)$ et $\partial_{i} \varphi_{n} \rightarrow D_{i} v$ dans $L^{2}(\Omega)$ pour $i=1, \ldots, N$. En écrivant (2.3) avec $\varphi=\varphi_{n}$, on obtient :

$$
\int_{\Omega}\left(\sum_{i=1}^{N} \sum_{j=1}^{N} a_{i, j}(x) \partial_{j} u(x) \partial_{i} \varphi_{n}(x)\right) \mathrm{d} x=\int_{\Omega} f(x) \varphi_{n}(x) \mathrm{d} x,
$$

et en passant à la limite, on obtient que $u$ satisfait le problème suivant, qu'on appelle formulation faible du problème 2.2. (on rappelle que l'on considère ici le cas $g=0$ )

$$
\begin{aligned}
& u \in H_{0}^{1}(\Omega), \\
& \int_{\Omega}\left(\sum_{i=1}^{N} \sum_{j=1}^{N} a_{i, j}(x) D_{j} u(x) D_{i} v(x)\right) \mathrm{d} x=\int_{\Omega} f(x) v(x) \mathrm{d} x, \forall v \in H_{0}^{1}(\Omega) .
\end{aligned}
$$

On vient ainsi de montrer que toute solution classique du problème 2.2 (lorsque $g=0$ ) est solution faible, c'est-à-dire vérifie 2.4.

Remarque 2.3 (Cas symétrique, formulation variationnelle) Dans le cas où $a_{i, j}=a_{j, i}$ p.p. pour $i \neq j, u$ est solution de (2.4) si et seulement si $u$ est solution du problème suivant, qu'on appelle formulation variationnelle :

$$
\begin{aligned}
& u \in H_{0}^{1}(\Omega), \\
& J(u) \leq J(v), \forall v \in H_{0}^{1}(\Omega),
\end{aligned}
$$

où la fonctionnelle $J$ est définie par : $J(v)=\frac{1}{2} \int_{\Omega}\left(\sum_{i=1}^{N} \sum_{j=1}^{N} a_{i, j} D_{i} v D_{j} v\right) \mathrm{d} x-\int_{\Omega} f(x) v(x) \mathrm{d} x$.

La démonstration de l'existence et de l'unicité des solutions des problèmes 2.4 et 2.5) utilise le lemme de LaxMilgram 11 que nous rappelons ici :

Lemme 2.4 (Lax-Milgram) Soient $H$ un espace de Hilbert réel muni du produit scalaire noté $(\cdot / \cdot)$, de norme associée notée $\|\cdot\|$, et a $(\cdot, \cdot)$ une application bilinéaire de $H \times H$ dans $\mathbb{R}$ qui est

1. Arthur Norton Milgram (1912-1961), mathématicien américain, qui a travaillé analyse fonctionnelle, en combinatoire, en géométrie différentielle, en topologie générale, en théorie des équations aux dérivées partielles et en théorie de Galois. 
- continue, ce qui équivalent à dire qu'il existe $c>0$ t.q., pour tout $(u, v) \in H^{2}$, on a $|a(u, v)| \leq c\|u\|\|v\|$,

- coercive sur $H$ (certains auteurs disent plutôt $H$-elliptique), c'est-à-dire qu'il existe $\alpha>0$, t.q., pour tout $u \in H, a(u, u) \geq \alpha\|u\|^{2}$,

et soit $T$ une forme linéaire continue sur $H$.

Alors il existe un unique u de $H$ tel que l'équation $a(u, v)=T(v)$ soit vérifiée pour tout $v$ de $H$ :

$$
\exists ! u \in H, \forall v \in H, \quad a(u, v)=T(v) .
$$

Si de plus la forme bilinéaire a est symétrique, alors u est l'unique élément de $H$ qui minimise la fonctionnelle $J: H \rightarrow \mathbb{R}$ définie par $J(v)=\frac{1}{2} a(v, v)-T(v)$ pour tout $v$ de $H$, c'est-à-dire :

$$
J(u)=\min _{v \in H} J(v) \text { et } J(u)<J(v) \text { si } u \neq v .
$$

Notons que dans le cas où la forme bilinéaire $a$ est symétrique, elle définit un produit scalaire sur $H$ équivalent au produit scalaire initial. Dans ce cas, le lemme de Lax-Milgram est une conséquence directe du théorème de représentation ${ }^{2}$ de Riesz ${ }^{3}$ dans un espace de Hilbert.

Pour appliquer le lemme de Lax-Milgram au problème [2.4], nous aurons besoin de l'inégalité de Poincaré 4 :

Lemme 2.5 (Inégalité de Poincaré) Soit $\Omega$ un ouvert borné de $\mathbb{R}^{N}, N \geq 1$ (ou qui est au moins borné dans une direction), alors il existe $C_{\Omega}$ ne dépendant que de $\Omega$ tel que

$$
\|u\|_{L^{2}(\Omega)} \leq C_{\Omega}\||\nabla u|\|_{L^{2}(\Omega)}, \forall u \in H_{0}^{1}(\Omega) .
$$

N.B. On désigne toujours par $|\cdot|$ la norme euclidienne dans $\mathbb{R}^{N}$. On a donc

$$
\||\nabla u|\|_{L^{2}(\Omega)}^{2}=\int_{\Omega}|\nabla u(x)|^{2} \mathrm{~d} x=\sum_{i=1}^{N} \int_{\Omega} D_{i} u(x)^{2} \mathrm{~d} x .
$$

Démonstration Par hypothèse sur $\Omega$, il existe $a>0$ tel que $\Omega \subset]-a, a\left[\times \mathbb{R}^{N-1}\right.$. Soit $u \in C_{c}^{\infty}(\Omega)$, on prolonge $u$ par 0 en dehors de $\Omega$, on a donc :

$$
u \in C_{c}^{\infty}\left(\mathbb{R}^{N}\right), u=0 \operatorname{sur} \Omega^{c} .
$$

Soit $x=\left(x_{1}, \ldots, x_{N}\right)^{t}=\left(x_{1}, y\right)^{t} \in \Omega$, avec $\left.x_{1} \in\right]-a, a\left[\right.$ et $y=\left(x_{2}, \ldots, x_{N}\right) \in \mathbb{R}^{N-1}$. On a :

$$
u\left(x_{1}, y\right)=\int_{-a}^{x_{1}} \partial_{1} u(t, y) \mathrm{d} t
$$

et donc, par l'inégalité de Cauchy-Schwarz,

$$
\left|u\left(x_{1}, y\right)\right|^{2} \leq\left(\int_{-a}^{a}\left|\partial_{1} u(t, y)\right| \mathrm{d} t\right)^{2} \leq 2 a \int_{-a}^{a}\left(\partial_{1} u(t, y)\right)^{2} \mathrm{~d} t .
$$

En intégrant entre $-a$ et $a$, on obtient :

$$
\int_{-a}^{a}\left|u\left(x_{1}, y\right)\right|^{2} \mathrm{~d} x_{1} \leq 4 a^{2} \int_{-a}^{a}\left(\partial_{1} u(t, y)\right)^{2} \mathrm{~d} t
$$

2. Théorème de représentation de Riesz : Soient $H$ un espace de Hilbert muni de son produit scalaire noté $(\cdot \mid \cdot)$ et $T \in H$ une forme linéaire continue sur $H$. Alors il existe un unique $y \in H$ tel que pour tout $x \in H$ on ait $T(x)=(y \mid x)$.

3. Frigyes Riesz (1880-1956), mathématicien hongrois. Il est l'un des fondateurs de l'analyse fonctionnelle.

4. Henri Poincaré (1854-1912) mathématicien, physicien théoricien et philosophe des sciences français, auteur de résultat important en optique et en calcul infinitésimal, et précurseur de la théorie des systèmes dynamiques. 
et donc, en intégrant par rapport à $y$,

$$
\int_{\Omega}|u(x)|^{2} \mathrm{~d} x \leq 4 a^{2} \int_{\Omega}\left(\partial_{1} u(x)\right)^{2} \mathrm{~d} x, \forall u \in C_{c}^{\infty}(\Omega)
$$

On procède ensuite par densité; pour $u \in H_{0}^{1}(\Omega)$, il existe une suite $\left(u_{n}\right)_{n \in \mathbb{N}} \subset C_{c}^{\infty}(\Omega)$ telle que $u_{n} \rightarrow u$ dans $H_{0}^{1}(\Omega)$. On a donc $u_{n} \rightarrow u$ dans $L^{2}(\Omega)$ et $\partial_{i} u \rightarrow D_{i} u$ dans $L^{2}(\Omega)$. On écrit alors (2.7) pour $u_{n}$ et en passant à la limite lorsque $n \rightarrow+\infty$, on obtient :

$$
\|u\|_{L^{2}(\Omega)}^{2} \leq 4 a^{2}\left\|D_{1} u\right\|_{L^{2}(\Omega)}^{2} \leq 4 a^{2} \sum_{i=1}^{N}\left\|D_{i} u\right\|_{L^{2}(\Omega)}^{2}=4 a^{2}\||\nabla u|\|_{L^{2}(\Omega)}^{2} .
$$

Théorème 2.6 (Existence et unicité de la solution de 2.4) Soit $\Omega$ un ouvert borné de $\mathbb{R}^{N}, f \in L^{2}(\Omega)$, et soient $\left(a_{i, j}\right)_{i, j=1, \ldots, N} \subset L^{\infty}(\Omega)$ et $\alpha>0$ tels que 2.1] soit vérifiée. Alors il existe une unique solution de [2.4].

Démonstration Pour appliquer le lemme de Lax-Milgram (lemme 2.4, on écrit le problème (2.4) sous la forme : $u \in H ; a(u, v)=T(v)$ pour tout $v \in H$, avec $H=H_{0}^{1}(\Omega)$ (qui est bien un espace de Hilbert, muni de la norme définie par $\left.\|u\|_{H^{1}(\Omega)}=\left(\|u\|_{L^{2}(\Omega)}^{2}+\||\nabla u|\|_{L^{2}(\Omega)}^{2}\right)^{\frac{1}{2}}\right)$, et avec $a$ et $T$ définies par

$$
a(u, v)=\int_{\Omega}\left(\sum_{i=1}^{N} \sum_{j=1}^{N} a_{i, j}(x) D_{j} u(x) D_{i} v(x)\right) \mathrm{d} x \text { et } T(v)=\int_{\Omega} f(x) v(x) \mathrm{d} x .
$$

On remarque tout d'abord que la forme linéaire $T$ est bien continue. En effet,

$$
|T(v)| \leq\|v\|_{L^{2}(\Omega)}\|f\|_{L^{2}(\Omega)} \leq\|v\|_{H^{1}(\Omega)}\|f\|_{L^{2}(\Omega)} .
$$

Quant à la forme $a$, elle est évidemment bilinéaire, et elle vérifie :

$$
|a(u, v)| \leq \sum_{i, j=1}^{N}\left\|a_{i, j}\right\|_{L^{\infty}(\Omega)}\left\|D_{i} u\right\|_{L^{2}(\Omega)}\left\|D_{j} v\right\|_{L^{2}(\Omega)} \leq C\|u\|_{H^{1}(\Omega)}\|v\|_{H^{1}(\Omega)},
$$

avec $C=\sum_{i, j=1}^{N}\left\|a_{i, j}\right\|_{L^{\infty}(\Omega)}$. Elle est donc continue.

Voyons si $a$ est coercive : il faut montrer qu'il existe $\beta \in \mathbb{R}_{+}$tel que $a(u, u) \geq \beta\|u\|_{H^{1}(\Omega)}^{2}$, pour tout $u \in H_{0}^{1}(\Omega)$. Par hypothèse sur $a$, on a :

$$
a(u, u)=\int_{\Omega}\left(\sum_{i=1}^{N} \sum_{j=1}^{N} a_{i, j}(x) D_{j} u(x) D_{i} u(x)\right) \mathrm{d} x \geq \alpha \int_{\Omega}\left(\sum_{i=1}^{N}\left|D_{i} u(x)\right|^{2}\right) \mathrm{d} x=\alpha \int_{\Omega}|\nabla u(x)|^{2} \mathrm{~d} x .
$$

On applique alors l'inégalité de Poincaré (2.6) :

$$
\|u\|_{H^{1}(\Omega)}^{2}=\|u\|_{L^{2}(\Omega)}^{2}+\sum_{i=1}^{N}\left\|D_{i} u\right\|_{L^{2}(\Omega)}^{2} \leq\left(C_{\Omega}^{2}+1\right) \sum_{i=1}^{N}\left\|D_{i} u\right\|_{L^{2}(\Omega)}^{2},
$$

d'où on obtient que :

$$
\sum_{i=1}^{N}\left\|D_{i} u\right\|_{L^{2}(\Omega)}^{2} \geq \frac{1}{C_{\Omega}^{2}+1}\|u\|_{H^{1}(\Omega)}^{2}
$$


et donc

$$
a(u, u) \geq \alpha \sum_{i=1}^{N}\left\|D_{i} u\right\|_{L^{2}(\Omega)}^{2} \geq \frac{\alpha}{C_{\Omega}^{2}+1}\|u\|_{H^{1}(\Omega)}^{2},
$$

ce qui démontre la coercivité de $a$. Par le lemme 2.4 de Lax-Milgram, on a donc bien existence et unicité de la solution du problème 2.4 .

Remarque 2.7 Le lemme 2.5 est encore vrai avec $1 \leq p \leq+\infty$ au lieu de $p=2$. Si $\Omega$ est un ouvert borné de $\mathbb{R}^{N}, N \geq 1$, et $1 \leq p \leq+\infty$, il existe $C_{p, \Omega}$ ne dépendant que de $p$ et $\Omega$ tel que

$$
\|u\|_{L^{p}(\Omega)} \leq C_{\Omega}\||\nabla u|\|_{L^{p}(\Omega)}, \forall u \in W_{0}^{1, p}(\Omega) .
$$

Ceci permet de définir une norme sur $W_{0}^{1, p}(\Omega)$ équivalente à la norme $W^{1, p}(\Omega)$, voir la définition 2.8 . (Pour $p=2$, cette équivalence de norme est en fait démontrée dans la démonstration du théorème 2.6.)

Définition 2.8 Soit $\Omega$ un ouvert borné de $\mathbb{R}^{N}(N \geq 1)$ et $1 \leq p \leq+\infty$. Pou $u \in W_{0}^{1, p}$, on pose

$$
\|u\|_{W_{0}^{1, p}(\Omega)}=\left(\int_{\Omega}|\nabla u(x)|^{p} \mathrm{~d} x\right)^{\frac{1}{p}} .
$$

Selon la remarque 2.7 c'est donc, sur $W_{0}^{1, p}(\Omega)$, une norme équivalente à la norme de $W^{1, p}(\Omega)$. Pour $p=2$, l'espace $W_{0}^{1,2}(\Omega)$ est aussi noté $H_{0}^{1}(\Omega)$ et la norme $\|\cdot\|_{W_{0}^{1,2}(\Omega)}$ est la norme $\|\cdot\|_{H_{0}^{1}(\Omega)}$.

Par le lemme de Lax-Milgram, on démontre de manière similaire l'existence et l'unicité dans le cas où le second membre de 2.4 est donné par un élément de $H^{-1}(\Omega)$ (dual de $H_{0}^{1}(\Omega)$, c'est-à-dire l'ensemble des formes linéaires continues sur $H_{0}^{1}(\Omega)$ ).

Théorème 2.9 (Existence et unicité, $\left.\boldsymbol{T} \in \boldsymbol{H}^{-1}\right)$ Soient $\Omega$ un ouvert borné de $\mathbb{R}^{N},\left(a_{i, j}\right)_{i, j=1, \ldots, N} \subset L^{\infty}(\Omega)$ et $\alpha>0$ tels que 2.1] soit vérifiée. Soit $T \in H^{-1}(\Omega)$, il existe alors une unique solution $u$ de :

$$
\begin{aligned}
& u \in H_{0}^{1}(\Omega), \\
& \int_{\Omega}\left(\sum_{i=1}^{N} \sum_{j=1}^{N} a_{i, j}(x) D_{j} u(x) D_{i} v(x)\right) \mathrm{d} x=T(v), \forall v \in H_{0}^{1}(\Omega) .
\end{aligned}
$$

Soit $\Omega$ un ouvert borné de $\mathbb{R}^{N}$ et $f \in L^{1}(\Omega)$. Il est intéressant de savoir si l'application $\varphi \mapsto \int_{\Omega} f(x) \varphi(x) \mathrm{d} x$ (définie, par exemple, pour $\varphi \in C_{c}^{\infty}(\Omega)$ ) se prolonge en un élément de $H^{-1}(\Omega)$ (et dans ce cas, le prolongement sera unique par densité de $C_{c}^{\infty}(\Omega)$ dans $H_{0}^{1}(\Omega)$ ). En dimension $N=1$, l'hypothèse $f \in L^{1}(\Omega)$ est suffisante. En dimension $N \geq 3$, l'hypothèse $f \in L^{q}(\Omega)$, avec $q=2 N /(N+2)$ est suffisante. En dimension $N=2$, l'hypothèse $f \in L^{q}(\Omega)$, avec $q>1$ est suffisante. Un résultat plus précis (pour $N=2$ ) est donné dans l'exercice 2.11 .

Nous n'avons traité ici que le cas de la condition aux limites de Dirichlet $\left.\right|^{5}$ homogène ( c'est-à-dire $g=0$ dans le problème 2.2. Le cas de la condition aux limites de Dirichlet non homogène est traité dans la section 2.5

L'existence et l'unicité de solutions faibles est possible avec d'autres conditions aux limites. L'exercice 2.6 traite le cas des conditions de Neumann 6 et l'exercice 2.9 les conditions dites de Fourier 7 (ou de Robin 8 selon les auteurs).

\footnotetext{
5. Johann Peter Gustav Lejeune Dirichlet (1805-1859), mathématicien prussien spécialiste en particulier de théorie des nombres et d'analyse mathématique

6. Carl Gottfried Neumann (1832-1925), mathématicien allemand qui a notamment travaillé sur le principe de Dirichlet et sur la théorie des équations intégrales.

7. Jean-Baptiste Joseph Fourier (1768-1830), mathématicien et physicien français, connu en particulier pour avoir déterminé, par le calcul, la diffusion de la chaleur en utilisant la décomposition d'une fonction quelconque en une série trigonométrique convergente (série de Fourier).

8. Victor Gustave Robin (1855-1897), mathématicien français, spécialiste de thermodynamique et en théorie du potentiel
} 
La résolution du problème de Neumann permet d'ailleurs de montrer une décomposition utile d'un élément de $L^{2}(\Omega)^{N}$, appelée décomposition de Hodge 9 , exercice 2.12 L'exercice 2.8 s'intéresse à des conditions aux limites apparaissant en mécanique du solide. Il est possible aussi de coupler un problème elliptique sur un ouvert $\Omega$ de $\mathbb{R}^{2}$ avec un problème elliptique unidimensionel sur le frontière de $\Omega$, ceci est l'objet de l'exercice 2.14

Les exercices 2.13, 2.15 et 2.10 montrent l'existence (et l'unicité ou une "unicité partielle") pour des systèmes elliptiques (problème des Stokes et équation de Schrödinger 10

Enfin, il est possible de traiter des problèmes elliptiques avec des coefficients $a_{i, j}$ non bornés. On introduit alors des espaces de Sobolev dit "à poids", exercice 2.4

\subsection{Analyse spectrale}

Soit $E$ un espace de Banach réel, et $T$ une application linéaire continue de $E$ dans $E$. On note :

- $\sigma(T)=\{\lambda \in \mathbb{R} ; T-\lambda$ Id est non bijective $\}$ l'ensemble des valeurs singulières de $T$,

- $\rho(T)=\{\lambda \in \mathbb{R} ; T-\lambda$ Id est bijective $\}=\mathbb{R} \backslash \sigma(T)$ l'ensemble des valeurs régulières de $T$,

- $\mathcal{V P}(T)=\{\lambda \in \mathbb{R} ; T-\lambda I d$ est non injective $\}$ l'ensemble des valeurs propres de $T$,

Ici et dans toute la suite de cet ouvrage, Id désigne l'application identité d'un ensemble dans lui-même. Lorsque $\operatorname{dim} E<+\infty$, on a $\mathcal{V P}(T)=\sigma(T)$. On a un résultat similaire en dimension infinie, à condition que l'opérateur $T$ soit linéaire continu et compact. Plus précisément, dans ce cas on a $: \mathcal{V P}(T) \backslash\{0\}=\sigma(T) \backslash\{0\}$. Le théorème suivant donne ce résultat dans les espaces de Hilbert séparables et pour un opérateur autoadjoint ${ }^{11}$

Proposition 2.10 (Opérateur linéaire continu compact autoajoint) Soit $E$ un espace de Hilbert séparable muni du produit scalaire $(\cdot, \cdot)_{E}$, et soit $T$ un opérateur linéaire continu compact autoadjoint dont le noyau $N(T)=\{u \in$ $E ; T(u)=0\}$ est réduit à $\{0\}$. Alors il existe une base hilbertienne de $E$ formée de vecteurs propres de $T$, c'està-dire d'éléments de $E$, notés $e_{n}, n \in \mathbb{N}$, vérifiant $\left(e_{n}, e_{m}\right)_{E}=\delta_{n, m}$ et tels que si $u \in E$, alors u peut s'écrire $u=\sum_{n \in N}\left(u, e_{n}\right)_{E} e_{n}$ (cette série étant convergente dans $E$ ), et les valeurs propres $\lambda_{n} \in \mathbb{R}$ associées, i.e. telles que Te $e_{n}=\lambda_{n} e_{n}$, sont telles que $\lambda_{n} \rightarrow 0$ lorsque $n \rightarrow+\infty$.

On va maintenant considérer, pour simplifier, le cas du Laplacien. Soit $\Omega$ un ouvert borné de $\mathbb{R}^{N}$. On rappelle que $\Delta u=\sum_{i=1}^{N} \partial_{i}^{2} u$ si $u$ est une fonction régulière. Pour étendre cette définition aux fonctions seulement localement intégrables, on pose, si $u \in L_{\text {loc }}^{1}(\Omega), \Delta u=\sum_{i=1}^{N} D_{i}^{2} u$. On définit maintenant un opérateur $A$ d'une partie de $L^{2}(\Omega)$ dans $L^{2}(\Omega)$ en définissant d'abord son domaine $D(A)$ :

$$
D(A)=\left\{u \in H_{0}^{1}(\Omega) ; \Delta u \in L^{2}(\Omega)\right\},
$$

Puis on pose $A u=-\Delta u$ si $u \in D(A)$. On a ainsi définit un opérateur linéaire $A: D(A) \subset L^{2}(\Omega) \rightarrow L^{2}(\Omega)$.

On a vu dans les paragraphes précédents que si $f \in L^{2}(\Omega)$, il existe une unique solution au problème (2.4) qui s'écrit, pour le Laplacien, c'est-à-dire avec les valeurs $a_{i, j}=\delta_{i, j}, i, j=1, \ldots, N$ :

$$
\begin{aligned}
& u \in H_{0}^{1}(\Omega), \\
& \int_{\Omega} \nabla u(x) \nabla v(x) \mathrm{d} x=\int_{\Omega} f(x) v(x) \mathrm{d} x, \forall v \in H_{0}^{1}(\Omega) .
\end{aligned}
$$

9. William Vallance Douglas Hodge (1903 -1975), mathématicien britanique spécialisé en géometrie

10. Erwin Rudolf Josef Alexander Schrödinger (1887-1961), physicien austro-irlandais à qui l'on doit de nombreux résultats de mécanique quantique.

11. On rappelle qu'un opérateur linéaire $T$ d'un espace de Hilbert muni du produit scalaire $(\cdot \mid \cdot)_{H}$ dans lui même est autoadjoint s'il est identique à son opérateur adjoint $T^{*}$, défini par $\left(T^{*} x \mid y\right)_{H}=(x \mid T y)_{H}$. Dans le cas d'un espace de Hilbert réel, on dit aussi que l'opérateur es symétrique 
Grâce à la densité de $C_{c}^{\infty}(\Omega)$ dans $H_{0}^{1}(\Omega)$, la fonction $u$ est solution de 2.9) si et seulement si $u \in D(A)$ et $-\Delta u=f$ p.p. ( c'est-à-dire $-\Delta u=f$ dans $L^{2}(\Omega)$ ). L'opérateur $A$ est donc inversible. Son inverse, l'opérateur $A^{-1}$, est défini de $L^{2}(\Omega)$ dans $L^{2}(\Omega)$ par $A^{-1} f=u$ où $u$ est solution de 2.9 . Cet opérateur est injectif mais non surjectif. Les deux opérateurs sont linéaires.

Pour montrer qu'il existe une base hilbertienne formée des vecteurs propres de $A^{-1}$, on va démontrer le théorème suivant :

Théorème 2.11 Soit $\Omega$ un ouvert borné de $\mathbb{R}^{N}$. Pour $f \in L^{2}(\Omega)$, on note $T f$ l'unique solution de (2.9). L'opérateur $T$ est linéaire continu compact et autoadjoint de $L^{2}(\Omega)$ dans $L^{2}(\Omega)$. De plus $N(T)=\left\{f \in L^{2}(\Omega), T f=0\right.$ p.p. $\}=\{0\}$.

Démonstration Il est immédiat de voir que $T$ est linéaire. On remarque tout d'abord que $N(T)=\{f \in E$, $T f=0$ p.p. $\}=\{0\}$. En effet, soit $f \in L^{2}(\Omega)$ telle que $T f=0$ p.p.. On a donc, d'après 22.9,

$$
\int_{\Omega} f v \mathrm{~d} x=0 \text { pour tout } v \in H_{0}^{1}(\Omega) .
$$

Comme $H_{0}^{1}(\Omega)$ est dense dans $L^{2}(\Omega)$ (on a même $C_{c}^{\infty}(\Omega)$ dense dans $L^{2}(\Omega)$ ), on en déduit $f=0$ p.p..

On montre maintenant la continuité de $T$. Soit $f \in L^{2}(\Omega)$ et $u=T f$. En prenant $v=u$ dans 2.9, on obtient

$$
\|u\|_{H_{0}^{1}(\Omega)}^{2}=\int_{\Omega} \nabla u \cdot \nabla u \mathrm{~d} x=\int_{\Omega} f u \mathrm{~d} x \leq\|f\|_{L^{2}(\Omega)}\|u\|_{L^{2}(\Omega)} .
$$

Par l'inégalité de Poincaré, il existe $C_{\Omega} \in \mathbb{R}_{+}$ne dépendant que de $\Omega$ tel que $\|u\|_{L^{2}(\Omega)}^{2} \leq C_{\Omega}\|u\|_{H_{0}^{1}(\Omega)}^{2}$, et donc :

$$
\|u\|_{H_{0}^{1}(\Omega)}^{2}=\sum_{i=1}^{N}\left\|D_{i} u\right\|_{L^{2}(\Omega)}^{2} \leq\|f\|_{L^{2}(\Omega)}\|u\|_{L^{2}(\Omega)} \leq C_{\Omega}\|f\|_{L^{2}(\Omega)}\|u\|_{H_{0}^{1}(\Omega)} .
$$

On en déduit que $\|u\|_{H_{0}^{1}(\Omega)} \leq C_{\Omega}\|f\|_{L^{2}(\Omega)}$ et donc :

$$
\|T f\|_{L^{2}(\Omega)}^{2}=\|u\|_{L^{2}(\Omega)}^{2} \leq C_{\Omega}\|u\|_{H_{0}^{1}(\Omega)}^{2} \leq C_{\Omega}^{2}\|f\|_{L^{2}(\Omega)}^{2},
$$

ce qui démontre la continuité de $T$.

Montrons maintenant que l'opérateur $T$ est compact, c'est-à-dire que l'image $T(B)$ d'un ensemble $B$ borné de $L^{2}(\Omega)$ est relativement compact dans $L^{2}(\Omega)$. On peut écrire $T$ sous la forme $T=I \circ T_{0}$ où $I$ est l'injection canonique de $H_{0}^{1}(\Omega)$ dans $L^{2}(\Omega)$, définie $\operatorname{par} I: v \in H_{0}^{1}(\Omega) \mapsto v \in L^{2}(\Omega)$, et $T_{0}$ est l'application qui à $f \in L^{2}(\Omega)$ associe $u=T f \in H_{0}^{1}(\Omega)$. L'application $T_{0}$ est continue de $L^{2}(\Omega)$ dans $H_{0}^{1}(\Omega)\left(\operatorname{car}\|T f\|_{H_{0}^{1}(\Omega)} \leq C_{\Omega}\|f\|_{L^{2}(\Omega)}\right)$ et l'injection $I$ est compacte par le théorème de Rellich (théorème 1.25 page 12, et donc l'opérateur $T$ est compact.

Montrons maintenant que l'opérateur $T$ est auto-adjoint, c'est-à-dire que

$$
(T f, g)_{L^{2}(\Omega)}=(f, T g)_{L^{2}(\Omega)}, \forall f, g \in L^{2}(\Omega) .
$$

Soient donc $f$ et $g \in L^{2}(\Omega), u$ l'unique solution de (2.9), et $v$ l'unique solution de 2.9] où on a remplacé $f$ par $g$ dans le second membre. On a, comme $v$ est solution de (2.9) où on a remplacé $f$ par $g$ :

$$
(T f, g)_{L^{2}(\Omega)}=\int_{\Omega} T f g \mathrm{~d} x=\int_{\Omega} u g \mathrm{~d} x=\int_{\Omega} \nabla u \cdot \nabla v \mathrm{~d} x .
$$

On montre de même que $(f, T g)_{L^{2}(\Omega)}=\int_{\Omega} \nabla u \cdot \nabla v \mathrm{~d} x$, ce qui démontre 2.10.

Voici maintenant la conséquence du théorème 2.11 et de la proposition 2.10 pour l'opérateur "Laplacien" avec condition de Dirichlet homogène. 
Théorème 2.12 (Base hilbertienne de $L^{2}(\Omega)$ formée de fonctions propres de $-\Delta$ ) Soit $\Omega$ un ouvert borné de $\mathbb{R}^{N}$, Au $=-\Delta u$ avec $D(A)=\left\{u \in H_{0}^{1}(\Omega) ; \Delta u \in L^{2}(\Omega)\right\}$. Il existe alors une base hilbertienne (dénombrable) de $L^{2}(\Omega)$, notée $\left(e_{n}\right)_{n \in \mathbb{N}^{*}}$, formée de vecteurs propres de A, associés aux valeurs

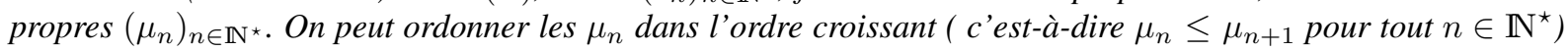
et l'on a $\mu_{1}>0$ et $\lim _{n \rightarrow+\infty} \mu_{n}=+\infty$.

Démonstration Pour $f \in L^{2}(\Omega)$, on note $T f$ l'unique solution de 2.9]. D'après le théorème 2.11 et la proposition 2.10, il existe donc une base hilbertienne $\left(e_{n}\right)_{n \in \mathbb{N}^{*}}$ de $L^{2}(\Omega)$ formées de fonctions propres de $T$. Les valeurs propres associées sont toutes strictement positives. En effet, si $f \in L^{2}(\Omega)$ et $f \neq 0$, alors $u=T f \neq 0$ et

$$
(T f, f)_{L^{2}(\Omega)}=(u, f)_{L^{2}(\Omega)}=\int_{\Omega} \nabla u \cdot \nabla u \mathrm{~d} x=\|u\|_{H_{0}^{1}(\Omega)}^{2}>0 .
$$

Si $\lambda_{n}$ est une valeur propre de $T$ est associée au vecteur propre $e_{n} \neq 0$, on a $T e_{n}=\lambda_{n} e_{n}$, et donc, comme $e_{n} \neq 0$,

$$
\lambda_{n}\left(e_{n}, e_{n}\right)_{L^{2}(\Omega)}=\left(\lambda_{n} e_{n}, e_{n}\right)_{L^{2}(\Omega)}=\left(T e_{n}, e_{n}\right)_{L^{2}(\Omega)}>0 .
$$

La suite $\left(\lambda_{n}\right)_{n \in \mathbb{N}}$ est donc formée de nombres strictement positifs. Quitte à changer l'ordre des $\lambda_{n}$, on peut supposer que cette suite est décroissance. Enfin, la proposition 2.10 donne que $\lim _{n \rightarrow+\infty} \lambda_{n}=0$.

Remarquons que les valeurs propres de $A$ sont donc les valeurs $\mu_{n}=\frac{1}{\lambda_{n}}$ pour tout $n \in \mathbb{N}^{\star}$, avec $\mu_{n}>0$ pour tout $n \in \mathbb{N}^{\star}$ et $\mu_{n} \rightarrow+\infty$ lorsque $n \rightarrow+\infty$.

En reprenant les notations du théorème 2.12. on peut alors caractériser le domaine de l'opérateur Laplacien (avec condition de Dirichlet homogène) $D(A)$ de la façon suivante :

$$
\text { Soit } u \in L^{2}(\Omega),[u \in D(A)] \Longleftrightarrow\left[\sum_{n \in \mathbb{N}} \mu_{n}^{2}\left(u, e_{n}\right)_{L^{2}(\Omega)}^{2}<+\infty .\right]
$$

De plus si $u \in D(A)$, alors $A u=\sum_{n=1}^{+\infty} \mu_{n}\left(u, e_{n}\right)_{L^{2}(\Omega)} e_{n}$. On peut ainsi définir les puissances de l'opérateur $A$ :

Définition 2.13 (Puissance de l'opérateur) Soit $\Omega$ un ouvert borné de $\mathbb{R}^{N}, A u=-\Delta u$ avec $D(A)=\{u \in$ $\left.H_{0}^{1}(\Omega) ; \Delta u \in L^{2}(\Omega)\right\}$. On note $\left(e_{n}\right)_{n \in \mathbb{N}^{\star}}$ une base hilbertienne de $L^{2}(\Omega)$ formée de vecteurs propres de $A$, associés aux valeurs propres $\left(\mu_{n}\right)_{n \in \mathbb{N}^{\star}}$. Soit $s \geq 0$. On définit

$$
D\left(A^{s}\right)=\left\{u \in L^{2}(\Omega): \sum_{n=1}^{+\infty} \mu_{n}^{2 s}\left(u, e_{n}\right)_{L^{2}(\Omega)}^{2}<+\infty\right\} .
$$

Et pour $u \in D\left(A^{s}\right)$, on peut alors définir $A^{s} u$ par :

$$
A^{s} u=\sum_{n=1}^{+\infty} \mu_{n}^{s}\left(u, e_{n}\right)_{L^{2}(\Omega)} e_{n} .
$$

Cette série étant convergente dans $L^{2}(\Omega)$.

Pour $s=0$, on a $D\left(A^{0}\right)=L^{2}(\Omega)$ et $A^{0} u=u: A^{0}$ est l'opérateur identité.

Pour $s=1$, on retrouve l'opérateur $A$.

Pour $s=\frac{1}{2}$, on a $D\left(A^{\frac{1}{2}}\right)=\left\{u \in L^{2}(\Omega) ; \sum_{n=1}^{+\infty} \mu_{n}\left(u, e_{n}\right)_{L^{2}(\Omega)}^{2}<+\infty\right\}$. On peut montrer que $D\left(A^{\frac{1}{2}}\right)=H_{0}^{1}(\Omega)$, et on a $A^{\frac{1}{2}} u=\sum_{n=1}^{+\infty} \sqrt{\mu_{n}}\left(u, e_{n}\right)_{L^{2}(\Omega)} e_{n}$.

Pour le cas $N=1, \Omega=] 0,1[$, Le théorème de décomposition spectrale est détaillé dans l'exercice 2.2 . 


\subsection{Régularité des solutions faibles}

Soit $\Omega$ un ouvert borné de $\mathbb{R}^{N}$ et $f \in L^{2}(\Omega)$. Sous les hypothèses $(2.1)$, on sait par les résultats précédents qu'il existe une unique solution au problème (2.4), et on se demande quelle est la régularité de cette solution en fonction des données du problème. Le problème est assez simple en dimension $N=1$, voir l'exercice 2.1, mais beaucoup plus difficile en dimension $N>1$. La réponse dépend de la régularité des coefficients de l'opérateur et de la régularité de la frontière de l'ouvert (on dit que la frontière de $\Omega$ est de classe $C^{k}$ si elle est localement le graphe d'une fonction de classe $C^{k}$ ).

Théorème 2.14 (Régularité de la solution du problème de Dirichlet)

Soit $\Omega$ un ouvert borné de $\mathbb{R}^{N}$ et $f \in L^{2}(\Omega)$. Sous les hypothèses [2.1), soit $u \in H_{0}^{1}(\Omega)$ la solution de (2.4).

1. Si $a_{i, j} \in C^{1}(\bar{\Omega})$ pour $i, j=1, \ldots, N$ et $\Omega$ est à frontière $C^{2}$, alors, pour tout $f \in L^{2}(\Omega)$, on a $u \in H^{2}(\Omega)$.

2. Si $a_{i, j} \in C^{\infty}(\bar{\Omega})$ pour $i, j=1, \ldots, N$, si $\Omega$ est à frontière $C^{\infty}$, et si $f \in H^{m}(\Omega)$ avec $m \geq 0$, alors $u \in H^{m+2}(\Omega)$.

En conséquence, si $f \in C^{\infty}(\bar{\Omega})$, alors $u \in C^{\infty}(\bar{\Omega})$ et donc u est solution classique. De même, si $f \in H^{m}(\Omega)$ avec $m>\frac{N}{2}$, alors $u \in C^{2}(\bar{\Omega})$ et donc u est encore solution classique.

Remarque 2.15 (Optimalité des hypothèses) Notons que la partie 1. du théorème précédent est fausse sans les hypothèses $a_{i, j} \in C^{1}(\bar{\Omega})$ et $\Omega$ est à frontière $C^{2}$.

Par contre dans le cas du Laplacien, c'est-à-dire $a_{i, j}=\delta_{i, j}$, si $\Omega$ est convexe, alors $u \in H^{2}(\Omega)$ dés que $f \in L^{2}(\Omega)$.

Idée de démonstration du théorème 2.14 première partie

On se ramène par la technique dite des "cartes locales" au cas $\Omega=\mathbb{R}_{+}^{N}=\left\{\left(x_{1}, y\right), y \in \mathbb{R}^{N-1}, x_{1}>0\right\}$, et au problème suivant :

$$
\begin{aligned}
& u \in H_{0}^{1}(\Omega), \\
& \int_{\Omega} \nabla u \cdot \nabla v \mathrm{~d} x=\int_{\Omega} f v \mathrm{~d} x, \forall v \in H_{0}^{1}(\Omega) .
\end{aligned}
$$

et on applique ensuite le théorème 2.18 Ce théorème montre que la solution de ce problème appartient à $H^{2}\left(\mathbb{R}_{+}^{N}\right)$.

La démonstration du théorème 2.18, due à Nirenberg que nous énonçons un peu plus loin nécessite les lemmes techniques suivants, que nous enonçons pour $N=2$, pour simplifier :

Lemme 2.16 Soit $\Omega=\mathbb{R}_{+}^{2}=\left\{\left(x_{1}, y\right), x_{1}>0, y \in \mathbb{R}\right\}$. Soit $g \in L^{2}(\Omega)$ et, pour $h>0, \Psi_{h} g$ défini par $: \Psi_{h} g=$ $\frac{1}{h}\left(g_{h}-g\right)$, où $g_{h} \in H_{0}^{1}(\Omega)$ est définie par $g_{h}(x)=g\left(x_{1}, x_{2}+h\right)$. Alors $\left\|\Psi_{h} g\right\|_{H^{-1}(\Omega)} \leq\|g\|_{L^{2}(\Omega)}$.

Démonstration Soit $g \in L^{2}(\Omega)$, par définition,

$$
\left\|\Psi_{h} g\right\|_{H^{-1}(\Omega)}=\sup \left\{\int_{\Omega} \Psi_{h} g v \mathrm{~d} x, v \in H_{0}^{1}(\Omega),\|v\|_{H^{1}(\Omega)} \leq 1\right\},
$$

et donc, par densité de $C_{c}^{\infty}(\Omega)$ dans $H_{0}^{1}(\Omega)$,

$$
\left\|\Psi_{h} g\right\|_{H^{-1}(\Omega)}=\sup \left\{\int_{\Omega} \Psi_{h} g v \mathrm{~d} x, v \in C_{c}^{\infty}(\Omega),\|v\|_{H^{1}(\Omega)} \leq 1\right\} .
$$


Soit $v \in C_{c}^{\infty}(\Omega)$ tel que $\|v\|_{H^{1}(\Omega)} \leq 1$.

$$
\begin{aligned}
\int_{\Omega} \Psi_{h} g v \mathrm{~d} x & =\frac{1}{h} \int_{\mathbb{R}_{+}} \int_{\mathbb{R}^{R}}\left(g\left(x_{1}, x_{2}+h\right)-g\left(x_{1}, x_{2}\right)\right) v\left(x_{1}, x_{2}\right) \mathrm{d} x_{1} \mathrm{~d} x_{2} \\
& =\frac{1}{h} \int_{\mathbb{R}_{+}} \int_{\mathbb{R}_{R}} g\left(x_{1}, \tilde{x}_{2}\right) v\left(x_{1}, \tilde{x}_{2}-h\right) \mathrm{d} x_{1} \mathrm{~d} \tilde{x}_{2}-\int_{\mathbb{R}_{+}} \int_{\mathbb{R}} g\left(x_{1}, x_{2}\right) v\left(x_{1}, x_{2}\right) \mathrm{d} x_{1} \mathrm{~d} x_{2} \\
& =-\int_{\mathbb{R}_{+}} \int_{\mathbb{R}} g\left(x_{1}, x_{2}\right) \frac{v\left(x_{1}, x_{2}-h\right)-v\left(x_{1}, x_{2}\right)}{-h} \mathrm{~d} x_{1} \mathrm{~d} x_{2} .
\end{aligned}
$$

Donc, par l'inégalité de Cauchy-Schwarz,

$$
\left|\int_{\Omega} \Psi_{h} g v \mathrm{~d} x\right| \leq\|g\|_{L^{2}(\Omega)}\left\|\frac{v(\cdot, \cdot-h)-v(\cdot, \cdot)}{-h}\right\|_{L^{2}(\Omega)} \leq\|g\|_{L^{2}(\Omega)}\|v\|_{H^{1}(\Omega)} \leq\|g\|_{L^{2}(\Omega)} .
$$

On en déduit que $\left\|\Psi_{h} g\right\|_{H^{-1}(\Omega)} \leq\|g\|_{L^{2}(\Omega)}$.

Lemme 2.17 Sous les hypothèses du lemme 2.16 soit $u \in L_{\mathrm{loc}}^{1}(\Omega)$, alors $\Psi_{h} u \rightarrow D_{2} u$ dans $\mathcal{D}^{\star}$ lorsque $h \rightarrow 0$.

Démonstration On pose $\mathcal{D}=C_{c}^{\infty}(\Omega)$. Soit $\varphi \in \mathcal{D}$; on veut montrer que

$$
\int_{\Omega} \Psi_{h} u \varphi \mathrm{d} x \rightarrow-\int_{\Omega} u \partial_{2} \varphi \mathrm{d} x=\left\langle D_{2} u, \varphi\right\rangle_{\mathcal{D}^{*}, \mathcal{D}} \text { lorsque } h \rightarrow 0 .
$$

Or

$$
\begin{aligned}
\int_{\Omega} \Psi_{h} u \varphi \mathrm{d} x & =\int_{\mathbb{R}_{+}} \int_{\mathbb{R}} \frac{u\left(x_{1}, x_{2}+h\right)-u\left(x_{1}, x_{2}\right)}{h} \varphi\left(x_{1}, x_{2}\right) \mathrm{d} x_{1} \mathrm{~d} x_{2} \\
& =-\int_{\mathbb{R}_{+}} \int_{\mathbb{R}} u\left(x_{1}, x_{2}\right) \frac{\varphi\left(x_{1}, x_{2}-h\right)-\varphi\left(x_{1}, x_{2}\right)}{-h} \mathrm{~d} x_{1} \mathrm{~d} x_{2} .
\end{aligned}
$$

Mais $\frac{\varphi\left(x_{1}, x_{2}-h\right)-\varphi\left(x_{1}, x_{2}\right)}{-h} \rightarrow \partial_{2} \varphi$ uniformément lorsque $h \rightarrow 0$, et le support de cette fonction est inclus dans un compact $K$ de $\Omega$, indépendant de $h$ si $|h|<1$. Donc $\lim _{h \rightarrow 0} \int_{\Omega} \Psi_{h} u \varphi \mathrm{d} x=-\int_{\Omega} u \partial_{2} \varphi \mathrm{d} x$.

Théorème 2.18 (Nirenberg) Soit $\Omega=\mathbb{R}_{+}^{N}=\left\{\left(x_{1}, y\right), y \in \mathbb{R}^{N-1}, x_{1}>0\right\}$ et $f \in L^{2}(\Omega)$, et soit $u \in H_{0}^{1}(\Omega)$ solution du problème suivant :

$$
\begin{aligned}
& u \in H_{0}^{1}(\Omega), \\
& \int_{\Omega} \nabla u \cdot \nabla v \mathrm{~d} x=\int_{\Omega} f v \mathrm{~d} x, \forall v \in H_{0}^{1}(\Omega) .
\end{aligned}
$$

Alors $u \in H^{2}\left(\mathbb{R}_{+}^{N}\right)$.

Démonstration On va effectuer la démonstration dans le cas $N=2$. Soit $u \in H_{0}^{1}(\Omega)$ solution de $[2.11$, $u$ vérifie donc :

$$
\int_{\Omega} \nabla u \cdot \nabla v \mathrm{~d} x+\int_{\Omega} u v \mathrm{~d} x=\int_{\Omega} g v \mathrm{~d} x, \forall v \in H_{0}^{1}(\Omega), \text { où } g=u+f \in L^{2}(\Omega) .
$$


On a donc

$$
(u, v)_{H_{0}^{1}(\Omega)}=\int_{\mathbb{R}_{+}^{2}} g v \mathrm{~d} x \leq\|g\|_{H^{-1}(\Omega)}\|v\|_{H^{1}(\Omega)},
$$

puisque, par définition, $\|g\|_{H^{-1}(\Omega)}=\sup \left\{\int_{\mathbb{R}_{+}^{2}} g v \mathrm{~d} x, v \in H_{0}^{1}(\Omega),\|v\|_{H^{1}(\Omega)} \leq 1\right\}$, où, comme d'habitude, on confond l'application $T_{g}$ qui à $v \in H_{0}^{1}(\Omega)$ associe $\int g v \mathrm{~d} x$, qui est donc un élement de $H^{-1}(\Omega)$, avec la (classe de) fonction(s) $g \in L^{2}(\Omega)$. On prend $v=u$ dans 2.12). On obtient $\|u\|_{H^{1}(\Omega)} \leq\|g\|_{H^{-1}(\Omega)}$.

Pour montrer la régularité sur $D_{2} u$, on introduit la fonction $\Psi_{h} u=\frac{1}{h}\left(u_{h}-u\right)$ où $u_{h} \in H_{0}^{1}(\Omega)$ est définie par $u_{h}(x)=u\left(x_{1}, x_{2}+h\right)$. Comme $u$ vérifie (2.11), $u_{h}$ vérifie $\int_{\Omega} \nabla u_{h} \cdot \nabla v \mathrm{~d} x=\int_{\Omega} f_{h} v \mathrm{~d} x$ où $f_{h}(x)=f\left(x_{1}, x_{2}+h\right)$, et donc $\Psi_{h} u=\frac{1}{h}\left(u_{h}-u\right)$ appartient à $H_{0}^{1}(\Omega)$ et vérifie

$$
\int_{\Omega} \nabla \Psi_{h} u \cdot \nabla v \mathrm{~d} x=\int_{\Omega} \Psi_{h} f v \mathrm{~d} x \text { pour tout } v \in H_{0}^{1}(\Omega) .
$$

On en déduit que $\left(\Psi_{h} u, v\right)_{H^{1}(\Omega)}=\int_{\Omega} \Psi_{h} g v \mathrm{~d} x$, et donc que $\left\|\Psi_{h} u\right\|_{H^{1}(\Omega)} \leq\left\|\Psi_{h} g\right\|_{H^{-1}(\Omega)}$. Par le lemme 2.16 . comme $g \in L^{2}(\Omega)$, on a donc

$$
\left\|\Psi_{h} u\right\|_{H^{1}(\Omega)} \leq\|g\|_{L^{2}(\Omega)} .
$$

Prenons maintenant $h=\frac{1}{n}$ et faisons $n \rightarrow+\infty$. Par ce qui précéde, la suite $\left(\Psi_{\frac{1}{n}} u\right)_{n \in \mathbb{N}}$ est bornée dans $H_{0}^{1}(\Omega)$, il existe donc une sous-suite encore notée $\left(\Psi_{\frac{1}{n}} u\right)_{n \in \mathbb{N}}$, et $w \in H_{0}^{1}(\Omega)$ telle que $\Psi_{\frac{1}{n}} u \rightarrow w$ dans $H_{0}^{1}(\Omega)$ faible ( c'est-à-dire $S\left(\Psi_{\frac{1}{n}} u\right) \rightarrow S(w)$ pour tout $S \in H^{-1}(\Omega)$ ). Donc $\Psi_{\frac{1}{n}} u \rightarrow w$ dans $\stackrel{D}{D}^{\star}$. Mais par le lemme 2.17 . $\Psi_{\frac{1}{n}} u \rightarrow D_{2} u$ dans $\mathcal{D}^{\star}$. Donc $D_{2} u=w \in H_{0}^{1}(\Omega)$, et par conséquent, $D_{1} D_{2} u \in L^{2}(\Omega)$ et $D_{2} D_{2} u \in L^{2}(\Omega)$. Pour conclure, il ne reste plus qu'à montrer que $D_{1} D_{1} u \in L^{2}(\Omega)$. Pour cela, on utilise l'équation satisfaite par $u$. En effet, comme $u$ est solution faible de [2.4, , on a $-\Delta u=f$ dans $\mathcal{D}^{\star}$, et donc $D_{1} D_{1} u=-f-D_{2} D_{2} u$ ce qui prouve que $D_{1} D_{1} u \in L^{2}(\Omega)$. Ceci termine la preuve.

\section{Remarque 2.19 (Plus de régularité...)}

Soit $\Omega$ un ouvert borné de $\mathbb{R}^{N}$ et $f \in L^{2}(\Omega)$. Sous les hypothèses $(2.1)$, soit $u \in H_{0}^{1}(\Omega)$ la solution de (2.4).

1. Supposons que $a_{i, j} \in C^{1}(\bar{\Omega})$ et que $\Omega$ est à frontière $C^{2}$. On a déjé vu que si $f \in L^{2}(\Omega)$ alors $u \in H^{2}(\Omega)$. On peut montrer que si $f \in L^{p}(\Omega)$ alors $u \in W^{2, p}(\Omega)(2 \leq p<+\infty)$.

2. Supposons maintenant qu'on ait seulement $a_{i, j} \in L^{\infty}(\Omega)$. On peut montrer [23] qu'il existe $p^{\star}>2$ tel que si $f \in L^{p}(\Omega)$ avec $2 \leq p \leq p^{\star}$, alors $u \in W_{0}^{1, p}(\Omega)$.

3. Toujours dans le cas $a_{i, j} \in L^{\infty}(\Omega)$, on peut montrer (ce résultat est dû à Stampacchia ${ }^{12}$ ) que si $f \in L^{p}(\Omega)$, avec $p>\frac{N}{2}$, alors $u \in L^{\infty}(\Omega)$.

4. Il est possible aussi de démontrer des résultat de régularité pour d'autres conditions aux limites. L'exercice 2.6 donne un exemple avec les conditions de Neumann, l'exercice 2.9 un exemple avec conditions de Fourier et l'exercice 2.10 traite l'exemple du système elliptique induit par l'équation de Schrödinger (qui est d'habitude présenté comme une équation dont l'inconnue prend ses valeurs dans $\mathbb{C}$ ).

\subsection{Positivité de la solution faible}

Question. (Positivité de la solution faible.) Soit $\Omega$ un ouvert borné de $\mathbb{R}^{N}, N \geq 1, a_{i, j} \in L^{\infty}(\Omega)$, pour $i, j=$ $1, \ldots, N$. On suppose que les fonctions $a_{i, j}$ vérifient $\left[2.1\right.$. Soit $f \in L^{2}(\Omega)$ et $u$ la solution de (2.4). On suppose que $f \geq 0$ p.p.. A-t-on $u \geq 0$ p.p.?

12. Guido Stampacchia, mathématicien italien (1922-1978), spécialiste de calcul des variations et des équations aux dérivées partielles, entre autres. 
Remarque 2.20 Soit $\Omega$ un ouvert borné de $\mathbb{R}^{N}, N \geq 1$. On suppose que $u \in C^{2}(\bar{\Omega}),-\Delta u=f$ dans $\Omega$ et $u=0$ sur le bord de $\Omega$ (la fonction $u$ est donc une solution classique avec $a_{i, j}=0$ si $i \neq j$ et $a_{i, j}=1$ si $i=j$ ). On suppose aussi que $f>0$ dans $\Omega$. On va montrer que $u \geq 0$ dans $\Omega$. Pour cela, on raisonne par l'absurde. On suppose qu'il existe $a \in \Omega$ t.q. $u(a)<0$. On choisit alors $x \in \Omega$ t.q. $u(x)=\min \{u(y), y \in \bar{\Omega}\}$ (un tel $x$ existe car $\bar{\Omega}$ est compact, $u$ continue et $u=0$ sur le bord de $\Omega$ ). On a alors

$$
\partial_{i} u(x)=0 \text { et } \partial_{i}^{2} u(x) \geq 0 \text { pour tout } i \in\{1, \ldots, N\} .
$$

Ceci donne $\Delta u(x) \geq 0$ en contradiction avec $\Delta u(x)=-f(x)<0$. On obtient donc finalement que $u(x) \geq 0$ pour tout $x \in \Omega$. Un argument supplémentaire permet de remplacer l'hypothèse $f>0$ par $f \geq 0$. En effet, supposons seulement $f \geq 0$. Pour $\varepsilon>0$, on pose $u_{\varepsilon}(x)=u(x)-\varepsilon x_{1}^{2}$ de sorte que $-\Delta u_{\varepsilon}=f+2 \varepsilon>0$ dans $\Omega$. Soit $x \in \bar{\Omega}$ t.q. $u(x)=\min \left\{u_{\varepsilon}(y), y \in \bar{\Omega}\right\}$. Si $x \in \Omega$, le raisonnement précédent montre que $\Delta u_{\varepsilon}(x) \geq 0$ en contraction avec $-\Delta u_{\varepsilon}(x)=f(x)+2 \varepsilon>0$. On a donc $x \in \partial \Omega$. On en déduit que

$$
u_{\varepsilon}(y) \geq u_{\varepsilon}(x) \geq-\varepsilon \max _{x \in \partial \Omega} x_{1}^{2} \text { pour tout } y \in \bar{\Omega} .
$$

En faisant $\varepsilon \rightarrow 0$, on obtient le résultat désiré, c'est-à-dire $u \geq 0$ dans $\bar{\Omega}$.

La question posée au début de ce paragraphe consiste donc à étendre cette propriété de positivité aux solutions faibles.

Nous donnons maintenant deux petits lemmes, dûs à G. Stampacchia.

Lemme 2.21 Soit $\Omega$ un ouvert borné de $\mathbb{R}^{N}(N \geq 1)$ et $\varphi \in C^{1}(\mathbb{R}, \mathbb{R})$. On suppose que $\varphi^{\prime}$ est bornée et $\varphi(0)=0$. Soit $u \in H_{0}^{1}(\Omega)$, alors $\varphi(u) \in H_{0}^{1}(\Omega)$ et $D_{i} \varphi(u)=\varphi^{\prime}(u) D_{i} u$ p.p. (pour tout $\left.i \in\{1, \ldots, N\}\right)$. (La notation $\varphi(u)$ désigne la fonction $\varphi \circ u$.)

Démonstration Il existe une suite $\left(u_{n}\right)_{n \in \mathbb{N}}$ de fonctions appartenant à $C_{c}^{\infty}(\Omega)$ t.q. $u_{n} \rightarrow u$ dans $H_{0}^{1}(\Omega)$ (quand $n \rightarrow+\infty)$, c'est-à-dire

$$
\begin{aligned}
& u_{n} \rightarrow u \text { dans } L^{2}(\Omega), \\
& D_{i} u_{n} \rightarrow D_{i} u \text { dans } L^{2}(\Omega), \text { pour tout } i \in\{1, \ldots, N\} .
\end{aligned}
$$

Après extraction éventuelle d'une sous suite, on peut même supposer qu'il existe $F \in L^{2}(\Omega)$ et, pour tout $i \in$ $\{1, \ldots, N\}, F_{i} \in L^{2}(\Omega)$ t.q.

$$
\begin{aligned}
& u_{n} \rightarrow u \text { p.p. et }\left|u_{n}\right| \leq F \text { p.p. et pour tout } n \in \mathbb{N} \\
& D_{i} u_{n} \rightarrow D_{i} u \text { p.p. et }\left|D_{i} u_{n}\right| \leq F_{i} \text { p.p. et pour tout } n \in \mathbb{N}, i \in\{1, \ldots, N\} .
\end{aligned}
$$

On a alors $\varphi\left(u_{n}\right) \in C_{c}^{1}(\Omega)$ et pour tout $n \in \mathbb{N}$ et tout $i \in\{1, \ldots, N\}$,

$$
D_{i} \varphi\left(u_{n}\right)=\partial_{i} \varphi\left(u_{n}\right)=\varphi^{\prime}\left(u_{n}\right) \partial_{i} u_{n} .
$$

On pose $M=\sup \left\{\left|\varphi^{\prime}(s)\right|, s \in \mathbb{R}\right\}$, de sorte que $|\varphi(s)| \leq M|s|$, pour tout $s \in \mathbb{R}$. On a donc

$$
\varphi\left(u_{n}\right) \rightarrow \varphi(u) \text { p.p. et }\left|\varphi\left(u_{n}\right)\right| \leq M F \text { p.p. et pour tout } n \in \mathbb{N} \text {. }
$$

Comme $M F \in L^{2}(\Omega)$, le théorème de convergence dominée (dans $L^{2}(\Omega)$ ) donne $\varphi\left(u_{n}\right) \rightarrow \varphi(u)$ dans $L^{2}(\Omega)$. On a donc aussi $D_{i} \varphi\left(u_{n}\right) \rightarrow D_{i} \varphi(u)$ dans $\mathcal{D}^{\star}(\Omega)$. On rappelle maintenant que $D_{i} \varphi\left(u_{n}\right)=\varphi^{\prime}\left(u_{n}\right) \partial_{i} u_{n}$. Comme

$$
\begin{aligned}
& \varphi^{\prime}\left(u_{n}\right) \rightarrow \varphi^{\prime}(u) \text { p.p., } \\
& \partial_{i} u_{n} \rightarrow D_{i} u \text { p.p., } \\
& \left|\varphi^{\prime}\left(u_{n}\right) \partial_{i} u_{n}\right| \leq M F_{i} \text { p.p. et pour tout } n \in \mathbb{N} .
\end{aligned}
$$


Le théorème de convergence dominée donne $\varphi^{\prime}\left(u_{n}\right) \partial_{i} u_{n} \rightarrow \varphi^{\prime}(u) D_{i} u$ dans $L^{2}(\Omega)$ et donc aussi dans $\mathcal{D}^{\star}(\Omega)$. Par unicité de la limite dans $\mathcal{D}^{\star}(\Omega)$ on a donc $D_{i} \varphi(u)=\varphi^{\prime}(u) D_{i} u$ p.p. (et pour tout $i$ ). Finalement, on obtient donc que $\varphi(u) \in H_{0}^{1}(\Omega)$ (comme limite, pour la norme de $H^{1}(\Omega)$, de fonctions de $H_{0}^{1}(\Omega)$ ) et $D_{i} \varphi(u)=\varphi^{\prime}(u) D_{i} u$ p.p., pour tout $i$.

Lemme 2.22 Soit $\Omega$ un ouvert borné de $\mathbb{R}^{N}(N \geq 1)$. Soit $u \in H_{0}^{1}(\Omega)$. On définit $u^{+}$par $u^{+}(x)=\max \{u(x), 0\}$ Pour $x \in \Omega$. Alors, $u^{+} \in H_{0}^{1}(\Omega)$ et $D_{i} u^{+}=1_{u \geq 0} D_{i} u=1_{u>0} D_{i} u$ p.p. (pour tout $i \in\{1, \ldots, N\}$ ). En particulier on a $D_{i} u=0$ p.p. (pour tout $i$ ) sur l'ensemble $\{u=0\}$.

Démonstration Pour $n \in \mathbb{N}^{\star}$, on définit $\varphi_{n} \in C^{1}(\mathbb{R}, \mathbb{R})$ par

$$
\begin{aligned}
& \varphi_{n}(s)=0 \text { si } s \leq 0, \\
& \varphi_{n}(s)=\frac{n}{2} s^{2} \text { si } 0<s<\frac{1}{n}, \\
& \varphi_{n}(s)=s-\frac{1}{2 n} \text { si } \frac{1}{n} \leq s .
\end{aligned}
$$

On a donc $\varphi_{n}(s) \rightarrow s^{+}$pour tout $s \in \mathbb{R}$ (quand $n \rightarrow+\infty$ ) et $\left|\varphi_{n}^{\prime}(s)\right| \leq 1$ pour tout $s$ et pour tout $n \in \mathbb{N}^{\star}$. Le lemme 2.21 donne $\varphi_{n}(u) \in H_{0}^{1}(\Omega)$ et $D_{i}\left(\varphi_{n}(u)\right)=\varphi_{n}^{\prime}(u) D_{i} u$ p.p. (et pour tout $i \in\{1, \ldots, N\}$ ). D'autre part, on a

$$
\varphi_{n}(u) \rightarrow u^{+} \text {p.p., }\left|\varphi_{n}(u)\right| \leq|u| \text { p.p. et pour tout } n \in \mathbb{N} .
$$

Le théorème de convergence dominée donne donc $\varphi_{n}(u) \rightarrow u^{+}$dans $L^{2}(\Omega)$ (et donc que $D_{i} \varphi_{n}(u) \rightarrow D_{i} u^{+}$dans $\mathcal{D}^{\star}(\Omega)$ ). Puis, on remarque que $\varphi_{n}^{\prime}(u) \rightarrow 1_{\{u>0\}}$ p.p. et donc

$$
\varphi_{n}^{\prime}(u) D_{i} u \rightarrow 1_{\{u>0\}} D_{i} u \text { p.p., }\left|\varphi_{n}^{\prime}(u) D_{i} u\right| \leq\left|D_{i} u\right| \text { p.p. et pour tout } n \in \mathbb{N},
$$

ce qui (toujours par le théorème de convergence dominée) donne $\varphi_{n}^{\prime}(u) D_{i} u \rightarrow 1_{\{u>0\}} D_{i} u$ dans $L^{2}(\Omega)$ (et donc dans $\mathcal{D}^{\star}(\Omega)$ ). Comme $D_{i}\left(\varphi_{n}(u)\right)=\varphi_{n}^{\prime}(u) D_{i} u$ on en déduit (par unicité de la limite dans $\mathcal{D}^{\star}(\Omega)$ ) que $D_{i} u^{+}=$ $1_{\{u>0\}} D_{i} u$ p.p.. La suite $\left(\varphi_{n}(u)\right)_{n \in \mathbb{N}}$ est donc une suite de $H_{0}^{1}(\Omega)$, elle converge dans $H^{1}(\Omega)$ vers $u^{+}$. On a bien montré, finalement, que $u^{+} \in H_{0}^{1}(\Omega)$ et $D_{i} u^{+}=1_{\{u>0\}} D_{i} u$ p.p. (et pour tout $i$ ).

En considérant la suite $\left(\psi_{n}(u)\right)_{n \in \mathbb{N}}$ avec $\psi_{n}$ définie par $\psi_{n}(s)=\varphi(s+1 / n)-1 /(2 n)$, un raisonnement analogue montre que $D_{i} u^{+}=1_{\{u \geq 0\}} D_{i} u$ (la différence essentielle entre $\varphi_{n}$ et $\psi_{n}$ est que $\varphi_{n}^{\prime}(0)=0$ alors que $\psi_{n}^{\prime}(0)=1$ ).

Remarque 2.23 Le lemme 2.22 peut se généraliser à toute fonction lipschitzienne s'annulant en 0 , on obtient ainsi le résultat suivant : Soit $\Omega$ un ouvert borné de $\mathbb{R}^{N}(N \geq 1)$ et $\varphi$ une fonction lipschitzienne de $\mathbb{R}$ dans $\mathbb{R}$, s'annulant en 0 . Soit $u \in H_{0}^{1}(\Omega)$. On a alors $\varphi(u) \in H_{0}^{1}(\Omega)$ et $D_{i} \varphi(u)=\varphi^{\prime}(u) D_{i} u$ p.p. (pour tout $i \in$ $\{1, \ldots, N\})$. Un exemple important consiste à prendre $\varphi(s)=(s-k)^{+}$pour tout $s \in \mathbb{R}$, avec $k$ donné dans $\mathbb{R}_{+}$. On obtient ainsi, pour $u \in H_{0}^{1}(\Omega),(u-k)^{+} \in H_{0}^{1}(\Omega)$ et $D_{i} \varphi(u)=1_{\{u>k\}} D_{i} u=1_{\{u \geq k\}} D_{i} u$ p.p..

On peut maintenant répondre à la question posée au début de ce paragraphe.

Théorème 2.24 (Positivité de la solution faible) Soit $\Omega$ un ouvert borné de $\mathbb{R}^{N}, N \geq 1, a_{i, j} \in L^{\infty}(\Omega)$, pour $i, j=1, \ldots, N$. On suppose que les $a_{i, j}$ vérifient 2.1. Soit $f \in L^{2}(\Omega)$ et u la solution de 2.4. On suppose que $f \geq 0$ p.p.. On a alors $u \geq 0$ p.p..

Démonstration On suppose que $f \leq 0$ p.p. et on va montrer que $u \leq 0$ p.p. (en changeant $f$ en $-f$ et $u$ et $-u$ on obtient le résultat désiré). Comme $u$ est solution de [2.4], on a

$$
\int_{\Omega} \sum_{i, j=1}^{n} a_{i, j}(x) D_{j} u(x) D_{i} v(x) \mathrm{d} x=\int_{\Omega} f(x) v(x) \mathrm{d} x \text { pour tout } v \in H_{0}^{1}(\Omega) .
$$


On choisit, dans cette égalité, $v=u^{+}$et on obtient

$$
\alpha \int_{\Omega}|\nabla u(x)|^{2} 1_{\{u \geq 0\}}(x) \mathrm{d} x \leq \int_{\Omega} \sum_{i, j=1}^{n} a_{i, j}(x) D_{j} u(x) D_{i} u^{+}(x) \mathrm{d} x=\int_{\Omega} f(x) u^{+}(x) \mathrm{d} x \leq 0 .
$$

On en déduit que $\alpha\left\|u^{+}\right\|_{H_{0}^{1}(\Omega)}^{2}=\alpha \int_{\Omega}\left|\nabla u^{+}(x)\right|^{2} d x \leq \int_{\Omega} f(x) u^{+}(x) \mathrm{d} x \leq 0$, et donc $u^{+}=0$ p.p., c'est-àdire $u \leq 0$ p.p..

\subsection{Condition de Dirichlet non homogène}

Nous n'avons considéré jusqu'ici que les problèmes elliptiques (linéaires) avec condition aux limites homogène (c.à.d que la solution est nulle au bord du domaine). On souhaite maintenant remplacer la condition " $u=0$ " sur le bord de $\Omega$ par " $u=g$ " sur le bord de $\Omega$. Ceci va être possible en se ramenant au problème de Dirichlet avec une condition aux limites homogène (c'est-à-dire en se ramenant aux théorèmes 2.6 et 2.9) à condition que $\Omega$ soit assez régulier pour que l'opérateur trace, noté $\gamma$ et introduit au chapitre 1, voir théorème 1.23, soit bien défini et que $g$ soit dans l'image de $\gamma$ ( c'est-à-dire $g=\gamma(G)$ avec $G \in H^{1}(\Omega)$ ).

Plus précisèment, soit $\Omega$ un ouvert borné de $\mathbb{R}^{N}(N \geq 1)$ à frontière lipschitzienne. On note $\partial \Omega$ cette frontière. Soient $a_{i, j} \in L^{\infty}(\Omega)$, pour $i, j=1, \ldots, N$,vérifiant l'hypothèse d'ellipticité uniforme 2.1). Soit $f$ une fonction de $\Omega$ dans $\mathbb{R}$ et $g$ une fonction de $\partial \Omega$ dans $\mathbb{R}$. On cherche une solution au problème (2.2). Le théorème 2.6 permet de démontrer le théorème suivant, où 2.13) est la formulation faible du problème 2.2.

Théorème 2.25 (Condition de Dirichlet non homogène (1)) Soit $\Omega$ un ouvert borné de $\mathbb{R}^{N}(N \geq 1)$ à frontière lipschitzienne, $f \in L^{2}(\Omega), g \in \operatorname{Im}(\gamma)$ (où $\gamma$ désigne l'opérateur trace de $H^{1}(\Omega)$ dans $L^{2}(\Omega)$ vu au théorème 1.23). Soient $\left(a_{i, j}\right)_{i, j=1, \ldots, N} \subset L^{\infty}(\Omega)$ et $\alpha>0$ tels que 2.1] soit vérifiée. Alors il existe une unique solution de (2.13).

$$
\begin{aligned}
& u \in H^{1}(\Omega), \gamma(u)=g, \\
& \int_{\Omega}\left(\sum_{i=1}^{N} \sum_{j=1}^{N} a_{i, j}(x) D_{j} u(x) D_{i} v(x)\right) \mathrm{d} x=\int_{\Omega} f(x) v(x) \mathrm{d} x, \forall v \in H_{0}^{1}(\Omega) .
\end{aligned}
$$

La démonstration fait partie de l'exercice 2.21. Elle consiste à chercher $u-G$ comme solution faible d'un problème elliptique posé dans $H_{0}^{1}(\Omega)$ avec un second membre dans $L^{2}(\Omega)$ et $G \in H^{1}(\Omega)$ t.q. $\gamma(G)=g$. Il est possible aussi de remplacer le second membre de 2.13 par $T(v)$ où $T \in H^{-1}(\Omega)$. On obtient alors le théorème 2.26 qui se démontre aussi en cherchant $u-G$ comme solution faible d'un problème elliptique posé dans $H_{0}^{1}(\Omega)$ avec un second membre dans $H^{-1}(\Omega)$ (voir l'exercice 2.21.

Théorème 2.26 (Condition de Dirichlet non homogène (2)) Soit $\Omega$ un ouvert borné de $\mathbb{R}^{N}(N \geq 1)$ à frontière lipschitzienne, $T \in H^{-1}(\Omega), g \in \operatorname{Im}(\gamma)$ (où $\gamma$ désigne l'opérateur trace de $H^{1}(\Omega)$ dans $L^{2}(\Omega)$ vu au théorème 1.23). Soient $\left(a_{i, j}\right)_{i, j=1, \ldots, N} \subset L^{\infty}(\Omega)$ et $\alpha>0$ tels que 2.1 soit vérifiée. Alors il existe une unique solution de (2.14).

$$
\begin{aligned}
& u \in H^{1}(\Omega), \gamma(u)=g, \\
& \int_{\Omega}\left(\sum_{i=1}^{N} \sum_{j=1}^{N} a_{i, j}(x) D_{j} u(x) D_{i} v(x)\right) \mathrm{d} x=T(v), \forall v \in H_{0}^{1}(\Omega) .
\end{aligned}
$$

Remarque 2.27 Sous les hypothèses du théorème 2.25, on peut aussi montrer, par une méthode voisine de celle donnée dans le théorème 2.24 que, si $f=0$ et $A \leq g \leq B$ p.p., avec $A, B \in \mathbb{R}$ (p.p. est à prendre ici au sens de la mesure de Lebesgue $N-1$ dimensionnelle sur $\partial \Omega$ ), on a alors $A \leq u \leq B$ p.p.o ù u est la solution de (2.13. $C$ 'est ce résultat que l'on appelle "principe du maximum". 
La suite de cette section donne quelques compléments sur l'image de l'opérateur trace (noté $\gamma$ ) défini sur $H^{1}(\Omega)$ lorque $\Omega$ est un ouvert borné de $\mathbb{R}^{N}$ à frontière lipschitzienne.

Définition $2.28\left(\right.$ Espace $H^{\mathbf{1} / 2}(\partial \Omega)$ ) Soit $\Omega$ un ouvert borné de $\mathbb{R}^{N}(N \geq 1)$ à frontière lipschitzienne. On note $H^{1 / 2}(\partial \Omega)$ l'ensemble des traces des fonctions $H^{1}(\Omega)$, c'est-à-dire $H^{1 / 2}(\partial \Omega)=\operatorname{Im} \gamma$ où $\gamma$ est l'opérateur trace de $H^{1}(\Omega)$ dans $L^{2}(\partial \Omega)$ vu au théorème 1.23 . On définit sur $H^{1 / 2}(\partial \Omega)$ une norme en posant

$$
\|u\|_{H^{1 / 2}(\partial \Omega)}=\inf \left\{\|\bar{u}\|_{H^{1}(\Omega)}, \gamma(\bar{u})=u\right\} .
$$

La proposition 2.30 montre que $H^{1 / 2}(\partial \Omega)$ est alors un espace de Hilbert et que l'application $u \mapsto u$ est continue de $H^{1 / 2}(\partial \Omega)$ dans $L^{2}(\partial \Omega)$. (On dit alors que $H^{1 / 2}(\partial \Omega)$ s'injecte continûment dans $L^{2}(\partial \Omega)$.) On note $H^{-1 / 2}(\partial \Omega)$ l'espace dual de $H^{1 / 2}(\partial \Omega)$ (c'est donc aussi un espace de Hilbert).

Remarque 2.29 (Compacité de $H^{1 / 2}(\partial \Omega)$ dans $L^{2}(\partial \Omega)$ ) Dans le cadre de la définition 2.28, on peut montrer (mais ceci n'est pas fait dans ce cours) la compacité de l'application $u \mapsto u$ de $H^{1 / 2}(\partial \Omega)$ dans $L^{2}(\partial \Omega)$.

Proposition 2.30 (Propriétés de l'espace $H^{1 / 2}(\partial \Omega)$ ) Soit $\Omega$ un ouvert borné de $\mathbb{R}^{N}(N \geq 1)$ à frontière lipschitzienne. On note $\gamma$ l'opérateur trace dé $H^{1 / 2}(\partial \Omega)$ fini sur $H^{1}(\Omega)$.

1. Soit $u \in H^{1 / 2}(\partial \Omega)$. Alors $\|u\|_{H^{1 / 2}(\partial \Omega)}=\|\bar{u}\|_{H^{1}(\Omega)}$ où $\bar{u}$ est l'unique solution faible de $-\Delta \bar{u}=0$ dans $\Omega$ avec $\gamma(\bar{u})=u$, c'est-à-dire l'unique solution de

$$
\begin{aligned}
& \bar{u} \in H^{1}(\Omega), \gamma(\bar{u})=u, \\
& \int_{\Omega} \nabla \bar{u}(x) \nabla v(x) \mathrm{d} x=0, \forall v \in H_{0}^{1}(\Omega) .
\end{aligned}
$$

2. L'espace est un espace de Hilbert.

3. L'espace $H^{1 / 2}(\partial \Omega)$ s'injecte continûment dans $L^{2}(\partial \Omega)$.

La démonstration de cette proposition fait l'object de l'exercice 2.22

Soit $\Omega$ un ouvert borné de $\mathbb{R}^{N}(N \geq 1)$ à frontière lipschitzienne. Avec la définition 2.28 (et la proposition 2.30), on voit que L'opérateur trace défini sur $H^{1}(\Omega)$ est un opérateur linéaire continu de $H^{1}(\Omega)$ dans $H^{1 / 2}(\partial \Omega)$ (et sa norme est égale à 1$)$. Si maintenant $u \in H^{1}(\Omega)^{N}$, on peut définir la trace de $u$ encore notée $\gamma(u)$ en prenant la trace de chacune des composantes de $u$. On a donc $\gamma(u) \in H^{1 / 2}(\partial \Omega)^{N} \subset L^{2}(\partial \Omega)^{N}$. On note $n(x)$ le vecteur normal à $\partial \Omega$, extérieur à $\Omega$. Comme $\Omega$ est à frontière lipschitzienne, le vecteur $n(x)$ est défini p.p. en $x \in \partial \Omega$ (p.p. signifie ici, comme d'habitude, p.p. pour la mesure de Lebesgue $(N-1)$-dimensionnelle sur $\partial \Omega$ ) et la fonction $x \mapsto n(x)$ définit un élément de $L^{\infty}(\partial \Omega)$. On obtient ainsi $\gamma(u) \cdot n \in L^{2}(\partial \Omega)$. Cette (classe de) fonction(s) $\gamma(u) \cdot n$ est appelée "trace normale de $u$ sur $\partial \Omega$ ".

L'exercice 2.23 montre qu' on peut définir $\gamma(u) \cdot n$ comme un élément de $H^{-1 / 2}(\Omega)$ sous l'hypothèse $u \in L^{2}(\Omega)^{N}$ avec $\operatorname{div}(u) \in L^{2}(\Omega)$ (cette hypothèse est donc plus faible que $u \in H^{1}(\Omega)^{N}$ ). Il est toutefois intéressant de noter que, sous cette hypothèse, $\gamma(u) \cdot n$ n'est pas toujours représenté par une fonction sur $\partial \Omega$ et ceci induit une difficulté lorsque l'on souhaite considérer la restriction de $\gamma(u) \cdot n$ à une partie du bord de $\Omega$, voir à ce propos l'exercice 2.24

\subsection{Exercices}

Exercice 2.1 (Régularité en dimension 1) $f \in L^{2}(] 0,1[)$. On rappelle (cf. cours) qu'il existe un et un seul $u$ solution de 


$$
\begin{aligned}
& u \in H_{0}^{1}(] 0,1[), \\
& \int_{0}^{1} D u(t) D v(t) \mathrm{d} t=\int_{0}^{1} f(t) v(t) \mathrm{d} t, \forall v \in H_{0}^{1}(] 0,1[) .
\end{aligned}
$$

On suppose maintenant que $f \in C([0,1], \mathbb{R})\left(\subset L^{2}(] 0,1[)\right.$. On pose $F(x)=\int_{0}^{x} f(t) \mathrm{d} t$, pour tout $x \in[0,1]$. Soit $u$ la solution de 2.15 . Montrer que, pour tout $\varphi \in C([0,1], \mathbb{R})$, on a

$$
\int_{0}^{1}(D u(t)+F(t)) \varphi(t) \mathrm{d} t=\int_{0}^{1} c \varphi(t) \mathrm{d} t
$$

avec un certain $c \in \mathbb{R}$ convenablement choisi (et indépendant de $\varphi$ ).

En déduire que $D u=-F+c$ p.p., puis que $u$ est deux fois continûment dérivable sur $] 0,1\left[\right.$ et $-u^{\prime \prime}(x)=f(x)$ pour tout $x \in] 0,1[$ (et que $u(0)=u(1)=0$ ).

Corrigé -

Soit $\varphi \in C([0,1], \mathbb{R})$. Pour $x \in[0,1]$ on pose

$$
\psi(x)=\int_{0}^{x} \varphi(t) d t-x \int_{0}^{1} \varphi(t) d t .
$$

On a donc $\psi \in C^{1}([0,1]), \psi(0)=\psi(1)=0$ et la dérivée faible de $\psi$ est égale p.p. à sa dérivée classique (voir la Définition 1.2, c'est-à-dire

$$
\left.D \psi(x)=\psi^{\prime}(x)=\varphi(x)-\int_{0}^{1} \varphi(s) \text { ds pour presque tout } x \in\right] 0,1[.
$$

On a donc $\psi \in L^{2}(\Omega)$ et $D \psi \in L^{2}(\Omega)$, ce qui prouve que $\psi \in H^{1}(] 0,1[)$. Comme $\psi(0)=\psi(1)=0$, on a même $\psi \in H_{0}^{1}(\Omega)$ (voir la section 1.5 . On peut donc prendre $v=\psi$ dans 2.15, on obtient

$$
\int_{0}^{1} D u(t) \varphi(t) \mathrm{d} t-\int_{0}^{1} \varphi(t) d t \int_{0}^{1} D u(t) d t=\int_{0}^{1} f(x) \psi(x) \mathrm{d} x .
$$

Comme $F$ est de classe $C^{1}$ et $F^{\prime}=f$, on a (en utilisant aussi $\psi(0)=\psi(1)=0$ )

$$
\int_{0}^{1} f(x) \psi(x) \mathrm{d} x=\int_{0}^{1} F^{\prime}(x) \psi(x) \mathrm{d} x=-\int_{0}^{1} F(x) \psi^{\prime}(x) \mathrm{d} x=-\int_{0}^{1} F(x) \varphi(x) \mathrm{d} x+\int_{0}^{1} F(x) \mathrm{d} x \int_{0}^{1} \varphi(t) d t .
$$

En posant $c=\int_{0}^{1} D u(t) d t+\int_{0}^{1} F(t) d t$, on a donc

$$
\int_{0}^{1}(D u(t)+F(t)) \varphi(t) \mathrm{d} t=c \int_{0}^{1} \varphi(t) d t \text { pour tout } \varphi \in C([0,1])
$$

Comme $D u+F-c \in L^{2}(] 0,1[)$ et que $C([0,1])$ est dense dans $L^{2}(] 0,1[)$, on en déduit

$$
D u=-F+c \text { p.p. dans }] 0,1[\text {. }
$$

On pose maintenant

$$
w(x)=\int_{0}^{x}(-F(t)+c) d t \text { pour } x \in[0,1] .
$$

Comme w est de classe $C^{1}$ (la fonction w est même de classe $C^{2}$ ) la dérivée par transposition de w est une dérivée faible et est égale p.p. à la dérivée classique de w. On a donc $D w=w^{\prime}=-F+c$ p.p.. On a donc Dw $=D u$ p.p. et on en déduit que $w-u$ est une fonction presque partout égale à une constante (voir l'exercice 1.2). En identifiant la (classe de) fonction(s) u a son représentant continu, on a donc u de classe $C^{2}, u^{\prime}=-F+c$ et $u^{\prime \prime}=-F^{\prime}=f$. On a aussi $u(0)=u(1)$ (car $u \in H_{0}^{1}(] 0,1[)$ et donc le représentant continu de u vérifie $\left.u(0)=u(1)=0\right)$.

\section{Exercice 2.2 (Décomposition spectrale en dimension 1)}

On reprend l'exercice précédent. On pose $E=L^{2}(] 0,1\left[\right.$ ) (muni de la norme $\|\cdot\|_{2}$ ). Pour $f \in E$, on rappelle qu'il existe un et un seul $u$ solution de 2.15. 
On note $T$ l'application de $E$ dans $E$ qui à $f$ associe $u$ (solution de (2.15), noter que $H_{0}^{1}(] 0,1[) \subset E$ ). On rappelle que $T$ est un opérateur linéaire compact autoadjoint de $E$ dans $E$.

1. Soit $\lambda \in \mathcal{V P}(T)$. Montrer qu'il existe $u \in C([0,1], \mathbb{R}) \cap C^{2}(] 0,1[, \mathbb{R}), u \neq 0$, tel que $-\lambda u^{\prime \prime}=u$, sur $] 0,1[$ et $u(0)=u(1)=0$.

2. Montrer que $\mathcal{V P}(T)=\left\{\frac{1}{k^{2} \pi^{2}}, k \in \mathbb{N}^{\star}\right\}$ et $\sigma(T)=\mathcal{V P}(T) \cup\{0\}$.

3. Soit $f \in E$. Pour $n \in \mathbb{N}^{\star}$, on pose $c_{n}=2 \int_{0}^{1} f(t) \sin (n \pi t) d t$. Montrer que :

$$
\left\|f-\sum_{p=1}^{n} c_{p} \sin (p \pi \cdot)\right\|_{2} \rightarrow 0, \text { quand } n \rightarrow \infty .
$$

(Comparer avec les séries de Fourier. )

4. Soit $\mu \in \mathbb{R}^{\star}$. En utilisant le fait que $T$ est compact, donner une C.N.S. sur $f \in E$ pour que le problème suivant ait une solution :

$$
\begin{aligned}
& u \in H_{0}^{1}(] 0,1[), \\
& \int_{0}^{1} D u(t) D v(t) d t+\mu \int_{0}^{1} u(t) v(t) d t=\int_{0}^{1} f(t) v(t) d t, \forall v \in H_{0}^{1}(] 0,1[) .
\end{aligned}
$$

Corrigé -

1. On a vu au théorème 2.11 que $N(T)=\{f \in E, T f=0$ p.p. $\}=\{0\}$, que les valeurs propres de $T$ sont toutes strictement positives et qu'il existe une base hilbertienne de $L^{2}(] 0,1[)$ formée de fonctions propres de $T$ (théorème 2.12. On cherche ici une telle base hilbertienne. Pour cela, on trouve tout d'abord les valeurs propres de T.

On rappelle que, pour $f \in E$, On a $T f \in H_{0}^{1}(] 0,1[$ et, en posant $u=T f$,

$$
\int_{0}^{1} D u(t) D v(t) \mathrm{d} t=\int_{0}^{1} f(t) v(t) d t \text { pour tout } v \in H_{0}^{1}(] 0,1[) .
$$

Soit $\lambda$ une valeur propre de $T$. On sait déjà que $\lambda>0$. Il existe $f \in E, f \neq 0$ t.q. $T f=\lambda f$. En posant $u=T f$, on a donc $u \in H_{0}^{1}(] 0,1[), u \neq 0$ et $f=u / \lambda$, ce qui donne

$$
\int_{0}^{1} D u(t) D v(t) \mathrm{d} t=\frac{1}{\lambda} \int_{0}^{1} u(t) v(t) d t \text { pour tout } v \in H_{0}^{1}([0,1[) .
$$

Comme $u \in H_{0}^{1}(] 0,1[)$, on a u continu sur $[0,1]$ (plus précisément, u admet un représentant continu et on identifie u à ce représentant) et $u(0)=u(1)=0$. L'exercice 2.1 montre alors que u est de classe $C^{2}$ et que

$$
\left.-\lambda u^{\prime \prime}(x)=u(x) \text { pour tout } x \in\right] 0,1[\text {. }
$$

2. Pour chercher les valeurs propres, la question précédente nous a ramené à la résolution d'une équation différentielle linéaire classique. Il est bien connu (c'est, par exemple, une conséquence du théorème d'existence et d'unicité de Cauchy-Lipschitz ${ }^{13}$ que l'ensemble de solutions de 2.16 est un espace vectoriel de dimension 2, engendré par le fonctions $x \mapsto \sin (x / \sqrt{\lambda})$ et $x \mapsto \cos (x / \sqrt{\lambda})$.

Si $\lambda$ est valeur propre de $T$, il existe donc (par la question précédente) $u \neq 0$ t.q. Tu $=\lambda u$, u de classe $C^{2}, u$ continu sur $[0,1], u(0)=u(1)=0$ et $u$ solution de 2.16. Il existe donc $A, B \in \mathbb{R}$ t.q.

$$
u(x)=A \sin \left(\frac{x}{\sqrt{\lambda}}\right)+B \cos \left(\frac{x}{\sqrt{\lambda}}\right) \text { pour tout } x \in[0,1] .
$$

Comme $u(0)=0$, on a nécessairement $B=0$. Puis, comme $u \neq 0$, on a nécessairement $A \neq 0$. Enfin, comme $u(1)=0$, on a nécessairement $\sin (1 / \sqrt{\lambda})=0$, ce qui donne l'existence de $k \in \mathbb{Z}$ t.q. $1 / \sqrt{\lambda}=k \pi$. Comme

13. Rudolph Otto Sigismund Lipschitz (1832-1903), mathématicien allemand connu en particulier pour ses travaux en analyse, équations différentielles et théorie des nombres. 
$\lambda>0$, on a donc $k \in \mathbb{N}^{\star}, 1 / \lambda=k^{2} \pi^{2}$ et $u(x)=A \sin (k \pi x)$ pour tout $x \in[0,1]$ avec $A \neq 0$ (la fonction $u$ vérifie bien $T u=\lambda u$, ce qu'on peut vérifier facilement en remarquant qu'il suffit d'écrire la formulation faible en prenant des fonctions $v$ dans $C_{c}^{\infty}(] 0,1[)$, car $C_{c}^{\infty}(] 0,1[)$ est dense dans $\left.H_{0}^{1}(] 0,1[)\right)$.

On a ainsi trouvé toutes les valeurs propres de $T, \mathcal{V P}(T)=\left\{\frac{1}{k^{2} \pi^{2}}, k \in \mathbb{N}^{\star}\right\}$. La section 2.2 donne alors que $\sigma(T) \backslash\{0\}=\mathcal{V} \mathcal{P}(T) \backslash\{0\}$. Enfin comme $T$ n'est pas surjectif(ce qui est toujours le cas pour un opérateur linéaire compact en dimension infinie), on a $0 \in \sigma(T)$ et donc $\sigma(T)=\mathcal{V} \mathcal{P}(T) \cup\{0\}$.

3. La question précédente nous a donné les valeurs propres de T mais aussi les sous espaces propres correspondants. Cette question est alors une application immédiate des résultats de la section 2.2 Pour $n \in \mathbb{N}^{\star}$, on pose $e_{n}(x)=$ $\sqrt{2} \sin (p \pi x)$ pour tout $x \in[0,1]$. La famille $\left\{e_{n}, n \in \mathbb{N}^{\star}\right\}$ est une base hilbertienne de $L^{2}(] 0,1[)$. On a donc, pour tout $f \in L^{2}(] 0,1[)$,

$$
\left\|f-\sum_{p=1}^{n} c_{p} \sin (p \pi \cdot)\right\|_{2} \rightarrow 0, \text { quand } n \rightarrow \infty,
$$

c'est-à-dire $f=\sum_{p=1}^{\infty} c_{p} \sin (p \pi \cdot)$, la convergence de la série étant à prendre dans l'espace $L^{2}(] 0,1[)$.

Cette série n'est pas la série de Fourier de f. En effet, la série de Fourier de $f$ est obtenue avec les fonctions $\sin (2 p \pi \cdot)$ et $\cos (2 p \pi \cdot)(p \in \mathbb{Z})$. La décomposition de $f$ en série de Fourier correspond aussi à l'opérateur $u \mapsto u^{\prime \prime}$, mais avec des conditions périodiques $\left(u(0)=u(1)\right.$ et $\left.u^{\prime}(0)=u^{\prime}(1)\right)$ au lieu des conditions de Dirichlet $(u(0)=u(1)=0)$.

4. Soit $f \in E$. La fonction u est solution du problème 2.17 si et seulement si $T(f-\mu u)=u$, c'est-à-dire

$$
T(u)+\frac{1}{\mu} u=\frac{T(f)}{\mu} .
$$

Comme $T$ est compact, ce problème à une solution si et seulement si $f$ est orthogonal (dans E) au sous espace propre de T associé à $(-1 / \mu)$.

Ceci peut se redémontrer à partir des questions précédentes. En effet, on pose $b_{n}=\left(f / e_{n}\right)_{E}$ (la famille $\left\{e_{n}\right.$, $\left.n \in \mathbb{N}^{\star}\right\}$ étant la base hilbertienne de $E$ donnée la question 3 ), de sorte que $f=\sum_{p=1}^{\infty} b_{n} e_{n}$ (cette série étant convergente dans $E$ ). On a alors aussi

$$
T(f)=\sum_{n=1}^{+\infty} \frac{b_{n}}{n^{2} \pi^{2}} e_{n}
$$

Cette série étant aussi convergente dans $E$.

Soit $u \in E$. On pose $a_{n}=\left(u / e_{n}\right)_{E}$, on a ainsi

$$
T(u)+\frac{1}{\mu} u=\sum_{n=1}^{+\infty} a_{n} \frac{\mu+n^{2} \pi^{2}}{\mu n^{2} \pi^{2}} e_{n},
$$

Cette série étant convergente dans E. La fonction u est donc solution de 2.17 si et seulement si

$$
a_{n}\left(\mu+n^{2} \pi^{2}\right)=\mu b_{n} \text { pour tout } n \in \mathbb{N}^{\star} .
$$

Si $\mu \neq-n^{2} \pi^{2}$ pour tout $n \in \mathbb{N}^{\star}$, il existe une et une seule solution à 2.17.

Si il existe $p \in \mathbb{N}^{\star}$ t.q. $\mu=p^{2} \pi^{2}$, l'équation 2.17 a une solution si et seulement si $b_{p}=0$, c'est-à-dire si et seulement si $f$ est orthogonal (dans $E$ ) à $e_{p}$. Ce qui est équivalent à dire que $f$ est orthogonal au sous espace propre de $T$ associé à la valeur propre $(-1 / \mu)$.

\section{Exercice 2.3 (1ere valeur propre de $-\Delta$ )}

On reprend les notations du théorème 2.12 Soit donc $\Omega$ un ouvert borné de $\mathbb{R}^{N}, A u=-\Delta u$ avec $D(A)=$ $\left\{u \in H_{0}^{1}(\Omega) ; \Delta u \in L^{2}(\Omega)\right\}$. On note $\left(e_{n}\right)_{n \in \mathbb{N}^{*}}$ une base hilbertienne de $L^{2}(\Omega)$ formée de vecteurs propres de $A$, associés aux valeurs propres $\left(\mu_{n}\right)_{n \in \mathbb{N}^{\star}}$. Les $\mu_{n}$ sont ordonnées dans l'ordre croissant ( c'est-à-dire $\mu_{n} \leq \mu_{n+1}$ pour tout $n \in \mathbb{N}^{\star}$ ) et l'on a $\mu_{1}>0$ et $\lim _{n \rightarrow+\infty} \mu_{n}=+\infty$. 
Pour $u \in H_{0}^{1}(\Omega) \backslash\{0\}$, on pose

$$
Q(u)=\frac{\int_{\Omega}|\nabla u(x)|^{2} \mathrm{~d} x}{\int_{\Omega} u^{2}(x) \mathrm{d} x}
$$

1. Montrer que

$$
\mu_{1}=\min _{v \in H_{0}^{1}(\Omega) \backslash\{0\}} Q(v) .
$$

2. Soit $u \in H_{0}^{1}(\Omega) \backslash\{0\}$ tel que $Q(u)=\mu_{1}$, montrer que $u \in D(A)$ et $A u=\mu_{1} u$ p.p..

3. On suppose maintenant que $\Omega$ est connexe. Montrer que $\mu_{1}$ est une valeur propre simple et que les fonctions propres associées à $\mu_{1}$ ont un signe constant ( c'est-à-dire que $A u=\mu_{1} u$ implique $u \geq 0$ p.p. ou $u \leq 0$ p.p.). [On rappelle que si $u \in H_{0}^{1}(\Omega)$ on a aussi $u^{+}, u^{-} \in H_{0}^{1}(\Omega)$, lemme 2.22 On pourra alors comparer $Q(u)$ avec $Q\left(u^{+}\right)$et $Q\left(u^{-}\right)$si $u^{+}$et $u^{-}$sont des fonctions non nulles p.p..]

\section{Exercice 2.4 (Problème elliptique à coefficients non bornés)}

Soit $\Omega$ un ouvert borné de $\mathbb{R}^{N}, N \geq 1$, et $p: \Omega \rightarrow \mathbb{R}$ une fonction mesurable t.q. $\inf \{p(x), x \in \Omega\}=a>0$. On pose $H^{1}(p, \Omega)=\left\{u \in L^{2}(\Omega)\right.$ t.q. $D_{i} u \in L_{\text {loc }}^{1}(\Omega)$ et $p D_{i} u \in L^{2}(\Omega)$ pour tout $\left.i \in\{1, \ldots, N\}\right\}$.

On rappelle que $D_{i} u$ désigne la dérivée, au sens des dérivées par transposition, de $u$ dans la direction $x_{i}$, la variable de $\mathbb{R}^{N}$ étant notée $x=\left(x_{1}, \ldots, x_{N}\right)^{t}$.

Pour $u \in H^{1}(p, \Omega)$, on définit $\|u\| \operatorname{par}\|u\|^{2}=\|u\|_{2}^{2}+\sum_{i=1}^{N}\left\|p D_{i} u\right\|_{2}^{2}$, avec $\|\cdot\|_{2}=\|\cdot\|_{L^{2}(\Omega)}$.

1. (Etude de l'espace fonctionnel.)

(a) Montrer que $H^{1}(p, \Omega) \subset H^{1}(\Omega)$.

(b) Montrer que $H^{1}(p, \Omega)$, muni de la norme $\|\cdot\|$, est un espace de Hilbert. [On pourra remarquer qu'une suite de Cauchy dans $H^{1}(p, \Omega)$ est aussi de Cauchy dans $H^{1}(\Omega)$.]

On pose $H_{0}^{1}(p, \Omega)=H^{1}(p, \Omega) \cap H_{0}^{1}(\Omega)$.

2. (Espace fonctionnel, suite.) Montrer que $H_{0}^{1}(p, \Omega)$ est un s.e.v. fermé de $H^{1}(p, \Omega)$.

3. (solution faible.) Soit $h \in L^{2}(\Omega)$, montrer qu'il existe un et un seul $u$ t.q.

$$
\begin{gathered}
u \in H_{0}^{1}(p, \Omega), \\
\int_{\Omega} p^{2}(x) \nabla u(x) \cdot \nabla v(x) \mathrm{d} x=\int_{\Omega} h(x) v(x) \mathrm{d} x, \forall v \in H_{0}^{1}(p, \Omega) .
\end{gathered}
$$

4. (Précisions...)

(a) On suppose ici que $p^{2} \in L_{\text {loc }}^{1}(\Omega)$. Montrer que $C_{c}^{\infty}(\Omega) \subset H_{0}^{1}(p, \Omega)$.

(b) On prend maintenant $N=1$ et $\Omega=] 0,1[$. Donner un exemple de fonction $p$ (avec $p: \Omega \rightarrow \mathbb{R}$ mesurable et t.q. $\inf \{p(x), x \in \Omega\}>0$ ) pour lequel $C_{c}^{\infty}(\Omega) \cap H_{0}^{1}(p, \Omega)=\{0\}$ (cette question est plus difficile).

Exercice 2.5 (Deux problèmes elliptiques emboités)

Soit $\Omega$ un ouvert borné de $\mathbb{R}^{d}, d \geq 1$, et $M$ et $N$ deux matrices de taille $d \times d$ à coefficients dans $L^{\infty}(\Omega)$. On suppose qu'il existe $\alpha>0$ t.q. pour presque tout $x \in \Omega$ et pour tout $\xi \in \mathbb{R}^{d}$, on a

$$
M(x) \xi \cdot \xi \geq \alpha|\xi|^{2} \text { et } N(x) \xi \cdot \xi \geq \alpha|\xi|^{2} .
$$


1. Soit $f \in L^{2}(\Omega)$. Montrer qu'il existe un unique $u$ t.q.

$$
\left\{\begin{array}{l}
u \in H_{0}^{1}(\Omega), \\
\int_{\Omega} N(x) \nabla u(x) \cdot \nabla v(x) \mathrm{d} x=\int_{\Omega}(M(x)+N(x)) \nabla w(x) \cdot \nabla v(x) \mathrm{d} x \text { pour tout } v \in H_{0}^{1}(\Omega),
\end{array}\right.
$$

avec $w$ solution de

$$
\left\{\begin{array}{l}
w \in H_{0}^{1}(\Omega), \\
\int_{\Omega} M(x) \nabla w(x) \cdot \nabla v(x) \mathrm{d} x=\int_{\Omega} f(x) v(x) \mathrm{d} x \text { pour tout } v \in H_{0}^{1}(\Omega) .
\end{array}\right.
$$

pour les questions suivantes, on note $T(f)$ cette unique solution de 2.20) avec $w$ solution de 2.21).

2. Montrer que $T$ est une application linéaire compacte de $L^{2}(\Omega)$ dans $L^{2}(\Omega)$ ( c'est-à-dire que $T$ est linéaire, continue et transforme les parties bornées de $L^{2}(\Omega)$ en parties relativement compactes de $L^{2}(\Omega)$ ).

3. On suppose dans cette question (et seulement dans cette question) qu'il existe $\lambda \in \mathbb{R}$ t.q. $M=\lambda N$. Montrer qu'il existe une matrice $A$, ne dépendant que de $M$ et $\lambda$, tel que, si $u=T(f)$,

$$
\int_{\Omega} A(x) \nabla u(x) \cdot \nabla v(x) \mathrm{d} x=\int_{\Omega} f(x) v(x) \mathrm{d} x \text { pour tout } v \in H_{0}^{1}(\Omega) .
$$

Donner l'expression de $A$ en fonction de $M$ et $\lambda$.

4. On suppose dans cette question que $d=2$ et $1<p \leq+\infty$. Montrer que pour tout $f \in L^{p}(\Omega)$ il existe un unique $u$ solution de 2.20) avec $w$ solution de 2.21].

Montrer que l'application qui à $f$ associe $u$ (solution de (2.20) avec $w$ solution de (2.21) est compacte de $L^{p}(\Omega)$ dans $L^{q}(\Omega)$ pour $1 \leq q<+\infty$

5. On suppose dans cette question que $d=3$ et $p=6 / 5$. Montrer que pour tout $f \in L^{p}(\Omega)$ il existe un unique $u$ solution de 2.20) avec $w$ solution de 2.21.

Montrer que l'application qui à $f$ associe $u$ (solution de (2.20) avec $w$ solution de 2.21) est continue de $L^{p}(\Omega)$ dans $L^{6}(\Omega)$ et compacte de $L^{p}(\Omega)$ dans $L^{q}(\Omega)$ pour $1 \leq q<6$.

Corrigé -

1. Le théorème 2.6 donne l'existence et l'unicité de w solution de 2.21. Pour $v \in H_{0}^{1}(\Omega)$, on pose alors

$$
S(v)=\int_{\Omega}(M(x)+N(x)) \nabla w(x) \cdot \nabla v(x) \mathrm{d} x .
$$

L'application S est linéaire continue de $H_{0}^{1}(\Omega)$ dans $\mathbb{R}$, c'est donc un élément de $H^{-1}(\Omega)$. Le théorème 2.9 donne alors l'existence et l'unicité de u solution de 2.20. Ce qui est bien le résultat demandé.

2. La solution $w$ de 2.21 dépend linéairement de f. Puis, la solution u de 2.20) dépend linéairement de $w$. On en déduit que u dépend linéairement de $f$ et donc que l'application $T$ est lináaire de $L^{2}(\Omega)$ dans $H_{0}^{1}(\Omega)$ et donc aussi linéaire de $L^{2}(\Omega)$ dans $L^{2}(\Omega)$.

Si $w$ est la solution de 2.21, on a, en prenant $v=w$ dans 2.21,

$$
\alpha\|w\|_{H_{0}^{1}(\Omega)}^{2} \leq\|f\|_{L^{2}(\Omega)}\|w\|_{L^{2}(\Omega)} .
$$

En utilisant l'inégalité de Poincaré(Lemme 2.5, il existe $C_{\Omega}$, ne dépendant que $\Omega$, tel que $\|w\|_{L^{2}(\Omega)} \leq C_{\Omega}\|w\|_{H_{0}^{1}(\Omega)}$. On a donc, avec $C_{1}=C_{\Omega} / \alpha$,

$$
\|w\|_{H_{0}^{1}(\Omega)} \leq C_{1}\|f\|_{L^{2}(\Omega)} .
$$


Comme $M$ et $N$ sont à coefficients dans $L^{\infty}(\Omega)$, il existe $\beta \in \mathbb{R}_{+}$(ne dépendant que de $M$ et $N$ ) tel que, pour tout $\xi \in \mathbb{R}^{d}$

$$
|(M+N) \xi| \leq \beta|\xi| p \cdot p .
$$

On a donc, pour tout $v \in H_{0}^{1}(\Omega)$ et $S$ définie dans la première question,

$$
|S(v)| \leq \beta\|w\|_{H_{0}^{1}(\Omega)}\|v\|_{H_{0}^{1}(\Omega)} .
$$

Si $u=T(f)$, on en déduit, en prenant $v=u$ dans 2.20,

et donc, avec 2.23 et $C_{2}=\beta C_{1} / \alpha$,

$$
\alpha\|u\|_{H_{0}^{1}(\Omega)}^{2} \leq \beta\|w\|_{H_{0}^{1}(\Omega)}\|u\|_{H_{0}^{1}(\Omega)},
$$

$$
\|u\|_{H_{0}^{1}(\Omega)} \leq C_{2}\|f\|_{L^{2}(\Omega)} .
$$

Ceci prouve que l'application $f \mapsto u$ est lináire continue de $L^{2}(\Omega)$ dans $H_{0}^{1}(\Omega)$. Comme l'application $u \mapsto u$ est compacte de $H_{0}^{1}(\Omega)$ dans $L^{2}(\Omega)$ (théorème 1.25 , on en déduit que T est une une application linéaire compacte de $L^{2}(\Omega)$ dans $L^{2}(\Omega)$.

3. On commence par remarquer que les hypothèses sur $M$ et $N$ imposent $\lambda>0$. Puis, si $u=T(f)$, 2.20 et 2.21 donnent, pour tout $v \in H_{0}^{1}(\Omega)$,

$$
\int_{\Omega} M(x) \nabla u(x) \cdot \nabla v(x) \mathrm{d} x=\int_{\Omega}(\lambda+1) M(x) \nabla w(x) \cdot \nabla v(x) \mathrm{d} x=(\lambda+1) \int_{\Omega} f(x) v(x) \mathrm{d} x .
$$

Ce qui donne bien que u est solution de 2.22 avec $A=M /(\lambda+1)$.

4. Soit $f \in L^{p}(\Omega)$. On note $p^{\prime}$ l'exposant conjugué de $p$, c'est-à-dire $p^{\prime}=p /(p-1)$. Le théorème d'injection de Sobolev (théorème 1.28 donne l'existence de $C_{p}$ (ne dépendant en fait que de $p$ ) tel que, pour tout $v \in H_{0}^{1}(\Omega)$, on a $v \in L^{p^{\prime}}(\Omega)$ et

$$
\|v\|_{L^{p^{\prime}(\Omega)}} \leq C_{p}\|v\|_{H_{0}^{1}(\Omega)} .
$$

Avec l'inégalité de Hölder, on en déduit que l'application $v \mapsto \int_{\Omega} f(x) v(x) \mathrm{d} x$ est un élément $H^{-1}(\Omega)$ et que

$$
\left|\int_{\Omega} f(x) v(x) \mathrm{d} x\right| \leq C_{p}\|f\|_{L^{p}(\Omega)}\|v\|_{H_{0}^{1}(\Omega)} .
$$

On peut alors reprendre (en les adaptant légèrement) les démonstrations des deux premières questions.

Le théorème 2.9 donne l'existence et l'unicité de $w$ solution de 2.21 et on a $\alpha\|w\|_{H_{0}^{1}(\Omega)} \leq C_{p}\|f\|_{L^{p}(\Omega)}$. Puis, le théorème 2.9 donne alors l'existence et l'unicité de u solution de 2.20 et, avec $\beta$ défini à la question 2 , on obtient

$$
\|u\|_{H_{0}^{1}(\Omega)} \leq \frac{\beta C_{p}}{\alpha^{2}}\|f\|_{L^{p}(\Omega)} .
$$

Ceci donne que l'application $f \mapsto u$ est linéaire continue de $L^{p}(\Omega)$ dans $H_{0}^{1}(\Omega)$. Puis, comme l'application $u \mapsto u$ est compacte de $H_{0}^{1}(\Omega)$ dans $L^{q}(\Omega)$ pour $1 \leq q<+\infty$ (voir la remarque 1.29 , on en déduit que l'application $f \mapsto u$ est une une application linéaire compacte de $L^{p}(\Omega)$ dans $L^{q}(\Omega)$ pour $1 \leq q<+\infty$.

5. La démonstration est ici très voisine de la précédente. On a ici $p=6 / 5$ et donc le conjugué de $p$ est $p^{\prime}=6=2^{\star}$. Soit $f \in L^{6 / 5}(\Omega)$. Le théorème d'injection de Sobolev (théorème 1.28 donne l'existence de $C$ (ne dépendant de rien) tel que, pour tout $v \in H_{0}^{1}(\Omega)$, on a $v \in L^{6}(\Omega)$ et

$$
\|v\|_{L^{6}(\Omega)} \leq C\|v\|_{H_{0}^{1}(\Omega)}
$$

Avec l'inégalité de Hölder, on en déduit que l'application $v \mapsto \int_{\Omega} f(x) v(x) \mathrm{d} x$ est un élément $H^{-1}(\Omega)$ et que

$$
\left|\int_{\Omega} f(x) v(x) \mathrm{d} x\right| \leq C\|f\|_{L^{6 / 5}(\Omega)}\|v\|_{H_{0}^{1}(\Omega)} .
$$

Le théorème 2.9 donne l'existence et l'unicité de $w$ solution de 2.21 et on a $\alpha\|w\|_{H_{0}^{1}(\Omega)} \leq C\|f\|_{L^{6 / 5}(\Omega)}$. Puis, le théorème 2.9 aonne alors l'existence et l'unicité de u solution de 2.20 et, avec $\beta$ défini à la question 2 , on obtient

$$
\|u\|_{H_{0}^{1}(\Omega)} \leq \frac{\beta C}{\alpha^{2}}\|f\|_{L^{6 / 5}(\Omega)} .
$$

Ceci donne que l'application $f \mapsto u$ est linéaire continue de $L^{6 / 5}(\Omega)$ dans $H_{0}^{1}(\Omega)$. Puis, comme l'application $u \mapsto u$ est continue de $H_{0}^{1}(\Omega)$ dans $L^{6}(\Omega)$ (théorème 1.28 et est compacte de $H_{0}^{1}(\Omega)$ dans $L^{q}(\Omega)$ pour $1 \leq q<6=2^{\star}$ (voir la remarque 1.29, on en déduit que l'application $f \mapsto u$ est une application linéaire continue de $L^{6 / 5}(\Omega)$ dans $L^{6}(\Omega)$ et linéaire compacte de $L^{6 / 5}(\Omega)$ dans $L^{q}(\Omega)$ pour $1 \leq q<6$. 


\section{Exercice 2.6 (Problème de Neumann)}

Soient $\Omega$ un ouvert borné connexe de $\mathbb{R}^{N}(N \geq 1)$, à frontière lipschitzienne. On pose $H=\left\{u \in H^{1}(\Omega)\right.$, $\left.\int_{\Omega} u(x) \mathrm{d} x=0\right\}$. On rappelle que sur un tel ouvert, une fonction $L_{\text {loc }}^{1}$ dont les dérivées (au sens des dérivées par transposition) sont nulles est nécessairement constante ( c'est-à-dire qu'il existe $C \in \mathbb{R}$ t.q. cette fonction soit égale à $C$ p.p.), voir l'exercice 1.4 .

1. (Inégalité de "Poincaré moyenne".) Montrer que $H$ est un s.e.v. fermé de $H^{1}(\Omega)$ et que, sur $H$, la norme $H^{1}$ est équivalente à la norme $\|\cdot\|_{m}$ définie par $\|u\|_{m}=\|(|\nabla u|)\|_{L^{2}(\Omega)}$.

[On pourra montrer, en raisonnant par l'absurde, qu'il existe $C$, ne dépendant que $\Omega$, t.q. $\|u\|_{L^{2}(\Omega)} \leq C\|u\|_{m}$, pour tout $u \in H$.]

2. (Caractérisation de $\left(H^{1}(\Omega)\right)^{\prime}$.) Soit $T \in\left(H^{1}(\Omega)\right)^{\prime}$, Montrer qu'il existe $a \in \mathbb{R}$ et $F \in\left(L^{2}(\Omega)\right)^{N}$ t.q.

$$
\langle T, u\rangle_{\left(H^{1}(\Omega)\right)^{\prime}, H^{1}(\Omega)}=a \int_{\Omega} u(x) \mathrm{d} x+\int_{\Omega} F(x) \cdot \nabla u(x) \mathrm{d} x, \forall u \in H^{1}(\Omega) .
$$

[On pourra considérer $T_{\left.\right|_{H}}$ et utiliser une injection convenable de $H$ dans $L^{2}(\Omega)^{N}$.]

Pour tout $x \in \Omega$, on se donne une matrice, notée $A(x)$, dont les coefficients sont notés $a_{i, j}(x), i, j=1, \ldots, N$. On suppose que $a_{i, j} \in L^{\infty}(\Omega)$ pour tout $i, j=1, \ldots, N$ et qu'il existe $\alpha>0$ t.q $A(x) \xi \cdot \xi \geq \alpha|\xi|^{2}$, pour tout $\xi \in \mathbb{R}^{N}$ et p.p. en $x \in \Omega$. Soient $a \in \mathbb{R}$ et $F \in\left(L^{2}(\Omega)\right)^{N}$. On cherche $u$ solution de

$$
\begin{aligned}
& u \in H^{1}(\Omega), \\
& \int_{\Omega} A(x) \nabla u(x) \cdot \nabla v(x) \mathrm{d} x=a \int_{\Omega} v(x) \mathrm{d} x+\int_{\Omega} F(x) \cdot \nabla v(x) \mathrm{d} x, \forall v \in H^{1}(\Omega) .
\end{aligned}
$$

3. (Existence et unicité.)

(a) Si $a \neq 0$, montrer que 2.25 n'a pas de solution.

(b) Si $a=0$, montrer que 2.25) a une solution et que cette solution est unique si l'on demande qu'elle appartienne à $H$.

(c) Dans cette question, on suppose que $a=0, a_{i, j} \in C^{\infty}(\bar{\Omega}, \mathbb{R})$ pour tout $i, j=1, \ldots, N, F \in C^{\infty}\left(\bar{\Omega}, \mathbb{R}^{N}\right)$, $\Omega$ est de classe $C^{\infty}$ et que la solution (appartenant à $H$ ) de $(2.25)$ est aussi dans $C^{\infty}(\bar{\Omega}, \mathbb{R})$, montrer que $-\operatorname{div}(A \nabla u)=-\operatorname{div} F$, dans $\Omega$, et que $A \nabla u \cdot \boldsymbol{n}=F \cdot \boldsymbol{n}$ sur $\partial \Omega$, où $\boldsymbol{n}$ est la normale à $\partial \Omega$, extérieure à $\Omega$.

4. (Dépendance par rapport aux paramètres.) On suppose $a=0$ et on note $u$ la solution (appartenant à $H$ ) de 2.25. On suppose que, pour tout $n \in \mathbb{N}, u_{n} \in H$ est la solution de 2.25) avec $A_{n}$ au lieu de $A$ et $F_{n}$ au lieu de $F$ (et $a=0)$. On suppose que

- $A_{n}=\left(a_{i, j}^{(n)}\right)_{i, j=1, \ldots, N}$ vérifie, pour tout $n$, les mêmes hypothèses que $A$ avec un $\alpha$ indépendant de $n$,

- $\left(a_{i, j}^{(n)}\right)_{n \in \mathbb{N}}$ est bornée dans $L^{\infty}(\Omega)$, pour tout $i, j=1, \ldots, N$,

$-a_{i, j}^{(n)} \rightarrow a_{i, j} p . p$., quand $n \rightarrow \infty$, pour tout $i, j=1, \ldots, N$,

$-F_{n} \rightarrow F$ dans $L^{2}(\Omega)^{N}$, quand $n \rightarrow \infty$.

Montrer que $\left(u_{n}\right)_{n \in \mathbb{N}}$ est bornée dans $H$, puis que $u_{n} \rightarrow u$ faiblement dans $H^{1}(\Omega)$ (quand $n \rightarrow \infty$ ) et enfin que $u_{n} \rightarrow u$ dans $H^{1}(\Omega)$.

5. (Régularité $H^{2}$ par la technique des réflexions, cette question est indépendante de la précédente.). On suppose que $a=0$ et qu'il existe $f \in L^{2}(\Omega)$ t.q. $\int_{\Omega} F(x) \cdot \nabla v(x) \mathrm{d} x=\int_{\Omega} f(x) \cdot v(x) \mathrm{d} x$, pour tout $v \in H^{1}(\Omega)$. On note $u$ la solution (appartenant à $H$ ) de 2.25 ) et on suppose que $N=2$ et que $\Omega=] 0,1[\times] 0,1\left[\right.$. On pose $\Omega_{s}=$ 
] - $1,1[\times] 0,1\left[\right.$. On définit $A, f$ et $u$ sur $\Omega_{s}$ en posant $a_{i, j}\left(x_{1}, x_{2}\right)=a_{i, j}\left(-x_{1}, x_{2}\right)$ si $\left.\left(x_{1}, x_{2}\right) \in\right]-1,0[\times] 0,1[$ et $i=j, a_{i, j}\left(x_{1}, x_{2}\right)=-a_{i, j}\left(-x_{1}, x_{2}\right)$ si $\left.\left(x_{1}, x_{2}\right) \in\right]-1,0[\times] 0,1\left[\right.$ et $i \neq j, f\left(x_{1}, x_{2}\right)=f\left(-x_{1}, x_{2}\right)$ si $\left.\left(x_{1}, x_{2}\right) \in\right]-1,0[\times] 0,1\left[\right.$ et $u\left(x_{1}, x_{2}\right)=u\left(-x_{1}, x_{2}\right)$ si $\left.\left(x_{1}, x_{2}\right) \in\right]-1,0[\times] 0,1[$. Montrer que $u$ est solution de (2.25, avec $\Omega_{s}$ au lieu de $\Omega$.

En utilisant ainsi plusieurs réflexions, montrer que $u \in H^{2}(\Omega)$ dans le cas $A(x)=\operatorname{Id}$ pour tout $x \in \Omega$.

Corrigé -

1. Pour $u \in H^{1}(\Omega)$, on pose $S(u)=\int_{\Omega} u(x) \mathrm{d} x$. L'application $S$ est bien définie sur $H^{1}(\Omega)\left(\right.$ car $H^{1}(\Omega) \subset L^{2}(\Omega) \subset$ $L^{1}(\Omega)$ ). Elle est linéaire. Enfin, elle est continue car

$$
S(u) \leq\|u\|_{L^{1}(\Omega)} \leq\|u\|_{L^{2}(\Omega)} \operatorname{mes}(\Omega)^{\frac{1}{2}} \leq\|u\|_{H^{1}(\Omega)} \operatorname{mes}(\Omega)^{\frac{1}{2}},
$$

où mes $(\Omega)$ est la mesure de Lebesgue ( $N$-dimensionnelle) de $\Omega$. Comme $H=\operatorname{Ker}(S)$, on en déduit que $H$ est s.e.v. fermé de $H^{1}(\Omega)$.

Pour tout $u \in H^{1}(\Omega)$, on a $\||\nabla u|\|_{L^{2}(\Omega)}^{2} \leq\||\nabla u|\|_{L^{2}(\Omega)}^{2}+\|u\|_{L^{2}(\Omega)}^{2}=\|u\|_{H^{1}(\Omega)}^{2}$. On a donc $\|u\|_{m} \leq\|u\|_{H^{1}(\Omega)}$ pour tout $u \in H$. Pour montrer que $\|\cdot\|_{m}$ est équivalente dans $H \grave{a}\|\cdot\|_{H^{1}(\Omega)}$, il suffit donc de montrer qu'il existe $C>0$ (ne dépendant que de $\Omega$ ) t.q.

$$
\|u\|_{L^{2}(\Omega)} \leq C\|u\|_{m} \text { pour tout } u \in H .
$$

(On aura alors $\|u\|_{H^{1}(\Omega)}^{2} \leq\left(C^{2}+1\right)\|u\|_{m}^{2}$ pour tout $u \in H$.)

Pour montrer 2.26, on raisonne par l'absurde. On suppose qu'il existe une suite déléments de $H,\left(u_{n}\right)_{n \in \mathbb{N}}$ telle que

$$
\left\|u_{n}\right\|_{L^{2}(\Omega)}>n\left\|u_{n}\right\|_{m} \text { pour tout } n \in \mathbb{N} .
$$

En remplaçant $u_{n}$ par $\frac{u_{n}}{\left\|u_{n}\right\|_{L^{2}(\Omega)}}$, on peut supposer $\left\|u_{n}\right\|_{L^{2}(\Omega)}=1$. On a alors aussi $\left\|u_{n}\right\|_{m} \leq 1 / n$, ce qui prouve que la suite $\left(u_{n}\right)_{n \in \mathbb{N}}$ est bornée dans $H^{1}(\Omega)$. Par les théorèmes de compacité vus au chapitre 1 ( section 1.6 , on en déduit que la suite $\left(u_{n}\right)_{n \in \mathbb{N}}$ est relativement compacte dans $L^{2}(\Omega)$. On peut supposer (après extraction d'une sous suite) qu'il existe $u \in L^{2}(\Omega)$ t.q. $u_{n} \rightarrow u$ dans $L^{2}(\Omega)$, quand $n \rightarrow+\infty$. Comme $\left\|u_{n}\right\|_{L^{2}(\Omega)}=1$ pour tout $n \in \mathbb{N}$, on a aussi $\|u\|_{L^{2}(\Omega)}=1$. On remarque aussi que les dérivées (par transposition) de $u_{n}$ convergent vers les dérivées de $u$ dans $\mathcal{D}^{\star}$. Or, de $\left\|u_{n}\right\|_{m} \leq 1 / n$ on déduit $\nabla u_{n} \rightarrow 0$ dans $L^{2}(\Omega)^{N}$. Comme la convergence $L^{2}$ entraîne la convergence dans $\mathcal{D}^{\star}$, on a donc $\nabla u=0$. Ceci montre que u est constante sur $\Omega$ (exercice 1.4. Comme $u_{n} \rightarrow u$ dans $H^{1}(\Omega)$ et que $u_{n} \in H$ pour tout $n \in \mathbb{N}$, on a aussi $u \in H$ et donc $\int_{\Omega} u(x) \mathrm{d} x=0$. On en déduit que $u=0$ p.p., ce qui est impossible car $\|u\|_{L^{2}(\Omega)}=1$.

2. Pour $v=\left(v_{1}, \ldots, v_{N}\right)^{t} \in L^{2}(\Omega)^{N}$, on pose $\|v\|_{L^{2}(\Omega)^{N}}=\int_{\Omega}|v(x)|^{2} \mathrm{~d} x$, de sorte que $L^{2}(\Omega)^{N}$ muni de cette norme est un espace de Hilbert. Pour $u \in H$, on pose $J(u)=\nabla u=\left(D_{1} u, \ldots, D_{N} u\right)^{t}$. L'application J est alors une isométrie de $H$ (muni de la norme $\|\cdot\|_{m}$ ) dans une partie de $L^{2}(\Omega)^{N}$, notée $\operatorname{Im}(J)$.

Soit $v \in \operatorname{Im}(J)$, il existe un unique $u \in H$ t.q. $v=J(u)$. On pose $S(v)=\langle T, u\rangle_{\left(H^{1}(\Omega)\right)^{\prime}, H^{1}(\Omega)}$. Comme J est une isométrie et que la norme $\|\cdot\|_{H^{1}(\Omega)}$ est équivalente dans $H$ à la norme $\|\cdot\|_{m}$, l'application $S$ est linéaire continue de $\operatorname{Im}(J)$, s.e.v. de $L^{2}(\Omega)^{N}$, dans $\mathbb{R}$. Par le théorème de Hahn-Banach, on peut donc prolonger $S$ en $\tilde{S}$, élément du dual topologique de $L^{2}(\Omega)^{N}$. Par le théorème de représentation de Riesz dans les espaces de Hilbert, il existe alors $F \in L^{2}(\Omega)^{N}$ t.q.

$$
\tilde{S}(v)=\int_{\Omega} F(x) \cdot v(x) \mathrm{d} x
$$

On a donc, pour tout $u \in H$,

$$
\langle T, u\rangle_{\left(H^{1}(\Omega)\right)^{\prime}, H^{1}(\Omega)}=\int_{\Omega} F(x) \cdot \nabla u(x) \mathrm{d} x .
$$

On pose maintenant

$$
a=\frac{1}{m e s(\Omega)}\left\langle T, 1_{\Omega}\right\rangle_{\left(H^{1}(\Omega)\right)^{\prime}, H^{1}(\Omega)},
$$

(où $1_{\Omega}$ désigne la fonction constante égale à 1 dans $\Omega$ ). 
Pour $u \in H^{1}(\Omega)$, on a $u=u-m+m$ (ou, plus rigoureusement, $u=u-m 1_{\Omega}+m 1_{\Omega}$ p.p.) avec

$$
m=\frac{1}{m e s(\Omega)} \int_{\Omega} u(x) \mathrm{d} x .
$$

Comme $u-m \in H$ et $\nabla(u-m)=\nabla u$ p.p. on a $\langle T, u\rangle_{\left(H^{1}(\Omega)\right)^{\prime}, H^{1}(\Omega)}=\int_{\Omega} F(x) \cdot \nabla u(x) \mathrm{d} x$ et donc

$\langle T, u\rangle_{\left(H^{1}(\Omega)\right)^{\prime}, H^{1}(\Omega)}=\langle T, u-m\rangle_{\left(H^{1}(\Omega)\right)^{\prime}, H^{1}(\Omega)}+m\left\langle T, 1_{\Omega}\right\rangle_{\left(H^{1}(\Omega)\right)^{\prime}, H^{1}(\Omega)}=\int_{\Omega} F(x) \cdot \nabla u(x) \mathrm{d} x+a \int_{\Omega} u(x) \mathrm{d} x$.

3.(a) On suppose que u est solution de 2.25. En prenant $v=1_{\Omega}$ dans 2.25, on a alors

$$
0=\operatorname{ames}(\Omega)+0
$$

Ce qui prouve que $a=0$.

(b) On applique le lemme de Lax-Milgram (lemme 2.4 dans l'espace de Hilbert H (muni de la norme $\|\cdot\|_{m}$ ) avec

et

$$
a(u, v)=\int_{\Omega} A(x) \nabla u(x) \nabla v(x) \mathrm{d} x,
$$

$$
T(v)=\int_{\Omega} F(x) \cdot \nabla v(x) \mathrm{d} x
$$

La continuité de a vient du fait que $a_{i, j} \in L^{\infty}(\Omega)$ pour tout $i, j$. La coercivité de a vient de l'existence de $\alpha>0$ donnée dans les hypothèses sur A. Enfin, la continuité de $T$ vient du fait que $F \in L^{2}(\Omega)^{N}$.

On obtient ainsi un unique $u \in H$ t.q. 2.25 soit vrai pour tout $v \in H$. Comme 2.25 est aussi vrai si $v$ est une fonction constante, on obtient aussi l'existence et l'unicité de $u \in H$ t.q. 2.25 soit vrai pour tout $v \in H^{1}(\Omega)$.

(c) On prend tout d'abord $v \in C_{c}^{\infty}(\Omega)$ dans 2.25 (avec a $=0$ ). La régularité de $A, F$, u et v nous permet d'intégrer par parties (la régularité de $\Omega$ ne sert à rien pour cette étape). On obtient

$$
\int_{\Omega}(-\operatorname{div}(A(x) \nabla u(x))+\operatorname{div}(F(x))) v(x) \mathrm{d} x=0 \text { pour tout } v \in C_{c}^{\infty}(\Omega) .
$$

On en déduit que $-\operatorname{div}(A(x) \nabla u(x))+\operatorname{div}(F(x))=0$ p.p. (par le lemme fondamental 1.1 puis, par continuité de la fonction $-\operatorname{div}(A \nabla u)+\operatorname{div}(F)$, que $-\operatorname{div}(A(x) \nabla u(x))+\operatorname{div}(F(x))=0$ pour tout $x \in \Omega$.

On prend maintenant des fonctions $v \in C^{\infty}(\bar{\Omega})$ dans 2.25 . On peut ici aussi intégrer par parties (on utilise ici la régularité de $\Omega$ ). On obtient

$$
\int_{\partial \Omega}(A(x) \nabla u(x)-F(x)) \cdot n(x) v(x) d \gamma(x)=0 \text { pour tout } v \in C^{\infty}(\bar{\Omega}),
$$

où $\partial \Omega$ est le bord de $\Omega$ et $d \gamma(x)$ désigne l'intégration par rapport à la mesure $(N-1)$-dimensionnelle sur $\partial \Omega$.

Par une technique de "cartes locales", on peut se ramener au cas du lemme fondamental (lemme 1.1 pour en déduire que $(A \nabla u-F) \cdot n=0$ p.p. sur $\partial \Omega$ puis partout sur $\partial \Omega$. Mais il est plus rapide de voir qu'il est possible de choisir v t.q. $v=(A \nabla u-F) \cdot n$ sur $\partial \Omega$. On obtient ainsi directement $(A \nabla u-F) \cdot n=0$ sur $\partial \Omega$.

\section{Exercice 2.7 (Norme $H^{2}$ sur $\mathbb{R}^{N}$ )}

Soit $N \geq 1$. Cet exercice montre que dans $\mathbb{R}^{N}$ la norme $H^{2}$ est équivalente à la somme de la norme $L^{2}$ de la fonction et de la norme $L^{2}$ de son laplacien. Cette équivalence est utilisée pour étudier un problème avec le bilaplacien.

1. Soit $f \in L^{2}\left(\mathbb{R}^{N}\right)$

(a) Soit $u \in H^{1}\left(\mathbb{R}^{n}\right)$ et $i \in\{1, \ldots, N\}$. Montrer que $\Delta u-u=\frac{\partial f}{\partial x_{i}}$ dans $\mathcal{D}^{\star}\left(\mathbb{R}^{N}\right)$ si et seulement si $u$ vérifie

$$
\int \nabla u(x) \cdot \nabla v(x) \mathrm{d} x+\int u(x) v(x) \mathrm{d} x=\int f(x) \frac{\partial v}{\partial x_{i}} \mathrm{~d} x \text { pour tout } v \in H^{1}\left(\mathbb{R}^{N}\right) .
$$


(b) Montrer qu'il existe un et un seul $u \in H^{1}\left(\mathbb{R}^{N}\right)$ solution de 2.27$)$ et que $\|u\|_{H^{1}} \leq\|f\|_{L^{2}}$.

2. Soit $u \in H^{2}\left(\mathbb{R}^{N}\right)$. Montrer qu'il existe $C_{1}$ et $C_{2}$ strictement positifs, ne dépendant (éventuellement) que de $N$, tels que

$$
C_{1}\left(\|u\|_{L^{2}}+\|\Delta u\|_{L^{2}}\right) \leq\|u\|_{H^{2}} \leq C_{2}\left(\|u\|_{L^{2}}+\|\Delta u\|_{L^{2}}\right) .
$$

3. On note $H^{-2}\left(\mathbb{R}^{N}\right)$ le dual (topologique) de $H^{2}\left(\mathbb{R}^{N}\right)$. Soit $f \in H^{-2}\left(\mathbb{R}^{N}\right)$ et $\lambda>0$.

(a) Soit $u \in H^{2}\left(\mathbb{R}^{N}\right)$. Montrer que $\Delta(\Delta) u+\lambda u=f$ dans $\mathcal{D}^{\star}\left(\mathbb{R}^{N}\right)$ si et seulement si $u$ est vérifie

$$
\int \Delta u(x) \Delta v(x) \mathrm{d} x+\lambda \int u(x) v(x) \mathrm{d} x=\langle f, v\rangle_{H^{-2}, H^{2}} .
$$

(b) Montrer qu'il existe un et un seul $u \in H^{2}\left(\mathbb{R}^{N}\right)$ solution de 2.28

\section{Exercice 2.8 (Modélisation d'un problème de contact)}

On pose $\left.B=\left\{x \in \mathbb{R}^{2},|x|<2\right\}, I=\right]-1,1\left[(\subset \mathbb{R})\right.$, et $\Omega=B \backslash[-1,1] \times\{0\}\left(\Omega\right.$ est donc un ouvert de $\left.\mathbb{R}^{2}\right)$. On note $\partial B=\bar{B}-B$. On rappelle que $|x|$ désigne la norme euclidienne de $x \in \mathbb{R}^{2}$ et $x \cdot y$ le produit scalaire correspondant de $x$ et $y\left(\in \mathbb{R}^{2}\right)$.

Soient $f \in L^{2}(\Omega)$ et $g \in L^{\infty}(I)$ t.q. $g \geq 0$ p.p. (sur $I$ ). On s'intéresse au problème suivant.

$$
\begin{aligned}
-\Delta u(x) & =f(x), x \in \Omega, \\
u(x) & =0, x \in \partial B \\
\frac{\partial u}{\partial y}\left(x, 0^{+}\right) & =\frac{\partial u}{\partial y}\left(x, 0^{-}\right), x \in I, \\
\frac{\partial u}{\partial y}\left(x, 0^{+}\right) & =g(x)\left(u\left(x, 0^{+}\right)-u\left(x, 0^{-}\right)\right), x \in I .
\end{aligned}
$$

1. (Recherche d'une formulation faible)

On suppose, dans cette question, que $f$ est une fonction continue sur $\bar{\Omega}$ et $g$ une fonction continue sur $I$. On note $\Omega_{+}=\Omega \cap\{(x, y), y>0\}$ et $\Omega_{-}=\Omega \cap\{(x, y), y<0\}$. Soit $u \in C^{2}(\Omega, \mathbb{R})$ t.q. $u_{\left.\right|_{\Omega_{+}}} \in C^{2}\left(\overline{\Omega_{+}}\right)$ et $u_{\left.\right|_{\Omega_{-}}} \in C^{2}\left(\overline{\Omega_{-}}\right)$. Noter alors que toutes les expressions dans 2.29- 2.32 ont bien un sens. On a, par exemple, $u\left(x, 0^{+}\right)=\lim _{y \rightarrow 0, y>0} u(x, y)$.

Montrer que $u$ est solution "classique" de 2.29)-2.32 (c'est-à-dire vérifie 2.29) pour tout $x \in \Omega, 2.30$ pour tout $x \in \partial B$ et 2.31, 2.32) pour tout $x \in I$ ) si et seulement si $u$ vérifie :

$$
\begin{aligned}
& u(x)=0, \forall x \in \partial B, \\
& \int_{\Omega} \nabla u(x) \cdot \nabla v(x) \mathrm{d} x+ \\
& \int_{I}^{\Omega} g(x)\left(u\left(x, 0^{+}\right)-u\left(x, 0^{-}\right)\right)\left(v\left(x, 0^{+}\right)-v\left(x, 0^{-}\right)\right) \mathrm{d} x=\int_{\Omega} f(x) v(x) \mathrm{d} x,
\end{aligned}
$$

pour tout $v \in C^{2}(\Omega, \mathbb{R})$ t.q. $v_{\left.\right|_{\Omega_{+}}} \in C^{2}\left(\overline{\Omega_{+}}\right), v_{\Omega_{-}} \in C^{2}\left(\overline{\Omega_{-}}\right)$et $v(x)=0$ pour tout $x \in \partial B$. Noter que $d x$ désigne l'intégration par rapport à la mesure de Lebesgue (1 ou 2 dimensionnelle).

2. (Construction de l'espace fonctionnel) On se donne une fonction $\rho \in C_{c}^{\infty}\left(\mathbb{R}^{2}, \mathbb{R}_{+}\right)$t.q. $\rho(x)=0$, si $|x| \geq 1$, et d'intégrale 1 (sur $\mathbb{R}^{2}$ ). Pour $n \in \mathbb{N}$, on définit $\rho_{n}$ par $\rho_{n}(x)=n^{2} \rho(n x)$, pour tout $x \in \mathbb{R}^{2}$. 
(a) (Trace sur $\partial B$, sans "cartes locales") Soit $u \in H^{1}(\Omega)$. Pour $n>5$, on pose $u_{n}(x)=\int_{\Omega} u(y) \rho_{n}(x(1-$ $\left.\left.\frac{1}{n}\right)-y\right) d y$, pour $x \in D$, avec $D=\left\{x \in B, \frac{3}{2}<|x|<2\right\}$. Montrer que $u_{n} \in C^{\infty}(\bar{D})$, et que $u_{n} \rightarrow u_{\left.\right|_{D}}$, dans $H^{1}(D)$, quand $n \rightarrow \infty$.

En déduire qu'il existe un opérateur linéaire continu $\gamma$ de $H^{1}(\Omega)$ dans $L^{2}(] 0,2 \pi[)$ t.q. $\gamma(u)(\theta)=$ $u(2 \cos \theta, 2 \sin \theta)$ p.p. en $\theta \in] 0,2 \pi\left[\right.$ si $u \in H^{1}(\Omega)$ et $u$ est continue $\operatorname{sur} \bar{B} \backslash[-1,1] \times\{0\}$.

(b) Montrer qu'il existe $\gamma_{+}$[resp. $\gamma_{-}$] linéaire continu de $H^{1}(\Omega)$ dans $L^{2}(I)$ t.q. $\gamma_{+}(u)(x)=u(x, 0+)$ [resp. $\left.\gamma_{-}(u)(x)=u(x, 0-)\right]$ p.p. en $x \in I$ si $u \in H^{1}(\Omega)$ et $u_{\left.\right|_{\Omega_{+}}}$est continue sur $\overline{\Omega_{+}}$[resp. $u_{\left.\right|_{\Omega_{-}}}$est continue sur $\left.\overline{\Omega_{-}}\right]$.

3. (Coerci(ti)vité)

On pose $H=\operatorname{Ker} \gamma$ (où $\gamma$ est défini à la question précédente).

Montrer qu'il existe $C$ t.q. $\|u\|_{L^{2}(\Omega)} \leq C\||\nabla u|\|_{L^{2}(\Omega)}$ pour tout $u \in H$. [On pourra, par exemple, remarquer que $u_{\Omega_{+}} \in H^{1}\left(\Omega_{+}\right)$et $\left.u_{\Omega_{\Omega_{-}}} \in H^{1}\left(\Omega_{-}\right)\right]$

4. (Existence et unicité de solutions faibles)

On rappelle que $H=\operatorname{Ker} \gamma$. Montrer qu'il existe un et un seul $u$ solution de (2.34).

$$
\left\{\begin{array}{l}
u \in H \\
\int_{\Omega} \nabla u(x) \cdot \nabla v(x) \mathrm{d} x+\int_{I} g(x)\left(\gamma_{+} u(x)-\gamma_{-} u(x)\right)\left(\gamma_{+} v(x)-\gamma_{-} v(x)\right) \mathrm{d} x \\
=\int_{\Omega} f(x) v(x) \mathrm{d} x, \forall v \in H .
\end{array}\right.
$$

5. Pour $n \in \mathbb{N}$, on note $u_{n}$ la solution de 2.34) avec $g$ t.q. $g(y)=n$, pour tout $y \in I$. Montrer que $u_{n} \rightarrow u$ (en un sens à préciser), quand $n \rightarrow \infty$, où $u$ est la (unique) solution (faible) de $-\Delta u=f$ dans $B, u=0$ sur $\partial B$.

\section{Exercice 2.9 (De Fourier à Dirichlet...)}

Soient $\sigma \geq 0, f \in L^{2}\left(\mathbb{R}_{+}^{N}\right)$ et $g \in L^{2}\left(\mathbb{R}^{N-1}\right)$. On s'intéresse au problème suivant :

$$
\begin{aligned}
& -\Delta u(x)+u(x)=f(x), x \in \mathbb{R}_{+}^{N}, \\
& -\partial_{1} u(0, y)+\sigma u(0, y)=g(y), y \in \mathbb{R}^{N-1} .
\end{aligned}
$$

1. Donner une définition de solution "classique" de 2.35 et de solution "faible" de 2.35.

2. Montrer l'existence et l'unicité de la solution faible de 2.35.

3. Montrer que si $g=0$ presque partout, la solution faible de 2.35 (trouvée à la question précédente) appartient à $H^{2}\left(R_{+}^{N}\right)$.

4. Toujours lorsque $g=0$ presque partout, on note $u_{n}$ la solution forte associée à $\sigma=n$. Montrer que $u_{n}$ converge dans $H^{1}\left(\mathbb{R}_{+}^{N}\right)$ vers $u$ solution faible de :

$$
\begin{aligned}
& -\Delta u(x)+u(x)=f(x), x \in \mathbb{R}_{+}^{N}, \\
& u(0, y)=0, y \in \mathbb{R}^{N-1} .
\end{aligned}
$$

\section{Exercice 2.10 (Equation de Schrödinger)}

Soit $N \geq 1$. On note $\Omega$ la boule unité de $\mathbb{R}^{N}$ (en fait, les résultats de cet exercice restent vrais si $\Omega$ un ouvert borné "assez régulier" de $\mathbb{R}^{N}$ ).

Pour $f_{1}, f_{2} \in L^{2}(\Omega)$, on s'intéresse au système : 


$$
\begin{aligned}
& -\Delta u_{1}+u_{2}=f_{1} \text { dans } \Omega, \\
& -\Delta u_{2}-u_{1}=f_{2} \text { dans } \Omega,
\end{aligned}
$$

avec diverses conditions aux limites.

1. On considère dans cette première question la condition aux limites :

$$
u_{1}=0, u_{2}=0 \text { sur } \partial \Omega .
$$

Soit $f_{1}, f_{2} \in L^{2}(\Omega)$, on dit que $\left(u_{1}, u_{2}\right)$ est solution faible du problème 2.37)- 2.38 si

$$
\begin{aligned}
& u_{1} \in H_{0}^{1}(\Omega), u_{2} \in H_{0}^{1}(\Omega), \\
& \int_{\Omega}^{\nabla} u_{1}(x) \cdot \nabla \varphi(x) \mathrm{d} x+\int_{\Omega} u_{2}(x) \varphi(x) d x=\int_{\Omega} f_{1}(x) \varphi(x) \mathrm{d} x, \forall \varphi \in H_{0}^{1}(\Omega), \\
& \int_{\Omega} \nabla u_{2}(x) \cdot \nabla \varphi(x) \mathrm{d} x-\int_{\Omega} u_{1}(x) \varphi(x) d x=\int_{\Omega} f_{2}(x) \varphi(x) \mathrm{d} x, \forall \varphi \in H_{0}^{1}(\Omega) .
\end{aligned}
$$

(a) Montrer que le problème (2.39) admet une et une seule solution. [Utiliser l'espace $V=H_{0}^{1}(\Omega) \times$ $H_{0}^{1}(\Omega)$.]

(b) Montrer que le problème (2.37)-2.38) admet une et une seule solution au sens suivant : $u_{1} \in H^{2}(\Omega) \cap$ $H_{0}^{1}(\Omega), u_{2} \in H^{2}(\Omega) \cap H_{0}^{1}(\Omega)$ et les équations 2.37) sont satisfaites p.p. sur $\Omega$. [Utiliser, en particulier, la question précédente et un théorème de régularité 2.14. Ne pas oublier de montrer aussi l'unicité.]

On suppose maintenant que $f_{1}, f_{2} \in C^{\infty}(\bar{\Omega})$. Montrer que $u_{1}, u_{2} \in C^{\infty}(\bar{\Omega})$.

(c) Pour $f=\left(f_{1}, f_{2}\right) \in L^{2}(\Omega) \times L^{2}(\Omega)$, soit $u=\left(u_{1}, u_{2}\right)$ la solution de (2.39), on note $u=T(f)$. Montrer que l'opérateur $T: f \mapsto u$ est un opérateur linéaire continu et compact de $L^{2}(\Omega) \times L^{2}(\Omega)$ dans lui-même.

2. On considère dans cette deuxième question la condition aux limites :

$$
\frac{\partial u_{1}}{\partial n}=0, \frac{\partial u_{2}}{\partial n}=0 \operatorname{sur} \partial \Omega
$$

où $n$ désigne le vecteur normal à $\partial \Omega$, extérieure à $\Omega$.

Pour résoudre le problème (2.37)- 2.40$)$, on va introduire un paramètre, $n \in \mathbb{N}^{\star}$, destiné à tendre vers l'infini.

Soit $f_{1}, f_{2} \in L^{2}(\Omega)$. Pour $n \in \mathbb{N}^{\star}$, on s'intéresse au système :

$$
\begin{aligned}
& -\Delta u_{1}+u_{2}+\frac{1}{n} u_{1}=f_{1} \text { dans } \Omega, \\
& -\Delta u_{2}-u_{1}+\frac{1}{n} u_{2}=f_{2} \text { dans } \Omega,
\end{aligned}
$$

avec la condition aux limites 2.40).

On dit que $\left(u_{1}, u_{2}\right)$ est solution faible du problème $2.41-(2.40)$ si

$$
\begin{aligned}
& u_{1} \in H^{1}(\Omega), u_{2} \in H^{1}(\Omega), \\
& \int_{\Omega} \nabla u_{1}(x) \cdot \nabla \varphi(x) \mathrm{d} x+\int_{\Omega}\left(u_{2}(x)+\frac{1}{n} u_{1}(x)\right) \varphi(x) d x=\int_{\Omega} f_{1}(x) \varphi(x) \mathrm{d} x, \forall \varphi \in H^{1}(\Omega), \\
& \int_{\Omega} \nabla u_{2}(x) \cdot \nabla \varphi(x) \mathrm{d} x+\int_{\Omega}\left(\frac{1}{n} u_{2}(x)-u_{1}(x)\right) \varphi(x) d x=\int_{\Omega} f_{2}(x) \varphi(x) \mathrm{d} x, \forall \varphi \in H^{1}(\Omega) .
\end{aligned}
$$


Noter aussi que $\left(u_{1}, u_{2}\right)$ est solution faible du problème (2.37)-(2.40) si $\left(u_{1}, u_{2}\right)$ est solution de (2.42) en remplaçant $\frac{1}{n}$ par 0 .

Soit $f_{1}, f_{2} \in L^{2}(\Omega)$.

(a) Soit $n \in \mathbb{N}^{\star}$.

Montrer que le problème 2.42) admet une et une seule solution, que l'on note $\left(u_{1}^{(n)}, u_{2}^{(n)}\right)$ dans la suite.

(b) Montrer que :

$$
\left\|u_{1}^{(n)}\right\|_{L^{2}(\Omega)}^{2}+\left\|u_{2}^{(n)}\right\|_{L^{2}(\Omega)}^{2} \leq\left\|f_{1}\right\|_{L^{2}(\Omega)}^{2}+\left\|f_{2}\right\|_{L^{2}(\Omega)}^{2} .
$$

En déduire que les suites $\left(u_{1}^{(n)}\right)_{n \in \mathbb{N}^{*}}$, et $\left(u_{2}^{(n)}\right)_{n \in \mathbb{N}^{\star}}$ sont bornées dans $H^{1}(\Omega)$.

(c) Montrer qu'il existe une et une seule solution au problème (2.42) obtenu en remplaçant $1 / n$ par 0 , c'est à dire une et une solution faible au problème 2.37]-22.40). [Pour l'existence, utiliser les suites $\left(u_{1}^{(n)}\right)_{n \in \mathbb{N}^{*}}$, et $\left(u_{2}^{(n)}\right)_{n \in \mathbb{N}^{\star}}$ de la question précédente et faire tendre $n$ vers $+\infty$. Montrer ensuite l'unicité.]

(d) Montrer que le problème 2.37)-(2.40) admet une et une seule solution au sens suivant : $u_{1} \in H^{2}(\Omega)$, $u_{2} \in H^{2}(\Omega)$, les équations (2.37) sont satisfaites p.p. sur $\Omega$ et les équations (2.40) sont satisfaites p.p. (pour la mesure de lebesgue $N$-1-dimensionnelle) sur $\partial \Omega$ en utilisant l'opérateur trace de $H^{1}(\Omega)$ dans $L^{2}(\partial \Omega)$ pour donner un sens à $\frac{\partial u_{1}}{\partial n}$ et $\frac{\partial u_{2}}{\partial n}$.

On suppose maintenant que $f_{1}, f_{2} \in C^{\infty}(\bar{\Omega})$. Montrer que $u_{1}, u_{2} \in C^{\infty}(\bar{\Omega})$.

(e) Pour $f=\left(f_{1}, f_{2}\right) \in L^{2}(\Omega) \times L^{2}(\Omega)$, soit $u=\left(u_{1}, u_{2}\right)$ la solution faible de 2.37)-2.40, on note $u=T(f)$. Montrer que l'opérateur $T: f \mapsto u$ est un opérateur linéaire continu et compact de $L^{2}(\Omega) \times L^{2}(\Omega)$ dans lui-même.

3. De manière similaire, résoudre le problème 2.37 avec la condition aux limites :

Exercice 2.11 (A la limite de $\boldsymbol{H}^{-1}$ )

Partie I, décomposition dans $H_{0}^{1}(\Omega)$

Soit $\Omega$ un ouvert de $\mathbb{R}^{N}, N \geq 1$.

1. Soit $\varphi \in C^{1}(\mathbb{R}, \mathbb{R})$ t.q. $\varphi^{\prime} \in L^{\infty}(\mathbb{R})$ et $\varphi(0)=0$. Soit $u \in H_{0}^{1}(\Omega)$. On note $\varphi(u)$ la fonction (de $\Omega$ dans $\mathbb{R}) x \mapsto \varphi\left(u(x)\right.$ ). Montrer que $\varphi(u) \in H_{0}^{1}(\Omega)$ et que $D_{i} \varphi(u)=\varphi^{\prime}(u) D_{i} u$ p.p. pour tout $i \in\{1, \ldots, N\}$ (où $\varphi^{\prime}(u)$ désigne la fonction $\left.x \mapsto \varphi^{\prime}(u(x))\right)$. [Reprendre la méthode vue en cours.]

On définit maintenant $\varphi$ de $\mathbb{R}$ dans $\mathbb{R}$ par

$$
\begin{aligned}
& \varphi(s)=s, \text { pour } 0 \leq s \leq 1, \\
& \varphi(s)=-\frac{s^{2}}{2}+2 s-\frac{1}{2}, \text { pour } 1<s \leq 2, \\
& \varphi(s)=\frac{3}{2}, \text { pour } 2<s, \\
& \varphi(s)=-\varphi(-s), \text { pour } s<0 .
\end{aligned}
$$

Pour $k \in \mathbb{N}^{\star}$, On définit $\varphi_{k}$ de $\mathbb{R}$ dans $\mathbb{R} \operatorname{par} \varphi_{k}(s)=k \varphi\left(\frac{s}{k}\right)$ pour $s \in \mathbb{R}$.

2. Montrer que, pour tout $s \in \mathbb{R}, \varphi_{k}(s) \rightarrow s$ et $\varphi_{k}^{\prime}(s) \rightarrow 1$ quand $k \rightarrow \infty$ et que $\left|\varphi_{k}(s)\right| \leq|s|, \varphi_{k}^{\prime}(s) \leq 1$. 
3. Soit $u \in H_{0}^{1}(\Omega)$. Montrer que $\varphi_{k}(u) \in H_{0}^{1}(\Omega)$, pour tout $k \in N^{\star}$, et que $\varphi_{k}(u) \rightarrow u$ dans $H_{0}^{1}(\Omega)$, quand $k \rightarrow \infty$.

4. En déduire que, pour tout $u \in H_{0}^{1}(\Omega)$ et pour tout $\varepsilon>0$, il existe $u_{1} \in L^{\infty}(\Omega)$ et $u_{2} \in H_{0}^{1}(\Omega)$ t.q. $u=u_{1}+u_{2}$ et $\left\|u_{2}\right\|_{H_{0}^{1}} \leq \varepsilon$.

\section{Partie II, Inégalité de Trudinger-Möser}

Soit $\Omega$ un ouvert borné de $\mathbb{R}^{2}$. On admet qu'il existe $C>0$, ne dépendant que de $\Omega$, t.q.

$$
\|u\|_{L^{q}(\Omega)} \leq C \sqrt{q}\|u\|_{H_{0}^{1}(\Omega)}, \forall u \in H_{0}^{1}(\Omega), \forall q \in[1, \infty[.
$$

1. Soit $u \in H_{0}^{1}(\Omega)$ t.q. $\|u\|_{H_{0}^{1}(\Omega)} \leq 1$. Montrer qu'il existe $\sigma>0$ et $a>0$, ne dépendant que de $C$ (donné ci dessus) t.q. $e^{\sigma u^{2}} \in L^{1}(\Omega)$ et $\left\|e^{\sigma u^{2}}\right\|_{L^{1}(\Omega)} \leq a$. [Développer $e^{s}$ en puissances de $s \ldots .$. ]

2. En utilisant la partie I (et la question précédente), Montrer que $e^{\sigma u^{2}} \in L^{1}(\Omega)$ pour tout $u \in H_{0}^{1}(\Omega)$ et tout $\sigma>0$. En déduire que $e^{\sigma u^{2}} \in L^{p}(\Omega)$ pour tout $u \in H_{0}^{1}(\Omega)$, tout $\sigma>0$ et tout $p \in[1, \infty[$.

\section{Partie III, sur la résolution du problème de Dirichlet}

Soit $\Omega$ un ouvert borné de $\mathbb{R}^{2}$. Soit $f \in L^{1}(\Omega)$ t.q. $f \sqrt{|\ln (|f|)|} \in L^{1}(\Omega)$.

1. (Préliminaire.) Soit $\sigma>0$. Montrer qu'il existe $\beta, \gamma \in \mathbb{R}_{+}^{\star}$, ne dépendant que de $\sigma$, t.q.

$$
s t \leq e^{\sigma s^{2}}+\beta t \sqrt{|\ln t|}+\gamma t, \forall s, t \in \mathbb{R}_{+}^{\star} .
$$

[On pourra, par exemple, remarquer que $s t \leq \max \left\{\beta t \sqrt{|\ln t|}, s e^{\left(s^{2} / \beta^{2}\right)}\right\}$ pour tout $\beta>0$ (et tous $s, t>0$ ). Puis, choisir $\beta$ (en fonction de $\sigma$ ) et conclure.]

2. Montrer que $f u \in L^{1}(\Omega)$ pour tout $u \in H_{0}^{1}(\Omega)$ et que l'application $T: u \mapsto \int_{\Omega} f(x) u(x) \mathrm{d} x$ est un élément de $H^{-1}(\Omega)$.

3. Montrer qu'il existe un et un seul $u \in H_{0}^{1}(\Omega)$ t.q. $-\Delta u=f$ dans $\mathcal{D}^{\star}(\Omega)$.

\section{Partie IV, contre-exemple}

Soit $\Omega$ un ouvert borné de $\mathbb{R}^{2}$ et $\left.\theta \in\right] 0, \frac{1}{2}[$. On suppose que $0 \in \Omega$ et on se donne $\delta \in] 0, \frac{1}{2}\left[\right.$ t.q. $B_{2 \delta}=\left\{x \in \mathbb{R}^{2}\right.$, $|x|<2 \delta\} \subset \Omega$.

1. Soit $\gamma \in] 0, \frac{1}{2}\left[\right.$. Montrer qu'il existe une fonction $u \in H_{0}^{1}(\Omega)$ telle que $u(x)=(\ln |x|)^{\gamma} p . p$. sur $B_{\delta}$. [On pose $v(x)=\left(\ln (|x|)^{\gamma}\right.$. On rappelle (voir exercice 1.5$)$ que $v \in H^{1}\left(B_{2 \delta}\right)$.

2. Montrer qu'il existe une fonction $f \in L^{1}(\Omega)$ telle que $f(\ln |f|)^{\theta} \in L^{1}(\Omega)$ et $f u \notin L^{1}(\Omega)$ pour certains $u \in H_{0}^{1}(\Omega)$.

3. Montrer qu'il existe une fonction $f \in L^{1}(\Omega)$ telle que $f(\ln |f|)^{\theta} \in L^{1}(\Omega)$ et telle qu'il n'existe pas $u \in H_{0}^{1}(\Omega)$ vérifiant $-\Delta u=f$ dans $\mathcal{D}^{\star}(\Omega)$.

\section{Exercice 2.12 (Décomposition de Hodge)}

Soient $\Omega$ un ouvert borné connexe à frontière lipschitzienne de $\mathbb{R}^{N}(N \geq 1)$ et $f \in\left(L^{2}(\Omega)\right)^{N}$.

Montrer qu'il existe une fonction $u \in H^{1}(\Omega)$ telle que

$$
\int_{\Omega} \nabla u(x) \cdot \nabla \varphi(x) \mathrm{d} x=\int_{\Omega} f(x) \cdot \nabla \varphi(x) \mathrm{d} x, \forall \varphi \in H^{1}(\Omega) .
$$

En déduire qu'il existe $u \in H^{1}(\Omega)$ et $g \in\left(L^{2}(\Omega)\right)^{N}$ telle que $f=\nabla u+g$, p.p. dans $\Omega$ et $\int_{\Omega} g(x) \cdot \nabla \varphi(x) \mathrm{d} x=0$ pour tout $\varphi \in H^{1}(\Omega)$.

On suppose maintenant que $g \in C^{1}(\bar{\Omega})$ et que $\Omega=\left(\left[0,1[)^{N}\right.\right.$. Montrer que $\operatorname{div} g=0$ sur $\Omega$ et que $g \cdot \boldsymbol{n}=0$ p.p. (pour la mesure de Lebesgue $(N-1)$-dimensionnelle) sur $\partial \Omega$, où $\boldsymbol{n}$ est un vecteur normal à $\partial \Omega$. 
Corrigé - L'exercice (corrigé) 2.6 donne l'existence d'une fonction $u \in H^{1}(\Omega)$ telle que

$$
\int_{\Omega} \nabla u(x) \cdot \nabla \varphi(x) \mathrm{d} x=\int_{\Omega} f(x) \cdot \nabla \varphi(x) \mathrm{d} x, \forall \varphi \in H^{1}(\Omega) .
$$

On peut aussi ajouter la condition $\int_{\Omega} u(x) \mathrm{d} x=0$ et on a alors existence et unicité de u (voir l'exercice 2.6.

On pose alors $g=f-\nabla u$. Les fonctions u et $g$ vérifient les conditions demandées.

On suppose maintenant que $g \in C^{1}(\bar{\Omega})$ et que $\Omega=(] 0,1[)^{N}$. On a

$$
\int_{\Omega} g(x) \cdot \nabla \varphi(x) \mathrm{d} x=0 \text { pour tout } \varphi \in H^{1}(\Omega) .
$$

On raisonne comme dans l'exercice 2.6 En prenant $\varphi \in C_{c}^{\infty}(\Omega)$, le lemme fondamental (lemme 1.1 nous permet de montrer que $\operatorname{div}(g)=0$ partout dans $\Omega$. Puis, en prenant $\varphi \in C^{1}(\bar{\Omega})$, une intégration par parties (plutôt plus facile que dans l'exercice 2.6 donne

$$
\int_{\partial \Omega} g(x) \cdot n(x) \varphi(x) d \gamma(x)=0 \text { pour tout } \varphi \in C^{1}(\bar{\Omega}) .
$$

De cette égalité, on déduit que $g \cdot n=0$ p.p. sur $\partial \Omega$.

Ceci peut se démontrer si $N=2$ de la manière suivante : $d \gamma(x)=d x_{1}$ ou $d x_{2}$, selon les parties de $\partial \Omega$ (avec $x=$ $\left.\left(x_{1}, x_{2}\right)^{t}\right)$. Avec $g=\left(g_{1}, g_{2}\right)^{t}$, on en déduit que $g_{1}(x)=0$ partout sur $\{0\} \times[0,1] \cup\{1\} \times[0,1]$ et $g_{2}(x)=0$ partout $\operatorname{sur}[0,1] \times\{0\} \cup[0,1] \times\{1\}$. (Ce qui donne bien $g \cdot n=0$ p.p. sur $\partial \Omega$.)

La généralisation au cas $N \geq 1$ ne pose pas de difficulté.

\section{Exercice 2.13 (Problème de Stokes, vitesse)}

Soient $\Omega$ un ouvert borné de $\mathbb{R}^{N}(N \geq 1)$ et $f=\left(f_{1}, \ldots, f_{N}\right)^{t} \in\left(L^{2}(\Omega)\right)^{N}$. On pose $H=\left\{u \in\left(H_{0}^{1}(\Omega)\right)^{N}\right.$; $\operatorname{div} u=0$ p.p. dans $\Omega\}$. On rappelle que $u$ est solution du problème de Stokes si :

$$
u=\left(u_{1}, \ldots, u_{N}\right)^{t} \in H, \sum_{i=1}^{N} \int_{\Omega} \nabla u_{i}(x) \cdot \nabla v_{i}(x) \mathrm{d} x=\int_{\Omega} f(x) \cdot v(x) \mathrm{d} x, \forall v=\left(v_{1}, \ldots, v_{N}\right)^{t} \in H .
$$

On se propose ici de montrer qu'il existe une et une seule solution de (2.43) par une méthode de pénalisation. Soit $n \in \mathbb{N}^{\star}$, on considère le problème suivant :

$$
\begin{aligned}
& u=\left(u_{1}, \ldots, u_{N}\right)^{t} \in\left(H_{0}^{1}(\Omega)\right)^{N} \\
& \int_{\Omega}\left(\nabla u_{i}(x) \cdot \nabla v(x)+n(\operatorname{div} u(x)) D_{i} v(x)\right) \mathrm{d} x=\int_{\Omega} f_{i}(x) v(x) \mathrm{d} x, \forall v=H_{0}^{1}(\Omega), \forall i \in\{1, \ldots, N\} .
\end{aligned}
$$

1. Montrer que 2.43) admet au plus une solution.

2. Montrer qu'il existe une et une seule solution à (2.44). [Utiliser le lemme de Lax-Milgram $\operatorname{sur}\left(H_{0}^{1}(\Omega)\right)^{N}$.] On note, dans la suite, $u^{(n)}$ cette solution.

3. Montrer que la suite $\left(u^{(n)}\right)_{n \in \mathbb{N}}$ est bornée dans $\left(H_{0}^{1}(\Omega)\right)^{N}$ et que la suite $\left(\sqrt{n} \operatorname{div} u^{(n)}\right)_{n \in \mathbb{N}}$ est bornée dans $L^{2}(\Omega)$.

4. Montrer que, après extraction éventuelle d'une sous suite, $u^{(n)} \rightarrow u$ faiblement dans $\left(H_{0}^{1}(\Omega)\right)^{N}$, quand $n \rightarrow \infty$, où $u$ est solution de (2.43). En déduire (avec la question 1) que 2.43) admet une unique solution, notée $u$, et que $u^{(n)} \rightarrow u$ faiblement dans $\left(H_{0}^{1}(\Omega)\right)^{N}$, quand $n \rightarrow \infty$ (sans extraction de sous suite).

\section{Exercice 2.14 (Conditions aux limites de Wentzel)}




\section{Notations et Rappels du cours}

On note $H_{p}^{1}(0,2 \pi)=\left\{u \in H^{1}(] 0,2 \pi[) ; u(0)=u(2 \pi)\right\}$ (on rappelle que, si $u \in H^{1}(] 0,2 \pi[$ ), $u$ admet toujours un représentant continu sur $[0,2 \pi]$ et on identifie $u$ avec ce représentant continu).

Soit $B=\left\{(x, y)^{t} \in \mathbb{R}^{2}, x^{2}+y^{2}<1\right\}$. On rappelle qu'il existe une application $\gamma: H^{1}(\Omega) \rightarrow L^{2}(\partial B)$, linéaire, continue et telle que $\gamma(u)=u$ p.p. sur $\partial B$ si $u \in H^{1}(\Omega) \cap C(\bar{B}, \mathbb{R})$.

Si $w \in L^{2}(\partial B)$, on définit $j(w) \in L^{2}(] 0,2 \pi[)$ par $j(w)(\theta)=w(\cos \theta, \sin \theta)$, pour $\theta \in[0,2 \pi[$. L'application $j$ est donc une isométrie de $L^{2}(\partial B)$ sur $L^{2}(] 0,2 \pi[)$, de sorte que $\bar{g}=j$ o $\gamma$ est linéaire continue de $H^{1}(\Omega)$ dans $L^{2}(] 0,2 \pi[)$.

On pose $H=\left\{u \in H^{1}(\Omega) ; \bar{g}(u) \in H_{p}^{1}(0,2 \pi)\right\}$. On munit $H$ du produit scalaire $(u / v)_{H}=(u / v)_{H^{1}(\Omega)}+$ $(\bar{g}(u) / \bar{g}(v))_{H_{p}^{1}(0,2 \pi)}$.

\section{Partie I (Préliminaire d'analyse fontionnelle)}

1. Montrer que $H_{p}^{1}(0,2 \pi)$ est une espace de Hilbert.

2. Montrer que $H$ est une espace de Hilbert.

\section{Partie II (Conditions aux limites de Wentzel)}

Pour $(x, y) \in \mathbb{R}^{2},(x, y) \neq(0,0)$, on définit $r$ et $\theta$ par $r=\left(x^{2}+y^{2}\right)^{\frac{1}{2}}$ et $\theta \in[0,2 \pi[$ tels que $x=r \cos \theta$ et $y=r \sin \theta$. Pour $u \in C^{1}\left(\mathbb{R}^{N} \backslash(0,0), \mathbb{R}\right)$, on pose $u_{r}(x, y)=\frac{x}{r} \frac{\partial u}{\partial x}(x, y)+\frac{y}{r} \frac{\partial u}{\partial y}(x, y)$ et $u_{\theta}(x, y)=-y \frac{\partial u}{\partial x}(x, y)+$ $x \frac{\partial u}{\partial y}(x, y)$. (Dans la suite, on pose $u_{\theta \theta}=\left(u_{\theta}\right)_{\theta}$, si $u \in C^{2}(\bar{B}, \mathbb{R})$.)

Pour $f$ et $g$ données, on s'intéresse au problème :

$$
\begin{aligned}
-\Delta u(x, y)+u(x, y) & =f(x, y),(x, y) \in B, \\
u_{r}(x, y)-u_{\theta \theta}(x, y)+u(x, y) & =g(x, y),(x, y) \in \partial B .
\end{aligned}
$$

Soient $f \in L^{2}(B)$ et $g \in L^{2}(\partial B)$, on appelle "solution faible" de 2.45-(2.46) une solution du problème suivant :

$$
u \in H,
$$

$$
\begin{array}{r}
\int_{B}\left(\sum_{i} D_{i} u(z) D_{i} v(z)+u(z) v(z)\right) d z+\int_{0}^{2 \pi}(D \bar{g}(u)(\theta) D \bar{g}(v)(\theta)+\bar{g}(u)(\theta) \bar{g}(v)(\theta)) d \theta \\
=\int_{B} f(z) v(z) d z+\int_{0}^{2 \pi} j(g)(\theta) j(\gamma(v))(\theta) d \theta, \forall v \in H .
\end{array}
$$

1. Soient $f \in L^{2}(B)$ et $g \in L^{2}(\partial B)$. Montrer qu'il existe une et une seule solution de 2.47)-(2.48).

2. (Question plus difficile) On retire, dans cette question, "uv" dans la 1ère intégrale de (2.48). Soient $f \in L^{2}(B)$ et $g \in L^{2}(\partial B)$. Montrer qu'il existe encore une et une seule solution de 2.47)-2.48.

3. Soient $f \in C(\bar{B}, \mathbb{R})$ et $g \in C(\partial B, \mathbb{R})$. Soit $u \in C^{2}(\bar{B}, \mathbb{R})$. Montrer que $u$ est solution au sens "classique" de 2.45 -2.46 (c.a.d. vérifie 2.45 pour tout $(x, y) \in \bar{B}$ et 2.46 pour tout $(x, y) \in \partial B$ ) si et seulement si $u$ est solution faible de (2.45)-2.46).

4. Pour $f \in L^{2}(B)$ et $g \in L^{2}(\partial B)$, on note $T(f, g)=(u, \gamma(u)) \in L^{2}(B) \times L^{2}(\partial B)$, où est l'unique solution faible de 2.45)-2.46. Montrer que $T$ est un opérateur linéaire compact autoadjoint de $L^{2}(B) \times L^{2}(\partial B)$ dans lui-même.

\section{Exercice 2.15 (problème de Stokes, vitesse et pression)}


Soient $\Omega$ un ouvert borné connexe de $\mathbb{R}^{N}(N \geq 1)$ à frontière lipschitzienne et $f=\left(f_{1}, \ldots, f_{N}\right)^{t} \in\left(L^{2}(\Omega)\right)^{N}$. On s'intéresse ici au problème de Stokes, c'est-à-dire à trouver $u=\left(u_{1}, \ldots, u_{N}\right)^{t}$ et $p$ solution de

$$
\begin{aligned}
& -\Delta u+\nabla p=f \text { dans } \Omega, \\
& \operatorname{div}(u)=0 \text { dans } \Omega, \\
& u=0 \text { sur } \partial \Omega .
\end{aligned}
$$

Noter que la première équation de 2.49 est vectorielle.

On pose $H=\left\{u \in\left(H_{0}^{1}(\Omega)\right)^{N} ; \operatorname{div} u=0\right.$ p.p. dans $\left.\Omega\right\}$. On appelle solution faible de 2.49] un couple $(u, p)$ solution de

$$
\begin{aligned}
& u=\left(u_{1}, \ldots, u_{N}\right)^{t} \in H, p \in L^{2}(\Omega), \\
& \sum_{i=1}^{N} \int_{\Omega} \nabla u_{i}(x) \cdot \nabla v_{i}(x) \mathrm{d} x-\int p(x) \operatorname{div} v(x) \mathrm{d} x=\int_{\Omega} f(x) \cdot v(x) \mathrm{d} x \\
& \text { pour tout } v=\left(v_{1}, \ldots, v_{N}\right)^{t} \in\left(H_{0}^{1}(\Omega)\right)^{N} .
\end{aligned}
$$

On pourra remarquer qu'une solution classique $(u, p)$ de 2.49) est solution de 2.50].

\section{Partie I, existence et unicité de $u$}

Montrer que, si $(u, p)$ est une solution classique de (2.49), $u$ est alors solution de

$$
u=\left(u_{1}, \ldots, u_{N}\right)^{t} \in H, \sum_{i=1}^{N} \int_{\Omega} \nabla u_{i}(x) \cdot \nabla v_{i}(x) \mathrm{d} x=\int_{\Omega} f(x) \cdot v(x) \mathrm{d} x, \forall v=\left(v_{1}, \ldots, v_{N}\right)^{t} \in H .
$$

On montre dans cette pemière partie que 2.51) a une et une seule solution et que si $(u, p)$ est solution de 2.50, $u$ est alors l'unique solution de 2.51.

1. Montrer que $H$ est un s.e.v. fermé de $\left(H_{0}^{1}(\Omega)\right)^{N}$.

2. Montrer que (2.51) admet une et une seule solution. [Utiliser le lemme de Lax-Milgram.]

3. Soit $(u, p)$ une solution de 2.50). Montrer que $u$ est l'unique solution de 2.51).

Soit $u$ la solution de (2.51). La suite de l'exercice consiste à trouver $p$ pour que $(u, p)$ soit solution de (2.50).

\section{Partie II, préliminaire d'analyse fonctionnelle}

Soit $E$ et $F$ deux espaces de Hilbert (réels). On note $(\cdot \mid \cdot)_{E}$ (resp. $\left.(\cdot \mid \cdot)_{F}\right)$ le produit scalaire dans $E$ (resp. $F$ ). Soit $A$ un opérateur linéaire continu de $E$ dans $F$. On note $A^{\star}$ l'opérateur adjoint de $A$. L'opérateur $A^{\star}$ est un opérateur linéaire continu de $F$ dans $E$. Pour tout $g \in F, A^{\star} g$ est l'unique élément de $E$ défini par

$$
\left(A^{\star} g \mid u\right)_{E}=(g \mid A u)_{F} \text { pour tout } u \in E .
$$

(Noter que l'existence et l'unicité de $A^{\star} g$ est donnée par le théorème de représentation de Riesz.)

1. Montrer que $\operatorname{Ker} A=\left(\operatorname{Im} A^{\star}\right)^{\perp}$.

(On rappelle que si $G \subset E, G^{\perp}=\left\{u \in E,(u \mid v)_{E}=0\right.$ pour tout $\left.v \in G\right\}$.)

2. Montrer que $(\operatorname{Ker} A)^{\perp}=\overline{\operatorname{Im} A^{\star}}$.

\section{Partie III, Existence et unicité partielle de $p$}

Dans cette partie, on va utiliser le lemme suivant (souvent attribué à J. Nečas, 1965) que nous admettons. 
Lemme 2.31 Soient $\Omega$ un ouvert borné connexe de $\mathbb{R}^{N}(N \geq 1)$ à frontière lipschitzienne et $q \in L^{2}(\Omega)$ telle que $\int_{\Omega} q(x) \mathrm{d} x=0$. Il existe alors $v \in\left(H_{0}^{1}(\Omega)\right)^{N}$ telle que $\operatorname{div}(v)=q$ p.p. dans $\Omega$ et

$$
\|v\|_{H_{0}^{1}(\Omega)^{N}} \leq C\|q\|_{L^{2}(\Omega)}
$$

où $C$ ne dépend que de $\Omega$.

On prend ici $E=H_{0}^{1}(\Omega)^{N}$ et $F=L^{2}(\Omega)$. Pour $u \in E$ on pose $A u=\operatorname{div} u$, de sorte que $A$ est un opérateur linéaire continu de $E$ dans $F$.

1. Soit $\left(p_{n}\right)_{n \in \mathbb{N}}$ une suite de $F$ et $v \in E$ telle que $A^{\star} p_{n} \rightarrow v$ dans $E$ quand $n \rightarrow+\infty$. Pour $n \in \mathbb{N}$, on pose $q_{n}=p_{n}-a_{n}$, où $a_{n}$ est la moyenne de $p_{n}$ dans $\Omega$.

(a) Montrer que $A^{\star} p_{n}=A^{\star} q_{n}$.

(b) Montrer que la suite $\left(q_{n}\right)_{n \in \mathbb{N}}$ est bornée dans $F$. [Utiliser le lemme 2.31]]

(c) Montrer que $v \in \operatorname{Im} A^{\star}$.

2. Montrer que $(\operatorname{Ker} A)^{\perp}=\operatorname{Im} A^{\star}$ et que $\operatorname{Ker} A=H$.

3. On rappelle que le produit scalaire dans $E$ est défini par

$$
(u / v)_{E}=\sum_{i=1}^{N} \int_{\Omega} \nabla u_{i}(x) \cdot \nabla v_{i}(x) \mathrm{d} x .
$$

On définit $T_{f} \in E \operatorname{par}\left(T_{f} \mid v\right)_{E}=\int_{\Omega} f(x) \cdot v(x) \mathrm{d} x$ pour tout $v \in E$. Soit $u$ la solution de 2.51.

(a) Montrer que $u-T_{f} \in H^{\perp}$. En déduire que $u-T_{f} \in \operatorname{Im} A^{\star}$.

(b) Montrer qu'il existe une fonction $p \in F$ telle que $(u, p)$ est solution de 2.50).

4. Soit $\left(u_{1}, p_{1}\right)$ et $\left(u_{2}, p_{2}\right)$ deux solutions de 2.50). Montrer que $u_{1}=u_{2}=u$ (où $u$ est l'unique solution de 2.51) ) et qu'il existe $a \in \mathbb{R}$ tel que $p_{1}-p_{2}=a$ p.p..

\section{Corrigé-}

Partie I, existence et unicité de $u$

Soit $(u, p)$ est une solution classique de 2.49. On remarque tout d'abord que $u \in H$. Puis, pour $v \in H$, on multiplie la première équation de 2.49 par $v$ et on intègre sur $\Omega$. Le fonctions $u$ et $v$ sont suffisamment régulières pour intégrer par parties et obtient ainsi l'équation 2.51. Ceci montre que u est alors solution de 2.51.

1. Pour $u \in H_{0}^{1}(\Omega)^{N}$, on pose $d(u)=\operatorname{div}(u)$. L'application d est linéaire continue de $H_{0}^{1}(\Omega)^{N}$ dans $L^{2}(\Omega)$. Comme $H=\operatorname{Ker} d$, on en déduit que $H$ est un s.e.v. fermé de $H_{0}^{1}(\Omega)^{N}$.

2. Il suffit ici d'appliquer le lemme de Lax-Milgram, lemme 2.4 (ou le théorème de Riesz dans les espaces de Hilbert) en remarquant que $H$ est un espace de Hilbert ( $H$ est muni de la norme naturelle de $H_{0}^{1}(\Omega)^{N}$ ), avec a et $T$ définis ainsi :

et

$$
a(u, v)=\sum_{i=1}^{N} \int_{\Omega} \nabla u_{i}(x) \cdot \nabla v_{i}(x) \mathrm{d} x,
$$

$$
T(v)=\int_{\Omega} f(x) \cdot v(x) \mathrm{d} x .
$$

3. Pour $v \in H$, on a $\operatorname{div}(v)=0$ p.p. dans $\Omega$ et donc $\int_{\Omega} p \operatorname{div}(v) \mathrm{d} x=0$. On en déduit que u est solution de 2.51. Par la question précédente, la fonction (vectorielle) u est donc l'unique solution de 2.51 .

Partie II, préliminaire d'analyse fonctionnelle 
1. Soit $u \in \operatorname{Ker} A$ (on a donc $A u=0$ ). Pour $v \in \operatorname{Im} A^{\star}$, il existe $g \in F$ tel que $v=A^{\star} g$, on a donc

$$
(v \mid u)_{E}=\left(A^{\star} g \mid u\right)_{E}=(g \mid A u)_{F}=0 .
$$

Ce qui montre que $u \in\left(\operatorname{Im} A^{\star}\right)^{\perp}$. On a donc $\operatorname{Ker} A \subset\left(\operatorname{Im} A^{\star}\right)^{\perp}$.

Récirpoquement, soit $u \in\left(\operatorname{Im} A^{\star}\right)^{\perp}$. On a alors, en posant $f=A u$,

$$
(A u \mid A u)_{F}=(f \mid A u)_{F}=\left(A^{\star} f \mid u\right)_{E}=0,
$$

car $A^{\star} f \in \operatorname{Im} A^{\star}$. Donc, $A u=0$, c'est-à-dire $u \in \operatorname{Ker} A$. Ceci donne $\left(\operatorname{Im} A^{\star}\right)^{\perp} \subset \operatorname{Ker} A$.

Finalement, on a bien montré que $\left(\operatorname{Im} A^{\star}\right)^{\perp}=\operatorname{Ker} A$.

2. Si $F$ est un s.e.v. fermé d'un espace de Hilbert $H$, on a toujours $H=F \oplus F^{\perp}$. D'autre part, si $G \subset H$, on a $G^{\perp}=\bar{G}^{\perp}$.

Si F est un s.e.v. d'un espace de Hilbert H, on a donc

Ceci permet de prouver que $\left(F^{\perp}\right)^{\perp}=\bar{F}$.

$$
H=\bar{F} \oplus F^{\perp} \text { et } H=F^{\perp} \oplus\left(F^{\perp}\right)^{\perp} .
$$

On applique ici ce résutat avec $F=\operatorname{Im} A^{\star}$, on obtient (avec la question précédente)

$$
\overline{\operatorname{Im} A^{\star}}=\left(\left(\operatorname{Im} A^{\star}\right)^{\perp}\right)^{\perp}=(\operatorname{Ker} A)^{\perp} .
$$

\section{Partie III, Existence et unicité partielle de $p$}

1.(a) Soit $v \in$ E. On a

$$
\left(A^{\star} p_{n} \mid v\right)_{E}=\left(p_{n} \mid A v\right)_{F}=\int_{\Omega} p_{n} \operatorname{div}(v) \mathrm{d} x
$$

et

$$
\left(A^{\star} q_{n} \mid v\right)_{E}=\left(q_{n} \mid A v\right)_{F}=\int_{\Omega} q_{n} \operatorname{div}(v) \mathrm{d} x=\int_{\Omega} p_{n} \operatorname{div}(v) \mathrm{d} x-a_{n} \int_{\Omega} \operatorname{div}(v) \mathrm{d} x .
$$

Comme $v \in H_{0}^{1}(\Omega)^{N}$, on a (en intégrant par parties) $\int_{\Omega} \operatorname{div}(v) \mathrm{d} x=0$ et donc

$$
\left(A^{\star} p_{n} \mid v\right)_{E}=\left(A^{\star} q_{n} \mid v\right)_{E} \text { pour tout } v \in H_{0}^{1}(\Omega)^{N} .
$$

Ceci montre bien que $A^{\star} p_{n}=A^{\star} q_{n}$

(b) Par le lemme 2.31 il existe $v_{n} \in H_{0}^{1}(\Omega)^{N}$ t.q. $\operatorname{div}\left(v_{n}\right)=q_{n}$ p.p. dans $\Omega$ et $\left\|v_{n}\right\|_{H_{0}^{1}(\Omega)^{N}} \leq C\left\|q_{n}\right\|_{L^{2}(\Omega)}$. On obtient alors

$$
\left(A^{\star} q_{n} \mid v_{n}\right)_{E}=\int_{\Omega} q_{n} \operatorname{div}\left(v_{n}\right) \mathrm{d} x=\int_{\Omega} q_{n}^{2} \mathrm{~d} x=\left\|q_{n}\right\|_{F}^{2} .
$$

La question précédente donne $A^{\star} p_{n}=A^{\star} q_{n}$. On a donc

$$
\left\|q_{n}\right\|_{F}^{2}=\left(A^{\star} p_{n} \mid v_{n}\right)_{E} \leq\left\|A^{\star} p_{n}\right\|_{E}\left\|v_{n}\right\|_{E} \leq C\left\|A^{\star} p_{n}\right\|_{E}\left\|q_{n}\right\|_{F}
$$

et donc

$$
\left\|q_{n}\right\|_{F} \leq C\left\|A^{\star} p_{n}\right\|_{E} .
$$

$L$ "hypothèse de convergence de $A^{\star} p_{n}$ donne que la suite $\left(A^{\star} p_{n}\right)_{n \in \mathbb{N}}$ est bornée (dans $E$ ). On en déduit que la suite $\left(q_{n}\right)_{n \in \mathbb{N}}$ est bornée dans $F$.

(c) Comme la suite $\left(q_{n}\right)_{n \in \mathbb{N}}$ est bornée dans l'espace de Hilbert $F$, on peut supposer, après extraction éventuelle d'une sous suite, que cette suite converge faiblement dans $F$. Il existe donc $q \in F$ t.q. $q_{n} \rightarrow q$ faiblement dans $F$, quand $n \rightarrow+\infty$. On va montrer que $v=A^{\star} q$.

Soit $w \in E$, On a $\lim _{n \rightarrow+\infty}\left(q_{n} \mid A w\right)_{F}=(q \mid A w)_{F}$. Mais

$$
\left(q_{n} \mid A w\right)_{F}=\left(A^{\star} q_{n} \mid w\right)_{E}=\left(A^{\star} p_{n} \mid w\right)_{E} .
$$

Comme $A^{\star} p_{n} \rightarrow v$ dans $E$, on a donc aussi $\lim _{n \rightarrow+\infty}\left(q_{n} \mid A w\right)_{F}=(v \mid w)_{E}$. On obtient donc

$$
(q \mid A w)_{F}=(v \mid w)_{E} \text { pour tout } w \in E .
$$

Ceci donne $\left(A^{\star} q \mid w\right)_{E}=(v \mid w)_{E}$ pour tout $w \in E$, et donc $v=A^{\star} q$. On a bien montré que $v \in \operatorname{Im} A^{\star}$. 
2. La question précédente montre que $\operatorname{Im} A^{\star}$ est fermé (dans $\left.E\right)$. Avec la partie II, on a donc $(\operatorname{Ker} A)^{\perp}=\operatorname{Im}\left(A^{\star}\right)$. On a déjà vu que $\operatorname{Ker} A=H$. On a donc $H^{\perp}=\operatorname{Im}\left(A^{\star}\right)$.

3.(a) On a $(u \mid v)_{E}=\int_{\Omega} f v \mathrm{~d} x=\left(T_{f} \mid v\right)_{E}$ pour tout $v \in H$. Ceci signifie bien que $u-T_{f} \in H^{\perp}$ et donc que $u-T_{f} \in \operatorname{Im} A^{\star}$.

(b) Comme $u-T_{f} \in \operatorname{Im} A^{\star}$, il existe $p \in F=L^{2}(\Omega)$ t.q. $u-T_{f}=A^{\star} p$. On a donc pour tout $v \in H_{0}^{1}(\Omega)^{N}$,

$$
(u \mid v)_{E}-\int_{\Omega} f v \mathrm{~d} x=\left(u-T_{f} \mid v\right)_{E}=\left(A^{\star} p \mid v\right)_{E}=(p \mid A v)_{F}=\int_{\Omega} p \operatorname{div}(v) \mathrm{d} x .
$$

Ce qui signifie bien que $(u, p)$ est solution de 2.50 .

4. On a déja montré à la question 3 de la partie I que $u_{1}=u_{2}=u$ où u est l'unique solution de 2.51.

On obtient alors que $\int_{\Omega} p_{1} \operatorname{div}(v) \mathrm{d} x=\int_{\Omega} p_{2} \operatorname{div}(v) \mathrm{d} x$ pour tout $v \in H_{0}^{1}(\Omega)^{N}$. En prenant $v=\left(v_{1}, \ldots, v_{N}\right)^{t}$ avec $v_{1} \in C_{c}^{\infty}(\Omega)$ et $v_{i}=0$ pour $i \geq 2$, on en déduit que $D_{1}\left(p_{1}-p_{2}\right)=0$ (dans $\left.\mathcal{D}^{\star}\right)$. De manière analogue on a $D_{i}\left(p_{1}-p_{2}\right)=0$ pour tout $i \in\{1, \ldots, N\}$. Ceci permet d'affirmer qu'il existe $a \in \mathbb{R}$ t.q. $p_{1}-p_{2}=$ a p.p. (voir l'exercice 1.4 .

\section{Exercice 2.16 (Continuité séquentielle de $\boldsymbol{L}^{2}$-faible dans $H_{0}^{1}$ )}

Soit $\Omega$ un ouvert borné de $\mathbb{R}^{N}(N>1)$. Pour tout $x \in \Omega$, on se donne une matrice, notée $A(x)$, dont les coefficients sont notés $a_{i, j}(x), i, j=1, \ldots, N$. On suppose que $a_{i, j} \in L^{\infty}(\Omega)$ pour tout $i, j=1, \ldots, N$ et qu'il existe $\alpha>0$ t.q $A(x) \xi \cdot \xi \geq \alpha|\xi|^{2}$, pour tout $\xi \in \mathbb{R}^{N}$ et p.p. en $x \in \Omega$.

Pour $f \in L^{2}(\Omega)$, on sait qu'il existe une unique solution au problème suivant :

$$
\left\{\begin{array}{l}
u \in H_{0}^{1}(\Omega), \\
\int_{\Omega} A(x) \nabla u(x) \cdot \nabla v(x) \mathrm{d} x=\int_{\Omega} f(x) v(x) \mathrm{d} x, \text { pour tout } v \in H_{0}^{1}(\Omega) .
\end{array}\right.
$$

Soit $\left(f_{n}\right)_{n \in \mathbb{N}}$ une suite bornée de $L^{2}(\Omega)$ et $f \in L^{2}(\Omega)$. On note $u$ la solution de 2.52) et, pour $n \in \mathbb{N}$, on note $u_{n}$ la solution de (2.52) avec $f_{n}$ au lieu de $f$. On suppose que $f_{n} \rightarrow f$ faiblement dans $L^{2}(\Omega)$.

1. Montrer que la suite $\left(u_{n}\right)_{n \in \mathbb{N}}$ est bornée dans $H_{0}^{1}(\Omega)$.

2. Montrer que $u_{n} \rightarrow u$ faiblement dans $H_{0}^{1}(\Omega)$ et que $u_{n} \rightarrow u$ dans $L^{2}(\Omega)$ (quand $n \rightarrow+\infty$ ).

3. Montrer que, quand $n \rightarrow+\infty$,

$$
\int_{\Omega} A(x) \nabla u_{n}(x) \cdot \nabla u_{n}(x) \mathrm{d} x \rightarrow \int_{\Omega} A(x) \nabla u(x) \cdot \nabla u(x) \mathrm{d} x .
$$

[Utiliser le fait que $\int_{\Omega} A(x) \nabla u_{n}(x) \cdot \nabla u_{n}(x) \mathrm{d} x=\int_{\Omega} f_{n}(x) u_{n}(x) \mathrm{d} x$ et passer à la limite sur le terme de droite de cette égalité.]

4. Montrer que $u_{n} \rightarrow u$ dans $H_{0}^{1}(\Omega)$. [On pourra considérer $\int_{\Omega} A(x) \nabla\left(u_{n}-u\right)(x) \cdot \nabla\left(u_{n}-u\right)(x) \mathrm{d} x$.]

Corrigé-

1. En prenant $v=u_{n}$ dans 2.52 (avec $f_{n}$ et $u_{n}$ au lieu de $f$ et $u$ ), on obtient

$$
\alpha\left\|u_{n}\right\|_{H_{0}^{1}(\Omega)}^{2} \leq\left\|f_{n}\right\|_{L^{2}(\Omega)}\left\|u_{n}\right\|_{L^{2}(\Omega)} \leq\left\|f_{n}\right\|_{L^{2}(\Omega)} C_{\Omega}\left\|u_{n}\right\|_{H_{0}^{1}(\Omega)},
$$

où $C_{\Omega}$ est donné par l'inégalité de Poincaré. On a donc, pour tout $n \in \mathbb{N}$,

$$
\left\|u_{n}\right\|_{H_{0}^{1}(\Omega)} \leq \frac{C_{\Omega}}{\alpha} \sup _{p \in \mathbb{N}}\left(\left\|f_{p}\right\|_{L^{2}(\Omega)}\right)=M<+\infty,
$$

la suite $\left(f_{n}\right)_{n \in \mathbb{N}}$ étant bornée dans $L^{2}(\Omega)$. 
2. Si $u_{n} \not \nrightarrow$ u faiblement dans $H_{0}^{1}(\Omega)$, il existe $\varepsilon>0, \psi \in H^{-1}(\Omega)$ et une sous suite, encore notée $\left(u_{n}\right)_{n \in \mathbb{N}}$, t.q.

$$
\left|\left\langle\psi, u_{n}-u\right\rangle_{H^{-1}(\Omega), H_{0}^{1}(\Omega)}\right| \geq \varepsilon \text { pour tout } n \in \mathbb{N} .
$$

Après une nouvelle extraction éventuelle, on peut supposer que $u_{n} \rightarrow \bar{u}$ faiblement dans $H_{0}^{1}(\Omega)$.

Soit $v \in H_{0}^{1}(\Omega)$. On a

$$
\int_{\Omega} A \nabla u_{n} \cdot \nabla v \mathrm{~d} x=\int_{\Omega} f_{n} v \mathrm{~d} x
$$

et donc, quand $n \rightarrow \infty$,

$$
\int_{\Omega} A \nabla \bar{u} \cdot \nabla v \mathrm{~d} x=\int_{\Omega} f v \mathrm{~d} x
$$

On en déduit que $\bar{u}=u$, ce qui est en contradiction avec 2.53 .

On a donc bien montré que $u_{n} \rightarrow u$ faiblement dans $H_{0}^{1}(\Omega)$ et, par le théorème de Rellich, que $u_{n} \rightarrow u$ dans $L^{2}(\Omega)$. 3.

$$
\int_{\Omega} A \nabla u_{n} \cdot \nabla u_{n} \mathrm{~d} x=\int_{\Omega} f_{n} u_{n} \mathrm{~d} x \rightarrow \int_{\Omega} f u d x=\int A \nabla u \cdot \nabla u \mathrm{~d} x,
$$

car $u_{n} \rightarrow u$ dans $L^{2}(\Omega)$ et $f_{n} \rightarrow f$ faiblement dans $L^{2}(\Omega)$.

4. On $a$

$$
\int_{\Omega} A\left(\nabla u_{n}-\nabla u\right) \cdot\left(\nabla u_{n}-\nabla u\right) \mathrm{d} x=\int_{\Omega} A \nabla u_{n} \cdot \nabla u_{n} \mathrm{~d} x-\int_{\Omega} A \nabla u_{n} \cdot \nabla u-\int_{\Omega} A \nabla u \cdot \nabla u_{n}+\int_{\Omega} A \nabla u \cdot \nabla u \mathrm{~d} x .
$$

Les quatre termes de droite de cette égalité tendent vers $\int_{\Omega} \nabla u \cdot \nabla u \mathrm{~d} x$ quand $n \rightarrow+\infty$. Le terme de gauche (qui est positif) tend donc vers 0 . Ceci donne $\alpha\left\|u_{n}-u\right\|_{H_{0}^{1}(\Omega)}^{2} \rightarrow 0$ (quand $n \rightarrow+\infty$ ) et donc, quand $n \rightarrow+\infty$,

$$
u_{n} \rightarrow u \quad \text { dans } H_{0}^{1}(\Omega) \text {. }
$$

Remarque sur la topologie faible : L'application $f \mapsto u$ (où u est solution de 2.52) est donc séquentiellement continue de $L^{2}(\Omega)$-faible dans $H_{0}^{1}(\Omega)$ (fort), c'est-à-dire qu'elle transforme les suites faiblement convergentes de $L^{2}(\Omega)$ en suites (fortement) convergentes de $H_{0}^{1}(\Omega)$. Elle est donc aussi séquentiellement continue de $L^{2}(\Omega)$-faible dans $L^{2}(\Omega)($ fort). Après avoir définie la topologie faible de $L^{2}(\Omega)$ (ce que nous ne faisons dans ce polycopié), on peut toutefois remarquer que cette application n'est pas continue de $L^{2}(\Omega)$-faible ( c'est-à-dire $L^{2}(\Omega)$ muni de la topologie faible) dans $L^{2}(\Omega)($ c'est-à-dire $L^{2}(\Omega)$ muni de la topologie associée à sa norme).

\section{Exercice 2.17 (Exercice liminaire à l'exercice 2.18}

Soit $\varphi$ une fonction décroissante de $\mathbb{R}_{+}$dans $\mathbb{R}_{+}$. On suppose qu'il existe $C>0$ et $\beta>1$ t.q.

$$
0 \leq x<y \Rightarrow \varphi(y) \leq C \frac{\varphi(x)^{\beta}}{y-x} .
$$

Montrer qu'il existe $a \in \mathbb{R}_{+}$t.q. $\varphi(a)=0$. [On pourra montrer l'existence d'une suite strictement croissante $\left(a_{k}\right)_{k \in \mathbb{N}^{\star}}$ t.q. $\varphi\left(a_{k}\right) \leq \frac{1}{2^{k}}$ pour tout $k \in \mathbb{N}^{\star}$ et $\lim _{k \rightarrow \infty} a_{k}<+\infty$. Pour cela, on pourra montrer qu'il existe $a_{0}$ t.q. $\varphi\left(a_{0}\right) \leq 1$ puis, par récurrence, définir $a_{k+1}$ par $\frac{C}{a_{k+1}-a_{k}} \frac{1}{2^{k \beta}}=\frac{1}{2^{k+1}}$.]

Corrigé - En prenant $x=0$ dans 2.54, on obtient $\lim _{y \rightarrow \infty} \varphi(y)=0$. Il existe donc $a_{0}$ t.q. $\varphi\left(a_{0}\right) \leq 1$.

On définit maintenant, par récurrence, une suite $\left(a_{k}\right)_{k \in \mathbb{N}}$ par

$$
\frac{C}{a_{k+1}-a_{k}}\left(\frac{1}{2^{k}}\right)^{\beta}=\frac{1}{2^{k+1}}
$$

on a alors, par récurrence, $\varphi\left(a_{k}\right) \leq \frac{1}{2^{k}}$.

En effet pour $k=0$ on a bien $\varphi\left(a_{0}\right) \leq 1$. 


$$
\begin{aligned}
\text { Puis, pour } k \geq 0 \text {, si } \varphi\left(a_{k}\right) & \leq \frac{1}{2^{k}} \text {, on } a \\
& \varphi\left(a_{k+1}\right) \leq \frac{C}{a_{k+1}-a_{k}} \varphi\left(a_{k}\right)^{\beta} \leq \frac{C}{a_{k+1}-a_{k}} \frac{1}{2^{k \beta}}=\frac{1}{2^{k+1}} .
\end{aligned}
$$

On montre maintenant que $\lim _{k \rightarrow \infty} a_{k}<\infty$. Pour cela, on remarque que

On a donc

$$
a_{k+1}-a_{k}=2 C \frac{2^{k}}{2^{k \beta}}=2 C \frac{1}{2^{k(\beta-1)}}=2 C b^{k} \text { avec } b=\frac{1}{2^{\beta-1}} .
$$

$$
a_{k}=a_{0}+\sum_{p=0}^{k-1} 2 C b^{p} \leq a_{0}+2 C \sum_{p=0}^{\infty} b^{p}=a_{0}+\frac{2 C}{1-b},
$$

$\operatorname{car} b=\frac{1}{2^{\beta-1}}<1$ car $\beta>1$. On prend donc $a=a_{0}+\frac{2 C}{1-b}$ et on a, comme $\varphi$ est décroissante,

$$
0 \leq \varphi(a) \leq \varphi\left(a_{k}\right) \text { pour tout } k \in \mathbb{N},
$$

et donc

$$
0 \leq \varphi(a) \leq \frac{1}{2^{k}} \text { pour tout } k \in \mathbb{N} .
$$

Ce qui donne $\varphi(a)=0$.

\section{Exercice 2.18 (Solutions bornées d'un problème elliptique)}

Soit $\Omega$ un ouvert borné de $\mathbb{R}^{N}(N>1)$. Pour tout $x \in \Omega$, on se donne une matrice, notée $A(x)$, dont les coefficients sont notés $a_{i, j}(x), i, j=1, \ldots, N$. On suppose que $a_{i, j} \in L^{\infty}(\Omega)$ pour tout $i, j=1, \ldots, N$ et qu'il existe $\alpha>0$ t.q $A(x) \xi \cdot \xi \geq \alpha|\xi|^{2}$, pour tout $\xi \in \mathbb{R}^{N}$ et p.p. en $x \in \Omega$.

Si $B$ est une partie borélienne de $\mathbb{R}^{N}$, on note $\operatorname{mes}(B)$ le mesure de Lebesgue $N$-dimensionnelle de $A$ ( c'est-àdire la "surface" si $N=2$ et le volume si $N=3$ ).

1. Soit $F \in L^{2}(\Omega)^{N}$. Montrer qu'il existe un et un seul $u$ solution de

$$
\left\{\begin{array}{l}
u \in H_{0}^{1}(\Omega), \\
\int_{\Omega} A(x) \nabla u(x) \cdot \nabla v(x) \mathrm{d} x=\int_{\Omega} F(x) \cdot \nabla v(x) \mathrm{d} x, \text { pour tout } v \in H_{0}^{1}(\Omega) .
\end{array}\right.
$$

Soit $p>N$. On suppose pour la suite de l'exercice que $F \in L^{p}(\Omega)^{N}$ (On rappelle que $L^{p}(\Omega)^{N} \subset L^{2}(\Omega)^{N}$ car $p>2$ ) et on note $u$ l'unique solution de 2.55.

Pour $k \in \mathbb{R}_{+}$, on définit la fonction $S_{k}$ de $\mathbb{R}$ dans $\mathbb{R}$ par

$$
\left\{\begin{array}{l}
S_{k}(s)=0 \text { si }-k \leq s \leq k \\
S_{k}(s)=s-k \text { si } s>k \\
S_{k}(s)=s+k \text { si } s<-k
\end{array}\right.
$$

On rappelle que si $v \in H_{0}^{1}(\Omega)$ on a $S_{k}(v) \in H_{0}^{1}(\Omega)$ et $\nabla S_{k}(v)=1_{A_{k}} \nabla v$ p.p., avec $A_{k}=\{|v|>k\}$ (voir la remarque 2.23.

2. Soit $k \in \mathbb{R}_{+}$, Montrer que

$$
\alpha\left\|\left|\nabla S_{k}(u)\right|\right\|_{L^{2}(\Omega)}=\alpha\left(\int_{A_{k}} \nabla u(x) \cdot \nabla u(x) \mathrm{d} x\right)^{\frac{1}{2}} \leq \operatorname{mes}\left(A_{k}\right)^{\frac{1}{2}-\frac{1}{p}}\||F|\|_{L^{p}(\Omega)} .
$$

[On pourra prendre $v=S_{k}(u)$ dans 2.55) et utiliser l'inégalité de Hölder.] 
3. On pose $1^{\star}=\frac{N}{N-1}$. On rappelle qu'il existe $C_{1}$ ne dépendant que de $N$ t.q.

$$
\|w\|_{L^{1^{\star}}(\Omega)} \leq C_{1}\|w\|_{W_{0}^{1,1}(\Omega)}=C_{1}\||\nabla w|\|_{L^{1}(\Omega)} \text { pour tout } w \in W_{0}^{1,1}(\Omega) .
$$

Soit $k, h \in \mathbb{R}_{+}$t.q. $k<h$. Montrer que

$$
(h-k) \operatorname{mes}\left(A_{h}\right)^{\frac{N-1}{N}} \leq\left(\int _ { A _ { h } } \left|S_{k}\left(\left.u(x)\right|^{1^{\star}} d x\right)^{\frac{1}{1^{\star}}} \leq C_{1}\left\|\left|\nabla S_{k}(u)\right|\right\|_{L^{1}(\Omega)} \leq C_{1}\left\|\left|\nabla S_{k}(u)\right|\right\|_{L^{2}(\Omega)} \operatorname{mes}\left(A_{k}\right)^{\frac{1}{2}} .\right.\right.
$$

En déduire qu'il existe $C_{2}$ ne dépendant que de $C_{1}, \alpha, F$ et $p$ t.q.

$$
(h-k) \operatorname{mes}\left(A_{h}\right)^{\frac{N-1}{N}} \leq C_{2} \operatorname{mes}\left(A_{k}\right)^{1-\frac{1}{p}} .
$$

4. Montrer que $u \in L^{\infty}(\Omega)$ ( c'est-à-dire qu'il existe $a \in \mathbb{R}_{+}$t.q. mes $\left(A_{a}\right)=0$ ). [On pourra poser $\varphi(k)=$ $\operatorname{mes}\left(A_{k}\right)^{\frac{N-1}{N}}$ et utiliser l'exercice 2.17]

5. Montrer qu'il existe $C_{3}$ ne dépendant que de $\Omega, \alpha$ et $p$ t.q.

$$
\|u\|_{L^{\infty}(\Omega)} \leq C_{3}\||F|\|_{L^{p}(\Omega)} .
$$

Corrigé -

1. Pour $u, v \in H_{0}^{1}(\Omega)$ on pose

$$
a(u, v)=\int_{\Omega} A \nabla u \cdot \nabla v \mathrm{~d} x \text { et } T(v)=\int_{\Omega} F \cdot \nabla v \mathrm{~d} x .
$$

Comme cela a été vu dans ce chapitre, la forme a est une forme bilinéaire continue coercive sur $H_{0}^{1}(\Omega)$. Puis, pour $v \in H_{0}^{1}(\Omega)$, on $a$

$$
T(v) \leq \int_{\Omega}|F \cdot \nabla v| \mathrm{d} x \leq\|\| F\left|\left\|_{L^{2}(\Omega)}\right\|\right| \nabla v\left|\left\|_{L^{2}(\Omega)}=\right\|\right| F \mid\left\|_{L^{2}(\Omega)}\right\| v \|_{H_{0}^{1}(\Omega)} .
$$

On en déduit que $T \in\left(H_{0}^{1}(\Omega)\right)^{\prime}$ et donc qu'il existe un et un seul u solution de 2.55.

2. En prenant $v=S_{k}(u)$ dans 2.55 on obtient

$$
\begin{aligned}
& \alpha\left\|\mid \nabla\left(S_{k}(u)\right)\right\|_{L^{2}(\Omega)}^{2}=\alpha \int_{A_{k}} \nabla u \cdot \nabla u \mathrm{~d} x=\int_{A_{k}} F \cdot \nabla u \mathrm{~d} x \\
& \left.\leq\||F|\|_{L^{2}\left(A_{k}\right)}\right)|| \nabla\left(S_{k}(u)\right) \mid \|_{L^{2}(\Omega)} \\
& \leq\left\||| F\left|\left\|_{L^{p}(\Omega)}\left(\operatorname{mes}\left(A_{k}\right)\right)^{\frac{1}{2}-\frac{1}{p}}\right\|\right| \nabla\left(S_{k}(u)\right) \mid\right\|_{L^{2}(\Omega)}, \\
& \operatorname{car} \int_{A_{k}}|F|^{2} d x \leq\left(\int_{\Omega}|F|^{p} d x\right)^{\frac{2}{p}} \text { mes }\left(A_{k}\right)^{1-\frac{2}{p}} \text {. On obtient ainsi } \\
& \alpha\left\||| \nabla\left(S_{k}(u)\right)\left|\left\|_{L^{2}(\Omega)} \leq\right\|\right||F|\right\|_{L^{p}(\Omega)} \operatorname{mes}\left(A_{k}\right)^{\frac{1}{2}-\frac{1}{p}} . \\
& (h-k)\left(m e s\left(A_{h}\right)\right)^{\frac{1}{1 \star}} \leq\left(\int_{\Omega}\left|S_{k}(u)\right|^{1^{\star}} d x\right)^{\frac{1}{1^{\star}}} \\
& \leq C_{1}\left\|\left|\nabla S_{k}(u)\right|\right\|_{L^{1}(\Omega)} \\
& \leq C_{1} \int_{A_{k}}\left|\nabla S_{k}(u)\right| \mathrm{d} x \leq C_{1}\left\|\left|\nabla S_{k}(u)\right|\right\|_{L^{2}(\Omega)} \operatorname{mes}\left(A_{k}\right)^{\frac{1}{2}} .
\end{aligned}
$$


Avec la question 2, on obtient

$$
(h-k)\left(\operatorname{mes}\left(A_{h}\right)\right)^{\frac{1}{1^{\star}}} \leq \frac{C_{1}}{\alpha}\||F|\|_{L p} m e s\left(A_{k}\right)^{1-\frac{1}{p}},
$$

et donc, avec $C_{2}=\frac{C_{1}}{\alpha}\||F|\|_{L^{p}(\Omega)}$,

$$
(h-k) \operatorname{mes}\left(A_{h}\right)^{\frac{N-1}{N}} \leq C_{2} \operatorname{mes}\left(A_{k}\right)^{1-\frac{1}{p}} .
$$

4. Pour $k \in \mathbb{R}_{+}$, on pose $\varphi(k)=\left(\operatorname{mes}\left(A_{k}\right)\right)^{\frac{N-1}{N}}$. On a alors, pour $h \geq k \geq 0$,

$$
(h-k) \varphi(h) \leq C_{2} \varphi(k)^{\frac{N}{N-1}} \frac{p-1}{p} .
$$

On pose $\beta=\frac{N}{N-1} \frac{p-1}{p}$.

On remarque que $\beta>1$ car $p>N$ (en effet, on a $\frac{N}{N-1} \frac{p-1}{p}>1 \Leftrightarrow N p-N>N p-p$ ).

On peut alors appliquer l'exercice 2.17 il donne l'existence de $a \in \mathbb{R}_{+}$t.q. $\varphi(a)=0$ et donc $\|u\|_{L^{\infty}(\Omega)} \leq a$.

5. On suppose tout d'abord que $\||F|\|_{L^{p}(\Omega)}=1$, ce qui donne, avec les notations des questions précédentes, $C_{2}=\frac{C_{1}}{\alpha}$.

On reprend alors le corrigé de l'exercice 2.17 Le choix de $a_{0}$ est t.q. $\varphi\left(a_{0}\right) \leq 1$. Comme

$$
\varphi(0) \leq \operatorname{mes}(\Omega)^{\frac{N-1}{N}}, \beta \frac{N-1}{N}=\frac{p-1}{p} \text { et } C_{2}=C_{1} / \alpha
$$

il suffit donc de prendre $a_{0}$ t.q.

$$
\frac{C_{1} m e s(\Omega)^{\frac{p-1}{p}}}{\alpha a_{0}} \leq 1
$$

On peut donc choisir $a_{0}=\frac{C_{1} m e s(\Omega)^{\frac{p-1}{p}}}{\alpha}$. On a alors $\|u\|_{L^{\infty}(\Omega)} \leq$ a avec

$$
a=a_{0}+\frac{2 C_{2}}{1-b}=a_{0}+\frac{C_{1}}{\alpha} \frac{2}{1-\frac{1}{2^{\beta-1}}} .
$$

On a donc $\|u\|_{L^{\infty}(\Omega)} \leq C_{3}$, avec $C_{3}=a_{0}+\frac{C_{1}}{\alpha} \frac{2}{1-\frac{1}{2^{\beta-1}}}$.

On remarque bien que $C_{3}$ ne dépend que $\Omega$, $\alpha$ et $p$ (noter que $N$ est implicitement dans $\Omega$ ).

On peut maintenant supposer que F est quelconque dans $L^{p}(\Omega)^{N}$ (la fonction u est toujours la solution de 2.55). Pour $\gamma>0$ la fonction $u / \gamma$ est solution de 2.55 avec $F / \gamma$ au lieu de $F$. Si $\||F|\|_{L^{p}(\Omega)}>0$, en choisissant $\gamma=\||F|\|_{L^{p}(\Omega)}$ (de sorte que $\||F / \gamma|\|_{L^{p}(\Omega)}=1$ ) on a donc $\|u / \gamma\|_{\leq} C_{3}$ ce qui donne

$$
\|u\|_{L^{\infty}(\Omega)} \leq C_{3}\||F|\|_{L^{p}(\Omega)} .
$$

(Noter aussi que l'inégalité est évidente si $\||F|\|_{L^{p}(\Omega)}=0$.)

\section{Exercice 2.19 (Solutions bornées d'un problème elliptique, suite)}

On reprend les premières hypothèses de l'exercice 2.18

Soit $\Omega$ un ouvert borné de $\mathbb{R}^{N}(N>1)$. Pour tout $x \in \Omega$, on se donne une matrice, notée $A(x)$, dont les coefficients sont notés $a_{i, j}(x), i, j=1, \ldots, N$. On suppose que $a_{i, j} \in L^{\infty}(\Omega)$ pour tout $i, j=1, \ldots, N$ et qu'il existe $\alpha>0$ t.q $A(x) \xi \cdot \xi \geq \alpha|\xi|^{2}$, pour tout $\xi \in \mathbb{R}^{N}$ et p.p. en $x \in \Omega$.

1. Soit $f \in L^{p}(\Omega)$ avec $p>1$ si $N=2$ et $p=2 N /(N+2)$ si $N \geq 3$. Montrer qu'il existe un et un seul $u$ solution de

$$
\left\{\begin{array}{l}
u \in H_{0}^{1}(\Omega), \\
\int_{\Omega} A(x) \nabla u(x) \cdot \nabla v(x) \mathrm{d} x=\int_{\Omega} f(x) v(x) \mathrm{d} x, \text { pour tout } v \in H_{0}^{1}(\Omega) .
\end{array}\right.
$$


2. Soit $p>N / 2$ et $f \in L^{p}(\Omega)$. Montrer qu'il existe un unique $u$ solution de 2.56]. [Se ramener à la question précédente.]

Montrer que $u \in L^{\infty}(\Omega)$ et qu'il existe $C$ ne dépendant que de $\Omega, \alpha$ et $p$ t.q.

$$
\|u\|_{L^{\infty}(\Omega)} \leq C\|f\|_{L^{p}(\Omega)} .
$$

[Se ramener à l'exercice 2.18]

\section{Exercice 2.20 (Diffusion évanescente et convection)}

\section{Partie I}

Soient $\Omega$ un ouvert borné de $\mathbb{R}^{N}(N \geq 1)$ et $w=\left(w_{1}, \ldots, w_{N}\right)^{t} \in\left(L^{\infty}(\Omega)\right)^{N}$ t.q. $\operatorname{div}(w)=0$ dans $\mathcal{D}^{\star}(\Omega)($ On rappelle que $\left.\operatorname{div}(w)=\sum_{i=1}^{N} D_{i} w_{i}\right)$. Soit $u \in H_{0}^{1}(\Omega)$.

1. Montrer que $u^{2} \in W_{0}^{1,1}(\Omega)$ et que $D_{i}\left(u^{2}\right)=2 u D_{i} u$, pour tout $i \in 1, \ldots, N$. [Utiliser la densité de $C_{c}^{\infty}(\Omega)$ dans $H_{0}^{1}(\Omega)$.]

2. Montrer que $\int_{\Omega} w(x) \cdot \nabla \varphi(x) \mathrm{d} x=0$, pour tout $\varphi \in W_{0}^{1,1}(\Omega)$. [Utiliser la densité de $C_{c}^{\infty}(\Omega)$ dans $W_{0}^{1,1}(\Omega)$.]

3. Montrer que $\int_{\Omega} w(x) \cdot \nabla\left(u^{2}\right)(x) \mathrm{d} x=2 \int_{\Omega} u(x) w(x) \cdot \nabla u(x) \mathrm{d} x=0$ (on rappelle que $w \cdot \nabla\left(u^{2}\right)=\sum_{i=1}^{N}$ $\left.w_{i} D_{i}\left(u^{2}\right)\right)$.

\section{Partie II}

Soit $\Omega$ un ouvert borné de $\mathbb{R}^{N}$, à frontière lipschiztienne (cette hypothèse donne l'existence de l'opérateur trace, noté $\gamma$, linéaire continu de $H^{1}(\Omega)$ dans $L^{2}(\partial \Omega)$ et t.q. $\gamma(u)=u$ sur $\partial \Omega$ si $u \in C(\bar{\Omega}) \cap H^{1}(\Omega)$ et $\operatorname{Ker}(\gamma)=$ $H_{0}^{1}(\Omega)$ ). Soient $a \in \mathbb{R}_{+}^{\star}$ et $w \in\left(L^{\infty}(\Omega)\right)^{N}$ t.q. $\operatorname{div}(w)=0$ dans $\mathcal{D}^{\star}(\Omega)$. Soient $f \in L^{2}(\Omega)$ et $g \in \operatorname{Im} \gamma$. On cherche $u$ solution du problème suivant :

$$
\begin{aligned}
& u \in H^{1}(\Omega), \gamma(u)=g\left(\text { dans } L^{2}(\partial \Omega)\right), \\
& \int_{\Omega} a \nabla u(x) \cdot \nabla v(x) \mathrm{d} x+\int_{\Omega} u(x) w(x) \cdot \nabla v(x) \mathrm{d} x=\int_{\Omega} f(x) v(x) \mathrm{d} x, \forall v \in H_{0}^{1}(\Omega) .
\end{aligned}
$$

1. Soit $G \in H^{1}(\Omega)$ t.q. $\gamma(G)=g\left(\right.$ dans $L^{2}(\partial \Omega)$ ). Montrer que $u$ est solution de 2.57) si et seulement si $u=G+\bar{u}$ avec $\bar{u}$ solution de 2.58 .

$$
\begin{aligned}
& \bar{u} \in H_{0}^{1}(\Omega), \\
& \int_{\Omega} a \nabla \bar{u}(x) \cdot \nabla v(x) \mathrm{d} x+\int_{\Omega} \bar{u}(x) w(x) \cdot \nabla v(x) \mathrm{d} x= \\
& \int_{\Omega} f(x) v(x) \mathrm{d} x-\int_{\Omega} a \nabla G(x) \cdot \nabla v(x) \mathrm{d} x-\int_{\Omega} G(x) w(x) \cdot \nabla v(x) \mathrm{d} x, \forall v \in H_{0}^{1}(\Omega) .
\end{aligned}
$$

2. Montrer que 2.57) admet une et une seule solution.

On note $u$ cette solution dans la suite de cette partie.

3. On suppose, dans cette question, que $g=0$ (de sorte que $u \in H_{0}^{1}(\Omega)$ ). Montrer que $a\|u\|_{H_{0}^{1}}^{2} \leq \int_{\Omega} f(x) u(x) \mathrm{d} x$.

4. Soit $b \in \mathbb{R}$. On suppose, dans cette question, que $f \leq 0$ p.p. dans $\Omega$ et que $g \leq b$ p.p. sur $\partial \Omega$ (pour la mesure $N-1$-dimensionnelle sur $\partial \Omega$ ). Montrer que $u \leq b$ p.p. dans $\Omega$. [On pourra admettre que $(u-b)^{+} \in H_{0}^{1}(\Omega)$ et que $\nabla(u-b)^{+}=1_{u>b} \nabla u$ p.p. (ce résultat est semblable à celui du lemme 2.22, utiliser (2.57) et la partie I.] 


\section{Partie III}

Dans cette partie on prend $N=2, \Omega=] 0,1\left[2, w=(-1,0)\right.$ et $g=0$. On suppose aussi que $f \in L^{\infty}(\Omega)$ et que $f \geq 0$ p.p. sur $\Omega$. On note $u_{n}$ la solution de (2.57) pour $a=1 / n\left(n \in \mathbb{N}^{\star}\right)$ et on s'intéresse à la limite de $u_{n}$ quand $n \rightarrow \infty$.

1. Soit $n \in \mathbb{N}^{\star}$. Montrer que $u_{n} \geq 0$ p.p..[Utiliser la Partie II, question 4.]

2. Soit $n \in \mathbb{N}^{\star}$. Montrer qu'il existe $C_{1}$, ne dépendant que de $f$, t.q. $\left\|u_{n}\right\|_{L^{\infty}(\Omega)} \leq C_{1}$. [On pourra, par exemple, chercher de quel problème de type 2.57) est solution la fonction $u_{n}+\beta \psi$, avec $\psi(x)=x_{1}$ et $\beta$ convenablement choisi, et utiliser la Partie II, question 4.]

3. Soit $n \in \mathbb{N}^{\star}$. Montrer qu'il existe $C_{2}$, ne dépendant que de $f$, t.q. $\left\|u_{n}\right\|_{H_{0}^{1}(\Omega)} \leq C_{2} \sqrt{n}$.

4. En utilisant la remarque 2.15 , montrer que $u_{n} \in H^{2}(\Omega)$ pour tout $n \in \mathbb{N}^{\star}$.

5. Soit $n \in \mathbb{N}^{\star}$. Si $u_{n} \in C^{1}(\bar{\Omega})$, déduire de la question 1 de la partie III que $\frac{\partial u_{n}}{\partial x_{1}}\left(0, x_{2}\right) \geq 0$ et $\frac{\partial u_{n}}{\partial x_{1}}\left(1, x_{2}\right) \leq$ 0 pour tout $\left.x_{2} \in\right] 0,1\left[\right.$ (de même, $\frac{\partial u_{n}}{\partial x_{2}}\left(x_{1}, 0\right) \geq 0$ et $\frac{\partial u_{n}}{\partial x_{2}}\left(x_{1}, 1\right) \leq 0$ pour tout $\left.x_{1} \in\right] 0,1[$ ). On admettra, dans la suite, que ce résultat est encore vrai, avec seulement $u_{n} \in H^{2}(\Omega)$, au sens $\gamma\left(D_{1} u_{n}\right)\left(0, x_{2}\right) \geq 0$ et $\gamma\left(D_{1} u_{n}\right)\left(1, x_{2}\right) \leq 0$ p.p. en $\left.x_{2} \in\right] 0,1\left[\right.$ (de même $\gamma\left(D_{2} u_{n}\right)\left(x_{1}, 0\right) \geq 0$ et $\gamma\left(D_{2} u_{n}\right)\left(x_{1}, 1\right) \leq 0$ p.p. en $\left.x_{1} \in\right] 0,1[)$.

6. En utilisant la question 2 de la partie III, montrer qu'on peut supposer (à une sous suite près) que $u_{n} \rightarrow u$ $\star$-faiblement dans $L^{\infty}(\Omega)$ quand $n \rightarrow+\infty$, c'est à dire :

$$
\int_{\Omega} u_{n}(x) \varphi(x) \mathrm{d} x \rightarrow \int_{\Omega} u(x) \varphi(x) \mathrm{d} x, \text { pour tout } \varphi \in L^{1}(\Omega) .
$$

Montrer que $u \geq 0$ p.p..

On cherche, dans la suite, l'équation et les conditions aux limites satisfaites par $u$.

7. Montrer que $D_{1} u=f$ dans $\mathcal{D}^{\star}(\Omega)$.

8. Soit $n \in \mathbb{N}^{\star}$ et $\varphi \in C^{1}(\bar{\Omega})$, montrer que

$$
\begin{aligned}
\frac{1}{n} \int_{\Omega} \nabla u_{n}(x) \nabla \varphi(x) \mathrm{d} x & +\frac{1}{n} \int_{0}^{1} \gamma\left(D_{1} u_{n}\right)\left(0, x_{2}\right) \varphi\left(0, x_{2}\right) \mathrm{d} x_{2}-\frac{1}{n} \int_{0}^{1} \gamma\left(D_{1} u_{n}\right)\left(1, x_{2}\right) \varphi\left(1, x_{2}\right) \mathrm{d} x_{2} \\
& +\frac{1}{n} \int_{0}^{1} \gamma\left(D_{2} u_{n}\right)\left(x_{1}, 0\right) \varphi\left(x_{1}, 0\right) \mathrm{d} x_{1}-\frac{1}{n} \int_{0}^{1} \gamma\left(D_{2} u_{n}\right)\left(x_{1}, 1\right) \varphi\left(x_{1}, 1\right) \mathrm{d} x_{1} \\
& -\int_{\Omega} u_{n}(x) \frac{\partial \varphi}{\partial x_{1}}(x) \mathrm{d} x=\int_{\Omega} f(x) \varphi(x) \mathrm{d} x .
\end{aligned}
$$

9. Soit $\varphi \in C^{1}(\bar{\Omega})$ t.q. $\varphi \geq 0$ sur $\partial \Omega$. Montrer que

$$
-\int_{\Omega} u(x) \frac{\partial \varphi}{\partial x_{1}}(x) \mathrm{d} x \leq \int_{\Omega} f(x) \varphi(x) \mathrm{d} x .
$$

10. On suppose, dans cette question, que $u \in C^{1}(\bar{\Omega})$ et que $f \in C(\bar{\Omega})$. Montrer que $\frac{\partial u}{\partial x_{1}}=f$ partout dans $\Omega$ et que $u\left(0, x_{2}\right)=0$ pour tout $\left.x_{2} \in\right] 0,1[$.

La fonction $u$ est-elle alors entièrement déterminée par $f$ ?

11. On remplaçe $w=(-1,0)$ par $w \in \mathbb{R}^{2} \backslash\{0\} \ldots$ De quel problème, dépendant de $w, u$ est elle solution? [distinguer les signes des 2 composantes de $w$.] 
Corrigé-

\section{Partie I}

1. Soit $u_{n} \in C_{c}^{\infty}(\Omega)$ t.q. $u_{n} \rightarrow u$ dans $H_{0}^{1}(\Omega)$. On a donc, quand $n \rightarrow+\infty, u_{n} \rightarrow u$ dans $L^{2}(\Omega)$ et $\partial_{i} u_{n} \rightarrow D_{i} u$ dans $L^{2}(\Omega)$ pour tout $i \in\{1, \ldots, N\}$. (On rappelle que $\partial_{i} u_{n}$ désigne la dérivée partielle classique de $u_{n}$ par rapport à sa $i$-ème variable.) On peut aussi supposer (après extraction éventuelle d'une sous suite) que $u_{n} \rightarrow$ u p.p. et qu'il existe $F \in L^{2}(\Omega)$ t.q. $\left|u_{n}\right| \leq F$ pour tout $n \in \mathbb{N}$. On en déduit, par convergence dominée, que $u_{n}^{2} \rightarrow u^{2}$ dans $L^{1}(\Omega)$ (quand $n \rightarrow+\infty$ ).

Soit $\varphi \in C_{c}^{\infty}(\Omega)$ et $i \in\{1, \ldots, N\}$, on a alors

$$
\left\langle D_{i}\left(u^{2}\right), \varphi\right\rangle_{\mathcal{D}^{\star}(\Omega), \mathcal{D}(\Omega)}=-\int_{\Omega} u^{2} \partial_{i} \varphi \mathrm{d} x=-\lim _{n \rightarrow+\infty} \int_{\Omega} u_{n}^{2} \partial_{i} \varphi \mathrm{d} x .
$$

Comme $u_{n}$ et $\varphi$ appartiennent à $\mathcal{D}(\Omega)$, on a, en intégrant par parties

$$
\int_{\Omega} u_{n}^{2} \partial_{i} \varphi \mathrm{d} x=-2 \int_{\Omega} \varphi u_{n} \partial_{i} u_{n} \mathrm{~d} x
$$

Comme $u_{n} \rightarrow u$ dans $L^{2}(\Omega)$ et $\partial_{i} u_{n} \rightarrow D_{i} u$ dans $L^{2}(\Omega)$, on a $u_{n} \partial_{i} u_{n} \rightarrow u D_{i} u$ dans $L^{1}(\Omega)$ et donc (comme $\varphi \in L^{\infty}(\Omega)$

$$
\lim _{n \rightarrow+\infty} \int_{\Omega} \varphi u_{n} \partial_{i} u_{n} \mathrm{~d} x=\int_{\Omega} \varphi u \partial_{i} u \mathrm{~d} x .
$$

En revenant à 2.60, on en déduit que

$$
\left\langle D_{i}\left(u^{2}\right), \varphi\right\rangle_{\mathcal{D}^{\star}(\Omega), \mathcal{D}(\Omega)}=-\int_{\Omega} u^{2} \partial_{i} \varphi \mathrm{d} x=2 \int_{\Omega} \varphi u D_{i} u \mathrm{~d} x .
$$

Ce qui prouve bien que $D_{i}\left(u^{2}\right)=2 u D_{i} u$ p.p. (les dérivées par transposition de $u$ et $u^{2}$ sont en fait des dérivées faibles et donc identifiées à des fonctions).

La démonstration précédente donne aussi que $u_{n}^{2} \rightarrow u^{2}$ dans $L^{1}(\Omega)$ et $\partial_{i}\left(u_{n}^{2}\right)=2 u_{n} \partial_{i} u_{n} \rightarrow 2 u D_{i} u=D_{i}\left(u^{2}\right)$ dans $L^{1}(\Omega)$. On a donc $u_{n}^{2} \rightarrow u^{2}$ dans $W^{1,1}(\Omega)$, ce qui donne, comme $u_{n} \in C_{c}^{\infty}(\Omega)$, que $u^{2} \in W_{0}^{1,1}(\Omega)$.

2. Soit $\varphi \in W_{0}^{1,1}(\Omega)$. il existe une suite $\left(\varphi_{n}\right)_{n \in \mathbb{N}}$ d'éléments de $C_{c}^{\infty}(\Omega)$ t.q. $\varphi_{n} \rightarrow \varphi$ dans $W^{1,1}(\Omega)$. Comme $\operatorname{div}(w)=$ 0 dans $\mathcal{D}^{\star}(\Omega)$, on a, pour tout $n \in \mathbb{N}$

$$
0=\left\langle\operatorname{div}(w), \varphi_{n}\right\rangle_{\mathcal{D}^{\star}(\Omega), \mathcal{D}(\Omega)}=-\sum_{i=1}^{N} \int_{\Omega} w_{i} \partial_{i} \varphi_{n} \mathrm{~d} x .
$$

Comme $\partial_{i} \varphi_{n} \rightarrow D_{i} \varphi$ dans $L^{1}(\Omega)$ (et que $\left.w \in L^{\infty}(\Omega)^{N}\right)$, on en déduit, quand $n \rightarrow+\infty$,

c'est-à-dire $\int_{\Omega} w(x) \cdot \nabla \varphi(x) \mathrm{d} x=0$.

$$
\sum_{i=1}^{N} \int_{\Omega} w_{i} D_{i} \varphi \mathrm{d} x=0
$$

3. On utilise le résulat de la question précédente avec $\varphi=u^{2}$ et le fait que $D_{i}\left(u^{2}\right)=2 u D_{i} u$, on obtient

$$
0=\int_{\Omega} w \cdot \nabla\left(u^{2}\right) \mathrm{d} x=\sum_{i=1}^{N} \int_{\Omega} 2 w_{i} u D_{i} u \mathrm{~d} x=2 \int_{\Omega} u w \cdot \nabla u \mathrm{~d} x .
$$

\section{Partie II}

1. On suppose que u est solution de 2.57 et on pose $\bar{u}=u-G$. On a alors $\bar{u} \in H^{1}(\Omega)$ et, comme $\gamma$ est un opérateur linéaire, $\gamma(\bar{u})=\gamma(u)-\gamma(G)=g-g=0$ (dans $L^{2}(\partial \Omega)$ ), ce qui prouve que $\bar{u} \in H_{0}^{1}(\Omega)$. Puis, si $v \in H_{0}^{1}(\Omega)$, en remplaçant u par $\bar{u}+G$ dans 2.57, on montre bien que $\bar{u}$ est solution de 2.58.

Réciproquement, on suppose que $\bar{u}$ est solution de 2.58. On pose alors $u=\bar{u}+G$, on a bien $u \in H^{1}(\Omega)$ et $\gamma(u)=\gamma(\bar{u})+\gamma(G)=0+g=g\left(\right.$ dans $\left.L^{2}(\partial \Omega)\right)$. Puis, si $v \in H_{0}^{1}(\Omega)$, en remplaçant $\bar{u}$ par $u-G$ dans 2.58, on montre bien que u est solution de 2.57.

On a bien ainsi montré l'équivalence désirée. 
2. En utilisant la lemme de Lax-Milgram (lemme 2.4 on va montrer que 2.58 admet une et une seule solution (grâce à la question précédente, on en déduit que 2.57 admet une et une seule solution).

Le problème 2.58 peut s'écrire

$$
\begin{aligned}
& \bar{u} \in H, \\
& \bar{a}(\bar{u}, v)=T(v) \text { pour tout } v \in H,
\end{aligned}
$$

avec $H=H_{0}^{1}(\Omega)$,

$$
\bar{a}(\bar{u}, v)=\int_{\Omega} a \nabla \bar{u}(x) \cdot \nabla v(x) \mathrm{d} x+\int_{\Omega} \bar{u}(x) w(x) \cdot \nabla v(x) \mathrm{d} x
$$

et

$$
T(v)=\int_{\Omega} f(x) v(x) \mathrm{d} x-\int_{\Omega} a \nabla G(x) \cdot \nabla v(x) \mathrm{d} x-\int_{\Omega} G(x) w(x) \cdot \nabla v(x) \mathrm{d} x .
$$

L'espace $H$ est bien un espace de Hilbert (avec sa norme naturelle). L'application $T$ est bien linéaire de $H$ dans $\mathbb{R}$ et, en utilisant l'inégalité de Hölder, on voit que T est continue (on utilise ici le fait que $f \in L^{2}(\Omega), G \in H^{1}(\Omega)$ et $w \in$ $\left.L^{\infty}(\Omega)^{d}\right)$. L'application a est bien bilinéaire de $H \times H$ dans $R$ et continue (grâce encore au fait que $\left.w \in L^{\infty}(\Omega)^{d}\right)$. Pour montrer la coercivité de $\bar{a}$, on utilise la question 3 de la partie I, elle donne, pour tout $u \in H$,

$$
\bar{a}(u, u)=\int_{\Omega} a \nabla u(x) \cdot \nabla u(x) \mathrm{d} x+\int_{\Omega} u(x) w(x) \cdot \nabla u(x) \mathrm{d} x=\int_{\Omega} a \nabla u(x) \cdot \nabla u(x) \mathrm{d} x=a\|u\|_{H_{0}^{1}(\Omega)} .
$$

Comme $a>0$, on en déduit bien que à est coercive. On peut donc appliquer le lemme 2.4 il donne l'existence et l'unicité de ù solution de 2.58. Grâce à la question précédente, on en déduit l'existence et l'unicité de u solution de 2.57 .

Petite précision : Noter que la non unicité de G n'est pas un problème pour l'unicité de de la solution de 2.57. En effet, on fixe $G$ et on note $\bar{u}$ l'unique solution de 2.58. Si u est solution de 2.57, $u-G$ est solution de 2.58 et donc $u=\bar{u}+G$. La fonction $\bar{u}+G$ est donc l'unique solution de 2.57]. En fait, la fonction $\bar{u}$ dépend du choix de $G$ mais la fonction $\bar{u}+G$ ne dépend pas de $G$.

3. Il sufffit de prendre $v=u$ dans 2.57. Avec la question 3 de la partie I on obtient

$$
a\|u\|_{H_{0}^{1}(\Omega)}^{2}=\int_{\Omega} f(x) u(x) \mathrm{d} x .
$$

4. Comme $(u-b)^{+} \in H_{0}^{1}(\Omega)$, on peut prendre $v=(u-b)^{+}$dans 2.57, on obtient, en utilisant $\nabla(u-b)^{+}=1_{u>b} \nabla u$ p.p. et $f \leq 0$ p.p.,

$$
\int_{u>b} a \nabla u(x) \cdot \nabla u(x) \mathrm{d} x+\int_{\Omega} u(x) w(x) \cdot \nabla(u-b)^{+}(x) \mathrm{d} x=\int_{\Omega} f(x)(u-b)^{+}(x) \mathrm{d} x \leq 0 .
$$

On remarque maintenant que $\int_{u>b} a \nabla u(x) \cdot \nabla u(x) \mathrm{d} x=\int_{\Omega} a \nabla(u-b)^{+} \cdot \nabla(u-b)^{+}=a\left\|(u-b)^{+}\right\|_{H_{0}^{1}(\Omega)}$ et que

$$
\begin{aligned}
\int_{\Omega} u(x) w(x) \cdot \nabla(u-b)^{+}(x) \mathrm{d} x & =\int_{\Omega}(u(x)-b) w(x) \cdot \nabla(u-b)^{+}(x) \mathrm{d} x+b \int_{\Omega} w(x) \cdot \nabla(u-b)^{+}(x) \mathrm{d} x \\
& =\int_{\Omega}(u(x)-b)^{+} w(x) \cdot \nabla(u-b)^{+}(x) \mathrm{d} x+b \int_{\Omega} w(x) \cdot \nabla(u-b)^{+}(x) \mathrm{d} x .
\end{aligned}
$$

La question 3 de la partie I donne $\int_{\Omega}(u(x)-b)^{+} w(x) \cdot \nabla(u-b)^{+}(x) \mathrm{d} x=0$. D'autre part, comme div $(w)=0$ dans $\mathcal{D}^{\star}(\Omega)$, on a $\int_{\Omega} w \cdot \nabla \varphi \mathrm{d} x=0$ pour tout $\varphi \in C_{c}^{\infty}(\Omega)$. Par densité de $C_{c}^{\infty}(\Omega)$ dans $H_{0}^{1}(\Omega)$ on a aussi (on utilise ici seulement le fait que $\left.w \in L^{2}(\Omega)^{N}\right) \int_{\Omega} w \cdot \nabla \varphi \mathrm{d} x=0$ pour tout $\varphi \in H_{0}^{1}(\Omega)$ et donc, en particulier pour $\varphi=(u-b)^{+}$. On en déduit que

$$
\int_{\Omega} u(x) w(x) \cdot \nabla(u-b)^{+}(x) \mathrm{d} x=0 .
$$

Revenant à 2.62, on obtient finalement $a\left\|(u-b)^{+}\right\|_{H_{0}^{1}(\Omega)} \leq 0$ et donc $(u-b)^{+}=0$ p.p., c'est-à-dire $u \leq b$ p.p. dans $\Omega$.

Remarque : On suppose maintenant $g=0$ et on note u la solution de 2.57. La démonstration précédente montre donc que $u \leq 0$ p.p. dans $\Omega$ si $f \leq 0$ p.p. dans $\Omega$. Si maintenant on suppose $f \geq 0$ On remarque que $(-u)$ est la solution de 2.57 avec $(-f)$ au lieu de $f$. On a donc $(-u) \leq 0$ p.p., c'est-à-dire $u \geq 0$ p.p.. 


\section{Partie III}

1. Le fait que $u_{n} \geq 0$ p.p. dans $\Omega$ est une conséquence directe de la remarque à la fin de la démonstration de la question 4 de la partie II.

2. La fonction $u_{n}$ vérfie

$$
\begin{aligned}
& u_{n} \in H_{0}^{1}(\Omega), \\
& \frac{1}{n} \int_{\Omega} \nabla u_{n}(x) \cdot \nabla v(x) \mathrm{d} x-\int_{\Omega} u_{n}(x) D_{1} v(x) \mathrm{d} x=\int_{\Omega} f(x) v(x) \mathrm{d} x, \forall v \in H_{0}^{1}(\Omega) .
\end{aligned}
$$

On pose $\bar{u}_{n}=u_{n}+\beta \psi\left(\right.$ de sorte que $\left.\nabla \bar{u}_{n}=\nabla u_{n}+\beta(1,0)^{t}\right)$. Les formules d'intégration par parties dans $H^{1}(\Omega)$ (théorème 1.24 donnent que, pour une fonction $v \in H_{0}^{1}(\Omega)$, on a

$$
\int_{\Omega} D_{1} v \mathrm{~d} x=0, \quad \int_{\Omega} \psi D_{1} v \mathrm{~d} x=-\int_{\Omega} v \partial_{1} \psi \mathrm{d} x=-\int_{\Omega} v \mathrm{~d} x .
$$

On en déduit que la fonction $\bar{u}_{n}$ est solution de

$$
\begin{aligned}
& \bar{u}_{n} \in H^{1}(\Omega), \gamma\left(u_{n}\right)=\beta x_{1}\left(\text { dans } L^{2}(\partial \Omega)\right), \\
& \frac{1}{n} \int_{\Omega} \nabla \bar{u}_{n}(x) \cdot \nabla v(x) \mathrm{d} x-\int_{\Omega} \bar{u}_{n}(x) D_{1} v(x) \mathrm{d} x=\int_{\Omega}(f(x)+\beta) v(x) \mathrm{d} x, \forall v \in H_{0}^{1}(\Omega) .
\end{aligned}
$$

On choisit $\beta=-\|f\|_{L^{\infty}(\Omega)}$. On a alors $f+\beta \leq 0$ p.p. dans $\Omega$ et $\beta x_{1} \leq 0$ sur $\partial \Omega$. On peut donc appliquer la question 4 de la partie 2 , elle donne $\bar{u}_{n} \leq 0$ p.p. dans $\Omega$ et donc $u_{n} \leq\|f\|_{L^{\infty}(\Omega)}$ p.p. dans $\Omega$. Avec la question précédente, ceci donne $0 \leq u_{n} \leq\|f\|_{L^{\infty}(\Omega)}$ p.p. dans $\Omega$. On peut donc choisir $C_{1}=\|f\|_{L^{\infty}(\Omega)}$.

3. La question 3 de la partie II donne

$$
\frac{1}{n}\left\|u_{n}\right\|_{H_{0}^{1}(\Omega)}^{2} \leq \int_{\Omega} f u_{n} \mathrm{~d} x
$$

Comme (avec $C_{1}$ donné à la question précédente) $\int_{\Omega} f u_{n} \mathrm{~d} x \leq \lambda_{N}(\Omega)\left\|u_{n}\right\|_{\infty}\|f\|_{\infty} \leq \lambda_{N}(\Omega) C_{1}\|f\|_{\infty}$, on a donc

$$
\left\|u_{n}\right\|_{H_{0}^{1}(\Omega)}^{2} \leq n \lambda_{N}(\Omega) C_{1}\|f\|_{\infty} .
$$

On peut donc prendre $C_{2}=\sqrt{\lambda_{N}(\Omega) C_{1}\|f\|_{\infty}}$.

4. Soit $n \in \mathbb{N}^{\star}$. La fonction $u_{n}$ est la solution faible de $-\Delta u_{n}=f-D_{1} u_{n}$. Comme $\Omega$ est convexe et que $f-D_{1} u_{n} \in$ $L^{2}(\Omega)\left(\right.$ car $u_{n} \in H_{0}^{1}(\Omega)$ ), la remarque 2.15 donne que $u \in H^{2}(\Omega)$.

5. Comme $u_{n} \in C^{1}(\bar{\Omega})$ et que $u_{n}=0$ sur $\partial \Omega$, on a, pour tout $\left.x_{2} \in\right] 0,1[$,

$$
\frac{\partial u_{n}}{\partial x_{1}}\left(0, x_{2}\right)=\lim _{x_{1} \rightarrow 0^{+}} \frac{u_{n}\left(x_{1}, x_{2}\right)}{x_{1}} .
$$

La question 1 de la partie III donne que $u_{n}\left(x_{1}, x_{2}\right) \geq 0$ pour tout $\left(x_{1}, x_{2}\right) \in \Omega$ (comme $u_{n}$ est continue, le fait que $u_{n} \geq 0$ p.p. dans $\Omega$ implique que $u_{n} \geq 0$ partout dans $\Omega$ ). On en déduit que $\frac{\partial u_{n}}{\partial x_{1}}\left(0, x_{2}\right) \geq 0$ pour tout $\left.x_{2} \in\right] 0,1[$. Les trois autres propriétés demandées se montrent de manière analogue.

6. La question 2 de la partie III donne que la suite $\left(u_{n}\right)_{n \in \mathbb{N}}$ est borné dans $L^{\infty}(\Omega)$. Il existe donc une sous suite de la suite $\left(u_{n}\right)_{n \in \mathbb{N}}$, encore notée $\left(u_{n}\right)_{n \in \mathbb{N}}$, et il existe $u \in L^{\infty}(\Omega)$ t.q. $u_{n} \rightarrow u \star$-faiblement dans $L^{\infty}(\Omega)$ quand $n \rightarrow+\infty$.

En prenant $\varphi=1_{u<0}$, on remarque que $\int_{\Omega} u_{n} \varphi \mathrm{d} x \geq 0$ (car $u_{n} \geq 0$ p.p.) et donc $\int_{\Omega} u \varphi \mathrm{d} x \geq 0$, c'est-à-dire

Ceci donne bien $u \geq 0$ p.p..

$$
\int_{u<0} u(x) \mathrm{d} x \geq 0
$$

7. Soit $\varphi \in C_{c}^{\infty}(\Omega)$. Pour tout $n \in \mathbb{N}^{\star}$ on a

$$
\frac{1}{n} \int_{\Omega} \nabla u_{n}(x) \cdot \nabla \varphi(x) \mathrm{d} x-\int_{\Omega} u_{n}(x) \partial_{1} \varphi(x) \mathrm{d} x=\int_{\Omega} f(x) \varphi(x) \mathrm{d} x .
$$

Comme $\left\|u_{n}\right\|_{H_{0}^{1}(\Omega)} \leq C_{2} \sqrt{n}$, on a

$$
\left|\frac{1}{n} \int_{\Omega} \nabla u_{n}(x) \cdot \nabla \varphi(x) \mathrm{d} x\right| \leq \frac{1}{n}\left\|u_{n}\right\|_{H_{0}^{1}(\Omega)}\|\varphi\|_{H_{0}^{1}(\Omega)} \leq \frac{C_{2}}{\sqrt{n}}\|\varphi\|_{H_{0}^{1}(\Omega)} .
$$


On a donc $\lim _{n \rightarrow+\infty} \frac{1}{n} \int_{\Omega} \nabla u_{n}(x) \cdot \nabla \varphi(x) \mathrm{d} x=0$.

D'autre part, on a $u_{n} \rightarrow u \star$-faiblement dans $L^{\infty}(\Omega)$, on en déduit que

$$
\lim _{n \rightarrow+\infty} \int_{\Omega} u_{n}(x) \partial_{1} \varphi(x) \mathrm{d} x=\int_{\Omega} u(x) \partial_{1} \varphi(x) \mathrm{d} x .
$$

Quand $n \rightarrow+\infty$ dans 2.63, on obtient donc

$$
-\int_{\Omega} u(x) \partial_{1} \varphi(x) \mathrm{d} x=\int_{\Omega} f(x) \varphi(x) \mathrm{d} x
$$

ce qui donne bien $D_{1} u=f$ dans $\mathcal{D}^{\star}(\Omega)$.

8. Comme $u_{n} \in H^{2}(\Omega)$, la dérivée par transposition $-\Delta u_{n}$ est un élément de $L^{2}(\Omega)$ et 2.63 donne

$$
-\frac{1}{n} \Delta u_{n}+D_{1} u_{n}=f \text { p.p.. }
$$

En multipliant cette équation par $\varphi$ (on utilise ici uniquement le fait que $\varphi \in L^{2}(\Omega)$ ) on a donc

$$
-\frac{1}{n} \int_{\Omega} \Delta u_{n} \varphi \mathrm{d} x+\int_{\Omega} \varphi D_{1} u_{n} \mathrm{~d} x=\int_{\Omega} f \varphi \mathrm{d} x .
$$

Comme les fonctions $D_{1} u_{n}$ et $D_{2} u_{n}$ sont dans $H^{1}(\Omega)$, on peut maintenant utiliser les formules d'intégration par parties (théorème 1.24. On obtient (comme $\varphi \in C^{1}(\bar{\Omega})$ )

$$
\begin{aligned}
-\frac{1}{n} \int_{\Omega}\left(D_{1} D_{1} u_{n}\right) \varphi \mathrm{d} x=\frac{1}{n} \int_{\Omega} D_{1} u_{n} \partial_{1} \varphi \mathrm{d} x & +\frac{1}{n} \int_{0}^{1} \gamma\left(D_{1} u_{n}\right)\left(0, x_{2}\right) \varphi\left(0, x_{2}\right) \mathrm{d} x_{2} \\
& -\frac{1}{n} \int_{0}^{1} \gamma\left(D_{1} u_{n}\right)\left(1, x_{2}\right) \varphi\left(1, x_{2}\right) \mathrm{d} x_{2} .
\end{aligned}
$$

$e t$

$$
\begin{aligned}
-\frac{1}{n} \int_{\Omega}\left(D_{2} D_{2} u_{n}\right) \varphi \mathrm{d} x=\frac{1}{n} \int_{\Omega} D_{2} u_{n} \partial_{2} \varphi \mathrm{d} x & +\frac{1}{n} \int_{0}^{1} \gamma\left(D_{2} u_{n}\right)\left(x_{1}, 0\right) \varphi\left(x_{1}, 0\right) \mathrm{d} x_{1} \\
& -\frac{1}{n} \int_{0}^{1} \gamma\left(D_{2} u_{n}\right)\left(x_{1}, 1\right) \varphi\left(x_{1}, 1\right) \mathrm{d} x_{1} .
\end{aligned}
$$

Une intégration par parties donne aussi (comme $u_{n} \in H_{0}^{1}(\Omega)$ )

$$
\int_{\Omega} \varphi D_{1} u_{n} \mathrm{~d} x=-\int_{\Omega} u_{n} \partial_{1} \varphi \mathrm{d} x .
$$

En utilisant ces trois intégrations par parties dans 2.64, on obtient l'égalité demandée.

9. Comme $\varphi \geq 0$ sur $\partial \Omega$, la question 5 et l'égalité de la question 8 donnent, pour tout $n \in \mathbb{N}^{\star}$,

$$
\frac{1}{n} \int_{\Omega} \nabla u_{n}(x) \nabla \varphi(x) \mathrm{d} x-\int_{\Omega} u_{n}(x) \frac{\partial \varphi}{\partial x_{1}}(x) \mathrm{d} x \leq \int_{\Omega} f(x) \varphi(x) \mathrm{d} x .
$$

On peut alors passer à la limite quand $n \rightarrow+\infty$, comme à la question 7 , et on obtient bien 2.59 .

10. La question 7 donne $D_{1} u=f$ dans $\mathcal{D}^{\star}(\Omega)$. Comme u est de classe $C^{1}$, $D_{1}$ u est représenté par la dérivée classique de $u$. Puis, comme $\partial_{1} u$ et $f$ sont continues sur $\Omega$, on en déduit que $\partial_{1} u=f$ partout dans $\Omega$. Comme $\partial_{1} u$ et $f$ sont continues sur $\bar{\Omega}$, on a même $\partial_{1} u=f$ partout dans $\bar{\Omega}$.

On prend maintenant $\varphi \in C^{1}(\bar{\Omega})$ t.q. $\varphi \geq 0$ sur $\partial \Omega, \varphi(x)=0$ si $x=\left(x_{1}, x_{2}\right) \in \partial \Omega, x_{1} \neq 0$.. Une intégration par parties dans 2.59 donne alors

$$
\int_{0}^{1} u\left(0, x_{2}\right) \varphi\left(0, x_{2}\right) \mathrm{d} x_{2} \leq 0 .
$$

Dans cette inégalité, la fonction $\varphi(0, \cdot)$ peut être égale (par exemple) à n'importe quelle fonction appartenant à $C_{c}^{\infty}(] 0,1[)$ et prenant ses valeurs dans $\mathbb{R}_{+}$. Comme $u(0, \cdot)$ est une fonction continue sur $] 0,1[$, On déduit donc de cette inégalité que $u\left(0, x_{2}\right) \leq 0$ pour tout $\left.x_{2} \in\right] 0,1[$.

La question 6 donne $u \geq 0$ p.p. sur $\Omega$. Comme u est continue sur $\bar{\Omega}$, on a donc $u \geq 0$ partout sur $\bar{\Omega}$. On obtient donc finalement $u\left(0, x_{2}\right)=0$ pour tout $\left.x_{2} \in\right] 0,1[$ (et même $[0,1]$ ).

La fonction u est bien entièrement déterminée par $f$, on a

$$
u\left(x_{1}, x_{2}\right)=\int_{0}^{x_{1}} f\left(t, x_{2}\right) \mathrm{d} t .
$$


11. On pose $w=(\alpha, \beta)$, avec $\alpha, \beta \neq 0$. En reprenant la même méthode que celle développée ci dessus pour le cas $w=(-1,0)$, la question équivalente à la question 10 donnera que u est solution du problème suivant :

$$
-w \cdot \nabla u=f \text { dans } \Omega,
$$

$u\left(0, x_{2}\right)=0$ pour tout $\left.x_{2} \in\right] 0,1\left[\right.$ si $\alpha<0$ et $u\left(1, x_{2}\right)=0$ pour tout $\left.x_{2} \in\right] 0,1[$ si $\alpha>0$.

$u\left(x_{1}, 0\right)=0$ pour tout $\left.x_{1} \in\right] 0,1\left[\right.$ si $\beta<0$ et $u\left(x_{1}, 1\right)=0$ pour tout $\left.x_{1} \in\right] 0,1[$ si $\beta>0$.

Exercice 2.21 (Condition de Dirichlet non homogène) Soit $\Omega$ un ouvert borné de $\mathbb{R}^{N}$ à frontière lipschitzienne et $g \in \operatorname{Im}(\gamma)$ (où $\gamma$ désigne l'opérateur trace vu au théorème 1.23 . Soient $\left(a_{i, j}\right)_{i, j=1, \ldots, N} \subset L^{\infty}(\Omega)$ et $\alpha>0$ tels que [2.1] soit vérifiée.

1. Soit $f \in L^{2}(\Omega)$. Montrer que 2.13 admet une unique solution.

2. Soit $T \in H^{-1}(\Omega)$. Montrer que 2.14) admet une unique solution.

3. On suppose dans cette question que $N=2$ et $1<p \leq+\infty$. Montrer que pour tout $f \in L^{p}(\Omega)$ il existe une unique solution au problème 2.13.

4. On suppose dans cette question que $N \geq 3$ et $p=2 N /(N+2)$. Montrer que pour tout $f \in L^{p}(\Omega)$ il existe une unique solution au problème 2.13.

Exercice $2.22\left(\right.$ Espace $\boldsymbol{H}^{\mathbf{1 / 2}}(\partial \Omega)$ ) Soit $\Omega$ un ouvert borné de $\mathbb{R}^{N}(N \geq 1)$ à frontière lipschitzienne. On note $\gamma$ l'opérateur trace défini sur $H^{1}(\Omega)$.

On rappelle que $H^{1 / 2}(\partial \Omega)=\operatorname{Im} \gamma$ et que $\|u\|_{H^{1 / 2}(\partial \Omega)}=\inf \left\{\|\bar{u}\|_{H^{1}(\Omega)}, \gamma(\bar{u})=u\right\}$.

1. Soit $u \in H^{1 / 2}(\partial \Omega)$. Montrer que $\|u\|_{H^{1 / 2}(\partial \Omega)}=\|\bar{u}\|_{H^{1}(\Omega)}$ où $\bar{u}$ est l'unique solution faible de $-\Delta \bar{u}=0$ dans $\Omega$ avec $\gamma(\bar{u})=u$, c'est-à-dire l'unique solution de

$$
\begin{aligned}
& \bar{u} \in H^{1}(\Omega), \gamma(\bar{u})=u, \\
& \int_{\Omega} \nabla \bar{u}(x) \nabla v(x) \mathrm{d} x=0, \forall v \in H_{0}^{1}(\Omega) .
\end{aligned}
$$

2. Montrer que l'espace $H^{1 / 2}(\partial \Omega)$ est un espace de Hilbert.

3. Montrer que l'espace $H^{1 / 2}(\partial \Omega)$ s'injecte continûment dans $L^{2}(\partial \Omega)$.

Exercice 2.23 (trace normale d'un élément de $\boldsymbol{H}_{\text {div }}$ ) Soit $\Omega$ un ouvert borné de $\mathbb{R}^{2}$ à frontière lipschitzienne. On pose $H_{\text {div }}(\Omega)=\left\{v=\left(v_{1}, v_{2}\right) \in L^{2}(\Omega)^{2}\right.$ t.q. $\left.\operatorname{div}(v) \in L^{2}(\Omega)\right\}$ et, pour $v \in H_{\operatorname{div}}(\Omega)$,

$$
\|v\|_{H_{\mathrm{div}}(\Omega)}=\left(\||v|\|_{L^{2}(\Omega)}^{2}+\|\operatorname{div}(v)\|_{L^{2}(\Omega)}^{2}\right)^{\frac{1}{2}} .
$$

1. Montrer que $H_{\text {div }}(\Omega)$, muni de la norme définie par (2.65), est un espace de Hilbert.

2. Soit $v \in H_{\text {div }}(\Omega)$.

(a) Montrer que

$$
\int_{\Omega} \nabla \varphi \cdot v \mathrm{~d} x+\int_{\Omega} \varphi \operatorname{div}(v) \mathrm{d} x=0,
$$

Pour tout $\varphi \in C_{c}^{\infty}(\Omega)$, puis pour tout $\varphi \in H_{0}^{1}(\Omega)$.

(b) Soit $u_{1}, u_{2} \in H^{1}(\Omega)$ t.q. $\gamma\left(u_{1}\right)=\gamma\left(u_{2}\right)$ (où $\gamma$ est l'opérateur trace défini sur $H^{1}(\Omega)$ ). Montrer que

$$
\int_{\Omega} \nabla u_{1} \cdot v \mathrm{~d} x+\int_{\Omega} u_{1} \operatorname{div}(v) \mathrm{d} x=\int_{\Omega} \nabla u_{2} \cdot v \mathrm{~d} x+\int_{\Omega} u_{2} \operatorname{div}(v) \mathrm{d} x .
$$


On rappelle que $H^{1 / 2}(\partial \Omega)=\operatorname{Im} \gamma$ et que $H^{1 / 2}(\partial \Omega)$ est un espace de Hilbert avec la norme définie dans l'exercice 2.22. On note $H^{-1 / 2}(\partial \Omega)$ l'espace dual de $H^{1 / 2}(\partial \Omega)$ ( c'est-à-dire l'ensemble des applications linéaires continues de $H^{1 / 2}(\partial \Omega)$ dans $\left.\mathbb{R}\right)$.

3. Soit $v \in H_{\text {div }}(\Omega)$. Montrer que l'on peut définir un élément de $H^{-1 / 2}(\partial \Omega)$, noté $T(v)$, en posant, pour $u \in$ $H^{1 / 2}(\Omega)$,

$$
\langle T(v), u\rangle_{H^{-1 / 2}(\partial \Omega), H^{1 / 2}(\partial \Omega)}=\int_{\Omega} \nabla \bar{u} \cdot v \mathrm{~d} x+\int_{\Omega} \bar{u} \operatorname{div}(v) \mathrm{d} x,
$$

avec $\bar{u} \in H^{1}(\Omega)$ t.q. $\gamma(\bar{u})=u$. (En particulier, le terme de droite de 2.66) est bien défini et ne dépend pas de $\bar{u}$ si $\bar{u} \in H^{1}(\Omega)$ et $\gamma(\bar{u})=u$.)

On a ainsi défini une application $T$ de $H_{\text {div }}(\Omega)$ dans $H^{-1 / 2}(\partial \Omega)$.

4. Montrer que l'application $T$ est linéaire continue de $H_{\text {div }}(\Omega)$ dans $H^{-1 / 2}(\partial \Omega)$.

5. On suppose dans cette question que $v \in H^{1}(\Omega)^{2}$ (on a donc aussi $v \in H_{\operatorname{div}}(\Omega)$ ). On note $\gamma(v)$ la fonction obtenue sur $\partial \Omega$ en prenant la trace de chacune des composantes de $v$. (On a donc $\gamma(v) \in H^{1 / 2}(\partial \Omega)^{2} \subset$ $L^{2}(\partial \Omega)^{2}$.) On note $n(x)$ le vecteur normal à $\partial \Omega$, extérieur à $\Omega$. Comme $\Omega$ est à frontière lipschitzienne, le vecteur $n(x)$ est défini p.p. en $x \in \partial \Omega$ (p.p. signifie ici, comme d'habitude, p.p. pour la mesure de Lebesgue 1-dimensionnelle sur $\partial \Omega$ ) et la fonction $x \mapsto n(x)$ définit un élément de $L^{\infty}(\partial \Omega)$. On obtient ainsi $\gamma(v) \cdot n \in$ $L^{2}(\partial \Omega)$. Cette (classe de) fonction $(\mathrm{s}) \gamma(u) \cdot n$ est appelée "trace normale de $u$ sur $\partial \Omega$ ". Montrer que

$$
\langle T(v), u\rangle_{H^{-1 / 2}(\partial \Omega), H^{1 / 2}(\partial \Omega)}=\int_{\partial \Omega} u \gamma(v) \cdot n d \lambda(x) .
$$

N.B. Cette question explique pourquoi l'application $T(v)$ est souvent notée $v \cdot n$ même si $v \in H_{\operatorname{div}(\Omega)}$ (et non à $\left.H^{1}(\Omega)^{2}\right)$. On peut aussi montrer que $H^{1 / 2}(\partial \Omega)$ est dense dans $L^{2}(\partial \Omega)$. Ceci permet de montrer que, lorsque $v \in H^{1}(\Omega), \gamma(v) \cdot n$ est l'unique élément de $L^{2}(\partial \Omega)$ vérifiant 2.67).

Exercice 2.24 (Pas de trace normale sur une partie du bord) On reprend ici les notations de l'exercice 2.23 . Soit maintenant $I$ une partie du bord de $\Omega$. Il semble naturel de poser

$$
H^{1 / 2}(I)=\left\{u \text { t.q. } u=\gamma(\bar{u}) \text { p.p. } \operatorname{sur} I \text { avec } \bar{u} \in H^{1}(\Omega)\right\} \text {. }
$$

(où p.p. signifie p.p. pour $\lambda$.) La norme sur $H^{1 / 2}(I)$ est alors

$$
\|u\|_{H^{1 / 2}(I)}=\inf \left\{\|\bar{u}\|_{H^{1}(\Omega)}, \gamma(\bar{u})=u \text { p.p. sur } I\right\} .
$$

$H^{1 / 2}(I)$ est alors un espace de Hilbert et on note $H^{-1 / 2}(I)$ son dual. L'objectif de cet exercice est de remarquer qu'en général il n'existe pas d'opérateur $T$ linéaire continu de $H_{\text {div }}(\Omega)$ dans $H^{-1 / 2}(I)$ t.q.

$$
\langle T(v), u\rangle_{H^{-1 / 2}(I), H^{1 / 2}(I)}=\int_{I} u v \cdot n d \lambda(x),
$$

pour tout $u \in H^{1 / 2}(I)$ et $v \in C^{1}\left(\bar{\Omega}, \mathbb{R}^{2}\right)$. Autrement dit on ne peut pas prolonger continûment l'opérateur naturel $v \mapsto v \cdot n$ (bien défini si $v$ est régulière) à l'espace $H_{\text {div }}(\Omega)$. Nous donnons dans cet exercice un exemple pour lequel ce prolongement est effectivement impossible.

Noter aussi que la définition de $H^{1 / 2}(I)$ (donnée par (2.68) ) et de sa norme (donnée par 2.69) dépend de $\Omega$ (et pas seulement de $I$ ). Cette dépendance est sans importance. Plus précisément, soit $\Omega_{1}$ et $\Omega_{2}$ deux ouverts bornés de $\mathbb{R}^{2}$ à frontières lipschitziennes. On suppose que $I$ est une partie de $\partial \Omega_{1}$ et une partie de $\partial \Omega_{2}$. Par un argument de cartes locales, permettant de ramener l'étude des traces de $H^{1}\left(\Omega_{i}\right), i=1,2$, à celles de $H^{1}\left(\mathbb{R}_{+}^{2}\right)$, on peut montrer 
que l'espace $H^{1 / 2}(I)$ ne dépend pas du fait que l'on considère $I$ comme une partie de $\partial \Omega_{1}$ ou une partie de $\partial \Omega_{2}$. De plus, les deux normes obtenues alors pour l'espace $H^{1 / 2}(I)$ sont équivalentes.

Pour construire notre exemple, on prend $\Omega=] 0, a\left[^{2}\right.$, avec $a>0$ t.q. $a \sqrt{2}<1$, et $\left.I=\right] 0, a[\times\{0\}$. L'objectif est de montrer qu'il n'existe pas d'opérateur $T$ linéaire continu de $H_{\text {div }}(\Omega)$ dans $H^{-1 / 2}(I)$ t.q.

$$
\langle T(v), u\rangle_{H^{-1 / 2}(I), H^{1 / 2}(I)}=\int_{I} u v \cdot n d \lambda(x), \text { pour tout } u \in H^{1 / 2}(I) \text { et } v \in C^{1}\left(\bar{\Omega}, \mathbb{R}^{2}\right) .
$$

Pour cela, on va raisonner par l'absurde. On suppose donc qu'il existe $T$ linéaire continu de $H_{\text {div }}(\Omega)$ dans $H^{-1 / 2}(I)$ vérifiant 2.70 .

Soit $0<\beta<1 / 2$. Pour $x \in \Omega$, on pose $\left(|\cdot|\right.$ désignant la norme euclidienne classique de $\mathbb{R}^{2}$ )

$$
u(x)=(-\ln (|x|))^{\beta} .
$$

On a $u \in C^{\infty}(\Omega)$ et on sait que $u \in H^{1}(\Omega)$ (voir exercice 1.5). La trace de $u$ sur $I$ est égale (p.p. pour $\lambda$ ) à la trace classique. On note $x_{1}, x_{2}$ les composantes de $x \in \mathbb{R}^{2}$. On prend maintenant $v=\left(v_{1}, v_{2}\right)$ avec

$$
v_{1}=-\frac{\partial u}{\partial x_{2}}, \quad v_{2}=\frac{\partial u}{\partial x_{1}} .
$$

1. Montrer que $\operatorname{div}(v)=\frac{\partial v_{1}}{\partial x_{1}}+\frac{\partial v_{2}}{\partial x_{2}}=0$ et donc que $v \in H_{\text {div }}(\Omega)$.

On définit $v^{(n)}$, pour $n$ t.q. $(a+1 / n) \sqrt{2}<1$, par

$$
v^{(n)}\left(x_{1}, x_{2}\right)=v\left(x_{1}+\frac{1}{n}, x_{2}\right)
$$

2. Montrer que $v^{(n)} \in C^{\infty}(\bar{\Omega})$ et $v^{(n)} \rightarrow v$ dans $H_{\text {div }}(\Omega)$ quand $n \rightarrow+\infty$.

On note $\chi$ la fonction identiquement égale à 1 sur $\partial \Omega$ (cette fonction est bien dans $H^{1 / 2}(I)$ car c'est la trace de la fonction qui vaut 1 sur tout $\Omega$ ).

3. Comme $v^{(n)} \cdot n=-v_{2}^{(n)}$ sur $I$, montrer que

$$
\left\langle T\left(v^{(n)}\right), \chi\right\rangle_{H^{-1 / 2}(I), H^{1 / 2}(I)}=\int_{I} \chi v^{(n)} \cdot n d \lambda(x)=\int_{0}^{a} \beta \frac{\left(-\ln \left(x_{1}+\frac{1}{n}\right)\right)^{\beta-1}}{x_{1}+\frac{1}{n}} \mathrm{~d} x_{1} .
$$

Montrer que le terme de gauche de cette égalité tend vers $\langle T(v), \chi\rangle_{H^{-1 / 2}(I), H^{1 / 2}(I)}$ (utiliser la continuité de $T$ ) et que le terme de droite tend vers $+\infty(\operatorname{car} \beta>0)$. En déduire la contradiction souhaitée.

4. Avec $v \cdot n$ pris au sens de l'exercice 2.23 , montrer que

$$
\langle v \cdot n, \chi\rangle_{H^{-1 / 2}(\partial \Omega), H^{1 / 2}(\partial \Omega)}=0,
$$

et que $\lim _{n \rightarrow+\infty} \int_{\partial \Omega} \chi v^{(n)} \cdot n d \lambda(x)=0$.

\section{Exercice 2.25 (Petite généralisation du théorème de Liouville)}

Le théorème de Liouville 14 s'énonce ainsi :

14. Joseph Liouville (1809-1882) est un mathématicien français, connu pour ses travaux en théorie des nombres et analyse complexe, et fondateur du Journal de Mathématiques Pures et Appliquées. 
Théorème 2.32 (Liouville) Si $f$ est une fonction définie et holomorphe ${ }^{15}$ sur tout le plan complexe, alors $f$ est constante dès lors qu'elle est bornée.

La démonstration de ce théorème se fait en général en utilisant les estimées de Cauchy d'une fonction holomorphe définie sur un voisinage d'un disque fermé, qui fournissent des bornes pour chacune des dérivées de cette fonction au centre du disque.

On rappelle que si $f=\mathcal{R} e(f)+i \mathcal{I} m(f)$ est une fonction holomorphe de $\mathbb{C}$ dans $\mathbb{R}$, alors elle ses parties réelle $\mathcal{R} e(f)$ et imaginaire $\mathcal{I} m(f)$ sont harmoniques. On montre ici le résultat suivant, qui généralise ce théorème au fonctions localement intégrables et bornées inférieurement de $\mathbb{R}^{d}$ dans $\mathbb{R}: f$ est constante dès lors qu'elle est bornée.

Théorème 2.33 (Liouville généralisé) Soient $d \geq 1$ et $u \in L_{\text {loc }}^{1}\left(\mathbb{R}^{d}\right)$ une fonction harmonique ( c'est-à-dire $\Delta u=$ 0 ) bornée inférieurement. alors u est constante, c'est-à-dire qu'il existe $C \in \mathbb{R}$ tel que $u=C$ p.p..

1. En régularisant $u$ avec une suite de noyaux régularisants, montrer qu'il suffit de prouver le théorème dans le cas $u \in C^{\infty}\left(\mathbb{R}^{d}\right)$, et qu'en ajoutant éventuellement une constante à $u$, on peut supposer $u \geq 0$.

On suppose donc maintenant que $u \in C^{\infty}\left(\mathbb{R}^{d}\right)$ et $u \geq 0$.

Pour $r>0$, on note $B_{r}=\left\{x \in \mathbb{R}^{d} ;|x|<r\right\}$ et $C_{r}=\left\{x \in \mathbb{R}^{d} ;|x|=r\right\}$ et pour tout $a \in \mathbb{R}^{d}$, on note $B_{a, r}=\left\{x \in \mathbb{R}^{d} ;|x-a|<r\right\}$.

2. Soit $r>0$. Montrer que l'intégration de $\Delta u$ sur $B_{r}$ donne

$$
\int_{C_{r}} \nabla u(x) \cdot n(x) d \gamma(x)=0,
$$

où $n(x)$ est la normale extérieure à $B_{r}$ et $\gamma$ la mesure de Hausdorff $d-1$ dimensionnelle sur $C_{r}$.

En se ramenant à $C_{1}$ et en utilisant une dérivation sous le signe $\int$, en déduire que la quantité

$$
\frac{1}{r^{d-1}} \int_{C_{r}} u(x) d \gamma(x)
$$

est indépendante de $r$.

3. Soit $r>0$. En utilisant le changement de variable $x \mapsto(r, y)$ avec $r=|x|$ et $y \in C_{1}$, montrer que la quantité

$$
\frac{1}{r^{d}} \int_{B_{r}} u(x) \mathrm{d} x
$$

est indépendante de $r$. En déduire que, pour tout $r>0$,

$$
\frac{1}{\left|B_{r}\right|} \int_{B_{r}} u(x) \mathrm{d} x=u(0) .
$$

De manière analogue, montrer que, pour tout $a \in \mathbb{R}^{d}$ et tout $r>0$,

$$
\frac{1}{\left|B_{r}\right|} \int_{B_{a, r}} u(x) \mathrm{d} x=u(a) .
$$

15. Une fonction holomorphe est une fonction à valeurs complexes, définie et dérivable en tout point d'un sous-ensemble ouvert du plan complexe $\mathbb{C}$. 
Noter que jusqu'a maintenant, seul le fait que $u \in L_{\text {loc }}^{1}\left(\mathbb{R}^{d}\right)$ a été utilisé. Le fait que $u$ est bornée inférieurement n'est utile que pour la dernière question.

4. Soit $a \in \mathbb{R}^{d}$. Comme $u \geq 0$, on a pour tout $r>\alpha=|a|$,

$$
\int_{B_{r-\alpha}} u(x) \mathrm{d} x \leq \int_{B_{a, r}} u(x) \mathrm{d} x \leq \int_{B_{r+\alpha}} u(x) \mathrm{d} x .
$$

En déduire que

$$
\frac{\left|B_{r-\alpha}\right|}{\left|B_{r}\right|} u(0) \leq u(a) \leq \frac{\left|B_{r+\alpha}\right|}{\left|B_{r}\right|} u(0) .
$$

Conclure que $u$ est constante. 


\section{Chapitre 3}

\section{Problèmes elliptiques non linéaires}

On présente dans ce chapitre deux types de méthodes pour obtenir des résulats d'existence de solution pour des problèmes elliptiques non linéaires : une méthode de compacité et une méthode de monotonie. On donnera également une méthode pour obtenir un résultat d'unicité.

\subsection{Méthodes de compacité}

\subsubsection{Degré topologique et théorème de Schauder}

Objectif. Soit $\Omega$ un ouvert borné de $\mathbb{R}^{N}, N \geq 1$, ou un ouvert borné d'un espace de Banach $E$. Soit $f \in$ $C\left(\bar{\Omega}, \mathbb{R}^{N}\right.$ ) (ou $f \in C(\bar{\Omega}, E)$ ) et $y \in \mathbb{R}^{N}$ (ou $y \in E$ ). On cherche à montrer qu'il existe $x \in \bar{\Omega}$ t.q. $f(x)=y$.

On commence par donner l'existence (et l'unicité) d'une application, appelée degré topologique, en dimension finie introduite en 1933 par Brouwer ${ }^{1}$ puis en dimension infinie par Leray et Schauder ${ }^{2}$. Cette application nous permet parfois d'obtenir le thèorème d'existence de solution recherché.

Théorème 3.1 (Degré topologique de Brouwer) Soit $N \geq 1$. On note $\mathcal{A}$ l'ensemble des triplets $(f, \Omega, y)$ où $\Omega$ est un ouvert borné de $\mathbb{R}^{N}, f \in C\left(\bar{\Omega}, \mathbb{R}^{N}\right)$ et $y \in \mathbb{R}^{N}$ tel que $y \notin\{f(x), x \in \partial \Omega\}$. Il existe une application $d$ de $\mathcal{A}$ dans $\mathbb{Z}$, appelée "degré topologique”, vérifiant les trois propriétés suivantes :

(d1) (Normalisation) $d(\mathrm{Id}, \Omega, y)=1$ si $y \in \Omega$.

(d2) (Degré d'une union) Si $\Omega_{1} \cup \Omega_{2} \subset \Omega, \Omega_{1} \cap \Omega_{2}=\emptyset$ et $y \notin\left\{f(x), x \in \bar{\Omega} \backslash \Omega_{1} \cup \Omega_{2}\right\}$, alors

$$
d(f, \Omega, y)=d\left(f, \Omega_{1}, y\right)+d\left(f, \Omega_{2}, y\right) .
$$

(d3) (Invariance par homotopie) Si $h \in C\left([0,1] \times \bar{\Omega}, \mathbb{R}^{N}\right), y \in C\left([0,1], \mathbb{R}^{N}\right)$ et si, pour tout $t \in[0,1]$, $y(t) \notin\{h(t, x), x \in \partial \Omega\}$, alors

$$
d(h(t, \cdot), \Omega, y(t))=d(h(0, \cdot), \Omega, y(0)) \text { pour tout } t \in[0,1] .
$$

Remarque 3.2 (Propriétés importantes) Des propriétés du degré topologique (d1)-(d3) données dans le théorème 3.1), et plus particulièrement de la propriété d'additivité $(\mathrm{d} 2)$, on déduit les propriétés suivantes :

1. Luitzen Egbertus Jan Brouwer (1881-1966), mathématicien et philosophe néerlandais, qui a travaillé en topologie, théorie des ensembles, théorie de la mesure et analyse complexe.

2. Juliusz Pawel Schauder, 1899-1943 est un mathématicien polonais d'origine juive, connu pour ses travaux en analyse fonctionnelle, équations aux dérivées partielles et physique mathématique. Assassiné par la Gestapo, après dénonciation d'un collègue allemand. 
1. Si $\Omega=\emptyset$, alors en prenant $\Omega_{1}=\Omega_{2}=\emptyset$ dans (d2), on obtient que $d(f, \emptyset, y)=0$.

2. Si $(f, \Omega, y) \in \mathcal{A}$ et $d(f, \Omega, y) \neq 0$, alors il existe $x \in \Omega$ tel que $f(x)=y$.

En effet, raisonnons par contraposée et supposons qu'il n'existe pas $x \in \Omega$ tel que $f(x)=y$. En prenant $\Omega_{1}=\Omega_{2}=\emptyset$, comme par hypothèse $y \notin\{f(x), x \in \partial \Omega\}$, on a $y \notin\left\{f(x), x \in \bar{\Omega} \backslash \Omega_{1} \cup \Omega_{2}\right\}$ et donc par (d2) et par l'item 1 ,

$$
d(f, \Omega, y)=d\left(f, \Omega_{1}, y\right)+d\left(f, \Omega_{2}, y\right)=0 .
$$

3. Soient $A$ une matrice $N \times N$ inversible, $\Omega$ un ouvert borné de $\mathbb{R}^{N}$ et $y \in \mathbb{R}^{N}$ tel que $A^{-1} y \in \Omega$; pour $x \in \mathbb{R}^{N}$, on pose $f(x)=A x$. On a alors $(f, \Omega, y) \in \mathcal{A}$ et le degré $d(f, \Omega, y)$ est égal au signe du déterminant de $A$, on a $\operatorname{donc} d(f, \Omega, y) \neq 0$.

La remarque 3.2 donne une méthode, dite méthode du degré topologique, ou argument de degré pour trouver des solutions à des problèmes non linéaires, dont voici la teneur. Soit $N \geq 1, \Omega$ un ouvert borné de $\mathbb{R}^{N}, f \in$ $C\left(\bar{\Omega}, \mathbb{R}^{N}\right)$ et $y \in \mathbb{R}^{N}$. On cherche à montrer qu'il existe $x \in \Omega$ telle que $f(x)=y$. Pour cela, on construit une application $h$ de $[0,1] \times \bar{\Omega}$ dans $\mathbb{R}^{N}$ t.q.

1. $h(1, \cdot)=f$,

2. $h(0, \cdot)=g$ où $g$ est une application linéaire inversible et telle que $y \in\{g(x), x \in \Omega\}$.

3. $h(t, x) \neq y$ pour tout $t \in[0,1]$ et tout $x \in \partial \Omega$.

On obtient alors $d(f, \Omega, y)=d(g, \Omega, y) \neq 0$ et donc qu'il existe $x \in \Omega$ t.q. $f(x)=y$.

Remarque 3.3 (Le cas $N=1$ ) Dans le cas $N=1$, la méthode du degré topologique que l'on vient de décrire n'est pas vraiment intéressante, car elle n'apporte rien de plus que le théorème des valeurs intermédiaires.

Une conséquence de cette méthode du degré topologique est le théorème de point fixe de Brouwer que nous rappelons maintenant (voir [9] pour l'article original en allemand, et [11, 30] pour des versions plus modernes).

Théorème 3.4 (Point fixe de Brouwer) Soit $N \geq 1, R>0$ et $f \in C\left(B_{R}, B_{R}\right)$ avec $B_{R}=\left\{x \in \mathbb{R}^{N},\|x\| \leq R\right\}$ (où $\mathbb{R}^{N}$ est muni d'une norme notée $\|\cdot\|$ ). Alors $f$ admet un point fixe, c'est-à-dire qu'il existe $x \in B_{R}$ t.q. $f(x)=x$.

Démonstration Si il existe $x \in \partial B_{R}$ ( c'est-à-dire t.q. $\|x\|=R$ ) t.q. $f(x)=x$, il n'y a plus rien à démontrer. On suppose donc maintenant $f(x) \neq x$ pour tout $x \in \partial B_{R}$. On pose alors $\Omega=\left\{x \in \mathbb{R}^{N},\|x\|<R\right\}$ (ce qui donne $\left.B_{R}=\bar{\Omega}\right)$ et, pour $t \in[0,1]$ et $x \in B_{R}, h(t, x)=x-t f(x)$. Il est facile de voir que $h(t, x) \neq 0$ pour tout $x \in \partial \Omega=\left\{x \in \mathbb{R}^{N},\|x\|=R\right\}$. On en déduit que $d(h(1, \cdot), \Omega, 0)=d(h(0, \cdot), \Omega, 0)=d(\operatorname{Id}, \Omega, 0)=1$ et donc qu'il existe $x \in \Omega$ t.q. $f(x)=x$.

Le théorème 3.1 a été généralisé dès 1934 à la dimension infinie par Leray et Schauder [22] sous une hypothèse fondamentale de compacité que nous donnons maintenant.

Définition 3.5 (Compacité) Soient $E$ un espace de Banach (réel), $B$ une partie de $E$ et $f$ une application de $B$ dans $E$. On dit que $f$ est compacte si $f$ vérifie les deux propriétés suivantes :

1. $f$ est continue,

2. $\{f(x), x \in C\}$ est relativement compacte (dans $E$ ) pour tout partie $C$ bornée de $B$.

Notons que dans l'article original de Leray-Schauder [22], le terme "compacte" n'est pas employé, l'expression "complètement continue" est utilisée en lieu et place.

On peut remarquer, dans la définition précédente, que si $f$ est linéaire (et $B=E$ ) la deuxième condition entraîne la première. Mais ceci est faux pour des applications non linéaires. 
Définition 3.6 Soit $E$ un espace de Banach (réel). On note $\mathcal{A}$ l'ensemble des triplets (Id $-f, \Omega, y)$ où $\Omega$ est un ouvert borné de $E$, $f$ est une application compacte de $\bar{\Omega}$ dans $E$ (ce qui est équivalent à dire que $f$ est continue et $\{f(x), x \in \bar{\Omega}\}$ est une partie relativement compacte de E) et $y \in E$ t.q. $y \notin\{x-f(x), x \in \partial \Omega\}$.

Théorème 3.7 (Degré topologique de Leray-Schauder) Soit E un espace de Banach (réel) et $\mathcal{A}$ donné par la définition 3.6 Il existe alors une application d de $\mathcal{A}$ dans $\mathbb{Z}$, appelée "degré topologique", vérifiant les trois propriétés suivantes :

(d1) (Normalisation) $d(\mathrm{Id}, \Omega, y)=1$ si $y \in \Omega$.

(d2) (Degré d'une union) $d(\operatorname{Id}-f, \Omega, y)=d\left(\operatorname{Id}-f, \Omega_{1}, y\right)+d\left(\operatorname{Id}-f, \Omega_{2}, y\right)$ si $\Omega_{1} \cup \Omega_{2} \subset \Omega, \Omega_{1} \cap \Omega_{2}=\emptyset$ et $y \notin\left\{x-f(x), x \in \bar{\Omega} \backslash \Omega_{1} \cup \Omega_{2}\right\}$.

(d3) (Invariance par homotopie) Si h est une application compacte de $[0,1] \times \bar{\Omega}$ dans $E$ (ce qui est équivalent à dire que h est continue et $\{h(t, x), t \in[0,1], x \in \bar{\Omega}\}$ est une partie relativement compacte de $E), y \in$ $C([0,1], E)$ et $y(t) \notin\{x-h(t, x), x \in \partial \Omega\}$ (pour tout $t \in[0,1]$ ), on a alors $d(\operatorname{Id}-h(t, \cdot), \Omega, y(t))=$ $d(\operatorname{Id}-h(0, \cdot), \Omega, y(0))$ pour tout $t \in[0,1]$.

Comme dans le cas de la dimension finie (voir la remarque 3.2), des propriétés du degré topologique (données dans le théorème 3.7), on déduit que $d(\operatorname{Id}-f, \Omega, y) \neq 0$ implique qu'il existe $x \in \Omega$ tel que $x-f(x)=y$. Pour donner l'analogue de la seconde propriété de la remarque 3.2 . nous avons besoin d'un deuxième théorème dû à Leray et Schauder que nous donnons maintenant.

Théorème 3.8 (Application linéaire compacte) Soit E un espace de Banach (réel), L une application linéaire compacte de $E$ dans $E$ et $\Omega$ un ouvert borné contenant 0 . On suppose que

$$
x \in E, L x=x \Rightarrow x \notin \partial \Omega .
$$

Alors $(\operatorname{Id}-L, \Omega, 0) \in \mathcal{A}$, où $\mathcal{A}$ est donné par la définition 3.6 et $d(\operatorname{Id}-L, \Omega, 0) \neq 0$.

Noter que, comme L est linéaire, l'hypothèse (3.1) est équivalente à dire $(x \in E, L x=x) \Rightarrow x=0$, ce qui est équivalent à dire que 1 n'est pas valeur propre de $L$.

On peut maintenant, comme en dimension finie, donner une méthode pour trouver des solutions à des problèmes non linéaires. Soit $E$ un espace de Banach, $\Omega$ un ouvert borné de $E$ contenant $0, f$ une application de $\bar{\Omega}$ dans $E$. On cherche à montrer qu'il existe $x \in \Omega$ t.q. $x-f(x)=0$ (quitte à changer $f$, on peut toujours se ramener à cette forme). Pour cela, on construit une application $h$ de $[0,1] \times \bar{\Omega}$ dans $E$, compacte et t.q.

1. $h(1, \cdot)=f$,

2. $h(0, \cdot)=L$ avec $L$ linéaire de $E$ de $E$,

3. $x-h(t, x) \neq 0$ pour tout $t \in[0,1]$ et tout $x \in \partial \Omega$.

On obtient alors $d(\operatorname{Id}-f, \Omega, 0)=d(\operatorname{Id}-L, \Omega, 0) \neq 0$ et donc qu'il existe $x \in \Omega$ t.q. $x-f(x)=0$.

Bien sûr, pour pouvoir construire une telle que fonction $h$, il faut que $f$ soit une application compacte et que $L$ soit une application linéaire compacte.

Comme en dimension finie, une première conséquence de l'existence du degré topologique est l'obtention d'un théorème de point fixe que nous donnons maintenant.

Théorème 3.9 (Point fixe de Schauder) Soit $E$ un espace de Banach, $R>0, B_{R}=\{x \in E,\|x\| \leq R\}$ et $f$ une application compacte de $B_{R}$ dans $B_{R}$ (c'est-à-dire $f$ continue et $\left\{f(x), x \in B_{R}\right\}$ relativement compacte dans $E$ ). Alors $f$ admet un point fixe, c'est-à-dire qu'il existe $x \in B_{R}$ t.q. $f(x)=x$. 
Démonstration La démonstration est très voisine de celle du théorème 3.4 Si il existe $x \in \partial B_{R}$ ( c'est-à-dire t.q. $\|x\|=R$ ) t.q. $f(x)=x$, il n'y a plus rien à démontrer. On suppose donc maintenant $f(x) \neq x$ pour tout $x \in \partial B_{R}$. On pose alors $\Omega=\{x \in E,\|x\|<R\}$ (ce qui donne $B_{R}=\bar{\Omega}$ ) et, pour $t \in[0,1]$ et $x \in B_{R}, h(t, x)=t f(x)$. Il est facile de voir que $x-h(t, x) \neq 0$ pour tout $x \in \partial \Omega=\left\{x \in \mathbb{R}^{N},\|x\|=R\right\}$. La compacité de $h$ se déduit de celle de $f$. On en déduit alors que $d(\operatorname{Id}-h(1, \cdot), \Omega, 0)=d(\operatorname{Id}-h(0, \cdot), \Omega, 0)=d(\operatorname{Id}, \Omega, 0)=1$ et donc qu'il existe $x \in \Omega$ t.q. $f(x)=x$.

Le théorème de Schauder est faux si on remplace l'hypothèse de compacité de $f$ par la simple hypothèse de continuité. Toutefois, la difficulté principale dans l'utilisation du théorème de Schauder (ou, plus généralement, dans l'utilisation du degré topologique) est souvent de montrer la continuité de $f$ (ou, dans l'utilisation du degré topologique, la continuité de l'application notée $h$ précédemment).

\subsubsection{Existence par le théorème de Schauder}

On rappelle tout d'abord la définition de fonction de Carathéodory.

Définition 3.10 3 Soit $N, p, q \in \mathbb{N}^{\star}$ et $\Omega$ un ouvert de $\mathbb{R}^{N}$. Soit a une application de $\Omega \times \mathbb{R}^{p}$ dans $\mathbb{R}^{q}$. On dit que a est fonction de Carathéodory si a $(\cdot, s)$ est borélienne pour tout $s \in \mathbb{R}^{p}$ et a $(x, \cdot)$ est continue pour presque tout $x \in \Omega$.

Remarque 3.11 Soit $N, p, q \in \mathbb{N}^{\star}, \Omega$ un ouvert de $\mathbb{R}^{N}$ et $a$ une fonction de Carathéodory de $\Omega \times \mathbb{R}^{p}$ dans $\mathbb{R}^{q}$. La fonction $a$ est alors borélienne de $\Omega \times \mathbb{R}^{p}$ dans $\mathbb{R}^{q}$ (ce qui pourrait être faux si $a$ était seulement borélienne par rapport à chacun de ses arguments). Si $v$ est une fonction borélienne de $\Omega$ dans $\mathbb{R}^{p}$, la fonction $x \mapsto a(x, v(x))$ est alors borélienne de $\Omega$ dans $\mathbb{R}^{q}$. Cette propriétéest plusieurs fois utilisée dans la suite (sans la rappeler) lorsque $v$ est dans $L^{r}(\Omega)$ (pour un $r \in[1,+\infty]$ ) en choisissant un représentant (borélien) de $v$ (la fonction $x \mapsto a(x, v(x)$ ) ne dépend pas du représentant choisi pour $v$, modulo la relation d'équivalence " $=$ p.p.").

On se place maintenant sous les hypothèses suivantes :

$$
\begin{aligned}
& N \geq 1, \Omega \text { est un ouvert borné de } \mathbb{R}^{N}, \\
& a: \Omega \times \mathbb{R} \rightarrow \mathbb{R} \text { est une fonction de Carathéodory, } \\
& \text { il existe } \alpha>0 \text { et } \beta>0 \text { t.q. } \alpha \leq a(\cdot, s) \leq \beta \text { p.p. et pour tout } s \in \mathbb{R}, \\
& f: \Omega \times \mathbb{R} \rightarrow \mathbb{R} \text { est une fonction de Carathéodory et } f \in L^{\infty}(\Omega \times \mathbb{R}) \text {. }
\end{aligned}
$$

Sous les hypothèses 3.2. on cherche à montrer l'existence de $u$, solution du problème suivant :

$$
\left\{\begin{array}{l}
u \in H_{0}^{1}(\Omega), \\
\int_{\Omega} a(x, u(x)) \nabla u(x) \cdot \nabla v(x) \mathrm{d} x=\int_{\Omega} f(x, u(x)) v(x) \mathrm{d} x, \text { pour tout } v \in H_{0}^{1}(\Omega) .
\end{array}\right.
$$

Théorème 3.12 (Existence, second membre borné) Sous les hypothèses (3.2), il existe u solution de 3.3.

Démonstration Pour $\bar{u} \in L^{2}(\Omega)$, le chapitre sur les équations elliptiques linéaires nous donne l'existence et l'unicité de $u$ solution de

$$
\left\{\begin{array}{l}
u \in H_{0}^{1}(\Omega), \\
\int_{\Omega} a(x, \bar{u}(x)) \nabla u(x) \cdot \nabla v(x) \mathrm{d} x=\int_{\Omega} f(x, \bar{u}(x)) v(x) \mathrm{d} x, \text { pour tout } v \in H_{0}^{1}(\Omega) .
\end{array}\right.
$$

3. Constantin Carathéodory (1873 -1950) mathématicien allemand d'origine grecque, dont les recherches portent sur le calcul des variations, et les équations aux dérivées partielles. 
Plus précisément, pour montrer l'existence et l'unicité de $u$ solution de (3.4), on applique le théorème 2.6. Pour cela, on met le problème (3.4) sous la forme (2.4) en posant $a_{i, j}=0$ si $i \neq j, a_{i, i}=a(\cdot, \bar{u})$ et $f=f(\cdot, \bar{u})$ (dans cette dernière égalité, la fonction $f$ du terme de gauche est celle de (2.4) et la fonction $f$ du terme de droite est celle de (3.4)). Le théorème 2.6 donne bien l'existence et l'unicité de $u$ solution de (3.4).

On pose $T(\bar{u})=u$; l'application $T$ est donc une application de $E$ dans $E$ avec $E=L^{2}(\Omega)$. Un point fixe de $T$ est une solution de 3.3 . Pour démontrer l'existence d'un tel point fixe, on utilise le théorème 3.9 .

Tout d'abord, en utilisant $\alpha$, l'inégalité de Poincaré et la borne $L^{\infty}$ de $f$, on montre facilement que l'image de $T$ est dans un borné de $H_{0}^{1}(\Omega)$ et donc (par le théorème 1.25 de Rellich) dans un compact de $L^{2}(\Omega)$. En prenant $R$ assez grand, l'application $T$ envoie donc $B_{R}=\left\{v \in L^{2}(\Omega),\|v\|_{2} \leq R\right\}$ dans $B_{R}$ et $\left\{T(\bar{u}), \bar{u} \in B_{R}\right\}$ est relativement compacte dans $L^{2}(\Omega)$. Pour utiliser le théorème 3.9. il reste à montrer la continuité de $T$.

Soit $\left(\bar{u}_{n}\right)_{n \in \mathbb{N}}$ une suite de $E$ t.q. $\bar{u}_{n} \rightarrow \bar{u}$ dans $E$, quand $n \rightarrow+\infty$. On pose $u_{n}=T\left(\bar{u}_{n}\right)$. Après extraction d'une sous suite, on peut supposer que $\bar{u}_{n} \rightarrow \bar{u}$ p.p. et qu'il existe $w \in H_{0}^{1}(\Omega)$ t.q. $u_{n} \rightarrow w$ faiblement dans $H_{0}^{1}(\Omega)$ (et donc aussi $u_{n} \rightarrow w$ dans $L^{2}(\Omega)$ ). On va montrer que $w$ est solution de (3.4). En effet, Soit $v \in H_{0}^{1}(\Omega)$, on a

$$
\int_{\Omega} a\left(x, \bar{u}_{n}(x)\right) \nabla u_{n}(x) \cdot \nabla v(x) \mathrm{d} x=\int_{\Omega} f\left(x, \bar{u}_{n}(x)\right) v(x) \mathrm{d} x, \text { pour tout } v \in H_{0}^{1}(\Omega) .
$$

En passant à la limite quand $n \rightarrow+\infty$ (en utilisant la convergence dominée et le passage à la limite sur le produit d'une convergence faible et d'une convergence forte dans $L^{2}$ ), on obtient

$$
\int_{\Omega} a(x, \bar{u}(x)) \nabla w(x) \cdot \nabla v(x) \mathrm{d} x=\int_{\Omega} f(x, \bar{u}(x)) v(x) \mathrm{d} x, \text { pour tout } v \in H_{0}^{1}(\Omega) .
$$

Ceci prouve que $w=T(\bar{u})$. On a donc prouvé, après extraction d'une sous suite, que $T\left(\bar{u}_{n}\right) \rightarrow T(\bar{u})$ dans $L^{2}(\Omega)$. Par un raisonnement classique par l'absurde on peut montrer que cette convergence reste vraie sans extraction de sous suite (voir l'exercice 3.1 pour un exemple de ce type de raisonnement). On a ainsi démontré la continuité de $T$. On peut donc appliquer le théorème 3.9 et conclure à l'existence d'un point fixe de $T$, ce qui termine cette démonstration.

\subsubsection{Existence par degré topologique}

On reprend le même problème que dans le paragraphe 3.1.2 en supprimant l'hypothèse $f$ bornée qui permettait une application simple du théorème de Schauder. On considère l'équation de diffusion-convection-réaction suivante :

$$
\left\{\begin{array}{l}
u \in H_{0}^{1}(\Omega) \\
\int a(x, u(x)) \nabla u(x) \cdot \nabla v(x)+\int_{\Omega} G(x) \varphi(u(x)) \cdot \nabla v(x) \mathrm{d} x= \\
\int_{\Omega} f(x, u(x)) v(x) \mathrm{d} x, \quad \forall v \in H_{0}^{1}(\Omega)
\end{array}\right.
$$

qui est la formulation faible du problème suivant :

$$
\left\{\begin{array}{l}
-\operatorname{div}(a(x, u) \nabla u)-\operatorname{div}(G(x) \varphi(u))=f(x, u), x \in \Omega, \\
u=0 \text { sur } \partial \Omega .
\end{array}\right.
$$

Notons que cette équation est non linéaire pour trois raisons : les termes de diffusion, convection et réaction sont non linéaires. Le premier terme du membre de gauche est le terme de diffusion, le second terme du membre de gauche est le terme de convection et le membre de droite est le terme de réaction. 
On se place sous les hypothèses suivantes :

$$
\begin{aligned}
& \Omega \text { est un ouvert borné de } \mathbb{R}^{N}, N \geq 1, \\
& a \text { est une fonction de Carathéodory (voir la définition } 3.10), \\
& \exists \alpha, \beta>0 ; \alpha \leq a(x, s) \leq \beta \quad \forall s \in \mathbb{R} \quad \text { p.p. } x \in \Omega, \\
& G \in C^{1}\left(\bar{\Omega}, \mathbb{R}^{N}\right) \text {, } \operatorname{div} G=0, \\
& \varphi \in C(\mathbb{R}, \mathbb{R}) \text { et il existe } C_{1} \geq 0:|\varphi(s)| \leq C_{1}|s| \quad \forall s \in \mathbb{R}, \\
& f \text { est une fonction de Carathéodory, et } \exists C_{2} \geq 0 \text { et } d \in L^{2}(\Omega) ;|f(x, s)| \leq d(x)+C_{2}|s|, \\
& \lim _{s \rightarrow \pm \infty} \frac{f(x, s)}{s}=0 .
\end{aligned}
$$

Remarque 3.13 (Alternative de Fredholm) Dans le cas où $a \equiv 1, \varphi=0$ et $f$ est de la forme $f(x, s)=d(x)+\lambda s$ où $\lambda$ est une valeur propre du Laplacien sur $\Omega$ avec condition de Dirichet ( c'est-à-dire qu'il existe $w \in H_{0}^{1}(\Omega)$, $w \neq 0$ t.q. $-\Delta w=\lambda w$ dans $\mathcal{D}^{\star}(\Omega)$ ) et $d$ un élément de $L^{2}(\Omega)$, le problème (3.6) devient $-\Delta u=\lambda u+d$, avec condition de Dirichlet. Ce problème n'admet une solution que si $d$ est orthogonal à l'espace propre associé à $\lambda$ (et dans ce cas on n'a pas unicité). Cette propriété est connue sous le nom d'alternative de Fredholm ${ }^{4}$ voir l'exercice 2.2 C'est pour assurer l'existence pour tout $d$ dans $L^{2}(\Omega)$ qu'on ajoute l'hypothèse de sous-linéarité sur $f$ (hypothèse $3.7 \mathrm{~g}$ )).

Remarque 3.14 (Coercivité) Lorsque $\operatorname{div} G \neq 0$, le problème peut se traiter de manière similaire à celle donnée dans la démonstration de théorème 3.15 à condition que $\operatorname{div} G \leq \lambda_{1}$ p.p. où $\lambda_{1}$ est la première valeur propre de $u \mapsto-\operatorname{div}(\alpha \nabla u)$ avec condition de Dirichlet (cette valeur propre est strictement positive). Sans cette condition, le problème devient plus difficile (voir l'exercice 3.5), même dans le cas linéaire, c'est-à-dire le cas où $a$ et $f$ ne dépendent pas de $u$ et où $\varphi(u)=u$. La difficulté principale est due à l'absence de coercivité de l'opérateur $u \mapsto-\operatorname{div}(\alpha \nabla u)-\operatorname{div}(G u)$.

Théorème 3.15 (Existence, second membre non borné) Sous les hypothèses (3.7), il existe une solution de (3.5).

Démonstration On donne ici une preuve par degré topologique. Cette méthode demande des estimations a priori c'est à dire des estimations sur $u$, sans connaître son existence. Supposons donc $u$ solution de (3.5), on peut (et on va) montrer qu'il existe $R>0$ tel que $\|u\|_{L^{2}} \leq R$. On établit les estimations à partir du problème non linéaire, et non pas à partir du problème linéarisé (ce qui serait le cas par Schauder, voir remarque 3.16; ceci présente de sérieux avantages. Par exemple dans le terme de convection non linéaire, on peut écrire (formellement)

$$
\begin{aligned}
\int_{\Omega} G \varphi(u) \cdot \nabla u \quad \mathrm{~d} x & =\int_{\Omega} G \cdot \nabla \phi(u) \mathrm{d} x \\
& =-\int_{\Omega} \operatorname{div} G \phi(u) \mathrm{d} x \\
& =0 \quad \operatorname{car} \operatorname{div} G=0
\end{aligned}
$$

où $\phi$ est la primitive de $\varphi$ s'annulant en 0 . Notons que l'estimation $\|u\|_{L^{2}(\Omega)} \leq R$ revient à montrer que toutes les solutions sont dans la boule $B_{R}$ (boule fermée de centre 0 et de rayon $R$ ), ce qui est une estimation uniforme sur toutes les solutions.

4. Erik Ivar Fredholm (1866-1927) mathématicien suédois connu pour ses travaux sur les équations intégrales et la théorie spectrale. 
On réécrit le problème sous la forme :

$$
\left\{\begin{array}{l}
u \in H_{0}^{1}(\Omega) \\
\int_{\Omega} a(u) \nabla u \cdot \nabla v \mathrm{~d} x=\langle F(u), v\rangle_{H^{-1}(\Omega), H_{0}^{1}(\Omega)}, \forall v \in H_{0}^{1}(\Omega),
\end{array}\right.
$$

où $F(u)$ est, pour $u \in L^{2}(\Omega)$, l'élément de $H^{-1}(\Omega)$ défini par

$$
\langle F(u), v\rangle_{H^{-1}(\Omega), H_{0}^{1}(\Omega)}=-\int_{\Omega} G \varphi(u) \cdot \nabla v \mathrm{~d} x+\int_{\Omega} f(u) v \mathrm{~d} x .
$$

Comme $G \in L^{\infty}(\Omega)^{N},|\varphi(s)| \leq C_{1}|s|$ et $|f(\cdot, s)| \leq d+C_{2}|s|$, il est facile de voir que l'application $F$ qui à $u$ associe $F(u)$ est continue de $L^{2}(\Omega)$ dans $H^{-1}(\Omega)$.

Pour $S \in H^{-1}(\Omega)$, le problème linéaire

$$
\left\{\begin{array}{l}
w \in H_{0}^{1}(\Omega) \\
\int_{\Omega} a(u) \nabla w \cdot \nabla v \mathrm{~d} x=\langle S, v\rangle_{H^{-1}(\Omega), H_{0}^{1}(\Omega)}
\end{array}\right.
$$

admet une unique solution $w \in H_{0}^{1}(\Omega)$. On note $B_{u}$ l'opérateur qui à $S$ dans $H^{-1}(\Omega)$ associe $w$ solution de (3.8). L'opérateur $B_{u}$ est linéaire continu de $H^{-1}(\Omega)$ dans $H_{0}^{1}(\Omega)$ et $H_{0}^{1}(\Omega)$ s'injecte compactement dans $L^{2}(\Omega)$. On en déduit que l'opérateur $B_{u}$ est compact de $H^{-1}(\Omega)$ dans $L^{2}(\Omega)$.

Le problème 3.5 est équivalent à résoudre le problème de point fixe $u=B_{u}(F(u))$. On va donc montrer, par degré topologique, que le problème suivant admet une solution

$$
\left\{\begin{array}{l}
u \in L^{2}(\Omega) \\
u=B_{u}(F(u)) .
\end{array}\right.
$$

Pour $t \in[0,1]$, on pose $h(t, u)=B_{u}(t F(u)) \in L^{2}(\Omega)$. L'application $h$ est ainsi définie de $[0,1] \times L^{2}(\Omega)$ dans $L^{2}(\Omega)$. Pour $R>0$, on pose $B_{R}=\left\{u \in L^{2}(\Omega)\right.$ t.q. $\left.\|u\|_{L^{2}(\Omega)}<R\right\}$. On va montrer que

1. il existe $R>0$ tel que, pour tout $t \in[0,1]$ et tout $u \in L^{2}(\Omega)$, si $u-h(t, u)=0$ alors $\|u\|_{L^{2}(\Omega)}<R$. (C'est cette estimation a priori qui est le point le plus difficile à montrer).

2. $h$ est continue de $[0,1] \times \bar{B}_{R}$ dans $\bar{B}_{R}$;

3. L'ensemble $\left\{h(t, u), t \in[0,1], u \in \bar{B}_{R}\right\}$ est relativement compact dans $L^{2}(\Omega)$.

Si on suppose qu'on a démontré les items 1 et 3 , on n'a pas de solution à l'équation $u-h(t, u)=0$ sur le bord de la boule $B_{R}$, et on peut donc définir le degré $d\left(\mathrm{Id}-h(t,),. B_{R}, 0\right)$. Ce degré ne dépend pas de $t$, on a donc :

$$
\begin{aligned}
d\left(\operatorname{Id}-h(t, \cdot), B_{R}, 0\right) & =d\left(\operatorname{Id}-h(0, \cdot), B_{R}, 0\right) \\
& =d\left(\operatorname{Id}, B_{R}, 0\right)=1 .
\end{aligned}
$$

On en déduit l'existence de $u \in B_{R}$ tel que $u-h(1, u)=0$, c'est à dire

$$
u=B_{u}(F(u)) \text {. }
$$

Donc $u$ est solution de 3.5 (et le théorème 3.15 est démontré).

Il reste donc à montrer les items 1 3. Commençons par démontrer l'item 3 (pour tout $R>0$ ). Soit $R>0$. On suppose que $\|u\|_{L^{2}} \leq R$. On a :

$$
F(u) \in H^{-1}(\Omega), \text { et }\langle F(u), v\rangle_{H^{-1}(\Omega), H_{0}^{1}(\Omega)}=-\int_{\Omega} G \varphi(u) \cdot \nabla v \mathrm{~d} x+\int_{\Omega} f(u) v \mathrm{~d} x .
$$


Estimons $\langle F(u), v\rangle_{H^{-1}(\Omega), H_{0}^{1}(\Omega)}$ :

$$
\begin{aligned}
\langle F(u), v\rangle_{H^{-1}(\Omega), H_{0}^{1}(\Omega)} & \leq\||G|\|_{\infty}\|\varphi(u)\|_{L^{2}(\Omega)}\|v\|_{H_{0}^{1}(\Omega)}+\|f(u)\|_{L^{2}(\Omega)}\|v\|_{L^{2}(\Omega)} \\
& \leq\||G|\|_{\infty} C_{1}\|u\|_{L^{2}(\Omega)}\|v\|_{H_{0}^{1}(\Omega)}+\|d\|_{L^{2}(\Omega)}\|v\|_{L^{2}(\Omega)}+C_{2}\|u\|_{L^{2}(\Omega)}\|v\|_{L^{2}(\Omega)} \\
& \leq\left(\||G|\|_{\infty} C_{1} R+C_{\Omega}\|d\|_{L^{2}(\Omega)}+C_{2} C_{\Omega} R\right)\|v\|_{H_{0}^{1}(\Omega)},
\end{aligned}
$$

où $C_{\Omega}$ ne dépend que de $\Omega$ (et est donnée par l'inégalité de Poincaré). Donc

$$
t\|F(u)\|_{H^{-1}} \leq\||G|\|_{\infty} C_{1} R+C_{\Omega}\|d\|_{L^{2}(\Omega)}+C_{2} C_{\Omega} R, \quad \forall t \in[0,1] .
$$

Posons $h(t, u)=B_{u}(t F(u))=w$ et montrons qu'il existe $\bar{R}$ dépendant que de $R, G, C_{\Omega}, C_{1}, C_{2}, \alpha$ tel que

$$
\|h(t, u)\|_{H_{0}^{1}(\Omega)} \leq \bar{R} .
$$

Par définition, $w$ est solution de

$$
\left\{\begin{array}{l}
\int_{\Omega} a(u) \nabla w \cdot \nabla v=\langle t F(u), v\rangle_{H^{-1}(\Omega), H_{0}^{1}(\Omega)} \quad \forall v \in H_{0}^{1}(\Omega), \\
w \in H_{0}^{1}(\Omega) .
\end{array}\right.
$$

En prenant $v=w$ dans 3.9 , on obtient :

$$
\alpha\|w\|_{H_{0}^{1}(\Omega)}^{2} \leq\|t F(u)\|_{H^{-1}(\Omega)}\|w\|_{H_{0}^{1}(\Omega)} \leq \widetilde{R}\|w\|_{H_{0}^{1}(\Omega)},
$$

avec $\widetilde{R}=\||G|\|_{\infty} C_{1} R+C_{\Omega}\|d\|_{L^{2}(\Omega)}+C_{2} C_{\Omega} R$. On a donc $\|h(t, u)\|_{H_{0}^{1}(\Omega)}=\|w\|_{H_{0}^{1}(\Omega)} \leq \frac{\widetilde{R}}{\alpha}=\bar{R}$.

On en déduit par le théorème de Rellich (théorème 1.25 , que l'ensemble $\left\{h(t, u), t \in[0,1], u \in \bar{B}_{R}\right\}$ est relativement compact dans $L^{2}(\Omega)$, ce qui montre bien l'item 3

Montrons maintenant l'item 2 Soit $\left(t_{n}\right)_{n \in \mathbb{N}} \subset[0,1]$ une suite telle que $t_{n} \rightarrow t$ lorsque $n \rightarrow+\infty$ et soit $\left(u_{n}\right)_{n \in \mathbb{N}} \subset L^{2}(\Omega)$ une suite telle que $u_{n} \rightarrow u$ dans $L^{2}(\Omega)$. On veut montrer que $h\left(t_{n}, u_{n}\right) \rightarrow h(t, u)$ dans $L^{2}(\Omega)$. Soit $w_{n}=h\left(t_{n}, u_{n}\right)$ et $w=h(t, u)$. Pour montrer que $w_{n} \rightarrow w$ dans $L^{2}(\Omega)$, on cherche à passer à la limite sur l'équation suivante :

$$
\left\{\begin{array}{l}
\int_{\Omega} a\left(u_{n}\right) \nabla w_{n} \cdot \nabla v \mathrm{~d} x=-t_{n} \int_{\Omega} G \varphi\left(u_{n}\right) \cdot \nabla v \mathrm{~d} x+t_{n} \int_{\Omega} f\left(u_{n}\right) v \mathrm{~d} x \\
w_{n} \in H_{0}^{1}(\Omega) .
\end{array}\right.
$$

On sait déjà que $\left(w_{n}\right)_{n \in \mathbb{N}}$ est bornée dans $H_{0}^{1}(\Omega)$, car la suite $\left(u_{n}\right)_{n \in \mathbb{N}}$ est bornée dans $L^{2}(\Omega)$ (c'est ce qu'on a montré à l'étape précédente : si $\left\|u_{n}\right\|_{L^{2}(\Omega)} \leq R$ alors $\left\|w_{n}\right\|_{H_{0}^{1}(\Omega)} \leq \bar{R}$.

La suite $\left(w_{n}\right)_{n \in \mathbb{N}}$ est bornée dans $H_{0}^{1}(\Omega)$, et à une sous suite près, (on ne renumérote pas) on a donc

$$
\begin{aligned}
& w_{n} \rightarrow \bar{w} \text { dans } H_{0}^{1} \text { faible et } w_{n} \rightarrow \bar{w} \text { dans } L^{2}(\Omega), \\
& u_{n} \rightarrow u \text { p.p. et } \exists H \in L^{2}(\Omega) ;\left|u_{n}\right| \leq H \text { p.p.. }
\end{aligned}
$$

Soit $v \in H_{0}^{1}(\Omega)$; comme $a\left(u_{n}\right) \rightarrow a(u)$ p.p. donc $a\left(u_{n}\right) \nabla v \rightarrow a(v) \nabla v$ p.p., et $\left|a\left(u_{n}\right) \nabla v\right| \leq \beta|\nabla v|$; on en déduit que $a\left(u_{n}\right) \nabla v \rightarrow a(u) \nabla v$ dans $L^{2}(\Omega)$. Mais $\nabla w_{n} \rightarrow \nabla \bar{w}$ dans $\left(L^{2}(\Omega)\right)^{N}$ faible. On a donc

$$
\int_{\Omega} a\left(u_{n}\right) \nabla w_{n} \cdot \nabla v \mathrm{~d} x \rightarrow \int_{\Omega} a(u) \nabla \bar{w} \cdot \nabla v \mathrm{~d} x \text { lorsque } n \rightarrow+\infty .
$$


On remarque ensuite que $\varphi\left(u_{n}\right) \rightarrow \varphi(u)$ p.p. et que $\left|\varphi\left(u_{n}\right)\right| \leq C_{1}\left|u_{n}\right| \leq C_{1} H$; donc par le théorème de convergence dominée de Lebesgue, $\varphi\left(u_{n}\right) \rightarrow \varphi(u)$ dans $L^{2}(\Omega)$ et $\int_{\Omega} G \varphi\left(u_{n}\right) \cdot \nabla v$ $d x \rightarrow \int_{\Omega} G \varphi(u) \cdot \nabla v \mathrm{~d} x$ lorsque $n \rightarrow+\infty$.

Enfin pour le dernier terme, comme $f\left(u_{n}\right) \rightarrow f(u)$ p.p. et $\left|f\left(u_{n}\right)\right| \leq|d|+C_{2} H$ p.p., on a donc par convergence dominée $f\left(u_{n}\right) \rightarrow f(u)$. On en déduit que $\int_{\Omega} f\left(u_{n}\right) v \mathrm{~d} x \rightarrow \int_{\Omega} f(u) v \mathrm{~d} x$ lorsque $n \rightarrow+\infty$.

En passant à la limite dans 3.10 , on obtient

$$
\int_{\Omega} a(u) \nabla \bar{w} \cdot \nabla v \mathrm{~d} x=-t \int_{\Omega} G \varphi(u) \cdot \nabla v \mathrm{~d} x+t \int_{\Omega} f(u) v \mathrm{~d} x,
$$

et donc $\bar{w}=h(t, u)=w$.

En raisonnant par l'absurde, on montre ensuite que (sans sous-suite) $w_{n} \rightarrow w$ dans $H_{0}^{1}$ faible et $w_{n} \rightarrow w$ dans $L^{2}(\Omega)$ où $w_{n}=h\left(t_{n}, u_{n}\right)$ et $w=h(t, u) ;$ l'application $h$ est donc continue. On a donc bien montré l'item 2

Il reste maintenant à démontrer l'item 1 On veut montrer qu'il existe $R>0$ tel que pour tout $t \in[0,1]$ et tout $u \in L^{2}(\Omega)$, si $u=h(t, u)$, alors $\|u\|_{L^{2}(\Omega)}<R$. Soit $t \in[0,1]$, et $u=h(t, u)=t B_{u}(F(u))$, c'est à dire

$$
\left\{\begin{array}{l}
\int_{\Omega} a(u) \nabla u \cdot \nabla v \mathrm{~d} x=-t \int_{\Omega} G \varphi(u) \cdot \nabla v \mathrm{~d} x+t \int_{\Omega} f(u) v \mathrm{~d} x, \quad \forall v \in H_{0}^{1}(\Omega) . \\
u \in H_{0}^{1}(\Omega) .
\end{array}\right.
$$

Pour $s \in \mathbb{R}$, on pose $\Phi(s)=\int_{0}^{s} \varphi(\xi) \mathrm{d} \xi$ ( $\Phi$ est donc une primitive de $\varphi$ ). Comme $u \in H_{0}^{1}(\Omega)$, il n'est pas difficile de montrer que $\Phi(u) \in W_{0}^{1,1}(\Omega)$ et que

$$
\int_{\Omega} G \varphi(u) \cdot \nabla u \mathrm{~d} x=\int_{\Omega} G \cdot \nabla \Phi(u) \mathrm{d} x .
$$

(Ceci est laissé en exercice, il suffit d'approcher $u$, dans $H_{0}^{1}(\Omega)$, par une suite de fonctions appartenant à $C_{c}^{\infty}(\Omega)$.) Comme $\operatorname{div}(G)=0$, on a alors

$$
\int_{\Omega} G \varphi(u) \cdot \nabla u \mathrm{~d} x=\int_{\Omega} G \cdot \nabla \Phi(u) \mathrm{d} x=\int_{\Omega} \operatorname{div} G \Phi(u) \mathrm{d} x=0 .
$$

On choisit maintenant $v=u$ dans 3.11. Par les hypothèses 3.7, on a donc :

$$
\alpha\|u\|_{H_{0}^{1}(\Omega)}^{2} \leq \int_{\Omega}|f(u) u| \mathrm{d} x
$$

On va déduire de cette dernière inégalité qu'il existe $R>0$ t.q $\|u\|_{L^{2}(\Omega)}<R$. C'est ici qu'on utilise l'hypothèse (3.7g) i.e. $\lim _{s \rightarrow \pm \infty} f(x, s) / s=0$.

Raisonnons par l'absurde. Supposons qu'un tel $R$ n'existe pas. Alors il existe une suite $\left(u_{n}\right)_{n \in \mathbb{N}^{\star}}$ d'éléments de $H_{0}^{1}(\Omega)$ telle que

$$
\left\|u_{n}\right\|_{L^{2}(\Omega)} \geq n \text { et } \alpha\left\|u_{n}\right\|_{H_{0}^{1}}^{2} \leq \int_{\Omega}\left|f\left(u_{n}\right) u_{n}\right| \mathrm{d} x .
$$

Montrons que ceci est impossible. Posons $v_{n}=\frac{u_{n}}{\left\|u_{n}\right\|_{L^{2}(\Omega)}}$. On a donc $\left\|v_{n}\right\|_{L^{2}(\Omega)}=1$ et

$$
\alpha\left\|v_{n}\right\|_{H_{0}^{1}(\Omega)}^{2} \leq \int_{\Omega}\left|\frac{f\left(u_{n}\right)}{\left\|u_{n}\right\|_{L^{2}(\Omega)}} v_{n}\right| \mathrm{d} x
$$


Or $|f(s)| \leq|d|+C_{2}|s|$, on a donc

$$
\begin{aligned}
\alpha\left\|v_{n}\right\|_{H_{0}^{1}(\Omega)}^{2} & \leq \int_{\Omega} \frac{|d|+C_{2}\left|u_{n}\right|}{\left\|u_{n}\right\|_{L^{2}}}\left|v_{n}\right| \mathrm{d} x \\
& \leq \int_{\Omega} \frac{\left|d \| v_{n}\right|}{\left\|u_{n}\right\|_{L^{2}}} \mathrm{~d} x+C_{2} \int_{\Omega}\left|v_{n}\right|^{2} \mathrm{~d} x \\
& \leq\|d\|_{L^{2}(\Omega)}+C_{2} .
\end{aligned}
$$

La suite $\left(v_{n}\right)_{n \in \mathbb{N}^{*}}$ est ainsi bornée dans $H_{0}^{1}(\Omega)$, et donc, à une sous-suite près, $v_{n} \rightarrow v$ dans $L^{2}(\Omega)$. Par passage à la limite on en déduit que $\|v\|_{L^{2}}=1$ (ce qui donne $v \neq 0$ ). On a aussi (toujours à une sous-suite près) :

$$
\begin{aligned}
& v_{n} \rightarrow v \quad \text { p.p., } \\
& \left|v_{n}\right| \leq H \quad \text { avec } \quad H \in L^{2}(\Omega) .
\end{aligned}
$$

Enfin, en utilisant l'inégalité de Poincaré, il existe $C_{\Omega}$, ne dépendant que de $\Omega$ t.q.

$$
\frac{\alpha}{C_{\Omega}}=\frac{\alpha}{C_{\Omega}}\left\|v_{n}\right\|_{L^{2}}^{2} \leq \alpha\left\|v_{n}\right\|_{H_{0}^{1}}^{2} \leq \int_{\Omega} \frac{\left|f\left(u_{n}\right)\right|}{\left\|u_{n}\right\|_{L^{2}(\Omega)}}\left|v_{n}\right| \mathrm{d} x .
$$

On pose

$$
X_{n}=\int_{\Omega} \frac{\left|f\left(u_{n}\right)\right|\left|v_{n}\right|}{\left\|u_{n}\right\|_{L^{2}(\Omega)}} \mathrm{d} x
$$

et on montre maintenant que $X_{n} \rightarrow 0$ lorsque $n \rightarrow+\infty$, ce qui est impossible car $X_{n} \geq \frac{\alpha}{C_{\Omega}}>0$. Montrons que $\frac{f\left(u_{n}\right)\left|v_{n}\right|}{\left\|u_{n}\right\|_{L^{2}(\Omega)}} \rightarrow 0$ p.p. avec domination (dans $L^{1}(\Omega)$ ); on aura alors par le théorème de convergence dominée que $X_{n} \rightarrow 0$ lorsque $n \rightarrow+\infty$. On montre tout d'abord la domination. On a

$$
\frac{\left|f\left(u_{n}\right)\right|}{\left\|u_{n}\right\|_{L^{2}(\Omega)}} \leq \frac{|d|+C_{2}\left|u_{n}\right|}{\left\|u_{n}\right\|_{L^{2}}} \leq|d|+C_{2}\left|v_{n}\right| \leq|d|+C_{2} H
$$

et donc $\left|\frac{f\left(u_{n}\right)}{\left\|u_{n}\right\|_{L^{2}(\Omega)}} v_{n}\right| \leq\left(|d|+C_{2} H\right) H \in L^{1}(\Omega)$.

On montre maintenant la convergence p.p.. On a $v_{n} \rightarrow v$ p.p. et donc $\exists A$; $\operatorname{mes}\left(A^{c}\right)=0$ et $v_{n}(x) \rightarrow v(x)$ pour tout $x \in A$. Soit $x \in A$,

1er cas : $\quad$ si $v(x)>0 ; v_{n}(x) \rightarrow v(x)$, mais $\lim _{n \rightarrow+\infty}\left\|u_{n}\right\|_{L^{2}(\Omega)}=+\infty$ donc $u_{n}(x)=v_{n}(x)\left\|u_{n}\right\|_{L^{2}(\Omega)} \rightarrow+\infty$. Par l'hypothèse $3.7 \mathrm{~g}), \lim _{s \rightarrow+\infty} \frac{f(s)}{s}=0$, et donc

$$
\frac{f\left(u_{n}(x)\right)}{\left\|u_{n}\right\|_{L^{2}(\Omega)}} v_{n}(x)=\frac{f\left(u_{n}(x)\right) u_{n}(x)}{u_{n}(x)\left\|u_{n}\right\|_{L^{2}(\Omega)}} v_{n}(x)=\frac{f\left(u_{n}(x)\right)}{u_{n}(x)}\left(v_{n}(x)\right)^{2} \rightarrow 0 \text { quand } n \rightarrow \infty .
$$

2ème cas : $\quad$ si $v(x)<0$; on a de même $\lim _{n \rightarrow+\infty} \frac{f\left(u_{n}(x)\right)}{\left\|u_{n}\right\|_{L^{2}(\Omega)}} v_{n}(x)=0$, car $\lim _{s \rightarrow-\infty} \frac{f(s)}{s}=0$.

3ème cas : $\quad$ si $v(x)=0$, on a

$$
\begin{aligned}
\left|\frac{f\left(u_{n}(x)\right)}{\left\|u_{n}\right\|_{L^{2}(\Omega)}} v_{n}(x)\right| & \leq \frac{|d(x)|+C_{2}\left|u_{n}(x)\right|}{\left\|u_{n}\right\|_{L^{2}(\Omega)}}\left|v_{n}(x)\right| \\
& \leq\left(|d(x)|+C_{2}\left|v_{n}(x)\right|\right)\left|v_{n}(x)\right| \\
& \rightarrow 0 \quad \text { car } \quad v(x)=0 .
\end{aligned}
$$


En résumé on a $\frac{f\left(u_{n}\right)}{\left\|u_{n}\right\|_{L^{2}(\Omega)}} v_{n} \rightarrow 0$ p.p. lorsque $n \rightarrow+\infty$. On a ainsi montré que $\lim _{n \rightarrow+\infty} X_{n}=0$, en contradiction avec $X_{n} \geq \alpha / C_{\Omega}$ pour tout $n \in \mathbb{N}^{\star}$.

On a donc montré qu'il existe $R>0$ t.q. $(u=h(t, u)) \Rightarrow\|u\|_{L^{2}(\Omega)}<R$, ce qui termine la démonstration de 1 . On a ainsi montré l'exsitence de solution à 3.5. Le théorème 3.15 est donc démontré.

Remarque 3.16 (Preuve du théorème d'existence 3.15 par Schauder) La preuve du théorème d'existence 3.15 par le théorème de Schauder est nettement plus compliquée que dans le cas $f$ bornée du théorème 3.12 Considèrons le problème linéaire suivant :

$$
\int_{\Omega} a(\bar{u}) \nabla u \cdot \nabla v \mathrm{~d} x+\int_{\Omega} G \varphi(\bar{u}) \cdot \nabla v \mathrm{~d} x=\int_{\Omega} f(\bar{u}) v \mathrm{~d} x \quad \text { pour tout } v \in H_{0}^{1}(\Omega) .
$$

Dans (3.13, on a noté, de manière abrégée, $a(\bar{u})$ et $f(\bar{u})$ les fonctions $x \mapsto a(x, \bar{u}(x))$ et $x \mapsto f(x, \bar{u}(x))$. Cette notation abrégée sera souvent utilisée par la suite.

Soit $T$ l'opérateur défini de $L^{2}(\Omega)$ dans $L^{2}(\Omega)$ par $T(\bar{u})=u$ où $u$ est solution de (3.13). Il est assez facile de montrer que $T$ est continu et même compact. Par contre il est difficile de montrer que $T$ envoie une boule de $L^{2}(\Omega)$ dans elle-même. Pour cela, il faut obtenir une estimation sur $u$ en fonction de $\bar{u}$, et ce n'est pas gagné. Prenons $v=u$ dans 3.13, comme on a fait dans le paragraphe 3.1.2. On obtient, grâce aux hypothèses 3.7,

$$
\alpha\|u\|_{H_{0}^{1}(\Omega)}^{2} \leq\||G|\|_{\infty} C_{1}\|\bar{u}\|_{L^{2}(\Omega)}\|u\|_{H_{0}^{1}(\Omega)}+\|d\|_{L^{2}(\Omega)}\|u\|_{L^{2}(\Omega)}+C_{2}\|\bar{u}\|_{L^{2}(\Omega)}\|u\|_{L^{2}(\Omega)} .
$$

Montrons que le dernier terme à lui tout seul empêche d'avoir facilement des estimations. Supposons $G=0$ et $d=0$, on a alors grâce à l'inégalité de Poincaré :

$$
\frac{\alpha}{C_{\Omega}^{2}}\|u\|_{L^{2}(\Omega)}^{2} \leq \alpha\|u\|_{H_{0}^{1}(\Omega)}^{2} \leq C_{2}\|\bar{u}\|_{L^{2}(\Omega)}\|u\|_{L^{2}(\Omega)}
$$

c'est à dire $\|u\|_{L^{2}(\Omega)} \leq \frac{C_{\Omega}^{2}}{\alpha} C_{2}\|\bar{u}\|_{L^{2}} \leq \frac{C_{\Omega}^{2}}{\alpha} C_{2} R$ si $\bar{u}$ est dans la boule de centre 0 et de rayon $R$ de $L^{2}(\Omega)$, c'est-à-dire si $\|\bar{u}\|_{L^{2}(\Omega)} \leq \stackrel{R}{R}$. On ne peut pas en conclure que $\|u\|_{L^{2}(\Omega)}<R$ (sauf si $C_{\Omega}^{2} C_{2}<\alpha$ et dans ce cas la seule solution de (3.5) ne peut être que $u=0$. En effet, si $u$ est solution de (3.5), le raisonnement précédent avec $\bar{u}=u$ donne $\|u\|_{L^{2}(\Omega)} \leq\left(C_{\Omega}^{2} C_{2} / \alpha\right)\|u\|_{L^{2}}$ et donc $u=0$ car $\left.C_{\Omega}^{2} C_{2} / \alpha<1\right)$.

La méthode ne fonctionne donc pas pas de manière directe. Toutefois, elle fonctionne avec un peu de travail supplémentaire en utilisant l' hypothèse de (3.7b). Une solution est, par exemple, de considérer une suite de problèmes avec second membre tronqué et de passer à la limite.

Il est maintenant naturel de se demander si, sous les hypothèses (3.7) (hypothèses du théorème 3.15), on peut montrer l'unicité de la solution. Dans le cas où $f$ ne dépend pas de $u$ et où l'équation est linéaire (c'est-à-dire que $a$ ne dépend pas de $u$ et $\varphi$ est linéaire), il suffit de prendre la différence de deux solutions comme fonction test dans les deux formulations faibles associées à ces deux solutions et de faire la différences des deux équations obtenues. On montre ainsi que les deux solutions sont égales (p.p.). Dans le cas général, la situation est plus compliquée.

Remarque 3.17 ( $f$ lipschitzienne ne donne pas l'unicité) Il est inutile de supposer $f$ lipschitzienne pour le résultat d'unicité, il suffit pour s'en convaincre de considérer l'exemple suivant, où f est lipchitzienne et pour lequel on n'a pas unicité. Prenons par exemple $a=1, \varphi=0$ et $f(u)=\lambda u$, où $\lambda$ est une valeur propre de $(-\Delta)$ avec condition de Dirichlet. Le problème est alors

$$
\left\{\begin{array}{l}
\int_{\Omega} \nabla u \cdot \nabla v \mathrm{~d} x=\lambda \int_{\Omega} u v \mathrm{~d} x \quad \forall \in H_{0}^{1}(\Omega) \\
u \in H_{0}^{1}(\Omega)
\end{array}\right.
$$


Ce problème admet deux solutions : $u_{1}=0$, et une fonction propre $u_{2} \neq 0$ associée à $\lambda$. Evidemment $f(u)=\lambda u$ ne satisfait pas la condition (3.7g) mais on peut modifier $f$ légérement pour que cette condition soir vérifiée, tout en gardant $u_{1}$ et $u_{2}$ comme solutions dés que $u_{2} \in L^{\infty}(\Omega)$ (le fait que $u_{2} \in L^{\infty}(\Omega)$ est toujours vrai si $N<6$; ceci peut se démontrer, par exemple, à partir de l'exercice 2.19. En effet, en prenant $\widetilde{f}$ qui est égale à $f$ sur $]-\gamma, \gamma[$ où $\gamma=\left\|u_{2}\right\|_{L^{\infty}}$ et qui est raccordée à 0 ensuite, de telle sorte que $\tilde{f} \in C_{c}(\mathbb{R}, \mathbb{R})$, on a les mêmes solutions pour $\tilde{f} \in C_{c}(\mathbb{R}, \mathbb{R})$, c'est-à-dire pour le problème :

$$
\left\{\begin{array}{l}
\int_{\Omega} \nabla u \cdot \nabla v \mathrm{~d} x=\int_{\Omega} \tilde{f}(u) v \mathrm{~d} x \\
u \in H_{0}^{1}(\Omega)
\end{array}\right.
$$

on a ainsi 2 solutions avec $\lim _{s \rightarrow \pm \infty} \tilde{f}(s) / s=0$. Le fait que $f$ soit lipschitzienne est donc inutile pour l'unicité.

Montrons l'unicité dans le cas où $f$ ne dépend pas de $s$, i.e. $f(x, s)=d(x)$, sous les hypothèses d'existence (3.7) et en supposant de plus que $a$ et $\varphi$ sont lipschitziennes, c'est-à-dire :

$$
\exists C_{3}>0 ; \forall s, s_{2} \in \mathbb{R},\left\{\begin{array}{l}
\left|a\left(x, s_{1}\right)-a\left(x, s_{2}\right)\right| \leq C_{3}\left|s_{1}-s_{2}\right| \text { p.p. } x \in \mathbb{R}, \\
\left|\varphi\left(s_{1}\right)-\varphi\left(s_{2}\right)\right| \leq C_{3}\left|s_{1}-s_{2}\right| .
\end{array}\right.
$$

Théorème 3.18 (Existence et unicité) Sous les hypothèses (3.7) et (3.14), il existe une et une seule solution à (3.5).

Démonstration : La technique utilisée ici apparaît pour la première fois dans un article d'Artola en 1986 [3]. Soient $u_{1}$ et $u_{2}$ deux solutions de (3.5). On a :

$$
\int_{\Omega} a\left(u_{1}\right) \nabla u_{1} \cdot \nabla v \mathrm{~d} x+\int_{\Omega} G \varphi\left(u_{1}\right) \cdot \nabla v \mathrm{~d} x=\int_{\Omega} f v \mathrm{~d} x
$$

et

$$
\int_{\Omega} a\left(u_{2}\right) \nabla u_{2} \cdot \nabla v \mathrm{~d} x+\int_{\Omega} G \varphi\left(u_{2}\right) \cdot \nabla v \mathrm{~d} x=\int_{\Omega} f v \mathrm{~d} x .
$$

L'idée est de prendre $v=T_{\varepsilon}\left(u_{1}-u_{2}\right)$ dans 3.15 et 3.16 , où $\varepsilon>0$ et $T_{\varepsilon}$ est la troncature au niveau $\varepsilon$, c'est-à-dire $T_{\varepsilon}(s)=\left\{\begin{array}{l}-\varepsilon \text { si } s<-\varepsilon, \\ s \text { si }-\varepsilon \leq s \leq \varepsilon, \\ \varepsilon \text { si } s>\varepsilon .\end{array}\right.$

Si $u_{1}$ et $u_{2} \in H_{0}^{1}(\Omega)$, alors $T_{\varepsilon}\left(u_{1}-u_{2}\right) \in H_{0}^{1}(\Omega)$ et $\nabla T_{\varepsilon}\left(u_{1}-u_{2}\right)=\nabla\left(u_{1}-u_{2}\right) 1_{\left\{0<\left|u_{1}-u_{2}\right|<\varepsilon\right\}}$ (ceci est une généralisation simple du lemme 2.22). En prenant $v=T_{\varepsilon}\left(u_{1}-u_{2}\right)$, et en faisant la différence de (3.15) et (3.16), on obtient :

$$
\begin{aligned}
\int_{\Omega}\left(a\left(u_{1}\right) \nabla u_{1} \cdot \nabla\left(T_{\varepsilon}\left(u_{1}-u_{2}\right)\right)-\right. & \left.a\left(u_{2}\right) \nabla u_{2} \cdot \nabla\left(T_{\varepsilon}\left(u_{1}-u_{2}\right)\right)\right) \mathrm{d} x \\
& =\int_{\Omega} G\left(\varphi\left(u_{2}\right)-\varphi\left(u_{1}\right)\right) \cdot \nabla\left(T_{\varepsilon}\left(u_{1}-u_{2}\right)\right) \mathrm{d} x
\end{aligned}
$$

soit encore, en posant $A_{\varepsilon}=\left\{0<\left|u_{1}-u_{2}\right|<\varepsilon\right\}$,

$$
\begin{aligned}
\int_{A_{\varepsilon}} a\left(u_{1}\right) \nabla\left(u_{1}-u_{2}\right) \cdot \nabla\left(u_{1}-u_{2}\right) \mathrm{d} x & =\int_{A_{\varepsilon}}\left(a\left(u_{2}\right)-a\left(u_{1}\right)\right) \nabla u_{2} \cdot \nabla\left(u_{1}-u_{2}\right) \mathrm{d} x \\
& +\int_{A_{\varepsilon}} G\left(\varphi\left(u_{2}\right)-\varphi\left(u_{1}\right)\right) \cdot \nabla\left(u_{1}-u_{2}\right) \mathrm{d} x .
\end{aligned}
$$


Par hypothèse, $a\left(u_{1}\right) \geq \alpha$ p.p., et donc :

$\alpha \int_{A_{\varepsilon}}\left|\nabla\left(u_{1}-u_{2}\right)\right|^{2} \mathrm{~d} x \leq \int_{A_{\varepsilon}} C_{3}\left|u_{2}-u_{1}\right|\left|\nabla u_{2}\right|\left|\nabla\left(u_{2}-u_{1}\right)\right| \mathrm{d} x+\int_{A_{\varepsilon}} C_{3}\left|u_{1}-u_{2}\right||G|\left|\nabla\left(u_{1}-u_{2}\right)\right| \mathrm{d} x$.

On a $\left|u_{1}-u_{2}\right| \leq \varepsilon$ p.p. dans $A_{\varepsilon}$. En appliquant l'inégalité de Cauchy Schwarz dans les deux dernières intégrales, on obtient donc :

$$
\begin{aligned}
\alpha \int_{A_{\varepsilon}}\left|\nabla\left(u_{1}-u_{2}\right)\right|^{2} \mathrm{~d} x & \leq C_{3} \varepsilon\left(\int_{A_{\varepsilon}}\left|\nabla u_{2}\right|^{2} \mathrm{~d} x\right)^{\frac{1}{2}}\left(\int_{A_{\varepsilon}}\left|\nabla\left(u_{2}-u_{1}\right)\right|^{2} \mathrm{~d} x\right)^{\frac{1}{2}} \\
& +C_{3} \varepsilon\left(\int_{A_{\varepsilon}}|G|^{2} \mathrm{~d} x\right)^{1 / 2}\left(\int_{A_{\varepsilon}}\left|\nabla\left(u_{1}-u_{2}\right)\right|^{2} \mathrm{~d} x\right)^{1 / 2} .
\end{aligned}
$$

On a donc

ou encore

$$
\alpha\left(\int_{A_{\varepsilon}}\left(\nabla\left(u_{1}-u_{2}\right)\right)^{2}\right)^{1 / 2} \leq C_{3} \varepsilon a_{\varepsilon}, \text { avec } a_{\varepsilon}=\left(\int_{A_{\varepsilon}}|G|^{2} \mathrm{~d} x\right)^{1 / 2}+\left(\int_{A_{\varepsilon}}\left|\nabla u_{2}\right|^{2} \mathrm{~d} x\right)^{1 / 2}
$$

$$
\alpha\left(\int_{\Omega}\left|\nabla\left(T_{\varepsilon}\left(u_{1}-u_{2}\right)\right)\right|^{2} \mathrm{~d} x\right)^{1 / 2}=\alpha\left\|\left|\nabla T_{\varepsilon}\left(u_{1}-u_{2}\right)\right|\right\|_{L^{2}(\Omega)} \leq C_{3} \varepsilon a_{\varepsilon}
$$

donc, en désignant par $m$ la mesure de Lebesgue sur $\mathbb{R}^{N}$,

$$
\left.\frac{\alpha}{m(\Omega)^{1 / 2}} \| \mid \nabla T_{\varepsilon}\left(u_{1}-u_{2}\right)\right)\left|\left\|_{L^{1}(\Omega)} \leq \alpha\right\|\right| \nabla T_{\varepsilon}\left(u_{1}-u_{2}\right) \mid \|_{L^{2}(\Omega)} \leq C_{3} \varepsilon a_{\varepsilon} .
$$

Comme $H_{0}^{1}(\Omega) \subset W_{0}^{1,1}(\Omega)$, on a $T_{\varepsilon}\left(u_{1}-u_{2}\right) \in H_{0}^{1}(\Omega) \subset W_{0}^{1,1}(\Omega)$ et l'inégalité de Sobolev donne

$$
\left\|T_{\varepsilon}\left(u_{1}-u_{2}\right)\right\|_{L^{1^{\star}}} \leq\left\|\left|\nabla T_{\varepsilon}\left(u_{1}-u_{2}\right)\right|\right\|_{L^{1}(\Omega)}, \text { avec } 1^{\star}=\frac{N}{N-1}
$$

et donc

$$
\frac{\alpha}{(m e s \Omega)^{1 / 2}}\left\|T_{\varepsilon}\left(u_{1}-u_{2}\right)\right\|_{L^{1^{\star}}} \leq C_{3} \varepsilon a_{\varepsilon} .
$$

Si $N=1$, on a $N /(N-1)=+\infty$ et conclut facilement que $u_{1}=u_{2}$ p.p.. Le cas $N \geq 2$ demande un léger développement supplémentaire. On remarque que

$$
\begin{aligned}
\left\|T_{\varepsilon}\left(u_{1}-u_{2}\right)\right\|_{L^{1^{\star}}} & =\left(\int_{\Omega}\left|T_{\varepsilon}\left(u_{1}-u_{2}\right)\right|^{1^{\star}} \mathrm{d} x\right)^{\frac{1}{1^{\star}}} \\
& \geq\left(\int_{B_{\varepsilon}} \varepsilon^{1^{\star}} \mathrm{d} x\right)^{1 / 1^{\star}} \\
& \geq \varepsilon\left(m\left(B_{\varepsilon}\right)\right)^{\frac{N-1}{N}}
\end{aligned}
$$

où $B_{\varepsilon}=\left\{x ;\left|u_{1}(x)-u_{2}(x)\right| \geq \varepsilon\right\}$. On a donc

$$
\varepsilon\left(m\left(B_{\varepsilon}\right)\right)^{\frac{N-1}{N}} \leq \frac{(m(\Omega))^{1 / 2}}{\alpha} C_{3} \varepsilon a_{\varepsilon}
$$


et on en déduit que $\left(m\left(B_{\varepsilon}\right)\right)^{\frac{N-1}{N}} \leq C_{4} a_{\varepsilon}$. Prenons, pour $n \in \mathbb{N}^{\star}, \varepsilon=1 / n$, on a $A_{1 /(n+1)} \subset A_{1 / n}$ et $\cap_{n \in \mathbb{N}} A_{1 / n}=\emptyset$, donc $m\left(A_{1 / n}\right) \rightarrow 0$ lorsque $n \rightarrow+\infty$ (par continuité décroissante d'une mesure). On rappelle que

$$
a_{1 / n}=\left(\int_{A_{1 / n}}|G|^{2} \mathrm{~d} x\right)^{1 / 2}+\left(\int_{A_{1 / n}}\left|\nabla u_{2}\right|^{2} \mathrm{~d} x\right)^{1 / 2} .
$$

Comme $G,\left|\nabla u_{2}\right| \in L^{2}(\Omega)$, on en déduit que $\lim _{n \rightarrow+\infty} a_{1 / n}=0$.

On a aussi $B_{1 /(n+1)} \supset B_{1 / n}$ et $\cup_{n \in \mathbb{N}} B_{1 / n}=\left\{\left|u_{1}-u_{2}\right|>0\right\}$. Donc $\lim _{n \rightarrow+\infty} m\left(B_{1 / n}\right)=m\left\{\left|u_{1}-u_{2}\right|>0\right\}$ (par continuité croissante d'une mesure). Comme

$$
\left(m\left(B_{1 / n}\right)\right)^{\frac{N-1}{N}} \leq C_{4} a_{1 / n},
$$

en passant à la limite lorsque $n$ tend vers $+\infty$, on obtient $m\left\{\left|u_{1}-u_{2}\right|>0\right\} \leq 0$, et donc $u_{1}=u_{2}$ p.p., ce qui termine la démonstration.

\subsection{Méthodes de monotonie}

\subsubsection{Introduction}

Pour le problème (3.3), dans le cas où $f$ (le second membre) dépend de $\nabla u$, on sait encore prouver l'existence d'une solution avec le théorème de Schauder, voir exercice 3.3 La question est plus difficile dans le cas où $a$ dépend de $\nabla u$. On se place sous les hypothèses suivantes:

$$
\left\{\begin{array}{l}
\Omega \text { ouvert borné de } \mathbb{R}^{N} \\
a \in C\left(\mathbb{R}^{N}, \mathbb{R}\right) \\
\exists \alpha, \beta \in \mathbb{R}_{+}^{*} ; \alpha \leq a(\xi) \leq \beta, \forall \xi \in \mathbb{R}^{N} \\
f \in L^{2}(\Omega)
\end{array}\right.
$$

On cherche à montrer l'existence d'une solution au problème suivant :

$$
\left\{\begin{array}{l}
u \in H_{0}^{1}(\Omega), \\
\int_{\Omega} a(\nabla u) \nabla u \cdot \nabla v \mathrm{~d} x=\int_{\Omega} f v \mathrm{~d} x, \forall v \in H_{0}^{1}(\Omega) .
\end{array}\right.
$$

Peut-on appliquer le théorème de Schauder? Pour l'appliquer, il faut l'utiliser dans $H_{0}^{1}(\Omega)$ pour que $a(\nabla u)$ ait un sens. Soit $\tilde{u} \in H_{0}^{1}(\Omega)$, par le lemme de Lax-Milgram, il existe un unique $u \in H_{0}^{1}(\Omega)$ solution de :

$$
\left\{\begin{array}{l}
u \in H_{0}^{1}(\Omega), \\
\int_{\Omega} a(\nabla \tilde{u}) \nabla u \cdot \nabla v \mathrm{~d} x=\int_{\Omega} f v \mathrm{~d} x, \forall v \in H_{0}^{1}(\Omega) .
\end{array}\right.
$$

Soit $T$ l'opérateur de $H_{0}^{1}(\Omega)$ dans $H_{0}^{1}(\Omega)$ défini $\operatorname{par} T(\tilde{u})=u$ solution de 3.17. L'opérateur $T$ est bien de $H_{0}^{1}(\Omega)$ dans $H_{0}^{1}(\Omega)$ et

(1) il existe $R>0$ t..q. $\|u\|_{H_{0}^{1}(\Omega)} \leq R$ pour tout $\tilde{u} \in H_{0}^{1}(\Omega)$,

(2) l'application $T$ est continue de $H_{0}^{1}(\Omega)$ dans $H_{0}^{1}(\Omega)$. En effet, si $\tilde{u}_{n} \rightarrow \tilde{u}$ dans $H_{0}^{1}(\Omega)$, on a $\nabla \tilde{u}_{n} \rightarrow \nabla \tilde{u}$ dans $L^{2}(\Omega)^{N}$ et il n'est pas très difficile de montrer que $T\left(\tilde{u}_{n}\right) \rightarrow T(\tilde{u})$ dans $H_{0}^{1}(\Omega)$.

Mais l'application $T$ n'est (en général) pas compacte (de $H_{0}^{1}(\Omega)$ dans $H_{0}^{1}(\Omega)$ ). Si on était en dimension finie, les points (1) et (2) suffiraient à montrer l'existence d'une solution. L'idée est donc de considérer des problèmes approchés en dimension finie et de passer à la limite en utilisant la monotonie de l'opérateur (qui est vraie sous des hypothèses données sur $a$ ci-après). 


\subsubsection{Opérateurs de Leray-Lions}

On considère ici un cas un peu simplifié des opérateurs de Leray-Lions 5 dont l'étude est exposée dans un célèbre article des auteurs en 1965 [21]. On se place sous les hypothèses suivantes :

$$
\begin{aligned}
& \Omega \text { ouvert borné de } \mathbb{R}^{N}, N \geq 1,1<p<+\infty, \\
& a: \mathbb{R}^{N} \rightarrow \mathbb{R}^{N} \text { continue, } \\
& \text { (coercivité) } \exists \alpha>\left.0|a(\xi) \cdot \xi \geq \alpha| \xi\right|^{p}, \forall \xi \in \mathbb{R}^{N}, \\
& \text { (croissance) } \exists C \in \mathbb{R} ;|a(\xi)| \leq C\left(1+|\xi|^{p-1}\right), \forall \xi \in \mathbb{R}^{N}, \\
& \text { (monotonie) }(a(\xi)-a(\eta)) \cdot(\xi-\eta) \geq 0 \quad \forall(\xi, \eta) \in\left(\mathbb{R}^{N}\right)^{2}, \\
& \sigma \in L^{\infty}(\Omega) ; \exists \sigma_{0}>0 ; \sigma \geq \sigma_{0} \quad \text { p.p., } \\
& f \in L^{\frac{p}{p-1}}(\Omega) .
\end{aligned}
$$

Ces hypothèses permettent en particulier de traiter certains modèles dits "LES" (Large Eddy Simulation) utilisés en mécanique des fluides. On s'intéresse alors au problème suivant :

$$
\left\{\begin{array}{l}
-\operatorname{div}(\sigma(x) a(\nabla u(x)))=f(x) \text { dans } \Omega, \\
u=0 \quad \text { sur } \partial \Omega .
\end{array}\right.
$$

Exemple 3.19 ( $\boldsymbol{p}$-laplacien et opérateur de Smagorinsky) Pour $\sigma \equiv 1$ et $a(\xi)=|\xi|^{p-2} \xi(1<p<+\infty)$, l'équation s'écrit $-\operatorname{div}\left(|\nabla u|^{p-2} \nabla u\right)=f$. L'opérateur $u \mapsto-\operatorname{div}\left(|\nabla u|^{p-2} \nabla u\right)$ s'appelle le $p$-laplacien. Pour $p=2$, on retrouve le Laplacien classique. Le cas $p=3$ donne l'opérateur de Smagorinsky ${ }^{6}$ qui apparait dans un modèle de LES.

Remarque 3.20 (Opérateur de Leray-Lions, cas général) Dans le cadre général des opérateurs dits de LerayLions, le terme $a(\nabla u)$ du problème (3.19) considéré ici remplacé par un terme de la forme $a(x, u, \nabla u)$, et la fonction $a$ est alors une fonction de trois variables.

Cherchons une forme faible adéquate de (3.19). Remarquons que si $w \in L^{p}(\Omega)^{N}$, alors, l'hypothèse (3.18d) de croissance sur $a$ donne

$$
\begin{aligned}
|a(w)| & \leq C\left(1+|w|^{p-1}\right) \\
& \leq C+C|w|^{p-1} \in L^{\frac{p}{p-1}}(\Omega)
\end{aligned}
$$

car $C \in L^{\infty}$ et $|w|^{p-1} \in L^{\frac{p}{p-1}}(\Omega)=L^{p^{\prime}}(\Omega)$ avec $p^{\prime}=\frac{p}{p-1}$ (ou encore $\frac{1}{p}+\frac{1}{p^{\prime}}=1$ ). Donc si $u \in W_{0}^{1, p}(\Omega)$, on a $\nabla u \in\left(L^{p}(\Omega)\right)^{N}$ et $a(\nabla u) \in\left(L^{p^{\prime}}(\Omega)\right)^{N}$.

Prenons alors $v \in W_{0}^{1, p}(\Omega)$, on a $\nabla v \in L^{p}(\Omega)^{N}$. On a donc

$$
a(\nabla u) \cdot \nabla v=\sum_{i=1}^{N} a_{i}(\nabla u) D_{i} v \in L^{1}(\Omega) .
$$

5. Jacques-Louis Lions (1928-2001) est un mathématicien français, membre de l'Académie des sciences, spécialiste la théorie des équations aux dérivées partielles et de la la théorie du contrôle.

6. Joseph Smagorinsky (1924-2005) est un physicien américain spécialiste des modèles de turbulence, de la météorologie dynamique et de la climatologie. 
Il est donc naturel de chercher $u \in W_{0}^{1, p}(\Omega)$ et de prendre les fonctions test dans $W_{0}^{1, p}(\Omega)$.

On rappelle aussi que si $f \in L^{p^{\prime}}(\Omega)$, l'application $v \mapsto \int_{\Omega} f(x) v(x) \mathrm{d} x$ est linéaire continue de $W_{0}^{1, p}(\Omega)$ dans $\mathbb{R}$. C'est donc un élément du dual (topologique) de $W_{0}^{1, p}(\Omega)$ (ce dual est noté $W^{-1, p^{\prime}}(\Omega)$ ). Par abus de langage, on note encore $f$ cet élément de $W^{-1, p^{\prime}}(\Omega)$, c'est-à-dire que pour $f \in L^{p^{\prime}}(\Omega)$, on a

$$
\langle f, v\rangle_{W^{-1, p^{\prime}}(\Omega), W_{0}^{1, p}(\Omega)}=\int_{\Omega} f(x) v(x) \mathrm{d} x \text { pour tout } v \in W_{0}^{1, p}(\Omega) .
$$

La forme faible de 3.19 que l'on considère est donc :

$$
\left\{\begin{array}{l}
u \in W_{0}^{1, p}(\Omega), \\
\int_{\Omega} \sigma a(\nabla u) \cdot \nabla v \\
d x=<f, v>_{W^{-1, p^{\prime}}(\Omega), W_{0}^{1, p}(\Omega)}, \forall v \in W_{0}^{1, p}(\Omega) .
\end{array}\right.
$$

Remarque 3.21 (Second membre plus général) Le cadre "naturel” du problème 3.20 n'est donc pas limité au cas $f \in L^{p^{\prime}}(\Omega)$. On peut remplacer dans (3.20) $f$ par n'importe quel élément de $W^{-1, p}(\Omega)$. Par exemple, si $F \in$ $L^{p^{\prime}}(\Omega)^{N}$, on peut remplacer, dans 3.20$),\langle f, v\rangle_{W^{-1, p^{\prime}}(\Omega), W_{0}^{1, p}(\Omega)} \operatorname{par} \int_{\Omega} F(x) \cdot \nabla v(x) \mathrm{d} x$. Sous cette hypothèse, le théorème d'existence et d'unicité donné ci après (théorème 3.22 reste vrai.

Théorème 3.22 (Existence et unicité) Sous les hypothèses (3.18), il existe $u \in W_{0}^{1, p}(\Omega)$ solution de (3.20). Si de plus a est strictement monotone, c'est à dire si

$$
(a(\xi)-a(\eta)) \cdot(\xi-\eta)>0 \text { pour tout }(\xi, \eta) \in\left(\mathbb{R}^{N}\right)^{2}, \xi \neq \eta
$$

alors la solution est unique.

Pour la démonstration de ce théorème, nous aurons besoin de quelques lemmes (classiques) d'intégration que nous rappelons ici. On pourra consulter l'ouvrage [15] pour leur démonstration :

Lemme 3.23 (Convergence forte contre convergence faible) Soit $1<p<+\infty$. On pose $p^{\prime}=\frac{p}{p-1}$. On suppose que $f_{n} \rightarrow f$ dans $L^{p}(\Omega)$ et $g_{n} \rightarrow g$ faiblement dans $L^{p^{\prime}}(\Omega)$. Alors

$$
\int_{\Omega} f_{n} g_{n} d x \rightarrow \int_{\Omega} f g d x \text { lorsque } n \rightarrow+\infty \text {. }
$$

Par contre, on rappelle que si $f_{n} \rightarrow f$ dans $L^{p}$ faible et $g_{n} \rightarrow g$ dans $L^{p^{\prime}}$ faible, on n'a pas en général convergence de $\int_{\Omega} f_{n} g_{n} \mathrm{~d} x$ vers $\int_{\Omega} f g \mathrm{~d} x$.

Lemme 3.24 Si $a \in C\left(\mathbb{R}^{N}, \mathbb{R}^{N}\right)$ vérifie l'hypothèse de croissance $3.18 \mathrm{~d}$ ) et si $u_{n} \rightarrow u$ dans $W_{0}^{1, p}(\Omega)$ alors $a\left(\nabla u_{n}\right) \rightarrow a(\nabla u)$ dans $L^{p^{\prime}}(\Omega)^{N}$.

Le lemme 3.24 se démontre par le théorème de convergence dominée de Lebesgue.

On rappelle aussi que les espaces $W_{0}^{1, p}(\Omega), L^{p}(\Omega)$ et $L^{p^{\prime}}(\Omega)$ sont réflexifs pour $1<p<+\infty$, et donc pour toute suite bornée d'un de ces espaces, on peut extraire une sous-suite faiblement convergente dans cet espace. On rappelle enfin que les espaces $W_{0}^{1, p}(\Omega), L^{p}(\Omega)$ et $L^{p^{\prime}}(\Omega)$ sont séparables, i.e. ils contiennent une partie dénombrable dense, ce qui va nous permettre l'approximation du problème par des problèmes de dimension finie. On aura également besoin du résultat suivant sur les opérateurs coercifs pour la démonstration théorème 3.22. 
Lemme 3.25 (Opérateur coercif dans $\mathbb{R}^{N}$ ) Soit $T: \mathbb{R}^{N} \rightarrow \mathbb{R}^{N}$ continue. On suppose que $T$ est coercif, c'est à dire que

$$
\frac{T(v) \cdot v}{|v|} \rightarrow+\infty \text { quand }|v| \rightarrow+\infty .
$$

Soit $b \in \mathbb{R}^{N}$. Alors, il existe $v \in \mathbb{R}^{N}$ t.q. $T(v)=$ b. L'opérateur $T$ est donc surjectif.

Démonstration On utilise le degré topologique de Brouwer (ce qui possible car on est en dimension finie). On pose $h(t, v)=t T(v)+(1-t) v$. Pour $t=0$, on a $h(0, v)=v$ (donc $h(0, v)=I$, où $I$ est l'opérateur $v \mapsto v$ ). Pour $t=1$ on a $h(1, v)=T(v)$. Pour appliquer le degré, on remarque d'abord que l'application $h:[0,1] \times \mathbb{R}^{N} \rightarrow \mathbb{R}^{N}$ est continue (car $T$ est continue).

On veut ensuite montrer qu'il existe $R>0$ t.q.

$$
t \in[0,1], v \in \mathbb{R}^{N} \text { et } h(t, v)=b \Rightarrow|v|<R .
$$

On suppose qu'on a démontré (3.22). Quitte à augmenter $R$, on peut aussi supposer que $|b|<R$. On pose $B_{R}=$ $\left\{x \in \mathbb{R}^{N}\right.$ t.q. $\left.|x|<R\right\}$. Par invariance par homotopie du degré, on a donc que $d\left(h(t, \cdot), B_{R}, b\right)$ ne dépend pas de $t$, et donc :

$$
d\left(T, B_{R}, b\right)=d\left(\operatorname{Id}, B_{R}, b\right) .
$$

Comme $b \in B_{R}$, on a $d\left(\operatorname{Id}, B_{R}, b\right)=1$ et donc $d\left(T, B_{R}, b\right)=1$. On en déduit l'existence de $v \in B_{R}$ tel que $T(v)=b$.

Il reste à démontrer qu'il existe $R>0$ vérifiant (3.22).

Soit $t \in[0,1]$ et $v \in \mathbb{R}^{N}$ t.q. $h(t, v)=b$, c'est-à-dire $t T(v)+(1-t) v=b$.

On a donc $t T(v) \cdot v+(1-t) v \cdot v=b \cdot v \leq|b||v|$ et donc, si $v \neq 0$,

$$
t \frac{T(v) \cdot v}{|v|}+(1-t)|v|=t \frac{T(v) \cdot v}{|v|}+(1-t) \frac{v \cdot v}{|v|} \leq|b| .
$$

Comme $\frac{T(w) \cdot w}{|w|} \rightarrow+\infty$ lorsque $|w| \rightarrow+\infty$, il existe $R>0$ t.q.

$$
|w| \geq R \Rightarrow \min \left(\frac{T(w) \cdot w}{|w|},|w|\right)>|b| .
$$

On en déduit que $|v|<R$. Ceci termine la démonstration.

Ce lemme se généralise à n'importe quel espace de dimension finie :

Lemme 3.26 (Opérateur coercif en dimension finie) Soit $E$ un espace de dimension finie, et $T: E \rightarrow E^{\prime}$ continue (noter que $\left.\operatorname{dim} E^{\prime}=\operatorname{dim} E<+\infty\right)$. On suppose que T est coercif, c'est-à-dire :

$$
\frac{\langle T(v), v\rangle_{E^{\prime}, E}}{\|v\|_{E}} \rightarrow+\infty \text { quand }\|v\|_{E} \rightarrow+\infty .
$$

Alors, pour tout $b \in E^{\prime}$ il existe $v \in E$ t.q. $T(v)=b$.

Démonstration On se ramène à $\mathbb{R}^{N}$. Soit $N=\operatorname{dim} E$. On choisit une base de $E$, notée $\left(e_{1}, \ldots, e_{N}\right)$, et on note $\left(e_{1}^{\star}, \ldots, e_{N}^{\star}\right)$ la base duale de $E^{\prime}$ ( c'est-à-dire t.q. $\left\langle e_{i}^{\star}, e_{j}\right\rangle_{E^{\prime}, E}=\delta_{i j}$ ). On définit une application $I$ de $\mathbb{R}^{N}$ dans $E$ et une application $J$ de $\mathbb{R}^{N}$ dans $E^{\prime}$ par

$$
I(\alpha)=\sum_{i=1}^{N} \alpha_{i} e_{i} \text { et } J(\beta)=\sum_{i=1}^{N} \beta_{i} e_{i}^{\star} \text { pour } \alpha, \beta \in \mathbb{R}^{N} .
$$


L'opérateur $I$ est une bijection linéaire de $\mathbb{R}^{N}$ dans $E$ et l'opérateur $J$ est une bijection linéaire de $\mathbb{R}^{N}$ dans $E^{\prime}$. Soit $\tilde{T}=J^{-1} \circ T \circ I$. L'opérateur $\tilde{T}$ est continu de $\mathbb{R}^{N}$ dans $\mathbb{R}^{N}$.

Soit $\alpha \in \mathbb{R}^{N}$. On pose $v=I(\alpha)$ et $\beta=\tilde{T}(\alpha)\left(\operatorname{donc} \beta=J^{-1}(T(v))\right.$ ). On a donc

$\tilde{T}(\alpha) \cdot \alpha=\beta \cdot \alpha=\sum_{i=1}^{N} \beta_{i} \alpha_{i}=\left\langle\sum_{j=1}^{N} \beta_{j} e_{j}^{\star}, \sum_{i=1}^{N} \alpha_{i} e_{i}\right\rangle_{E^{\prime}, E}=\langle J(\beta), I(\alpha)\rangle_{E^{\prime}, E}=J(\beta)\left(\sum_{i=1}^{N} \alpha_{i} e_{i}\right)=\langle T(v), v\rangle_{E^{\prime}, E}$.

En prenant comme norme sur $\mathbb{R}^{N},\|\alpha\|=\|I(\alpha)\|_{E}$, l'hypothèse de coercivité sur $T$ donne alors

$$
\lim _{\|\alpha\| \rightarrow+\infty} \frac{\tilde{T}(\alpha) \cdot \alpha}{\|\alpha\|}=+\infty
$$

Par équivalence des normes en dimension finie, on a donc aussi

$$
\lim _{|\alpha| \rightarrow+\infty} \frac{\tilde{T}(\alpha) \cdot \alpha}{|\alpha|}=+\infty
$$

On peut donc appliquer le lemme 3.25 à $\tilde{T}$.

Soit $b \in E^{\prime}$. On pose $\beta=J^{-1}(b)$. Le lemme 3.25 donne l'existence de $\alpha \in \mathbb{R}^{N}$ t.q. $\tilde{T}(\alpha)=\beta$. On pose $v=I(\alpha)$ et on a alors $T(v)=T \circ I(\alpha)=J \circ \tilde{T}(\alpha)=J(\beta)=b$. On a ainsi montré l'existence de $v$ dans $E$ t.q. $T(v)=b$.

Démonstration du théorème 3.22

Etape 1 Existence de la solution à un problème en dimension finie

L'espace $W_{0}^{1, p}(\Omega)$ est séparable. Il existe donc une famille dénombrable $\left(f_{n}\right)_{n \in \mathbb{N}^{\star}}$ dense dans $W_{0}^{1, p}(\Omega)$. Soit $E_{n}=\operatorname{Vect}\left\{f_{1} \ldots f_{n}\right\}$ l'espace vectoriel engendré par les $n$ premières fonctions de cette famille. On a donc $\operatorname{dim} E_{n} \leq n$ et $E_{n} \subset E_{n+1}$ pour tout $n \in \mathbb{N}^{\star}$ et on a $\overline{\cup_{n \in \mathbb{N}} E_{n}}=W_{0}^{1, p}$. On en déduit que pour tout $v \in W_{0}^{1, p}(\Omega)$ il existe une suite $\left(v_{n}\right)_{n \in \mathbb{N}^{\star}}$ telle que $v_{n} \in E_{n}$ pour tout $n \in \mathbb{N}^{\star}$ et $v_{n} \rightarrow v$ dans $W_{0}^{1, p}(\Omega)$ lorsque $n \rightarrow+\infty$.

Dans cette première étape, on fixe $n \in \mathbb{N}^{\star}$ et on cherche $u_{n}$ solution du problème suivant, posé en dimension finie :

$$
\left\{\begin{array}{l}
u_{n} \in E_{n}, \\
\int_{\Omega} \sigma a\left(\nabla u_{n}\right) \cdot \nabla v \mathrm{~d} x=<f, v>_{W^{-1, p^{\prime}}, W_{0}^{1, p}} \forall v \in E_{n} .
\end{array}\right.
$$

L'application $v \mapsto<f, v>_{W^{-1, p^{\prime}}, W_{0}^{1, p}}$ est une application linéaire $E_{n}$ dans $\mathbb{R}$ (elle est donc aussi continue car $\left.\operatorname{dim} E_{n}<+\infty\right)$. On note $b_{n}$ cette application. On a donc $b_{n} \in E_{n}^{\prime}$ et

$$
\left\langle b_{n}, v\right\rangle_{E_{n}^{\prime}, E_{n}}=<f, v>_{W^{-1, p^{\prime}}, W_{0}^{1, p}} .
$$

Soit $u \in E_{n}$. On note $T_{n}(u)$ l'application de $E_{n}$ dans $\mathbb{R}$ qui a $v \in E_{n}$ associe $\int_{\Omega} \sigma a(\nabla u) \cdot \nabla v \mathrm{~d} x$. Cette application est linéaire, c'est donc aussi un élément de $E_{n}^{\prime}$ et on a

$$
\left\langle T_{n}(u), v\right\rangle_{E_{n}^{\prime}, E_{n}}=\int_{\Omega} \sigma a(\nabla u) \cdot \nabla v \mathrm{~d} x .
$$

On a ainsi défini une application $T$ de $E_{n}$ dans $E_{n}^{\prime}$. On va montrer que $T$ est continue et coercive. On pourra ainsi en déduire, par le lemme 3.26, que $T$ est surjectif, et donc qu'il existe $u_{n} \in E_{n}$ vérifiant $T\left(u_{n}\right)=b_{n}$, c'est-à-dire $u_{n}$ solution du problème (3.23). 
(a) Continuité de $T_{n}$ On rappelle que $n$ est fixé. Pour simplifier les notations, on oublie ici l'indice $n$, c'est-àdire que l'on pose $E=E_{n}$ et $T=T_{n}$. On munit $E$ de la norme définie par $\|\cdot\|_{E}=\|\cdot\|_{W_{0}^{1, p}}$. Soit $u, \bar{u} \in E$. On a :

$$
\begin{array}{r}
\|T(u)-T(\bar{u})\|_{E^{\prime}}=\max _{v \in E,\|v\|_{E}=1}<T(u)-T(\bar{u}), v>_{E^{\prime}, E} \\
=\max _{v \in E,\|v\|_{W_{0}^{1, p}=1}} \int_{\Omega} \sigma(a(\nabla u)-a(\nabla \bar{u})) \cdot \nabla v \mathrm{~d} x \\
\leq \max _{v \in W_{0}^{1, p},\|v\|_{W_{0}^{1, p}}=1} \int_{\Omega} \sigma(a(\nabla u)-a(\nabla \bar{u})) \cdot \nabla v \mathrm{~d} x .
\end{array}
$$

On pose $\beta=\|\sigma\|_{L^{\infty}(\Omega)}$, on obtient alors

$$
\begin{aligned}
\|T(u)-T(\bar{u})\|_{E^{\prime}} & \leq \max _{v \in W_{0}^{1, p},\|v\|_{W_{0}^{1, p}=1}} \beta\||a(\nabla u)-a(\nabla \bar{u})|\|_{L^{p^{\prime}}(\Omega)}\||\nabla v|\|_{L^{p}(\Omega)} \\
& \leq \beta\||a(\nabla u)-a(\nabla \bar{u})|\|_{L^{p^{\prime}}(\Omega)} .
\end{aligned}
$$

Donc si $\left(u_{n}\right)_{n \in \mathbb{N}}$ est une suite de $E$ t.q. $u_{n} \rightarrow \bar{u}$ dans $E$, on a

$$
\left\|T\left(u_{n}\right)-T(\bar{u})\right\|_{E^{\prime}} \leq \beta\left\|\left|a\left(\nabla u_{n}\right)-a(\nabla \bar{u})\right|\right\|_{L^{p^{\prime}}(\Omega)} .
$$

Par le lemme 3.24 on a $a\left(\nabla u_{n}\right) \rightarrow a(\nabla \bar{u})$ dans $\left(L^{p^{\prime}}(\Omega)\right)^{N}$. On a ainsi montré que $T\left(u_{n}\right) \rightarrow T(\bar{u})$ dans $E^{\prime}$, et donc que $T$ est continue (de $E$ dans $E^{\prime}$ ).

(b) Coercivité de $T_{n}$ On rappelle qu'on a posé $E=E_{n}$ et $T=T_{n}$. On veut montrer que

$$
\frac{<T(u), u>_{E^{\prime}, E}}{\|u\|_{E}} \rightarrow+\infty \text { lorsque }\|u\|_{E} \rightarrow+\infty \text {. }
$$

Par définition, et grâce aux hypothèses (3.18,

$$
<T(u), u>_{E^{\prime}, E}=\int_{\Omega} \sigma a(\nabla u) \cdot \nabla u \mathrm{~d} x \geq \sigma_{0} \alpha \int_{\Omega}|\nabla u|^{p} \mathrm{~d} x .
$$

On a donc

$$
<T(u), u>_{E^{\prime}, E} \geq C\|u\|_{W_{0}^{1, p}}^{p}=C\|u\|_{E}^{p} \quad \text { avec } C=\sigma_{0} \alpha
$$

Finalement,

$$
\frac{<T(u), u>_{E^{\prime}, E}}{\|u\|_{E}} \geq C\|u\|_{E}^{p-1} \rightarrow+\infty \text { lorsque }\|u\|_{E} \rightarrow+\infty,
$$

car on a supposé $p>1$ (le cas $p=1$ est plus difficile et demande des outils supplémentaires, mais il est intéressant en géométrie pour le problème des surfaces minimales par exemple).

On a ainsi montré que $T$ est coercive. On peut donc appliquer le lemme 3.26. Il donne l'existence d'une solution au problème en dimension finie (3.23) (cette technique permet aussi par exemple de démontrer l'existence de solution pour le problème (3.20) approché par éléments finis $P 1$ ).

Etape 2 Existence de la solution à un problème en dimension infinie On a montré l'existence d'une solution au problème (3.23). On va maintenant tenter (et réussir!) un passage à la limite sur ce problème lorsque $n \rightarrow+\infty$ pour montrer l'existence d'une solution au problème 3.20 . Pour cela nous allons :

(a) obtenir une estimation sur $u_{n}$, qui nous permettra d'obtenir de la compacité, et donc d'effectuer

(b) un passage à la limite sur les problèmes (3.23) de manière à avoir l'existence d'une solution $u$ du problème (3.20) comme limite des solutions $u_{n}$ des problèmes 3.23); pour cela, il nous faudra une 
(c) astuce pour montrer que la limite du terme non linéaire est bien le terme qu'on veut...

(a) Estimation sur $u_{n}$

On prend $v=u_{n}$ dans 3.23 . On obtient :

$$
\sigma_{0} \alpha \int_{\Omega}\left|\nabla u_{n}\right|^{p} \mathrm{~d} x \leq\|f\|_{W^{-1, p^{\prime}}}\left\|u_{n}\right\|_{W_{0}^{1, p}}
$$

par coercivité de $a$. On a donc : $\sigma_{0} \alpha\left\|u_{n}\right\|_{W_{0}^{1, p}}^{p} \leq\|f\|_{W^{-1, p^{\prime}}}\left\|u_{n}\right\|_{W_{0}^{1, p}}$, d'où

$$
\left\|u_{n}\right\|_{W_{0}^{1, p}}^{p-1} \leq \frac{1}{\sigma_{0} \alpha}\|f\|_{W^{-1, p^{\prime}}}
$$

\section{(b) Passage à la limite}

La suite $\left(u_{n}\right)_{n \in \mathbb{N}}$ est donc bornée dans $W_{0}^{1, p}(\Omega)$, qui est réflexif. On en déduit qu'il existe une sous-suite encore notée $\left(u_{n}\right)_{n \in \mathbb{N}}$ telle que $u_{n} \rightarrow u$ faiblement dans $W_{0}^{1, p}(\Omega)$.

Par hypothèse, $\left|a\left(\nabla u_{n}\right)\right| \leq C\left(1+\left|\nabla u_{n}\right|^{p-1}\right)$, donc la suite $\left(a\left(\nabla u_{n}\right)\right)_{n \in \mathbb{N}}$ est bornée dans $\left(L^{p^{\prime}}(\Omega)\right)^{N}$, qui est réflexif. Donc il existe $\zeta \in\left(L^{p^{\prime}}(\Omega)\right)^{N}$ telle que, à une sous-suite près,

$$
a\left(\nabla u_{n}\right) \rightarrow \zeta \quad \text { faiblement dans } \quad\left(L^{p^{\prime}}(\Omega)\right)^{N} .
$$

Soit $v \in W_{0}^{1, p}(\Omega)$, on sait que $\overline{\bigcup_{n \in \mathbb{N}} E_{n}}=W_{0}^{1, p}$, donc il existe $\left(v_{n}\right)_{n \in \mathbb{N}}$ t.q. $v_{n} \in E_{n}$ pour tout $n \in \mathbb{N}^{\star}$ et

$$
\begin{array}{ll}
v_{n} & \rightarrow v \text { dans } W_{0}^{1, p}(\Omega), \\
\nabla v_{n} & \rightarrow \nabla v \text { dans }\left(L^{p}(\Omega)\right)^{N} .
\end{array}
$$

On utilise alors (3.23) avec $v=v_{n}$ on obtient :

$$
\int_{\Omega} \sigma a\left(\nabla u_{n}\right) \cdot \nabla v_{n} \mathrm{~d} x=<f, v_{n}>
$$

Mais $<f, v_{n}>\rightarrow<f, v>$, car $v_{n}$ converge faiblement vers $v$ dans $W_{0}^{1, p}(\Omega)$.

De plus $a\left(\nabla u_{n}\right) \rightarrow \zeta$ faiblement dans $\left(L^{p^{\prime}}(\Omega)\right)^{N}$ et $\nabla v_{n} \rightarrow \nabla v$ (fortement) dans $\left(L^{p}(\Omega)\right)^{N}$. Donc par le lemme 3.23 , on a

$$
\int_{\Omega} \sigma \zeta \cdot \nabla v \mathrm{~d} x=<f, v>_{W^{-1, p^{\prime}}, W_{0}^{1, p}} \quad \forall v \in W_{0}^{1, p}(\Omega) .
$$

On a ainsi prouvé l'existence de $u \in W_{0}^{1, p}(\Omega)$ t.q. $u$ est la limite faible dans $W_{0}^{1, p}(\Omega)$ de la suite $\left(u_{n}\right)_{n \in \mathbb{N}}$ et t.q. la limite faible dans $\left(L^{p^{\prime}}(\Omega)\right)^{N}$ de la suite $\left(a\left(\nabla u_{n}\right)\right)_{n \in \mathbb{N}}$, notée $\zeta$, vérifie 3.24). Si $\zeta$ était égal à $a(\nabla u)$, on aurait terminé. Ceci serait facile à établir si $a$ était linéaire. En effet, supposons qu'il existe une matrice carrée de taille $N$, notée $A$, telle que $a(\xi)=A \xi$ pour tout $\xi \in \mathbb{R}^{N}$ (et donc $p=p^{\prime}$ ). Comme $u_{n} \rightarrow u$ dans $\mathcal{D}^{\star}(\Omega)$ on a aussi $D_{i} u_{n} \rightarrow D_{i} u$ dans $\mathcal{D}^{\star}(\Omega)$ (pour tout $i$ ). Comme la suite $\left(D_{i} u_{n}\right)_{n \in \mathbb{N}}$ est bornée dans $L^{2}(\Omega)$, on en déduit que $D_{i} u_{n} \rightarrow D_{i} u$ faiblement dans $L^{2}(\Omega)$ (pour tout $i$ ) et donc $A \nabla u_{n} \rightarrow A \nabla u$ faiblement dans $L^{2}(\Omega)^{N}$. Ce qui prouve que $\zeta=a(\nabla u)$. Malheureusement, la situation est plus compliqués quand $a$ est non linéaire.

(c) Limite du terme non linéaire Pour terminer, il reste à démontrer que

$$
\int_{\Omega} \sigma \zeta \cdot \nabla v \mathrm{~d} x=\int_{\Omega} \sigma a(\nabla u) \cdot \nabla v \mathrm{~d} x \text { pour tout } v \in W_{0}^{1, p}(\Omega) .
$$

On peut le démontrer par deux manières différentes, selon les hypothèses : 
1. Par l'astuce de Minty 7 , qui utilise uniquement la monotonie de $a$, c'est-à-dire $(a(\xi)-a(\eta)) \cdot(\xi-\eta) \geq 0$. On a dans ce cas uniquement $u_{n} \rightarrow u$ faiblement dans $W_{0}^{1, p}(\Omega)$.

2. Par la méthode de Leray-Lions, qui utilise la stricte monotonie de $a$, c'est-à-dire $(a(\xi)-a(\eta)) \cdot(\xi-\eta)>0$ pour tout $\xi, \eta, \xi \neq \eta$. On a alors en plus $u_{n} \rightarrow u$ dans $W_{0}^{1, p}(\Omega)$ ( c'est-à-dire une convergence forte et non seulement faible).

\section{(A) Etape commune à Minty et Leray-Lions.}

Pour montrer (3.25), on commence par étudier la limite de $\int_{\Omega} \sigma a\left(\nabla u_{n}\right) \cdot \nabla u_{n}$

$d x$. L'astuce consiste à utiliser l'équation! En effet $\int_{\Omega} \sigma a\left(\nabla u_{n}\right) \cdot \nabla u_{n} \mathrm{~d} x=<f, u_{n}>\rightarrow<f, u>\operatorname{car} u_{n} \rightarrow u$ dans $W_{0}^{1, p}(\Omega)$ faible. Mais on sait (étape précédente) que $u$ satisfait [3.24], et donc : $\left\langle f, u>_{E^{\prime}, E}=\int_{\Omega} \sigma \zeta \cdot \nabla u\right.$ $d x$.

$$
\begin{aligned}
\text { Donc } \lim _{n \rightarrow+\infty} \int_{\Omega} \sigma a\left(\nabla u_{n}\right) \cdot \nabla u_{n} & \\
d x & =<f, u>_{E^{\prime}, E} \\
& =\int_{\Omega} \sigma \zeta \cdot \nabla u
\end{aligned}
$$

$d x$.

On distingue maintenant les méthodes de Minty et de Leray-Lions (qui sont toutefois très voisines).

(B) Démonstration de 3.25

\section{- lére méthode : Astuce de Minty}

Soit $v \in W_{0}^{1, p}(\Omega)$; il existe $\left(v_{n}\right)_{n \in \mathbb{N}}$ tel que $v_{n} \in E_{n}$ pour tout $n \in \mathbb{N}$ et $v_{n} \rightarrow v$ dans $W_{0}^{1, p}(\Omega)$ lorsque $n \rightarrow+\infty$. On va passer à la limite dans le terme $\int_{\Omega} \sigma a\left(\nabla u_{n}\right) \cdot \nabla v_{n}$

$d x$ grâce à l'hypothèse de monotonie. En effet,

$$
\begin{aligned}
& 0 \leq \int_{\Omega} \sigma\left(a\left(\nabla u_{n}\right)-a\left(\nabla v_{n}\right)\right) \cdot\left(\nabla u_{n}-\nabla v_{n}\right) \mathrm{d} x= \\
& \int_{\Omega} \sigma a\left(\nabla u_{n}\right) \cdot \nabla u_{n} \mathrm{~d} x-\int_{\Omega} \sigma a\left(\nabla u_{n}\right) \cdot \nabla v_{n} \mathrm{~d} x-\int_{\Omega} \sigma a\left(\nabla v_{n}\right) \cdot \nabla u_{n} \mathrm{~d} x+\int_{\Omega} \sigma a\left(\nabla v_{n}\right) \cdot \nabla v_{n} \mathrm{~d} x \\
& \begin{array}{lllllll} 
& T_{1, n} & - & T_{2, n} & - & T_{3, n}
\end{array} \quad+\quad T_{4, n} .
\end{aligned}
$$

On a vu que en (A) que $T_{1, n} \rightarrow \int_{\Omega} \sigma \zeta \cdot \nabla u \mathrm{~d} x$ lorsque $n \rightarrow \infty$.

On a

$$
\lim _{n \rightarrow+\infty} T_{2, n}=\int_{\Omega} \sigma \zeta \cdot \nabla v \mathrm{~d} x
$$

par produit d'une convergence forte dans $\left(L^{p}\right)^{N}$ et d'une convergence faible dans $\left(L^{p^{\prime}}(\Omega)^{N}\right)$ (lemme 3.23 . De même,

$$
\lim _{n \rightarrow+\infty} T_{3, n}=\int_{\Omega} \sigma a(\nabla v) \cdot \nabla u \mathrm{~d} x
$$

7. George James Minty Jr. (1929-1986) mathématicien américain, specialisé en analyse et mathématiques discrètes. 
par produit d'une convergence forte dans $\left(L^{p^{\prime}}(\Omega)^{N}\right)$ et d'unc convergence faible dans $\left(L^{p}(\Omega)\right)^{N}$. Enfin, on a aussi

$$
\lim _{n \rightarrow+\infty} T_{4, n}=\int_{\Omega} \sigma a(\nabla v) \cdot \nabla v \mathrm{~d} x
$$

lorsque $n \rightarrow+\infty$ et ce dernier terme est le plus simple car on a le produit d'une convergence forte dans $\left(L^{p^{\prime}}(\Omega)^{N}\right)$ et d'une convergence forte dans $\left(L^{p}(\Omega)\right)^{N}$.

Le passage à la limite dans l'inégalité donne donc :

$$
\int_{\Omega} \sigma(\zeta-a(\nabla v)) \cdot(\nabla u-\nabla v) \mathrm{d} x \geq 0 \text { pour tout } v \in W_{0}^{1, p}(\Omega) .
$$

On choisit maintenant astucieusement la fonction test $v$. On prend $v=u+\frac{1}{n} w$, avec $w \in W_{0}^{1, p}(\Omega)$ et $n \in \mathbb{N}^{\star}$. On obtient ainsi :

$$
-\frac{1}{n} \int_{\Omega} \sigma\left(\zeta-a\left(\nabla u+\frac{1}{n} \nabla w\right)\right) \cdot \nabla w \mathrm{~d} x \geq 0
$$

et donc

$$
\int_{\Omega} \sigma\left(\zeta-a\left(\nabla u+\frac{1}{n} \nabla w\right)\right) \cdot \nabla w d x \leq 0 .
$$

Mais $u+\frac{1}{n} w \rightarrow u$ dans $W_{0}^{1, p}(\Omega)$, donc par le lemme 3.24. $a\left(\nabla u+\frac{1}{n} \nabla w\right) \rightarrow a(\nabla u)$ dans $\left(L^{p^{\prime}}(\Omega)\right)^{N}$. En passant à la limite lorsque $n \rightarrow+\infty$, on obtient alors

$$
\int_{\Omega} \sigma(\zeta-a(\nabla u)) \cdot \nabla w d x \leq 0 \quad \forall w \in W_{0}^{1, p}(\Omega) .
$$

Par linéarité (on peut changer $w$ en $-w$ ), on a donc : $\int_{\Omega} \sigma(\zeta-a(\nabla u)) \cdot \nabla w \mathrm{~d} x=0, \forall w \in W_{0}^{1, p}(\Omega)$. On en déduit que

$$
\int_{\Omega} \sigma \zeta \cdot \nabla w d x=\int_{\Omega} \sigma a(\nabla u) \cdot \nabla w d x \quad \forall w \in W_{0}^{1, p}(\Omega) .
$$

On a donc bien démontré que $u$ est solution de 3.20 .

Notons que l'on a montré ce résultat par approximation, c'est à dire en montrant d'abord l'existence de solution à un problème approché qui se pose en dimension finie, puis en passant à la limite. Ceci est également possible en utilisant un problème approché obtenu avec des schémas numériques, par exemple avec un schéma numérique utilisant des éléments finis $P 1$.

- 2éme méthode : Astuce de Leray-Lions On suppose maintenant la stricte monotonie de $a$, c'est-à-dire :

$$
(a(\xi)-a(\eta)) \cdot(\xi-\eta)>0 \text { si } \xi \neq \eta .
$$

On va montrer que $u$ est solution de 3.20 (on le sait déjà, grâce à la première méthode) mais aussi que $a(\nabla u)=\zeta$ et surtout que $u_{n} \rightarrow u$ dans $W_{0}^{1, p}(\Omega)$.

Comme $\overline{\cup_{n \in \mathbb{N}} E_{n}}=W_{0}^{1, p}$, il existe une suite $\left(v_{n}\right)_{n \in \mathbb{N}}$ telle que $v_{n} \in E_{n}$ pour tout $n \in \mathbb{N}$ et $v_{n} \rightarrow u$ dans $W_{0}^{1, p}(\Omega)$. Par hypothèse de monotonie, on a :

$$
\int_{\Omega} \sigma\left(a\left(\nabla u_{n}\right)-a\left(\nabla v_{n}\right)\right) \cdot\left(\nabla u_{n}-\nabla v_{n}\right) \geq 0 .
$$


Avec le même raisonnement que pour Minty (mais maintenant $v_{n} \rightarrow u$ au lieu de $v_{n} \rightarrow v$ ), on a :

$$
\lim _{n \rightarrow+\infty} \int_{\Omega} \sigma\left(a\left(\nabla u_{n}\right)-a\left(\nabla v_{n}\right)\right) \cdot\left(\nabla u_{n}-\nabla v_{n}\right) \mathrm{d} x=\int_{\Omega} \sigma(\zeta-a(\nabla u)) \cdot \underbrace{(\nabla u-\nabla u)}_{=0} d x,
$$

donc si on pose $F_{n}(x)=\sigma\left(a\left(\nabla u_{n}\right)-a\left(\nabla v_{n}\right)\right) \cdot\left(\nabla u_{n}-\nabla v_{n}\right)$, on a $F_{n} \geq 0$ p.p. et $\int_{\Omega} F_{n} \mathrm{~d} x \rightarrow 0$ lorsque $n \rightarrow+\infty$. Donc $F_{n} \rightarrow 0$ dans $L^{1}$, et donc, après extraction d'une sous-suite, $F_{n} \rightarrow 0$ p.p.. On peut aussi supposer, après extraction d'une sous-suite, que $\nabla v_{n} \rightarrow \nabla u$ p.p..

Soit $x \in \Omega$ t.q. $\sigma(x) \geq \sigma_{0}, F_{n}(x) \rightarrow 0$ (on a donc $\left.a\left(\nabla u_{n}(x)\right)-a\left(\nabla v_{n}(x)\right) \cdot\left(\nabla u_{n}(x)-\nabla v_{n}(x)\right) \rightarrow 0\right)$ et $\nabla v_{n}(x) \rightarrow \nabla u(x)$ lorsque $n \rightarrow+\infty$.

Etape A : La suite $\left(\nabla u_{n}(x)\right)_{n \in \mathbb{N}}$ est bornée dans $\mathbb{R}^{N}$. En effet, grâce aux hypothèses (3.18b) (3.18d) de coercivité et croissance sur $a$, on a

$$
\begin{aligned}
& \frac{1}{\sigma_{0}} F_{n}(x) \geq\left(a\left(\nabla u_{n}(x)\right)-a\left(\nabla v_{n}(x)\right) \cdot\left(\nabla u_{n}(x)-\nabla v_{n}(x)\right)\right. \\
& \geq \alpha\left|\nabla u_{n}(x)\right|^{p}-C\left(1+\left|\nabla u_{n}(x)\right|^{p-1}\right)\left|\nabla v_{n}(x)\right|-C\left(1+\left|\nabla v_{n}(x)\right|^{p-1}\right)\left|\nabla u_{n}(x)\right|+\alpha\left|\nabla v_{n}(x)\right|^{p} .
\end{aligned}
$$

On en déduit que la suite $\left(F_{n}(x)\right)_{n \in \mathbb{N}}$ est non bornée si la suite $\left(\nabla u_{n}(x)\right)_{n \in \mathbb{N}}$ est non bornée. Or $F_{n}(x) \rightarrow$ 0 lorsque $n \rightarrow+\infty$. Donc il faut que la suite $\left(\nabla u_{n}(x)\right)_{n \in \mathbb{N}}$ soit bornée.

Etape B : Soit $\xi \in \mathbb{R}^{N}$ limite d'une sous suite de la suite $\left(\nabla u_{n}(x)\right)_{n \in \mathbb{N}}$. On rappelle que $x$ est fixé. On a donc pour cette sous suite (encore notée $\left.\left(\nabla u_{n}(x)\right)_{n \in \mathbb{N}}\right) \lim _{n \rightarrow+\infty} a\left(\nabla u_{n}(x)\right)=a(\xi)$. Comme $\lim _{n \rightarrow+\infty} F_{n}(x)=0$, on en déduit

$$
(a(\xi)-a(\nabla u(x)) \cdot(\xi-\nabla u(x))=0 .
$$

Or, le ler terme de cette égalité est strictement positif si $\xi \neq \nabla u(x)$. On a donc donc $\xi=\nabla u(x)$. On a donc (sans extraction de sous suite pour cette étape) $\nabla u_{n}(x) \rightarrow \nabla u(x)$ quand $n \rightarrow+\infty$.

En résumé, on a ainsi montré que $\nabla u_{n} \rightarrow \nabla u$ p.p. (à une sous suite près, car on extrait une sous suite pour avoir $F_{n} \rightarrow 0$ p.p. et $\nabla v_{n} \rightarrow \nabla u$ p.p.). On en déduit que $a\left(\nabla u_{n}\right) \rightarrow a(\nabla u)$ p.p.. Ceci est suffisant pour montrer que $\zeta=a(\nabla u)$. En effet, on sait déjà que $a\left(\nabla u_{n}\right) \rightarrow \zeta$ dans $\left(L^{p^{\prime}}\right)^{N}(\Omega)$ faible. On en déduit alors que $\zeta=a(\nabla u)$ par le lemme d'intégration (voir [15, Exercice 6.18] pour la démonstration) suivant :

Lemme 3.27 (Convergence par norme dominée) Soit $\Omega$ un ouvert borné. On suppose que $f_{n} \rightarrow f$ p.p. et que $\left(f_{n}\right)_{n \in \mathbb{N}}$ bornée dans $L^{q}, q>1$. Alors $f_{n} \rightarrow f$ dans $L^{r}(\Omega)$ pour tout $r$ t.q. $1 \leq r<q$.

Du lemme 3.27 on déduit que la suite $\left(a\left(\nabla u_{n}\right)\right)_{n \in \mathbb{N}}$ converge dans $L^{r}(\Omega)^{N}$, pour $1 \leq r<p^{\prime}$, vers $a(\nabla u)$. Comme cette même suite converge faiblement dans $\left(L^{p^{\prime}}\right)^{N}(\Omega)$ vers $\zeta$, on peut conclure (par exemple en utilisant l'unicité de la limite faible dans $L^{r}(\Omega)^{N}$ ) que les limites sont égales, c'est-à-dire

$$
\zeta=a(\nabla u) \text { p.p.. }
$$

Il reste à montrer que $u_{n} \rightarrow u$ dans $W_{0}^{1, p}(\Omega)$. On sait déjà que $u_{n} \rightarrow u$ faiblement dans $W_{0}^{1, p}(\Omega)$ et donc (fortement) dans $L^{p}(\Omega)$. Comme, après extraction d'une sous suite, on a $\nabla u_{n} \rightarrow \nabla u$ p.p., le lemme 3.27 nous donne $\nabla u_{n} \rightarrow \nabla u$ dans $L^{r}(\Omega)^{N}$ pour tout $1 \leq r<p$ et donc $u_{n} \rightarrow u$ dans $W_{0}^{1, r}(\Omega)$ pour tout $1 \leq r<p$. En raisonnant par contradiction on a même $u_{n} \rightarrow u$ dans $W_{0}^{1, r}(\Omega)$ pour tout $1 \leq r<p$ sans extraction de sous suite. Mais ceci ne donne pas la convergence dans $W_{0}^{1, p}(\Omega)$. Pour démontrer cette convergence dans $W_{0}^{1, p}(\Omega)$, on réutilise l'étape $(\mathrm{A})$ commune à Minty et Leray-Lions. Cette étape a donné

$$
\lim _{n \rightarrow+\infty} \int_{\Omega} \sigma a\left(\nabla u_{n}\right) \cdot \nabla u_{n} \mathrm{~d} x=\int_{\Omega} \sigma \zeta \cdot \nabla u \mathrm{~d} x .
$$


Mais on sait maintenant que $\zeta=a(\nabla u)$ p.p., on a donc

$$
\lim _{n \rightarrow+\infty} \int_{\Omega} \sigma a\left(\nabla u_{n}\right) \cdot \nabla u_{n} \mathrm{~d} x=\int_{\Omega} \sigma a(\nabla u) \cdot \nabla u \mathrm{~d} x
$$

On rappelle maintenant un lemme classique d'intégration (encore...), valable dans tout espace mesuré mais que l'on donne ici dans le cas qui nous intéresse.

\section{Lemme 3.28 (Convergence $L^{\mathbf{1}}$ pour une suite de fonctions positives)}

Soit $\Omega$ un ouvert borné de $\mathbb{R}^{N}$. Soit $f \in L^{1}(\Omega)$. On suppose que la suite $\left(f_{n}\right)_{n \in \mathbb{N}}$ de fonctions de $L^{1}(\Omega)$ vérifie:

1. $f_{n} \geq 0$ p.p., pour tout $n \in \mathbb{N}$,

2. $f_{n} \rightarrow f$ p.p. quand $n \rightarrow+\infty$,

3. $\lim _{n \rightarrow+\infty} \int_{\Omega} f_{n}(x) \mathrm{d} x=\int_{\Omega} f(x) \mathrm{d} x$.

Alors $f_{n} \rightarrow f$ dans $L^{1}(\Omega)$.

Après extraction éventuelle d'une sous suite, on peut supposer $\nabla u_{n} \rightarrow \nabla u$ p.p.. On applique alors le lemme 3.28 à la suite $\left(f_{n}\right)_{n \in \mathbb{N}}$ définie par $f_{n}=\sigma a\left(\nabla u_{n}\right) \cdot \nabla u_{n}$. Le lemme 3.28 donne la convergence $L^{1}(\Omega)$ de cette suite et donc l'équiintégrabilité de la suite $\left(f_{n}\right)_{n \in \mathbb{N}}$. Avec l'hypothèse de coercivité sur $a$ et l'hypothèse sur $\sigma$, on obtient l'équiintégrabilité de la suite $\left(\left|\nabla u_{n}\right|^{p}\right)_{n \in \mathbb{N}}$. Il reste à appliquer le théorème de Vitali (voir le poly d'intégration) pour conclure que $\nabla u_{n} \rightarrow \nabla u$ dans $L^{p}(\Omega)^{N}$ et donc que $u_{n} \rightarrow u$ dans $W_{0}^{1, p}(\Omega)$. Comme d'habitude, un argument par contradiction montre que cette convergence dans $W_{0}^{1, p}(\Omega)$ a lieu sans extraction sous suite dès que $u_{n}$ converge faiblement vers $u$ dans $W_{0}^{1, p}(\Omega)$ (et pour avoir cette convergence, on a dû extraire une sous suite). Dans l'étape 3 ci dessous, on va démontrer l'unicité de la solution de (3.20) si $a$ est strictement monotone. Ceci permet de conclure que la suite $\left(u_{n}\right)_{n \in \mathbb{N}}$ converge (sans extraction de sous suite) dans $W_{0}^{1, p}(\Omega)$ vers l'unique solution de 3.20).

On a ainsi terminé la partie "existence" du théorème 3.22

Etape 3 : Unicité On suppose que $a$ est strictement monotone. Soient $u_{1}$ et $u_{2}$ deux solutions.

$$
\int_{\Omega} \sigma a\left(\nabla u_{i}\right) \cdot \nabla v \mathrm{~d} x=<f, v>\quad i=1,2 \quad \forall v \in W_{0}^{1, p}(\Omega) .
$$

On fait la différence des deux équations, et on prend $v=u_{1}-u_{2}$. On obtient :

$$
\int_{\Omega} \sigma\left(a\left(\nabla u_{1}\right)-a\left(\nabla u_{2}\right)\right) \cdot \nabla\left(u_{1}-u_{2}\right) d x=0
$$

Or $Y=\sigma\left(a\left(\nabla u_{1}\right)-a\left(\nabla u_{2}\right)\right) \cdot \nabla\left(u_{1}-u_{2}\right) \geq 0$, et $Y>0$ si $\nabla u_{1} \neq \nabla u_{2}$; on a donc $\nabla u_{1}=\nabla u_{2}$ p.p. et donc $u_{1}=u_{2}$ car $u_{1}$ et $u_{2} \in W_{0}^{1, p}(\Omega)$.

Remarque 3.29 Dans le cas des opérateurs de Leray-Lions, lorsque $a$ dépend de $x, \nabla u$ mais aussi de $u$, on arrive encore à montrer des résulats d'unicité si $p \leq 2$. 


\subsection{Exercices}

Exercice 3.1 (Continuité d'une application de $L^{p}$ dans $L^{q}$ )

Soit $(E, T, m)$ un espace mesuré fini, $p, q \in[1, \infty[$ et $g$ une application continue de $\mathbb{R}$ dans $\mathbb{R}$ t.q. :

$$
\exists C \in \mathbb{R}_{+}^{\star} ;|g(s)| \leq C|s|^{\frac{p}{q}}+C, \forall s \in \mathbb{R} .
$$

1. Soit $u \in \mathcal{L}_{\mathbb{R}}^{p}(E, T, m)$. Montrer que $g \circ u \in \mathcal{L}_{\mathbb{R}}^{q}(E, T, m)$.

On pose $L^{r}=L_{\mathbb{R}}^{r}(E, T, m)$, pour $r=p$ et $r=q$. Pour $u \in L^{p}$, on pose $G(u)=\left\{h \in \mathcal{L}_{\mathbb{R}}^{q}(E, T, m) ; h=g \circ v\right.$ p.p.\}, avec $v \in u$ (de sorte que $G(u) \in L^{q}$ ).

2. Montrer que la définition précédente a bien un sens, c'est à dire que $G(u)$ ne dépend pas du choix de $v$ dans $u$.

3. Soit $\left(u_{n}\right)_{n \in \mathbb{N}} \subset L^{p}$. On suppose que $u_{n} \rightarrow u$ p.p., quand $n \rightarrow+\infty$, et qu'il existe $F \in L^{p}$ t.q. $\left|u_{n}\right| \leq F$ p.p., pour tout $n \in \mathbb{N}$. Montrer que $G\left(u_{n}\right) \rightarrow G(u)$ dans $L^{q}$.

4. Montrer que $G$ est continue de $L^{p}$ dans $L^{q}$.

5. On considère ici $(E, T, m)=([0,1], \mathcal{B}(\mathbb{R}), \lambda)$ et on prend $p=q=1$. On suppose que $g$ ne vérifie pas 3.26$)$. On va construire $u \in L^{1}$ t.q. $G(u) \notin L^{1}$.

(a) Soit $n \in \mathbb{N}^{\star}$, montrer qu'il existe $\alpha_{n} \in \mathbb{R}$ tel que : $\left|g\left(\alpha_{n}\right)\right| \geq n\left|\alpha_{n}\right|$ et $\left|\alpha_{n}\right| \geq n$.

(b) On choisit une suite $\left(\alpha_{n}\right)_{n \in \mathbb{N}^{\star}}$ vérifiant les conditions données à la question précédente. Montrer qu'il existe $\alpha>0$ t.q.

$$
\sum_{n=1}^{+\infty} \frac{\alpha}{\left|\alpha_{n}\right| n^{2}}=1
$$

(c) Soit $\left(a_{n}\right)_{n \in \mathbb{N}^{\star}}$ une suite définie par : $a_{1}=1$ et $a_{n+1}=a_{n}-\frac{\alpha}{\left|\alpha_{n}\right| n^{2}}$ (où $\alpha_{n}$ et $\alpha$ sont définies dans les 2 questions précédentes). On pose $u=\sum_{n=1}^{+\infty} \alpha_{n} 1_{\left[a_{n+1}, a_{n}[\right.}$. Montrer que $u \in L^{1}$ et $G(u) \notin L^{1}$.

Corrigé -

1. La fonction u est mesurable de $E$ (muni de la tribu $T$ ) dans $\mathbb{R}$ (muni de la tribu $\mathcal{B}(\mathbb{R})$ ) et g est borélienne ( c'est-àdire mesurable de $\mathbb{R}$ dans $\mathbb{R}$, muni de la tribu $\mathcal{B}(\mathbb{R})$ ). On en déduit, par composition, que $g \circ$ u est mesurable (de $E$ dans $\mathbb{R}$ ).

Pour $s \in[-1,1]$, on $a|g(s)| \leq 2 C$ et donc $|g(s)|^{q} \leq 2^{q} C^{q}$. Pour $s \in \mathbb{R} \backslash[-1,1]$, on a $|g(s)| \leq 2 C|s|^{\frac{p}{q}}$ et donc $|g(s)|^{q} \leq 2^{q} C^{q}|s|^{p}$. On a donc, pour tout $s \in \mathbb{R},|g(s)|^{q} \leq 2^{q} C^{q}+2^{q} C^{q}|s|^{p}$. On en déduit que, pour tout $x \in E$, $|g \circ u(x)|^{q}=|g(u(x))|^{q} \leq 2^{q} C^{q}+2^{q} C^{q}|u(x)|^{p}$, et donc :

$$
\int|g \circ u|^{q} d m \leq 2^{q} C^{q}\|u\|_{p}^{p}+2^{q} C^{q} m(E)
$$

ce qui donne $g \circ u \in \mathcal{L}_{\mathbb{R}}^{q}(E, T, m)$.

2. Soient $v, w \in u$. Il existe $A \in T$ t.q. $m(A)=0$ et $v=w$ sur $A^{c}$. On a donc aussi $g \circ v=g \circ w$ sur $A^{c}$ et donc $g \circ v=g \circ w$ p.p.. On en déduit que $\left\{h \in \mathcal{L}_{\mathbb{R}}^{q}(E, T, m) ; h=g \circ v\right.$ p.p. $\}=\left\{h \in \mathcal{L}_{\mathbb{R}}^{q}(E, T, m) ; h=g \circ w\right.$ p.p. $\}$.

$G(u)$ ne dépend donc pas du choix de $v$ dans $u$.

3. Pour tout $n \in \mathbb{N}$, on choisit un représentant de $u_{n}$, encore notée $u_{n}$. On choisit aussi des représentants de $u$ et $F$, notés toujours u et $F$. Comme $u_{n} \rightarrow$ up.p. quand $n \rightarrow+\infty$ et que g est continu, il est facile de voir que go $u_{n} \rightarrow g \circ u$ p.p.. On a donc $G\left(u_{n}\right) \rightarrow G(u)$ p.p..

On remarque aussi que $\left|g \circ u_{n}\right| \leq C\left|u_{n}\right|^{\frac{p}{q}}+C \leq C|F|^{\frac{p}{q}}+C$ p.p. et donc $\left|G\left(u_{n}\right)\right| \leq C|F|^{\frac{p}{q}}+C$ p.p., pour tout $n \in \mathbb{N}$. 
Comme $F \in L^{p}$, on $a|F|^{\frac{p}{q}} \in L^{q}$. Les fonctions constantes sont aussi dans $L^{q}$ (car $\left.m(E)<\infty\right)$. On a donc $C|F|^{\frac{p}{q}}+C \in L^{q}$. On peut alors appliquer le théorème de convergence dominée dans $L^{q}$, il donne que $G\left(u_{n}\right) \rightarrow G(u)$ dans $L^{q}$ quand $n \rightarrow+\infty$.

4. On raisonne par l'absurde. On suppose que $G$ n'est pas continue de $L^{p}$ dans $L^{q}$. Il existe donc $u \in L^{p}$ et $\left(u_{n}\right)_{n \in \mathbb{N}} \subset$ $L^{p}$ t.q. $u_{n} \rightarrow$ udans $L^{p}$ et $G\left(u_{n}\right) \nrightarrow \rightarrow G(u)$ dans $L^{q}$ quand $n \rightarrow+\infty$.

Comme $G\left(u_{n}\right) \nrightarrow \rightarrow G(u)$, il existe $\varepsilon>0$ et $\varphi: \mathbb{N} \rightarrow \mathbb{N}$ t.q. $\varphi(n) \rightarrow \infty$ quand $n \rightarrow \infty$ et :

$$
\left\|G\left(u_{\varphi(n)}\right)-G(u)\right\|_{q} \geq \varepsilon \text { pour tout } n \in \mathbb{N} .
$$

(La suite $\left(G\left(u_{\varphi(n)}\right)\right)_{n \in \mathbb{N}}$ est une sous suite de la suite $\left.\left(G\left(u_{n}\right)\right)_{n \in \mathbb{N} \text {. }}\right)$

Comme $u_{\varphi(n)} \rightarrow u$ dans $L^{p}$, on peut appliquer la "réciproque partielle de la convergence dominée dans $L^{p}$ ”. On obtient l'existence de $\psi: \mathbb{N} \rightarrow \mathbb{N}$ et de $F \in L^{p}$ t.q. $\psi(n) \rightarrow \infty$ quand $n \rightarrow \infty, u_{\varphi \circ \psi(n)} \rightarrow$ u p.p. et $\left|u_{\varphi \circ \psi(n)}\right| \leq F$ p.p., pour tout $n \in \mathbb{N}$. (La suite $\left(u_{\varphi \circ \psi(n)}\right)_{n \in \mathbb{N}}$ est une sous suite de la suite $\left.\left(u_{\varphi(n)}\right)_{n \in \mathbb{N}}\right)$.

On peut maintenant appliquer la question 2 à la suite $\left(u_{\varphi \circ \psi(n)}\right)_{n \in \mathbb{N}}$. Elle donne que $G\left(u_{\varphi \circ \psi(n)}\right) \rightarrow G(u)$ dans $L^{q}$ quand $n \rightarrow+\infty$. Ce qui est en contradiction avec 3.27.

5.(a) On raisonne par l'absurde. On suppose que $|g(s)|<n|s|$ pour tout s t.q. $|s| \geq n$. On pose $M=\max \{|g(s)|$, $s \in[-n, n]\}$. On a $M<\infty$ car g est continue sur le compact $[-n, n]$ (noter que $n$ est fixé). en posant $C=$ $\max \{n, M\}$, on a donc :

$$
|g(s)| \leq C|s|+C, \text { pour tout } s \in \mathbb{R},
$$

en contradiction avec l'hypothèse que g ne vérifie pas 3.26 .

Il existe donc s, t.q. $|s| \geq n$ et $|g(s)| \geq n|s|$. Ceci prouve l'existence de $\alpha_{n}$.

(b) Comme $\alpha_{n} \geq n$, on a $\frac{1}{\left|\alpha_{n}\right| n^{2}} \leq \frac{1}{n^{3}}$ et donc :

On choisit alors $\alpha=\frac{1}{\beta}$.

$$
0<\beta=\sum_{n \in \mathbb{N}^{\star}} \frac{1}{\left|\alpha_{n}\right| n^{2}}<\infty
$$

(c) Pour $n \geq 2$, on a $a_{n}=1-\sum_{p=1}^{n-1} \frac{\alpha}{\left|\alpha_{p}\right| p^{2}}$.

Grâce au choix de $\alpha$, on a donc $a_{n}>0$ pour tout $n \in \mathbb{N}^{\star}$, et $a_{n} \downarrow 0$, quand $n \rightarrow+\infty$.

La fonction u est bien mesurable et, par le théorème de convergence monotone, on obtient :

$$
\int|u| d \lambda=\sum_{n \in \mathbb{N}^{\star}}\left|\alpha_{n}\right|\left(a_{n}-a_{n+1}\right)=\sum_{n \in \mathbb{N}^{\star}} \frac{\alpha}{n^{2}}<\infty .
$$

Donc, $u \in \mathcal{L}^{1}$ et aussi $u \in L^{1}$ en confondant, comme d'habitude, u avec sa classe. on remarque ensuite que $g \circ u=\sum_{n=1}^{+\infty} g\left(\alpha_{n}\right) 1_{\left[a_{n+1}, a_{n}[\text {. On a donc : }\right.}$

$$
\int|g \circ u| d \lambda=\sum_{n \in \mathbb{N}^{\star}}\left|g\left(\alpha_{n}\right)\right|\left(a_{n}-a_{n+1}\right) \geq \sum_{n \in \mathbb{N}^{\star}} \frac{\alpha}{n}=\infty .
$$

ceci montre que $g \circ u \notin \mathcal{L}^{1}$ et donc $G(u) \notin L^{1}$.

\section{Exercice 3.2 (Existence par Schauder)}

Soit $\Omega$ un ouvert borné de $\mathbb{R}^{N}(N \geq 1), g \in L^{2}(\Omega), a$ une fonction continue de $\mathbb{R}$ dans $\mathbb{R}$ et $h$ une fonction continue de $\mathbb{R} \times \mathbb{R}^{N}$ dans $\mathbb{R}$. On suppose que

$-0<\alpha=\inf _{s \in \mathbb{R}} a(s) \leq \sup _{s \in \mathbb{R}} a(s)=\beta<+\infty$,

— il existe $\delta \in\left[0,1\left[\right.\right.$ et $C_{1} \in \mathbb{R}$ t.q. $|h(s, \xi)| \leq C_{1}\left(1+|s|^{\delta}+|\xi|^{\delta}\right)$ pour tout $(s, \xi) \in \mathbb{R} \times \mathbb{R}^{N}$. 
1. Soit $\bar{u} \in H_{0}^{1}(\Omega)$. Montrer que $h(\bar{u}, \nabla \bar{u}) \in L^{2}(\Omega)$ et qu'il existe un unique $u$ solution de

$$
\left\{\begin{array}{l}
u \in H_{0}^{1}(\Omega), \\
\int_{\Omega} a(\bar{u}(x)) \nabla u(x) \cdot \nabla v(x) \mathrm{d} x+\int_{\Omega} h(\bar{u}(x), \nabla \bar{u}(x)) v(x) \mathrm{d} x=\int_{\Omega} g(x) v(x) \mathrm{d} x, \forall v \in H_{0}^{1}(\Omega) .
\end{array}\right.
$$

Dans la suite, on note $T$ l'application qui à $\bar{u}$ associe $u$, unique solution de 3.28). L'application $T$ est donc de $H_{0}^{1}(\Omega)$ dans $H_{0}^{1}(\Omega)$.

2. (Estimation $\operatorname{sur} u$ ) Soit $\bar{u} \in H_{0}^{1}(\Omega)$ et $u=T(\bar{u})$. Montrer qu'il existe $C_{2}$ ne dépendant que $\Omega, \alpha, g$ et $C_{1}$ t.q.

$$
\|u\|_{H_{0}^{1}(\Omega)} \leq C_{2}\left(1+\|\bar{u}\|_{H_{0}^{1}(\Omega)}^{\delta}\right) .
$$

[On pourra prendre $v=u$ dans 3.28 .]

En déduire qu'il existe $R \in \mathbb{R}_{+}^{\star}$ t.q.

$$
\|\bar{u}\|_{H_{0}^{1}(\Omega)} \leq R \Rightarrow\|u\|_{H_{0}^{1}(\Omega)} \leq R
$$

3. (Continuité de $T$ ) Soit $\left(\bar{u}_{n}\right)_{n \in \mathbb{N}}$ une suite de $H_{0}^{1}(\Omega)$ et $\bar{u} \in H_{0}^{1}(\Omega)$. On suppose que $\bar{u}_{n} \rightarrow \bar{u}$ dans $H_{0}^{1}(\Omega)$ (quand $n \rightarrow+\infty)$. On pose $f_{n}=h\left(\bar{u}_{n}, \nabla \bar{u}_{n}\right), f=h(\bar{u}, \nabla \bar{u}), u_{n}=T\left(\bar{u}_{n}\right)$ et $u=T(\bar{u})$.

(a) Montrer que $f_{n} \rightarrow f$ dans $L^{2}(\Omega)$.

(b) Montrer que $u_{n} \rightarrow u$ faiblement dans $H_{0}^{1}(\Omega)$.

(c) Montrer que $\int_{\Omega} a\left(\bar{u}_{n}(x)\right) \nabla u_{n}(x) \cdot \nabla u_{n}(x) \mathrm{d} x \rightarrow \int_{\Omega} a(\bar{u}(x)) \nabla u(x) \cdot \nabla u(x) \mathrm{d} x$.

En déduire que $u_{n} \rightarrow u$ dans $H_{0}^{1}(\Omega)$. [On pourra s'inspirer de l'exercice 2.16]

4. (Compacité de $T$ ) Soit $\left(\bar{u}_{n}\right)_{n \in \mathbb{N}}$ une suite bornée de $H_{0}^{1}(\Omega)$. On pose $f_{n}=h\left(\bar{u}_{n}, \nabla \bar{u}_{n}\right)$ et $u_{n}=T\left(\bar{u}_{n}\right)$. Montrer qu'il existe une sous suite de la suite $\left(\bar{u}_{n}\right)_{n \in \mathbb{N}}$, encore notée $\left(\bar{u}_{n}\right)_{n \in \mathbb{N}}$, et il existe $f \in L^{2}(\Omega)$ et $b \in L^{\infty}(\Omega)$ t.q.

$$
\begin{aligned}
& f_{n} \rightarrow f \text { faiblement dans } L^{2}(\Omega), \\
& a\left(\bar{u}_{n}\right) \rightarrow b \text { p.p.. }
\end{aligned}
$$

Montrer que la suite $\left(u_{n}\right)_{n \in \mathbb{N}}$ est convergente dans $H_{0}^{1}(\Omega)$. [On pourra raisonner comme dans l'exercice 2.16] 5. Montrer qu'il existe $u \in H_{0}^{1}(\Omega)$ t.q. $u=T(u)$ (et donc $u$ solution de (3.28) avec $\bar{u}=u$ ).

Corrigé-

1. On remarque que $|h(\bar{u}, \nabla \bar{u})| \leq C_{1}\left(1+|\bar{u}|^{\delta}+|\nabla \bar{u}|^{\delta}\right) \in L^{\frac{2}{\delta}}(\Omega) \subset L^{2}(\Omega) \operatorname{car} \delta \leq 1$.

Pour $u, v \in H_{0}^{1}(\Omega)$, on pose

$$
A(u, v)=\int_{\Omega} a(\bar{u}) \nabla u \cdot \nabla v \mathrm{~d} x
$$

Comme $0<\alpha \leq a(\bar{u}) \leq \beta$, il est facile de montrer de A est bilinéaire continue et coercive sur $\left(H_{0}^{1}(\Omega)\right)^{2}$.

On conclut alors qu'il existe bien une unique solution à 3.28.

2. En prenant $v=u$ dans 3.28, on obtient

$$
\alpha\|u\|_{H_{0}^{1}(\Omega)}^{2} \leq\|g\|_{L^{2}(\Omega)}\|u\|_{L^{2}(\Omega)}+C_{1}|\Omega|^{1 / 2}\|u\|_{L^{2}(\Omega)}+\left(C_{1}\left\||\bar{u}|^{\delta}\right\|_{L^{2}(\Omega)}+C_{1}\left\||\nabla \bar{u}|^{\delta}\right\|_{L^{2}(\Omega)}\right)\|u\|_{L^{2}(\Omega)} .
$$

Avec $C_{\Omega}$ donnée par l'inégalité de Poincaré, on en déduit

$$
\frac{\alpha}{C_{\Omega}}\|u\|_{H_{0}^{1}(\Omega)} \leq\|g\|_{L^{2}(\Omega)}+C_{1}\left(|\Omega|^{1 / 2}+\left\||\bar{u}|^{\delta}\right\|_{L^{2}(\Omega)}+\left\||\nabla \bar{u}|^{\delta}\right\|_{L^{2}(\Omega)}\right) .
$$


Mais en utilisant l'inégalité de Hölder (ou l'inégalité de Jensen pour la fonction (concave) de $\mathbb{R}_{+}$dans $\mathbb{R}_{+}$définie par $s \mapsto s^{\delta}$ ) on obtient

et

$$
\left\||\bar{u}|^{\delta}\right\|_{L^{2}(\Omega)}^{2}=\int_{\Omega}|\bar{u}|^{2 \delta} d x \leq\left(\int_{\Omega} \bar{u}^{2}\right)^{\delta}|\Omega|^{1-\delta} .
$$

$$
\left\||\nabla \bar{u}|^{\delta}\right\|_{L^{2}(\Omega)}^{2}=\int_{\Omega}|\nabla \bar{u}|^{2 \delta} d x \leq\left(\int_{\Omega}|\nabla \bar{u}|^{2}\right)^{\delta}|\Omega|^{1-\delta} .
$$

(Si $\delta \in\left[1 / 2,1\left[\right.\right.$, ces deux inégalités correspondent à l'injection classique de $L^{2}(\Omega)$ dans $L^{2 \delta}(\Omega)$.)

De ces deux majorations (et avec l'inégalité de Poincaré), on déduit l'existence de $\bar{C}$ ne dépendant que de $\Omega$ et $\delta$ t.q.

$$
\left\||\bar{u}|^{\delta}\right\|_{L^{2}(\Omega)} \leq \bar{C}\|\bar{u}\|_{H_{0}^{1}(\Omega)}^{\delta} \text { et }\left\||\nabla \bar{u}|^{\delta}\right\|_{L^{2}(\Omega)} \leq \bar{C}\|\bar{u}\|_{H_{0}^{1}(\Omega)}^{\delta} .
$$

En revenant à 3.29 , on obtient l'existence de $C_{2}$ ne dépend que $\alpha, g$, $\Omega$ et $C_{1}$ t.q.

$$
\|u\|_{H_{0}^{1}(\Omega)} \leq C_{2}\left(1+\|\bar{u}\|_{H_{0}^{1}(\Omega)}^{\delta}\right) \text {. }
$$

Pour conclure, on remarque qu'il existe $R \in \mathbb{R}_{+}^{\star}$ t.q. $R>C_{2}\left(1+R^{\delta}\right)$. En effet, on a

$$
R-C_{2}-C_{2} R^{\delta}=R\left(1-\frac{C_{2}}{R}-C_{2} R^{\delta-1}\right) .
$$

il suffit donc de prendre $R>2 C_{2}$ et $R>\left(2 C_{2}\right)^{\frac{1}{1-\delta}}$. (c'est ici que l'hypothèse $\delta<1$ est utilisée.)

On a alors

$$
\|\bar{u}\|_{H_{0}^{1}(\Omega)} \leq R \Rightarrow\|u\|_{H_{0}^{1}(\Omega)} \leq C_{2}\left(1+R^{\delta}\right) \leq R
$$

3.(a) Si $f_{n} \nrightarrow \nrightarrow f$ dans $L^{2}(\Omega)$, il existe $\varepsilon>0$ et une sous suite, encore notée $\left(f_{n}\right)_{n \in \mathbb{N}}$ t.q.

$$
\left\|f_{n}-f\right\|_{L^{2}(\Omega)} \geq \varepsilon \text { pour tout } n \in \mathbb{N} \text {. }
$$

Après extraction éventuelle d'une sous suite, on peut supposer que

$$
\bar{u}_{n} \rightarrow \bar{u} \text { p.p., avec un domination par une fonction } G \text { appartenant à } L^{2}(\Omega) .
$$

et

$$
\nabla \bar{u}_{n} \rightarrow \nabla \bar{u} \text { p.p., avec un domination par une fonction } H \text { appartenant à } L^{2}(\Omega) \text {. }
$$

On a alors $f_{n} \rightarrow f$ p.p. et $\left|f_{n}\right| \leq C_{1}\left(1+|G|^{\delta}+|H|^{\delta}\right)$ p.p. (et pour tout $\left.n \in \mathbb{N}\right)$. Comme $0 \leq \delta \leq 1$, on a $|G|^{\delta}+|H|^{\delta} \in L^{2}(\Omega)$. Le théorème de convergence dominée (dans $L^{2}(\Omega)$ ) donne alors $f_{n} \rightarrow f$ dans $L^{2}(\Omega)$, en contradiction avec 3.30 .

(b) La question 2 donne que la suite $\left(u_{n}\right)_{n \in \mathbb{N}}$ est bornée dans $H_{0}^{1}(\Omega)$. Si $u_{n} \nrightarrow \nrightarrow$ u faiblement dans $H_{0}^{1}(\Omega)$, il existe $\varepsilon>0, \psi \in H^{-1}(\Omega)$ et une sous suite, encore noté $\left(u_{n}\right)_{n \in \mathbb{N}}$, t.q.

$$
\left|\left\langle\psi, u_{n}-u\right\rangle_{H^{-1}(\Omega), H_{0}^{1}(\Omega)}\right| \geq \varepsilon \text { pour tout } n \in \mathbb{N} .
$$

Puis, après extraction éventuelle d'une sous suite, on peut supposer

$$
\begin{array}{ll}
u_{n} \rightarrow w & \text { faiblement dans } H_{0}^{1}(\Omega), \\
\bar{u}_{n} \rightarrow \bar{u} & \text { p.p.. }
\end{array}
$$

Soit $v \in H_{0}^{1}(\Omega)$. Comme $u_{n}=T\left(\bar{u}_{n}\right)$, on a

$$
\int_{\Omega} a\left(\bar{u}_{n}\right) \nabla u_{n} \cdot \nabla v \mathrm{~d} x+\int_{\Omega} f_{n} v \mathrm{~d} x=\int_{\Omega} g v \mathrm{~d} x .
$$

Comme $a\left(\bar{u}_{n}\right) \rightarrow a(\bar{u})$ p.p. et $\left|a\left(\bar{u}_{n}\right)\right| \leq \beta$ p.p., on a, par convergence dominée, $a\left(\bar{u}_{n}\right) \nabla v \rightarrow a(\bar{u}) \nabla v$ dans $L^{2}(\Omega)^{N}$. En passant à la limite quand $n \rightarrow+\infty$ dans l'égalité précédente, on obtient donc

$$
\int_{\Omega} a(\bar{u}) \nabla w \cdot \nabla v \mathrm{~d} x+\int_{\Omega} f v \mathrm{~d} x=\int_{\Omega} g v \mathrm{~d} x .
$$

Ce qui prouve que $w=u$, en contradiction avec 3.31. On a bien ainsi montré que $u_{n} \rightarrow u$ faiblement dans $H_{0}^{1}(\Omega)$ quand $n \rightarrow+\infty$. 
(c) Pour tout $n \in \mathbb{N}$ on a

$$
\int_{\Omega} a\left(\bar{u}_{n}\right) \nabla u_{n} \cdot \nabla u_{n} \mathrm{~d} x=\int_{\Omega} g u_{n} \mathrm{~d} x-\int_{\Omega} f_{n} u_{n} \mathrm{~d} x .
$$

Comme $u_{n} \rightarrow$ u dans $L^{2}(\Omega)$ et que $f_{n} \rightarrow f$ dans $L^{2}(\Omega)$, on en déduit

$$
\lim _{n \rightarrow+\infty} \int_{\Omega} a\left(\bar{u}_{n}\right) \nabla u_{n} \cdot \nabla u_{n} \mathrm{~d} x=\int_{\Omega} g u \mathrm{~d} x-\int_{\Omega} f u \mathrm{~d} x .
$$

et donc, comme $u=T(\bar{u})$,

$$
\lim _{n \rightarrow+\infty} \int_{\Omega} a\left(\bar{u}_{n}\right) \nabla u_{n} \cdot \nabla u_{n} \mathrm{~d} x=\int_{\Omega} a(\bar{u}) \nabla u \cdot \nabla u \mathrm{~d} x .
$$

On remarque maintenant que

$$
\alpha\left\|u_{n}-u\right\|_{H_{0}^{1}(\Omega)}^{2} \leq \int_{\Omega} a\left(\bar{u}_{n}\right)\left(\nabla u_{n}-\nabla u\right) \cdot\left(\nabla u_{n}-\nabla u\right) \mathrm{d} x .
$$

Pour montrer que $u_{n} \rightarrow u$ dans $H_{0}^{1}(\Omega)$, il suffit donc de montrer que

$$
\lim _{n \rightarrow+\infty} \int_{\Omega} a\left(\bar{u}_{n}\right)\left(\nabla u_{n}-\nabla u\right) \cdot\left(\nabla u_{n}-\nabla u\right) \mathrm{d} x=0 .
$$

Pour montrer 3.32, on utilise le fait (facile à montrer en raisonnant par l'absurde) que

$$
a\left(\bar{u}_{n}\right) \nabla u \rightarrow a(\bar{u}) \nabla u \text { dans } L^{2}(\Omega)^{N}, \text { quand } n \rightarrow+\infty .
$$

On en déduit que (en utilisant le fait que $\nabla u_{n} \rightarrow \nabla u$ faiblement dans $L^{2}(\Omega)^{N}$ )

$$
\begin{aligned}
& \lim _{n \rightarrow+\infty} \int_{\Omega} a\left(\bar{u}_{n}\right) \nabla u \cdot \nabla u_{n} \mathrm{~d} x=\int_{\Omega} a(\bar{u}) \nabla u \cdot \nabla u \mathrm{~d} x \\
& \text { et } \lim _{n \rightarrow+\infty} \int_{\Omega} a\left(\bar{u}_{n}\right) \nabla u \cdot \nabla u \mathrm{~d} x=\int_{\Omega} a(\bar{u}) \nabla u \cdot \nabla u \mathrm{~d} x .
\end{aligned}
$$

Ceci permet de montrer 3.32 et donc de conclure que $u_{n} \rightarrow u$ dans $H_{0}^{1}(\Omega)$. Ce qui prouve la continuité de l'opérateur $T$ de $H_{0}^{1}(\Omega)$ dans $H_{0}^{1}(\Omega)$.

4. La suite $\left(f_{n}\right)_{n \in \mathbb{N}}$ est bornée dans $L^{2}(\Omega)$ et la suite $\left(\bar{u}_{n}\right)_{n \in \mathbb{N}}$ est bornée dans $H_{0}^{1}(\Omega)$ et donc relativement compacte dans $L^{2}(\Omega)$. On peut donc supposer, après extraction d'une sous suite, qu'il existe $f \in L^{2}(\Omega)$ et $\zeta \in L^{2}(\Omega)$ t.q.

$$
\begin{aligned}
& f_{n} \rightarrow \text { f faiblement dans } L^{2}(\Omega), \\
& \bar{u}_{n} \rightarrow \zeta \text { p.p. }
\end{aligned}
$$

En posant $b=a(\zeta)$, on a donc $b \in L^{\infty}(\Omega)$ et $a\left(\bar{u}_{n}\right) \rightarrow$ b p.p..

(On peut montrer que $\zeta \in H_{0}^{1}(\Omega)$ mais il est faux de dire que $f=h(\zeta, \nabla \zeta)$ p.p..)

Comme $\alpha \leq b \leq \beta$ p.p., il existe un et un seul u solution de

$$
\left\{\begin{array}{l}
u \in H_{0}^{1}(\Omega), \\
\int_{\Omega} b \nabla u \cdot \nabla v \mathrm{~d} x=\int_{\Omega}(g-f) v \mathrm{~d} x \text { pour tout } v \in H_{0}^{1}(\Omega) .
\end{array}\right.
$$

On va montrer que la suite $\left(u_{n}\right)_{n \in \mathbb{N}}$ converge dans $H_{0}^{1}(\Omega)$ vers $u$, solution de 3.34 (on travaille ici avec la suite extraite qui vérifie 3.33).

On sait déjà que la suite $\left(u_{n}\right)_{n \in \mathbb{N}}$ est bornée dans $H_{0}^{1}(\Omega)$. En raisonnant par l'absurde, il est alors assez facile de montrer que $u_{n} \rightarrow$ faiblement dans $H_{0}^{1}(\Omega)$. En effet, supposons que (après extraction de sous suite) $u_{n} \rightarrow w$ faiblement dans $H_{0}^{1}(\Omega)$. On a alors, pour tout $n \in \mathbb{N}$ et tout $v \in H_{0}^{1}(\Omega)$,

$$
\int_{\Omega} a\left(\bar{u}_{n}\right) \nabla u_{n} \cdot \nabla v \mathrm{~d} x=\int_{\Omega}\left(g-f_{n}\right) v \mathrm{~d} x
$$

En passant à la limite quand $n \rightarrow+\infty$ dans cette équation, grâce aux convergences données dans 3.33, on obtient

$$
\int_{\Omega} b \nabla w \cdot \nabla v \mathrm{~d} x=\int_{\Omega}(g-f) v \mathrm{~d} x
$$


Ceci prouve que $w=u$. On en déduit bien que $u_{n} \rightarrow u$ faiblement dans $H_{0}^{1}(\Omega)$ quand $n \rightarrow+\infty$. On a donc aussi $u_{n} \rightarrow u$ (fortement) dans $L^{2}(\Omega)$.

Il reste à montrer la convergence (forte) de $u_{n}$ vers $u$ dans $H_{0}^{1}(\Omega)$. Pour cela on remarque tout d'abord que

$$
\int_{\Omega} a\left(\bar{u}_{n}\right) \nabla u_{n} \cdot \nabla u_{n} \mathrm{~d} x=\int_{\Omega}\left(g-f_{n}\right) u_{n} \mathrm{~d} x \rightarrow \int_{\Omega}(g-f) u \mathrm{~d} x \text { quand } n \rightarrow+\infty .
$$

Comme u est solution de 3.34 on a donc

$$
\lim _{n \rightarrow+\infty} \int_{\Omega} a\left(\bar{u}_{n}\right) \nabla u_{n} \cdot \nabla u_{n} \mathrm{~d} x=\int_{\Omega} b \nabla u \cdot \nabla u \mathrm{~d} x .
$$

En utilisant cette convergence et 3.33 on montre alors que

$$
\lim _{n \rightarrow+\infty} \int_{\Omega} a\left(\bar{u}_{n}\right) \nabla\left(u_{n}-u\right) \cdot \nabla\left(u_{n}-u\right) \mathrm{d} x=0 .
$$

Comme $\alpha\left\|u_{n}-u\right\|_{H_{0}^{1}(\Omega)}^{2} \leq \int_{\Omega} a\left(\bar{u}_{n}\right) \nabla\left(u_{n}-u\right) \cdot \nabla\left(u_{n}-u\right) \mathrm{d} x$ on conclut bien que $u_{n} \rightarrow u$ dans $H_{0}^{1}(\Omega)$.

5. Il suffit ici d'appliquer le théorème de Schauder. L'opérateur T est continu et compact de $H_{0}^{1}(\Omega)$ dans $H_{0}^{1}(\Omega)$. Il existe $R>0$ t.q. T envoie la boule de centre 0 et de rayon $R\left(\right.$ de $\left.H_{0}^{1}(\Omega)\right)$ dans elle-même. Le théorème de Schauder permet alors de dire qu'il existe $u$ dans cette boule (et donc dans $H_{0}^{1}(\Omega)$ ) t.q. $u=T(u)$. La fonction u ainsi trouvée est solution de 3.28 avec $\bar{u}=u$.

\section{Exercice 3.3 (Existence par Schauder, généralisation de l'exercice 3.2)}

Soit $\Omega$ un ouvert borné de $\mathbb{R}^{N}(N \geq 1)$, a une fonction de $\Omega \times \mathbb{R}$ dans $\mathbb{R}$ et $f$ une fonction de $\Omega \times \mathbb{R} \times \mathbb{R}^{N}$ dans $\mathbb{R}$ vérifiant :

- $a$ est mesurable par rapport à $x \in \Omega$, pour tout $s \in \mathbb{R}$, et continue par rapport à $s \in \mathbb{R}$, p.p. en $x \in \Omega$.

- Il existe $\alpha, \beta \in \mathbb{R}_{+}^{\star}$ t.q. $\alpha \leq a(x, s) \leq \beta$ pour tout $s \in \mathbb{R}$ et p.p. en $x \in \Omega$.

- $f$ est mesurable par rapport à $x \in \Omega$, pour tout $s, p \in \mathbb{R} \times \mathbb{R}^{N}$, et continue par rapport à $(s, p) \in \mathbb{R} \times \mathbb{R}^{N}$, p.p. en $x \in \Omega$.

- Il existe $d \in L^{2}(\Omega), C \in \mathbb{R}$ et $\delta \in\left[0,1\left[\right.\right.$ t.q. $|f(\cdot, s, p)| \leq C\left(d+|s|^{\delta}+|p|^{\delta}\right)$ p.p., pour tout $s, p \in \mathbb{R} \times \mathbb{R}^{N}$. Montrer qu'il existe un et un seul $u$ solution du problème suivant :

$$
\left\{\begin{array}{l}
u \in H_{0}^{1}(\Omega), \\
\int_{\Omega} a(x, u(x)) \nabla u(x) \cdot \nabla v(x) \mathrm{d} x=\int_{\Omega} f(x, u(x), \nabla u(x)) v(x) \mathrm{d} x, \text { pour tout } v \in H_{0}^{1}(\Omega) .
\end{array}\right.
$$

[On pourra construire une application de $H_{0}^{1}(\Omega)$ dans $H_{0}^{1}(\Omega)$ et utiliser le théorème de Schauder.]

Corrigé-

On suit la même démarche que pour l'exercice 3.2 (les modifications sont mineures). On construit un opérateur, noté T, de $H_{0}^{1}(\Omega)$ dans $H_{0}^{1}(\Omega)$. On montre que, si R est bien choisi, T envoie la boule de centre 0 et de rayon $R$ dans elle même. Enfin, on montre la continuité et la compacité de $T$ et on conclut alors par le théorème de Schauder.

Construction de $T$

Soit $\bar{u} \in H_{0}^{1}(\Omega)$. En choisissant un représentant de la classe de fonctions $\bar{u}$, la fonction $x \mapsto a(x, \bar{u}(x))$ est borélienne et ne change que sur un ensemble de mesure nulle si on change le représentant de $\bar{u}$, ceci est dû au fait que a est une fonction de Carathéodory (ce qui est la première hypothèse sur a). Avec la seconde hypothèse sur a, on a donc a $(\cdot, \bar{u}) \in L^{\infty}(\Omega)$ et $\alpha \leq a(\cdot, \bar{u}) \leq \beta$ p.p..

De même, en choisissant des représentants de $\bar{u}$ et $\nabla \bar{u}$, la fonction $x \mapsto f(x, \bar{u}(x), \nabla \bar{u})$ est borélienne et ne change que sur un ensemble de mesure nulle si on change les représentants de $\bar{u}$ et $\nabla \bar{u}$, ceci est aussi dû au fait que $f$ est une fonction de Carathéodory (ce qui est la première hypothèse sur $f$ ). Avec la seconde hypothèse sur $f$, on a

$$
|f(\cdot, \bar{u}, \nabla \bar{u})| \leq C\left(d+|\bar{u}|^{\delta}+|\nabla \bar{u}|^{\delta}\right) p \cdot p .
$$


et donc $f(\cdot, \bar{u}, \nabla \bar{u}) \in L^{2}(\Omega)$ car $d, \bar{u},|\nabla \bar{u}| \in L^{2}(\Omega)$ et $\delta \leq 1$.

On peut maintenant appliquer le théorème 2.6 il donne l'existence et l'unicité de u solution de

$$
\left\{\begin{array}{l}
u \in H_{0}^{1}(\Omega), \\
\int_{\Omega} a(x, \bar{u}(x)) \nabla u(x) \cdot \nabla v(x) \mathrm{d} x=\int_{\Omega} f(x, \bar{u}(x), \nabla \bar{u}(x)) v(x) \mathrm{d} x, \text { pour tout } v \in H_{0}^{1}(\Omega) .
\end{array}\right.
$$

On pose $u=T(\bar{u})$. On a ainsi construit une application $T$ de $H_{0}^{1}(\Omega)$ dans $H_{0}^{1}(\Omega)$. Pour conclure, il suffit maintenant de montrer que $T$ admet un point fixe.

\section{Estimations sur $T$}

Soit $\bar{u} \in H_{0}^{1}(\Omega)$ et $u=T(\bar{u})$. En prenant $v=u$ dans 3.36 , on obtient

$$
\alpha\|u\|_{H_{0}^{1}(\Omega)}^{2} \leq C\left(\|d\|_{L^{2}(\Omega)}+\left\||\bar{u}|^{\delta}\right\|_{L^{2}(\Omega)}+C_{1}\left\||\nabla \bar{u}|^{\delta}\right\|_{L^{2}(\Omega)}\right)\|u\|_{L^{2}(\Omega)}
$$

Avec $C_{\Omega}$ donnée par l'inégalité de Poincaré, on en déduit

$$
\frac{\alpha}{C_{\Omega}}\|u\|_{H_{0}^{1}(\Omega)} \leq C\left(\|d\|_{L^{2}(\Omega)}+\left\||\bar{u}|^{\delta}\right\|_{L^{2}(\Omega)}+\left\||\nabla \bar{u}|^{\delta}\right\|_{L^{2}(\Omega)}\right) .
$$

Mais comme dans l'exercice 3.2 on a (en utilisant l'inégalité de Hölder)

$$
\left\||\bar{u}|^{\delta}\right\|_{L^{2}(\Omega)}^{2} \leq\left(\int_{\Omega} \bar{u}^{2}\right)^{\delta}|\Omega|^{1-\delta} \text { et }\left\||\nabla \bar{u}|^{\delta}\right\|_{L^{2}(\Omega)}^{2} \leq\left(\int_{\Omega}|\nabla \bar{u}|^{2}\right)^{\delta}|\Omega|^{1-\delta} .
$$

On en déduit (avec l'inégalité de Poincaré) l'existence de $\bar{C}$ ne dépendant que de $\Omega$ et $\delta$ t.q.

$$
\left\||\bar{u}|^{\delta}\right\|_{L^{2}(\Omega)} \leq \bar{C}\|\bar{u}\|_{H_{0}^{1}(\Omega)}^{\delta} \text { et }\left\||\nabla \bar{u}|^{\delta}\right\|_{L^{2}(\Omega)} \leq \bar{C}\|\bar{u}\|_{H_{0}^{1}(\Omega)}^{\delta} .
$$

Avec 3.29 , on obtient l'existence de $C_{2}$ ne dépend que $\alpha$, $\Omega$ et $C$ t.q.

$$
\|u\|_{H_{0}^{1}(\Omega)} \leq C_{2}\left(1+\|\bar{u}\|_{H_{0}^{1}(\Omega)}^{\delta}\right) .
$$

Pour conclure cette étape, on remarque qu'il existe $R \in \mathbb{R}_{+}^{\star}$ t.q. $R>C_{2}\left(1+R^{\delta}\right)($ car $\delta<1)$ et donc

$$
\|\bar{u}\|_{H_{0}^{1}(\Omega)} \leq R \Rightarrow\|u\|_{H_{0}^{1}(\Omega)} \leq C_{2}\left(1+R^{\delta}\right) \leq R .
$$

Ce qui montre que $T$ envoie $B_{R}$ dans $B_{R}$ où $B_{R}$ est la boule (fermée) de centre 0 et de rayon $R$.

\section{Continuité de $T$}

Soit $\left(\bar{u}_{n}\right)_{n \in \mathbb{N}}$ une suite de $H_{0}^{1}(\Omega)$ et $\bar{u} \in H_{0}^{1}(\Omega)$. On suppose que $\bar{u}_{n} \rightarrow \bar{u}$ dans $H_{0}^{1}(\Omega)$ (quand $\left.n \rightarrow+\infty\right)$. On pose $u_{n}=T\left(\bar{u}_{n}\right)$ et $u=T(\bar{u})$. On veut montrer que $u_{n} \rightarrow u$ dans $H_{0}^{1}(\Omega)$ quand $n \rightarrow+\infty$. On pose aussi $g_{n}=$ $f\left(\cdot, \bar{u}_{n}, \nabla \bar{u}_{n}\right), g=f(\cdot, \bar{u}, \nabla \bar{u}), A_{n}=a\left(\cdot, \bar{u}_{n}\right)$ et $A=a(\cdot, \bar{u})$.

On commence par remarquer que $g_{n} \rightarrow g$ dans $L^{2}(\Omega)$ (quand $n \rightarrow+\infty$ ). La démonstration est ici identique à celle de $f_{n} \rightarrow f$ dans l'exercice 3.2 la seule modification est dans la domination de $g_{n}$ qui ici est $\left|g_{n}\right| \leq C\left(d+|G|^{\delta}+|H|^{\delta}\right)$ p.p. (au lieu de $\left|f_{n}\right| \leq C_{1}\left(1+|G|^{\delta}+|H|^{\delta}\right)$ p.p. dans l'exercice 3.2.

On montre maintenant que $u_{n} \rightarrow u$ faiblement dans $H_{0}^{1}(\Omega)$. On suit encore le même raisonnement que dans l'exercice 3.2 L'inégalité 3.38 donne que la suite $\left(u_{n}\right)_{n \in \mathbb{N}}$ est bornée dans $H_{0}^{1}(\Omega)$. Si $u_{n} \not \rightarrow$ u faiblement dans $H_{0}^{1}(\Omega)$, il existe $\varepsilon>0, \psi \in H^{-1}(\Omega)$ et une sous suite, encore noté $\left(u_{n}\right)_{n \in \mathbb{N}}$, t.q.

$$
\left|\left\langle\psi, u_{n}-u\right\rangle_{H^{-1}(\Omega), H_{0}^{1}(\Omega)}\right| \geq \varepsilon \text { pour tout } n \in \mathbb{N} .
$$

Puis, après extraction éventuelle d'une sous suite, on peut supposer

$$
\begin{array}{ll}
u_{n} \rightarrow w & \text { faiblement dans } H_{0}^{1}(\Omega), \\
\bar{u}_{n} \rightarrow \bar{u} & \text { p.p.. }
\end{array}
$$

Soit $v \in H_{0}^{1}(\Omega)$. Comme $u_{n}=T\left(\bar{u}_{n}\right)$, on a

$$
\int_{\Omega} A_{n} \nabla u_{n} \cdot \nabla v \mathrm{~d} x=\int_{\Omega} g_{n} v \mathrm{~d} x
$$

Comme $A_{n} \rightarrow$ A p.p. (puisque a est p.p. continue par rapport à son deuxième argument) et $\left|A_{n}\right| \leq \beta$ p.p., on a, par convergence dominée, $A_{n} \nabla v \rightarrow A \nabla v$ dans $L^{2}(\Omega)^{N}$. En passant à la limite quand $n \rightarrow+\infty$ dans l'égalité précédente, on obtient donc

$$
\int_{\Omega} A \nabla w \cdot \nabla v \mathrm{~d} x=\int_{\Omega} g v \mathrm{~d} x
$$


Ce qui prouve que $w=u$ (grâce à l'unicité de la solution de 3.36 ) et donc $u_{n} \rightarrow$ u faiblement dans $H_{0}^{1}(\Omega)$, en contradiction avec 3.39. On a bien ainsi montré que $u_{n} \rightarrow u$ faiblement dans $H_{0}^{1}(\Omega)$ quand $n \rightarrow+\infty$ (sans extraction de sous-suite).

on veut montrer maintenant que $u_{n} \rightarrow u$ dans $H_{0}^{1}(\Omega)$ (et pas seulement faiblement). En prenant $v=u_{n}$ dans 3.40 , on a

$$
\int_{\Omega} A_{n} \nabla u_{n} \cdot \nabla u_{n} \mathrm{~d} x=\int_{\Omega} g_{n} u_{n} \mathrm{~d} x .
$$

Comme $u_{n} \rightarrow u$ dans $L^{2}(\Omega)$ et que $g_{n} \rightarrow g$ dans $L^{2}(\Omega)$, on en déduit

et donc, comme $u=T(\bar{u})$, 3.36 donne

$$
\lim _{n \rightarrow+\infty} \int_{\Omega} A_{n} \nabla u_{n} \cdot \nabla u_{n} \mathrm{~d} x=\int_{\Omega} g u \mathrm{~d} x .
$$

$$
\lim _{n \rightarrow+\infty} \int_{\Omega} A_{n} \nabla u_{n} \cdot \nabla u_{n} \mathrm{~d} x=\int_{\Omega} A \nabla u \cdot \nabla u \mathrm{~d} x .
$$

On remarque maintenant que

$$
\alpha\left\|u_{n}-u\right\|_{H_{0}^{1}(\Omega)}^{2} \leq \int_{\Omega} A_{n}\left(\nabla u_{n}-\nabla u\right) \cdot\left(\nabla u_{n}-\nabla u\right) \mathrm{d} x .
$$

Comme dans l'exercice 3.2 on utilise le fait que

$$
A_{n} \nabla u \rightarrow A \nabla u \text { dans } L^{2}(\Omega)^{N} \text {, quand } n \rightarrow+\infty
$$

et que $\nabla u_{n} \rightarrow \nabla$ ufaiblement dans $L^{2}(\Omega)$. Ceci donne

et donc, avec 3.41 ,

$$
\begin{aligned}
& \lim _{n \rightarrow+\infty} \int_{\Omega} A_{n} \nabla u \cdot \nabla u_{n} \mathrm{~d} x=\lim _{n \rightarrow+\infty} \int_{\Omega} A \nabla u \cdot \nabla u \mathrm{~d} x \\
& \text { et } \lim _{n \rightarrow+\infty} \int_{\Omega} A_{n} \nabla u \cdot \nabla u \mathrm{~d} x=\lim _{n \rightarrow+\infty} \int_{\Omega} A \nabla u \cdot \nabla u \mathrm{~d} x,
\end{aligned}
$$

$$
\lim _{n \rightarrow+\infty} \int_{\Omega} A_{n}\left(\nabla u_{n}-\nabla u\right) \cdot\left(\nabla u_{n}-\nabla u\right) \mathrm{d} x=0 .
$$

On conclut bien, avec 3.42 , que $u_{n} \rightarrow u$ dans $H_{0}^{1}(\Omega)$. Ce qui prouve la continuité de l'opérateur $T$ de $H_{0}^{1}(\Omega)$ dans $H_{0}^{1}(\Omega)$.

\section{Compacité de $T$}

On suit toujours le raisonnement fait pour l'exercice 3.2 Soit $\left(\bar{u}_{n}\right)_{n \in \mathbb{N}}$ une suite bornée de $H_{0}^{1}(\Omega)$. On pose $g_{n}=$ $f\left(\cdot, \bar{u}_{n}, \nabla \bar{u}_{n}\right)$ et $u_{n}=T\left(\bar{u}_{n}\right)$.

La suite $\left(g_{n}\right)_{n \in \mathbb{N}}$ est bornée dans $L^{2}(\Omega)$ et la suite $\left(\bar{u}_{n}\right)_{n \in \mathbb{N}}$ est bornée dans $H_{0}^{1}(\Omega)$ et donc relativement compacte dans $L^{2}(\Omega)$. On peut donc supposer, après extraction d'une sous suite, qu'il existe $g \in L^{2}(\Omega)$ et $\zeta \in L^{2}(\Omega)$ t.q.

$$
\begin{aligned}
& g_{n} \rightarrow \text { g faiblement dans } L^{2}(\Omega), \\
& \bar{u}_{n} \rightarrow \zeta \text { p.p. }
\end{aligned}
$$

En posant $b=a(\cdot, \zeta)$, on a donc $b \in L^{\infty}(\Omega)$ et $a\left(\cdot, \bar{u}_{n}\right) \rightarrow$ b p.p..

Comme $\alpha \leq b \leq \beta$ p.p., il existe un et un seul u solution de

$$
\left\{\begin{array}{l}
u \in H_{0}^{1}(\Omega) \\
\int_{\Omega} b \nabla u \cdot \nabla v \mathrm{~d} x=\int_{\Omega} g v \mathrm{~d} x \text { pour tout } v \in H_{0}^{1}(\Omega) .
\end{array}\right.
$$

On va montrer que la suite $\left(u_{n}\right)_{n \in \mathbb{N}}$ converge dans $H_{0}^{1}(\Omega)$ vers $u$, solution de 3.44 (on travaille ici avec la suite extraite qui vérifie 3.43).

On sait déjà que la suite $\left(u_{n}\right)_{n \in \mathbb{N}}$ est bornée dans $H_{0}^{1}(\Omega)$. En raisonnant par l'absurde, il est alors assez facile de montrer que $u_{n} \rightarrow u$ faiblement dans $H_{0}^{1}(\Omega)$. En effet, supposons que (après extraction de sous suite) $u_{n} \rightarrow w$ faiblement dans $H_{0}^{1}(\Omega)$. On a alors, pour tout $n \in \mathbb{N}$ et tout $v \in H_{0}^{1}(\Omega)$,

$$
\int_{\Omega} a\left(x, \bar{u}_{n}\right) \nabla u_{n} \cdot \nabla v \mathrm{~d} x=\int_{\Omega} g_{n} v \mathrm{~d} x .
$$


En passant à la limite quand $n \rightarrow+\infty$ dans cette équation, grâce aux convergences données dans 3.43 , on obtient

$$
\int_{\Omega} b \nabla w \cdot \nabla v \mathrm{~d} x=\int_{\Omega} g v \mathrm{~d} x .
$$

Ceci prouve que $w=u$. On en déduit bien que $u_{n} \rightarrow u$ faiblement dans $H_{0}^{1}(\Omega)$ quand $n \rightarrow+\infty$. On a donc aussi $u_{n} \rightarrow u$ (fortement) dans $L^{2}(\Omega)$.

Il reste à montrer la convergence (forte) de $u_{n}$ vers u dans $H_{0}^{1}(\Omega)$. Pour cela on remarque tout d'abord que

$$
\int_{\Omega} a\left(x, \bar{u}_{n}\right) \nabla u_{n} \cdot \nabla u_{n} \mathrm{~d} x=\int_{\Omega} g_{n} u_{n} \mathrm{~d} x \rightarrow \int_{\Omega} g u \mathrm{~d} x \text { quand } n \rightarrow+\infty .
$$

Comme $u$ est solution de 3.44 on a donc

$$
\lim _{n \rightarrow+\infty} \int_{\Omega} a\left(x, \bar{u}_{n}\right) \nabla u_{n} \cdot \nabla u_{n} \mathrm{~d} x=\int_{\Omega} b \nabla u \cdot \nabla u \mathrm{~d} x .
$$

En utilisant cette convergence et 3.43 on montre alors que

$$
\lim _{n \rightarrow+\infty} \int_{\Omega} a\left(x, \bar{u}_{n}\right) \nabla\left(u_{n}-u\right) \cdot \nabla\left(u_{n}-u\right) \mathrm{d} x=0 .
$$

Comme $\alpha\left\|u_{n}-u\right\|_{H_{0}^{1}(\Omega)}^{2} \leq \int_{\Omega} a\left(x, \bar{u}_{n}\right) \nabla\left(u_{n}-u\right) \cdot \nabla\left(u_{n}-u\right) \mathrm{d} x$ on conclut bien que $u_{n} \rightarrow u$ dans $H_{0}^{1}(\Omega)$. (On notera que la convergence est obtenue seulement pour la suite extraite qui vérifie 3.43.)

On a bien montré la compacité de T.

\section{Conclusion}

Il suffit ici d'appliquer le théorème de Schauder. L'opérateur T est continu et compact de $H_{0}^{1}(\Omega)$ dans $H_{0}^{1}(\Omega)$. Il existe $R>0$ t.q. T envoie la boule de centre 0 et de rayon $R\left(\right.$ de $\left.H_{0}^{1}(\Omega)\right)$ dans elle-même. Le théorème de Schauder permet alors de dire qu'il existe $u$ dans cette boule (et donc dans $H_{0}^{1}(\Omega)$ ) t.q. $u=T(u)$. La fonction u ainsi trouvée est solution de 3.35 .

\section{Exercice 3.4 (Degré d'une application affine)}

Soit $E$ un espace de Banach (réel). Pour $R>0$, on pose $B_{R}=\left\{v \in E\right.$ t.q. $\left.\|v\|_{E}<R\right\}$.

1. Soit $f$ une application constante de $E$ dans $E$. Il existe donc $a \in E$ t.q. $f(v)=a$ pour tout $v \in E$. Soit $R>0$ t.q. $\|a\|_{E} \neq R$. Montrer que $d\left(\operatorname{Id}-f, B_{R}, 0\right)$ est bien défini et que $d\left(\operatorname{Id}-f, B_{R}, a\right)=1$ si $R>\|a\|_{E}$ et $d\left(\mathrm{Id}-f, B_{R}, a\right)=0$ si $R<\|a\|_{E}$.

2. Soit $L$ une application linéaire compact de $E$ dans $E$. On suppose que 1 n'est pas valeur propre de $L$. Soit $a \in E$. On définit $f$ de $E$ dans $E$ en posant $f(v)=L v+a$ pour tout $v \in E$.

(a) Montrer que l'équation $u-f(u)=0$ a au plus une solution.

(b) Montrer que l'équation $u-f(u)=0$ a une unique solution. On note $b$ cette solution. Montrer que $d(\operatorname{Id}-$ $\left.f, B_{R}, 0\right) \neq 0$ si $R>\|b\|_{E}$ et $d\left(\operatorname{Id}-f, B_{R}, 0\right)=0$ si $R<\|b\|_{E}$.

Corrigé-

1. Il est clair que $f$ est continue et compacte et que $u-f(u)=0$ si et seulement si $u=a$. Si $\|a\|_{E} \neq R, d\left(\operatorname{Id}-f, B_{R}, a\right)$ est donc bien défini.

Si $R<\|a\|_{E}$, l'équation $u-f(u)=0$ n'a pas de solution dans $B_{R}$ et donc $d\left(\operatorname{Id}-f, B_{R}, 0\right)=0$.

Si $R>\|a\|_{E}$, on pose $h(t, v)=t f(v)$. La fonction h est continue et compacte de $[0,1] \times E$ dans $E$ et l'équation $u=t f(u)$ n'a pas de solution sur $\partial B_{R}$ pour $t \in[0,1]$ (car l'unique solution de $u=t f(u)$ est ta). On a donc $d\left(\operatorname{Id}-f, B_{R}, 0\right)=d\left(\operatorname{Id}-h(1, \cdot), B_{R}, 0\right)=d\left(\operatorname{Id}-h(0, \cdot), B_{R}, 0\right)=d\left(\operatorname{Id}, B_{R}, 0\right)=1$.

2.(a) Soit $u_{1}, u_{2} \in E$ t.q. $u_{1}-f\left(u_{1}\right)=0$ et $u_{2}-f\left(u_{2}\right)=0$. En posant $u=u_{1}-u_{2}$ on a donc $u-L u=0$. Comme 1 n'est pas valeur propre de $L$, on a donc $u=0$. Ce qui prouve bien que $u-f(u)=0$ a au plus une solution. 
(b) On pose $h(t, u)=L u+$ ta. La fonction h est continue et compacte de $[0,1] \times E$ dans $E$. Soit $R>0$. L'équation $u=h(0, u) n$ 'a pas de solution sur $\partial B_{R}$ (car 1 n'est pas valeur propre de $\left.L\right)$.

Si l'équation $u=h(t, u)$ n'a pas de solution pour $t \in] 0,1]$ sur $\partial B_{R}$, on a

$$
d\left(\mathrm{Id}-f, B_{R}, 0\right)=d\left(\mathrm{Id}-h(1,0), B_{R}, 0\right)=d\left(\mathrm{Id}-L, B_{R}, 0\right) \neq 0,
$$

d'après le théorème 3.8 Il existe donc $u \in B_{R}$ t.q. $u-f(u)=0$.

D'autre part, si l'équation $u=h(t, u)$ a une solution sur $\partial B_{R}$ pour un $t$ dans $\left.] 0,1\right]$. On note c cette solution et on remarque $(c / t)-f(c / t)=0$.

Dans tous les cas, on a donc montré qu'il existe $u \in E$ t.q. $u-f(u)=0$.

Enfin, il est facile de voir que $d\left(\operatorname{Id}-f, B_{R}, 0\right)=d\left(\operatorname{Id}-L, B_{R}, 0\right) \neq 0$ si $R>\|b\|_{E}$ et $d\left(\operatorname{Id}-f, B_{R}, 0\right)=0$ si $R<\|b\|_{E}$.

\section{Exercice 3.5 (Convection-diffusion, Dirichlet, existence)}

Soit $\Omega$ un ouvert borné de $\mathbb{R}^{N}, N=2$ ou $3, p>N, W \in L^{p}(\Omega)^{N}, \varphi$ une fonction lipschitzienne de $\mathbb{R}$ dans $\mathbb{R}$ t.q. $\varphi(0)=0$ et $f \in L^{2}(\Omega)$.

On s'intéresse ici au problème suivant

$$
\left\{\begin{array}{l}
-\Delta u+\operatorname{div}(W \varphi(u))=f \operatorname{dans} \Omega \\
u=0 \operatorname{sur} \partial \Omega
\end{array}\right.
$$

Le but de cet exercice est de montrer l'existence de solution faible au problème (3.45). L'unicité (et la positivité si $f \geq 0$ p.p.) de la solution faible est montré dans l'exercice 3.6 .

1. Soit $u \in H_{0}^{1}(\Omega)$, montrer que $W \varphi(u) \in L^{2}(\Omega)^{N}$. [Utiliser le théorème d'injection de Sobolev, théorème 1.28]

Cette première question permet de définir la formulation faible du problème (3.45). Elle consiste à chercher $u$ solution de 3.46.

$$
\left\{\begin{array}{l}
u \in H_{0}^{1}(\Omega) \\
\int_{\Omega} \nabla u(x) \cdot \nabla v(x) \mathrm{d} x-\int_{\Omega} \varphi(u(x)) W(x) \cdot \nabla v(x) \mathrm{d} x=\int_{\Omega} f(x) v(x) \mathrm{d} x \text { pour tout } v \in H_{0}^{1}(\Omega) .
\end{array}\right.
$$

Pour montrer l'existence d'une solution à (3.46) on va utiliser la méthode du degré topologique en construisant une application $h$ de $[0,1] \times L^{q}(\Omega)$ dans $L^{q}(\Omega)$ avec $q=2 p /(p-2)$ (de sorte que $1 / p+1 / q=1 / 2$ ). On pose donc pour la suite $q=2 p /(p-2)$. Si $N=3$, on pose $2^{\star}=6$ et si $N=2$, on choisit pour $2^{\star}$ un nombre strictement supérieur à $q$ (de sorte que, pour $N=2$ ou $3, H_{0}^{1}(\Omega)$ s'injecte continûment dans $L^{2^{\star}}(\Omega)$ et compactement dans $\left.L^{q}(\Omega)\right)$.

2. (Construction des opérateurs $B$ et $h$ ) Soit $\tilde{u} \in L^{q}$. Montrer qu'il existe un unique $u$ solution de

$$
\left\{\begin{array}{l}
u \in H_{0}^{1}(\Omega), \\
\int_{\Omega} \nabla u(x) \cdot \nabla v(x) \mathrm{d} x-\int_{\Omega} \varphi(\tilde{u}(x)) W(x) \cdot \nabla v(x) \mathrm{d} x=\int_{\Omega} f(x) v(x) \mathrm{d} x \text { pour tout } v \in H_{0}^{1}(\Omega) .
\end{array}\right.
$$

On note $B$ l'opérateur qui à $\tilde{u}$ dans $L^{q}(\Omega)$ associe $u$ solution de 3.47. Puis, pour $t \in[0,1]$ et $\tilde{u} \in L^{q}(\Omega)$, on pose $h(t, \tilde{u})=B(t \tilde{u})$.

3. Montrer que $h$ est continu et compact de $[0,1] \times L^{q}(\Omega)$ dans $L^{q}(\Omega)$. 
4. (Estimations a priori) Soit $u \in L^{q}(\Omega)$ t.q. $u=h(t, u)$. On a donc

$$
\left\{\begin{array}{l}
u \in H_{0}^{1}(\Omega), \\
\int_{\Omega} \nabla u(x) \cdot \nabla v(x) \mathrm{d} x-\int_{\Omega} \varphi(t u(x)) W(x) \cdot \nabla v(x) \mathrm{d} x=\int_{\Omega} f(x) v(x) \mathrm{d} x \text { pour tout } v \in H_{0}^{1}(\Omega) .
\end{array}\right.
$$

Pour $s \in \mathbb{R}$, on pose $\psi(s)=\int_{0}^{s} \frac{1}{(1+|\xi|)^{2}} d \xi$.

(a) Montrer que $\psi(u) \in H_{0}^{1}(\Omega)$. En prenant $v=\psi(u)$ dans [3.48, , montrer qu'il existe $C_{l}$ ne dépendant que $\Omega$, $W, \varphi$ et $f$ t.q.

$$
\|\ln (1+|u|)\|_{H_{0}^{1}(\Omega)} \leq C_{l} .
$$

(b) Pour $v \in L^{2^{\star}}(\Omega)$, montrer que pour tout $A \geq 0$ on a

$$
\int_{\Omega}|v(x)|^{q} d x \leq\left(\int_{\Omega}|v(x)|^{2^{\star}} \mathrm{d} x\right)^{\frac{q}{2^{\star}}} \lambda_{N}(\{|v| \geq A\})^{1-\frac{q}{2^{\star}}}+A^{q} \lambda_{N}(\Omega) .
$$

On rappelle que $\lambda_{N}$ est la mesure de Lebesgue sur les boréliens de $\mathbb{R}^{N}$.

(c) En utilisant $(a)$ et $(b)$, montrer qu'il existe $C>0$, ne dépendant que de $\Omega, W, \varphi$ et $f$ t.q. $\|u\|_{H_{0}^{1}(\Omega)}<C$.

5. (Degré topologique) Montrer l'existence d'une solution à 3.46 .

6. On retire dans cette question l'hypothèse $\varphi(0)=0$ et on se donne un élément $T$ de $H^{-1}(\Omega)$. Montrer qu'il existe $u$ solution de (3.46) avec $\int_{\Omega} f(x) v(x) \mathrm{d} x$ remplacé par $\langle T, v\rangle_{H^{-1}(\Omega), H_{0}^{1}(\Omega)}$.

Corrigé -

1. Le théorème 1.28 donne que $u \in L^{6}(\Omega)$ si $N=3$ et que $u \in L^{r}(\Omega)$ pour tout $r \in[1,+\infty[$ si $N=2$. Comme $\varphi$ est lipschitzienne et $\varphi(0)=0$, il existe $C_{1}$ t.q. $|\varphi(s)| \leq C_{1}|s|$ pour tout $s \in \mathbb{R}$. On a donc aussi $\varphi(u) \in L^{6}(\Omega)$ si $N=3$ et $\varphi(u) \in L^{r}(\Omega)$ pour tout $r \in[1,+\infty[$ si $N=2$.

Pour $N=3$, on a $W \in L^{3}(\Omega)^{3}$ et $\varphi(u) \in L^{6}(\Omega)$, ce qui donne $W \varphi(u) \in L^{2}(\Omega)^{3}$ car $1 / 6+1 / 3=1 / 2$.

Pour $N=2$, on a $W \in L^{p}(\Omega)^{2}$ et $\varphi(u) \in L^{2 p /(p-2)}(\Omega)$, ce qui donne $W \varphi(u) \in L^{2}(\Omega)^{2}$ car $1 / p+(p-2) / 2 p=$ $1 / 2$.

2. L'application $v \mapsto \int_{\Omega} \varphi(\tilde{u}(x)) W(x) \cdot \nabla v(x) \mathrm{d} x+\int_{\Omega} f(x) v(x) \mathrm{d} x$ est linéaire continue de $H_{0}^{1}(\Omega)$ dans $\mathbb{R}$. L'existence et l'unicité de u solution de 3.47 est donc une conséquence du théorème 2.9

3. On montre tout d'abord la continuité de $h$. Soit $\left(t_{n}, \tilde{u}_{n}\right)_{n \in \mathbb{N}}$ une suite de $[0,1] \times L^{q}(\Omega)$ t.q. $t_{n} \rightarrow t$ et $\tilde{u}_{n} \rightarrow \tilde{u}$ dans $L^{q}(\Omega)$ quand $n \rightarrow+\infty$. On pose $u_{n}=h\left(t_{n}, \tilde{u}_{n}\right)$ et $u=h(t, \tilde{u})$. On veut montrer que $u_{n} \rightarrow u$ dans $L^{q}(\Omega)$. On raisonne par l'absurde. Si $u_{n} \not \rightarrow$ dans $L^{q}(\Omega)$, il existe $\varepsilon>0$ et une sous suite, encore notée $\left(u_{n}\right)_{n \in \mathbb{N} \text {, t.q. }}$.

$$
\left\|u_{n}-u\right\|_{L^{q}(\Omega)} \geq \varepsilon \text { pour tout } n \in \mathbb{N} .
$$

Après une éventuelle extraction de sous suite (ce qui ne change pas 3.49 ), on peut aussi supposer que

$$
\tilde{u}_{n} \rightarrow \tilde{u} \text { p.p. et }\left|\tilde{u}_{n}\right| \leq H \text { p.p. pour tout } n \in \mathbb{N},
$$

avec $H \in L^{q}(\Omega)$. On en déduit (par convergence dominée dans $L^{q}(\Omega)$ car $\varphi$ est continue et $\left.|\varphi(s)| \leq C_{1}|s|\right)$ que $\varphi\left(t_{n} \tilde{u}_{n}\right) \rightarrow \varphi(t \tilde{u})$ dans $L^{q}(\Omega)$ et donc $\varphi\left(t_{n} \tilde{u}_{n}\right) W \rightarrow \varphi(t \tilde{u}) W$ dans $L^{2}(\Omega)^{N}$ (en remarquant que $|H W| \in L^{2}(\Omega)$, $\operatorname{car}|W| \in L^{p}(\Omega)$ et $\left.1 / p+1 / q=1 / 2\right)$.

Comme la suite $\left(\varphi\left(t_{n} \tilde{u}_{n}\right) W\right)_{n \in \mathbb{N}}$ est bornée dans $L^{2}(\Omega)^{N}$ et que $u_{n}$ est solution de 3.47 avec $t_{n} \tilde{u}_{n}$ au lieu de $\tilde{u}$, on montre (en prenant $v=u_{n}$ dans 3.47 que la suite $\left(u_{n}\right)_{n \in \mathbb{N}}$ est bornée dans $H_{0}^{1}(\Omega)$. On peut donc supposer (toujours après extraction de sous suite) qu'il existe $\bar{u}$ t.q. $u_{n} \rightarrow \bar{u}$ faiblement dans $H_{0}^{1}(\Omega)$. On a donc aussi (par compacité de 
l'injection de $H_{0}^{1}(\Omega)$ dans $\left.L^{q}(\Omega)\right) u_{n} \rightarrow \bar{u}$ dans $L^{q}(\Omega)$. On montre alors que $\bar{u}$ est solution de 3.47 avec tũ au lieu de $\tilde{u}$ (et donc que $\bar{u}=u$ ). Il suffit pour cela de passer à limite, pour tout $v \in H_{0}^{1}(\Omega)$, dans l'équation suivante

$$
\int_{\Omega} \nabla u_{n}(x) \cdot \nabla v(x) \mathrm{d} x-\int_{\Omega} \varphi\left(t_{n} \tilde{u}_{n}(x)\right) W(x) \cdot \nabla v(x) \mathrm{d} x=\int_{\Omega} f(x) v(x) \mathrm{d} x .
$$

Ce passage à limite découle facilement du fait que $\nabla u_{n} \rightarrow \nabla \bar{u}$ faiblement dans $L^{2}(\Omega)^{N}$ et $\varphi\left(t_{n} \tilde{u}_{n}\right) W \rightarrow \varphi(t \tilde{u}) W$ dans $L^{2}(\Omega)^{N}$.

On obtient ainsi que $\bar{u}=B(t \tilde{u})=u$, en contradiction avec 3.49 (car $u_{n} \rightarrow \bar{u}$ dans $L^{q}(\Omega)$ ). On a ainsi montré que $u_{n} \rightarrow u$ dans $L^{q}(\Omega)$. En fait, un raisonnement semblable par contradiction montrerait même que $u_{n} \rightarrow u$ faiblement dans $H_{0}^{1}(\Omega)$ mais ceci est inutile pour la suite.

On montre maintenant la compacité de h (ce qui est un peu plus facile). On suppose que test quelconque dans $[0,1]$ et que ũ reste dans un borné de $L^{q}(\Omega)$. On pose $u=h(t, \tilde{u})$. La fonction u est donc solution de 3.47 avec tu au lieu de $\tilde{u}$. Grâce $|\varphi(s)| \leq C_{1}|s|$, la fonction $\varphi(\tilde{u})$ reste dans un borné de $L^{q}(\Omega)$ et donc $\varphi(\tilde{u}) W$ reste dans un borné de $L^{2}(\Omega)^{N}$. En prenant maintenant $v=u$ dans 3.47 (avec tũ au lieu de $\tilde{u}$ ), on en déduit que u reste dans un borné de $H_{0}^{1}(\Omega)$. Comme $H_{0}^{1}(\Omega)$ s'injecte compactement dans $L^{q}(\Omega)$, on en déduit que u reste dans un compact de $L^{q}(\Omega)$. Ce qui prouve bien la compacité de $h$.

Remarque : un moyen probablement un peu plus rapide pour montrer la continuité et la compacité de hest de remarquer que hest la composée de B, qui est un opérateur continu et compact et $L^{q}(\Omega)$ dans $L^{q}(\Omega)$, avec l'application $(t, u) \mapsto$ tu qui est continue de $[0,1] \times L^{q}(\Omega)$ dans $L^{q}(\Omega)$.

4.(a) La fonction $\psi\left(\right.$ de $\mathbb{R}$ dans $\mathbb{R}$ ) est de classe $C^{1}$ sur $\mathbb{R}$ et est lipschitzienne (car $\left|\psi^{\prime}(s)\right| \leq 1$ pour tout $\left.s \in \mathbb{R}\right)$. Lemme 2.21 donne alors que $\psi(u) \in H_{0}^{1}(\Omega)$ et $\nabla \psi(u)=(\nabla u) /(1+|u|)^{2}$. On remarque aussi $|\psi(s)| \leq 1$ pour tout $s$.

En prenant $v=\psi(u)$ dans 3.48 et en utilisant $|\varphi(s)| \leq C_{1}|s|$ et $|\psi(s)| \leq 1$, on obtient

$$
\begin{gathered}
\int_{\Omega} \frac{|\nabla u(x)|^{2}}{(1+|u(x)|)^{2}} \mathrm{~d} x \leq C_{1} \int_{\Omega} \frac{|t u(x)|}{(1+|u(x)|)^{2}}|W(x)||\nabla u(x)| \mathrm{d} x+\|f\|_{L^{1}(\Omega)} \\
\leq C_{1} \int_{\Omega}|W(x)| \frac{|\nabla u(x)|}{1+|u(x)|} \mathrm{d} x+\|f\|_{L^{1}(\Omega)} .
\end{gathered}
$$

En utilisant $a b \leq \frac{a^{2}}{2 C_{1}}+2 C_{1} b^{2}$ pour $a, b \in \mathbb{R}$, on obtient

$$
\frac{1}{2} \int_{\Omega} \frac{|\nabla u(x)|^{2}}{(1+|u(x)|)^{2}} \mathrm{~d} x \leq 2 C_{1}^{2}\||W|\|_{L^{2}(\Omega)}^{2}+\|f\|_{L^{1}(\Omega)} .
$$

On remarque maintenant que (toujours par le lemme $2.21 \ln (1+|u|) \in H_{0}^{1}(\Omega)$ et l'inégalité précédente donne

$$
\|\ln (1+|u|)\|_{H_{0}^{1}(\Omega)}^{2} \leq 2\left(2 C_{1}^{2}\||W|\|_{L^{2}(\Omega)}^{2}+\|f\|_{L^{1}(\Omega)}\right) .
$$

Ce qui donne la majoration désirée avec $C_{l}^{2}=2\left(2 C_{1}^{2}\||W|\|_{L^{2}(\Omega)}^{2}+\|f\|_{L^{1}(\Omega)}\right)$.

(b) On a

$$
\int_{\Omega}|v(x)|^{q} d x=\int_{\{|v| \geq A\}}|v(x)|^{q} d x+\int_{\{|v|<A\}}|v(x)|^{q} d x \leq \int_{\{|v| \geq A\}}|v(x)|^{q} d x+A^{q} \lambda_{N}(\Omega) .
$$

Puis, l'inégalité de Hölder (avec $2^{\star} / q$ et son conjugué) donne

$$
\int_{\{|v| \geq A\}}|v(x)|^{q} d x=\int_{\Omega}|v(x)|^{q} 1_{\{|v| \geq A\}} \mathrm{d} x \leq\left(\int_{\Omega}|v(x)|^{2^{\star}} d x\right)^{\frac{q}{2^{\star}}} \lambda_{N}(\{|v| \geq A\})^{1-\frac{q}{2^{\star}}} .
$$

Ce qui donne bien l'inégalité désirée.

(c) On prend $v=u$ dans 3.48, on obtient, avec l'inégalité de Hölder,

$$
\|u\|_{H_{0}^{1}(\Omega)}^{2} \leq C_{1}\|u\|_{L^{q}(\Omega)}\|W \mid\|_{L^{p}(\Omega)}\|u\|_{H_{0}^{1}(\Omega)}+\|f\|_{L^{2}} C_{\Omega}\|u\|_{H_{0}^{1}(\Omega)},
$$

où $C_{\Omega}$ ne dépend que $\Omega$ et est donné par l'inégalité de Poincaré.

On commence par utiliser l'inégalité donnée dans 4(b) (élevée à la puissance $1 / q$ ). Pour tout $A>0$ on a

$$
\|u\|_{L^{q}(\Omega)} \leq 2\|u\|_{L^{2^{\star}}} \lambda_{N}(\{|u| \geq A\})^{\frac{1}{q}-\frac{1}{2^{\star}}}+2 A \lambda_{N}(\Omega)^{\frac{1}{q}} .
$$


Comme l'injection de $H_{0}^{1}(\Omega)$ dans $L^{2^{\star}}(\Omega)$ est continue, il existe $\bar{C}_{\Omega}$ ne dépendant que de $\Omega$ t.q. $\|u\|_{L^{2^{\star}}(\Omega)} \leq$ $\bar{C}_{\Omega}\|u\|_{H_{0}^{1}(\Omega)}$. On a donc

$$
\|u\|_{L^{q}(\Omega)} \leq 2 \bar{C}_{\Omega}\|u\|_{H_{0}^{1}(\Omega)} \lambda_{N}(\{|u| \geq A\})^{\frac{1}{q}-\frac{1}{2^{\star}}}+2 A \lambda_{N}(\Omega)^{\frac{1}{q}} .
$$

On utilise maintenant 4(a) (et l'inégalité de Poincaré). Pour tout $A \geq 0$ on a

$$
\ln (1+A) \lambda_{N}(\{|u| \geq A\})^{\frac{1}{2}} \leq\|\ln (1+|u|)\|_{L^{2}(\Omega)} \leq C_{\Omega}\|\ln (1+|u|)\|_{H_{0}^{1}(\Omega)} \leq C_{\Omega} C_{l} .
$$

Comme $\lim _{A \rightarrow+\infty} \ln (1+A)=+\infty$, il existe donc A ne dépendant (comme $C_{l}$, noter aussi que pet $q$ sont donnés par $W$ ) que de $\Omega, W$, $\varphi$ et $f$ t.q.

Avec ce choix de A, 3.50 donne

$$
\lambda_{N}(\{|u| \geq A\})^{\frac{1}{q}-\frac{1}{2^{\star}}} \leq \frac{1}{4 \bar{C}_{\Omega} C_{1}\||W|\|_{L^{p}(\Omega)}} .
$$

On en déduit

$$
\|u\|_{H_{0}^{1}(\Omega)}^{2} \leq \frac{1}{2}\|u\|_{H_{0}^{1}(\Omega)}^{2}+\left(2 A C_{1}\||W|\|_{L^{p}(\Omega)} \lambda_{N}(\Omega)^{\frac{1}{q}}+\|f\|_{L^{2}(\Omega)} C_{\Omega}\right)\|u\|_{H_{0}^{1}(\Omega)} .
$$

$\|u\|_{H_{0}^{1}(\Omega)} \leq 2\left(2 A C_{1}\||W|\|_{L^{p}(\Omega)} \lambda_{N}(\Omega)^{\frac{1}{q}}+\|f\|_{L^{2}(\Omega)} C_{\Omega}\right)$.

Ce qui est une estimation sur $\|u\|_{H_{0}^{1}(\Omega)}$ ne dépendant que $\Omega, W$, $\varphi$ et $f$.

5. Comme $H_{0}^{1}(\Omega)$ s'injecte continûment dans $L^{q}(\Omega)$, la question $4(c) R>0$ t.q.

$$
t \in[0,1], u \in L^{q}(\Omega), u=h(t, u) \Rightarrow\|u\|_{L^{q}(\Omega)}<R .
$$

La question 3 donne la continuité et la compacité de $h$ de $[0,1] \times L^{q}(\Omega)$ dans $L^{q}(\Omega)$. On peut donc appliquer l'invariance par homotopie du degré topologique sur la boule (ouverte) de $L^{p}(\Omega)$ de centre 0 et de rayon $R$ avec comme point cible 0 . On obtient

$$
d\left(\mathrm{Id}-h(1, \cdot), B_{R}, 0\right)=d\left(\operatorname{Id}-h(0, \cdot), B_{R}, 0\right) .
$$

L'application $\tilde{u} \mapsto h(0, \tilde{u})$ est constante $(h(0, \tilde{u})$ est, pour tout $\tilde{u}$, la solution faible de $-\Delta u=f$ dans $\Omega$ avec $u=0$ sur $\partial \Omega)$. La solution de $v=h(0, v)$ est unique et appartient à $B_{R}$. Ceci suffit pour dire que $d\left(\operatorname{Id}-h(0, \cdot), B_{R}, 0\right) \neq 0$ (on peut ramener la constante à 0 par homotopie en remarquant, par exemple, que $d\left(\operatorname{Id}-t h(0, \cdot), B_{R}, 0\right)$ ne dépend pas de $t \in[0,1]$, voir l'exercice 3.4 .

6. On commence par remplacer $\int_{\Omega} f(x) v(x) \mathrm{d} x$ par $\langle T, v\rangle_{H^{-1}(\Omega), H_{0}^{1}(\Omega)}$ dans 3.46. La démonstration est alors très semblable à la précédente. Les seuls points demandant une petite modification sont dans les questions $4(a)$ et $4(c)$. Dans la question $4(a)$, On a majoré $\int_{\Omega}|f \psi(u)| d x$ par $\|f\|_{L^{1}(\Omega)}$. Il faut maintenant majorer

$$
\left|\langle T, \psi(v)\rangle_{H^{-1}(\Omega), H_{0}^{1}(\Omega)}\right| .
$$

Cette majoration se fait en remarquant que

$$
\begin{aligned}
&\left|\langle T, \psi(v)\rangle_{H^{-1}(\Omega), H_{0}^{1}(\Omega)}\right| \leq\|T\|_{H^{-1}(\Omega)}\|\psi(u)\|_{H_{0}^{1}(\Omega)} \leq\|T\|_{H^{-1}(\Omega)}\left\|\frac{|\nabla u|}{(1+|u|)^{2}}\right\|_{L^{2}(\Omega)} \leq \\
& \leq\|T\|_{H^{-1}(\Omega)}\left\|\frac{|\nabla u|}{1+|u|}\right\|_{L^{2}(\Omega)} \leq 4\|T\|_{H^{-1}(\Omega)}^{2}+\frac{1}{4}\left\|\frac{|\nabla u|}{1+|u|}\right\|_{L^{2}(\Omega)}^{2} .
\end{aligned}
$$

Dans la question $4(c)$, on a majoré $\int_{\Omega}|f u| \mathrm{d} x$ par $C_{\Omega}\|f\|_{L^{2}(\Omega)}\|u\|_{H_{0}^{1}(\Omega)}$. Il faut maintenant majorer

Ce qui est facile car

$$
\left|\langle T, u\rangle_{H^{-1}(\Omega), H_{0}^{1}(\Omega)}\right| \text {. }
$$

$$
\left|\langle T, u\rangle_{H^{-1}(\Omega), H_{0}^{1}(\Omega)}\right| \leq\|T\|_{H^{-1}(\Omega)}\|u\|_{H_{0}^{1}(\Omega)} .
$$

Il reste maintenant à retirer l'hypothèse $\varphi(0)=0$. Ceci est assez facile car il suffit de se ramener au cas précédent en remplaçant $\varphi$ par $\varphi-\varphi(0)$ et en ajoutant au second membre de $3.46-\int_{\Omega} \varphi(0) W(x) \cdot \nabla v(x) \mathrm{d} x$. On se ramène bien au cas précédent car l'application $v \mapsto \int_{\Omega} \varphi(0) W(x) \cdot \nabla v(x) \mathrm{d} x$ est bien un élément de $H^{-1}(\Omega)\left(\right.$ car $\left.W \in L^{2}(\Omega)^{N}\right)$. 


\section{Exercice 3.6 (Convection-diffusion, Dirichlet, unicité)}

On reprend ici les mêmes hypothèses que dans l'exercice 3.5. c'est-à-dire :

Soit $\Omega$ un ouvert borné de $\mathbb{R}^{N}, N=2$ ou $3, p>N, W \in L^{p}(\Omega)^{N}, \varphi$ une fonction lipschitzienne de $\mathbb{R}$ dans $\mathbb{R}$ t.q. $\varphi(0)=0$ et $f \in L^{2}(\Omega)$.

L'exercice 3.5 a montré qu'il existait $u$ solution faible de (3.45), c'est-à-dire $u$ solution de

$$
\left\{\begin{array}{l}
u \in H_{0}^{1}(\Omega), \\
\int_{\Omega} \nabla u(x) \cdot \nabla v(x) \mathrm{d} x-\int_{\Omega} \varphi(u(x)) W(x) \cdot \nabla v(x) \mathrm{d} x=\int_{\Omega} f(x) v(x) \mathrm{d} x \text { pour tout } v \in H_{0}^{1}(\Omega) .
\end{array}\right.
$$

L'objectif de cet exercice est de montrer l'unicité de la solution de (3.51) et de montrer que $u \geq 0$ p.p. si $f \geq 0$ p.p..

1. Montrer l'unicité de la solution de 3.51).

2. On retire dans cette question (et seulement dans cette question) l'hypothèse $\varphi(0)=0$ et on se donne un élément $T$ de $H^{-1}(\Omega)$. Montrer que le problème (3.46) avec $\int_{\Omega} f(x) v(x) \mathrm{d} x$ remplacé par $\langle T, v\rangle_{H^{-1}(\Omega), H_{0}^{1}(\Omega)}$ a une unique solution (l'existence a été montrée dans l'exercice 3.5).

3. On suppose $f \leq 0$ p.p.. Soit $u$ la solution de 3.51]. Montrer que $u \leq 0$ p.p.. [Pour $n \in \mathbb{N}^{\star}$, on pourra prendre $v=S_{n}(u)$ dans 3.51) avec $S_{n} \in C(\mathbb{R}, \mathbb{R})$ définie par $S_{n}(s)=\max (0, \min (s, 1 / n))$ et faire tendre $n$ vers $+\infty$.]

\section{Corrigé-}

1. La démonstration d'unicité faite pour le théorème 3.18 n'a pas utilisée complètement les hypothèses sur G (qui étaient $G \in C^{1}\left(\bar{\Omega}, \mathbb{R}^{N}\right)$ et $\operatorname{div} G=0$ ). Elle a utilisé seulement le fait que $G \in L^{2}(\Omega)^{N}$. Ici nous avons $W \in L^{p}(\Omega)^{N}$. Comme $p>N$, ceci donne bien $W \in L^{2}(\Omega)^{N}$ et la démonstration faite pour le théorème 3.18 est donc aussi valable ici. Nous la rappelons rapidement.

Soient $u_{1}$ et $u_{2}$ deux solutions de 3.51. On a donc :

$$
\begin{aligned}
& \int_{\Omega} \nabla u_{1} \cdot \nabla v \mathrm{~d} x-\int_{\Omega} \varphi\left(u_{1}\right) W \cdot \nabla v \mathrm{~d} x=\int_{\Omega} f v \mathrm{~d} x, \\
& \int_{\Omega} \nabla u_{2} \cdot \nabla v \mathrm{~d} x-\int_{\Omega} \varphi\left(u_{2}\right) W \cdot \nabla v \mathrm{~d} x=\int_{\Omega} f v \mathrm{~d} x .
\end{aligned}
$$

et

Pour $n \in \mathbb{N}^{\star}$ on définit $T_{n} \in C(\mathbb{R}, \mathbb{R})$ par $T_{n}(s)=\max (-1 / n, \min (s, 1 / n)$ ). Le lemme 2.22 (ou plutôt sa généralisation, voir la remarque 2.23 donne $T_{n}\left(u_{1}-u_{2}\right) \in H_{0}^{1}(\Omega)$ et $\nabla T_{n}\left(u_{1}-u_{2}\right)=\nabla\left(u_{1}-u_{2}\right) 1_{A_{n}}$ avec $A_{n}=\left\{0<\left|u_{1}-u_{2}\right|<1 / n\right\}$.

On prend $v=T_{n}\left(u_{1}-u_{2}\right)$ dans 3.52 et 3.53 , on obtient

$$
\int_{\Omega} \nabla\left(u_{1}-u_{2}\right) \cdot \nabla T_{n}\left(u_{1}-u_{2}\right) \mathrm{d} x=\int_{\Omega}\left(\varphi\left(u_{1}\right)-\varphi\left(u_{2}\right)\right) W \cdot \nabla\left(T_{n}\left(u_{1}-u_{2}\right)\right) \mathrm{d} x .
$$

Avec $C_{1}$ t.q. $\left|\varphi\left(s_{1}\right)-\varphi\left(s_{2}\right)\right| \leq C_{1}\left|s_{1}-s_{2}\right|$ pour tout $s_{1}, s_{2} \in \mathbb{R}$, ceci donne

$$
\int_{A_{n}}\left|\nabla\left(u_{1}-u_{2}\right)\right|^{2} \mathrm{~d} x \leq \int_{A_{n}} C_{1}\left|u_{1}-u_{2}\right||W|\left|\nabla\left(u_{1}-u_{2}\right)\right| \mathrm{d} x .
$$

On a $\left|u_{1}-u_{2}\right| \leq 1 / n$ p.p. dans $A_{n}$. En appliquant l'inégalité de Cauchy Schwarz dans la dernière intégrale, on obtient donc:

$$
\int_{A_{n}}\left|\nabla\left(u_{1}-u_{2}\right)\right|^{2} \mathrm{~d} x \leq \frac{C_{1}}{n}\left(\int_{A_{n}}|W|^{2} \mathrm{~d} x\right)^{1 / 2}\left(\int_{A_{n}}\left|\nabla\left(u_{1}-u_{2}\right)\right|^{2} \mathrm{~d} x\right)^{1 / 2} .
$$


On a donc

$$
\left\|\left|\nabla T_{n}\left(u_{1}-u_{2}\right)\right|\right\|_{L^{2}(\Omega)}=\left(\int_{A_{n}}\left|\nabla\left(u_{1}-u_{2}\right)\right|^{2}\right)^{1 / 2} \leq \frac{C_{1}}{n} a_{n}, \text { avec } a_{n}=\left(\int_{A_{n}}|W|^{2} \mathrm{~d} x\right)^{1 / 2} .
$$

On utilise maintenant l'inégalité de Sobolev et Hölder pour obtenir, avec $1^{\star}=\frac{N}{N-1}$ et en désignant par m la mesure de Lebesgue sur $\mathbb{R}^{N}$,

$$
\left\|T_{n}\left(u_{1}-u_{2}\right)\right\|_{L^{1 \star}} \leq\left\|\left|\nabla T_{n}\left(u_{1}-u_{2}\right)\right|\right\|_{L^{1}(\Omega)} \leq m(\Omega)^{1 / 2}\left\|\left|\nabla T_{n}\left(u_{1}-u_{2}\right)\right|\right\|_{L^{2}(\Omega)} \leq \frac{C_{1} m(\Omega)^{1 / 2}}{n} a_{n} .
$$

On pose $B_{n}=\left\{\left|u_{1}-u_{2}\right| \geq 1 / n\right\}$, de sorte que

$$
\frac{1}{n}\left(m\left(B_{n}\right)\right)^{\frac{N-1}{N}} \leq\left(\int_{B_{n}}\left|T_{n}\left(u_{1}-u_{2}\right)\right|^{1^{\star}} \mathrm{d} x\right)^{\frac{1}{1^{\star}}} \leq\left\|T_{n}\left(u_{1}-u_{2}\right)\right\|_{L^{1^{\star}}} .
$$

On a donc

$$
\left(m\left(B_{n}\right)\right)^{\frac{N-1}{N}} \leq C_{1} m(\Omega)^{1 / 2} a_{n}
$$

Pour $n \in \mathbb{N}^{\star}$, on a $A_{n+1} \subset A_{n}$. Comme $\cap_{n \in \mathbb{N}^{\star}} A_{n}=\emptyset$, la continuité décroissante de $m$ donne que $\lim _{n \rightarrow+\infty}$ $m\left(A_{n}\right)=0$. Comme $W \in L^{2}(\Omega)^{N}$ on en déduit que $\lim _{n \rightarrow+\infty} a_{n}=0$ et donc, grâce à 3.54, que $\lim _{n \rightarrow+\infty}$ $m\left(B_{n}\right)=0$.

On remarque enfin que $B_{n+1} \supset B_{n}$, pour tout $n \in \mathbb{N}^{\star}$, et $\cup_{n \in \mathbb{N}} B_{n}=\left\{\left|u_{1}-u_{2}\right|>0\right\}$. Donc $\lim _{n \rightarrow+\infty} m\left(B_{n}\right)=$ $m\left\{\left|u_{1}-u_{2}\right|>0\right\}$ (par continuité croissante d'une mesure). On obtient donc $m\left\{\left|u_{1}-u_{2}\right|>0\right\}=0$ et donc $u_{1}=u_{2}$ p.p..

2. La démonstration est identique à la précédente. Il suffit de remplacer, dans 3.52 et 3.53 , $\int_{\Omega} f(x) v(x) \mathrm{d} x$ par $\langle T, v\rangle_{H^{-1}(\Omega), H_{0}^{1}(\Omega)}$.

3. La démonstration est voisine de celle de la première question. Pour $n \in \mathbb{N}^{\star}$, on prend $v=S_{n}(u)$ dans 3.51] avec $S_{n} \in C(\mathbb{R}, \mathbb{R})$ définie par $S_{n}(s)=\max (0, \min (s, 1 / n))$. Ceci est possible car $S_{n}(u) \in H_{0}^{1}(\Omega)$. On sait aussi que $\nabla S_{n}(u)=1_{E_{n}} \nabla u$ avec $E_{n}=\{0<u<1 / n\}$ (voir la remarque 2.23. Comme $S_{n}(u) \geq 0$ p.p. et $f \leq 0$ p.p., on obtient

$$
\int_{\Omega} \nabla u \cdot \nabla S_{n}(u) \mathrm{d} x-\int_{\Omega} \varphi(u) W \cdot \nabla S_{n}(u) \mathrm{d} x \leq 0
$$

On a donc

$$
\int_{\Omega}\left|\nabla S_{n}(u)\right|^{2} \mathrm{~d} x=\int_{\Omega} \nabla u \cdot \nabla S_{n}(u) \mathrm{d} x \leq \int_{\Omega} \varphi(u) W \cdot \nabla S_{n}(u) \mathrm{d} x .
$$

Il existe $C_{1}>0$ t.q. $\left|\varphi\left(s_{1}\right)-\varphi\left(s_{2}\right)\right| \leq C_{1}\left|s_{1}-s_{2}\right|$ pour tout $s_{1}, s_{2} \in \mathbb{R}$, ceci donne, avec l'inégalité de Cauchy Schwarz dans la dernière intégrale

$$
\left\|\left|\nabla S_{n}(u)\right|\right\|_{L^{2}(\Omega)} \leq \frac{C_{1}}{n}\left(\int_{E_{n}}|W(x)|^{2} \mathrm{~d} x\right)^{\frac{1}{2}} .
$$

On pose $\gamma_{n}=\left(\int_{E_{n}}|W(x)|^{2} \mathrm{~d} x\right)^{\frac{1}{2}}$.

On utilise maintenant l'inégalité de Sobolev et Hölder pour obtenir, avec $1^{\star}=\frac{N}{N-1}$ et en désignant par m la mesure de Lebesgue sur $\mathbb{R}^{N}$,

$$
\left\|S_{n}(u)\right\|_{L^{1^{\star}}} \leq\left\|\left|\nabla S_{n}(u)\right|\right\|_{L^{1}(\Omega)} \leq m(\Omega)^{1 / 2}\left\|\left|\nabla S_{n}(u)\right|\right\|_{L^{2}(\Omega)} \leq \frac{C_{1} m(\Omega)^{1 / 2}}{n} \gamma_{n} .
$$

On pose $D_{n}=\{u \geq 1 / n\}$, de sorte que

On a donc

$$
\frac{1}{n}\left(m\left(D_{n}\right)\right)^{\frac{N-1}{N}} \leq\left(\int_{D_{n}}\left|S_{n}(u)\right|^{1^{\star}} \mathrm{d} x\right)^{\frac{1}{1^{\star}}} \leq\left\|S_{n}(u)\right\|_{L^{1^{\star}}}
$$

$$
\left(m\left(D_{n}\right)\right)^{\frac{N-1}{N}} \leq C_{1} m(\Omega)^{1 / 2} \gamma_{n} .
$$


On conclut comme à la première question. Pour $n \in \mathbb{N}^{\star}$, on a $E_{n+1} \subset E_{n}$. Comme $\cap_{n \in \mathbb{N} \star} E_{n}=\emptyset$, la continuité décroissante de $m$ donne que $\lim _{n \rightarrow+\infty} m\left(E_{n}\right)=0$. Comme $W \in L^{2}(\Omega)^{N}$ on en déduit que $\lim _{n \rightarrow+\infty} \gamma_{n}=0$ et donc, grâce à 3.56, que $\lim _{n \rightarrow+\infty} m\left(D_{n}\right)=0$.

On remarque enfin que $D_{n+1} \supset D_{n}$, pour tout $n \in \mathbb{N}^{\star}$, et $\cup_{n \in \mathbb{N}} D_{n}=\{u>0\}$. Donc $\lim _{n \rightarrow+\infty} m\left(D_{n}\right)=$ $m\{u>0\}$ (par continuité croissante d'une mesure). On obtient donc $m\{u>0\}=0$ et donc $u \leq 0$ p.p.

\section{Exercice 3.7 (Existence par minimisation)}

Soit $\Omega$ un ouvert borné de $\mathbb{R}^{N}$ ( $N \geq 1$ ), $a$ une fonction de $\Omega$ dans $\mathbb{R}$ et $f$ une fonction de $\Omega \times \mathbb{R}$ dans $\mathbb{R}$ vérifiant :

$-a \in L^{\infty}(\Omega)$.

- Il existe $\alpha \in \mathbb{R}_{+}^{\star}$ t.q. $\alpha \leq a$ p.p..

$-f$ est mesurable par rapport à $x \in \Omega$, pour tout $s \in \mathbb{R}$, et continue par rapport à $s \in \mathbb{R}$, p.p. en $x \in \Omega$.

- Il existe $d \in L^{2}(\Omega), C \in \mathbb{R}$ et $\delta \in\left[0,1\left[\right.\right.$ t.q. $|f(\cdot, s)| \leq C|s|^{\delta}+d$ p.p., pour tout $s \in \mathbb{R}$.

Pour tout $s \in \mathbb{R}$ et p.p. en $x \in \Omega$, on pose $F(x, s)=\int_{0}^{s} f(x, t) d t$.

1. Soit $u \in H_{0}^{1}(\Omega)$. Montrer que $F(\cdot, u) \in L^{1}(\Omega)$.

Pour $u \in H_{0}^{1}(\Omega)$, on pose $E(u)=\frac{1}{2} \int_{\Omega} a(x) \nabla u(x) \cdot \nabla u(x) \mathrm{d} x-\int_{\Omega} F(x, u(x)) \mathrm{d} x$.

2. Montrer que $E(u) \rightarrow+\infty$ quand $\|u\|_{H_{0}^{1}(\Omega)} \rightarrow+\infty$.

3. Montrer qu'il existe $u \in H_{0}^{1}(\Omega)$ t.q. $E(u) \leq E(v)$ pour tout $v \in H_{0}^{1}(\Omega)$.

4. Montrer qu'il existe $u$ solution du problème suivant :

$$
\left\{\begin{array}{l}
u \in H_{0}^{1}(\Omega), \\
\int_{\Omega} a(x) \nabla u(x) \cdot \nabla v(x) \mathrm{d} x=\int_{\Omega} f(x, u(x)) v(x) \mathrm{d} x, \text { pour tout } v \in H_{0}^{1}(\Omega) .
\end{array}\right.
$$

\section{Exercice 3.8 (Minimisation avec contrainte)}

Soit $\Omega$ un ouvert borné de $\mathbb{R}^{N}(N \geq 2)$ et $\left.p \in\right] 1, \frac{N+2}{N-2}[$. On cherche une solution non nulle au problème suivant :

$$
\left\{\begin{array}{l}
-\Delta u=|u|^{p-1} u \text { dans } \Omega \\
u=0 \text { sur } \partial \Omega
\end{array}\right.
$$

1. Pour $v \in H_{0}^{1}(\Omega)$, on pose $E(v)=\frac{1}{2} \int_{\Omega}|\nabla v(x)|^{2} d x$ et $F(v)=\int_{\Omega}|v|^{p+1} \mathrm{~d} x$. On pose aussi $A=\left\{v \in H_{0}^{1}(\Omega)\right.$, $F(v)=1$ \}. Montrer qu'il existe $u \in A$ t.q. $u \geq 0$ p.p. et $E(u) \leq E(v)$ pour tout $v \in A$.

2. Montrer qu'il existe $u$ non nulle, $u \geq 0$ p.p., solution faible de 3.58.

Exercice 3.9 (Convergence faible et non linéarité) Remarque liminaire : Soit $\varphi \in C(\mathbb{R}, \mathbb{R})$. Lorsque qu'une suite $\left(u_{n}\right)_{n \in \mathbb{N}}$ tend faiblement vers $u$ dans un espace $L^{p}$ et que la suite $\varphi\left(u_{n}\right)$ tend faiblement vers $f$ dans un espace $L^{q}$, il est en général faux que $f=\varphi(u)$ p.p.. On ajoute l'hypothèse que $\int u_{n} \varphi\left(u_{n}\right)$ converge vers $\int u f$. Si $\varphi$ est croissante, l'astuce de Minty permet alors de montrer que $f=\varphi(u)$ p.p.. Si $\varphi$ est strictement croissante, on obtient même une convergence forte de $u_{n}$ vers $u$ (c'est l'“astuce de Leray-Lions"). Cet exercice détaille ces idées dans le cadre $p=q=2$ avec une mesure finie.

Soit $(X, T, m)$ un espace mesuré fini ( c'est-à-dire $m(X)<+\infty)$. On note $L^{2}$ l'espace $L_{\mathbb{R}}^{2}(X, T, m)$. Soit $\left(u_{n}\right)_{n \in \mathbb{N}}$ et $\left(v_{n}\right)_{n \in \mathbb{N}}$ deux suites bornées de $L^{2}$ et $u, v \in L^{2}$. On suppose que les suites $\left(u_{n}\right)_{n \in \mathbb{N}}$ et $\left(v_{n}\right)_{n \in \mathbb{N}}$ convergent faiblement dans $L^{2}$ vers $u$ et $v$. On rappelle que ceci signifie que

$$
\lim _{n \rightarrow+\infty} \int u_{n} w d m=\int u w d m \text { et } \lim _{n \rightarrow+\infty} \int v_{n} w d m=\int v w d m \text { pour tout } w \in L^{2} .
$$


1. On suppose, dans cette question seulement, que $v_{n}=u_{n}$ p.p., pour tout $n \in \mathbb{N}$ (et donc $u=v$ p.p.). Montrer que $u_{n} \rightarrow u$ dans $L^{2}$ (quand $n \rightarrow+\infty$ ) si et seulement si $\int u_{n}^{2} d m \rightarrow \int u^{2} d m$ (quand $n \rightarrow+\infty$ ).

On suppose pour toute la suite de l'exercice que $\int u_{n} v_{n} d m \rightarrow \int u v d m$ (quand $n \rightarrow+\infty$ ) et qu'il existe une fonction $\varphi$ de $\mathbb{R}$ dans $\mathbb{R}$ t.q.

$-\varphi$ continue et il existe $C \in \mathbb{R}$ t.q. $|\varphi(s) \leq C+C| s \mid$ pour tout $s \in \mathbb{R}$.

$-v_{n}=\varphi\left(u_{n}\right)$ p.p., pour tout $n \in \mathbb{N}$.

2. Soit $w \in L^{2}$, montrer que, quand $n \rightarrow+\infty$,

$$
\int\left(\varphi\left(u_{n}\right)-\varphi(w)\right)\left(u_{n}-w\right) d m \rightarrow \int(v-\varphi(w))(u-w) d m .
$$

3. On suppose que $\varphi$ est croissante.

(a) Soit $\bar{w} \in L^{2}$ et $t \in \mathbb{R}$. Montrer que

$$
\int(v-\varphi(u+t \bar{w})) t \bar{w} d m \leq 0
$$

[Utiliser 3.59].] En déduire que $\int(v-\varphi(u)) \bar{w} d m=0$.

(b) Montrer que $v=\varphi(u)$ p.p..

4. On suppose que $\varphi$ strictement croissante. Pour $n \in \mathbb{N}$, on pose $G_{n}=\left(\varphi\left(u_{n}\right)-\varphi(u)\right)\left(u_{n}-u\right)$.

(a) Montrer que $G_{n} \rightarrow 0$ dans $L^{1}$ quand $n \rightarrow+\infty$ (utiliser (3.59)).

(b) Montrer qu'il existe une sous suite de la suite $\left(G_{n}\right)_{n \in \mathbb{N}}$, notée $\left(G_{\psi(n)}\right)_{n \in \mathbb{N}}$ (avec $\psi$ strictement croissante de $\mathbb{N}$ dans $\mathbb{N}$ ) t.q. $G_{\psi(n)} \rightarrow 0$ p.p.. En déduire que $u_{\psi(n)} \rightarrow u$ p.p. (utiliser la croissance stricte de $\varphi$ ).

(c) Montrer que $u_{n} \rightarrow u$ dans $L^{p}$ pour tout $p \in[1,2[$.

Corrigé -

1. On remarque que

$$
\left\|u_{n}-u\right\|_{2}^{2}=\int u_{n}^{2} d m+\int u^{2} d m-2 \int u_{n} u d m .
$$

Comme $u_{n} \rightarrow u$ faiblement dans $L^{2}$, on a $\lim _{n \rightarrow+\infty} \int u_{n} u d m=\int u^{2} d m$. On déduit alors facilement de 3.60 que $u_{n} \rightarrow u$ dans $L^{2}$ si et seulement si $\lim _{n \rightarrow+\infty} \int u_{n}^{2} d m=\int u^{2} d m$.

2. On commence par remarquer que $\varphi(w) \in L^{2}$ (grâce aux hypothèses sur $\varphi$ et $m(X)<+\infty$ ). On a alors

$$
\int\left(\varphi\left(u_{n}\right)-\varphi(w)\right)\left(u_{n}-w\right)=\int\left(v_{n} u_{n}-v_{n} w-\varphi(w) u_{n}+\varphi(w) w\right) d m
$$

Les convergences faibles de $u_{n}$ et $v_{n}$ vers $u$ et $v$ donnent

$$
\lim _{n \rightarrow+\infty} \int u_{n} \varphi(w) d m=\int u \varphi(w) d m \text { et } \lim _{n \rightarrow+\infty} \int v_{n} w d m=\int v w d m .
$$

Enfin on a, par hypothèse, $\lim _{n \rightarrow+\infty} \int u_{n} v_{n} d m=\int u v d m$. On en déduit que bien que

$$
\lim _{n \rightarrow+\infty}\left(\varphi\left(u_{n}\right)-\varphi(w)\right)\left(u_{n}-w\right) d m=\int(v-\varphi(w))(u-w) d m .
$$

3.(a) On utilise ici 3.59 avec $w=u+t \bar{w}$. On obtient, quand $n \rightarrow+\infty$,

$$
\int\left(\varphi\left(u_{n}\right)-\varphi(u+t \bar{w})\right)\left(u_{n}-u-t \bar{w}\right) d m \rightarrow-\int(v-\varphi(u+t \bar{w})) t \bar{w} d m
$$

Comme $\varphi$ est croissante, on a $\left(\varphi\left(u_{n}\right)-\varphi(u+t \bar{w})\right)\left(u_{n}-u-t \bar{w}\right) \geq 0$ p.p. et donc $\int\left(\varphi\left(u_{n}\right)-\varphi(u+t \bar{w})\right)\left(u_{n}-\right.$ $u-t \bar{w}) d m \geq 0$. On en déduit, quand $n \rightarrow+\infty, \int(v-\varphi(u+t \bar{w})) t \bar{w} d m \leq 0$. 
En prenant $t=\frac{1}{m}\left(m \in \mathbb{N}^{\star}\right)$, on a donc $\int\left(v-\varphi\left(u+\frac{\bar{w}}{m}\right)\right) \bar{w} \leq 0$. En appliquant le théorème de convergence dominée (remarquer que $\left|\left(v-\varphi\left(u+\frac{\bar{w}}{m}\right)\right) \bar{w}\right| \leq F$ p.p. avec $F=(|v|+C+C|u|+C|\bar{w}|)|\bar{w}| \in L^{1}$ ), on obtient, quand $m \rightarrow \infty$,

$$
\int(v-\varphi(u)) \bar{w} d m \leq 0 .
$$

De même, en prenant $t=-\frac{1}{m}$, on montre $\int(v-\varphi(u)) \bar{w} d m \geq 0$. On a donc $\int(v-\varphi(u)) \bar{w} d m=0$.

(b) On choisit $\bar{w}=1_{A}-1_{A^{c}}$, avec $A=\{v-\varphi(u) \geq 0\}$. La question précédente donne alors $\int|v-\varphi(u)| d m=0$ et donc $v=\varphi(u)$ p.p.

4.(a) En prenant $w=u$ dans 3.59 , on obtient $\lim _{n \rightarrow+\infty} \int G_{n} d m=0$. Comme $\varphi$ est croissante, on a $G_{n} \geq 0$ p.p., pour tout $n \in \mathbb{N}$ et donc $\left\|G_{n}\right\|_{1}=\int G_{n} d m$. On en déduit bien que $G_{n} \rightarrow 0$ dans $L^{1}$.

(b) Comme $G_{n} \rightarrow 0$ dans $L^{1}$, il existe une sous suite de la suite $\left(G_{n}\right)_{n \in \mathbb{N}}$, notée $\left(G_{\psi(n)}\right)_{n \in \mathbb{N}}$ (avec $\psi$ strictement croissante de $\mathbb{N}$ dans $\mathbb{N})$ t.q. $G_{\psi(n)} \rightarrow 0$ p.p. (c'est la réciproque partielle du théorème de convergence dominée). Il existe donc $A \in T$ t.q. $m(A)=0$ et $G_{\psi(n)}(x) \rightarrow 0$ (quand $n \rightarrow+\infty$ ) si $x \in A^{c}$.

Soit $x \in A^{c}$. On pose $a=u(s)$. Pour $s \in \mathbb{R}$, on pose $f(s)=(\varphi(s)-\varphi(a))|s-a|$. Comme $\varphi$ est strictement croissante continue, la fonction $f$ est aussi strictement croissante continue. Elle admet donc une fonction réciproque, notée g, qui est continue. Comme $\mid f\left(u_{\psi(n)}(x) \mid=G_{\psi(n)}(x) \rightarrow 0\right.$, on a $f\left(u_{\psi(n)}(x)\right) \rightarrow 0$ et donc $u_{\psi(n)}(x)=$ $g\left(f\left(u_{\psi(n)}(x)\right)\right) \rightarrow g(0)=a$. On a donc $\lim _{n \rightarrow+\infty} u_{\psi(n)}(x)=u(x)$ pour tout $x \in A^{c}$. Ce qui prouve bien que $u_{\psi(n)} \rightarrow$ u p.p..

(c) On montre que $u_{n} \rightarrow u$ dans $L^{p}$ pour tout $p \in[1,2[$ en raisonnant pas l'absurde. On suppose qu'il existe $p \in[1,2[$ t.q. $\left(u_{n}\right)_{n \in \mathbb{N}}$ ne converge par vers $u$ dans $L^{p}$. Il existe alors $\varepsilon>0$ et une sous suite de la suite $\left(u_{n}\right)_{n \in \mathbb{N}}$ qui reste en dehors de la boule (de $\left.L^{p}\right)$ de centre u et de rayon $\varepsilon$. Par le raisonnement de la question précédente, de cette sous suite, un peut extraire une sous suite, notée $\left(u_{n}\right)_{\psi(n)}$ qui converge p.p. vers $u$. Comme la suite $\left(u_{n}\right)_{n \in \mathbb{N}}$ est bornée dans $L^{2}$, on peut alors montrer que cette sous suite converge dans $L^{p}$ vers u (c'est une conséquence du théorème de Vitali). En contradiction avec le fait que cette sous suite reste en dehors de la boule (de $L^{p}$ ) de centre u et de rayon $\varepsilon$. On a ainsi montré que $u_{n} \rightarrow u$ dans $L^{p}$ pour tout $p \in[1,2[$.

\section{Exercice 3.10 (Opérateur de Leray-Lions)}

Soit $\Omega$ un ouvert borné de $\mathbb{R}^{N}(N \geq 1)$, $a$ une fonction de $\Omega \times \mathbb{R} \times \mathbb{R}^{N}$ dans $\mathbb{R}^{N}$ et $\left.p \in\right] 1,+\infty[$ vérifiant (avec $\left.p^{\prime}=p /(p-1)\right)$ :

- $a$ est mesurable par rapport à $x \in \Omega$, pour tout $s, \xi \in \mathbb{R} \times \mathbb{R}^{N}$, et continue par rapport à $(s, \xi) \in \mathbb{R} \times \mathbb{R}^{N}$, p.p. en $x \in \Omega$.

- (Coercivité) Il existe $\alpha \in \mathbb{R}_{+}^{\star}$ t.q. $\alpha(x, s, \xi) \cdot \xi \geq \alpha|\xi|^{p}$ pour tout $(s, \xi) \in \mathbb{R} \times \mathbb{R}^{N}$ et p.p. en $x \in \Omega$.

- (Croissance) Il existe $d \in L^{p^{\prime}}(\Omega)$ et $C \in \mathbb{R}$ t.q. $|a(\cdot, s, \xi)| \leq C\left(d+|s|^{p-1}+|\xi|^{p-1}\right)$ p.p., pour tout $s, \xi \in \mathbb{R} \times \mathbb{R}^{N}$.

- (Monotonie) $\left(a(x, s, \xi)-a(x, s, \eta) \cdot(\xi-\eta)>0\right.$ pour tout $(s, \xi, \eta) \in \mathbb{R} \times \mathbb{R}^{N} \times \mathbb{R}^{N}$, $\xi \neq \eta$, et p.p. en $x \in \Omega$.

Soit $f \in W^{-1, p^{\prime}}(\Omega)$. Montrer qu'il existe $u$ solution du problème suivant :

$$
\left\{\begin{array}{l}
u \in W_{0}^{1, p}(\Omega), \\
\int_{\Omega} a(x, u(x), \nabla u(x)) \cdot \nabla v(x) \mathrm{d} x=\langle f, v\rangle_{W^{-1, p^{\prime}}(\Omega), W_{0}^{1, p}(\Omega)}, \text { pour tout } v \in W_{0}^{1, p}(\Omega) .
\end{array}\right.
$$

[Reprendre la démonstration du cours.] 


\section{Chapitre 4}

\section{Problèmes paraboliques}

\subsection{Aperçu des méthodes}

\section{Equation de la chaleur dans $\mathbb{R}^{N}$, solutions classiques}

Soit $N \geq 1$ et $u_{0} \in C\left(\mathbb{R}^{N}, \mathbb{R}\right)$. On s'intéresse ici à chercher des solutions classiques au problème suivant

$$
\begin{aligned}
& \partial_{t} u(x, t)-\Delta u(x, t)=0, x \in \mathbb{R}^{N}, t \in \mathbb{R}_{+}^{\star}, \\
& u(x, 0)=u_{0}(x), x \in \mathbb{R}^{N}
\end{aligned}
$$

où $\partial_{t} u$ désigne la dérivée partielle de $u$ par rapport au temps $t$, et $\Delta u$ désigne le laplacien de $u$ :

$$
\Delta u=\sum_{i=1}^{N} \partial_{i}^{2} u,
$$

où $\partial_{i}^{2} u$ désigne la dérivée partielle seconde de $u$ par rapport à la $i$-ème variable d'espace $x_{i}$. Par "solution classique", on entend une fonction $u \in C^{2}\left(\mathbb{R}^{N} \times \mathbb{R}_{+}^{\star}, \mathbb{R}\right) \cap C\left(\mathbb{R}^{N} \times \mathbb{R}_{+}, \mathbb{R}\right)$ solution de (4.1) au sens classique de la dérivation et de la condition initiale.

On commence par un petit calcul formel (le terme "formel" signifiant souvent en mathématiques "non nécessairement justifié"). On suppose qu'on peut utiliser pour la condition initiale et pour la solution la transformée de Fourier (en espace). On retient comme formule pour la transformée de Fourier d'une fonction intégrable sur $\mathbb{R}^{N}$ la formule suivante :

$$
\hat{f}(\xi)=\frac{1}{(2 \pi)^{\frac{N}{2}}} \int e^{-i x \cdot \xi} f(x) \mathrm{d} x .
$$

Si $u$ est solution de 4.1, , on obtient ainsi (si on est autorisé à utiliser la transformée de Fourier)

$$
\hat{\partial}_{t} u(\xi, t)+|\xi|^{2} \hat{u}(\xi, t)=0 \text {, pour } \xi \in \mathbb{R}^{N} \text { et } t>0 \text {, et } \hat{u}(\xi, 0)=\hat{u}_{0}(\xi) \text {, pour } \xi \in \mathbb{R}^{N}
$$

ce qui donne

$$
\hat{u}(\xi, t)=e^{-|\xi|^{2} t} \hat{u}_{0}(\xi), \text { pour } \xi \in \mathbb{R}^{N} \text { et } t \geq 0 .
$$

En choisissant $g(t) \in L^{1}\left(\mathbb{R}^{N}\right)$ t.q. $\widehat{g(t)}(\xi)=e^{-|\xi|^{2} t}$, on a donc $\hat{u}(\cdot, t)=\widehat{g(t)} \hat{u}_{0}$ pour tout $t \geq 0$ et donc (en utilisant le fait que la transformée de Fourier transforme la convolution en produit),

$$
\hat{u}(\cdot, t)=(2 \pi)^{-\frac{N}{2}} g{\widehat{(t) \star u_{0}}}_{\text {pour } t \geq 0 .}
$$


ou encore

$$
u(\cdot, t)=(2 \pi)^{-\frac{N}{2}} g(t) \star u_{0} \text { pour } t \geq 0 .
$$

Il reste à calculer $g(t)$. Comme $\widehat{g(t)} \in L^{1}\left(\mathbb{R}^{N}\right)$ pour $t>0$, le théorème d'inversion de Fourier nous donne $g(t)=\widehat{\widehat{g(t)}}(-\cdot)$, c'est-à-dire

$$
g(t)(x)=\frac{1}{(2 \pi)^{\frac{N}{2}}} \int e^{i x \cdot \xi} e^{-|\xi|^{2} t} d \xi \text { pour } x \in \mathbb{R}^{N} \text { et } t>0 .
$$

Le changement de variable $\xi=\eta / \sqrt{2 t}$ donne alors

$$
g(t)(x)=\frac{1}{(2 \pi)^{\frac{N}{2}}} \frac{1}{(2 t)^{\frac{N}{2}}} \int e^{i x \cdot \frac{\eta}{\sqrt{2 t}}} e^{-\frac{|\eta|^{2}}{2}} d \eta \text { pour } x \in \mathbb{R}^{N} \text { et } t>0 .
$$

Finalement, on obtient

$$
g(t)(x)=\frac{1}{(2 t)^{\frac{N}{2}}} e^{-\left|\frac{x}{\sqrt{2 t}}\right|^{2} \frac{1}{2}}=\frac{1}{(2 t)^{\frac{N}{2}}} e^{-\frac{|x|^{2}}{4 t}} \text { pour } x \in \mathbb{R}^{N} \text { et } t>0,
$$

Ce qui donne

$$
u(x, t)=\frac{1}{(4 \pi t)^{\frac{N}{2}}} \int e^{-\frac{|x-y|^{2}}{4 t}} u_{0}(y) d y \text { pour } x \in \mathbb{R}^{N} \text { et } t>0 .
$$

Il est maintenant possible de donner des conditions sur $u_{0}$ pour lesquelles (4.2) donne une solution classique de (4.1). Voici deux exemples de conditions suffisantes pour lesquelles la fonction $u$ donnée par (4.2) est une solution classique de 4.1) :

Exemple 1 :

$$
u_{0} \in\left(L^{1}\left(\mathbb{R}^{N}\right)+L^{\infty}\left(\mathbb{R}^{N}\right)\right) \cap C\left(\mathbb{R}^{N}, \mathbb{R}\right) .
$$

Exemple 2 :

$$
u_{0} \in C\left(\mathbb{R}^{N}, \mathbb{R}\right) \text { et il existe } \exists C \in \mathbb{R} \text { et } p \in \mathbb{N} \text { t.q. }\left|u_{0}(x)\right| \leq C\left(1+|x|^{p}\right) \text { pour tout } x \in \mathbb{R}^{N} .
$$

On a ainsi obtenu des résultats d'existence d'une solution classique pour (4.1). A-t-on alors unicité de la solution? On n'a pas de résultat d'unicité si on ne met des hypothèses que sur $u_{0}$. Plus précisément, on peut construire une solution classique non nulle de 4.1) avec $u_{0}=0$. Il n'y a donc jamais unicité de la solution classique de 4.1). Par contre, si on rajoute une hypothèse convenable de croissance sur la solution, on a un résultat d'unicité.

Théorème 4.1 Sous l'hypothèse (4.3), il existe une et une seule fonction u vérifiant :

1. $u$ est solution classique de 4.1.

2. $\forall T>0, \exists C_{T} \in \mathbb{R}, P_{T} \in \mathbb{N}$ t.q. $|u(x, t)| \leq C_{T}\left(1+|x|^{P_{T}}\right), \forall x \in \mathbb{R}^{N}, \forall t \in[0, T]$.

Comme nous l'avons déjà dit, sans la deuxième condition sur $u$ donnée dans le théorème 4.1 il n'y a pas unicité de la solution puisque l'on peut trouver $u \in C^{\infty}\left(\mathbb{R}^{N} \times[0,+\infty[), u \neq 0\right.$ et t.q.

$$
\left\{\begin{array}{l}
\partial_{t} u-\Delta u=0 \text { dans } \mathbb{R}^{N} \times \mathbb{R}_{+}, \\
u(x, 0)=0 \quad \forall x \in \mathbb{R}^{N} .
\end{array}\right.
$$

Un exemple est donné dans le livre de Smoller [29]. La démonstration de l'unicité dans le théorème 4.1]peut se faire en utilisant la transformée Fourier dans l'espace $\mathcal{S}^{\prime}$ (où $\mathcal{S}^{\prime}$ est le dual de l'ensemble $\mathcal{S}$ des fonctions $C^{\infty}$ à décroissance rapide ainsi que toutes leurs dérivées, muni de sa topologie naturelle). Pour cela, on remarque que, sous l'hypothèse de croissance donnée dans le théorème 4.1. on a $u \in \mathcal{S}^{\prime}$. Notons que ce raisonnement par analyse de Fourier est limité à $\mathbb{R}^{N}$ et essentiellement au cas du laplacien. 


\section{Solutions presque classiques, équation de diffusion, $\Omega$ ouvert borné}

Soit $\Omega$ un ouvert borné de $\mathbb{R}^{N}$. Pour $u_{0}$ donné, on s'intéresse maintenant au problème

$$
\left\{\begin{array}{l}
\partial_{t} u-\Delta u=0 \quad \text { dans } \quad \Omega \times \mathbb{R}_{+}^{\star}, \\
u(x, t)=0 \text { pour } x \in \partial \Omega \text { et } t>0 \\
u(x, 0)=u_{0}(x) \text { pour } x \in \Omega
\end{array}\right.
$$

Pour cela on définit un opérateur $\mathcal{A}$ d'une partie de $L^{2}(\Omega)$, notée $D(\mathcal{A})$, dans $L^{2}(\Omega)$ en posant

$$
D(\mathcal{A})=\left\{u \in H_{0}^{1}(\Omega) ; \Delta u \in L^{2}(\Omega)\right\}
$$

et, pour $u \in D(\mathcal{A}), \mathcal{A} u=-\Delta u$. Dans la définition de $D(\mathcal{A}), \Delta u$ est une dérivée faible de $u$. Le fait que $u \in D(\mathcal{A})$ signifie donc simplement que $u \in H_{0}^{1}(\Omega)$ et qu'il existe $f \in L^{2}(\Omega)$ t.q. $\int_{\Omega} \nabla u \cdot \nabla v \mathrm{~d} x=\int_{\Omega} f v \mathrm{~d} x$ pour tout $v \in C_{c}^{\infty}(\Omega)$ (ou, de manière équivalente, pour tout $v \in H_{0}^{1}(\Omega)$ ).

On suppose maintenant que $u_{0} \in L^{2}(\Omega)$ et va chercher une solution de (4.4) au sens suivant :

$$
\left\{\begin{array}{l}
u \in C^{1}(] 0,+\infty\left[, L^{2}(\Omega)\right) \cap C\left(\left[0,+\infty\left[, L^{2}(\Omega)\right)\right.\right. \\
u(t) \in D(\mathcal{A}) \text { et } u^{\prime}(t)+\mathcal{A} u(t)=0 \text { p.p., pour tout } t>0 \\
u(0)=u_{0} \text { p.p. }
\end{array}\right.
$$

où $u(t)$ désigne la fonction $x \mapsto u(x, t)$. On rappelle (voir le chapitre 2) qu'il existe une base hilbertienne de $L^{2}(\Omega)$ (c'est à dire une famille orthonormale dense dans $L^{2}(\Omega)$ ), notée $\left(e_{n}\right)_{n \in \mathbb{N}^{*}}$, formée de fonctions propres de l'opérateur $\mathcal{A}$. Pour tout $n \in \mathbb{N}^{\star}$, la fonction $e_{n}$ est une solution faible de

$$
\left\{\begin{array}{l}
-\Delta e_{n}=\lambda_{n} e_{n} \text { dans } \Omega \\
e_{n}=0 \text { sur } \partial \Omega
\end{array}\right.
$$

avec $\lambda_{n}>0$ et $\lambda_{n} \uparrow+\infty$ lorsque $n \rightarrow+\infty$. Pour tout $n \in \mathbb{N}^{\star}$, on a donc $e_{n} \in D(\mathcal{A})$ et $\mathcal{A} e_{n}=\lambda_{n} e_{n}$. Soit $u_{0} \in L^{2}(\Omega)$. En notant $(u \mid v)_{2}$ le produit scalaire de $u$ et $v$ dans $L^{2}(\Omega)$, on a

$$
u_{0}=\sum_{n=1}^{+\infty}\left(u_{0} \mid e_{n}\right)_{2} e_{n}
$$

(cette série étant convergente dans $L^{2}(\Omega)$ ).

On a aussi $\sum_{n=1}^{+\infty}\left(u_{0} \mid e_{n}\right)_{2}^{2}=\left\|u_{0}\right\|_{2}^{2}<+\infty$.

On pose, pour $t \geq 0$,

$$
u(t)=\sum_{n=1}^{+\infty} e^{-\lambda_{n} t}\left(u_{0} \mid e_{n}\right)_{2} e_{n}
$$

qui est une série convergente dans $L^{2}(\Omega)$. On a donc ainsi $u(t) \in L^{2}\left(\mathbb{R}^{N}\right)$ pour tout $t \geq 0$ et on a même (comme la suite $\left(\lambda_{n}\right)_{n \in \mathbb{N}^{*}}$ est bornée inférieurement par $\lambda_{1}$ avec $\left.\lambda_{1}>0\right) u \in C\left(\left[0,+\infty\left[, L^{2}(\Omega)\right)\right.\right.$ et $u(0)=u_{0}$ p.p.. D'autre part, comme $\lambda_{n} \uparrow+\infty$ quand $n \rightarrow+\infty$, il est facile de montrer que la fonction $u$ est dérivable de $] 0,+\infty[$ dans $L^{2}(\Omega)$ et que la dérivée de $u$ est obtenue est dérivant la série terme à terme (ceci est une conséquence du fait que la série dérivée terme à terme est, sur $] 0,+\infty\left[\right.$, localement uniformément convergente dans $L^{2}(\Omega)$ ). On a donc, pour tout $t>0$,

$$
u^{\prime}(t)=\frac{d u}{d t}=\sum_{n=1}^{+\infty}\left(-\lambda_{n}\right) e^{-\lambda_{n} t}\left(u_{0} \mid e_{n}\right)_{2} e_{n}
$$


(La série écrite dans le terme de droite est convergente dans $L^{2}(\Omega)$ ).

On rappelle que (selon le chapitre $2 p u(t) \in D(\mathcal{A})$ pour tout $t>0$ car la série $\sum_{n=1}^{+\infty} \lambda_{n} e^{-\lambda_{n} t}\left(u_{0} \mid e_{n}\right)_{2} e_{n}$ est convergente dans $L^{2}(\Omega)$. Le chapitre 2 donne aussi, pour tout $t>0$,

$$
\mathcal{A} u(t)=\sum_{n=1}^{+\infty} \lambda_{n} e^{-\lambda_{n} t}\left(u_{0} \mid e_{n}\right)_{2} e_{n} .
$$

On a donc $u \in C^{1}(] 0,+\infty\left[, L^{2}(\Omega)\right)$ et $u^{\prime}(t)+\mathcal{A}(u(t))=0$ p.p. pour tout $\left.t \in\right] 0,+\infty[$. On a ainsi trouvé une solution au problème 4.5 .

On veut montrer maintenant que cette solution est unique. Pour cela, nous allons montrer que si $u$ est solution du problème avec donnée initiale nulle, c'est-à-dire

$$
\left\{\begin{array}{l}
u \in C^{1}(] 0,+\infty\left[, L^{2}(\Omega)\right) \cap C\left(\left[0,+\infty\left[, L^{2}(\Omega)\right),\right.\right. \\
u(t) \in D(\mathcal{A}) \text { et } u^{\prime}(t)+\mathcal{A} u(t)=0 \text { p.p., pour tout } t>0, \\
u(0)=0 \text { p.p., }
\end{array}\right.
$$

alors $u$ reste nulle pour tout temps, c'est-à-dire $u(t)=0$ p.p. pour tout $t \geq 0$.

Soit $t>0$. Comme $u^{\prime}(t)+\mathcal{A} u(t)=0$ p.p., en prenant le produit scalaire avec $u(t) \in L^{2}(\Omega)$, on obtient $\left(u^{\prime}(t) \mid u(t)\right)_{2}+(\mathcal{A} u(t) \mid u(t))_{2}=0$. Comme $(\mathcal{A} u(t) \mid u(t))_{2}=\int_{\Omega}|\nabla u(t)|^{2} \mathrm{~d} x$, on a donc

$$
\left(u^{\prime}(t) \mid u(t)\right)_{2}=-\int_{\Omega}|\nabla u(t)|^{2} \mathrm{~d} x \leq 0 .
$$

Soit $\psi$ l'application définie par $t \mapsto \psi(t)=\|u(t)\|_{L^{2}(\Omega)}^{2}$. L'application $\psi$ est continue sur $\mathbb{R}_{+}$, dérivable sur $\mathbb{R}_{+}^{\star}$ et $\psi^{\prime}(t)=2\left(u^{\prime}(t) \mid u(t)\right)_{2}$ pour tout $t>0$. On a donc $\psi^{\prime}(t) \leq 0$ pour tout $t>0$. L'application $\psi$ est donc décroissante sur $\mathbb{R}_{+}$et, comme $\psi(0)=0$, on en déduit que $\psi(t) \leq 0$ pour tout $t \geq 0$. Donc $\psi(t)=0$ pour tout $t \geq 0$. On a bien finalement $u(t)=0$ p.p. pour tout $t \geq 0$.

Nous avons ainsi montré l'existence et l'unicité de la solution de (4.5). Cette solution est dite "presque classique" car la dérivation en temps est prise au sens classique (puisque $u$ est de classe $C^{1}$ à valeurs dans $L^{2}(\Omega)$ ), la condition intiale est prise au sens classique dans $L^{2}(\Omega)$. Par contre le laplacien de $u(t)$ est pris au sens des dérivées faibles. Il faut un travail supplémentaire (sur la régularité des fonctions $e_{n}$ ) pour montrer que l'équation $\partial_{t} u-\Delta u$ est vérifiée au sens classique sur $\mathbb{R}^{N} \times \mathbb{R}_{+}^{\star}$. Ceci est fait pour $N=1$ dans l'exercice 4.1

Remarque 4.2 (Généralisation) On peut aussi montrer l'existence et l'unicité (pour $u_{0} \in L^{2}(\Omega)$ ) de la solution de (4.5) en remplaçant, dans la définition de $\mathcal{A}, \Delta u \operatorname{par} \sum_{i, j=1}^{N} D_{j}\left(a_{i j} D_{i} u\right)$, avec $a_{i j} \in L^{\infty}(\Omega)$, sous une hypothèse de coercivité, c'est-à-dire si il existe $\alpha>0$ t.q. $\alpha|\xi|^{2} \leq \sum a_{i j} \xi_{i} \xi_{j}$ p.p. dans $\Omega$ et pour tout $\xi \in \mathbb{R}^{N}$. On peut aussi remplacer $u^{\prime}(t)+\mathcal{A} u(t)=0$ par $u^{\prime}(t)+\mathcal{A} u(t)=f(t)$ si $f \in C\left(\left[0, \infty\left[, L^{2}(\Omega)\right)\right.\right.$.

\section{Solutions presque classiques par semi-groupes}

Soit $E$ un espace de Banach réel et $\mathcal{A}: D(\mathcal{A}) \subset E \rightarrow E$ un opérateur linéaire. L'ensemble $D(\mathcal{A})$ est donc le s.e.v. de $E$ sur lequel $\mathcal{A}$ est défini.

Définition 4.3 (Opérateur $m$-accrétif) On dit que $\mathcal{A}$ est m-accrétif si $\mathcal{A}$ vérifie :

(i) $D(\mathcal{A})$ est dense dans $E$,

(ii) $\forall \lambda>0,(\mathrm{Id}+\lambda \mathcal{A})$ est inversible, d'inverse continue et $\left\|(I+\lambda \mathcal{A})^{-1}\right\|_{\mathcal{L}(E, E)} \leq 1$. 
On rappelle que $\mathcal{L}(E, E)$ désigne l'ensemble des opérateurs linéaires continus de $E$ dans $E$, et que

$$
\|T\|_{\mathcal{L}(E, E)}=\sup _{u \in E, u \neq 0} \frac{\|T(u)\|_{E}}{\|u\|_{E}}, \text { pour tout } T \in \mathcal{L}(E, E) .
$$

Remarque 4.4 (Opérateur maximal monotone) Soit $E$ est un espace de Hilbert réel et $\mathcal{A}: D(\mathcal{A}) \subset E \rightarrow E$ un opérateur linéaire. L'opérateur $\mathcal{A}$ est $m$-accrétif si et seulement si il vérifie :

$$
\left\{\begin{array}{l}
(\mathcal{A} u \mid u)_{E} \geq 0 \quad \forall u \in D(\mathcal{A}) \\
(\operatorname{Id}+\mathcal{A}) \text { surjectif. }
\end{array}\right.
$$

Dans ce cas, on dit que $\mathcal{A}$ est maximal monotone.

Exemple : Le laplacien Soit $\Omega$ est un ouvert borné de $\mathbb{R}^{N}, E=L^{2}(\Omega)$ et $\mathcal{A}$ défini par $D(\mathcal{A})=\{u \in$ $\left.H_{0}^{1}(\Omega) ; \Delta u \in L^{2}(\Omega)\right\}$ et $\mathcal{A} u=-\Delta u$ si $u \in D(\mathcal{A})$. L'opérateur $\mathcal{A}$ est alors un opérateur $m$-accrétif (ou maximal monotone puisqu'on est dans le cas d'un Hilbert).

Remarque 4.5 (Graphe d'un opérateur $\boldsymbol{m}$-accrétif) Le graphe d'un opérateur $m$-accrétif est fermé. En ce sens, il est maximal, d'où le " $m$ " dans $m$-accrétif.

On admettra le théorème suivant dû à Hille ${ }^{1}$ et Yosida ${ }^{2}$ (voir par exemple [29] pour sa démonstration A VERIFIER) :

Théorème 4.6 (Hille-Yosida) Soit $E$ un espace de Banach, $\mathcal{A}: D(\mathcal{A}) \subset E \rightarrow E$ un opérateur linéaire m-accrétif et $u_{0} \in D(\mathcal{A})$. Alors il existe un unique $u: \mathbb{R}_{+} \rightarrow$ E tel que

$$
\begin{aligned}
& u \in C^{1}([0,+\infty[, E), \\
& u(t) \in D(\mathcal{A}), \forall t \geq 0, \\
& u^{\prime}(t)+\mathcal{A}(u(t))=0 \text { sur } \mathbb{R}_{+}^{\star}, \\
& u(0)=u_{0} .
\end{aligned}
$$

La démonstration de ce théorème peut s'effectuer par une discrétisation en temps : on considére le problème elliptique $\frac{u_{n+1}-u_{n}}{\delta t}+\mathcal{A} u_{n+1}=0$, qui s'écrit encore $u_{n+1}=(\operatorname{Id}+\delta t \mathcal{A})^{-1} u_{n}$, où $\delta t>0$ est le pas de la discrétisation. On effectue ensuite un passage à la limite $\delta t \rightarrow 0$.

Définition 4.7 (Semi-groupe) Soit $E$ un espace de Banach, $\mathcal{A}: D(\mathcal{A}) \subset E \rightarrow E$ un opérateur linéaire $m$ accrétif. Pour $u_{0} \in D(\mathcal{A})$ et $t \geq 0$, on pose $S(t) u_{0}=u(t)$, où u est l'unique fonction vérifiant [4.6]. Alors l'opérateur $S(t)$ est un opérateur linéaire continu de $D(\mathcal{A}) \subset E$ dans $E$ qui vérifie

$$
\left\{\begin{array}{l}
S(t+s)=S(t) \circ S(s) \text { pour } t, s \geq 0 \\
S(0)=\mathrm{Id} \\
\left\|S(t) u_{0}\right\|_{E} \leq\left\|u_{0}\right\|_{E}
\end{array}\right.
$$

On dit que $\{S(t), t \geq 0\}$ est un semi-groupe de contraction.

1. Carl Einar Hille (1894-1980) américain d'originie suédoise, spécialiste d'analyse.

2. Kōsaku Yosida (1909-1990), mathématicien japonais, spécialiste de l'analyse fonctionnelle. 
Soit $t \geq 0$, comme $\overline{D(\mathcal{A})}=E$, l'opérateur $S(t)$ se prolonge de manière unique à tout $E$ en un opérateur $\overline{S(t)}$ et $\overline{S(t)}$ dans $\mathcal{L}(E, E)$.

Définition 4.8 (Solution "mild") Soit $E$ un espace de Banach, $\mathcal{A}: D(\mathcal{A}) \subset E \rightarrow E$ un opérateur linéaire maccrétif. Soit $u_{0} \in E$, la fonction $u(t)=\overline{S(t)} u_{0}$, définie de manière unique, s'appelle solution mild du problème

$$
\left\{\begin{array}{l}
\partial_{t} u+\mathcal{A} u=0 \\
u(0)=u_{0}
\end{array}\right.
$$

Dans le cas du laplacien, on peut se demander en quel sens la solution mild satisfait (4.4). On peut montrer que la solution mild est solution en un sens faible que nous verrons dans la section 4.3 . De plus, cette solution mild est, dans ce cas particulier, l'unique solution faible. Cependant, cette situation n'est pas complètement générale. La solution mild, obtenue par densité, est toujours unique (dès que $u_{0} \in E$, quelque soit l'espace de Banach $E$ et l'opérateur $m$-accrétif $\mathcal{A}$ ). Pour les problèmes issus d'équations aux dérivées partielles, cette solution mild est en général une solution faible du problème que l'on veut résoudre; toutefois le problème de l'unicité de la solution faible est beaucoup plus difficile. On peut avoir non unicité de la solution faible quand on prend une "solution faible" dans un sens qui semble pourtant raisonnable. Il n'y a pas alors d'équivalence entre la notion de solution mild et de solution faible, car la solution mild est unique et, malheureusement, il n'y a pas unicité de la solution faible.

Pour essayer d'éclairer cette difficulté, nous nous intéressons, dans le paragraphe qui suit, à un problème un peu plus simple. Nous nous intéressons à un problème elliptique pour lequel nous montrons que la solution obtenue par densité, à la manière de la solution mild introduite ci-dessus, est unique alors qu'on n'a pas unicité des solutions faibles en prenant une définition "naturelle" de solution faible. Ceci montre en particulier que dans le cas parabolique, il n'y a pas non plus d'équivalence entre solution mild et solution faible (par exemple en considérant les solutions stationnaires).

Le Laplacien avec donnée $L^{1} \quad$ On considère un ouvert borné $\Omega$ de $\mathbb{R}^{N}, N \geq 2$, des fonctions $a_{i j}$, pour $i, j=$ $1, \ldots, N$, appartenant à $L^{\infty}(\Omega)$ et satisfaisant l'hypothèse de coercivité habituelle :

$$
\exists \alpha>0 ; \quad \sum_{i, j} \alpha_{i j} \xi_{i} \xi_{j} \geq \alpha|\xi|^{2} \text { p.p. dans } \Omega, \quad \forall \xi \in \mathbb{R}^{N} .
$$

On note $A$ la fonction à valeurs matricielle donnée par les fonctions $a_{i j}, i, j=1, \ldots, N$. On sait (par le lemme de Lax-Milgram) que pour tout $f \in L^{2}(\Omega)$, il existe un unique $u$ solution de

$$
\left\{\begin{array}{l}
u \in H_{0}^{1}(\Omega), \\
\int_{\Omega} A \nabla u \cdot \nabla v \\
d x=\int f v, \quad \forall v \in H_{0}^{1}(\Omega) .
\end{array}\right.
$$

On considère le même problème avec une donnée moins régulière $f \in L^{1}(\Omega)$.

Etape 1 - Estimation, cas régulier. Pour tout $1 \leq q<\frac{N}{N-1}$, on montre qu'il existe $C_{q} \in \mathbb{R}$ tel que si $u$ est (l'unique) solution de (4.7) avec $f \in L^{2}(\Omega)$ alors $\|u\|_{W_{0}^{1, q}(\Omega)} \leq C_{q}\|f\|_{L^{1}(\Omega)}$. On pose alors $T_{q}(f)=u$.

Etape 2 - Solution mild Pour tout $1 \leq q<\frac{N}{N-1}$, l'application $T_{q}$ de $L^{2}(\Omega)$ dans $W_{0}^{1, q}(\Omega)$ est continue de $L^{2}(\Omega)$ muni de la norme de $L^{1}(\Omega)$ dans $W_{0}^{1, q}(\Omega)$ (muni de sa norme naturelle). On peut donc prolonger $T_{q}$ de manière unique par densité sur tout $L^{1}(\Omega)\left(\operatorname{car} L^{2}(\Omega)\right.$ est dense dans $L^{1}(\Omega)$ ). On appelle $\bar{T}_{q}$ ce prolongement. On remarque alors que $\overline{T_{q_{1}}} f=\overline{T_{q_{2}}} f$ pour tout $q_{1}, q_{2}$ t.q. $1 \leq q_{1}<q_{2}<\frac{N}{N-1}$. On peut ainsi définir un opérateur (linéaire) $T$ de $L^{1}$ dans $\bigcap_{1 \leq q<\frac{N}{N-1}} W_{0}^{1, q}(\Omega) \operatorname{par} T(f)=\overline{T_{q}}(f)$ pour tout $1 \leq q<\frac{N}{N-1}$. 
Définition 4.9 (Solution mild pour un problème elliptique à donnée $L^{1}$ ) Sous les hypothèses précédentes sur $\Omega$ et $A$, soit $f \in L^{1}(\Omega)$. La fonction $T f$ est la solution mild de

$$
\left\{\begin{array}{l}
-\operatorname{div}(A \nabla u)=f \text { dans } \Omega, \\
u=0 \text { sur } \partial \Omega .
\end{array}\right.
$$

Etape 3 - Quel sens donner à la solution mild? Il est naturel de se demander quel rapport il y a entre la solution mild $u=T f$ et une solution faible du problème de Dirichlet homogène. Il est assez facile de montrer que $u$ est solution faible dans le sens (naturel) suivant :

$$
\left\{\begin{array}{l}
u \in \bigcap_{1 \leq q<\frac{N}{N-1}} W_{0}^{1, q}(\Omega), \\
\int_{\Omega} A \nabla u \cdot \nabla v \mathrm{~d} x=\int_{\Omega} f v \mathrm{~d} x \quad \forall v \in \bigcup_{r>N} W_{0}^{1, r}(\Omega) .
\end{array}\right.
$$

Remarque : un résultat analogue d'existence de solution mild et donc d'existence de solution faible est encore vrai si $f$ est une mesure sur $\Omega$ (dans (4.9), on remplace alors $f v d x$ par $v d f$ ). Pour montrer ce résultat, on utilise la densité de $L^{2}(\Omega)$ dans l'ensemble des mesures sur $\Omega$ au sens de la convergence faible- $\star$ dans $C(\bar{\Omega})^{\prime}$ (car l'ensemble des mesures sur $\Omega$ peut être vu comme une partie du dual de $C(\bar{\Omega})$ ). Il faut aussi utiliser le fait que les éléments de $W_{0}^{1, r}(\Omega)$ sont des fonctions continues pour $r>N$.

Etape 4 - Unicité mild, non unicité faible La solution mild est unique. La solution de (4.9) est-elle unique? pas toujours... . Montrons d'abord que si $N=2$, la solution faible est unique. Ceci se montre par régularité sur le problème dual, qui entraîne l'unicité sur le problème primal. Soit $u$ solution de

$$
\left\{\begin{array}{l}
u \in \bigcap_{1 \leq q<2} W_{0}^{1, q}(\Omega) \\
\int_{d x=0} A \nabla u \cdot \nabla v \\
d x=0 v \in \bigcup_{r>2} W_{0}^{1, r}(\Omega) .
\end{array}\right.
$$

On veut montrer que $u=0$. On ne peut pas prendre comme fonction test $v=u$ dans 4.9 ) ou 4.10 , en raison du manque de régularité de $u$. Pour contourner ce problème, on va raisonner en utilisant la régularité des solutions du problème dual. On remarque d'abord que $u$ est solution de (4.10) et que (4.10) peut se re-écrire ainsi :

$$
\left\{\begin{array}{l}
u \in \bigcap_{1 \leq q<2} W_{0}^{1, q}, \\
\int A^{t} \nabla v \cdot \nabla u \\
d x=0, \quad \forall v \in \bigcup_{r>2} W_{0}^{1, r}(\Omega) .
\end{array}\right.
$$

Or on sait (par le lemme de Lax-Milgram) que si $g \in L^{2}(\Omega)$ il existe un unique $v$ solution de

$$
\left\{\begin{array}{l}
v \in H_{0}^{1}(\Omega), \\
\int A^{t} \nabla v \cdot \nabla w \mathrm{~d} x=\int g w \mathrm{~d} x, \quad \forall w \in H_{0}^{1}(\Omega) .
\end{array}\right.
$$

(4.12) est le problème dual de 4.7).)

On aimerait pouvoir prendre $w=u$ dans 4.12), car on aurait alors, grâce à 4.11, $\int_{\Omega} g u \mathrm{~d} x=0$ pour tout $g \in L^{2}(\Omega)$, ce qui permettrait de conclure que $u=0$. Mais pour l'instant, on ne peut pas car $u$ et $v$ ne sont pas dans les bons espaces. L'astuce consiste à considérer un second membre $g$ plus régulier dans (4.12) et d'utiliser le résultat de régularité suivant [23]. 
Théorème 4.10 (Meyers) Il existe $p^{\star}>2$, ne dépendant que de A et $\Omega$, t.q. si $g \in L^{\infty}(\Omega)$ et v solution de (4.12) alors $v \in W_{0}^{1, p^{\star}}(\Omega)$.

Soit donc $g \in L^{\infty}(\Omega)$ et $v$ solution de (4.12). On a alors $v \in W_{0}^{1, p^{\star}}(\Omega)$ grâce au théorème de Meyers. On note $\left(p^{\star}\right)^{\prime}$ l'exposant conjugué de $p^{\star}$, de sorte que $\left(p^{\star}\right)^{\prime}=\frac{p^{\star}}{p^{\star}-1}<2$. Par densité de $C_{c}^{\infty}(\Omega)$ dans $W_{0}^{1,\left(p^{\star}\right)^{\prime}}(\Omega)$, on déduit de 4.12) que

$$
\int A^{t} \nabla v \cdot \nabla w \mathrm{~d} x=\int g w \mathrm{~d} x, \quad \forall w \in W_{0}^{1,\left(p^{\star}\right)^{\prime}}(\Omega) .
$$

Or, comme $\left(p^{\star}\right)^{\prime}=\frac{p^{\star}}{p^{\star}-1}<2$, toute solution $u$ solution de 4.11) appartient à $W_{0}^{1,\left(p^{\star}\right)^{\prime}}(\Omega)$. On peut donc prendre $w=u$ dans 4.13. Mais comme $v \in W_{0}^{1, p^{\star}}(\Omega)$ avec $p^{\star}>2$, on a également, par 4.11), $\int_{\Omega} A^{t} \nabla v \cdot \nabla u \mathrm{~d} x=0$. On a donc $\int_{\Omega} g u \mathrm{~d} x=0$, pour tout $g \in L^{\infty}(\Omega)$. On en déduit que $u=0$ en prenant successivement $g=1_{\{u>0\}}$ puis $g=1_{\{u<0\}}$.

Pour $N=3$, le théorème de régularité de Meyers est encore vrai, la démonstration d'unicité est donc encore juste si $A$ est telle que $p^{\star}>N=3$ (afin de pouvoir prendre $v$ comme fonction test dans (4.9)). Mais il existe des fonctions matricielle $A=\left(a_{i j}\right)_{i, j=1, N}$ (à coefficients dans $L^{\infty}(\Omega)$ et coercive) pour lesquelles $p^{\star}<3$. L'unicité n'est plus assuré et on peut effectivement construire des cas pour lesquels la solution de (4.9) n'est pas unique (voir [25]).

Etape 5 Que peut-on rajouter à solution faible pour avoir l'unicité ? Pour $f \in L^{1}(\Omega)$, on montre qu'il existe une

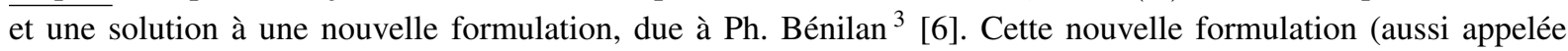
formulation entropique), est la suivante :

$$
\left\{\begin{array}{l}
u \in W_{0}^{1, q}(\Omega), \text { pour tout } 1 \leq q<\frac{N}{N-1}, \quad T_{k}(u) \in H_{0}^{1}(\Omega), \forall k>0, \\
\int_{\Omega} A \nabla u \cdot \nabla T_{k}(u-\varphi) \mathrm{d} x=\int f T_{k}(u-\varphi) \mathrm{d} x \quad \forall \varphi \in C_{c}^{\infty}(\Omega), \forall k \geq 0 .
\end{array}\right.
$$

où $T_{k}$ est la fonction troncature, définie par: $T_{k}(s)=\max (\min (k, s),-k)$. L'article de Ph. Bénilan et al. démontre qu'il existe une unique solution au problème (4.14) (qui est donc la solution mild de (4.8)). Ph. Bénilan conjecturait que le fait d'ajouter la condition $T_{k}(u) \in H_{0}^{1}(\Omega)$ (pour tout $k>0$ ) à la formulation (4.9) devait suffire à assurer l'unicité. De fait, dans les articles de Serrin et Prignet [27, 25], le contre exemple consiste à construire une solution non nulle de (4.9) avec $f=0$, mais cette solution ne vérifie pas $T_{k}(u) \in H_{0}^{1}(\Omega)$ pour $k>0$. La conjecture de Bénilan reste donc une conjecture...

Une autre question ouverte consiste à trouver une formulation semblable à 4.14 donnant l'unicité lorsque $f$ est une mesure sur $\Omega$.

Remarque 4.11 (Limitation de la méthode semi-groupe) Une difficulté importante de cette méthode par semigroupe est sa généralisation au cas où $A$ dépend de $t$.

\section{Méthode de Faedo-Galerkin (discrétisation en espace)}

Soit $\Omega$ un ouvert borné de $\mathbb{R}^{N}, T>0, u_{0}$ une fonction de $\Omega$ dans $\mathbb{R}$ et $f$ une fonction de $\left.\Omega \times\right] 0, T[$ dans $\mathbb{R}$. On considère l'équation de la chaleur

$$
\left\{\begin{array}{l}
\left.\partial_{t} u-\Delta u=f \text { dans } \Omega \times\right] 0, T[ \\
u=0 \text { sur } \partial \Omega \times] 0, T[ \\
u(., 0)=u_{0}
\end{array}\right.
$$

3. Philippe Bénilan (1941-2001), mathématicien français, spéialiste des EDP, professeur à l'université de Besançon. 
Pour $u_{0} \in L^{2}(\Omega)$ et $f$ dans un espace convenablement choisi, on va chercher $u \in L^{2}(] 0, T\left[, H_{0}^{1}(\Omega)\right)$ solution faible de ce problème. La méthode de Faedo ${ }^{4}$-Galerkin ${ }^{5}$ consiste à construire par approximation une suite de problèmes dont la solution existe et de montrer la convergence des solutions des problèmes approchées vers une fonction qui satisfait une formulation faible de (4.15).

Comme $H_{0}^{1}(\Omega)$ est séparable, il existe une suite $\left(E_{n}\right)_{n \in \mathbb{N}}$ d'espaces inclus dans $H_{0}^{1}(\Omega)$, de dimension finie, par exemple $\operatorname{dim} E_{n}=n$, et tels que $E_{n} \subset E_{n+1}$ et

$$
\overline{\bigcup_{n \in \mathbb{N}} E_{n}}=H_{0}^{1}(\Omega)
$$

Soit $\left\{e_{1} \ldots e_{n}\right\}$ une base de $E_{n}$. On cherche $u_{n}(t)=\sum_{i=1}^{n} \alpha_{i}(t) e_{i}$ t.q.

$$
\sum_{i=1}^{n} \int_{\Omega} \alpha_{i}^{\prime}(t) e_{i} e_{k} \mathrm{~d} x+\sum_{i=1}^{n} \int_{\Omega} \alpha_{i}(t) \nabla e_{i} \cdot \nabla e_{k} d x=\int_{\Omega} f(t) e_{k} \mathrm{~d} x, \text { pour tout } k=\{1 \ldots n\} \text { et } t>0,
$$

et les $\alpha_{i}(0)$ sont choisis pour que $u_{n}(0)=\sum_{i=1}^{n} \alpha_{i}(0) e_{i} \rightarrow u_{0}$ dans $L^{2}(\Omega)$ quand $n \rightarrow+\infty$. Ceci donne, pour tout $n \in \mathbb{N}^{\star}$, un système de $n$ équations différentielles avec condition initiale pour lequel on montre l'existence d'une solution. On cherche alors des estimations sur la solution $u_{n}$ qui permettent de passer à la limite quand $n \rightarrow+\infty$. Cette technique, qu'on va développer plus loin, permet de montrer que pour $f \in L^{2}(] 0, T\left[, H^{-1}\right)$ la limite $u$ des $u_{n}$ satisfait :

$$
\left\{\begin{array}{l}
u \in L^{2}(] 0, T\left[, H_{0}^{1}(\Omega)\right), \partial_{t} u \in L^{2}(] 0, T\left[, H^{-1}(\Omega)\right), u \in C\left([0, T], L^{2}(\Omega)\right), u(0)=u_{0} \text { p.p. } \\
\int_{0}^{T}\left\langle\partial_{t} u, v\right\rangle_{H^{-1}, H_{0}^{1}} \mathrm{~d} t+\int_{0}^{T}\left(\int_{\Omega} \nabla u \cdot \nabla v \mathrm{~d} x\right) \mathrm{d} t=\int_{0}^{T}\langle f, v\rangle_{H^{-1}, H_{0}^{1}} \mathrm{~d} t, \forall v \in L^{2}(] 0, T\left[, H_{0}^{1}(\Omega)\right) .
\end{array}\right.
$$

\section{Discrétisation en espace et en temps}

On discrétise le problème (4.15) par un schéma numérique, par exemple par éléments finis $P 1$ en espace et par un schéma d'Euler 6 implicite en temps. On obtient une solution approchée notée $u_{n}$. On obtient alors des estimations sur $u_{n}$. On passe ensuite à la limite lorsque $n \rightarrow+\infty$.

Pour $f \in L^{2}\left(0, T, L^{2}(\Omega)\right)$, on obtient ainsi pour le problème de la chaleur 4.15) que la limite $u$ du schéma numérique satisfait :

$$
\left\{\begin{aligned}
u \in L^{2}(] 0, T\left[, H_{0}^{1}(\Omega)\right) & \\
-\int_{0}^{T} \int_{\Omega} u \varphi_{t} \mathrm{~d} x \mathrm{~d} t+ & \int_{0}^{T} \int_{\Omega} \nabla u \cdot \nabla \varphi \mathrm{d} x \mathrm{~d} t-\int_{\Omega} u_{0}(x) \varphi(x, 0) \mathrm{d} x \\
& =\int_{0}^{T} \int_{\Omega} f \varphi \mathrm{d} x \mathrm{~d} t, \quad \forall \varphi \in C_{0}^{\infty}([0, T[\times \Omega))
\end{aligned}\right.
$$

On démontrera plus loin le lemme suivant, qui donne l'équivalence des formulations (4.16) et 4.17).

Lemme 4.12 (Équivalence des formulations faibles) Soit $\Omega$ un ouvert borné de $\mathbb{R}^{N}, T>0, u_{0} \in L^{2}(\Omega)$ et $f \in L^{2}\left(0, T, L^{2}(\Omega)\right)$. Alors, la fonction u est solution de (4.16) si et seulement si u est solution de (4.17).

4. Alessandro Faedo, 1913-2001 Mathématicien et homme politique italien, connu pour ses travaux en analyse numérique; il a été l'un des élèves de Leonida Tonelli et, après sa mort, il lui a succédé à la chaire d'analyse mathématique de l'université de Pise

5. Boris Grigoryevich Galerkin, 1871-1945, mathématician et ingénieur soviétique, connu pour sa méthode d'intégration approchée et spécialiste de mécanique des structures.

6. Leonhard Euler (1707-1783) mathématicien physicien, astronome, geographe et enginieur suisse qui fonda théorie des graphes et la topology and fit des découvertes fondamentales dans de nombreuses branches des mathématiques 


\section{Existence par degré topologique}

Dans le cas de problèmes non linéaires, plutôt que d'approcher ou de discrétiser, on peut se ramener à un problème linéaire et appliquer un argument de type degré topologique, en utilisant les résultats connus dans le cas linéaire. C'est ce qu'on fera par exemple sur le problème (voir le problème 4.33)

$$
\left\{\begin{array}{l}
\left.\partial_{t} u+\operatorname{div}(v(t, x) f(u))-\Delta u=0 \text { dans } \Omega \times\right] 0, T[ \\
u=0 \operatorname{sur} \partial \Omega \times] 0, T[ \\
u(\cdot, 0)=u_{0} .
\end{array}\right.
$$

\subsection{Intégration de fonctions à valeurs vectorielles}

On a utilisé dans les formulations faible de la section 4.1 les espaces $L^{2}(] 0, T\left[, L^{2}(\Omega)\right), L^{2}(] 0, T\left[, H_{0}^{1}(\Omega)\right)$ et $L^{2}(] 0, T\left[, H^{-1}(\Omega)\right)$. Ce sont des espaces pour lesquels on utilise l'intégration de fonctions à valeurs dans un espace de Banach de dimension infinie (comme, par exemple, $L^{2}(\Omega)$ ). Il nous faut donc préciser comment on définit une telle intégrale. Rappelons d'abord comment on définit l'intégrale sur $\mathbb{R}$. On commence par définir la notion de mesurabilité. On rappelle que si $(X, T, m)$ est un espace mesuré, une fonction $f: X \rightarrow \mathbb{R}$ est mesurable si $f^{-1}(B) \in T$ pour tout $B \in \mathcal{B}(\mathbb{R})$. Une généralisation facile de cette notion de mesurabilité aux espaces de Banach est donc la suivante : une fonction $f$ de $X$ dans $E$ est mesurable si $f^{-1}(B) \in T$ pour tout $B \in \mathcal{B}(E)$ (où $\mathcal{B}(E)$ est la tribu engendrée par les ouverts de $E$ ). En fait cette notion n'a aucun intérêt du point de vue de l'intégrale si $E$ n'est pas séparable. Par ailleurs, une fonction réelle $f: X \rightarrow \mathbb{R}$ est mesurable si et seulement si $f$ est une limite simple de fonctions étagées, i.e. si et seulement si il existe une suite $\left(f_{n}\right)_{n \in \mathbb{N}}$ de fonctions étagées t.q. $f_{n} \rightarrow f$ simplement (cette équivalence n'est plus vraie si $f$ est à valeurs dans $E$ où est $E$ est un espace de Banach non séparable). C'est cette dernière notion (limite simple de fonctions étagées) qui est intéressante pour définir l'intégrale. Plus précisément, comme l'intégrale ne voit pas les changements d'une fonction sur un ensemble de mesure nulle, nous allons définir la notion de " $m$-mesurabilité" de la manière suivante :

Définition 4.13 (Fonction $\boldsymbol{m}$-mesurable) Soit $(X, T, m)$ un espace mesuré. On dit qu'une fonction $f$, définie de $X$ à valeurs dans $\mathbb{R}$ ou à valeurs dans un Banach $E$ est $m$-mesurable si elle est limite presque partout de fonctions étagées.

Proposition 4.14 (Mesurabilité et $\boldsymbol{m}$-mesurabilité) Soit $(X, T, m)$ un espace mesuré.

1. Une fonction $f$ de $X$ à valeurs dans $\mathbb{R}$ est m-mesurable si et seulement si il existe $g$ mesurable t.q. $f=g$ p.p..

2. Soit $E$ est un espace de Banah séparable et $f$ une fonction de $X$ dans E. Alors,

(a) $f$ est mesurable au sens " $f^{-1}(B) \in T$ pour tout $B \in B(E)$ " si et seulement si $f$ est limite simple de fonctions étagées.

(b) Comme dans le cas réel, la fonction $f$ de X dans E est m-mesurable si et seulement si il existe $g$ mesurable t.q. $f=g$ p.p..

Soit $(X, T, m)$ un ensemble mesuré, $E$ un espace de Banach et $f$ une fonction $m$-mesurable de $X$ dans $E$. Il est facile de voir que la fonction $x \mapsto\|f(x)\|_{E}$ est $m$-mesurable de $X$ dans $\mathbb{R}_{+}$. la quantité $\int\|f(x)\|_{E} d m(x)$ est donc parfaitement définie dans $\mathbb{R}_{+} \cup\{+\infty\}$. Ceci permet de définir l'espace $\mathcal{L}_{E}^{1}(X, T, m)$.

Définition 4.15 (L'espace $\mathcal{L}_{E}^{1}(X, T, m)$ ) Soit $(X, T, m)$ un ensemble mesuré, E un espace de Banach et $f$ une fonction m-mesurable de $X$ dans $E$. La fonction $f$ appartient à $\mathcal{L}_{E}^{1}(X, T, m)$ si

$$
\int\|f(x)\|_{E} d m(x)<+\infty
$$


On veut maintenant définir l'intégrale d'un élément de $\mathcal{L}_{E}^{1}(X, T, m)$ où $(X, T, m)$ est un espace mesuré et $E$ un espace de Banach. Soit $f \in \mathcal{L}_{E}^{1}(X, T, m)$. On ne peut pas utiliser la même technique que pour $E=\mathbb{R}$ ( c'est-àdire décomposer $f$ en $f^{+}$et $f^{-}$et commencer par intégrer des fonctions mesurables positives). Par contre, comme $f$ est $m$-mesurable, on sait qu'il existe une suite $\left(f_{n}\right)_{n \in \mathbb{N}}$ de fonctions étagées t.q. $f_{n} \rightarrow f$ p.p.. Il existe donc $A \in T$ t.q. $m\left(A^{c}\right)=0$ et $f_{n}(x) \rightarrow f(x)$ pour tout $x \in A$. On pose alors

$$
A_{n}=\left\{x \in A ;\left\|f_{n}(x)\right\|_{E} \leq 2\|f(x)\|_{E}\right\} \text { et } g_{n}=f_{n} 1_{A_{n}} .
$$

(le nombre 2 pourrait être remplacé ici par n'importe quel nombre $>1$ et $A$ pourrait être remplacé par un autre ensemble vérifiant les mêmes propriétés. Cela ne changerait pas la définition de l'intégrale donnée dans la définition 4.16,) La suite $\left(g_{n}\right)_{n \in \mathbb{N}}$ vérifie :

$$
\begin{aligned}
& g_{n} \text { étagée pour tout } n \in \mathbb{N}, \\
& g_{n} \rightarrow f \text { p.p. } \\
& \int\left\|g_{n}-f\right\|_{E} d m \rightarrow 0 \text { par convergence dominée. }
\end{aligned}
$$

La définition de l'intégrale d'une fonction étagée est immédiate. On pose

$$
I_{n}=\int g_{n} d m
$$

On peut alors montrer que $\left(I_{n}\right)_{n \in \mathbb{N}}$ est une suite de Cauchy dans $E$. Cette suite converge donc dans $E$. On peut aussi montrer que la limite de cette suite ne dépend que de $f$ (et non du choix de $f_{n}$ et de $A$ ). Il est donc naturel de définir l'intégrale de $f$ comme la limite de cette suite. C'est ce qui est fait dans la définition 4.16

Définition 4.16 (Intégrale à valeurs dans $\boldsymbol{E}$ ) Soit $f: X \rightarrow E$ où $(X, T, m)$ est un espace mesuré et $E$ un espace de Banach.

1. (Rappel) $f \in \mathcal{L}_{E}^{1}(X, T, m)$ si f est m-mesurable et $\int\|f\|_{E} d m<+\infty$.

2. (Intégrale) Soit $f \in \mathcal{L}_{E}^{1}(X, T, m)$. Soit $\left(f_{n}\right)_{n \in \mathbb{N}}$ suite de fonctions étagées t.q. $f_{n} \rightarrow f$ p.p.. Soit $A \in T$ t.q. $m\left(A^{c}\right)=0$ et $f_{n}(x) \rightarrow f(x)$ pour tout $x \in A$. On pose $A_{n}=\left\{x \in A\right.$ t.q. $\left.\left\|f_{n}(x)\right\|_{E} \leq 2\|f(x)\|\right\}$ et $g_{n}=f_{n} 1_{A_{n}}$. On définit l'intégrale de $f$ par :

$$
\int f d_{m}=\lim _{n \rightarrow+\infty} \int g_{n} d m \in E
$$

Avec la relation d'équivalence $=$ p.p., on définit alors $L_{E}^{1}(X, T, m)$. Il est alors facile aussi de deviner les définitions de $\mathcal{L}_{E}^{p}(X, T, m)$ et $L_{E}^{p}(X, T, m)$ pour tout $1 \leq p \leq+\infty$. Un élément de $L_{E}^{p}(X, T, m)$ est donc un ensemble d'éléments de $\mathcal{L}_{E}^{p}(X, T, m)$ (et deux fonctions appartenant à cet ensemble sont égales p.p.). Comme d'habitude en intégration, si $F \in L_{E}^{p}(X, T, m)$, on confond $F$ et $f$ si $f \in F$ (de sorte que l'on raisonne comme si $\left.F \in \mathcal{L}_{E}^{p}(X, T, m)\right)$.

Proposition 4.17 Soit $1 \leq p \leq+\infty,(X, T, m)$ un espace mesuré $\sigma$-fini et $E$ un espace de Banach. Alors

1. $L_{E}^{p}(X, T, m)$ (avec sa norme naturelle) est complet. C'est donc un espace de Banach.

2. Si $p<+\infty$ et E est séparable, $L_{E}^{p}(X, T, m)$ est séparable.

3. Si $p=2$ et E est un Hilbert, alors $L_{E}^{2}(X, T, m)$ est aussi un espace de Hilbert, dont le produit scalaire est défini par:

$$
(u \mid v)_{L_{E}^{2}(X, T, m)}=\int_{X}(u(x) \mid v(x))_{E} d m(x) .
$$

Par conséquent, de toute suite bornée de $L_{E}^{2}(X, T, m)$ on peut extraire une sous-suite faiblement convergente dans $L_{E}^{2}(X, T, m)$. 
4. Si $1<p<+\infty$ et si E est un espace de Banach réflexif séparable, l'espace $L_{E}^{p}(X, T, m)$ est alors un espace de Banach réflexif séparable.

5. (Dualité dans $L_{E}^{p}(X, T, m)$ ) Soit $1 \leq p \leq+\infty, p^{\prime}=p /(p-1)$ et $v \in L_{E^{\prime}}^{p^{\prime}}(X, T, m)$. Pour $u \in L_{E}^{p}(X, T, m)$, l'application $T_{v}: u \mapsto \int\langle v, u\rangle_{E^{\prime}, E} d m$ est bien définie, linéaire et continue de $L_{E}^{p}(X, T, m)$ dans $\mathbb{R}$. (On a donc $\left.T_{v} \in\left(L_{E}^{p}(X, T, m)\right)^{\prime}\right)$.

De plus, l'application $T: v \mapsto T_{v}$ est une isométrie de $L_{E^{\prime}}^{p^{\prime}}(X, T, m)$ sur son image (qui est donc une partie de $\left.L_{E}^{p}(X, T, m)^{\prime}\right)$.

Si $E^{\prime}$ est séparable et $p<\infty$, l'image de T est $\left(L_{E}^{p}(X, T, m)\right)^{\prime}$ en entier.

6. (Convergence dominée) Soit $1 \leq p<+\infty$ et $\left(u_{n}\right)_{n \in \mathbb{N}}$ une suite de $L_{E}^{p}(X, T, m)$. Si

(a) $u_{n} \rightarrow u \quad$ p.p.,

(b) $\left\|u_{n}\right\|_{E} \leq G$ p.p. pour tout $n \in \mathbb{N}$ avec $G \in \mathcal{L}_{\mathbb{R}}^{p}(X, T, m)$,

alors $u_{n} \rightarrow u$ dans $L_{E}^{p}(X, T, m)$.

Démonstration de la proposition 4.17

Les propriétés 1-4 ne sont démontrées pas ici. La propriété 5 est partiellement démontrée dans l'exercice 4.2 La propriété 6 est plus facile. Il suffit de remarquer que

$$
\left\|u_{n}-u\right\|_{E} \leq\left\|u_{n}\right\|_{E}+\|u\|_{E} \leq 2 G .
$$

On a donc $\left\|u_{n}-u\right\|_{E}^{p} \leq 2^{p}|G|^{p} \in \mathcal{L}_{\mathbb{R}}^{1}(X, T, m)$. Par le théorème de convergence dominée pour les fonctions à valeurs dans $\mathbb{R}$, on en déduit que $\int\left\|u_{n}-u\right\|_{E}^{p} d m \rightarrow 0$ et donc que $u_{n} \rightarrow u$ dans $L_{E}^{p}(X, T, m)$.

Remarque 4.18 Soit $E$ une espace de Banach. On dit que $E$ est uniformément convexe si

$$
\forall \eta>0, \exists \varepsilon>0 \text { t.q. }\left(\|x\|_{E}=1,\|y\|_{E}=1,\|x-y\| \geq \eta\right) \Rightarrow\left\|\frac{x+y}{2}\right\| \leq 1-\varepsilon .
$$

Les espaces $L^{p}(\Omega)$ avec $1<p<+\infty$ et $\Omega$ ouvert de $\mathbb{R}^{N}$ sont uniformément convexes. Une conséquence importante du fait que $E$ soit un Banach uniformément convexe est que si $\left(x_{n}\right)_{n \in \mathbb{N}}$ est une suite de $E$ t.q. $x_{n} \rightarrow x$ faiblement dans $E$ et $\left\|x_{n}\right\|_{E} \rightarrow\|x\|_{E}$, alors $x_{n} \rightarrow x$ dans $E$. Cette propriété permet éventuellement de simplifier certaines démonstrations de la proposition 4.17 (mais n'est pas nécessaire).

Différentes notions de dérivée pour une fonction à valeurs vectorielles.

Plaçons nous maintenant dans le cadre qui va nous intéresser pour les équations paraboliques, c'est à dire :

$$
(X, T, m)=(] 0, T[, \mathcal{B}(] 0, T[), \lambda) .
$$

On va d'abord définir proprement la dérivée par rapport au temps, $\partial_{t} u=d u / d t$, pour une fonction de $] 0, T[$ dans $E$ qui n'est pas dérivable au sens classique ( c'est-à-dire au sens de l'existence de la limite, dans $E$, du quotient différentiel habituel).

On note maintenant $L_{E}^{p}(] 0, T[)$ ou $L^{p}(] 0, T[, E)$ l'espace $L_{E}^{p}(] 0, T[, \mathcal{B}(] 0, T[), \lambda)$ et $L_{E, l o c}^{1}(] 0, T[)$ ou $L_{\text {loc }}^{1}(] 0, T[$, $E$ ) l'ensemble des (classes de) fonctions à valeurs dans $E$ localement intégrables sur $] 0, T[$ (avec la mesure de Lebesgue).

Lemme 4.19 Soit $u \in L_{1 o c}^{1}(] 0, T[, E)$ on suppose que $\int_{0}^{T} u(t) \varphi(t) \mathrm{d} t=0$ pour tout $\varphi \in C_{c}^{\infty}(] 0, T[, \mathbb{R})$. Alors $u=0 p \cdot p$. 
Démonstration: Semblable au cas $E=\mathbb{R}$.

On peut ainsi définir la dérivée par transposition de $u$.

Définition 4.20 (Dérivée par transposition) Soit $E$ un espace de Banach, $1 \leq p \leq+\infty$ et $u \in L_{E}^{p}(] 0, T[)$. On note $\mathcal{D}$ l'espace $C_{c}^{\infty}(] 0, T[, \mathbb{R})$ et $\mathcal{D}_{E}^{\star}$ l'ensemble des applications linéaires de $\mathcal{D}$ dans $E$. On définit $\partial_{t} u \in \mathcal{D}_{E}^{\star}$ par:

$$
\left\langle\partial_{t} u, \varphi\right\rangle_{\mathcal{D}_{E}^{\star}, \mathcal{D}}=-\int_{0}^{T} u(t) \varphi^{\prime}(t) \mathrm{d} t \in E .
$$

Remarque 4.21 Si $u \in C^{1}(] 0, T[, E)$ on a

$$
-\int_{0}^{T} u(t) \varphi^{\prime}(t)=\int_{0}^{T} u^{\prime}(t) \varphi(t)=\left\langle\partial_{t} u, \varphi\right\rangle_{\mathcal{D}_{E}^{\star}, \mathcal{D}}
$$

On confond alors $u^{\prime}$ (dérivée classique) avec $\partial_{t} u$ (dérivée par transposition), c'est à dire la fonction $u^{\prime}$ qui appartient à $C([0, T], E)$ avec l'application linéaire de $\mathcal{D}$ dans $E$ notée $\partial_{t} u$. (Grâce au lemme 4.19, la fonction $u^{\prime}$ est entièrement déterminée par $\partial_{t} u$, ce qui justifie la confusion entre $u^{\prime}$ et $\partial_{t} u$.)

Définition 4.22 (Dérivée faible) Soit $E$ et $F$ deux espaces de Banach et $1 \leq p, q \leq+\infty$. On suppose qu'il existe un espace vectoriel $G$ t.q. $E \subset G$ et $F \subset G$. Soit $u \in L_{E}^{p}(] 0, T\left[\right.$ ) (on a donc $\partial_{t} u \in \mathcal{D}_{E}^{\star}$ ). On dit que $\partial_{t} u \in L_{F}^{q}(] 0, T[)$ si il existe une fonction $v \in L_{F}^{q}(] 0, T[)$ telle que

$$
\left\langle\partial_{t} u, \varphi\right\rangle_{\mathcal{D}_{E}^{\star}, \mathcal{D}}=-\underbrace{\int_{0}^{T} u(t) \varphi^{\prime}(t) \mathrm{d} t}_{\in E}=\underbrace{\int_{0}^{T} v(t) \varphi(t) \mathrm{d} t}_{\in F} .
$$

Cette égalité n'a de sens que s'il existe un espace vectoriel $G$ tel que $E \subset G$ et $F \subset G$. Dans ce cas, on confond $\partial_{t} u \in \mathcal{D}_{E}^{\star}$ et $v \in L_{F}^{q}(] 0, T[$ ). (Ici aussi le lemme 4.19 est utile pour faire cette confusion car, grâce au lemme 4.19. si la fonction $v$ existe elle est alors unique.)

En résumé, on a donc trois notions de dérivée d'une fonction $u$ de $] 0, T[$ à valeurs dans $E$ (espace de Banach).

(1) Dérivée classique : $\left.u^{\prime}:\right] 0, T[\rightarrow E$ (existe rarement...).

(2) Dérivée par transposition : $\partial_{t} u \in \mathcal{D}_{E}^{\star}$ (existe dès que $u \in L_{E}^{p}(] 0, T[)$ ).

(3) Dérivée faible : $\partial_{t} u \in L_{F}^{q}(] 0, T[)$ où $F$ est un Banach tel que $E \subset G$ et $F \subset G$ avec $G$ espace vectoriel.

Exemples Soit $\Omega$ un ouvert borné de $\mathbb{R}^{N}$.

1. $E=H_{0}^{1}(\Omega), F=H^{-1}(\Omega)$. On a alors $E, F \subset G=\mathcal{D}^{\star}(\Omega)$.

2. $1 \leq p<+\infty, q=p /(p-1), E=W_{0}^{1, p}(\Omega), F=W^{-1, q}(\Omega)$. On a aussi $E, F \subset G=\mathcal{D}^{\star}(\Omega)$.

3. $E=H^{1}(\Omega) F=\left(H^{1}(\Omega)\right)^{\prime}$. Pour cet exemple, on a $F \not \subset \mathcal{D}^{\star}(\Omega)$. En effet, on prend par exemple $T$ définie, pour $v \in H^{1}(\Omega)$, par

$$
T(v)=\int_{\partial \Omega} v d \gamma(x)
$$

L'application $T$ est bien une application linéaire continue sur $H^{1}(\Omega)$, donc $T \in H^{1}(\Omega)^{\prime}$. Mais $T=0$ sur $\mathcal{D}(\Omega)$, et donc, comme $T$ n'est pas l'application nulle, $F$ ne s'injecte pas dans $\mathcal{D}^{\star}(\Omega)$.

Dans cet exemple, pour injecter $E$ et $F$ dans le même espace $G$, on commence par identifier $\left(L^{2}(\Omega)\right)^{\prime}$ (dual topologique de $L^{2}(\Omega)$ ) avec $L^{2}(\Omega)$. Ceci est possible car si $T \in L^{2}(\Omega)^{\prime}$, par le théorème de représentation de Riesz, il existe une et une seul fonction $u \in L^{2}(\Omega)$ telle que $T(v)=(v / u)_{2}$ pour tout $v \in L^{2}(\Omega)$. 
On confond alors $u$ et $T$. Par cette identification, on a donc $L^{2}(\Omega)^{\prime}=L^{2}(\Omega)$. On remarque maintenant que, si $E, H$ sont deux espaces de Banach tels que $E \subset H$, et avec injection continue de $E$ dans $H$ et densité de $E$ dans $H$, on a alors $H^{\prime} \subset E^{\prime}$. On prend ici $E=H^{1}(\Omega)$ et $H=L^{2}(\Omega)$ et on a donc (grâce à l'identification entre $L^{2}(\Omega)$ et $\left.L^{2}(\Omega)^{\prime}\right) H^{\prime}=L^{2}(\Omega) \subset H^{1}(\Omega)^{\prime}$. Finalement, on a donc $E, F \subset G$ en prenant $G=\left(H^{1}(\Omega)\right)^{\prime}$. On a ainsi mis $E$ et $F$ dans le même espace $G=\left(H^{1}(\Omega)\right)^{\prime}$ grâce à l'identification de $L^{2}(\Omega)$ avec $\left(L^{2}(\Omega)\right)^{\prime}$.

4. Dans l'exemple précédent, on a deux espaces de Banach $E$ et $H$ avec $E \subset H$, injection continue de $E$ dans $H$ et densité de $E$ dans $H$. On a alors $H^{\prime} \subset E^{\prime}$. L'espace $H$ est une espace de Hilbert. En identifiant $H$ avec $H^{\prime}$, on a donc $E \subset E^{\prime}$. Mais si l'objectif est seulement d'avoir $E \subset E^{\prime}$, il n'est pas nécessaire d'avoir $H^{\prime} \subset E^{\prime}$ et on peut retirer l'hypothèse de densité de $E$ dans $H$. Plus précisément, soit $E$ un espace de Banach et $H$ un espace de Hilbert avec $E \subset H$ et injection continue de $E$ dans $H$. On identifie $H$ avec $H^{\prime}$. Soit maintenant $u \in E$, comme $u \in H$, on a donc identification entre $u$ et $T_{u}$ qui est l'application $v \mapsto(v \mid u)_{H}$ de $H$ dans $\mathbb{R}$. On peut alors aussi identifier $u$ (qui est dans $E$ ) avec la restriction de $T_{u}$ à $E$ (qui est un élément de $E^{\prime}$ ). Cette identification est légitime car si $u_{1}$ et $u_{2}$ sont deux éléments différents de $E$, les restrictions de $T_{u_{1}}$ et $T_{u_{2}}$ à $E$ sont des applications différentes. On a ainsi $E \subset E^{\prime}$. Dans cet exemple, il faut toutefois noter que (en l'absence de densité de $E$ dans $H$ ) des éléments différents de $H^{\prime}$ ont la même restriction à $E$ (et peuvent donc correspondre au même élément de $E$ ).

Un exemple intéressant de cette situation est obtenu en prenant $H=L^{2}(\Omega)^{N}$ (avec $\Omega$ ouvert borné de $\mathbb{R}^{N}$, $N>1)$ et $E=\{u \in H: \operatorname{div}(u)=0\}$.

Remarque 4.23 (Comparaison de $L^{p}(] 0, T\left[, L^{p}(\Omega)\right)$ et $\left.L^{p}(\Omega \times] 0, T[)\right)$

Soient $T>0, \Omega$ un ouvert de $\mathbb{R}^{N}$ et $1 \leq p<+\infty$.

Soit $u \in L^{p}(] 0, T\left[, L^{p}(\Omega)\right)$. Il existe alors $v \in L^{p}(\Omega \times] 0, T[)$ tel que $u(t)=v(\cdot, t)$ p.p. (dans $\Omega$ ) et pour presque tout $t \in] 0, T[$. (Noter que cette égalité est vraie quel que soit les représentants choisis pour $u$ et $v$.)

Récripoquement, si $v \in L^{p}(\Omega \times] 0, T[)$, il existe $u \in L^{p}(] 0, T\left[, L^{p}(\Omega)\right)$ tel que $u(t)=v(\cdot, t)$ p.p. (dans $\left.\Omega\right)$ et pour presque tout $t \in] 0, T[$.

En conservant ces notations, on peut alors comparer $d u / d t$ (qui s'applique à un élément de $C_{c}^{\infty}(] 0, T[, \mathbb{R})$ ) et $\partial v / \partial t$ (qui s'applique à un élément de $C_{c}^{\infty}(\Omega \times] 0, T[, \mathbb{R})$ ). La réponse est que pour tout $\varphi \in C_{c}^{\infty}(] 0, T[, \mathbb{R})$ et tout $\psi \in C_{c}^{\infty}(\Omega, \mathbb{R})$ on a

$$
\begin{aligned}
\int_{\Omega}\left\langle\frac{d u}{d t}, \varphi\right\rangle_{\mathcal{D}_{E}^{\star}, \mathcal{D}}(x) \psi(x) \mathrm{d} x=-\int_{\Omega} & \left(\int_{0}^{T} u(t) \varphi^{\prime}(t) d t\right)(x) \psi(x) \mathrm{d} x \\
& =-\int_{\Omega} \int_{0}^{T} v(x, t) \varphi^{\prime}(t) \psi(x) \mathrm{d} x \mathrm{~d} t=\left\langle\frac{\partial v}{\partial t}, \varphi \psi\right\rangle_{\mathcal{D}^{\star}(\Omega \times] 0, T[), \mathcal{D}(\Omega \times] 0, T[)}
\end{aligned}
$$

Proposition 4.24 (Commutation de l'action et intégrale) Soit $E$ un espace de Banach, $u \in L_{E}^{1}(] 0, T[), \psi \in E^{\prime}$ et $\varphi \in C_{c}^{\infty}(] 0, T[)$. Alors,

$$
<\psi, \int_{0}^{T} u(t) \varphi(t) \mathrm{d} t>_{E^{\prime}, E}=\int_{0}^{T}<\psi, u(t)>_{E^{\prime}, E} \varphi(t) \mathrm{d} t .
$$

La preuve de la proposition 4.24 est laissée en exercice.

Lemme 4.25 Soit $E$ un espace de Banach, $1 \leq p \leq+\infty$ et $u \in L_{E}^{p}(] 0, T[)$. On suppose que $\partial_{t} u \in L_{E}^{p}(] 0, T[)$ (on a alors $u \in W_{E}^{1, p}(] 0, T[)$. Alors, $u \in C([0, T], E)$, et même $u \in C^{0,1-1 / p}([0, T], E)$. Plus précisément, il existe $a \in E$ t.q. $u(t)=a+\int_{0}^{t} \partial_{t} u(s) d s$ pour presque tout $\left.t \in\right] 0, T[$ et u est alors identifié à la fonction (continue $\operatorname{sur}[0, T]) t \mapsto a+\int_{0}^{t} \partial_{t} u(s) d s$. 
Démonstration La démonstration est semblable au cas $E=\mathbb{R}$. On pose $\partial_{t} u=v \in L_{E}^{p}(] 0, T[)$ et on définit $w$ $\operatorname{par} w(t)=\int_{0}^{t} v(s) d s$, de sorte que $w \in C([0, T], E)$. On montre assez facilement que $w_{t}=v=\partial_{t} u$ et donc $(w-u)_{t}=0$. On a donc

$$
\int_{0}^{T}(w-u) \varphi_{t} \mathrm{~d} t=0 \text { pour tout } \varphi \in C_{c}^{\infty}(] 0, T[, \mathbb{R}) .
$$

On choisit maintenant une fonction $\varphi_{0} \in C_{c}^{\infty}(] 0, T[, \mathbb{R})$ t.q. $\int_{0}^{T} \varphi_{0}(s) d s=1$. Pour $\psi \in C_{c}^{\infty}(] 0, T[, \mathbb{R})$ on définit $\varphi$ par

$$
\varphi(t)=\int_{0}^{t} \psi(s) d s-\int_{0}^{t} \varphi_{0}(s) d s \int_{0}^{T} \psi(s) d s
$$

de sorte que $\varphi \in C_{c}^{\infty}(] 0, T[, \mathbb{R})$ et on peut prendre $\varphi$ dans 4.18 . On en déduit (la preuve est laissée en exercice) qu'il existe $a \in E$ t.q. $w-u=a$ p.p. Donc $u \in C([0, T], E)$. Avec l'inégalité de Hölder, on montre ensuite que $u \in C^{0,1-1 / p}$.

Nous allons maintenant montrer un lemme plus difficile donnant aussi la continuité de $u$. On suppose que $E$ est un espace de Banach et $F$ un espace de Hilbert t.q. $E \subset F$, avec injection continue, et $E$ dense dans $F$. On a donc aussi $F^{\prime} \subset E^{\prime}$. Comme $F$ est un espace de Hilbert, on peut identifier $F$ avec son dual par le théorème de représentation de Riesz, c'est-à-dire que l'on identifie $v \in F$ avec l'application $T_{v}: u \mapsto(v \mid u)_{F}$ (qui est un élément de $F^{\prime}$ ). L'application $v \mapsto T_{v}$ est une isométrie (bijective) de $F$ dans $F^{\prime}$. Avec cette identification, on a donc $E \subset F=F^{\prime} \subset E^{\prime}$. Donc, tout élément de $E$ est alors un élément de $E^{\prime}$. Pour $u, v \in E$, on a

$$
<v, u\rangle_{E^{\prime}, E}=(v \mid u)_{F},
$$

c'est-à-dire $\left\langle T_{v}, u>_{E^{\prime}, E}=(v \mid u)_{F}\right.$, où $T_{v}$ est l'élément de $F^{\prime}$ identifié à $v \in F$. Avec cette identification de $F$ avec $F^{\prime}$, on va maintenant donner un résultat de continuité de $u$ dans $F$ si $u \in L^{2}(] 0, T[, E)$ et $\partial_{t} u \in$ $L^{2}(] 0, T\left[, E^{\prime}\right)$. Il faut faire très attention que cette dernière hypothèse n'a de sens que par l'identification de $F$ avec $F^{\prime}$ (si on change d'espace $F$, on change le sens de $\partial_{t} u \in L^{2}(] 0, T\left[, E^{\prime}\right)$, alors que $E^{\prime}$ n'est pas changé!).

Lemme 4.26 (CS de Continuité) Soit $E$ un espace de Banach et $F$ un espace de Hilbert tels que $E \subset F$, avec injection continue, et $E$ dense dans $F$. On identifie $F$ avec $F^{\prime}$ (de sorte que $E \subset F=F^{\prime} \subset E^{\prime}$ ). Soit $u \in$ $L_{E}^{2}(] 0, T[)$, on suppose que $\partial_{t} u \in L_{E^{\prime}}^{2}(] 0, T[)$. Alors $u \in C([0, T], F)$ et, pour tout $t_{1}, t_{2} \in[0, T]$ on a

$$
\left\|u\left(t_{1}\right)\right\|_{F}^{2}-\left\|u\left(t_{2}\right)\right\|_{F}^{2}=2 \int_{t_{2}}^{t_{1}}<\partial_{t} u, u>_{E^{\prime}, E} \mathrm{~d} t .
$$

Démonstration On va montrer que $u \in C([0, T[), F)$.

Soit $\rho \in C_{c}^{\infty}(]-2,-1[)$ tel que $\int_{\mathbb{R}} \rho \mathrm{d} x=1$ et $\rho \geq 0$. Pour $n \in \mathbb{N}^{\star}$, on pose $\rho_{n}=n \rho(n \cdot)$ de sorte que le support de $\rho_{n}$ est inclus dans $]-\frac{2}{n},-\frac{1}{n}\left[\right.$ et que $\int \rho_{n} \mathrm{~d} x=1$. On définit $u_{n}$ par convolution avec $\rho_{n}$, c'est-àdire $u_{n}=\tilde{u} \star \rho_{n}$ avec

$$
\begin{aligned}
& \tilde{u}=u \text { sur }] 0, T[ \\
& u=0 \text { sur }] 0, T\left[^{c} .\right.
\end{aligned}
$$

On a donc $u_{n} \rightarrow u$ dans $L_{E}^{2}(] 0, T[), u_{n} \in C_{c}^{\infty}(\mathbb{R}, E)$ et $u_{n}^{\prime}=\tilde{u} \star \rho_{n}^{\prime}$.

Soit $t \in \mathbb{R}$, on a

$$
u_{n}^{\prime}(t)=\int_{0}^{T} u(s) \rho_{n}^{\prime}(t-s) d s .
$$


On remarque maintenant que $\rho_{n}(t-s)=0$ si $\left.t-s \notin\right] \frac{-2}{n}, \frac{-1}{n}[$ c'est à dire $s \notin] t+\frac{1}{n}, t+\frac{2}{n}[$.

Soit $\varepsilon>0$ et $t \in\left[0, T-\varepsilon\left[\right.\right.$. Pour $n \geq n_{0}$ avec $n_{0}$ t.q. $\frac{2}{n_{0}}<\varepsilon$ on a $t+\frac{2}{n}<T$ et donc

$$
\rho_{n}(t-\cdot) \in C_{c}^{\infty}(] 0, T[) .
$$

On a alors

$$
\begin{aligned}
& u_{n}^{\prime}(t)=<\partial_{t} u, \rho_{n}(t-\cdot)>_{\mathcal{D}_{E}^{\star}, \mathcal{D}} \\
& =\int_{0}^{T} \partial_{t} u(s) \rho_{n}(t-s) d s \in E^{\prime}, \operatorname{car} \partial_{t} u \in L_{E^{\prime}}^{2}(] 0, T[) .
\end{aligned}
$$

Donc, $u_{n}^{\prime}=\tilde{\partial_{t} u} \star \rho_{n} \operatorname{sur}[0, T-\varepsilon[$, avec

$$
\tilde{\partial}_{t} u=\left\{\begin{array}{l}
\left.\partial_{t} u \operatorname{sur}\right] 0, T[ \\
0 \text { sur }] 0, T\left[{ }^{c}\right.
\end{array}\right.
$$

Mais $\tilde{\partial_{t} u} \star \rho_{n} \rightarrow \tilde{\partial}_{t} u$ dans $L^{2}\left(\mathbb{R}, E^{\prime}\right)$. Donc $u_{n}^{\prime}=\tilde{\partial_{t} u} \star \rho_{n} \rightarrow \partial_{t} u$ dans $L_{E^{\prime}}^{2}(] 0, T-\varepsilon[)$.

En résumé, $\left\{\begin{array}{l}u_{n} \rightarrow u \text { dans } L_{E}^{2}(] 0, T[) \\ u_{n}^{\prime} \rightarrow \partial_{t} u \text { dans } L_{E^{\prime}}^{2}(] 0, T-\varepsilon[) \quad \forall \varepsilon>0 .\end{array}\right.$

On va maintenant montrer que $\left(u_{n}(t)\right)_{n \in \mathbb{N}}$ est une suite de Cauchy dans $F$ pour tout $t \in[0, T$, et même, pour tout $\varepsilon>0$, uniformément si $t \in[0, T-\varepsilon]$.

Soit $\varepsilon>0$ et $\varphi \in C^{1}([0, T], \mathbb{R})$ définie par $\varphi(t)=\left\|u_{n}(t)-u_{m}(t)\right\|_{F}^{2}$. On a donc, pour $\left.t \in\right] 0, T[$,

$$
\varphi^{\prime}(t)=2\left((\underbrace{\left.u_{n}^{\prime}-u_{m}^{\prime}\right)(t)}_{\in E} \mid \underbrace{\left(u_{n}-u_{m}\right)(t)}_{\in E})_{F} .\right.
$$

Soient $t_{1}, t_{2} \in[0, T-\varepsilon]$, on a

$$
\begin{aligned}
\varphi\left(t_{2}\right)-\varphi\left(t_{1}\right) & =\int_{t_{1}}^{t_{2}} 2\left(u_{n}^{\prime}(s)-u_{n}^{\prime}(s) \mid u_{n}(s)-u_{m}(s)\right)_{F} d s \\
& =2 \int_{t_{1}}^{t_{2}}<\left(u_{n}\right)_{t}(s)-\left(u_{m}\right)_{t}(s), u_{n}(s)-u_{m}(s)>_{E^{\prime}, E} d s .
\end{aligned}
$$

Donc,

$$
\begin{aligned}
\varphi\left(t_{2}\right)-\varphi\left(t_{1}\right) & \leq 2\left(\int_{0}^{T-\varepsilon}\left\|\left(u_{n}\right)_{t}-\left(u_{m}\right)_{t}\right\|_{E^{\prime}}^{2} d s\right)^{1 / 2}\left(\int_{0}^{T}\left\|u_{n}-u_{m}\right\|_{E}^{2} d s\right)^{1 / 2} \\
& \leq 2\left\|\left(u_{n}\right)_{t}-\left(u_{m}\right)_{t}\right\|_{L_{E^{\prime}}^{2}}\left\|u_{n}-u_{m}\right\|_{L_{E}^{2}} .
\end{aligned}
$$

Soit $\eta>0$, comme $u_{n} \rightarrow u$ dans $L_{E}^{2}\left(\left[0, T[)\right.\right.$ et que la suite $\left(\left(u_{n}\right)_{t}\right)_{n \in \mathbb{N}^{\star}}$ est bornée dans $L_{E^{\prime}}^{2}(] 0, T-\varepsilon[)$, il existe $n_{0}$ t.q.

$$
\varphi\left(t_{2}\right)-\varphi\left(t_{1}\right) \leq \eta \text { pour } m, n \geq n_{0} .
$$

On a donc

$$
\varphi\left(t_{2}\right) \leq \varphi\left(t_{1}\right)+\eta \quad \text { pour } n, m \geq n_{0} .
$$

On intègre cette inégalité pour $\left.t_{1} \in\right] 0, T-\varepsilon[$. On obtient

$$
\begin{aligned}
(T-\varepsilon) \varphi\left(t_{2}\right) & \leq \int_{0}^{T-\varepsilon}\left\|u_{n}-u_{m}\right\|_{F}^{2} d t_{1}+T \eta \\
& \leq\left\|u_{n}-u_{m}\right\|_{L_{F}^{2}}^{2}+T \eta
\end{aligned}
$$


En utilisant une nouvelle fois que $u_{n} \rightarrow u$ dans $L_{E}^{2}(] 0, T[)$, il existe $n_{1}$ t.q.

$$
(T-\varepsilon) \varphi\left(t_{2}\right) \leq(T+1) \eta \text { pour } n, m \geq n_{1} .
$$

On a donc $\varphi(t) \leq \frac{(T+1) \eta}{T-\varepsilon}$ pour $n, m \geq n_{1}$ et $t \in[0, T-\varepsilon]$. On a ainsi montré que, pour tout $t \in[0, T-\varepsilon]$,

$$
n, m \geq n_{1} \Rightarrow\left\|u_{n}(t)-u_{m}(t)\right\|_{F}^{2} \leq \frac{(T+1) \eta}{T-\varepsilon} .
$$

Ceci montre bien que la suite $\left(u_{n}(t)\right)_{n \in \mathbb{N}}$ est de Cauchy dans $F$ uniformément par rapport à $t$, si $t \in[0, T-\varepsilon]$. Il existe donc une fonction $w$ de $\left[0, T\left[\right.\right.$ dans $F$ t.q. $u_{n}(t) \rightarrow w(t)$ dans $F$ pour tout $t \in[0, T[$. Comme cette convergence est uniforme sur $[0, T-\varepsilon]$ pour tout $\varepsilon>0$, la fonction $w$ est continue sur $[0, T-\varepsilon]$ pour tout $\varepsilon>0$. On a donc $w \in C([0, T[, F)$.

Comme $u_{n} \rightarrow u$ dans $L_{E}^{2}(] 0, T[$ ) (et donc p.p. sur $] 0, T[$ après extraction éventuelle d'une sous suite), on a donc $u=w$ p.p., et donc $u \in C([0, T[, F)$ (car on identifie, comme d'habitude, la classe de fonctions $u$ avec son représentant continu qui est justement $w)$.

De manière analogue, en décentrant les noyaux régularisants de l'autre côté, on montre que $u \in C(] 0, T], F)$. On a donc $u \in C([0, T], F)$.

Enfin, on a aussi pour $t_{1}, t_{2} \in[0, T]$,

$$
\begin{aligned}
\left\|u_{n}\left(t_{1}\right)\right\|_{F}^{2}-\left\|u_{n}\left(t_{2}\right)\right\|_{F}^{2} & =2 \int_{t_{2}}^{t_{1}}\left(u_{n}^{\prime}(s) \mid u_{n}(s)\right)_{F} d s \\
& =2 \int_{t_{2}}^{t_{1}}<\left(u_{n}\right)_{t}(s), u_{n}(s)>_{E^{\prime}, E} d s
\end{aligned}
$$

en passant à la limite sur $n$, on obtient

$$
\left\|u\left(t_{1}\right)\right\|_{F}^{2}-\left\|u\left(t_{2}\right)\right\|_{F}^{2}=2 \int_{t_{2}}^{t_{1}}<\partial_{t} u(s), u(s)>_{E^{\prime}, E} d s .
$$

Remarque 4.27 Un exemple classique du lemme 4.26 consiste à prendre $E=H_{0}^{1}(\Omega)$ (où $\Omega$ est un ouvert de $\mathbb{R}^{N}$, $N \geq 1), F=L^{2}(\Omega), F^{\prime}$ identifié à $F$, et donc $E^{\prime}=H^{-1}(\Omega)$. Le choix de $E^{\prime}$ pour l'espace dans lequel est $\partial_{t} u$ est crucial dans la démonstration du lemme 4.26 mais des généralisations de ce lemme sont possibles. L'exercice 4.4 montre que $u \in L^{2}(] 0,1\left[, H^{1}(] 0,+\infty[)\right.$ et $\partial_{t} u \in L^{2}(] 0,1\left[, H^{-1}(] 0,+\infty[)\right.$ (l'espace $L^{2}$ étant identifié à lui même) sont des hypothèses suffisantes pour obtenir $u \in C\left([0, T], L^{2}(\Omega)\right)$. Le lemme 4.26 ne s'applique pas ici directement car $H^{-1}(] 0,+\infty[)$ est le dual de $H_{0}^{1}(] 0,+\infty[)$ et n'est donc pas le dual de $H^{1}(] 0,+\infty[)$. Un argument de prolongement permet de se ramener au lemme 4.26 (exercice 4.4).

\section{3 Étude de l'équation de la chaleur}

Notation : Si $E$ est un espace de Banach et $T>0$, on notera souvent $L^{2}(] 0, T[, E)$ (au lieu de $L_{E}^{2}(] 0, T[)$ ) l'espace $L_{E}^{2}(] 0, T[, \mathcal{B}(] 0, T[, \lambda)$.

On s'intéresse dans ce paragraphe au problème suivant :

Soit $\Omega$ un ouvert borné de $\mathbb{R}^{N}, T \in \mathbb{R}_{+}^{\star}, f$ une fonction de $\left.\Omega \times\right] 0, T\left[\right.$ dans $\mathbb{R}$ et $u_{0}$ une fonction de $\Omega$ dans $\mathbb{R}$. On cherche $u$ tel que

$$
\begin{cases}\partial_{t} u-\Delta u=f & \text { dans } \Omega \times] 0, T[, \\ u=0 & \text { sur } \partial \Omega \times] 0, T[, \\ u(\cdot, 0)=u_{0} . & \end{cases}
$$


On va donner pour ce problème linéaire un résultat d'existence par la méthode de Faedo-Galerkin et d'unicité de solution faible.

Théorème 4.28 (Faedo-Galerkin) Soit $\Omega$ un ouvert borné de $\mathbb{R}^{N}, T>0$ et $u_{0} \in L^{2}(\Omega)$, on identifie $L^{2}(\Omega)$ avec son dual et on suppose que $f \in L^{2}(] 0, T\left[, H^{-1}(\Omega)\right)$. Alors, il existe un et un seul u tel que

$$
\left\{\begin{array}{l}
u \in L^{2}(] 0, T\left[, H_{0}^{1}(\Omega)\right), \partial_{t} u \in L^{2}(] 0, T\left[, H^{-1}(\Omega)\right), \\
\int_{0}^{T}\left\langle\partial_{t} u(s), v(s)\right\rangle_{H^{-1}, H_{0}^{1}} d s+\int_{0}^{T} \int_{\Omega} \nabla u(s) \cdot \nabla v(s) \mathrm{d} x \mathrm{~d} s=\int_{0}^{T}\langle f(s), v(s)\rangle_{H^{-1}, H_{0}^{1}} \mathrm{~d} s \\
u(0)=u_{0} \text { p.p.. }
\end{array}\right.
$$

(On rappelle que $u(s)$ (resp. $v(s)$ ) désigne la fonction $x \mapsto u(x, t)$ (resp. $v(x, t)$ ).

On a de plus les estimations suivantes sur $u$ et $\partial_{t} u$ :

$$
\begin{aligned}
& \|u\|_{L^{2}(] 0, T\left[, H_{0}^{1}(\Omega)\right)} \leq\left\|u_{0}\right\|_{2}+\|f\|_{L^{2}(] 0, T\left[, H^{-1}(\Omega)\right)} \\
& \left\|\partial_{t} u\right\|_{L^{2}(] 0, T\left[, H^{-1}(\Omega)\right)} \leq\left\|u_{0}\right\|_{2}+2\|f\|_{L^{2}(] 0, T\left[, H^{-1}(\Omega)\right)} \\
& \|u(t)\|_{2}^{2} \leq\left\|u_{0}\right\|_{2}^{2}+\left\|\partial_{t} u\right\|_{L^{2}(] 0, T\left[, H^{-1}(\Omega)\right)}^{2}+\|u\|_{L^{2}(] 0, T\left[, H_{0}^{1}(\Omega)\right)}^{2}, \text { pour tout } t \in[0, T[.
\end{aligned}
$$

\section{Démonstration}

On rappelle que l'on a identifié $L^{2}(\Omega)$ avec $\left.L^{2}(\Omega)\right)^{\prime}$, de sorte que $H_{0}^{1}(\Omega) \subset L^{2}(\Omega)=L^{2}(\Omega)^{\prime} \subset H^{-1}(\Omega)$. Comme on cherche $u$ t.q. $u \in L^{2}(] 0, T\left[, H_{0}^{1}(\Omega)\right)$ et $\partial_{t} u \in L^{2}(] 0, T\left[, H^{-1}(\Omega)\right)$, on a nécessairement $u \in C\left([0, T], L^{2}(\Omega)\right)$ (d'après le lemme 4.26). La fonction $u$ est donc définie en tout $t \in[0, T]$, ce qui permet de donner un sens à la condition $u(0)=u_{0}$ p.p..

L'idée, pour démontrer ce théorème, est de résoudre d'abord le problème dans des espaces de dimension finie; on pourrait le faire par exemple avec des espaces d'éléments finis, mais c'est plus simple en utilisant une base hilbertienne formée de fonctions propres du Laplacien, c'est-à-dire une base hilbertienne de $L^{2}(\Omega)$, notée $\left\{e_{n}, n \in \mathbb{N}^{\star}\right\}$ t.q. $e_{n}$ est (pour tout $n$ ) une solution faible de

$$
\left\{\begin{array}{l}
-\Delta e_{n}=\lambda_{n} e_{n} \quad \text { dans } \Omega \\
e_{n}=0 \quad \text { sur } \partial \Omega
\end{array}\right.
$$

avec $\lambda_{n} \in \mathbb{R}$.

Etape 1, remarques liminaires. La famille $\left(e_{n}\right)_{n \in \mathbb{N}^{\star}}$ est une base hilbertienne de $L^{2}(\Omega)$ et vérifie

$$
\left\{\begin{array}{l}
e_{n} \in H_{0}^{1}(\Omega), \\
\int_{\Omega} \nabla e_{n} \cdot \nabla v \mathrm{~d} x=\lambda_{n} \int_{\Omega} e_{n} v \mathrm{~d} x, \text { pour tout } v \in H_{0}^{1}(\Omega),
\end{array}\right.
$$

avec $\lambda_{n}>0$ pour tout $n \in \mathbb{N}^{\star}$ et $\lambda_{n} \uparrow+\infty$ quand $n \rightarrow+\infty$.

Comme $\left(e_{n}\right)_{n \in \mathbb{N}^{\star}}$ est une base hilbertienne de $L^{2}(\Omega)$, on a, pour tout $w \in L^{2}(\Omega), w=\sum_{n \in \mathbb{N}^{\star}}\left(w \mid e_{n}\right)_{2} e_{n}$ au sens de la convergence $L^{2}(\Omega)$ ( c'est-à-dire que $\sum_{i=1}^{n}\left(w \mid e_{i}\right) e_{i} \rightarrow w$ dans $L^{2}(\Omega)$ quand $n \rightarrow+\infty$ ).

On va montrer maintenant que la famille $\left(\lambda_{n}^{-1 / 2} e_{n}\right)_{n \in \mathbb{N}^{\star}}$ est une base hilbertienne de $H_{0}^{1}(\Omega)$. On rappelle que $H_{0}^{1}(\Omega)$ est un espace de Hilbert, la norme de $u$ dans $H_{0}^{1}(\Omega)$ est définie par $\|u\|_{H_{0}^{1}(\Omega)}=\||\nabla u|\|_{L^{2}(\Omega)}$ et donc le produit scalaire de $u$ et $v$ dans $H_{0}^{1}(\Omega)$ est donné par $(u \mid v)_{H_{0}^{1}(\Omega)}=\int_{\Omega} \nabla u \cdot \nabla v \mathrm{~d} x$.

On remarque tout d'abord que pour tout $n, m \geq 1$, on a

$$
\int_{\Omega} \nabla e_{n} \cdot \nabla e_{m} \mathrm{~d} x=\lambda_{n} \int_{\Omega} e_{n} e_{m} \mathrm{~d} x=\lambda_{n} \delta_{n, m} .
$$


On en déduit que $\left(e_{n} \mid e_{m}\right)_{H_{0}^{1}(\Omega)}=0$ si $n \neq m$ et

$$
\left\|\frac{e_{n}}{\sqrt{\lambda_{n}}}\right\|_{H_{0}^{1}(\Omega)}^{2}=\int_{\Omega} \frac{\nabla e_{n} \cdot \nabla e_{n}}{\lambda_{n}} \mathrm{~d} x=1 .
$$

Puis, on remarque que l'espace vectoriel engendré par la famille $\left(e_{n}\right)_{n \in \mathbb{N}^{\star}}$, noté $e v\left\{e_{n}, n \in \mathbb{N}^{\star}\right\}$, est dense dans $H_{0}^{1}(\Omega)$. En effet soit $v \in H_{0}^{1}(\Omega)$ t.q. $\left(v \mid e_{n}\right)_{H_{0}^{1}(\Omega)}=0$ pour tout $n \in \mathbb{N}^{\star}$. On a donc, pour tout $n \in \mathbb{N}^{\star}$,

$$
0=\left(v \mid e_{n}\right)_{H_{0}^{1}(\Omega)}=\int_{\Omega} \nabla e_{n} \cdot \nabla v \mathrm{~d} x=\lambda_{n} \int_{\Omega} e_{n} v \mathrm{~d} x .
$$

Comme $\left(e_{n}\right)_{n \in \mathbb{N}^{\star}}$ est une base hilbertienne de $L^{2}(\Omega)$ (et $\lambda_{n} \neq 0$ pour tout $n \in \mathbb{N}^{\star}$ ), on en déduit que $v=0$ p.p.. Ceci montre que l'orthogonal dans $H_{0}^{1}(\Omega)$ de $e v\left\{e_{n}, n \in \mathbb{N}^{\star}\right\}$ est réduit à $\{0\}$ et donc que $e v\left\{e_{n}, n \in \mathbb{N}^{\star}\right\}$ est dense dans $H_{0}^{1}(\Omega)$. Finalement, on obtient ainsi que la famille $\left(\lambda_{n}^{-1 / 2} e_{n}\right)_{n \in \mathbb{N}^{\star}}$ est une base hilbertienne de $H_{0}^{1}(\Omega)$.

\section{Etape 2, solution approchée.}

Soit $n \in \mathbb{N}^{\star}$. On pose $E_{n}=e v\left\{e_{p}, p=1, \ldots n\right\}$. On cherche une solution approchée $u_{n}$ sous la forme $u_{n}(t)=$ $\sum_{i=1}^{n} \alpha_{i}(t) e_{i}$ avec $\alpha_{i} \in C([0, T], \mathbb{R})$. En supposant que les $\alpha_{i}$ sont dérivables pour tout $t$ (ce qui n'est pas vrai, en général), on a donc

$$
u_{n}^{\prime}(t)=\sum_{i=1}^{n} \alpha_{i}^{\prime}(t) e_{i},
$$

de sorte que, pour tout $\varphi \in H_{0}^{1}(\Omega)$ et tout $\left.t \in\right] 0, T\left[\right.$, on a (compte tenu de l'injection de $L^{2}(\Omega)$ dans $H_{0}^{1}(\Omega)$ ),

$$
\left\langle u_{n}^{\prime}(t), \varphi\right\rangle_{H^{-1}(\Omega), H_{0}^{1}(\Omega)}=\sum_{i=1}^{n} \alpha_{i}^{\prime}(t) \int_{\Omega} e_{i} \varphi \mathrm{d} x .
$$

D'autre part, pour tout $t \in[0, T]$ on a

$$
-\Delta u_{n}(t)=-\sum_{i=1}^{n} \alpha_{i}(t) \Delta e_{i}=\sum_{i=1}^{n} \lambda_{i} \alpha_{i}(t) e_{i} \text { dans } \mathcal{D}^{\star}(\Omega) \text { et dans } H^{-1}(\Omega),
$$

c'est-à-dire , pour tout $\varphi \in H_{0}^{1}(\Omega)$,

$$
\left\langle-\Delta u_{n}(t), \varphi\right\rangle_{H^{-1}(\Omega), H_{0}^{1}(\Omega)}=\int_{\Omega} \nabla u_{n}(t) \cdot \nabla \varphi \mathrm{d} x=\sum_{i=1}^{n} \lambda_{i} \alpha_{i}(t) \int_{\Omega} e_{i} \varphi \mathrm{d} x .
$$

Enfin, comme $f \in L^{2}(] 0, T\left[, H^{-1}\right)$, on a pour tout $\varphi \in H_{0}^{1}(\Omega),\langle f(\cdot), \varphi\rangle_{H^{-1}(\Omega), H_{0}^{1}(\Omega)} \in L_{\mathbb{R}}^{1}(] 0, T[)$. La quantité $\langle f(t), \varphi\rangle_{H^{-1}(\Omega), H_{0}^{1}(\Omega)}$ est donc définie pour presque tout $t$ et on obtient finalement, pour presque tout $t$ et pour tout $\varphi \in H_{0}^{1}(\Omega)$,

$$
\left\langle u_{n}^{\prime}(t)-\Delta u_{n}(t)-f(t), \varphi\right\rangle_{H^{-1}(\Omega), H_{0}^{1}(\Omega)}=\sum_{i=1}^{n}\left(\alpha_{i}^{\prime}(t)+\lambda_{i} \alpha_{i}(t)\right) \int_{\Omega} e_{i} \varphi \mathrm{d} x-\langle f(t), \varphi\rangle_{H^{-1}(\Omega), H_{0}^{1}(\Omega)} .
$$

Pour obtenir $u_{n}$, une idée naturelle est de choisir les fonctions $\alpha_{i}$ pour que

$$
\left\langle u_{n}^{\prime}(t)-\Delta u_{n}(t)-f(t), \varphi\right\rangle_{H^{-1}(\Omega), H_{0}^{1}(\Omega)}=0
$$


pour tout $\varphi \in E_{n}$. En posant $f_{i}(t)=\left\langle f(t), e_{i}\right\rangle_{H^{-1}(\Omega), H_{0}^{1}(\Omega)}$, ceci est équivalent à demander pour tout $i \in$ $\{1, \ldots, n\}$,

$$
\alpha_{i}^{\prime}(t)+\lambda_{i} \alpha_{i}(t)=f_{i}(t) .
$$

En tenant compte de la condition initiale et en posant $\alpha_{i}^{(0)}=\left(u_{0} \mid e_{i}\right)_{2}$, ceci suggère donc de prendre

$$
\alpha_{i}(t)=\alpha_{i}^{(0)} e^{-\lambda_{i} t}+\int_{0}^{t} e^{-\lambda_{i}(t-s)} f_{i}(s) d s .
$$

Les fonctions $\alpha_{i}$ ainsi définies appartiennent à $C([0, T], \mathbb{R})$ et on a donc $u_{n} \in C\left([0, T], E_{n}\right) \subset C\left([0, T], H_{0}^{1}(\Omega)\right)$ avec $u_{n}(t)=\sum_{i=1}^{n} \alpha_{i}(t) e_{i}$.

Etape 3, précision sur la dérivée en temps. Soit $n \in \mathbb{N}^{\star}$ et $u_{n}$ la solution approchée donnée par l'étape précédente. Les fonctions $\alpha_{i}$ ne sont pas nécessairement dérivables. On va préciser ici ce que vaut la dérivée (par transposition) de $u_{n}$. On va noter cette dérivée $\left(u_{n}\right)_{t}$. Par définition de la dérivation par transposition, $\left(u_{n}\right)_{t}$ est un élément de $\mathcal{D}_{E}^{\star}$ avec $E=H_{0}^{1}(\Omega)$. Soit $\varphi \in C_{c}^{\infty}(] 0, T[, \mathbb{R})$ on a

$$
\left\langle\left(u_{n}\right)_{t}, \varphi\right\rangle_{\mathcal{D}_{E}^{\star}, \mathcal{D}}=-\int_{0}^{T} u_{n}(t) \varphi^{\prime}(t) \mathrm{d} t \in E_{n} \subset H_{0}^{1}(\Omega) .
$$

Comme $u_{n}=\sum_{i=1}^{n} \alpha_{i} e_{i}$, on a donc

$$
\left\langle\left(u_{n}\right)_{t}, \varphi\right\rangle_{\mathcal{D}_{E}^{\star}, \mathcal{D}}=-\sum_{i=1}^{n} \int_{0}^{T} \alpha_{i}(t) e_{i} \varphi^{\prime}(t) \mathrm{d} t=-\sum_{i=1}^{n}\left(\int_{0}^{T} \alpha_{i}(t) \varphi^{\prime}(t) \mathrm{d} t\right) e_{i} .
$$

On utilise maintenant 4.20,

$$
\int_{0}^{T} \alpha_{i}(t) \varphi^{\prime} \mathrm{d} t=T_{i}+S_{i}
$$

avec

$$
\begin{gathered}
T_{i}=\int_{0}^{T} \alpha_{i}^{(0)} e^{-\lambda_{i} t} \varphi^{\prime}(t) d t=\int_{0}^{T} \alpha_{i}^{(0)} \lambda_{i} e^{-\lambda_{i} t} \varphi(t) d t . \\
S_{i}=\int_{0}^{T}\left(\int_{0}^{t} e^{-\lambda_{i}(t-s)} f_{i}(s) d s\right) \varphi^{\prime}(t) \mathrm{d} t .
\end{gathered}
$$

Pour transformer $S_{i}$ on utilise le théorème de Fubini :

$$
\begin{array}{r}
S_{i}=\int_{0}^{T}\left(\int_{0}^{T} 1_{[0, t]}(s) e^{-\lambda_{i}(t-s)} f_{i}(s) d s\right) \varphi^{\prime}(t) \mathrm{d} t=\int_{0}^{T}\left(\int_{0}^{T} 1_{[s, T]}(t) e^{-\lambda_{i}(t-s)} \varphi^{\prime}(t) \mathrm{d} t\right) f_{i}(s) d s \\
=\int_{0}^{T}\left(\int_{s}^{T} e^{-\lambda_{i}(t-s)} \varphi^{\prime}(t) \mathrm{d} t\right) f_{i}(s) d s= \\
=\int_{0}^{T}\left(\int_{s}^{T} \lambda_{i} e^{-\lambda_{i}(t-s)} \varphi(t) \mathrm{d} t\right) f_{i}(s) d s-\int_{0}^{T} \varphi(s) f_{i}(s) d s . \\
=\int_{0}^{T}\left(\int_{0}^{t} \lambda_{i} e^{-\lambda_{i}(t-s)} f_{i}(s) d s\right) \varphi(t) d t-\int_{0}^{T} f_{i}(t) \varphi(t) d t .
\end{array}
$$

On en déduit que $T_{i}+S_{i}=\int_{0}^{T} \lambda_{i} \alpha_{i}(t) \varphi(t) \mathrm{d} t-\int_{0}^{T} f_{i}(t) \varphi(t) \mathrm{d} t$, et donc

$$
\left\langle\left(u_{n}\right)_{t}, \varphi\right\rangle_{\mathcal{D}^{\star}, \mathcal{D}}=-\sum_{i=1}^{n} \int_{0}^{T} \lambda_{i} \alpha_{i}(t) e_{i} \varphi(t) \mathrm{d} t+\sum_{i=1}^{n} \int_{0}^{T} f_{i}(t) e_{i} \varphi(t) d t .
$$


Comme cette égalité est vraie pour tout $\varphi \in C_{c}^{\infty}(] 0, T[, \mathbb{R})$, on a finalement

$$
\left(u_{n}\right)_{t}=-\sum_{i=1}^{n} \lambda_{i} \alpha_{i} e_{i}+\sum_{i=1}^{n} f_{i} e_{i} \in L^{2}(] 0, T\left[, E_{n}\right)
$$

Ce qui peut aussi s'écrire, avec $f^{(n)}=\sum_{i=1}^{n} f_{i} e_{i}$,

$$
\left(u_{n}\right)_{t}=\Delta u_{n}+f^{(n)} \in L^{2}(] 0, T\left[, E_{n}\right) \subset L^{2}(] 0, T\left[, H_{0}^{1}(\Omega)\right) \subset L^{2}(] 0, T\left[, H^{-1}(\Omega)\right) .
$$

Soit maintenant $v \in L^{2}(] 0, T\left[, H_{0}^{1}(\Omega)\right)$. Comme $\left(u_{n}\right)_{t} \in L^{2}(] 0, T\left[, H^{-1}(\Omega)\right)$, on a $\left\langle\left(u_{n}\right)_{t}, v\right\rangle_{H^{-1}(\Omega), H_{0}^{1}(\Omega)} \in$ $L^{1}(] 0, T[)$ et

$$
\int_{0}^{T}\left\langle\left(u_{n}\right)_{t}(t), v(t)\right\rangle_{H^{-1}(\Omega), H_{0}^{1}(\Omega)} d t=-\int_{0}^{T} \int_{\Omega} \nabla u_{n} \cdot \nabla v \mathrm{~d} x \mathrm{~d} t+\sum_{i=1}^{n} \int_{0}^{T} \int_{\Omega} f_{i} e_{i} v \mathrm{~d} x \mathrm{~d} t .
$$

Ceci donne, en revenant à la définition de $f_{i}$,

$$
\begin{array}{r}
\int_{0}^{T}\left\langle\left(u_{n}\right)_{t}(t), v(t)\right\rangle_{H^{-1}(\Omega), H_{0}^{1}(\Omega)} d t+\int_{0}^{T} \int_{\Omega} \nabla u_{n} \cdot \nabla v \mathrm{~d} x \mathrm{~d} t=\sum_{i=1}^{n} \int_{0}^{T}\left\langle f(t), e_{i}\right\rangle_{H^{-1}(\Omega), H_{0}^{1}(\Omega)}\left(\int_{\Omega} e_{i} v \mathrm{~d} x\right) \mathrm{d} t \\
=\int_{0}^{T}\left\langle f(t), \sum_{i=1}^{n}\left(v \mid e_{i}\right)_{2} e_{i}\right\rangle_{H^{-1}(\Omega), H_{0}^{1}(\Omega)} d t .
\end{array}
$$

On note $P_{n}$ l'opérateur de projection orthogonale dans $L^{2}(\Omega)$ sur le s.e.v. $E_{n}$. L'opérateur $P_{n}$ peut donc être vu comme un opérateur de $L^{2}(\Omega)$ dans $H_{0}^{1}(\Omega)$ (car $E_{n} \subset H_{0}^{1}(\Omega)$ ). On note alors $P_{n}^{t}$ l'opérateur transposé qui est donc un opérateur de $H^{-1}(\Omega)$ dans $\left(L^{2}(\Omega)\right)^{\prime}$ qui est lui même identifié à $L^{2}(\Omega)$ et est aussi un s.e.v. de $H^{-1}(\Omega)$. On obtient alors (pour tout $v \in L^{2}(] 0, T\left[, H_{0}^{1}(\Omega)\right)$ )

$$
\int_{0}^{T}\left\langle\left(u_{n}\right)_{t}, v\right\rangle_{H^{-1}, H_{0}^{1}} d t+\int_{0}^{T} \int_{\Omega} \nabla u_{n} \cdot \nabla v \mathrm{~d} x \mathrm{~d} t=\int_{0}^{T}\left\langle f, P_{n} v\right\rangle_{H^{-1}, H_{0}^{1}} d t=\int_{0}^{T}\left\langle P_{n}^{t} f, v\right\rangle_{H^{-1}, H_{0}^{1}} d t .
$$

On a aussi $u_{n} \in C\left([0, T], H_{0}^{1}(\Omega)\right)$ et $u_{n}(0)=P_{n} u_{0}$.

\section{Etape 4, estimations sur la solution approchée.}

Pour $n \in \mathbb{N}^{\star}$, on a $u_{n} \in C\left([0, T], H_{0}^{1}(\Omega)\right) \subset L^{2}(] 0, T\left[, H_{0}^{1}(\Omega)\right)$ et $\left(u_{n}\right)_{t}=\Delta u_{n}+f^{(n)} \in L^{2}(] 0, T\left[, H^{-1}(\Omega)\right)$. D'après la section 4.2 , on a donc

$$
\frac{1}{2}\left\|u_{n}(T)\right\|_{2}^{2}-\frac{1}{2}\left\|u_{0}\right\|_{2}^{2}=\int_{0}^{T}\left\langle\left(u_{n}\right)_{t}, u_{n}\right\rangle_{H^{-1}, H_{0}^{1}} \mathrm{~d} t .
$$

En prenant $v=u_{n}$ dans 4.21], on en déduit

$$
\frac{1}{2}\left\|u_{n}(T)\right\|_{2}^{2}-\frac{1}{2}\left\|u_{0}\right\|_{2}^{2}+\int_{0}^{T} \int_{\Omega}\left|\nabla u_{n}\right|^{2} \mathrm{~d} x \mathrm{~d} t=\int_{0}^{T}\left\langle f, P_{n} u_{n}\right\rangle_{H^{-1}, H_{0}^{1}} d t
$$

et donc

$$
\left\|u_{n}\right\|_{L^{2}(] 0, T\left[, H_{0}^{1}(\Omega)\right)}^{2}=\int_{0}^{T} \int_{\Omega}\left|\nabla u_{n}\right|^{2} \mathrm{~d} x \mathrm{~d} t \leq \frac{1}{2}\left\|u_{0}\right\|_{2}^{2}+\int_{0}^{T}\left\langle f, P_{n} u_{n}\right\rangle_{H^{-1}, H_{0}^{1}} d t .
$$


On en déduit, en remarquant que $P_{n} u_{n}=u_{n}$,

$$
\begin{aligned}
& \left\|u_{n}\right\|_{L^{2}(] 0, T\left[, H_{0}^{1}(\Omega)\right)}^{2} \leq \frac{1}{2}\left\|u_{0}\right\|_{2}^{2}+\int_{0}^{T}\left\langle f, u_{n}\right\rangle_{H^{-1}, H_{0}^{1}} d t \\
& \quad \leq \frac{1}{2}\left\|u_{0}\right\|_{2}^{2}+\|f\|_{L^{2}(] 0, T\left[, H^{-1}(\Omega)\right)}\left\|u_{n}\right\|_{L^{2}(] 0, T\left[, H_{0}^{1}(\Omega)\right)} \\
& \leq \frac{1}{2}\left\|u_{0}\right\|_{2}^{2}+\frac{1}{2}\|f\|_{L^{2}(] 0, T\left[, H^{-1}(\Omega)\right)}^{2}+\frac{1}{2}\left\|u_{n}\right\|_{L^{2}(] 0, T\left[, H_{0}^{1}(\Omega)\right)}^{2} .
\end{aligned}
$$

On a donc

$$
\left\|u_{n}\right\|_{L^{2}(] 0, T\left[, H_{0}^{1}(\Omega)\right)}^{2} \leq\left\|u_{0}\right\|_{2}^{2}+\|f\|_{L^{2}(] 0, T\left[, H^{-1}(\Omega)\right)}^{2} .
$$

Ce qui donne aussi

$$
\left\|u_{n}\right\|_{L^{2}(] 0, T\left[, H_{0}^{1}(\Omega)\right)} \leq\left\|u_{0}\right\|_{2}+\|f\|_{L^{2}(] 0, T\left[, H^{-1}(\Omega)\right)} .
$$

Comme $\left(u_{n}\right)_{t}=\Delta u_{n}+P_{n}^{t} f$ (égalité (4.21) ) et que $\left\|P_{n} w\right\|_{H_{0}^{1}(\Omega)} \leq\|w\|_{H_{0}^{1}(\Omega)}$ pour tout $w \in H_{0}^{1}(\Omega)$, on obtient aussi une borne sur $\left(u_{n}\right)_{t}$ :

$$
\left\|\left(u_{n}\right)_{t}\right\|_{L^{2}(] 0, T\left[, H^{-1}(\Omega)\right)} \leq\left\|u_{n}\right\|_{L^{2}(] 0, T\left[, H_{0}^{1}(\Omega)\right)}+\|f\|_{L^{2}(] 0, T\left[, H^{-1}(\Omega)\right)}
$$

et donc

$$
\left\|\left(u_{n}\right)_{t}\right\|_{L^{2}(] 0, T\left[, H^{-1}(\Omega)\right)} \leq\left\|u_{0}\right\|_{2}+2\|f\|_{L^{2}(] 0, T\left[, H^{-1}(\Omega)\right)} \cdot
$$

La suite $\left(u_{n}\right)_{n \in \mathbb{N}^{\star}}$ est donc bornée dans $L^{2}(] 0, T\left[, H_{0}^{1}(\Omega)\right)$ et la suite $\left(\left(u_{n}\right)_{t}\right)_{n \in \mathbb{N}^{\star}}$ est bornée dans $L^{2}(] 0, T[$, $\left.H^{-1}(\Omega)\right)$.

Etape 5, passage à la limite. Grâce aux estimations obtenues à l'étape précédente, on peut supposer, après extraction éventuelle d'une sous suite, que, quand $n \rightarrow+\infty$,

$$
\begin{gathered}
u_{n} \rightarrow u \text { faiblement dans } L^{2}(] 0, T\left[, H_{0}^{1}(\Omega)\right), \\
\left(u_{n}\right)_{t} \rightarrow w \text { faiblement dans } L^{2}(] 0, T\left[, H^{-1}(\Omega)\right)
\end{gathered}
$$

et les estimations sur $u_{n}$ et $\left(u_{n}\right)_{t}$ donnent aussi les estimations suivantes sur $u$ et $w$ :

$$
\begin{aligned}
\|u\|_{L^{2}(] 0, T\left[, H_{0}^{1}(\Omega)\right)} & \leq\left\|u_{0}\right\|_{2}+\|f\|_{L^{2}(] 0, T\left[, H^{-1}(\Omega)\right)}, \\
\|w\|_{L^{2}(] 0, T\left[, H^{-1}(\Omega)\right)} & \leq\left\|u_{0}\right\|_{2}+2\|f\|_{L^{2}(] 0, T\left[, H^{-1}(\Omega)\right)} .
\end{aligned}
$$

Nous allons montrer tout d'abord que $w=\partial_{t} u$ (puis nous montrerons que $u$ est solution de $\partial_{t} u=\Delta u+f$ au sens demandé par 4.19).

Par définition de $\partial_{t} u$, on a, pour tout $\varphi \in C_{c}^{\infty}(] 0, T[, \mathbb{R})$,

$$
\int_{0}^{T} \partial_{t} u(t) \varphi(t) d t=-\int_{0}^{T} u(t) \varphi^{\prime}(t) d t .
$$

Pour démontrer que $\partial_{t} u=w$, il suffit donc de montrer que l'on a, pour tout $\varphi \in C_{c}^{\infty}(] 0, T[, \mathbb{R})$,

$$
\int_{0}^{T} w(t) \varphi(t) d t=-\int_{0}^{T} u(t) \varphi^{\prime}(t) d t .
$$

On rappelle que le terme de gauche de l'égalité (4.22) est dans $H^{-1}(\Omega)$ alors que le terme de droite est dans $H_{0}^{1}(\Omega)$. Cette égalité utilise donc le fait que $H_{0}^{1}(\Omega) \subset H^{-1}(\Omega)$, cette inclusion étant due au fait que nous avons identifié $L^{2}(\Omega)^{\prime}$ avec $L^{2}(\Omega)$. 
Soit donc $\varphi \in C_{c}^{\infty}(] 0, T[, \mathbb{R})$. Nous allons montrer 4.22]. Pour $\psi \in H_{0}^{1}(\Omega)$, on considère l'application $S$ de $L^{2}(] 0, T\left[, H_{0}^{1}(\Omega)\right)$ dans $\mathbb{R}$ définie par

$$
S(v)=\int_{\Omega}\left(-\int_{0}^{T} v(t) \varphi^{\prime}(t) d t\right) \psi(x) \mathrm{d} x \text { pour } v \in L^{2}(] 0, T\left[, H_{0}^{1}(\Omega)\right) .
$$

L'application $S$ est linéaire continue de $L^{2}(] 0, T\left[, H_{0}^{1}(\Omega)\right)$ dans $\mathbb{R}$. Comme $u_{n} \rightarrow u$ faiblement $L^{2}(] 0, T$, $H_{0}^{1}(\Omega)$ ), on a donc $S\left(u_{n}\right) \rightarrow S(u)$ quand $n \rightarrow+\infty$. Or, pour $v=u_{n}$ et pour $v=u$, on a

$$
S(v)=-\left(\int_{0}^{T} v(t) \varphi^{\prime}(t) d t \mid \psi\right)_{2}=-\left\langle\int_{0}^{T} v(t) \varphi^{\prime}(t) d t, \psi\right\rangle_{H^{-1}, H_{0}^{1}}
$$

On a donc, quand $n \rightarrow+\infty$,

$$
-\left\langle\int_{0}^{T} u_{n}(t) \varphi^{\prime}(t) d t, \psi\right\rangle_{H^{-1}, H_{0}^{1}} \rightarrow-\left\langle\int_{0}^{T} u(t) \varphi^{\prime}(t) d t, \psi\right\rangle_{H^{-1}, H_{0}^{1}} .
$$

On utilise maintenant le fait que $-\int_{0}^{T} u_{n}(t) \varphi^{\prime}(t) d t=\int_{0}^{T}\left(u_{n}\right)_{t}(t) \varphi(t) d t$ (par définition de $\left(u_{n}\right)_{t}$ ). On a donc

$$
\left\langle\int_{0}^{T}\left(u_{n}\right)_{t}(t) \varphi(t) d t, \psi\right\rangle_{H^{-1}, H_{0}^{1}} \rightarrow-\left\langle\int_{0}^{T} u(t) \varphi^{\prime}(t) d t, \psi\right\rangle_{H^{-1}, H_{0}^{1}} .
$$

On considère maintenant l'application $\bar{S}$ de $L^{2}(] 0, T\left[, H^{-1}(\Omega)\right)$ dans $\mathbb{R}$ définie par

$$
\bar{S}(v)=\left\langle\int_{0}^{T} v(t) \varphi(t) d t, \psi\right\rangle_{H^{-1}, H_{0}^{1}} \text { pour } v \in L^{2}(] 0, T\left[, H^{-1}(\Omega)\right) .
$$

L'application $\bar{S}$ est linéaire continue de $L^{2}(] 0, T\left[, H^{-1}(\Omega)\right)$ dans $\mathbb{R}$. Comme $\left(u_{n}\right)_{t} \rightarrow w$ faiblement $L^{2}(] 0, T[$, $H^{-1}(\Omega)$, on a donc $\bar{S}\left(\left(u_{n}\right)_{t}\right) \rightarrow \bar{S}(w)$ quand $n \rightarrow+\infty$, c'est-à-dire

$$
\left\langle\int_{0}^{T}\left(u_{n}\right)_{t}(t) \varphi(t) d t, \psi\right\rangle_{H^{-1}, H_{0}^{1}} \rightarrow\left\langle\int_{0}^{T} w(t) \varphi(t) d t, \psi\right\rangle_{H^{-1}, H_{0}^{1}} .
$$

On en déduit que pour tout $\psi \in H_{0}^{1}(\Omega)$, on a

$$
-\left\langle\int_{0}^{T} u(t) \varphi^{\prime}(t) d t, \psi\right\rangle_{H^{-1}, H_{0}^{1}}=\left\langle\int_{0}^{T} w(t) \varphi(t) d t, \psi\right\rangle_{H^{-1}, H_{0}^{1}} .
$$

On a donc bien montré que $-\int_{0}^{T} u(t) \varphi^{\prime}(t) d t=\int_{0}^{T} w(t) \varphi(t) d t$ pour tout $\varphi \in C_{c}^{\infty}(] 0, T[, \mathbb{R})$, c'est-à-dire que $\partial_{t} u=w$.

Nous savons donc que $u_{n} \rightarrow u$ faiblement dans $L^{2}(] 0, T\left[, H_{0}^{1}(\Omega)\right)$ et que $\partial_{t} u_{n} \rightarrow \partial_{t} u$ faiblement dans $L^{2}(] 0, T[$, $H^{-1}(\Omega)$ ). Pour montrer que $u$ est solution de $\partial_{t} u=\Delta u+f$ au sens demandé par [4.19], il suffit maintenant de passer à la limite dans 4.21). Soit $v \in L^{2}(] 0, T\left[, H_{0}^{1}(\Omega)\right)$, on a pour tout $n \in \mathbb{N}^{\star}$, selon [4.21,

$$
\int_{0}^{T}\left\langle\left(u_{n}\right)_{t}, v\right\rangle_{H^{-1}, H_{0}^{1}} d t+\int_{0}^{T}\left(u_{n} \mid v\right)_{H_{0}^{1}} \mathrm{~d} t=\int_{0}^{T}\left\langle f, P_{n} v\right\rangle_{H^{-1}, H_{0}^{1}} d t .
$$

Les deux termes de gauche de cette égalité passent à limite quand $n \rightarrow+\infty$ grâce aux convergences de $u_{n}$ et $\left(u_{n}\right)_{t}$. Pour le terme de droite, on utilise l'étape liminaire. On remarque que $P_{n} v(t) \rightarrow v(t)$ dans $H_{0}^{1}(\Omega)$ pour 
presque tout $t$ et $\left\|P_{n} v(t)\right\|_{H_{0}^{1}(\Omega)} \leq\|v(t)\|_{H_{0}^{1}(\Omega)}$ pour presque tout $t$. Cela permet de passer à la limite dans le terme de droite, par le théorème de convergence dominée. On obtient ainsi

$$
\int_{0}^{T}\left\langle\partial_{t} u, v\right\rangle_{H^{-1}, H_{0}^{1}} d t+\int_{0}^{T}(u \mid v)_{H_{0}^{1}} \mathrm{~d} t=\int_{0}^{T}\langle f, v\rangle_{H^{-1}, H_{0}^{1}} d t
$$

Ce qui est bien le sens souhaité dans la formulation 4.19.

Etape 6, condition initiale. Comme $u \in L^{2}(] 0, T\left[, H_{0}^{1}(\Omega)\right)$ et $\partial_{t} u \in L^{2}(] 0, T\left[, H^{-1}(\Omega)\right)$, on sait que $u \in$ $C\left([0, T], L^{2}(\Omega)\right)$ (voir la section 4.2). Pour terminer la démonstration du fait que $u$ est solution de (4.19], il reste donc seulement à montrer que $u(0)=u_{0}$ p.p. ( c'est-à-dire $u(0)=u_{0}$ dans $L^{2}(\Omega)$ ).

On sait que $u(t) \rightarrow u(0)$ dans $L^{2}(\Omega)$ quand $t \rightarrow 0$. On sait aussi que $u_{n}(0)=\sum_{i=1}^{n} \alpha_{i}^{(0)} e_{i} \rightarrow u_{0}$ dans $L^{2}(\Omega)$ quand $n \rightarrow+\infty$. Pour en déduire que $u(0)=u_{0}$, il suffit de montrer que la suite $\left(u_{n}\right)_{n \in \mathbb{N}^{\star}}$ est relativement compacte dans $C\left([0, T], H^{-1}(\Omega)\right)$. En effet, si la suite $\left(u_{n}\right)_{n \in \mathbb{N}^{\star}}$ est relativement compacte dans $C\left([0, T], H^{-1}(\Omega)\right)$, il existe $w \in C\left([0, T], H^{-1}(\Omega)\right)$ et une sous suite, encore notée $\left(u_{n}\right)_{n \in \mathbb{N}}$, t.q. $u_{n}(t) \rightarrow w(t)$ dans $H^{-1}(\Omega)$ uniformément par rapport à $t \in[0, T]$ (et donc aussi dans $L^{2}(] 0, T\left[, H^{-1}(\Omega)\right)$ ). En particulier, on a donc $w(0)=u_{0}$. Mais on sait déjà que $u_{n} \rightarrow u$ faiblement dans $L^{2}(] 0, T\left[, H_{0}^{1}(\Omega)\right)$ et donc aussi faiblement dans $L^{2}(] 0, T\left[, H^{-1}(\Omega)\right)$. Par unicité de la limite, on a donc $u=w$ p.p. sur $] 0, T[$ et donc $u(t)=w(t)$ pour tout $t \in[0, T]$ car $u$ et $w$ sont continues sur $[0, T]$. On obtient ainsi, finalement, $u(0)=w(0)=u_{0}$.

Il reste à montrer que la suite $\left(u_{n}\right)_{n \in \mathbb{N}^{\star}}$ est relativement compacte dans $C\left([0, T], H^{-1}(\Omega)\right)$. Par le théorème d'Ascoli, il suffit de montrer que

1. Pour tout $t \in[0, T],\left(u_{n}(t)\right)_{n \in \mathbb{N}^{\star}}$ est relativement compacte dans $H^{-1}(\Omega)$.

2. $\left\|u_{n}(t)-u_{n}(s)\right\|_{H^{-1}} \rightarrow 0$, quand $s \rightarrow t$, uniformément par rapport à $n \in \mathbb{N}^{\star}$ (et pour tout $t \in[0, T]$ ).

Par démontrer le deuxième item, on utilise la fait que $\left(u_{n}\right)_{t} \in L^{1}\left([0, T], H^{-1}(\Omega)\right)$. La section 4.2 nous donne que pour tout $t_{1}, t_{2} \in[0, T], t_{1}>t_{2}$, et tout $n \in \mathbb{N}^{\star}$, on a (dans $H^{-1}(\Omega)$ )

$$
u_{n}\left(t_{1}\right)-u_{n}\left(t_{2}\right)=\int_{t_{2}}^{t_{1}}\left(u_{n}\right)_{t}(s) d s
$$

et donc

$$
\begin{aligned}
& \left\|u_{n}\left(t_{1}\right)-u_{n}\left(t_{2}\right)\right\|_{H^{-1}} \leq \int_{t_{2}}^{t_{1}}\left\|\left(u_{n}\right)_{t}(s)\right\|_{H^{-1}} d s \leq\left(\int_{0}^{T}\left\|\left(u_{n}\right)_{t}(s)\right\|_{H^{-1}}^{2} d s\right)^{\frac{1}{2}} \sqrt{t_{1}-t_{2}} \\
& \leq\left\|\left(u_{n}\right)_{t}\right\|_{L^{2}(] 0, T\left[, H^{-1}\right)} \sqrt{t_{1}-t_{2}} \text {. }
\end{aligned}
$$

Comme la suite $\left(\left(u_{n}\right)_{t}\right)_{n \in \mathbb{N}^{\star}}$ est bornée dans $L^{2}(] 0, T\left[, H^{-1}(\Omega)\right)$, on en déduit bien que $\left\|u_{n}(t)-u_{n}(s)\right\|_{H^{-1}} \rightarrow 0$, quand $s \rightarrow t$, uniformément par rapport à $n \in \mathbb{N}^{\star}$ (et pour tout $t \in[0, T]$ ).

Pour démontrer de premier item, on utilise encore la section 4.2 . Comme $u_{n} \in L^{2}(] 0, T\left[, H_{0}^{1}(\Omega)\right)$ et $\left(u_{n}\right)_{t} \in$ $L^{2}(] 0, T\left[, H^{-1}(\Omega)\right)$, on a, pour tout $t, s \in[0, T]$,

$$
\left\|u_{n}(t)\right\|_{2}^{2}=\left\|u_{n}(s)\right\|_{2}^{2}+2 \int_{s}^{t}\left\langle\left(u_{n}\right)_{t}(\xi), u_{n}(\xi)\right\rangle_{H^{-1}, H_{0}^{1}} d \xi
$$

et donc

$\left\|u_{n}(t)\right\|_{2}^{2} \leq\left\|u_{n}(s)\right\|_{2}^{2}+2 \int_{s}^{t}\left|\left\langle\left(u_{n}\right)_{t}(\xi), u_{n}(\xi)\right\rangle_{H^{-1}, H_{0}^{1}}\right| d \xi \leq\left\|u_{n}(s)\right\|_{2}^{2}+2\left\|\left(u_{n}\right)_{t}\right\|_{L^{2}(] 0, T\left[, H^{-1}\right)}\left\|u_{n}\right\|_{L^{2}(] 0, T\left[, H_{0}^{1}\right)}$. 
En intégrant cette inégalité par rapport à $s$ sur $[0, T]$, on en déduit

$$
T\left\|u_{n}(t)\right\|_{2}^{2} \leq\left\|u_{n}\right\|_{L^{2}(] 0, T\left[, L^{2}(\Omega)\right)}^{2}+2 T\left\|\left(u_{n}\right)_{t}\right\|_{L^{2}(] 0, T\left[, H^{-1}\right)}\left\|u_{n}\right\|_{L^{2}(] 0, T\left[, H_{0}^{1}\right)} .
$$

Ceci montre que la suite $\left(u_{n}(t)\right)_{n \in \mathbb{N}^{\star}}$ est bornée dans $L^{2}(\Omega)$ pour tout $t \in[0, T]$ (et même uniformément par rapport à $t)$. On en déduit que la suite $\left(u_{n}(t)\right)_{n \in \mathbb{N}^{\star}}$ est relativement compacte dans $H^{-1}(\Omega)$ pour tout $t \in[0, T]$. On peut donc appliquer le théorème d'Ascoli et conclure, comme cela est indiqué au début de cette étape, que $u(0)=u_{0}$ p.p.. Ceci termine la démonstration du fait que $u$ est solution de 4.19) et donc la démonstration de la partie "existence" du théorème 4.28 .

Dans la démonstration, nous avons obtenu les estimations suivantes

$$
\begin{gathered}
\|u\|_{L^{2}(] 0, T\left[, H_{0}^{1}(\Omega)\right)} \leq\left\|u_{0}\right\|_{2}+\|f\|_{L^{2}(] 0, T\left[, H^{-1}(\Omega)\right)}, \\
\left\|\partial_{t} u\right\|_{L^{2}(] 0, T\left[, H^{-1}(\Omega)\right)} \leq\left\|u_{0}\right\|_{2}+2\|f\|_{L^{2}(] 0, T\left[, H^{-1}(\Omega)\right)} .
\end{gathered}
$$

Enfin, comme $u \in L^{2}(] 0, T\left[, H_{0}^{1}(\Omega)\right)$ et $\partial_{t} u \in L^{2}(] 0, T\left[, H^{-1}(\Omega)\right)$, le lemme 4.26 donne, pour tout $t$,

$$
\|u(t)\|_{2}^{2}=\left\|u_{0}\right\|_{2}^{2}+2 \int_{0}^{t}\left\langle\partial_{t} u(s), u(s)\right\rangle_{H^{-1}, H_{0}^{1}} d s,
$$

on en déduit que

$$
\|u(t)\|_{2}^{2} \leq\left\|u_{0}\right\|_{2}^{2}+\left\|\partial_{t} u\right\|_{L^{2}(] 0, T\left[, H^{-1}(\Omega)\right)}^{2}+\|u\|_{L^{2}(] 0, T\left[, H_{0}^{1}(\Omega)\right)}^{2}, \text { pour tout } t \in[0, T[.
$$

Etape 7, unicité. On montre maintenant la partie "unicité" du théorème 4.28 Soit $u_{1}, u_{2}$ deux solutions de (4.19). On pose $u=u_{1}-u_{2}$. En faisant la différence des équations satisfaites par $u_{1}$ et $u_{2}$ et en prenant, pour $t \in[0, T]$, $v=u 1_{0, t[}$ comme fonction test, on obtient

$$
\int_{0}^{t}\left\langle\partial_{t} u(s), u(s)\right\rangle_{H^{-1}, H_{0}^{1}} d s+\int_{0}^{t} \int_{\Omega} \nabla u(s) \cdot \nabla u(s) \mathrm{d} x d s=0 .
$$

Comme $u \in L^{2}(] 0, T\left[, H_{0}^{1}(\Omega)\right)$ et $\partial_{t} u \in L^{2}(] 0, T\left[, H^{-1}(\Omega)\right)$, on a, d'après la section 4.2 .

$$
\frac{1}{2}\left(\|u(t)\|_{2}^{2}-\left(\|u(0)\|_{2}^{2}\right)=\int_{0}^{t}\left\langle\partial_{t} u(s), u(s)\right\rangle_{H^{-1}, H_{0}^{1}} d s .\right.
$$

On en déduit, pour tout $t \in[0, T]$,

$$
\left(\|u(t)\|_{2}^{2}-\left(\|u(0)\|_{2}^{2}\right)+2 \int_{0}^{t} \int_{\Omega} \nabla u(s) \cdot \nabla u(s) \mathrm{d} x d s=0 .\right.
$$

Enfin, comme $u(0)=0$, on obtient bien, finalement, $u(t)=0$ p.p. dans $\Omega$, pour tout $t \in[0, T]$. Ce qui montre la partie "unicité" du théorème 4.28 .

Nous allons maintenant donner quelques propriétés complémentaires sur la solution de 4.19)

Proposition 4.29 (Dépendance continue) Soit $\Omega$ un ouvert borné de $\mathbb{R}^{N}$ et $T>0$. Pour $u_{0} \in L^{2}(\Omega)$ et $f \in L^{2}(] 0, T\left[, H^{-1}(\Omega)\right)$, on note $T\left(u_{0}, f\right)$ la solution du problème 4.19]. L'opérateur T est linéaire continu de $L^{2}(\Omega) \times L^{2}(] 0, T\left[, H^{-1}(\Omega)\right)$ dans $L^{2}(] 0, T\left[, H_{0}^{1}(\Omega)\right)$ et dans $C\left([0, T], L^{2}(\Omega)\right)$. 
Démonstration Il suffit de reprendre les estimations vues dans le théorème 4.28, pour $u$ solution de 4.19]. :

$$
\begin{aligned}
& \|u\|_{L^{2}(] 0, T\left[, H_{0}^{1}\right)} \leq\|f\|_{L^{2}(] 0, T\left[, H^{-1}\right)}+\left\|u_{0}\right\|_{2}, \\
& \|u(t)\|_{2}^{2} \leq\left\|u_{0}\right\|_{2}^{2}+\left\|\partial_{t} u\right\|_{L^{2}(] 0, T\left[, H^{-1}\right)}^{2}+\|u\|_{L^{2}(] 0, T\left[, H_{0}^{1}\right)}^{2} \text { pour tout } t \in[0, T], \\
& \left\|\partial_{t} u\right\|_{L^{2}(] 0, T\left[, H^{-1}\right)} \leq\left\|u_{0}\right\|_{2}+2\|f\|_{L^{2}(] 0, T\left[H^{-1}\right)} .
\end{aligned}
$$

On en déduit bien la continuité de $T$ dans les espaces annoncés.

Proposition 4.30 (Positivité et principe du maximum) Soit $\Omega$ un ouvert borné de $\mathbb{R}^{N}$, T>0 et $u_{0} \in L^{2}(\Omega)$. On note u la solution de (4.19) avec $f=0$.

1. On suppose $u_{0} \geq 0$ p.p.. On a alors $u(t) \geq 0$ p.p. et pour tout $t \in[0, T]$.

2. On suppose que $u_{0} \in L^{\infty}(\Omega)$. Soit $A, B \in \mathbb{R}$ t.q. $A \leq 0 \leq B$ et $A \leq u_{0} \leq B$ p.p.. On a alors $A \leq u(t) \leq B$ p.p. et pour tout $t \in[0, T]$.

Démonstration Pour démontrer le premier item, on montre plutôt (ce qui est équivalent) que $u_{0} \leq 0$ p.p. implique $u(t) \leq 0$ p.p. pour tout $t \in[0, T]$. On suppose donc que $u_{0} \leq 0$ p.p.. On utilise alors le lemme 4.31 avec $\varphi(s)=s^{+}$. Il donne que $u^{+} \in L^{2}(] 0, T\left[, H_{0}^{1}(\Omega)\right)$. Pour $t \in[0, T]$, on peut donc prendre $v=u^{+} 1_{0, t[}$ dans l'équation de 4.19 , et on obtient

$$
\int_{0}^{t}\left\langle\partial_{t} u(s), u^{+}(s)\right\rangle_{H^{-1}, H_{0}^{1}} d s+\int_{0}^{t} \int_{\Omega} \nabla u(s) \cdot \nabla u^{+}(s) \mathrm{d} x d s=0 .
$$

En utilisant encore le lemme 4.31, on a donc

$$
\frac{1}{2}\left\|u^{+}(t)\right\|_{2}^{2}-\frac{1}{2}\left\|u^{+}(0)\right\|_{2}^{2}+\int_{0}^{t}\left\|u^{+}(s)\right\|_{H_{0}^{1}}^{2} d s=0 .
$$

Comme $u^{+}(0)=u_{0}^{+}=0$ p.p., on en déduit bien que $\left\|u^{+}(t)\right\|_{2}=0$ et donc $u(t) \leq 0$ p.p..

La démonstration du deuxième item est semblable en utilisant le lemme 4.31 avec $\varphi(s)=(s-B)^{+}$et $\varphi(s)=$ $(s-A)^{-}$.

Lemme 4.31 Soit $\Omega$ un ouvert borné de $\mathbb{R}^{N}$ et $T>0$. Soit $\varphi$ une fonction lipchitzienne de $\mathbb{R}$ dans $\mathbb{R}$ t.q. $\varphi(0)=0$. On définit $\Phi$ par

$$
\Phi(\xi)=\int_{0}^{\xi} \varphi(\bar{\xi}) d \bar{\xi} \text { pour } \xi \in \mathbb{R} .
$$

Soit $u \in L^{2}(] 0, T\left[, H_{0}^{1}(\Omega)\right)$ t.q. $\partial_{t} u \in L^{2}(] 0, T\left[, H^{-1}(\Omega)\right)$. On a alors $\varphi(u)=L^{2}(] 0, T\left[, H_{0}^{1}(\Omega)\right)$, $\Phi(u) \in$ $C\left([0, T], L^{1}(\Omega)\right)$ et, pour tout $t_{1}, t_{2} \in[0, T]$,

$$
\int_{\Omega} \Phi\left(u\left(t_{2}\right)\right) \mathrm{d} x-\int_{\Omega} \Phi\left(u\left(t_{1}\right)\right) \mathrm{d} x=\int_{t_{1}}^{t_{2}}\left\langle\partial_{t} u(s), \varphi(u(s)\rangle_{H^{-1}, H_{0}^{1}} d s .\right.
$$

On a aussi pour presque tout $t \in] 0, T\left[, \varphi(u(t)) \in H_{0}^{1}(\Omega)\right.$ et $\nabla \varphi(u(t))=\varphi^{\prime}(u(t)) \nabla u$ p.p., c'est-à-dire, en étant plus précis, $\nabla \varphi(u)(x, t)=\varphi^{\prime}(u(x, t)) \nabla u(x, t)$ pour presque tout $x \in \Omega$. Dans cette égalité, on peut prendre pour $\varphi^{\prime}(u(x, t)) n^{\prime i m p o r t e ~ q u e l l e ~ v a l e u r ~ s i ~} \varphi$ n'est pas dérivable au point $u(x, t)$. En particulier ceci montre que, pour tout $a \in \mathbb{R}, \nabla u=0$ p.p. sur l'ensemble $\{u=a\}$. 
Démonstration Ce lemme est la version "parabolique" des lemmes 2.21 et 2.22 vus précédemment. La démonstration consiste à considérer d'abord (comme dans le lemme 2.21) que $\varphi$ est de classe $C^{1}$ et à régulariser $u$. Puis à approcher $\varphi$ par des fonctions de classe $C^{1}$ (au moins lorsque $\varphi$ est dérivable sauf en un nombre fini de points, le cas général étant plus difficile). Cette preuve n'est pas détaillée ici.

On donne maintenant l'équivalence entre la formulation faible $4.19 \mathrm{p}$ et une autre formulation faible, la formulation (4.23). Cette deuxième formulation est, en particulier, intéressante lorsque l'on cherche à prouver la convergence des solutions approchées obtenues par une discrétisation en espace et en temps.

Proposition 4.32 (Equivalence entre deux formulations faibles) Soit $\Omega$ un ouvert borné de $\mathbb{R}^{N}, T>0, u_{0} \in$ $L^{2}(\Omega)$ et $f \in L^{2}(] 0, T\left[, H^{-1}(\Omega)\right.$ ) (on identifie, comme d'habitude $L^{2}(\Omega)$ avec $L^{2}(\Omega)^{\prime}$ ). Alors u est solution de (4.19) si et seulement si u vérifie :

$$
\left\{\begin{array}{l}
u \in L^{2}(] 0, T\left[, H_{0}^{1}(\Omega)\right), \\
-\int_{0}^{T} \int_{\Omega} u \varphi_{t} \mathrm{~d} x \mathrm{~d} t-\int_{\Omega} u_{0}(x) \varphi(x, 0) \mathrm{d} x+\int_{0}^{T} \int_{\Omega} \nabla u \cdot \nabla \varphi \mathrm{d} x \mathrm{~d} t \\
=\int_{0}^{T}<f(s), \varphi(\cdot, s)>_{H^{-1}, H_{0}^{1}} d s \text { pour tout } \varphi \in C_{c}^{\infty}([0, T[\times \Omega, \mathbb{R}) .
\end{array}\right.
$$

Démonstration On montre tout d'abord que " $u$ solution de 4.19$] \Rightarrow u$ est solution de 4.23 ". On suppose donc que $u$ est solution de 4.19]. Soit $\varphi \in C_{c}^{\infty}\left(\Omega \times\left[0, T[, \mathbb{R})\right.\right.$. Pour $n \in \mathbb{N}^{\star}$, on pose

$$
\varphi_{n}(x, t)=\sum_{i=0}^{n-1} 1_{] t_{i}, t_{i+1}[}(t) \varphi\left(x, t_{i}\right),
$$

où $t_{i}=(i / n) T$. Comme $\varphi$ est une fonction régulière, il est clair que $\varphi_{n} \in L^{2}(] 0, T\left[, H_{0}^{1}(\Omega)\right)$ et que $\varphi_{n} \rightarrow \varphi$ dans $L^{2}(] 0, T\left[, H_{0}^{1}(\Omega)\right)$ quand $n \rightarrow+\infty$. Comme $\varphi_{n} \in L^{2}(] 0, T\left[, H_{0}^{1}(\Omega)\right)$ et que $u$ est solution de 4.19], on a

$$
\int_{0}^{T}\left\langle\partial_{t} u, \varphi_{n}\right\rangle_{H^{-1}, H_{0}^{1}} d t+\int_{0}^{T} \int_{\Omega} \nabla u \cdot \nabla \varphi_{n} \mathrm{~d} x \mathrm{~d} t=\int_{0}^{T}\left\langle f, \varphi_{n}\right\rangle_{H^{-1}, H_{0}^{1}} \mathrm{~d} t .
$$

On pose $T_{n}=\int_{0}^{T}\left\langle\partial_{t} u, \varphi_{n}\right\rangle_{H^{-1}, H_{0}^{1}} d t$. Comme $\varphi_{n} \rightarrow \varphi$ dans $L^{2}(] 0, T\left[, H_{0}^{1}(\Omega)\right)$ quand $n \rightarrow+\infty$, l'égalité précédente donne

$$
\lim _{n \rightarrow+\infty} T_{n}=\int_{0}^{T}\left\langle\partial_{t} u, \varphi\right\rangle_{H^{-1}, H_{0}^{1}} d t=-\int_{0}^{T} \int_{\Omega} \nabla u \cdot \nabla \varphi \mathrm{d} x \mathrm{~d} t+\int_{0}^{T}\langle f, \varphi\rangle_{H^{-1}, H_{0}^{1}} \mathrm{~d} t .
$$

On va maintenant calculer $\lim _{n \rightarrow+\infty} T_{n}$ en utilisant 4.24]. On a

$$
\begin{aligned}
T_{n}=\int_{0}^{T}\left\langle\partial_{t} u(t), \sum_{i=0}^{n-1} 1_{] t_{i}, t_{i+1}}(t) \varphi\left(\cdot, t_{i}\right)\right\rangle_{H^{-1}, H_{0}^{1}} d t & =\sum_{i=0}^{n-1} \int_{t_{i}}^{t_{i+1}}\left\langle\partial_{t} u(t), \varphi\left(\cdot, t_{i}\right)\right\rangle_{H^{-1}, H_{0}^{1}} d t \\
& =\sum_{i=0}^{n-1}\left\langle\int_{t_{i}}^{t_{i+1}} \partial_{t} u(t) d t, \varphi\left(\cdot, t_{i}\right)\right\rangle_{H^{-1}, H_{0}^{1}}
\end{aligned}
$$

Comme $u, \partial_{t} u \in L^{2}(] 0, T\left[, H^{-1}(\Omega)\right)$ (on rappelle que $H_{0}^{1}(\Omega) \subset H^{-1}(\Omega)$ par l'identification de $L^{2}(\Omega)$ avec son dual), on a (d'après la section 4.2$] u \in C\left([0, T], H^{-1}(\Omega)\right)$ et

$$
\int_{t_{i}}^{t_{i+1}} \partial_{t} u(t) \mathrm{d} t=u\left(t_{i+1}\right)-u\left(t_{i}\right) \in H^{-1}(\Omega)
$$


On a donc

$$
T_{n}=\sum_{i=0}^{n-1}\left\langle u\left(t_{i+1}\right)-u\left(t_{i}\right), \varphi\left(\cdot, t_{i}\right)\right\rangle_{H^{-1}, H_{0}^{1}}=\sum_{i=0}^{n-1} \int_{\Omega}\left(u\left(x, t_{i+1}\right)-u\left(x, t_{i}\right)\right) \varphi\left(x, t_{i}\right) \mathrm{d} x .
$$

La dernière égalité venant de la manière avec laquelle un élément de $H_{0}^{1}(\Omega)$ est considéré comme un élément de $H^{-1}(\Omega)$. Une intégration par parties discrète donne alors (en remarquant que $\varphi\left(\cdot, t_{n}\right)=0$ )

$$
T_{n}=-\int_{\Omega} u(x, 0) \varphi(x, 0) \mathrm{d} x+\sum_{i=1}^{n} \int_{\Omega}\left(\varphi\left(x, t_{i-1}\right)-\varphi\left(x, t_{i}\right)\right) u\left(x, t_{i}\right) \mathrm{d} x .
$$

Puis, comme $\varphi$ est une fonction régulière,

$$
\begin{aligned}
& T_{n}=-\int_{\Omega} u_{0}(x) \varphi(x, 0) \mathrm{d} x-\sum_{i=1}^{n} \int_{\Omega}\left(\int_{t_{i-1}}^{t_{i}} \varphi_{t}(x, t) d t\right) u\left(x, t_{i}\right) \mathrm{d} x \\
& =-\int_{\Omega} u_{0}(x) \varphi(x, 0) \mathrm{d} x-\int_{0}^{T} \int_{\Omega} \varphi_{t}(x, t)\left(\sum_{i=1}^{n} 1_{] t_{i-1}, t_{i}}\left[u\left(x, t_{i}\right)\right) \mathrm{d} x \mathrm{~d} t\right. \\
& =-\int_{\Omega} u_{0}(x) \varphi(x, 0) \mathrm{d} x-\int_{0}^{T} \int_{\Omega} \varphi_{t}(x, t)\left(u(x, t)+R_{n}(x, t)\right) \mathrm{d} x \mathrm{~d} t
\end{aligned}
$$

avec $R_{n}(x, t)=\sum_{i=1}^{n} 1_{] t_{i-1}, t_{i}}\left[u\left(x, t_{i}\right)-u(x, t)\right.$. Pour tout $t \in[0, T]$, on a

$$
\left\|R_{n}(\cdot, t)\right\|_{L^{2}(\Omega)} \leq \max \left\{\left\|u\left(s_{1}\right)-u\left(s_{2}\right)\right\|_{L^{2}(\Omega)}, s_{1}, s_{2} \in[0, T],\left|s_{1}-s_{2}\right| \leq \frac{T}{n}\right\} .
$$

Comme $u \in C\left([0, T], L^{2}(\Omega)\right)$, on en déduit que $\lim _{n \rightarrow+\infty}\left\|R_{n}(\cdot, t)\right\|_{L^{2}(\Omega)}=0$ uniformément par rapport à $t \in[0, T]$ et donc que

$$
\lim _{n \rightarrow+\infty} \int_{0}^{T} \int_{\Omega} \varphi_{t}(x, t) R_{n}(x, t) \mathrm{d} x \mathrm{~d} t=0
$$

En résumé, on a donc

$$
\lim _{n \rightarrow+\infty} T_{n}=-\int_{\Omega} u_{0}(x) \varphi(x, 0) \mathrm{d} x-\int_{0}^{T} \int_{\Omega} \varphi_{t}(x, t) u(x, t) \mathrm{d} x \mathrm{~d} t .
$$

Avec 4.25 on a donc, pour tout $\varphi \in C_{c}^{\infty}([0, T[\times \Omega, \mathbb{R})$,

$$
-\int_{0}^{T} \int_{\Omega} \varphi_{t}(x, t) u(x, t) \mathrm{d} x \mathrm{~d} t-\int_{\Omega} u_{0}(x) \varphi(x, 0) \mathrm{d} x+\int_{0}^{T} \int_{\Omega} \nabla u \cdot \nabla \varphi \mathrm{d} x \mathrm{~d} t=\int_{0}^{T}\langle f, \varphi\rangle_{H^{-1}, H_{0}^{1}} \mathrm{~d} t .
$$

Ceci montre que $u$ est bien solution de 4.23 .

On montre maintenant que " $u$ solution de $4.23 \Rightarrow u$ est solution de 4.19 ". On suppose donc que $u$ est solution de 4.23). On veut montrer que $u$ est solution de 4.19). On va raisonner en deux étapes. On va d'abord montrer que $\partial_{t} u \in L^{2}(] 0, T\left[, H^{-1}(\Omega)\right)$ et $\partial_{t} u=\Delta u+f$ (remarquer que $\Delta u, f \in L^{2}(] 0, T\left[, H^{-1}(\Omega)\right)$ puis que $u(0)=u_{0}$.

Etape 1 On montre ici que $\partial_{t} u \in L^{2}(] 0, T\left[, H^{-1}(\Omega)\right)$ et $\partial_{t} u=\Delta u+f$. On utilise la définition de $\partial_{t} u$. On a $\partial_{t} u \in \mathcal{D}_{E}^{\star}$, avec $E=H_{0}^{1}(\Omega)$, et pour tout $\phi \in C^{\infty}(] 0, T[, \mathbb{R})$

$$
\left\langle\partial_{t} u, \phi\right\rangle_{\mathcal{D}_{E}^{\star}, \mathcal{D}}=-\int_{0}^{T} u(t) \phi^{\prime}(t) \mathrm{d} t \in H_{0}^{1}(\Omega) .
$$


Pour montrer que $\partial_{t} u \in L^{2}(] 0, T\left[, H^{-1}(\Omega)\right)$ et $\partial_{t} u=\Delta u+f$, il s'agit donc de montrer que, pour tout $\phi \in$ $C_{c}^{\infty}(] 0, T[, \mathbb{R})$,

$$
-\int_{0}^{T} u(t) \phi^{\prime}(t) \mathrm{d} t=\int_{0}^{T}(\Delta u(t)+f(t)) \phi(t) \mathrm{d} t .
$$

Noter que le membre de gauche de cette égalité est dans $H_{0}^{1}(\Omega)$ et donc dans $H^{-1}(\Omega)$ (grâce à l'identification entre $L^{2}(\Omega)$ et son dual) et que le membre de droite est dans $H^{-1}(\Omega)$. Pour montrer l'égalité de ces deux termes, il suffit de montrer que

$$
\left\langle-\int_{0}^{T} u(t) \phi^{\prime}(t) \mathrm{d} t, \psi\right\rangle_{H^{-1}, H_{0}^{1}}=\left\langle\int_{0}^{T}(\Delta u(t)+f(t)) \phi(t) \mathrm{d} t, \psi\right\rangle_{H^{-1}, H_{0}^{1}} \text { pour tout } \psi \in H_{0}^{1}(\Omega),
$$

c'est-à-dire que

$$
-\int_{0}^{T}\left\langle u(t) \phi^{\prime}(t), \psi\right\rangle_{H^{-1}, H_{0}^{1}} d t=\int_{0}^{T}\langle(\Delta u(t)+f(t)) \phi(t), \psi\rangle_{H^{-1}, H_{0}^{1}} d t \text { pour tout } \psi \in H_{0}^{1}(\Omega) .
$$

Par densité de $C_{c}^{\infty}(\Omega, \mathbb{R})$ dans $H_{0}^{1}(\Omega)$, il suffit de considérer $\psi \in C_{c}^{\infty}(\Omega, \mathbb{R})$. En utilisant la manière donc $H_{0}^{1}(\Omega)$ est inclus dans $H^{-1}(\Omega)$, on a

$$
-\int_{0}^{T}\left\langle u(t) \phi^{\prime}(t), \psi\right\rangle_{H^{-1}, H_{0}^{1}} d t=-\int_{0}^{T} \int_{\Omega} u(x, t) \phi^{\prime}(t) \psi(x) \mathrm{d} x \mathrm{~d} t .
$$

D'autre part, on a

$$
\begin{array}{r}
\int_{0}^{T}\langle(\Delta u(t)+f(t)) \phi(t), \psi\rangle_{H^{-1}, H_{0}^{1}} d t=\int_{0}^{T} \phi(t)\langle\Delta u(t)+f(t), \psi\rangle_{H^{-1}, H_{0}^{1}} d t \\
=-\int_{0}^{T} \phi(t) \int_{\Omega} \nabla u(x, t) \cdot \nabla \psi(x) \mathrm{d} x \mathrm{~d} t+\int_{0}^{T} \phi(t)\langle f, \psi\rangle_{H^{-1}, H_{0}^{1}} d t .
\end{array}
$$

En résumé, pour terminer l'étape 1, il suffit donc de montrer que

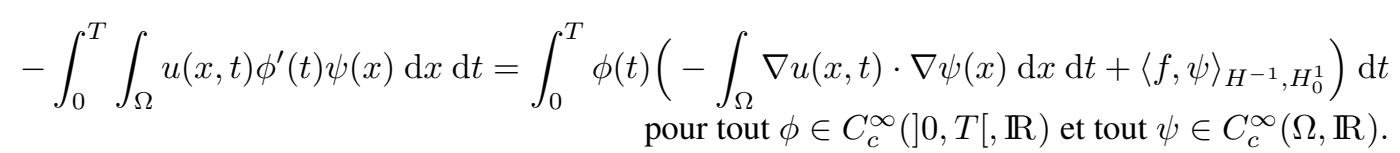

Comme cela a été déjà dit, ceci donnera $\partial_{t} u \in L^{2}(] 0, T\left[, H^{-1}(\Omega)\right)$ et $\partial_{t} u=\Delta u+f$.

Pour montrer 4.26), on utilise 4.23). Soit $\phi \in C_{c}^{\infty}(] 0, T[, \mathbb{R})$ et $\psi \in C_{c}^{\infty}(\Omega, \mathbb{R})$. On choisit dans 4.23, $\varphi(x, t)=\phi(t) \psi(x)$ (ce qui est possible car on a bien $\varphi \in C_{c}^{\infty}(] 0, T[\times \Omega, \mathbb{R})$. On obtient

$$
-\int_{0}^{T} \int_{\Omega} u(x, t) \phi^{\prime}(t) \psi(x) \mathrm{d} x \mathrm{~d} t+\int_{0}^{T} \int_{\Omega}(\nabla u(x, t) \cdot \nabla \psi(x)) \phi(t) \mathrm{d} x \mathrm{~d} t=\int_{0}^{T}\langle f(t), \phi(t) \psi\rangle_{H^{-1}, H_{0}^{1}} \mathrm{~d} t .
$$

Ceci donne (4.26) et termine donc l'étape 1 , c'est-à-dire $\partial_{t} u \in L^{2}(] 0, T\left[, H^{-1}(\Omega)\right)$ et $\partial_{t} u=\Delta u+f$ (ce qui l'équation demandée dans (4.19)).

Etape 2 Comme $u \in L^{2}(] 0, T\left[, H_{0}^{1}(\Omega)\right)$ et $\partial_{t} u \in L^{2}(] 0, T\left[, H^{-1}(\Omega)\right.$ ), on a (d'après la section 4.2$), u \in C([0, T]$, $L^{2}(\Omega)$ ). On montre dans cette deuxième étape que $u(0)=u_{0}$. Pour $n \in \mathbb{N}^{\star}$, on choisit une fonction $r_{n}$ de $C_{c}^{\infty}\left(\left[0, T[, \mathbb{R})\right.\right.$ décroissante et t.q. $\left|r_{n}^{\prime}(t)\right| \leq 2 n$ pour tout $t, r_{n}(0)=1$ et $r_{n}(t)=0$ si $t \geq 1 / n$. 
Soit $\psi \in C_{c}^{\infty}(\Omega)$ et $n \in \mathbb{N}^{\star}$ t.q. $1 / n<T$. On prend $\varphi(x, t)=r_{n}(t) \psi(x)$ dans 4.23 . On obtient

$$
\begin{array}{r}
-\int_{0}^{\frac{1}{n}} \int_{\Omega} u(x, t) r_{n}^{\prime}(t) \psi(x) \mathrm{d} x \mathrm{~d} t-\int_{\Omega} u_{0}(x) \psi(x) \mathrm{d} x+\int_{0}^{\frac{1}{n}} \int_{\Omega} \nabla u(x, t) \cdot \nabla \psi(x) r_{n}(t) \mathrm{d} x \mathrm{~d} t \\
=\int_{0}^{\frac{1}{n}}\langle f(t), \psi\rangle_{H^{-1}, H_{0}^{1}} r_{n}(t) d t
\end{array}
$$

c'est-à-dire

$$
T_{n}=\int_{\Omega} u_{0}(x) \psi(x) \mathrm{d} x-R_{n}+S_{n}
$$

avec

$$
\begin{gathered}
T_{n}=-\int_{0}^{\frac{1}{n}} \int_{\Omega} u(x, t) r_{n}^{\prime}(t) \psi(x) \mathrm{d} x \mathrm{~d} t \\
R_{n}=\int_{0}^{\frac{1}{n}} \int_{\Omega} \nabla u(x, t) \cdot \nabla \psi(x) r_{n}(t) \mathrm{d} x \mathrm{~d} t \text { et } S_{n}=\int_{0}^{\frac{1}{n}}\langle f(t), \psi\rangle_{H^{-1}, H_{0}^{1}} r_{n}(t) d t .
\end{gathered}
$$

On montre tout d'abord que $R_{n}$ et $S_{n}$ tendent vers 0 . En effet, on a

$$
\begin{aligned}
\left|R_{n}\right| \leq \int_{0}^{\frac{1}{n}} \int_{\Omega}|\nabla u(x, t)||\nabla \psi(x)| \mathrm{d} x \mathrm{~d} t & \\
\leq\left(\int_{0}^{\frac{1}{n}} \int_{\Omega}|\nabla u(x, t)|^{2} \mathrm{~d} x \mathrm{~d} t\right)^{\frac{1}{2}} & \left(\int_{0}^{\frac{1}{n}} \int_{\Omega}|\nabla \psi(x)|^{2} \mathrm{~d} x \mathrm{~d} t\right)^{\frac{1}{2}} \\
& \leq\|u\|_{L^{2}(] 0, T\left[, H_{0}^{1}\right)} \frac{1}{\sqrt{n}}\|\psi\|_{H_{0}^{1}} .
\end{aligned}
$$

On a donc $\lim _{n \rightarrow+\infty} R_{n}=0$. On a aussi $\lim _{n \rightarrow+\infty} S_{n}=0$ car

$$
\left|S_{n}\right| \leq\|f\|_{L^{2}(] 0, T\left[, H^{-1}\right)} \frac{1}{\sqrt{n}}\|\psi\|_{H_{0}^{1}}
$$

On remarque maintenant que

$$
\begin{array}{r}
T_{n}=-\int_{0}^{\frac{1}{n}} \int_{\Omega} u(x, 0) r_{n}^{\prime}(t) \psi(x) \mathrm{d} x \mathrm{~d} t-\int_{0}^{\frac{1}{n}} \int_{\Omega}(u(x, t)-u(x, 0)) r_{n}^{\prime}(t) \psi(x) \mathrm{d} x \mathrm{~d} t \\
=\int_{\Omega} u(x, 0) \psi(x) \mathrm{d} x-\int_{0}^{\frac{1}{n}} \int_{\Omega}(u(x, t)-u(x, 0)) r_{n}^{\prime}(t) \psi(x) \mathrm{d} x \mathrm{~d} t
\end{array}
$$

(On a utilisé ici $r_{n}(0)=1$ et $r_{n}(1 / n)=0$.) On majore de dernier terme de cette égalité :

$$
\left|\int_{0}^{\frac{1}{n}} \int_{\Omega}(u(x, t)-u(x, 0)) r_{n}^{\prime}(t) \psi(x) \mathrm{d} x \mathrm{~d} t\right| \leq \frac{1}{n} 2 n\|\psi\|_{L^{2}(\Omega)} \max _{t \in\left[0, \frac{1}{n}\right]}\|u(\cdot, t)-u(\cdot, 0)\|_{L^{2}(\Omega)} .
$$

Comme $u \in C\left([0, T], L^{2}(\Omega)\right)$ ce denier terme tend vers 0 quand $n \rightarrow+\infty$ et on a donc, finalement,

$$
\lim _{n \rightarrow+\infty} T_{n}=\int_{\Omega} u(x, 0) \psi(x) \mathrm{d} x .
$$

Avec (4.27), comme $\lim _{n \rightarrow+\infty} R_{n}=\lim _{n \rightarrow+\infty} S_{n}=0$, on en déduit

$$
\int_{\Omega} u(x, 0) \psi(x) \mathrm{d} x=\int_{\Omega} u_{0}(x) \psi(x) \mathrm{d} x \text { pour tout } \psi \in C_{c}^{\infty}(\Omega) .
$$


Ceci permet de conclure que $u(0)=u_{0}$ et termine la démonstration de la proposition 4.32

Nous donnons maintenant un théorème de compacité qui sera très utile pour la résolution de problèmes non linéaires comme ceux de la section 4.4. C'est l'équivalent parabolique des théorèmes de compacité vus pour les problèmes elliptiques. Soit $\Omega$ est un ouvert borné de $\mathbb{R}^{N}$. Pour $f \in H^{-1}(\Omega)$, on note $T(f)$ la solution faible de l'équation $-\Delta u=f$ avec $u \in H_{0}^{1}(\Omega)$. On a déjà montré que l'opérateur $T$ était compact de $H^{-1}(\Omega)$ dans $L^{2}(\Omega)$.

Proposition 4.33 (Compacité) Soit $\Omega$ un ouvert borné de $\mathbb{R}^{N}$ et $T>0$. On identifie $L^{2}(\Omega)^{\prime}$ avec $L^{2}(\Omega)$. Pour $f \in L^{2}(] 0, T\left[, H^{-1}(\Omega)\right)$ et $u_{0} \in L^{2}(\Omega)$ on note $T\left(f, u_{0}\right)$ la solution de 4.19 .

L'opérateur T est compact de $L^{2}(] 0, T\left[, H^{-1}(\Omega)\right) \times L^{2}(\Omega)$ dans $L^{2}(] 0, T\left[, L^{2}(\Omega)\right)$.

Démonstration La proposition 4.29 donne déjà la continuité de $T$. Il reste donc à montrer que $T$ transforme les parties bornées de $L^{2}(] 0, T\left[, H^{-1}(\Omega)\right) \times L^{2}(\Omega)$ en parties relativement compactes de $L^{2}(] 0, T\left[, L^{2}(\Omega)\right)$. Soit donc $A$ une partie bornée de $L^{2}(] 0, T\left[, H^{-1}(\Omega)\right) \times L^{2}(\Omega)$. On pose $B=\left\{T\left(f, u_{0}\right),\left(f, u_{0}\right) \in A\right\}$. Les estimations vues dans le théorème 4.28 montrent que $B$ est une partie bornée de $L^{2}(] 0, T\left[, H_{0}^{1}(\Omega)\right)$ et que $\left\{\partial_{t} u, u \in B\right\}$ est une partie bornée de $L^{2}(] 0, T\left[, H^{-1}(\Omega)\right)$. Le lemme de compacité donné ci-après (lemme 4.34), dû à J. L. Lions, donne alors la relative compacité de $A$ dans $L^{2}(] 0, T\left[, L^{2}(\Omega)\right)$.

Lemme 4.34 (Compacité espace-temps, cadre $L^{\mathbf{2}}$ ) Soit $\Omega$ un ouvert borné de $\mathbb{R}^{N}$ et $\left(u_{n}\right)_{n \in \mathbb{N}}$ une suite de $L^{2}(] 0, T\left[, L^{2}(\Omega)\right)$. On identifie $L^{2}(\Omega)^{\prime}$ avec $L^{2}(\Omega)$. On suppose que

1. la suite $\left(u_{n}\right)_{n \in \mathbb{N}}$ est bornée dans $L^{2}(] 0, T\left[, H_{0}^{1}(\Omega)\right)$,

2. la suite $\left(\left(\partial_{t} u_{n}\right)\right)_{n \in \mathbb{N}}$ est bornée dans $L^{2}(] 0, T\left[, H^{-1}(\Omega)\right)$.

Alors, la suite $\left(u_{n}\right)_{n \in \mathbb{N}}$ est relativement compacte dans $L^{2}(] 0, T\left[, L^{2}(\Omega)\right)$.

Démonstration Ce lemme sera démontré plus loin dans un cadre plus général, voir théorème 4.38 .

Remarque 4.35 (Opérateurs elliptiques généraux) Les résultats de ce paragraphe, c'est-à-dire le théorème 4.28 et les propositions 4.29 , 4.30 , 4.32 et 4.33 , sont encore vrais en remplaçant l'opérateur $\Delta u \operatorname{par} \operatorname{div}(A \nabla u)$ si la matrice $A$ est à coefficients dans $L^{\infty}(\Omega)$ et qu'il existe $\alpha>0$ t.q. $A \xi \cdot \xi \geq \alpha|\xi|^{2}$ p.p. et pour tout $\xi \in \mathbb{R}^{N}$ (exercice 4.5). Il est aussi possible de considérer le cas où les coefficients de la matrice $A$ dépendent aussi de $t$. Une possibilité est alors de faire une discrétisation en temps et de remplacer sur chaque intervalle de temps la matrice $A$ par sa moyenne sur l'intervalle considéré. On résout alors le problème sur chacun de ces intervalles temporels. Il suffit ensuite d'obtenir des estimations sur la solution approchée et de passer à la limite sur le pas de discrétisation.

\subsection{Existence et unicité pour des problèmes paraboliques non linéaires}

Comme dans le cas elliptique, l'existence de la solution d'un problème parabolique non linéaire peut se prouver par point fixe de Schauder ou par degré topologique. Nous donnons ci après deux exemples.

\subsubsection{Premier exemple : diffusion non linéaire}

Considérons d'abord l'exemple suivant : soit $\Omega$ un ouvert borné de $\mathbb{R}^{N}$ et $A: \mathbb{R} \rightarrow M_{N}(\mathbb{R})$ (où $M_{N}(\mathbb{R})$ désigne l'ensemble des matrices $N \times N$ à coefficiens réels) t.q.

$$
\begin{aligned}
& \forall s \in \mathbb{R}, A(s)=\left(a_{i, j}(s)\right)_{i, j=1, \ldots, N} \text { où } a_{i, j} \in L^{\infty}(\mathbb{R}) \cap C(\mathbb{R}, \mathbb{R}), \\
& \exists \alpha>0 ; A(s) \xi \cdot \xi \geq \alpha|\xi|^{2}, \forall \xi \in \mathbb{R}^{N}, \forall s \in \mathbb{R}, \\
& f \in L^{2}(] 0, T\left[, H^{-1}(\Omega)\right) \text { et } u_{0} \in L^{2}(\Omega) .
\end{aligned}
$$


Alors on peut montrer par le théorème de Schauder qu'il existe $u$ solution de (toujours avec $L^{2}(\Omega)$ identifié à $\left.L^{2}(\Omega)^{\prime}\right)$ :

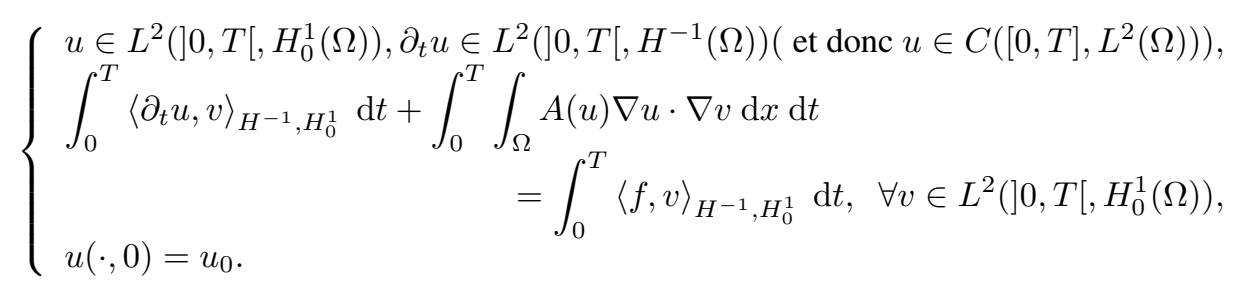

Pour utiliser le théorème de Schauder, on utilise la résolution de problèmes linéaires : soit $\bar{u} \in L^{2}(] 0, T\left[, L^{2}(\Omega)\right)$, on définit l'opérateur $T$ de $L^{2}(] 0, T\left[, L^{2}(\Omega)\right)$ dans $L^{2}(] 0, T\left[, L^{2}(\Omega)\right)$ par $T(\bar{u})=u$ où $u$ est la solution du problème 4.32) où on a remplacé $A(u)$ par $A(\bar{u})$, c'est-à-dire :

$$
\left\{\begin{array}{l}
u \in L^{2}(] 0, T\left[, H_{0}^{1}(\Omega)\right), \partial_{t} u \in L^{2}(] 0, T\left[, H^{-1}(\Omega)\right), \\
\int_{0}^{T}\left\langle\partial_{t} u, v\right\rangle_{H^{-1}, H_{0}^{1}} \mathrm{~d} t+\int_{0}^{T} \int_{\Omega} A(\bar{u}) \nabla u \cdot \nabla v \mathrm{~d} x \mathrm{~d} t \\
=\int_{0}^{T}\langle f, v\rangle_{H^{-1}, H_{0}^{1}} \mathrm{~d} t, \forall v \in L^{2}(] 0, T\left[, H_{0}^{1}(\Omega)\right), \\
u(\cdot, 0)=u_{0} .
\end{array}\right.
$$

On montre ensuite qu'il existe $R>0$ tel que l'image de $T$ est incluse dans $B_{R}$ où $B_{R}$ est la boule (de $L^{2}(] 0, T$, $\left.L^{2}(\Omega)\right)$ ) de rayon $R$ et de centre 0 . On montre que $T$ est continu et que $T$ est compact (par le lemme de compacité espace-temps 4.34. On conclut avec le théorème de Schauder. Ceci est laissé à titre d'exercice (exercice 4.6). Notons qu'on peut aussi montrer l'unicité si $A$ est lipschitzienne.

\subsubsection{Deuxième exemple : diffusion convection non linéaire}

Etudions maintenant un deuxième exemple en utilisant de degré topologique pour l'existence de la solution. Considérons l'équation de convection-diffusion suivante (avec une convection éventuellement non linéaire) :

$$
\begin{aligned}
& \partial_{t} u+\operatorname{div}(\boldsymbol{b} f(u))-\Delta u=0, \\
& \text { avec } \boldsymbol{b} \in L^{2}(] 0, T\left[,\left(L^{2}(\Omega)^{N}\right) \text { et } u_{0} \in L^{2}(\Omega) .\right.
\end{aligned}
$$

On suppose que $f$ est une fonction lipschitzienne bornée de $\mathbb{R}$ dans $\mathbb{R}$ (et $f(u)$ désigne, comme d'habitude, la fonction $(x, t) \mapsto f(u(x, t)))$.

Une formulation faible du problème s'écrit (avec $L^{2}(\Omega)$ identifié à $\left.L^{2}(\Omega)^{\prime}\right)$ :

$$
\left\{\begin{array}{c}
u \in L^{2}(] 0, T\left[, H_{0}^{1}(\Omega)\right), \partial_{t} u \in L^{2}(] 0, T\left[, H^{-1}(\Omega)\right)\left(\text { et donc } u \in C\left([0, T], L^{2}(\Omega)\right)\right) \\
\int_{0}^{T}\left\langle\partial_{t} u, v\right\rangle_{H^{-1}, H_{0}^{1}} \mathrm{~d} t-\int_{0}^{T} \int_{\Omega} \boldsymbol{b} f(u) \cdot \nabla v \mathrm{~d} x \mathrm{~d} t \\
\quad+\int_{0}^{T^{2}} \int_{\Omega} \nabla u \cdot \nabla v \mathrm{~d} x \mathrm{~d} t=0, \forall v \in L^{2}(] 0, T\left[, H_{0}^{1}(\Omega)\right), \\
u(\cdot, 0)=u_{0}
\end{array}\right.
$$

Montrons l'existence d'une solution au problème 4.33 par degré topologique. Pour cela on va montrer que le problème (4.33) peut s'écrire sous la forme $u-h(1, u)=0$, où l'application $h$, définie de $[0,1] \times E$ dans $E$, avec $E=L^{2}(] 0, T\left[, L^{2}(\Omega)\right)$, vérifie : 
1. $h$ est compacte,

2. il existe $R \in \mathbb{R}_{+}$t.q.

$$
u-h(s, u)=0, s \in[0,1], u \in E \Rightarrow u \notin \partial B_{R},
$$

3. l'application définie de $E$ dans $E \operatorname{par} u \mapsto u-h(0, u)$ est linéaire.

(Ceci est suffisant pour obtenir l'existence d'une solution au problème 4.33.)

Soit $s \in[0,1]$ et $u \in E$. On note $h(s, u)$ la solution du problème suivant :

$$
\left\{\begin{array}{l}
w \in L^{2}(] 0, T\left[, H_{0}^{1}(\Omega)\right), w_{t} \in L^{2}(] 0, T\left[, H^{-1}(\Omega)\right), \\
\begin{array}{rl}
\int_{0}^{T}\left\langle w_{t}, v\right\rangle_{H^{-1}, H_{0}^{1}} \mathrm{~d} t-\int_{0}^{T} \int_{\Omega} s \boldsymbol{b} f(u) \cdot \nabla v \mathrm{~d} x \mathrm{~d} t \\
\quad+\int_{0}^{T} \int_{\Omega} \nabla w \cdot \nabla v \mathrm{~d} x \mathrm{~d} t=0, \forall v \in L^{2}(] 0, T\left[, H_{0}^{1}(\Omega)\right), \\
w(\cdot, 0)=s u_{0} .
\end{array}
\end{array}\right.
$$

Notons que $h(s, u)=w$ est bien définie car $\boldsymbol{b} f(u) \in L^{2}(] 0, T\left[,\left(L^{2}(\Omega)^{N}\right)\right.$.

De plus, il est facile de voir que le point 3. ci-dessus est vérifié, car $h(0, u)=0$ et donc $u-h(0, u)=u$. Il reste à montrer les points 1 et 2 , c'est-à-dire que $h$ est continue, que $\{h(s, u), s \in[0,1], u \in B\}$ est une partie relativement compacte de $E$, si $B$ est une partie bornée de $E$, et qu'il existe $R>0$ t.q. $u-h(s, u)=0$ n'a pas de solution avec $s \in[0,1]$ et $u \in \partial B_{R}$.

Montrons d'abord que $h$ est continue. Soit $\left(s_{n}\right)_{n \in \mathbb{N}}$ une suite de $[0,1]$ et $\left(u_{n}\right)_{n \in \mathbb{N}}$ une suite de $E$ t.q. $s_{n} \rightarrow s$ dans $\mathbb{R}$ et $u_{n} \rightarrow u$ dans $E$ lorsque $n \rightarrow+\infty$. On pose $w_{n}=h\left(s_{n}, u_{n}\right), w=h(s, u)$ et on veut montrer que $w_{n} \rightarrow w$ dans $E$. Les fonctions $w_{n}$ vérifient :

$$
\left\{\begin{array}{l}
w_{n} \in L^{2}(] 0, T\left[, H_{0}^{1}(\Omega)\right),\left(w_{n}\right)_{t} \in L^{2}(] 0, T\left[, H^{-1}(\Omega)\right), \\
\int_{0}^{T}\left\langle\left(w_{n}\right)_{t}, v\right\rangle_{H^{-1}, H_{0}^{1}} \mathrm{~d} t-\int_{0}^{T} \int_{\Omega} s_{n} \boldsymbol{b} f\left(u_{n}\right) \cdot \nabla v \mathrm{~d} x \mathrm{~d} t \\
\quad+\int_{0}^{T} \int_{\Omega} \nabla w_{n} \cdot \nabla v \mathrm{~d} x \mathrm{~d} t=0, \forall v \in L^{2}(] 0, T\left[, H_{0}^{1}(\Omega)\right), \\
w_{n}(\cdot, 0)=s_{n} u_{0} .
\end{array}\right.
$$

En prenant $v=w_{n}$ dans (4.35), on montre que la suite $\left(w_{n}\right)_{n \in \mathbb{N}}$ est bornée dans $L^{2}\left(10, T\left[, H_{0}^{1}(\Omega)\right)\right.$ et que la suite $\left(\left(w_{n}\right)_{t}\right)_{n \in \mathbb{N}}$ est bornée dans $L^{2}(] 0, T\left[, H^{-1}(\Omega)\right)$. On en déduit par le lemme 4.34 que $w_{n} \rightarrow \tilde{w}$ dans $E$, après extraction éventuelle d'une sous suite. Mais les bornes sur $w_{n}$ et $\left(w_{n}\right)_{t}$ donnent aussi $w_{n} \rightarrow \tilde{w}$ faiblement dans $L^{2}(] 0, T\left[, H_{0}^{1}(\Omega)\right)$ et $\left(w_{n}\right)_{t} \rightarrow \tilde{w}_{t}$ faiblement dans $L^{2}(] 0, T\left[, H^{-1}(\Omega)\right)$. Enfin, on peut supposer, toujours après extraction éventuelle d'une sous suite que $u_{n} \rightarrow u$ p.p.. En passant à la limite dans l'équation satisfaite par $w_{n}$ et en passant à la limite sur la condition initiale, on montre alors que $\tilde{w}=w$ (comme cela a été fait dans la démonstration du théorème 4.28). Ceci permet de conclure (sans extraction de sous suite, grâce à la partie unicité du théorème 4.28) que $w_{n} \rightarrow w$ dans $E$ et donne donc la continuité de $h$.

On pose maintenant $A=\left\{h(s, u), u \in L^{2}(] 0, T\left[, L^{2}(\Omega)\right), s \in[0,1]\right\}$. Il est facile de voir que $A$ est borné dans $L^{2}(] 0, T\left[, H_{0}^{1}(\Omega)\right)$ et que $\left\{w_{t}, w \in A\right\}$ est borné dans $\left.L^{2}(] 0, T\left[, H^{-1}(\Omega)\right)\right\}$. Le lemme 4.34 donne alors la relative compacité de $A$ dans $E$. Enfin l'existence de $R>0$ t.q. $u-h(s, u)=0$ n'ait pas de solution avec $s \in[0,1]$ et $u \in \partial B_{R}$ vient du fait que $A$ est borné dans $L^{2}(] 0, T\left[, H_{0}^{1}(\Omega)\right)$ et donc aussi dans $E$.

En conclusion, on peut utiliser l'invariance par homotopie du degré. On obtient $d\left(\operatorname{Id}-h(1, \cdot), B_{R}, 0\right)=d(\mathrm{Id}-$ $\left.h(0, \cdot), B_{R}, 0\right)=d\left(\operatorname{Id}, B_{R}, 0\right)=1$. Donc, il existe $u \in B_{R}$ t.q. $u-h(1, u)=0$, c'est-à-dire $u$ solution de (4.33).

On montre maintenant l'unicité de la solution de (4.33). On va montrer cette unicité dans un cadre un peu plus général; notons qu'on pourrait aussi montrer l'existence dans ce cadre, il suffit de reprendre la preuve préédente 
avec dans (4.34) $(s a(u)+(1-s) \alpha) \nabla w$ au lieu de $\nabla w)$ en remplaçant $\Delta u \operatorname{par} \operatorname{div}(a(u) \nabla u)$, c'est-à-dire en considérant l'équation

$$
\partial_{t} u+\operatorname{div}(b f(u))-\operatorname{div}(a(u) \nabla u)=0,
$$

avec une fonction $a$ lipschitzienne de $\mathbb{R}$ dans $\mathbb{R}$ et vérifant $\alpha \leq a(s) \leq \beta$ pour tout $s \in \mathbb{R}$ et deux nombres strictement positifs $\alpha, \beta$.

Soit $u_{1}, u_{2}$ deux solutions de (4.33) (avec $\operatorname{div}(a(u) \nabla u)$ au lieu de $\Delta u$ ). On pose $u=u_{1}-u_{2}$ et on va montrer que $u=0$ p.p..

Pour $\varepsilon>0$ on définit la fonction $T_{\varepsilon}$ de $\mathbb{R}$ dans $\mathbb{R} \operatorname{par} T_{\varepsilon}(s)=\max \{-\varepsilon, \min \{s, \varepsilon\}\}$. On note aussi $\phi_{\varepsilon}$ la primitive de $T_{\varepsilon}$ s'annulant en 0 . En prenant $v=T_{\varepsilon}(u)$ dans les formulations faibles satisfaites par $u_{1}$ et $u_{2}$, on obtient

$$
\begin{aligned}
\int_{0}^{T}\left\langle\partial_{t} u, T_{\varepsilon}(u)\right\rangle_{H^{-1}, H_{0}^{1}} \mathrm{~d} t & -\int_{0}^{T} \int_{\Omega} b\left(f\left(u_{1}\right)-f\left(u_{2}\right)\right) \cdot \nabla T_{\varepsilon}(u) \mathrm{d} x \mathrm{~d} t \\
& +\int_{0}^{T} \int_{\Omega} a\left(u_{1}\right) \nabla u \cdot \nabla T_{\varepsilon}(u) \mathrm{d} x \mathrm{~d} t=\int_{0}^{T} \int_{\Omega}\left(a\left(u_{2}\right)-a\left(u_{1}\right)\right) \nabla u_{2} \cdot \nabla T_{\varepsilon}(u) \mathrm{d} x \mathrm{~d} t .
\end{aligned}
$$

Comme $\nabla T_{\varepsilon}(u)=\nabla u 1_{0<|u|<\varepsilon}$ p.p., on en déduit que

$$
\begin{aligned}
& \int_{\Omega} \phi_{\varepsilon}(u(x, T)) \mathrm{d} x-\int_{\Omega} \phi_{\varepsilon}(u(x, 0)) \mathrm{d} x+\alpha \int_{0}^{T} \int_{\Omega} \nabla u \cdot \nabla u 1_{0<|u|<\varepsilon} d x \mathrm{~d} t \\
& \leq \int_{0}^{T} \int_{\Omega}|b|\left|f\left(u_{1}\right)-f\left(u_{2}\right)\right||\nabla u| 1_{0<|u|<\varepsilon} d x \mathrm{~d} t+\int_{0}^{T} \int_{\Omega}\left|a\left(u_{1}\right)-a\left(u_{2}\right)\right|\left|\nabla u_{2}\right||\nabla u| 1_{0<|u|<\varepsilon} d x \mathrm{~d} t .
\end{aligned}
$$

Comme $a$ et $f$ sont des fonctions lipschitziennes, il existe $L$ t.q.

$$
\left|f\left(s_{1}\right)-f\left(s_{2}\right)\right| \leq L\left|s_{1}-s_{2}\right| \text { et }\left|a\left(s_{1}\right)-a\left(s_{2}\right)\right| \leq L\left|s_{1}-s_{2}\right|, \text { pour tout } s_{1}, s_{2} \in \mathbb{R} .
$$

On utilise alors le fait que $u_{0}=0$ p.p. et $\phi_{\varepsilon} \geq 0$ pour déduire de 4.36 , avec $A_{\varepsilon}=\{0<|u|<\varepsilon\}$ et $y=(x, t)$,

$$
\begin{aligned}
\alpha \int_{0}^{T} \int_{\Omega}\left|\nabla T_{\varepsilon}(u)\right|^{2} \mathrm{~d} x \mathrm{~d} t \leq L \varepsilon\left(\int_{A_{\varepsilon}}|b|^{2} \mathrm{~d} y\right)^{\frac{1}{2}}( & \left.\int_{0}^{T} \int_{\Omega}\left|\nabla T_{\varepsilon}(u)\right|^{2} \mathrm{~d} x \mathrm{~d} t\right)^{\frac{1}{2}} \\
& +L \varepsilon\left(\int_{A_{\varepsilon}}\left|\nabla u_{2}\right|^{2} \mathrm{~d} y\right)^{\frac{1}{2}}\left(\int_{0}^{T} \int_{\Omega}\left|\nabla T_{\varepsilon}(u)\right|^{2} \mathrm{~d} x \mathrm{~d} t\right)^{\frac{1}{2}} .
\end{aligned}
$$

On a donc $\alpha\left\|\left|\nabla T_{\varepsilon}(u)\right|\right\|_{L^{2}(Q)} \leq a_{\varepsilon} \varepsilon$, avec $\left.Q=\right] 0, T[\times \Omega$ et

$$
a_{\varepsilon}=L\left(\int_{A_{\varepsilon}}|b|^{2} \mathrm{~d} y\right)^{\frac{1}{2}}+L\left(\int_{A_{\varepsilon}}\left|\nabla u_{2}\right|^{2} \mathrm{~d} y\right)^{\frac{1}{2}} .
$$

Comme $\cap_{\varepsilon>0} A_{\varepsilon}=\emptyset$ la continuité décroissante d'une mesure donne que la mesure de Lebesgue $(N+1$ dimensionnelle) de $A_{\varepsilon}$ tend vers 0 quand $\varepsilon \rightarrow 0$ et on a donc, comme $b, \nabla u_{2} \in L^{2}(Q)^{N}$, (noter que $L^{2}(Q)$ peut être identifié à $\left.L^{2}(] 0, T\left[, L^{2}(\Omega)\right)\right)$

$$
\lim _{\varepsilon \rightarrow 0} \int_{A_{\varepsilon}}|b|^{2} \mathrm{~d} y=\lim _{\varepsilon \rightarrow 0} \int_{A_{\varepsilon}}\left|\nabla u_{2}\right|^{2} \mathrm{~d} y=0,
$$

ce qui donne $\lim _{\varepsilon \rightarrow 0} a_{\varepsilon}=0$. Il nous reste maintenant à utiliser, par exemple, l'injection de $W_{0}^{1,1}(\Omega)$ dans $L^{1^{\star}}(\Omega)$. elle donne pour $t \in] 0, T[$,

$$
\left\|T_{\varepsilon}(u(t))\right\|_{L^{1^{\star}}(\Omega)} \leq\left\|\left|\nabla T_{\varepsilon}(u(t))\right|\right\|_{L^{1}(\Omega)} .
$$


On désigne par "mes" le mesure de le Lebesgue dans $\mathbb{R}^{N}$. On remarque maintenant que pour $\left.t \in\right] 0, T[$

$$
\varepsilon \operatorname{mes}\{|u(t)| \geq \varepsilon\}^{\frac{1}{1^{\star}}} \leq\left(\int_{\Omega}\left|T_{\varepsilon}(u)\right|^{1^{\star}} \mathrm{d} x\right)^{\frac{1}{1^{\star}}} .
$$

On a donc, avec 4.37,

$$
\varepsilon \operatorname{mes}\{|u(t)| \geq \varepsilon\}^{\frac{1}{1^{\star}}} \leq\left\|\left|\nabla T_{\varepsilon}(u(t))\right|\right\|_{L^{1}(\Omega)}=\int_{\Omega}\left|\nabla T_{\varepsilon}(u(x, t))\right| \mathrm{d} x,
$$

et, en intégrant par rapport à $t$, sachant que $1 / 1^{\star}=(N-1) / N$ et utilisant l'inégalité de Cauchy-Schwarz,

$$
\begin{array}{r}
\varepsilon \int_{0}^{T} \operatorname{mes}\{|u(t)| \geq \varepsilon\}^{\frac{N-1}{N}} \mathrm{~d} t \leq \int_{0}^{T} \int_{\Omega}\left|\nabla T_{\varepsilon}(u(x, t))\right| \mathrm{d} x \mathrm{~d} t \leq\left\|\left|\nabla T_{\varepsilon}(u)\right|\right\|_{L^{2}(Q)}(T \operatorname{mes}(\Omega))^{\frac{1}{2}} \\
\leq \frac{(T \operatorname{mes}(\Omega))^{\frac{1}{2}}}{\alpha} a_{\varepsilon} \varepsilon .
\end{array}
$$

On a donc

$$
\int_{0}^{T} \operatorname{mes}\{|u(t)| \geq \varepsilon\}^{\frac{N-1}{N}} \mathrm{~d} t \leq \frac{(T \operatorname{mes}(\Omega))^{\frac{1}{2}}}{\alpha} a_{\varepsilon} .
$$

quand $\varepsilon \rightarrow 0$, par convergence dominée, on en déduit (comme $\lim _{\varepsilon \rightarrow 0} a_{\varepsilon}=0$ )

$$
\int_{0}^{T} \operatorname{mes}\{|u(t)|>0\}^{\frac{N-1}{N}} \mathrm{~d} t \leq 0 .
$$

On en déduit que mes $\{|u(t)|>0\}=0$ p.p. en $t \in] 0, T[$ et donc $u=0$ p.p., ce qui termine cette preuve d'unicité.

Remarque 4.36 (Hypothèses supplémentaires sur $b$ et $\boldsymbol{u}_{\mathbf{0}}$ ) Voici trois propriétés supplémentaires (dont la démonstration est laissée en exercice) au résultat d'existence et unicité de solution que nous venons de donner dans cette section pour ce problème de convection diffusion, sous hypothèses supplémentaires sur $\boldsymbol{b}$ et $u_{0}$. Ce complément sera utile pour l'étude des équations hyperboliques, théorème 5.24.

1. On ajoute l'hypothèse $\operatorname{div} \boldsymbol{b}=0$ et on suppose $u_{0} \in L^{\infty}(\Omega)$. Il existe donc $A \leq 0$ et $B \geq 0$ t.q. $A \leq u_{0} \leq B$ p.p.. Soit $u$ la solution de 4.33, on a alors $A \leq u \leq B$ p.p.. Pour démontrer ce résultat, il suffit de prendre $v=(u-B)^{+}$(pour montrer $u \leq B$ p.p.) et $v=(A-u)^{+}$(pour montrer $u \geq A$ p.p.) dans la formulation faible (4.33).

2. On ajoute toujours l'hypothèse $\operatorname{div} \boldsymbol{b}=0$. Dans l'étude de ce problème de convection diffusion, on a supposé $f$ lipschitzienne et bornée. En fait si $u_{0} \in L^{\infty}(\Omega)$, et donc $A \leq u_{0} \leq B$ p.p.. avec $A \leq 0 \leq B$, on peut remplacer cette hypothèse sur $f$ par " $f$ localement lipschitzienne" (exemple : $f(s)=s^{2}$ ). La démonstration consiste à remplacer dans 4.33 $f$ par $\bar{f}$, où $\bar{f}$ est définie $\operatorname{par} \bar{f}(s)=f(\max \{A, \min \{s, B\}\})$. La solution obtenue, $u$, vérifie alors $A \leq u \leq B$ p.p. (d'après l'item précédent) et est donc solution de 4.33) (avec $f$ ).

3. Enfin si $\operatorname{div} \boldsymbol{b} \in L^{\infty}(\Omega),\|\operatorname{div} \boldsymbol{b}\|_{\infty}=C$, si $f$ lipschitzienne de constante de Lipschitz $L$ (mais $f$ non nécessairement bornée) et $u_{0} \in L^{\infty}(\Omega),\left\|u_{0}\right\|_{\infty}=M$, on peut aussi montrer un résultat d'existence et d'unicité de solution à 4.33). La solution $u$ vérifie alors $\|u(t)\|_{\infty} \leq M \exp (C L t)$ pour tout $t \in[0, T]$.

\subsection{Compacité en temps}

Dans cette section, on démontre le lemme de compacité dans $L^{2} 4.34$ comme conséquence du théorème 4.42 . énoncé dans un cadre plus général, et utile pour la résolution de nombreux problèmes paraboliques.

Commençons par la version abstraite du lemme 4.34 de compacité dans $L^{2}$, qu'on obtient en remplaçant l'espace $L^{2}$ par un espace de Hilbert quelconque. 
Lemme 4.37 (Compacité en temps, cadre hilbertien) On suppose que $X$ et $H$ sont des espaces de Hilbert, que $X$ s'injecte compactement dans $H$, et que $X$ est dense dans $H$ On identifie $H$ avec $H^{\prime}$ (par le théorème de représentation de Riesz). On suppose maintenant que $\left(u_{n}\right)_{n \in \mathbb{N}}$ est une suite bornée de $L^{2}(] 0, T[, X)(T>0$ est donné) et que $\left(\left(u_{n}\right)_{t}\right)_{n \in \mathbb{N}}$ est une suite bornée de $L^{2}(] 0, T\left[, X^{\prime}\right)$. Alors, la suite $\left(u_{n}\right)_{n \in \mathbb{N}}$ est relativement compacte dans $L^{2}(] 0, T[, H)$.

Notons dans le lemme 4.34 on a $X=H_{0}^{1}(\Omega)$ et $H=L^{2}(\Omega)$ ). Ce résultat de compacité dans le cadre hilbertien (attribué à J.L. Lions) a ensuite été généralisé par Aubin dans le cadre d'espaces de Banach [4] :

Théorème 4.38 (Compacité en temps, cadre $\boldsymbol{L}^{\boldsymbol{p}}, \boldsymbol{p}>\mathbf{1}$ ) Soit $X, B, Y$ trois espaces de Banach et $1<p<$ $+\infty$. On suppose que $X$ s'injecte compactement dans $B$ et que $B$ s'injecte continûment dans $Y$. On suppose maintenant que $\left(u_{n}\right)_{n \in \mathbb{N}}$. est une suite bornée de $L^{p}(] 0, T[, X)\left(T>0\right.$ est donné) et que $\left(\left(u_{n}\right)_{t}\right)_{n \in \mathbb{N}}$ est une suite bornée de $L^{p}(] 0, T[, Y)$. Alors, la suite $\left(u_{n}\right)_{n \in \mathbb{N}}$ est relativement compacte dans $L^{p}(] 0, T[, B)$.

Ce résultat a ensuite été généralisé par J. Simon [28] pour avoir aussi le cas $p=1$. C'est ce dernier résultat que nous donnons dans ce paragraphe ainsi que sa démonstration (qui contient donc les démonstrations des lemmes 4.344 .34 et du théorème 4.38

Nous donnons enfin quelques généralisations du résultat de Simon utiles en particulier lors de l'étude mathématique de schémas numériques.

Nous donnons d'abord le théorème fondamental de compacité de Kolmogorov (sous deux formes légèment différentes) dont nous déduisons ensuite les théorèmes 4.42 et 4.51 .

Théorème 4.39 (Kolmogorov (1)) Soit $B$ un espace de Banach, $1 \leq p<+\infty, T>0$ et $A \subset L^{p}((0, T), B)$. Le sous ensemble A est relativement compact dans $L^{p}((0, T), B)$ si A verifie les conditions suivantes :

1. Pour tout $f \in A$, il existe $P f \in L^{p}(\mathbb{R}, B)$ tel que $P f=f$ p.p. dans $(0, T)$ et $\|P f\|_{L^{p}(\mathbb{R}, B)} \leq C$, où $C$ ne dépend que de $A$.

2. Pour tout $\varphi \in C_{c}^{\infty}(\mathbb{R}, \mathbb{R})$, la famille $\left\{\int_{\mathbb{R}}(P f) \varphi \mathrm{d} t, f \in A\right\}$ est relativement compacte dans $B$.

3. $\|P f(\cdot+h)-P f\|_{L^{p}(\mathbb{R}, B)} \rightarrow 0$, lorsque $h \rightarrow 0$, uniformément par rapport à $f \in A$.

Ces trois conditions sont donc des conditions suffisantes pour la compacité relative; de fait, ces conditions sont aussi nécessaires mais ceci n'est pas la partie intéressante du théorème.

Démonstration tu théorème 4.39

Soit $\left(\rho_{n}\right)_{n \in \mathbb{N}^{\star}}$ une suite de noyaux régularisants, c'est-à-dire :

$$
\begin{aligned}
& \rho_{n}(x)=n \rho(n x), \forall n \in \mathbb{N}^{\star}, \forall x \in \mathbb{R}, \\
& \operatorname{avec} \rho \in C_{c}^{\infty}(\mathbb{R}, \mathbb{R}), \int_{\mathbb{R}} \rho \mathrm{d} x=1, \rho \geq 0, \rho(-\cdot)=\rho .
\end{aligned}
$$

On pose $K=[0, T]$ et, pour $n \in \mathbb{N}^{\star}, A_{n}=\left\{\left(P f \star \rho_{n}\right)_{\left.\right|_{K}}, f \in A\right\}$. La preuve se fait en deux étapes. À l'étape 1 , on utilise le théorème d'Ascoli et l'hypothèse 2 du théorème 4.39 , pour montrer que pour $n \in \mathbb{N}^{\star}$, l'ensemble $A_{n}$ est relativement compact dans l'espace des fonctions continues $C(K, B)$ muni de sa topologie habituelle. On en déduit que $A_{n}$ est aussi relativement compact dans $L^{p}((0, T), B)$. À l'étape 2 , on montre que les hypothèses 1 et 3 du théorème 4.39 donnent que $P f \star \rho_{n} \rightarrow P f$ in $L^{p}(\mathbb{R}, B)$, lorsque $n \rightarrow+\infty$, uniformément par rapport à $f \in A$. Ceci permet de conclure que la famille $A$ est relativement compacte dans $L^{p}((0, T), B)$.

Etape 1. Soit $n \in \mathbb{N}^{\star}$; pour montrer que $A_{n}$ est relativement compact dans $C(K, B)$, on utilise le théorème d'Ascoli; on doit donc démontrer que les propriétés suivantes sont vérifiées :

(a) pour tout $t \in K$, l'ensemble $\left\{P f \star \rho_{n}(t), f \in A\right\}$ est relativement compact dans $B$, 
(b) la suite $\left\{P f \star \rho_{n}, f \in A\right\}$ est équicontinue de $K$ dans $B$ ( c'est-à-dire que la continuité est uniforme par rapport à $f \in A$ ).

On montre d'abord la propriété (a). Soit $t \in K$;

$$
P f \star \rho_{n}(t)=\int_{\mathbb{R}} P f(s) \rho_{n}(t-s) d s=\int_{\mathbb{R}} P f(s) \varphi(s) d s .
$$

L'hypothèse 2 du théorème 4.39 donne la propriété (a), à savoir que $\left\{P f \star \rho_{n}(t), f \in A\right\}$ est relativement compact dans $B$.

Voyons maintenant la propriété (b). Soient $t_{1}, t_{2} \in K$ et $q=p /(p-1)$; grâce à l'inégalité de Hölder, on a

$$
\begin{aligned}
\left\|P f \star \rho_{n}\left(t_{2}\right)-P f \star \rho_{n}\left(t_{1}\right)\right\|_{B} & \leq \int_{\mathbb{R}}\|P f(s)\|_{B}\left|\rho_{n}\left(t_{2}-s\right)-\rho_{n}\left(t_{1}-s\right)\right| d s \\
& \leq\|P f\|_{L^{p}(\mathbb{R}, B)}\left\|\rho_{n}\left(t_{2}-\cdot\right)-\rho_{n}\left(t_{1}-\cdot\right)\right\|_{L^{q}(\mathbb{R}, \mathbb{R})} .
\end{aligned}
$$

Comme $\rho_{n}$ est uniformément continue à support compact, on déduit facilement de cette inégalité (et de l'hypothèse 1 du théorème 4.39) et que la famille $A_{n}$ est uniformément equicontinue de $K$ sur $B$. Ceci donne la propriété (b) ce qui permet de conclure que $A_{n}$ est relativement compact dans $C(K, B)$. Cette compacité relative est équivalente à dire que pour tout $\varepsilon>0$, il existe un nombre fini de boules de rayon $\varepsilon$ (pour la norme naturelle sur $C(K, B)$ ) recouvrant l'ensemble $A_{n}$. Puisque $\|\cdot\|_{L^{p}((0, T), B)} \leq T^{1 / p}\|\cdot\|_{C(K, B)}$, on obtient aussi la compacité relative de $A_{n}$ dans $L^{p}((0, T), B)$.

\section{Etape 2.}

Soit $t \in \mathbb{R}$, comme $\int_{\mathbb{R}} \rho_{n}(s) d s=1$ et $\bar{s}=n s$, on a

$$
P f \star \rho_{n}(t)-P f(t)=\int_{\mathbb{R}}(P f(t-s)-P f(t)) \rho_{n}(s) d s=\int_{-1}^{1}\left(P f\left(t-\frac{\bar{s}}{n}\right)-P f(t)\right) \rho(\bar{s}) d \bar{s} .
$$

Puis, par l'inégalité de Hölder, on a, avec $q=p /(p-1)$,

$$
\left.\left\|P f \star \rho_{n}(t)-\operatorname{Pf}(t)\right\|_{B}^{p} \leq\left(\int_{-1}^{1} \| P f\left(t-\frac{s}{n}\right)-P f(t)\right) \|_{B}^{p} d s\right)\|\rho\|_{L^{q}}^{p} .
$$

En intégrant par rapport à $t \in \mathbb{R}$ et en utilisant le théorème de Fubini-Tonelli, on obtient

$$
\left\|P f \star \rho_{n}-P f\right\|_{L^{p}((0, T), B)}^{p} \leq 2 \sup \left\{\|P f(\cdot+h)-P f\|_{L^{p}(\mathbb{R}, B)}^{p},|h| \leq 1\right\}\|\rho\|_{L^{q}}^{p} .
$$

Enfin, la troisième hypothèse du théorème 4.39 entraîne que $\left\|P f \star \rho_{n}-P f\right\|_{L^{p}((0, T), B)} \rightarrow 0$ lorque $n \rightarrow+\infty$, uniformément par rapport à $f \in A$. En conséquence, la compacité relative de $A_{n}$ dans $L^{p}((0, T), B)$ pour tout $n \in \mathbb{N}$ (démontrée à l'étape 1 ) donne lala compacité relative de $A$ dans $L^{p}((0, T), B)$. Ceci conclut la preuve du théorème 4.39 .

Donnons maintenant une forme alternative du théorème prćédent qui a l'avantage de ne pas demander de prolongement de $f$ en dehors $[0, T]$.

Théorème 4.40 (Kolmogorov (2)) Soit $B$ un espace de Banach, $1 \leq p<+\infty, T>0$ et $A \subset L^{p}((0, T), B)$. Le sous ensemble A est relativement compact dans $L^{p}((0, T), B)$ si A satisfait les conditionssuivantes :

1. le sous ensemble A est borné dans $L^{p}((0, T), B)$.

2. Pour tout $\varphi \in C_{c}^{\infty}(\mathbb{R}, \mathbb{R})$, la famille $\left\{\int_{0}^{T} f \varphi \mathrm{d} t, f \in A\right\}$ est relativement compacte dans $B$. 
3. Il existe une fonction croissante de $(0, T)$ to $\mathbb{R}_{+}$telle que $\lim _{h \rightarrow 0^{+}} \eta(h)=0$ et, pour tout $h \in(0, T)$ et $f \in A$,

$$
\int_{0}^{T-h}\|f(t+h)-f(t)\|_{B}^{p} \mathrm{~d} t \leq \eta(h) .
$$

\section{Démonstration :}

La preuve utilise le théorème 4.39 avec $P f=0$ sur $[0, T]^{c}$. Les deux premiers items des hypothèses du Theorem 4.39 sont clairement satisfaites. La seule difficulté est de prouver le troisième item. On le fait en deux étapes.

Étape 1. On va d'abord montrer que $\lim _{\delta \rightarrow 0} \int_{0}^{\delta}\|f(t)\|_{B}^{p} \mathrm{~d} t \rightarrow 0$ lorsque $\delta \rightarrow 0^{+}$, uniformément par rapport à $f \in A$.

Soit $\delta, h \in(0, T)$ tels que $\delta+h \leq T$. Pour tout $t \in(0, \delta)$ one has $\|f(t)\|_{B} \leq\|f(t+h)\|_{B}+\|f(t+h)-f(t)\|_{B}$ et donc

$$
\|f(t)\|_{B}^{p} \leq 2^{p}\|f(t+h)\|_{B}^{p}+2^{p}\|f(t+h)-f(t)\|_{B}^{p} .
$$

En intégrant cette inégalité pour $t \in(0, \delta)$, on otient

$$
\int_{0}^{\delta}\|f(t)\|_{B}^{p} \mathrm{~d} t \leq 2^{p} \int_{0}^{\delta}\|f(t+h)\|_{B}^{p} \mathrm{~d} t+2^{p} \int_{0}^{\delta}\|f(t+h)-f(t)\|_{B}^{p} \mathrm{~d} t .
$$

Soient maintenant $h_{0} \in(0, T)$ et $\delta \in\left(0, T-h_{0}\right)$. For all $h \in\left(0, h_{0}\right)$, Inequality 4.39) gives, using $\eta(h) \leq \eta\left(h_{0}\right)$,

$$
\int_{0}^{\delta}\|f(t)\|_{B}^{p} \mathrm{~d} t \leq 2^{p} \int_{0}^{\delta}\|f(t+h)\|_{B}^{p} \mathrm{~d} t+2^{p} \eta\left(h_{0}\right) .
$$

En intégrant cette inégalité pour $h \in\left(0, h_{0}\right)$, on a :

$$
h_{0} \int_{0}^{\delta}\|f(t)\|_{B}^{p} \mathrm{~d} t \leq 2^{p} \int_{0}^{h_{0}}\left(\int_{0}^{\delta}\|f(t+h)\|_{B}^{p} \mathrm{~d} t\right) d h+2^{p} h_{0} \eta\left(h_{0}\right) .
$$

Puis, grâce au théorème de Fubini-Tonelli,

$$
\begin{aligned}
\int_{0}^{h_{0}}\left(\int_{0}^{\delta}\|f(t+h)\|_{B}^{p} \mathrm{~d} t\right) d h=\int_{0}^{\delta}\left(\int_{0}^{h_{0}}\|f(t+h)\|_{B}^{p} d h\right) \mathrm{d} t & \leq \int_{0}^{\delta}\left(\int_{0}^{T}\|f(s)\|_{B}^{p} d s\right) \leq \delta\|f\|_{L^{p}((0, T), B)}^{p}
\end{aligned}
$$

d'où l'on déduit que

$$
h_{0} \int_{0}^{\delta}\|f(t)\|_{B}^{p} \mathrm{~d} t \leq \delta 2^{p}\|f\|_{L^{p}((0, T), B)}^{p}+2^{p} h_{0} \eta\left(h_{0}\right) .
$$

Soit maintenant $\varepsilon>0$. On choisit d'abord $h_{0} \in(0, T)$ tel que $2^{p} \eta\left(h_{0}\right) \leq \varepsilon$. Puis, on choisit $\bar{\delta}=\min \{T-$ $\left.h_{0}, \varepsilon h_{0} /\left(2^{p} C\right)\right\}$, avec $C=\sup _{f \in A}\|f\|_{L^{p}((0, T), B)}^{p}$. On obtient alors, pour tout $f \in A$,

$$
0 \leq \delta \leq \bar{\delta} \Rightarrow \int_{0}^{\delta}\|f(t)\|_{B}^{p} \leq 2 \varepsilon
$$

ce qui entraîne que $\int_{0}^{\delta}\|f(t)\|_{B}^{p} \mathrm{~d} t \rightarrow 0$ as $\delta \rightarrow 0^{+}$, uniformément par rapport à $f \in A$. On a ainsi terminé l'étape 1 . 
Noter que des arguments similaires donnent que $\int_{T-\delta}^{T}\|f(t)\|_{B}^{p} \mathrm{~d} t \rightarrow 0$ lorsque $\delta \rightarrow 0^{+}$, uniformément par rapport à $f \in A$ (ceci se prouve en utilisant $g$ définie par $g(t)=f(T-t)$ ) au lieu de $f$.

Étape 2. On montre maintenant que le troisième item des hypothèses du théorème 4.39 est satisfait, ce qui conclut la preuve du théorème 4.40 .

On rappelle que $P f(t)=0$ if $t \in[0, T]^{c}$, et donc, pour tout $h \in(0, T)$ et $f \in A$,

$$
\begin{aligned}
\int_{\mathbb{R}}\|P f(t+h)-P f(t)\|_{B}^{p} \mathrm{~d} t & \leq \int_{-h}^{0}\|f(t+h)\|_{B}^{p} \mathrm{~d} t+\int_{0}^{T-h}\|f(t+h)-f(t)\|_{B}^{p} \mathrm{~d} t+\int_{T-h}^{T}\|f(t)\|_{B}^{p} \mathrm{~d} t \\
& \leq \int_{0}^{h}\|f(t)\|_{B}^{p} \mathrm{~d} t+\int_{0}^{T-h}\|f(t+h)-f(t)\|_{B}^{p} \mathrm{~d} t+\int_{T-h}^{T}\|f(t)\|_{B}^{p} d t .
\end{aligned}
$$

Soit $\varepsilon>0$. Il existe $h_{1}>0$ tel que $\eta\left(h_{1}\right) \leq \varepsilon$. Grâce à l'étape 1 , il existe $h_{2}>0$ tel que pour tout $f \in A$,

$$
0 \leq h \leq h_{2} \Rightarrow \int_{0}^{h}\|f(t)\|_{B}^{p} \mathrm{~d} t \leq \varepsilon \text { and } \int_{T-h}^{T}\|f(t)\|_{B}^{p} \mathrm{~d} t \leq \varepsilon .
$$

En définissant $h_{3}=\min \left\{h_{1}, h_{2}\right\}$, on a pour tout $f \in A$,

$$
0 \leq h \leq h_{3} \Rightarrow \int_{\mathbb{R}}\|\operatorname{Pf}(t+h)-\operatorname{Pf}(t)\|_{B}^{p} \mathrm{~d} t \leq 3 \varepsilon
$$

ce qui conclut l'étape 2 et la preuve du théorème 4.40 .

On déduit alors du théoreme 4.40 un corollaire qui sera utile pour la preuve du théom̀e 4.42

Corollaire 4.41 (Compacité en temps) Soit $B$ un espace de Banach, $1 \leq p<+\infty, T>0 A \subset L^{p}((0, T), B)$. Soit $X$ be un espace de Banach inclus dans $B$ avec injection compacte. On suppose que $A$ verifie les conditions ssuivantes:

1. A est borné dans $L^{p}((0, T), B)$.

2. A est borné dans $L^{1}((0, T), X)$.

Noter que ces deux conditions sont vérifiées si A est borné dans $L^{p}((0, T), X)$.

3. Il existe une fonction croissante de $(0, T)$ dans $\mathbb{R}_{+}$telle que $\lim _{h \rightarrow 0^{+}} \eta(h)=0$, pour tout $h \in(0, T) f \in A$,

$$
\int_{0}^{T-h}\|f(t+h)-f(t)\|_{B}^{p} \mathrm{~d} t \leq \eta(h) .
$$

Alors l'ensemble A est relativement compacte dans $L^{p}((0, T), B)$.

Démonstration Pour appliquer le théorème 4.40, on doit vérifier que, pour tout $\varphi \in C_{c}^{\infty}(\mathbb{R}, \mathbb{R})$, la famille $\left\{\int_{0}^{T} f \varphi \mathrm{d} t, f \in A\right\}$ est relativement compacte dans $B$.

Soit $\varphi \in C_{c}^{\infty}(\mathbb{R}, \mathbb{R})$. Si $f \in A$, on a, en notant $\|\varphi\|_{u}=\max _{t \in \mathbb{R}}|\varphi(t)|$,

$$
\left\|\int_{0}^{T} f \varphi \mathrm{d} t\right\|_{X} \leq\|\varphi\|_{u}\|f\|_{L^{1}((0, T), X)}
$$

Comme $A$ est borné dans $L^{1}((0, T), X)$, la famille $\left\{\int_{0}^{T} f \varphi \mathrm{d} t, f \in A\right\}$ est borné dans $X$ et donc relativement compacte dans $B$.

Nous donnons maintenant un théorème, essentiallement dû à J. P. Aubin (pour $p>1$ ) et J. Simon (pour $p=1$ ), qu'on a utilisé dans la section prćédente pour prouver l'existence d'une solution pour des équations paraboliques. 
Théorème 4.42 (Aubin-Simon) Soit $1 \leq p<+\infty$, et soient $X, B, Y$ trois espaces de Banach tels que

(i) $X \subset B$ avec injection compacte,

(ii) $X \subset Y$ et si $\left(f_{n}\right)_{n \in \mathbb{N}}$ est une suite d'éléments de $X$ telle que la suite $\left(\left\|f_{n}\right\|_{X}\right)_{n \in \mathbb{N}}$ est bornée dans $\mathbb{R}$, $f_{n} \rightarrow f$ in $B$ et $f_{n} \rightarrow 0$ in $Y$ (as $\left.n \rightarrow+\infty\right)$, alors $f=0$.

Soit $T>0$ et $\left(u_{n}\right)_{n \in \mathbb{N}}$ une suite d'éléments de $L^{p}((0, T), X)$ telle que

1. $\left(u_{n}\right)_{n \in \mathbb{N}}$ est bornée dans $L^{p}((0, T), X)$,

2. $\left(\frac{d u_{n}}{d t}\right)_{n \in \mathbb{N}}$ est bornée dans $L^{1}((0, T), Y)$.

Alors il existe $u \in L^{p}((0, T), B)$ tel que à une sous-suite près, $u_{n} \rightarrow u$ in $L^{p}((0, T), B)$.

Remarque 4.43 Noter que les hypothèses (3.7b) du théorèm 4.42 est plus faible que l'hypothèse des articles originaux d'Aubin et Simon, qui est :

$$
B \subset Y \text { avec injection continue. }
$$

L'hypothèse (3.7b ne demande en particulier pas que $B \subset Y$, ce qui est intéressant par exemple dans les applications à des solutions de schémas numériques. On peut aussi noter que l'hypothèse (3.7a) peut aussi s'écrire

Si la suite $\left(\left\|w_{n}\right\|_{X}\right)_{n \in \mathbb{N}}$ est bornée, alors, à une sous-suite près, il existe $w \in B$ tel que $w_{n} \rightarrow w$ in $B$.

Cette reformulation conduit à une généralisation facile qui sert à prouver la convergence des approximations numériques de la solution des équations paraboliques : dans cette généralisation, $X$ est remplacé par une suite $\left(X_{n}\right)_{n \in \mathbb{N}}$, voir Theorem 4.51 ci-dessous.

Preuve du théorème 4.42 La preuve utilise le corollaire 4.41 avec $A=\left\{u_{n}, n \in \mathbb{N}\right\}$. L'ensemble $A$ est borné dans $L^{p}((0, T), B)$ (car $X$ s'injecte continûment dans $B$ ) et donc dans $L^{1}((0, T), X)$, de sorte que nous n'avons plus qu'à prouver le troisième point des hypothèses du corollaire 4.41 . c'.à.d.

$$
\int_{0}^{T-h}\left\|u_{n}(\cdot+h)-u_{n}\right\|_{B}^{p} \mathrm{~d} t \rightarrow 0 \text { as } h \rightarrow 0, \text { uniformly w.r.t. } n \in \mathbb{N} .
$$

Soit $0<h<T$ et $\varepsilon>0$. Pour $t \in(0, T-h)$, on a en utilisant le lemme de Lions 4.44 donné plus loin,

$$
\begin{aligned}
\left\|u_{n}(t+h)-u_{n}(t)\right\|_{B} & \leq \varepsilon\left\|u_{n}(t+h)-u_{n}(t)\right\|_{X}+C_{\varepsilon}\left\|u_{n}(t+h)-u_{n}(t)\right\|_{Y} \\
& \leq \varepsilon\left\|u_{n}(t+h)\right\|_{X}+\varepsilon\left\|u_{n}(t)\right\|_{X}+C_{\varepsilon}\left\|u_{n}(t+h)-u_{n}(t)\right\|_{Y},
\end{aligned}
$$

et donc

$$
\left\|u_{n}(t+h)-u_{n}(t)\right\|_{B}^{p} \leq(3 \varepsilon)^{p}\left\|u_{n}(t+h)\right\|_{X}^{p}+(3 \varepsilon)^{p}\left\|u_{n}(t)\right\|_{X}^{p}+\left(3 C_{\varepsilon}\right)^{p}\left\|u_{n}(t+h)-u_{n}(t)\right\|_{Y}^{p} .
$$

L'intégration de cette inégalité par rapport à $t$ (entre 0 et $T-h$ ) conduit à

$$
\int_{0}^{T-h}\left\|u_{n}(t+h)-u_{n}(t)\right\|_{B}^{p} \mathrm{~d} t \leq 2(3 \varepsilon)^{p}\left\|u_{n}\right\|_{L^{p}((0, T), X)}^{p}+\left(3 C_{\varepsilon}\right)^{p} \int_{0}^{T-h}\left\|u_{n}(t+h)-u_{n}(t)\right\|_{Y}^{p} \mathrm{~d} t .
$$

On utilise maintenant le deuxième point de la remarque 4.46 (également donné dans la section 4.2], qui nous donne que $u_{n} \in C([0, T], Y)$ et $u_{n}\left(t_{1}\right)-u_{n}\left(t_{2}\right)=\int_{t_{1}}^{t_{2}} \frac{d u_{n}}{d t}(s) d s$ pour tout $t_{1}, t_{2} \in[0, T]$. Ceci nous permet de majorer 
le deuxième terme du second membre de 4.42 :

$$
\begin{aligned}
\int_{0}^{T-h}\left\|u_{n}(t+h)-u_{n}(t)\right\|_{Y}^{p} \mathrm{~d} t & \leq \int_{0}^{T-h}\left(\int_{t}^{t+h}\left\|\frac{d u_{n}}{d t}(s)\right\|_{Y} d s\right)^{p} \mathrm{~d} t \\
& \leq M^{p-1} \int_{0}^{T-h}\left(\int_{t}^{t+h}\left\|\frac{d u_{n}}{d t}(s)\right\|_{Y} d s\right) \mathrm{d} t \\
& \leq M^{p-1} \int_{0}^{T-h}\left(\int_{0}^{T} 1_{[t, t+h]}(s)\left\|\frac{d u_{n}}{d t}(s)\right\|_{Y} d s\right) \mathrm{d} t
\end{aligned}
$$

où $M$ est un majorant de la norme $L^{1}((0, T), Y)$ de $\frac{d u_{n}}{d t}\left(\right.$ i.e $\left\|\frac{d u_{n}}{d t}\right\|_{L^{1}((0, T), Y)} \leq M$ for all $\left.n\right)$.

En utilisant le fait que $1_{[t, t+h]}(s)=1_{[s-h, s]}(t)$ et le théorme de Fubini-Tonelli, on obtient

$$
\int_{0}^{T-h}\left\|u_{n}(t+h)-u_{n}(t)\right\|_{Y}^{p} d t \leq h M^{p} .
$$

Donc, en tenant compte de 4.43, 4.42 donne

$$
\int_{0}^{T-h}\left\|u_{n}(t+h)-u_{n}(t)\right\|_{B}^{p} \mathrm{~d} t \leq 2(3 \varepsilon)^{p}\left\|u_{n}\right\|_{L^{p}((0, T), X)}^{p}+\left(3 C_{\varepsilon}\right)^{p} h M^{p} .
$$

On peut alors conclure :.soit $\eta>0$, on choisit $\varepsilon>0$ pour majorer le premier terme du second membre de (4.44) par $\eta$ (indépendamment de $n \in \mathbb{N}$ ). Comme $C_{\varepsilon}$ est donné, il existe $h_{0} \in(0, T)$ tel que le second terme du second membre de 4.44) est majoré par $\eta$ (indépendamment de $n \in \mathbb{N}$ ) si $0<h<h_{0}$. Finalement, on obtient

$$
0<h<h_{0} \Rightarrow \int_{0}^{T-h}\left\|u_{n}(t+h)-u_{n}(t)\right\|_{B}^{p} \mathrm{~d} t \leq 2 \eta,
$$

ce qui conclut la preuve du théorème 4.42

Lemme 4.44 (Lions) Soient $X, B, Y$ trois espaces de Banach tels que

(i) $X \subset B$ avec injection compacte,

(ii) $X \subset Y$ et si $\left(u_{n}\right)_{n \in \mathbb{N}}$ est une suite d'éléments de $X$ telle que la suite $\left(\left\|u_{n}\right\|_{X}\right)_{n \in \mathbb{N}}$ est bornée, $u_{n} \rightarrow u$ in $B$ et $u_{n} \rightarrow 0$ dans $Y$ (as $\left.n \rightarrow+\infty\right)$, alors $u=0$.

Alors pour tout $\varepsilon>0$, il existe $C_{\varepsilon}$ tel que, pour $w \in X$,

$$
\|w\|_{B} \leq \varepsilon\|w\|_{X}+C_{\varepsilon}\|w\|_{Y} .
$$

Remarque 4.45 (Sur les hypothèses du lemme de Lions) Comme dans le théorème 4.42, l'hypothèse (ii) du lemme 4.44 peut être remplacée par l'hypothèse plus forte

(ii)' $B \subset Y$ avec injection continue.

Preuve du lemme 4.44 On raisonne par contradiction : on suppose qu'il existe $\varepsilon>0$ et une suite $\left(u_{n}\right)_{n \in \mathbb{N}}$ telle que $\left(u_{n}\right)_{n \in \mathbb{N}}$ telle que $u_{n} \in X$ et $1=\left\|u_{n}\right\|_{B}>\varepsilon\left\|u_{n}\right\|_{X}+n\left\|u_{n}\right\|_{Y}$, pour tout $n \in \mathbb{N}$. Alors $\left(u_{n}\right)_{n \in \mathbb{N}}$ est bornée dans $X$ et donc relativement compacte dans $B$. On peut donc supposer (à une sous-suite près) que $u_{n} \rightarrow u$ dans $B$ et $\|u\|_{B}=1$. De plus $u_{n} \rightarrow 0$ in $Y$ (car $\left\|u_{n}\right\|_{Y} \leq 1 / n$ ). L'hypothèse $(i i)$ du lemme 4.44 donne donc que $u=0$, ce qui contredit $\|u\|_{B}=1$. 
Remarque 4.46 (Sur la dérivée faible de $u$ ) On rappelle ici (points 1 et 2) certains résultats essentiellement donnés dans la section 4.2 (avec une preuve différente pour le deuxième point). Soient $X, Y$ deux espaces Banach et $Z$ un espace vectoriel tel que $X, Y \subset Z$ (bien sûr, un cas simple est $Z=Y$ ). Soientt $p, q \in[1, \infty]$.

1. En supposant que $u \in L^{p}((0, T), X)$, la dérivée faible de $u$, notée $d u / d t$, est définie par son action sur les fonctions test, c'est-à-dire son action sur $\varphi$ pour tout $\varphi \in C_{c}^{\infty}((0, T))$ (noter que $\varphi$ prend ses valeurs dans $\mathbb{R}$ ). En fait, si $\varphi \in \mathcal{C}_{c}^{\infty}((0, T))$ (et $\varphi^{\prime}$ est la dérivée classique de $\varphi$ ), la fonction $\varphi^{\prime} u$ appartient à $L^{p}((0, T), X)$ et donc à $L^{1}((0, T), X)$ et l'action de $d u / d t$ sur $\varphi$ est définie comme

$$
\left\langle\frac{d u}{d t}, \varphi\right\rangle=-\int_{0}^{T} \varphi^{\prime}(t) u(t) \mathrm{d} t
$$

Noter que $\left\langle\frac{d u}{d t}, \varphi\right\rangle \in X$.

Puis $d u / d t \in L^{q}((0, T), Y)$ signifie qu'il existe $v \in L^{q}((0, T), Y)$ (et ce $v$ est unique) de telle sorte que

$$
-\int_{0}^{T} \varphi^{\prime}(t) u(t) \mathrm{d} t=\int_{0}^{T} \varphi(t) v(t) \mathrm{d} t \text { for all } \varphi \in C_{c}^{\infty}((0, T)) .
$$

Noter que $\int_{0}^{T} \varphi^{\prime}(t) u(t) \mathrm{d} t \in X \subset Z$ et $\int_{0}^{T} \varphi(t) v(t) \mathrm{d} t \in Y \subset Z$, donc l'égalité dans (4.45) tient dans $Z$. Enfin, on identifie la dérivée faible de $u$ (qui est une forme linéaire $\operatorname{sur} \mathcal{C}_{c}^{\infty}((0, T))$ ) avec la fonction $v$ (qui appartient à $\left.L^{q}((0, T), Y)\right)$.

2. Si $u \in L^{p}((0, T), X)$ et $d u / d t \in L^{q}((0, T), Y)$, il est assez facile de prouver que $u \in C([0, T], Y)$ et que

$$
u(t)=u(0)+\int_{0}^{t} \frac{d u}{d t}(s) d s, \text { for all } t \in[0, T] .
$$

En effet, on pose $v=d u / d t$ et on définit $w \in C([0, T], Y)$ par

$$
w(t)=\int_{0}^{t} v(s) d s \text { pour tout } t \in[0, T] .
$$

Pour $n \in \mathbb{N}^{\star}$, soit $u_{n}=u \star \rho_{n}$ avec $\rho_{n}$ défini par (4.38) et en ayant prolongé $u$ par 0 en dehors de $[0, T]$. Comme $u \in L^{p}(\mathbb{R}, X)$, on a $u_{n} \rightarrow u$ dans $L^{p}(\mathbb{R}, X)$ et donc, à une sous-suite près, $u_{n}(t) \rightarrow u(t)$ dans $X$ (lorque $n \rightarrow+\infty$ ) pour p.p. $t \in(0, T)$.

Soit $t_{1}, t_{2} \in(0, T)$ tel que $0<t_{1}<t_{2}<T$ et $u_{n}\left(t_{i}\right) \rightarrow u\left(t_{i}\right)$ dans $X$ (lorsque $n \rightarrow+\infty$ ) pour $i=1,2$. Pour $n \in \mathbb{N}^{\star}$ tel que $1 / n<t_{1}$ et $1 / n<T-t_{2}$, on définit $\varphi_{n}$ par

$$
\varphi_{n}(t)=\int_{t_{2}}^{t} \rho_{n}\left(t_{2}-s\right) d s-\int_{t_{1}}^{t} \rho_{n}\left(t_{1}-s\right) d s
$$

On a $\varphi_{n} \in C_{c}^{\infty}((0, T), \mathbb{R})$ et $\varphi_{n}^{\prime}(t)=\rho_{n}\left(t_{2}-t\right)-\rho_{n}\left(t_{1}-t\right)$ pour $t \in(0, T)$. De plus,

$$
u_{n}\left(t_{2}\right)-u_{n}\left(t_{1}\right)=\int_{0}^{T} u(s) \varphi_{n}^{\prime}(s) d s=-\int_{0}^{T} v(s) \varphi_{n}(s) d s .
$$

Remarquons maintenant que (en utilisant $\rho(-\cdot)=\rho$ et avec un changement de variable)

$$
\left.\varphi_{n}(t)=\int_{t_{2}}^{t} \rho_{n}\left(s-t_{2}\right)\right) d s-\int_{t_{1}}^{t} \rho_{n}\left(s-t_{1}\right) d s=-\int_{t_{1}}^{t_{2}} \rho_{n}(t-s) d s=-1_{\left(t_{1}, t_{2}\right)} \star \rho_{n}(t) .
$$


De plus, $\varphi_{n} \rightarrow-1_{\left(t_{1}, t_{2}\right)}$ p.p. et puisque $\left|\varphi_{n}\right| \leq 1$ p.p. (pour tous les $n$ ), le théorème de convergence dominée donne

$$
-\int_{0}^{T} v(s) \varphi_{n}(s) d s \rightarrow \int_{t_{1}}^{t_{2}} v(s) d s \text { in } Y .
$$

On obtient alors, en faisant $n \rightarrow+\infty$,

$$
u\left(t_{2}\right)-u\left(t_{1}\right)=\int_{t_{1}}^{t_{2}} v(s) d s=w\left(t_{2}\right)-w\left(t_{1}\right) .
$$

Ceci prouve qu'il existe $c \in \mathbb{R}$ tel que $u=w+c$ p.p. $\operatorname{sur}(0, T)$ puis que $u \in C((0, T), Y)$ (puisque, comme d'habitude, nous identifions $u$ avec la fonction continue $w+c)$.

3. Dans le théorème 4.42, grâce au point précédent, on a $u_{n} \in C([0, T], Y)$ pour tous les $n \in \mathbb{N}$. Cependant, la limite $u$ n'est pas nécessairement continue. Un simple contre-exemple est obtenu avec $p=1, X=B=Y$ et, par exemple, $T=2$. Ensuite, il est assez facile de construire une suite $\left(u_{n}\right)_{n}$ bornée dans $W^{1,1}((0, T))$ dont la limite est la fonction caractéristique de $[1,2]$.

Remarque 4.47 (Un cas classique plus simple) Il existe un cas classique où le lemme 4.44 est plus simple. Soit $B$ un espace Hilbert et $X$ un espace Banach $X \subset B$. On définit sur $X$ la norme duale de $\|\cdot\|_{X}$ pour le produit scalaire de $B$, à savoir

$$
\|u\|_{Y}=\sup \left\{(u \mid v)_{B}, ; v \in X,\|v\|_{X} \leq 1\right\}
$$

Ensuite, pour tout $\varepsilon>0$ et $u \in X$,

$$
\|u\|_{B} \leq \varepsilon\|u\|_{X}+\frac{1}{\varepsilon}\|u\|_{Y}
$$

La preuve est simple, puisque

$$
\|u\|_{B}=(u \mid u)_{B}^{\frac{1}{2}} \leq\left(\|u\|_{Y}\|u\|_{X}\right)^{\frac{1}{2}} \leq \varepsilon\|u\|_{X}+\frac{1}{\varepsilon}\|u\|_{Y}
$$

Noter que la compacité de $X$ en $B$ n'est pas nécessaire ici (mais, même dans ce cas simple du lemme 4.44,1a compacité de $X$ en $B$ est nécessaire pour le Théorème 4.42,)

Nous donnons maintenant une généralisation du corollaire 4.41 et du théorème 4.42 en utilisant une suite de sous-espaces de $B$ au lieu des espaces $X$ et $Y$.

Définition 4.48 (Suite compactement incluse) Soit B un espace Banach et $\left(X_{n}\right)_{n \in \mathbb{N}}$ une suite d'espaces Banach inclus dans $B$. On dira que la suite $\left(X_{n}\right)_{n \in \mathbb{N}}$ est compactement incluse dans $B$ si toute suite $\left(u_{n}\right)_{n \in \mathbb{N}}$ satisfaisant

- $u_{n} \in X_{n}$ for all $n \in \mathbb{N}$,

- la suite $\left(\left\|u_{n}\right\|_{X_{n}}\right)_{n \in \mathbb{N}}$ est bornée

est relativement compacte dans $B$.

Proposition 4.49 (Compacité en temps avec une suite de sous-espaces) Soient $1 \leq p<+\infty$ et $T>0$. Soit $\left(f_{n}\right)_{n \in \mathbb{N}}$ une suite de $L^{p}((0, T), B)$ satisfaisant aux conditions suivantes

1. La suite $\left(f_{n}\right)_{n \in \mathbb{N}}$ est bornée dans $L^{p}((0, T), B)$.

2. La suite $\left(\left\|f_{n}\right\|_{L^{1}\left((0, T), X_{n}\right)}\right)_{n \in \mathbb{N}}$ est bornée. 
3. Il existe une fonction croissante de $(0, T) \grave{a} \mathbb{R}_{+}$telle que $\lim _{h \rightarrow 0^{+}} \eta(h)=0$ et, pour tous $h \in(0, T)$ et $n \in \mathbb{N}$,

$$
\int_{0}^{T-h}\left\|f_{n}(t+h)-f_{n}(t)\right\|_{B}^{p} \mathrm{~d} t \leq \eta(h)
$$

alors la suite $\left(f_{n}\right)_{n \in \mathbb{N}}$ est relativement compacte dans $L^{p}((0, T), B)$.

Preuve Comme dans le corollaire 4.41, pour appliquer le Théorème 4.40, il suffit de prouver que, pour tous les $\varphi \in C_{c}^{\infty}(\mathbb{R}, \mathbb{R})$, le La suite $\left\{\int_{0}^{T} f_{n} \varphi \mathrm{d} t, n \in \mathbb{N}\right\}$ est relativement compacte en $B$.

Let $\varphi \in C_{c}^{\infty}(\mathbb{R}, \mathbb{R})$. Pour $n \in \mathbb{N}$, on a, avec $\|\varphi\|_{u}=\max _{t \in \mathbb{R}}|\varphi(t)|$,

$$
\left\|\int_{0}^{T} f_{n} \varphi \mathrm{d} t\right\|_{X_{n}} \leq\|\varphi\|_{u}\left\|f_{n}\right\|_{L^{1}\left((0, T), X_{n}\right)} .
$$

Puisque la suite $\left(\left\|f_{n}\right\|_{L^{1}\left((0, T), X_{n}\right)}\right)_{n \in \mathbb{N}}$ est bornée, la suite $\left\{\left\|\int_{0}^{T} f_{n} \varphi \mathrm{d} t\right\|_{X_{n}}, n \in \mathbb{N}\right\}$ est également bornée. Par conséquent, la suite $\left\{\int_{0}^{T} f_{n} \varphi \mathrm{d} t, n \in \mathbb{N}\right\}$ est relativement compacte en $B$.

Voici maintenant une généralisation du Théorème 4.42 utilisant une suite de sous-espaces de $B$.

Définition 4.50 (Suite compacte-continue) Soit B un espace de Banach, $\left(X_{n}\right)_{n \in \mathbb{N}}$ une suite d'espaces de Banach incluse dans $B$ et $\left(Y_{n}\right)_{n \in \mathbb{N}}$ une suite d'espaces Banach. On dira que la suite $\left(X_{n}, Y_{n}\right)_{n \in \mathbb{N}}$ est compactecontinue dans $B$ si les conditions suivantes sont remplies

1. La suite $\left(X_{n}\right)_{n \in \mathbb{N}}$ est compactement incluse dans $B$ (voir définition 4.48).

2. $X_{n} \subset Y_{n}$ (pour tous les $n \in \mathbb{N}$ ) et si la suite $\left(u_{n}\right)_{n \in \mathbb{N}}$ est telle que $u_{n} \in X_{n}$ (for all $n \in \mathbb{N}$ ), $\left(\left\|u_{n}\right\|_{X_{n}}\right)_{n \in \mathbb{N}}$ bornée et $\left\|u_{n}\right\|_{Y_{n}} \rightarrow 0$ (lorsque $n \rightarrow+\infty$ ), alors toute sous-suite de $\left(u_{n}\right)_{n \in \mathbb{N}}$ convergeant dans $B$ converge (en $B)$ vers 0 .

Théorème 4.51 (Aubin-Simon avec une suite de sous-espaces) Let $1 \leq p<+\infty$. Soit B un espace Banach, $\left(X_{n}\right)_{n \in \mathbb{N}}$ une suite d'espaces Banach incluse dans $B$ et $\left(Y_{n}\right)_{n \in \mathbb{N}}$ une suite d'espaces Banach. On suppose que la suite $\left(X_{n}, Y_{n}\right)_{n \in \mathbb{N}}$ est compacte-continue dans $B$ (voir définition 4.50).

Soit $T>0$ et $\left(f_{n}\right)_{n \in \mathbb{N}}$ une suite d'éléments de $L^{p}((0, T), B)$ satisfaisant aux conditions suivantes

1. la suite $\left(f_{n}\right)_{n \in \mathbb{N}}$ est bornée dans $L^{p}((0, T), B)$,

2. $f_{n} \in L^{p}\left((0, T), X_{n}\right)$ (pour tout $\left.n \in \mathbb{N}\right)$ et la suite $\left(\left\|f_{n}\right\|_{L^{p}\left((0, T), X_{n}\right)}\right),_{n \in \mathbb{N}}$ est bornée,

3. $\frac{d f_{n}}{d t} \in L^{1}\left((0, T), Y_{n}\right)$ (for all $\left.n \in \mathbb{N}\right)$ et la suite $\left(\left\|\frac{d f_{n}}{d t}\right\|_{L^{1}\left((0, T), Y_{n}\right)}\right)_{n \in \mathbb{N}}$ est bornée,

alors il existe $f \in L^{p}((0, T), B)$ telle que, à une sous-suite près, $f_{n} \rightarrow f$ dans $L^{p}((0, T), B)$.

Démonstration La preuve utilise la proposition 4.49 . Il suffit de prouver la troisième hypothèse sur $\left(f_{n}\right)_{n \in \mathbb{N}}$ de la Proposition 4.49 , c.à.d.

$$
\int_{0}^{T-h}\left\|f_{n}(\cdot+h)-f_{n}\right\|_{B}^{p} \mathrm{~d} t \rightarrow 0 \text { as } h \rightarrow 0, \text { uniformément par rapport à } n \in \mathbb{N} .
$$

En effet, pour tous les $n \in \mathbb{N}$, comme $f_{n} \in L^{p}((0, T), B)$, on a $\int_{0}^{T-h}\left\|f_{n}(\cdot+h)-f_{n}\right\|_{B}^{p} \mathrm{~d} t \rightarrow 0$ lorsque $h \rightarrow 0$. La seule difficulté est de prouver l'uniformité de cette convergence par rapport à $n \in \mathbb{N}$. Ainsi, il suffit de prouver que pour tous les $\eta>0$ il existe $n_{0} \in \mathbb{N}$ et $0<h_{0}<T$ tel que

$$
n \geq n_{0}, 0<h \leq h_{0} \Rightarrow \int_{0}^{T-h}\left\|f_{n}(\cdot+h)-f_{n}\right\|_{B}^{p} \mathrm{~d} t \leq \eta .
$$


Soit $\varepsilon>0$; le lemma 4.52 donne l'existence de $n_{0} \in \mathbb{N}$ et de $C_{\varepsilon} \in \mathbb{R}$ tel que

$$
n \geq n_{0}, u \in X_{n} \Rightarrow\|u\|_{B} \leq \varepsilon\|u\|_{X_{n}}+C_{\varepsilon}\|u\|_{Y_{n}} .
$$

On a donc, pour $n \geq n_{0}, 0<h<T$ et $t \in(0, T-h)$, on a

$$
\begin{array}{r}
\left\|f_{n}(t+h)-f_{n}(t)\right\|_{B} \leq \varepsilon\left\|f_{n}(t+h)-f_{n}(t)\right\|_{X_{n}}+C_{\varepsilon}\left\|f_{n}(t+h)-f_{n}(t)\right\|_{Y_{n}} \\
\leq \varepsilon\left\|f_{n}(t+h)\right\| X_{X_{n}}+\varepsilon\left\|f_{n}(t)\right\|_{X_{n}}+C_{\varepsilon}\left\|f_{n}(t+h)-f_{n}(t)\right\|_{Y_{n}},
\end{array}
$$

puis

$$
\left\|f_{n}(t+h)-f_{n}(t)\right\|_{B}^{p} \leq(3 \varepsilon)^{p}\left\|f_{n}(t+h)\right\|_{X_{n}}^{p}+(3 \varepsilon)^{p}\left\|f_{n}(t)\right\|_{X_{n}}^{p}+\left(3 C_{\varepsilon}\right)^{p}\left\|f_{n}(t+h)-f_{n}(t)\right\|_{Y_{n}}^{p} .
$$

L'intégration de cette inégalité par rapport à $t$ conduit à

$$
\int_{0}^{T-h}\left\|f_{n}(t+h)-f_{n}(t)\right\|_{B}^{p} \mathrm{~d} t \leq 2(3 \varepsilon)^{p}\left\|f_{n}\right\|_{L^{p}\left((0, T), X_{n}\right)}^{p}+\left(3 C_{\varepsilon}\right)^{p} \int_{0}^{T-h}\left\|f_{n}(t+h)-f_{n}(t)\right\|_{Y_{n}}^{p} \mathrm{~d} t
$$

Rappelons (voir la remarque 4.46 que $f_{n} \in C\left([0, T], Y_{n}\right)$ et $f_{n}\left(t_{1}\right)-f_{n}\left(t_{2}\right)=\int_{t_{1}}^{t_{2}} \frac{d f_{n}}{d t}(s) d s$ for all $t_{1}, t_{2} \in[0, T]$. On peut donc majorer le second terme du second membre de 4.47) :

$$
\begin{aligned}
\int_{0}^{T-h}\left\|f_{n}(t+h)-f_{n}(t)\right\|_{Y_{n}}^{p} \mathrm{~d} t & \leq \int_{0}^{T-h}\left(\int_{t}^{t+h}\left\|\frac{d f_{n}}{d t}(s)\right\|_{Y_{n}} d s\right)^{p} \mathrm{~d} t \\
& \leq \int_{0}^{T-h} M^{p-1}\left(\int_{t}^{t+h}\left\|\frac{d f_{n}}{d t}(s)\right\|_{Y_{n}} d s\right) \mathrm{d} t \\
& \leq M^{p-1} \int_{0}^{T-h}\left(\int_{0}^{T} 1_{[t, t+h]}(s)\left\|\frac{d f_{n}}{d t}(s)\right\|_{Y_{n}} d s\right) \mathrm{d} t
\end{aligned}
$$

où $M$ est une borne de la norme $L^{1}\left((0, T), Y_{n}\right)$ de $\frac{d f_{n}}{d t}$.

En utilisant $1_{[t, t+h]}(s)=1_{[s-h, s]}(t)$ et le théorème de Fubini-Tonelli, on obtient

$$
\int_{0}^{T-h}\left\|f_{n}(t+h)-f_{n}(t)\right\|_{Y_{n}}^{p} d t \leq h M^{p}
$$

Grâce à cette dernière inégalité, (4.47) donne

$$
\int_{0}^{T-h}\left\|f_{n}(t+h)-f_{n}(t)\right\|_{B}^{p} \mathrm{~d} t \leq 2(3 \varepsilon)^{p}\left\|f_{n}\right\|_{L^{p}\left((0, T), X_{n}\right)}^{p}+\left(3 C_{\varepsilon}\right)^{p} h M^{p} .
$$

On peut maintenant conclure. Soit $\eta>0$; on choisit $\varepsilon>0$ de manière à majorer par $\eta$ le premier terme du second membre de 4.49) (indépendamment de $n \in \mathbb{N}$ ). Ce choix de $\varepsilon$ impose $n_{0}$ et $C_{\varepsilon}$. Pour ce $C_{\varepsilon}$ donné, il existe $h_{0} \in(0, T)$ tel que le deuxième terme du second membre de 4.49 ) est majoré par $\eta$ (indépendamment de $n \in \mathbb{N}$ ) si $0<h<h_{0}$. Enfin, on obtient

$$
n \geq n_{0}, 0<h<h_{0} \Rightarrow \int_{0}^{T-h}\left\|f_{n}(t+h)-f_{n}(t)\right\|_{B}^{p} \mathrm{~d} t \leq 2 \eta .
$$

Ceci conclut la preuve du Théorème 4.51 . 
Lemme 4.52 (Aubin-Simon pour une suite de sous-espaces) Soit $B$ un espace de Banach, $\left(X_{n}\right)_{n \in \mathbb{N}}$ une suite d'espaces Banach incluse dans $B$ et $\left(Y_{n}\right)_{n \in \mathbb{N}}$ être une suite d'espaces Banach. Nous supposons que la suite $\left(X_{n}, Y_{n}\right)_{n \in \mathbb{N}}$ est compacte-continue en $B$ (voir définition 4.50 ).

Ensuite, pour tout $\varepsilon>0$, il existe $n_{0} \in \mathbb{N}$ et $C_{\varepsilon}$ tel que, pour $n \geq n_{0}$ et $w \in X_{n}$, on a

$$
\|w\|_{B} \leq \varepsilon\|w\|_{X_{n}}+C_{\varepsilon}\|w\|_{Y_{n}} .
$$

Démonstration. La preuve peut se faire par contradiction. Supposons qu'il existe $\varepsilon>0$, une fonction croissante $\varphi$ de $\mathbb{N}$ à $\mathbb{N}$ et, pour tous les $n \in \mathbb{N}, u_{\varphi(n)} \in X_{\varphi(n)}$ telles que

$$
1=\left\|u_{\varphi(n)}\right\|_{B}>\varepsilon\left\|u_{\varphi(n)}\right\|_{X_{n}}+n\left\|u_{\varphi(n)}\right\|_{Y_{n}} .
$$

Afin de définir $u_{n}$ pour tous les $n$, on pose $u_{n}=0$ pour $n \notin \operatorname{Im}(\varphi)$. La suite $\left(u_{n}\right)_{n \in \mathbb{N}}$ est telle que $u_{n} \in X_{n}$ (for all $n \in \mathbb{N}),\left(\left\|u_{n}\right\|_{X_{n}}\right)_{n \in \mathbb{N}}$ bornée et $\left\|u_{n}\right\|_{Y_{n}} \rightarrow 0$ quand $n \rightarrow+\infty$; la deuxième hypothèse de la définition 4.50 donne que toute sous-suite (de la suite $\left(u_{n}\right)_{n \in \mathbb{N}}$ ) convergeant dans $B$ converge (dans $B$ ) vers 0 . Mais, comme $\left(\left\|u_{n}\right\|_{X_{n}}\right)_{n \in \mathbb{N}}$ est borné, la première hypothèse de la définition 4.50 donne que la sous-suite $\left(u_{\varphi(n)}\right)_{n \in \mathbb{N}}$ admet une sous-suite convergeant dans $B$. La limite de cette sous-suite doit être 0 et sa norme dans $B$ doit être égale à 1 , ce qui est impossible. Ceci conclut la preuve du lemma 4.52

Il est également possible de remplacer dans le Théorème 4.51 la dérivée temporelle par une dérivée discrète. Ceci est intéressant pour prouver la convergence de la solution approximative d'un problème parabolique en utilisant un schéma numérique. C'est le but du Théorème 4.53

Théorème 4.53 (Aubin-Simon pour une suite de sous-espaces et une dérivée en temps discrète) Soit $1 \leq p<$ $+\infty$, soit B un espace de Banach, $\left(X_{n}\right)_{n \in \mathbb{N}}$ une suite d'espaces Banach inclus dans $B$ et $\left(Y_{n}\right)_{n \in \mathbb{N}}$ une suite d'espaces de Banach. On suppose que la suite $\left(X_{n}, Y_{n}\right)_{n \in \mathbb{N}}$ est compacte-continue dans $B$ (voir définition 4.50). Soit $T>0$ et $\left(u_{n}\right)_{n \in \mathbb{N}}$ une suite de $L^{p}((0, T), B)$ satisfaisant aux conditions suivantes

1. Pour tous les $n \in \mathbb{N}$, il existe $N \in \mathbb{N}^{\star}$ et $k_{1}, \ldots, k_{N}$ dans $\mathbb{R}_{+}^{\star}$ tel que $\sum_{i=1}^{N} k_{i}=T$ et $u_{n}(t)=v_{i}$ pour $t \in\left(t_{i-1}, t_{i}\right), i \in\{1, \ldots, N\}, t_{0}=0, t_{i}=t_{i-1}+k_{i}, v_{i} \in X_{n}$. (Bien entendu, les valeurs $N, k_{i}$ et $v_{i}$ dépendent de n).

La dérivée discrète en temps $\partial_{t} u_{n}$ est défine p.p. par

$$
\partial_{t} u_{n}(t)=\frac{v_{i}-v_{i-1}}{k_{i}} \text { for } t \in\left(t_{i-1}, t_{i}\right) .
$$

2. La suite $\left(u_{n}\right)_{n \in \mathbb{N}}$ est bornée dans $L^{p}((0, T), B)$,

3. La suite $\left(\left\|u_{n}\right\|_{L^{p}\left((0, T), X_{n}\right)}\right)_{n \in \mathbb{N}}$ est bornée.

4. La suite $\left(\left\|\partial_{t} u_{n}\right\|_{L^{1}\left((0, T), Y_{n}\right)}\right)_{n \in \mathbb{N}}$ est bornée.

Alors, il existe $u \in L^{p}((0, T), B)$ telle que, à une sous-suite près, $u_{n} \rightarrow u$ dans $L^{p}((0, T), B)$.

\section{Démonstration.}

Le début de la preuve suit de près celle du Théorème 4.51. Comme pour cette dernière, on utilise la proposition 4.49 et il ne reste qu'à prouver la troisièmehypothèse sur $\left(u_{n}\right)_{n \in \mathbb{N}}$ de lcette proposition, c.à.d.

$$
\int_{0}^{T-h}\left\|u_{n}(\cdot+h)-u_{n}\right\|_{B}^{p} \mathrm{~d} t \rightarrow 0 \text { as } h \rightarrow 0, \text { uniformément w.r.t. } n \in \mathbb{N} .
$$

Ici aussi, pour tous les $n \in \mathbb{N}$, puisque $u_{n} \in L^{p}((0, T), B)$, on a $\int_{0}^{T-h}\left\|u_{n}(\cdot+h)-u_{n}\right\|_{B}^{p} \mathrm{~d} t \rightarrow 0$ lorsque $h \rightarrow 0$. La seule difficulté est de prouver l'uniformité de cette convergence par rapport à $n \in \mathbb{N}$. Ainsi, il suffit de prouver 
que pour tous les $\eta>0$ il existe $n_{0} \in \mathbb{N}$ et $0<h_{0}<T$ tels que

$$
n \geq n_{0}, 0<h \leq h_{0} \Rightarrow \int_{0}^{T-h}\left\|u_{n}(\cdot+h)-u_{n}\right\|_{B}^{p} \mathrm{~d} t \leq \eta .
$$

Soit $\varepsilon>0$. le lemme 4.52 donne l'existence de $n_{0} \in \mathbb{N}$ et $C_{\varepsilon} \in \mathbb{R}$ tel que

$$
n \geq n_{0}, u \in X_{n} \Rightarrow\|u\|_{B} \leq \varepsilon\|u\|_{X_{n}}+C_{\varepsilon}\|u\|_{Y_{n}} .
$$

Pour $n \geq n_{0}, 0<h<T$ et $t \in(0, T-h)$, on a

$$
\begin{aligned}
\left\|u_{n}(t+h)-u_{n}(t)\right\|_{B} & \leq \varepsilon\left\|u_{n}(t+h)-u_{n}(t)\right\|_{X_{n}}+C_{\varepsilon}\left\|u_{n}(t+h)-u_{n}(t)\right\|_{Y_{n}} \\
& \leq \varepsilon\left\|u_{n}(t+h)\right\|_{X_{n}}+\varepsilon\left\|u_{n}(t)\right\|_{X_{n}}+C_{\varepsilon}\left\|u_{n}(t+h)-u_{n}(t)\right\|_{Y_{n}},
\end{aligned}
$$

et donc

$$
\left\|u_{n}(t+h)-u_{n}(t)\right\|_{B}^{p} \leq(3 \varepsilon)^{p}\left\|u_{n}(t+h)\right\|_{X_{n}}^{p}+(3 \varepsilon)^{p}\left\|u_{n}(t)\right\|_{X_{n}}^{p}+\left(3 C_{\varepsilon}\right)^{p}\left\|u_{n}(t+h)-u_{n}(t)\right\|_{Y_{n}}^{p} .
$$

L'intégration de cette inégalité par rapport à $t$ conduit à

$$
\int_{0}^{T-h}\left\|u_{n}(t+h)-u_{n}(t)\right\|_{B}^{p} \mathrm{~d} t \leq 2(3 \varepsilon)^{p}\left\|u_{n}\right\|_{L^{p}\left((0, T), X_{n}\right)}^{p}+\left(3 C_{\varepsilon}\right)^{p} \int_{0}^{T-h}\left\|u_{n}(t+h)-u_{n}(t)\right\|_{Y_{n}}^{p} \mathrm{~d} t .
$$

La preuve diffère maintenant de celle du Théorème 4.51. car on a affaire à la dérivée discrète de $u_{n}$ au lieu de la dérivée de $u_{n}$. Nous remarquons que pour p.p. $t \in(0, T-h)$ ( $n$ et $h$ sont fixes)

$$
u_{n}(t+h)-u_{n}(t)=\sum_{i ; t_{i} \in(t, t+h)}\left(v_{i+1}-v_{i}\right)=\sum_{i=1}^{N-1}\left(v_{i+1}-v_{i}\right) 1_{(t, t+h)}\left(t_{i}\right)=\sum_{i=1}^{N-1} \frac{v_{i+1}-v_{i}}{k_{i+1}} k_{i+1} 1_{(t, t+h)}\left(t_{i}\right),
$$

où $1_{(t, t+h)}\left(t_{i}\right)=1$ si $t_{i} \in(t, t+h)$ et 0 si $t_{i} \notin(t, t+h)$.

Soit $M$ une borne de la norme $L^{1}\left((0, T), Y_{n}\right)$ de $\partial_{t} u_{n}: M \geq \sum_{i=1}^{N-1}\left\|\frac{v_{i+1}-v_{i}}{k_{i+1}}\right\|_{Y_{n}} k_{i+1}$ for all $n$. On obtient alors

$$
\begin{aligned}
\left\|u_{n}(t+h)-u_{n}(t)\right\|_{Y_{n}}^{p} & \leq\left(\sum_{i=1}^{N-1}\left\|\frac{v_{i+1}-v_{i}}{k_{i+1}}\right\|_{Y_{n}} k_{i+1} 1_{(t, t+h)}\left(t_{i}\right)\right)^{p} \\
& \leq M^{p-1}\left(\sum_{i=1}^{N-1}\left\|\frac{v_{i+1}-v_{i}}{k_{i+1}}\right\|_{Y_{n}} k_{i+1} 1_{(t, t+h)}\left(t_{i}\right)\right)
\end{aligned}
$$

L'intégration de cette inégalité par rapport à $t \in(0, T-h)$ donne, puisque $1_{(t, t+h)}\left(t_{i}\right)=1_{\left(t_{i}-h, t_{i}\right)}(t)$,

$$
\int_{0}^{T-h}\left\|u_{n}(t+h)-u_{n}(t)\right\|_{Y_{n}}^{p} \mathrm{~d} t \leq h M^{p}
$$

En utilisant cette inégalité dans 4.51, on a

$$
\int_{0}^{T-h}\left\|u_{n}(t+h)-u_{n}(t)\right\|_{B}^{p} \mathrm{~d} t \leq 2(3 \varepsilon)^{p}\left\|u_{n}\right\|_{L^{p}\left((0, T), X_{n}\right)}^{p}+\left(3 C_{\varepsilon}\right)^{p} M^{p} h .
$$

On peut alors conclure comme dans le Théorème 4.51: soit $\eta>0$, on choisit $\varepsilon>0$ pour que le premier terme du second membre de (4.52) soit majoré par $\eta$ (indépendamment de $n \in \mathbb{N}$ ). Ce choix de $\varepsilon$ impose $n_{0}$ et $C_{\varepsilon}$. 
Pour $C_{\varepsilon}$ donné, il existe $h_{0} \in(0, T)$ tel que le deuxième terme du second membre de 4.52 soit borné par $\eta$ (indépendamment de $n \in \mathbb{N}$ ) si $0<h<h_{0}$. Enfin, on obtient

$$
n \geq n_{0}, 0<h<h_{0} \Rightarrow \int_{0}^{T-h}\left\|u_{n}(t+h)-u_{n}(t)\right\|_{B}^{p} \mathrm{~d} t \leq 2 \eta .
$$

Ceci conclut la preuve du Théorème 4.53 .

Sous les hypothèses du théorème 4.51 ou du théorème 4.53 , une autre question intéressante est de prouver une régularité supplémentaire pour $u$, à savoir que $u \in L^{p}((0, T), X)$ où $X$ est un espace lié aux espaces $X_{n}$ (et inclus dans $B$ ). La définition qui suit introduit la notion de suite $B$-limite-incluse, qui précise le lien entre l'espace $X$ et les espaces $X_{n}$. Le résultat de régularité pour une telle suite est énoncé le théorème 4.55

Définition 4.54 (Suite $B$-limite-incluse) Soient B être un espace Banach, $\left(X_{n}\right)_{n \in \mathbb{N}}$ une suite d'espaces Banach inclus dans $B$ et $X$ un espace Banach inclus dans $B$. La suite $\left(X_{n}\right)_{n \in \mathbb{N}}$ est dite B-limite-incluse dans $X$ s'il existe $C \in \mathbb{R}$ tel que si $u$ est la limite dans $B$ d'une sous-suite d'une suite $\left(u_{n}\right)_{n \in \mathbb{N}}$ vérifiant $u_{n} \in X_{n}$ et $\left\|u_{n}\right\|_{X_{n}} \leq 1$, alors $u \in X$ et $\|u\|_{X} \leq C$.

Théorème 4.55 (Régularité de la limite) Soient $1 \leq p<+\infty, T>0, B$ un espace Banach, $X$ un espace Banach inclus dans $B$, et $\left(X_{n}\right)_{n \in \mathbb{N}}$ une suite d'espaces Banach incluse dans $B$ et B-limite-incluse dans $X$ au sens de la définition 4.54 Pour $n \in \mathbb{N}$, soit $u_{n} \in L^{p}\left((0, T), X_{n}\right)$ telle que la suite $\left(\left\|u_{n}\right\|_{L^{p}\left((0, T), X_{n}\right)}\right)_{n \in \mathbb{N}}$ est bornée et que $u_{n} \rightarrow u$ dans $L^{p}((0, T), B)$ quand $n \rightarrow+\infty$. Alors $u \in L^{p}((0, T), X)$.

\section{Démonstration.}

Puisque $u_{n} \rightarrow u$ dans $L^{p}((0, T), B)$ lorsque $n \rightarrow+\infty$, nous pouvons supposer, à une sous-suite près, que $u_{n} \rightarrow u$ dans $B$ p.p.. Comme la suite $\left(X_{n}\right)_{n \in \mathbb{N}}$ est $B$-limite comprise dans $X$, on obtient, avec $C$ donné par la définition 4.54 .

$$
\|u\|_{X} \leq C \liminf _{n \rightarrow+\infty}\left\|u_{n}\right\|_{X_{n}} \text { p.p.. }
$$

Par le lemme de Fatou, on a

$$
\int_{0}^{T}\|u(t)\|_{X}^{p} \mathrm{~d} t \leq C^{p} \int_{0}^{T} \liminf _{n \rightarrow+\infty}\left\|u_{n}(t)\right\|_{X_{n}}^{p} \mathrm{~d} t \leq C^{p} \liminf _{n \rightarrow+\infty} \int_{0}^{T}\left\|u_{n}(t)\right\|_{X_{n}}^{p} \mathrm{~d} t .
$$

Enfin, puisque la suite $\left(\left\|u_{n}\right\|_{L^{p}\left((0, T), X_{n}\right)}\right)_{n \in \mathbb{N}}$ est bornée, on a bien $u \in L^{p}((0, T), X)$.

\subsection{Exercices}

Exercice 4.1 (Solution classique en dimension 1) Corrigé 4.1

Soit $u_{0} \in L^{2}(] 0,1[)$. On s'intéresse ici au problème suivant :

$$
\left\{\begin{array}{l}
\left.\partial_{t} u(x, t)-u_{x x}(x, t)=0, x \in\right] 0,1[, t \in] 0,+\infty[ \\
u(0, t)=u(1, t)=0, t \in] 0,+\infty[ \\
u(x, 0)=u_{0}(x), x \in[0,1]
\end{array}\right.
$$

La notation $\partial_{t} u$ désigne la dérivée de $u$ par rapport à $t$ et $\partial_{x}^{2} u$ désigne la dérivée seconde de $u$ par rapport à $x$. On dit que $u$ est une solution classique de (4.53) si $u$ vérifie les trois conditions suivantes

(c1) $u$ est de classe $C^{2}$ sur $] 0,1[\times] 0,+\infty\left[\right.$ et vérifie au sens classique $\partial_{t} u-\partial_{x}^{2} u=0$ en tout point de $] 0,1[\times] 0,+\infty[$,

(c2) pour tout $t>0$, les fonctions $u, \partial_{x} u$ et $\partial_{x}^{2} u$ sont continues sur $[0,1] \times[t,+\infty[$ et $u(0, t)=u(1, t)=0$, 
(c3) $u(\cdot, t) \rightarrow u_{0}$ dans $L^{2}(] 0,1[)$ quand $t \rightarrow 0(t>0)$.

1. Montrer que le problème 4.53 admet au plus une solution classique. [On pourra reprendre la méthode développée à la section 4.1.]

2. Montrer que le problème (4.53) admet une solution classique. [On pourra reprendre la méthode développée à la section 4.1 en explicitant une base hilbertienne convenable de $L^{2}$ (]0,1[), voir l'exercice 2.2.]

\section{Exercice 4.2 (Dual de $\boldsymbol{L}_{\boldsymbol{E}}^{\boldsymbol{p}}$ ) Corrigé 4.2}

Soit $(X, T, m)$ un espace mesuré, $E$ un espace de Banach et $1<p<+\infty$. On pose $p^{\prime}=p /(p-1)$.

1. Soit $v \in L_{E^{\prime}}^{p^{\prime}}(X, T, m)$ et $u \in L_{E}^{p}(X, T, m)$. Montrer que l'application $x \mapsto\langle v(x), u(x)\rangle_{E^{\prime}, E}$ est $m$-mesurable de $X$ dans $\mathbb{R}$ puis que $\langle v, u\rangle_{E^{\prime}, E} \in L_{\mathbb{R}}^{1}(X, T, m)$ et

$$
\int\left|\langle v, u\rangle_{E^{\prime}, E}\right| d m \leq\|v\|_{L_{E^{\prime}}^{p^{\prime}}}\|u\|_{L_{E}^{p}}
$$

2. Soit $v \in L_{E^{\prime}}^{p^{\prime}}(X, T, m)$.

(a) Montrer que l'application $u \mapsto \int\langle v, u\rangle_{E^{\prime}, E} d m$ est bien définie, linéaire et continue de $L_{E}^{p}(X, T, m)$ dans $\mathbb{R}$. On note $T_{v}$ cette application (on a donc $T_{v} \in L_{E}^{p}(X, T, m)^{\prime}$.)

(b) Montrer que $\left\|T_{v}\right\|_{L_{E}^{p}(X, T, m)^{\prime}} \leq\|v\|_{L_{E^{\prime}}^{p^{\prime}}(X, T, m)}$.

(c) (Question plus difficile) Montrer que $\left\|T_{v}\right\|_{L_{E}^{p}(X, T, m)^{\prime}}=\|v\|_{L_{E^{\prime}}^{p^{\prime}}(X, T, m)}$.

\section{Exercice 4.3 (Dérivée faible pour une union de domaines)}

On suppose que $\Omega_{1}$ et $\Omega_{2}$ sont deux disjoints de $\mathbb{R}^{N}(N \geq 1)$ et on note $\Omega$ l'intérieur de l'adhérence de $\Omega_{1} \cup \Omega_{2}$ (l'ouvert $\Omega$ peut donc être différent de $\Omega_{1} \cup \Omega_{2}$ ).

Soit $f \in L^{2}(] 0, T\left[, L^{2}(\Omega)\right)$. Pour $i=1,2$, on note $f_{i}$ la fonction obtenue en restreignant $f$ à $\Omega_{i}$, on a donc $f_{i} \in L 2(] 0, T\left[, L^{2}\left(\Omega_{i}\right)\right)$.

On identifie (comme d'habitude) $L^{2}(\Omega)$ avec son dual et $L^{2}\left(\Omega_{i}\right)$ avec son dual (pour $i=1,2$ ).

On suppose $d f_{i} / d t \in L^{2}(] 0, T\left[, H^{1}\left(\Omega_{i}\right)^{\prime}\right)$ (pour $\left.i=1,2\right)$. Montrer que $d f / d t \in L^{2}(] 0, T\left[, H^{1}(\Omega)^{\prime}\right)$.

Corrigé - Il s'agit de montrer qu'il existe $u \in L^{2}(] 0, T\left[, H^{1}(\Omega)^{\prime}\right)$ tel que, pour tout $\varphi \in C_{c}^{\infty}(\Omega, \mathbb{R})$,

$$
-\int_{0}^{T} f(t) \varphi^{\prime}(t) \mathrm{d} t=\int_{0}^{T} u(t) \varphi(t) \mathrm{d} t
$$

Le terme de gauche de cette égalité est dans $L^{2}(\Omega)$ et le terme de droite est dans $H^{1}(\Omega)^{\prime}$. Comme on a identifié $L^{2}(\Omega)$ avec son dual et que $H^{1}(\Omega)$ est dense dans $L^{2}(\Omega)$, on a $L^{2}(\Omega) \subset H^{1}(\Omega)^{\prime}$ et montrer cette égalité consiste donc à montrer que pour tout $\psi \in H^{1}(\Omega)$ on a

$$
\int_{\Omega}\left(-\int_{0}^{T} f(t) \varphi(t) \mathrm{d} t\right)(x) \psi(x) \mathrm{d} x=\left\langle\int_{0}^{T} u(t) \varphi(t) \mathrm{d} t, \psi\right\rangle_{H^{1}(\Omega)^{\prime}, H^{1}(\Omega)} .
$$

On cherche donc $u \in L^{2}(] 0, T\left[, H^{1}(\Omega)^{\prime}\right)$ vérifiant 4.54 pour tout $\varphi \in C_{c}^{\infty}(\Omega, \mathbb{R})$ et tout $\psi \in H^{1}(\Omega)$.

Pour $i=1,2$, on sait que $d f_{i} / d t \in L^{2}(] 0, T\left[, H^{1}\left(\Omega_{i}\right)^{\prime}\right)$, il existe donc $u_{i} \in L^{2}(] 0, T\left[, H^{1}\left(\Omega_{i}\right)^{\prime}\right)$ (que peut confondre avec $\left.d f_{i} / d t\right)$ tel que, pour tout $\varphi \in C_{c}^{\infty}(\Omega, \mathbb{R})$ et tout $\psi \in H^{1}\left(\Omega_{i}\right)$ on a

$$
\int_{\Omega_{i}}\left(-\int_{0}^{T} f_{i}(t) \varphi(t) \mathrm{d} t\right)(x) \psi(x) \mathrm{d} x=\left\langle\int_{0}^{T} u_{i}(t) \varphi(t) \mathrm{d} t, \psi\right\rangle_{H^{1}\left(\Omega_{i}\right)^{\prime}, H^{1}\left(\Omega_{i}\right)} .
$$


Soient $\varphi \in C_{c}^{\infty}(\Omega, \mathbb{R})$ et $\psi \in H^{1}(\Omega)$. On note $\psi_{i}$ la restriction de $\psi$ a $\Omega_{i}$. On a donc $\psi_{i} \in H^{1}\left(\Omega_{i}\right)$ et $\|\psi\|_{H^{1}(\Omega)}^{2} \leq$ $\sum_{i=1}^{2}\left\|\psi_{i}\right\|_{H^{1}\left(\Omega_{i}\right)}^{2}$. En utilisant 4.55 (et la proposition 4.24 on a

$$
\begin{gathered}
\int_{\Omega}\left(-\int_{0}^{T} f(t) \varphi(t) \mathrm{d} t\right)(x) \psi(x) \mathrm{d} x=\sum_{i=1}^{2} \int_{\Omega_{i}}\left(-\int_{0}^{T} f_{i}(t) \varphi(t) \mathrm{d} t\right)(x) \psi_{i}(x) \mathrm{d} x \\
=\sum_{i=1}^{2}\left\langle\int_{0}^{T} u_{i}(t) \varphi(t) \mathrm{d} t, \psi_{i}\right\rangle_{H^{1}\left(\Omega_{i}\right)^{\prime}, H^{1}\left(\Omega_{i}\right)}=\int_{0}^{T} \sum_{i=1}^{2}\left\langle u_{i}(t) \varphi(t), \psi_{i}\right\rangle_{H^{1}\left(\Omega_{i}\right)^{\prime}, H^{1}\left(\Omega_{i}\right)} \mathrm{d} t \\
=\int_{0}^{T} \sum_{i=1}^{2}\left\langle u_{i}(t), \psi_{i}\right\rangle_{H^{1}\left(\Omega_{i}\right)^{\prime}, H^{1}\left(\Omega_{i}\right)} \varphi(t) \mathrm{d} t=\int_{0}^{T}\langle u(t), \psi\rangle_{H^{1}\left(\Omega_{i}\right)^{\prime}, H^{1}\left(\Omega_{i}\right)} \varphi(t) \mathrm{d} t \\
=\left\langle\int_{0}^{T} u(t) \varphi(t) \mathrm{d} t, \psi\right\rangle_{H^{1}(\Omega)^{\prime}, H^{1}(\Omega)},
\end{gathered}
$$

où $u(t)$ est défini (pour presque tout $t$ ) par

$$
\langle u(t), \psi\rangle_{H^{1}(\Omega)^{\prime}, H^{1}(\Omega)}=\sum_{i=1}^{2}\langle u(t), \psi\rangle_{H^{1}\left(\Omega_{i}\right)^{\prime}, H^{1}\left(\Omega_{i}\right)} .
$$

Comme $u_{i} \in L^{2}(] 0, T\left[, H^{1}\left(\Omega_{i}\right)^{\prime}\right)$ et $\|\psi\|_{H^{1}(\Omega)}^{2} \leq \sum_{i=1}^{2}\left\|\psi_{i}\right\|_{H^{1}\left(\Omega_{i}\right)}^{2}$, on a bien $u \in L^{2}(] 0, T\left[, H^{1}(\Omega)^{\prime}\right)$ et cela termine la démonstration.

Exercice 4.4 (Sur la continuité à valeurs $\boldsymbol{L}^{2}$ ) On pose $T>0$ et $\left.\Omega=\right] 0,+\infty[$. On identifie, comme d'habitude, $L^{2}(\Omega)$ avec son dual. On suppose que $u \in L^{2}(] 0, T\left[, H^{1}(\Omega)\right)$ et $\partial_{t} u \in L^{2}(] 0, T\left[, H^{-1}(\Omega)\right)$.

On rappelle la définition de "dérivée par transposition de $u$ ". C'est l'élément de $\mathcal{D}_{H^{1}(\Omega)}^{\star}$, noté $\partial_{t} u$, tel que, pour tout $\varphi \in \mathcal{D}=C_{c}^{\infty}(\Omega)$,

$$
\left\langle\partial_{t} u, \varphi\right\rangle_{\mathcal{D}_{H^{1}(\Omega)}^{\star}, \mathcal{D}}=-\int_{0}^{T} u(\cdot, t) \varphi^{\prime}(t) \mathrm{d} t .
$$

La notation $u(\cdot, t)$ désigne la fonction $x \mapsto u(\cdot, t))$. Noter que $d \int_{0}^{T} u(\cdot, t) \varphi^{\prime}(t) \mathrm{d} t \in H^{1}(\Omega)$.

On rappelle aussi que $\partial_{t} u \in L^{2}(] 0, T\left[, H^{-1}(\Omega)\right)$ signifie qu'il existe une fonction $v$ (encore notée $\partial_{t} u$ et appelée "dérivée faible de $u$ ") appartenant à $L^{2}(] 0, T\left[, H^{-1}(\Omega)\right)$ telle que

$$
\left\langle\partial_{t} u, \varphi\right\rangle_{\mathcal{D}_{H^{1}(\Omega)}^{\star}, \mathcal{D}}=-\int_{0}^{T} u(\cdot, t) \varphi^{\prime}(t) \mathrm{d} t=\int_{0}^{T} v(\cdot, t) \varphi(t) \mathrm{d} t .
$$

Noter que $\int_{0}^{T} v(\cdot, t) \varphi(t) \mathrm{d} t \in H^{-1}(\Omega)$.

Cette égalité a bien un sens car $H^{1}(\Omega) \subset L^{2}(\Omega)=L^{2}(\Omega)^{\prime} \subset H^{-1}(\Omega)$.

On montre dans cet exercice que $u \in C\left([0, T], L^{2}(\Omega)\right)$. Le lemme 4.26 ne s'applique pas directement car $H^{-1}(\Omega)$ n'est pas le dual de $H^{1}(\Omega)$ (noté $\left(H^{1}(\Omega)^{\prime}\right)$. On peut d'ailleurs remarquer que l'application qui à $T$ élément de $H^{1}(\Omega)^{\prime}$ associe sa restriction a $H_{0}^{1}(\Omega)$, qui est donc un élément de $H^{-1}(\Omega)$, n'est pas injective.

La méthode proposée ici consiste à se ramener au lemme 4.26 (avec $E=H^{1}(\mathbb{R})$ et $F=L^{2}(\mathbb{R})$ ) en utilisant un prolongement convenable de $u$.

On se donne trois nombres $\alpha$, $\beta$ et $\gamma$ strictement positifs vérifiant $\alpha-\beta=1$ et $(\alpha+1)-\beta / \gamma=0$ (un exemple possible est $\alpha=2, \beta=1, \gamma=1 / 3)$. On définit $\bar{u}$ de $\mathbb{R} \times] 0, T[$ par

$$
\begin{array}{r}
\bar{u}(x, t)=u(x, t) \text { si } x \geq 0, \\
\bar{u}(x, t)=\alpha u(-x, t)-\beta u(-\gamma x, t) \text { si } x<0 .
\end{array}
$$

1. Montrer que $\bar{u} \in L^{2}(] 0, T\left[, H^{1}(\mathbb{R})\right)$. 
Corrigé - La condition $\alpha-\beta=1$ donne que $u(t)$ est continue au point $x=0$ (pour presque tout $t)$. Cette continuité en 0 permet alors de montrer que $\bar{u} \in L^{2}(] 0, T\left[, H^{1}(\mathbb{R})\right)$.

2. Cette question donne une égalité essentielle pour calculer $\partial_{t} \bar{u}$, c'est-à-dire $\int_{0}^{T} \bar{u}(\cdot, t) \varphi^{\prime}(t) \mathrm{d} t$. Soit $\varphi \in C_{c}^{\infty}(] 0, T[)$ et $\psi \in C_{c}^{\infty}(\mathbb{R})$. Pour $x>0$, on pose

$$
\bar{\psi}(x)=\psi(x)+\alpha \psi(-x)-\frac{\beta}{\gamma} \psi\left(-\frac{x}{\gamma}\right) .
$$

(a) Montrer que

$$
\left\langle\int_{0}^{T} \bar{u}(\cdot, t) \varphi^{\prime}(t) \mathrm{d} t, \psi\right\rangle_{H^{-1}(\mathbb{R}), H^{1}(\mathbb{R})}=\int_{0}^{T}\left(\int_{0}^{\infty} u(x, t) \bar{\psi}(x) \mathrm{d} x\right) \varphi^{\prime}(t) d t .
$$

[Utiliser la proposition 4.24 puis des changements de variables.]

Corrigé - Comme $\bar{u} \in L^{1}(] 0, T\left[, H^{1}(\mathbb{R})\right), \int_{0}^{T} \bar{u}(\cdot, t) \varphi^{\prime}(t) \mathrm{d} t \in H^{1}(\mathbb{R})$. Grâce à l'identification de $L^{2}(\mathbb{R})$ avec son dual, $H^{1}(\mathbb{R}) \subset L^{2}(\mathbb{R})=L^{2}(\mathbb{R})^{\prime} \subset H^{1}(\mathbb{R})^{\prime}=H^{-1}(\mathbb{R})$ (les inclusions étant avec continuité).

On calcule maintenant $\left\langle\int_{0}^{T} \bar{u}(\cdot, t) \varphi^{\prime}(t) \mathrm{d} t, \psi\right\rangle_{H^{-1}(\mathbb{R}), H^{1}(\mathbb{R})}$ en utilisant la proposition 4.24 le fait que $\bar{u}(\cdot, t) \in$ $H^{-1}(\mathbb{R})$ (par l'identification de $L^{2}(\mathbb{R})$ avec son dual) et des changements de variables.

$$
\begin{gathered}
\left\langle\int_{0}^{T} \bar{u}(\cdot, t) \varphi^{\prime}(t) \mathrm{d} t, \psi\right\rangle_{H^{-1}(\mathbb{R}), H^{1}(\mathbb{R})}=\int_{0}^{T}\left\langle\bar{u}(\cdot, t) \varphi^{\prime}(t), \psi\right\rangle_{H^{-1}(\mathbb{R}), H^{1}(\mathbb{R})} d t \\
\quad=\int_{0}^{T}\langle\bar{u}(\cdot, t), \psi\rangle_{H^{-1}(\mathbb{R}), H^{1}(\mathbb{R})} \varphi^{\prime}(t) \mathrm{d} t=\int_{0}^{T}\left(\int_{\mathbb{R}} \bar{u}(x, t) \psi(x) \mathrm{d} x\right) \varphi^{\prime}(t) \mathrm{d} t \\
=\int_{0}^{T}\left(\int_{0}^{\infty} u(x, t) \psi(x) \mathrm{d} x+\int_{-\infty}^{0} \alpha u(-x, t) \psi(x) \mathrm{d} x-\int_{-\infty}^{0} \beta u(-\gamma x, t) \psi(x) \mathrm{d} x\right) \varphi^{\prime}(t) d t \\
=\int_{0}^{T}\left(\int_{0}^{\infty} u(x, t) \psi(x) \mathrm{d} x+\int_{0}^{\infty} \alpha u(x, t) \psi(-x) \mathrm{d} x-\int_{0}^{\infty} \frac{\beta}{\gamma} u(x, t) \psi\left(-\frac{x}{\gamma}\right) \mathrm{d} x\right) \varphi^{\prime}(t) d t \\
=\int_{0}^{T}\left(\int_{0}^{\infty} u(x, t) \bar{\psi}(x) \mathrm{d} x\right) \varphi^{\prime}(t) d t .
\end{gathered}
$$

(b) Montrer que $\bar{\psi} \in H_{0}^{1}(\Omega)$. En déduire que

$$
\left\langle\int_{0}^{T} \bar{u}(\cdot, t) \varphi^{\prime}(t) \mathrm{d} t, \psi\right\rangle_{H^{-1}(\mathbb{R}), H^{1}(\mathbb{R})}=\int_{0}^{T}\langle v(\cdot, t), \bar{\psi}\rangle_{H^{-1}(\Omega), H_{0}^{1}(\Omega)} \varphi(t) \mathrm{d} t
$$

où $v=\partial_{t} u\left(\right.$ et donc $\left.v \in L^{2}(] 0, T\left[, H^{-1}(\Omega)\right)\right)$.

Corrigé - La fonction $\bar{\psi}$ appartient à $C_{c}^{\infty}(\bar{\Omega})$ ( c'est-à-dire qu'elle est la restriction à $\Omega$ d'un élément de $C_{c}^{\infty}(\mathbb{R})$ ) et sa trace en $x=0$ est nulle grâce au fait que $(\alpha+1)-\beta / \gamma=0$. La fonction $\bar{\psi}$ appartient donc à $H_{0}^{1}(\Omega)$ et il est facile de montrer qu'il existe $C$, ne dépendant que de $\alpha$, $\beta$ et $\gamma$, tel que $\|\bar{\psi}\|_{H_{0}^{1}(\Omega)} \leq C\|\psi\|_{H^{1}(\mathbb{R})}$.

Dans l'égalité obtenue à la question précédente, on utilise le fait que $u(\cdot, t) \in H^{-1}(\Omega)$ (par l'identification de 
$L^{2}(\Omega)$ avec son dual), de nouveau la proposition 4.24 et le fait que $\partial_{t} u=v \in L^{2}(] 0, T\left[, H^{-1}(\Omega)\right)$. On obtient

$$
\begin{aligned}
\left\langle\int_{0}^{T} \bar{u}(\cdot, t) \varphi^{\prime}(t) \mathrm{d} t, \psi\right\rangle_{H^{-1}(\mathbb{R}), H^{1}(\mathbb{R})}=\int_{0}^{T}\left(\int_{0}^{\infty} u(x, t) \bar{\psi}(x) \mathrm{d} x\right) \varphi^{\prime}(t) d t \\
=\int_{0}^{T}\langle u(\cdot, t), \bar{\psi}\rangle_{H^{-1}(\Omega), H_{0}^{1}(\Omega)} \varphi^{\prime}(t) d t=\int_{0}^{T}\left\langle u(\cdot, t) \varphi^{\prime}(t), \bar{\psi}\right\rangle_{H^{-1}(\Omega), H_{0}^{1}(\Omega)} d t \\
=\left\langle\int_{0}^{T} u(\cdot, t) \varphi^{\prime}(t) d t, \bar{\psi}\right\rangle_{H^{-1}(\Omega), H_{0}^{1}(\Omega)}=\left\langle-\int_{0}^{T} v(\cdot, t) \varphi(t) d t, \bar{\psi}\right\rangle_{H^{-1}(\Omega), H_{0}^{1}(\Omega)} \\
=-\int_{0}^{T}\langle v(\cdot, t), \bar{\psi}\rangle_{H^{-1}(\Omega), H_{0}^{1}(\Omega)} \varphi(t) \mathrm{d} t .
\end{aligned}
$$

3. Montrer que $\left.\partial_{t} \bar{u} \in L^{2}(] 0, T\left[, H^{-1}(\mathbb{R})\right)\right)$. En déduire que $\bar{u} \in C\left([0, T], L^{2}(\mathbb{R})\right)$ et que $u \in C\left([0, T], L^{2}(\Omega)\right)$.

Corrigé - Pour $\varphi \in C_{c}^{\infty}(] 0, T[)$ et $\psi \in C_{c}^{\infty}(\mathbb{R})$, on note $\psi \otimes \varphi$ la fonction $(x, t) \mapsto \psi(x) \varphi(t)$.

On note $S$ l'application de $C_{c}^{\infty}(\mathbb{R}) \times C_{c}^{\infty}(] 0, T[)$ dans $\mathbb{R}$ définie par

$$
S(\psi \otimes \varphi)=\int_{0}^{T}\langle v(\cdot, t), \bar{\psi}\rangle_{H^{-1}(\Omega), H_{0}^{1}(\Omega)} \varphi(t) \mathrm{d} t,
$$

où $\bar{\psi}$ est définie comme à la question 2

Par linéarité, $S$ se prolonge sur l'espace vectoriel, noté $G$, engendré par les fonctions $\psi \otimes \varphi$.

Si $\phi \in G, \phi(x, t)=\sum_{i=1}^{n} a_{i} \varphi_{i}(t) \psi_{i}(x)$, avec $n \in \mathbb{N}, \varphi \in C_{c}^{\infty}(] 0, T[)$ et $\psi_{i} \in C_{c}^{\infty}(\mathbb{R})$ (pour tout $i$ ), et

$$
S(\phi)=\int_{0}^{T}\left\langle v(\cdot, t), \sum_{i=1}^{n} a_{i} \varphi_{i}(t) \bar{\psi}_{i}\right\rangle_{H^{-1}(\Omega), H_{0}^{1}(\Omega)} \mathrm{d} t .
$$

On a, pour tout $t$, avec $C$ donné à la question $2 b$

On en déduit que

$$
\left\|\sum_{i=1}^{n} a_{i} \varphi_{i}(t) \bar{\psi}_{i}\right\|_{H_{0}^{1}(\Omega)} \leq C\left\|\sum_{i=1}^{n} a_{i} \varphi_{i}(t) \psi_{i}\right\|_{H^{1}(\mathbb{R})}=\|\phi(\cdot, t)\|_{H^{1}(\mathbb{R})} .
$$

$$
\begin{aligned}
|S(\phi)| \leq \int_{0}^{T}\|v(\cdot, t)\|_{H^{-1}(\Omega)}\left\|\sum_{i=1}^{n} a_{i} \varphi_{i}(t) \bar{\psi}_{i}\right\|_{H_{0}^{1}(\Omega)} d t \leq C \int_{0}^{T}\|v(\cdot, t)\|_{H^{-1}(\Omega)}\|\phi(\cdot, t)\|_{H^{1}(\mathbb{R})} d t & \\
& \leq C\|v\|_{L^{2}(] 0, T\left[, H^{-1}(\Omega)\right)}\|\phi\|_{L^{2}(] 0, T\left[H^{1}(\mathbb{R})\right)} .
\end{aligned}
$$

L'application $S$ se prolonge donc en une application linéaire continue de $L^{2}(] 0, T\left[, H^{1}(\mathbb{R})\right)$ dans $\mathbb{R}$ (ce prolongement est même unique car $G$ est dense dans $\left.L^{2}(] 0, T\left[, H^{1}(\mathbb{R})\right)\right)$. Ceci montre qu'il existe $w \in L^{2}(] 0, T\left[,\left(H^{1}(\mathbb{R})^{\prime}\right)(q u i\right.$ est le dual de $\left.L^{2}(] 0, T\left[, H^{1}(\mathbb{R})\right)\right)$ tel que

$$
S(\phi)=\int_{0}^{T}\langle w(\cdot, t), \phi(\cdot, t)\rangle_{H^{-1}(\mathbb{R}), H^{1}(\mathbb{R})} d t .
$$

En particulier ceci donne pour tout $\varphi \in C_{c}^{\infty}(] 0, T[)$ et $\psi \in C_{c}^{\infty}(\mathbb{R})$,

$$
\begin{gathered}
\left\langle\int_{0}^{T} \bar{u}(\cdot, t) \varphi^{\prime}(t) \mathrm{d} t, \psi\right\rangle_{H^{-1}(\mathbb{R}), H^{1}(\mathbb{R})}=-S(\psi \otimes \varphi)=-\int_{0}^{T}\langle w(\cdot, t), \varphi(t) \psi\rangle_{H^{-1}(\mathbb{R}), H^{1}(\mathbb{R})} d t \\
=\left\langle-\int_{0}^{T} w(t) \varphi(t) d t, \psi\right\rangle_{H^{-1}(\mathbb{R}), H^{1}(\mathbb{R})} .
\end{gathered}
$$

On a donc $\int_{0}^{T} \bar{u}(\cdot, t) \varphi^{\prime}(t) \mathrm{d} t=-\int_{0}^{T} w(t) \varphi(t) d t$ pour tout $\varphi \in C_{c}^{\infty}(] 0, T[)$,

c'est-à-dire $\partial_{t} \bar{u}=w \in L^{2}(] 0, T\left[,\left(H^{1}(\mathbb{R})^{\prime}\right)\right.$.

Le lemme 4.26 donne alors $\bar{u} \in C\left([0, T], L^{2}(\mathbb{R})\right)$ et donc $u \in C\left([0, T], L^{2}(\Omega)\right)$.

N.B. Par un argument de cartes locales, le résultat démontré dans cet exercice reste vrai si $\Omega$ est un ouvert borné de $\mathbb{R}^{N}, N \geq 1$, à bord fortement lipschitzien (remarque 1.17] [18]. 


\section{Exercice 4.5 (Diffusion non homogène et non isotrope) Corrigé 4.3}

On reprend les hypothèses du théorème 4.28 Soit donc $\Omega$ un ouvert borné de $\mathbb{R}^{N}, T>0$ et $u_{0} \in L^{2}(\Omega)$. On identifie $L^{2}(\Omega)$ avec son dual et on suppose que $f \in L^{2}(] 0, T\left[, H^{-1}(\Omega)\right)$.

On se donne aussi une application, notée $A$, de $\Omega$ dans $\mathcal{M}_{N}(\mathbb{R})$ (ensemble des matrices carrés à $N$ lignes et $N$ colonnes, à coefficients dans $\mathbb{R}$ ). On suppose que les coefficients de $A$ appartiennent à $L^{\infty}(\Omega)$ et qu'il existe $\alpha>0$ t.q.

$$
A \xi \cdot \xi \geq \alpha|\xi|^{2} \text { p.p., pour tout } \xi \in \mathbb{R}^{N} .
$$

En suivant la démonstration donnée dans ce chapitre, montrer qu'il existe un et un seul $u$ tel que

$$
\left\{\begin{aligned}
& u \in L^{2}(] 0, T\left[, H_{0}^{1}(\Omega)\right), \partial_{t} u \in L^{2}(] 0, T\left[, H^{-1}(\Omega)\right), \\
& \int_{0}^{T}<\partial_{t} u(s), v(s)>_{H^{-1}, H_{0}^{1}} d s+\int_{0}^{T}\left(\int_{\Omega} A \nabla u(s) \cdot \nabla v(s) \mathrm{d} x\right) d s= \\
& \quad \int_{0}^{T}<f(s), v(s)>_{H^{-1}, H_{0}^{1}} d s \text { pour tout } v \in L^{2}(] 0, T\left[, H_{0}^{1}(\Omega)\right), \\
& u(0)=u_{0} \text { p.p.. }
\end{aligned}\right.
$$

\section{Exercice 4.6 (Existence par le théorème de Schauder) Corrigé 4.4}

Soit $\Omega$ un ouvert borné de $\mathbb{R}^{N}$ et $A: \mathbb{R} \rightarrow M_{N}(\mathbb{R})$ (où $M_{N}(\mathbb{R})$ désigne les matrices $N \times N$ à coefficients réels) t.q.

$$
\begin{array}{r}
\forall s \in \mathbb{R}, A(s)=\left(a_{i, j}(s)\right)_{i, j=1, \ldots, N} \text { où } a_{i, j} \in L^{\infty}(\mathbb{R}) \cap C(\mathbb{R}, \mathbb{R}), \\
\exists \alpha>0 ; A(s) \xi \cdot \xi \geq \alpha|\xi|^{2}, \forall \xi \in \mathbb{R}^{N}, \forall s \in \mathbb{R}, \\
f \in L^{2}(] 0, T\left[, H^{-1}(\Omega)\right) \text { et } u_{0} \in L^{2}(\Omega) .
\end{array}
$$

On identifie $L^{2}(\Omega)$ à $L^{2}(\Omega)^{\prime}$, comme d'habitude. On veut, dans cet exercice, montrer l'existence d'une solution au problème 4.32 .

Soit $\bar{u} \in L^{2}(] 0, T\left[, L^{2}(\Omega)\right)$, on définit l'opérateur $T$ de $L^{2}(] 0, T\left[, L^{2}(\Omega)\right)$ dans $L^{2}(] 0, T\left[, L^{2}(\Omega)\right) \operatorname{par} T(\bar{u})=u$ où $u$ est la solution (donnée par l'exercice 4.5 ) du problème

$$
\left\{\begin{array}{l}
u \in L^{2}(] 0, T\left[, H_{0}^{1}(\Omega)\right), \partial_{t} u \in L^{2}(] 0, T\left[, H^{-1}(\Omega)\right), \\
\int_{0}^{T}\left\langle\partial_{t} u, v\right\rangle_{H^{-1}, H_{0}^{1}} \mathrm{~d} t+\int_{0}^{T} \int_{\Omega} A(\bar{u}) \nabla u \cdot \nabla v \mathrm{~d} x \mathrm{~d} t \\
\quad=\int_{0}^{T}\langle f, v\rangle_{H^{-1}, H_{0}^{1}} \mathrm{~d} t, \forall v \in L^{2}(] 0, T\left[, H_{0}^{1}(\Omega)\right), \\
u(\cdot, 0)=u_{0} .
\end{array}\right.
$$

1. Montrer que $T$ est continu de $L^{2}(] 0, T\left[, L^{2}(\Omega)\right)$ dans $L^{2}(] 0, T\left[, L^{2}(\Omega)\right)$.

2. Montrer que $T$ est compact de $L^{2}(] 0, T\left[, L^{2}(\Omega)\right)$ dans $L^{2}(] 0, T\left[, L^{2}(\Omega)\right)$.

3. Montrer qu'il existe $R>0$ t.q. $\|T(\bar{u})\|_{L^{2}(] 0, T\left[, L^{2}(\Omega)\right)} \leq R$ pour tout $u \in L^{2}(] 0, T\left[, L^{2}(\Omega)\right)$.

4. Montrer qu'il existe $u$ solution de 4.32).

5. On suppose maintenant de plus que $a_{i, j}$ est, pour tout $i, j$, une fonction lipschitzienne. Montrer que (4.32) admet une unique solution.

Exercice 4.7 (Existence par schéma numérique) Corrigé 4.5 
On se propose, dans cet exercice, de montrer l'existence d'une solution faible au problème 4.60-4.62 en passant à la limite sur un solution approchée donnée par un schéma numérique.

$$
\begin{gathered}
\left.\frac{\partial u}{\partial t}(x, t)-\frac{\partial^{2} \varphi(u)}{\partial x^{2}}(x, t)=v(x, t), x \in\right] 0,1[, t \in] 0, T[ \\
\left.\frac{\partial \varphi(u)}{\partial x}(0, t)=\frac{\partial \varphi(u)}{\partial x}(1, t)=0, t \in\right] 0, T[ \\
\left.u(x, 0)=u_{0}(x), x \in\right] 0,1[
\end{gathered}
$$

où $\varphi, v, T, u_{0}$ sont donnés et sont t.q.

1. $T>0, v \in L^{\infty}(] 0,1[\times] 0, T[)$,

2. $\varphi$ croissante, lipschitzienne de $\mathbb{R}$ dans $\mathbb{R}$,

3. $u_{0} \in L^{\infty}(] 0,1[)$ et $\varphi\left(u_{0}\right)$ lipschitzienne de $[0,1]$ dans $\mathbb{R}$.

Un exemple important est donné par $\varphi(s)=\alpha_{1} s$ si $s \leq 0, \varphi(s)=0$ si $0 \leq s \leq L$ et $\varphi(s)=\alpha_{2}(s-L)$ si $s \geq L$, avec $\alpha_{1}, \alpha_{2}$ et $L$ donnés dans $\mathbb{R}_{+}^{\star}$. Noter pour cet exemple que $\varphi^{\prime}=0$ sur $] 0, L[$.

Les ensembles ]0,1[ et ]0,1[×]0,T[ sont munis de leur tribu borélienne et de la mesure de Lebesgue sur cette tribu. On appelle "solution faible" de 4.60, 4.61, 4.62) une solution de

$$
\begin{gathered}
u \in L^{\infty}(] 0,1[\times] 0, T[) \\
\int_{0}^{T} \int_{0}^{1}\left(u(x, t) \frac{\partial \psi}{\partial t}(x, t)+\varphi(u(x, t)) \frac{\partial^{2} \psi}{\partial x^{2}}(x, t)+v(x, t) \psi(x, t)\right) \mathrm{d} x \mathrm{~d} t \\
+\int_{0}^{1} u_{0}(x) \psi(x, 0) \mathrm{d} x=0, \quad \forall \psi \in C_{T}^{2,1}\left(\mathbb{R}^{2}\right),
\end{gathered}
$$

où $C_{T}^{2,1}\left(\mathbb{R}^{2}\right)=\left\{\psi: \mathbb{R}^{2} \rightarrow \mathbb{R}, 2\right.$ fois continûment dérivable par rapport à $x$ et une fois continûment dérivable par rapport à $t$, t.q. $\frac{\partial \psi}{\partial x}(0, t)=\frac{\partial \psi}{\partial x}(1, t)=0$ pour tout $t \in[0, T]$ et $\psi(x, T)=0$ pour tout $\left.x \in[0,1]\right\}$.

1. (Question indépendante des questions suivantes.) On suppose, dans cette question seulement, que $\varphi$ est de classe $C^{2}, v$ est continue sur $[0,1] \times[0, T]$ et $u_{0}$ est continue sur $[0,1]$. Soit $w \in C^{2}\left(\mathbb{R}^{2}, \mathbb{R}\right)$ et $u$ la restriction de $w$ à $] 0,1[\times] 0, T[$. Montrer que $u$ est solution de (4.63), (4.64) si et seulement si $u$ vérifie (4.60), (4.61) et (4.62) au sens classique ( c'est-à-dire pour tout $(x, t) \in[0,1] \times[0, T]$ ).

2. (Un résulat de compacité.) Soit $A \subset C\left([0, T], L^{2}(] 0,1[)\right)$. On munit $C\left([0, T], L^{2}(] 0,1[)\right)$ de la norme usuelle, c'est-à-dire

$$
\|u\|=\sup \left\{\|u(t)\|_{L^{2}(] 0,1[)}, t \in[0, T]\right\} .
$$

On suppose que $A$ vérifie les 2 hypothèses suivantes

(a) Pour tout $t \in[0, T],\{u(t), u \in A\}$ est un borné de $L^{\infty}(] 0,1[)$ et il existe $C$ t.q.

$$
\int_{\mathbb{R}}|u(t)(x+\eta)-u(t)(x)|^{2} \mathrm{~d} x \leq C \eta,
$$

pour tout $\eta \in \mathbb{R}_{+}^{\star}$ et tout $u \in A$, avec $u(t)$ prolongée par 0 hors de $[0,1]$.

(b) Il existe $C \in \mathbb{R}$ t.q. $\|u(t)-u(s)\|_{L^{2}(] 0,1[)} \leq C|t-s|$, pour tout $t, s \in[0, T]$ et tout $u \in A$.

Montrer que $A$ est relativement compacte dans $C\left([0, T], L^{2}(] 0,1[)\right)$. 
3. (Passage à la limite sur une non linéarité, cette question est indépendante de la question précédente.)

Soit $\left(u_{n}\right)_{n \in \mathbb{N}}$ une suite bornée de $L^{\infty}(] 0,1[\times] 0, T[)$. Soient $u \in L^{\infty}(] 0,1[\times] 0, T[)$ et $f \in L^{1}(] 0,1[\times] 0, T[)$.

On suppose que, quand $n \rightarrow \infty$,

(a) $u_{n} \rightarrow u \quad \star$-faiblement dans $L^{\infty}(] 0,1[\times] 0, T[)$,

(b) $\varphi\left(u_{n}\right) \rightarrow f$ dans $L^{1}(] 0,1[\times] 0, T[)$ et p.p..

Montrer que $\varphi(u)=f$ p.p. sur $] 0,1[\times] 0, T[$.

[Indication : Utiliser l'astuce de Minty...]

On cherche maintenant une solution approchée de 44.60), 4.61, ,4.62).

Soient $N, M \in \mathbb{N}^{\star}$. On pose $h=\frac{1}{N}$ et $k=\frac{T}{M}$. On va construire une solution approchée de (4.60), 4.61, (4.62) à partir de la famille $\left\{u_{i}^{n}, i=1, \ldots, N, n=0, \ldots, M\right\}$ vérifiant les équations suivantes

$$
\begin{gathered}
u_{i}^{0}=\frac{1}{h} \int_{(i-1) h}^{i h} u_{0}(x) \mathrm{d} x, i=1, \ldots, N, \\
\frac{u_{i}^{n+1}-u_{i}^{n}}{k}-\frac{\varphi\left(u_{i-1}^{n+1}\right)-2 \varphi\left(u_{i}^{n+1}\right)+\varphi\left(u_{i+1}^{n+1}\right)}{h^{2}}=v_{i}^{n}, i=1, \ldots, N, n=0, \ldots, M-1,
\end{gathered}
$$

avec $u_{0}^{n+1}=u_{1}^{n+1}, u_{N+1}^{n+1}=u_{N}^{n+1}$, pour tout $n=0, \ldots, M-1$ et $v_{i}^{n}=\frac{1}{k h} \int_{n k}^{(n+1) k} \int_{(i-1) h}^{i h} v(x, t) \mathrm{d} x \mathrm{~d} t$, pour tout $i=1, \ldots, N$, pour tout $n=0, \ldots, M$.

4. (Existence et unicité de la solution approchée.)

Soit $n \in\{0, \ldots, M-1\}$. On suppose connu $\left\{u_{i}^{n}, i=1, \ldots, N\right\}$.

(a) On suppose que $\varphi$ est bijective de $\mathbb{R}$ dans $\mathbb{R}$. Soit $\left\{u_{i}^{n+1}, i=1, \ldots, N\right.$. On pose $r_{i}=\varphi\left(u_{i}^{n+1}\right)$ pour tout $i=1, \ldots, N\}$. Montrer que $\left\{u_{i}^{n+1}, i=1, \ldots, N\right\}$ est solution de (4.66) si et seulement si $\left(r_{i}\right)_{i=1, \ldots, N}$ est un point fixe de l'application (bien définie...) qui à $w=\left(w_{i}\right)_{i=1, \ldots, N}$ associe $r=\left(r_{i}\right)_{i=1, \ldots, N}$ défini $\left(\right.$ avec $w_{0}=w_{1}$ et $\left.w_{N+1}=w_{N}\right)$ par

$$
\varphi^{-1}\left(r_{i}\right)+\frac{2 k}{h^{2}} r_{i}=\frac{k}{h^{2}}\left(w_{i-1}+w_{i+1}\right)+u_{i}^{n}+k v_{i}^{n}, i=1, \ldots, N
$$

Montrer que cette application est strictement contractante (de $\mathbb{R}^{N}$ dans $\mathbb{R}^{N}$ ) pour la norme dite "du sup". En déduire qu'il existe une unique famille $\left\{u_{i}^{n+1}, i=1, \ldots, N\right\}$ solution de (4.66).

(b) (plus difficile) On ne suppose plus que $\varphi$ est bijective de $\mathbb{R}$ dans $\mathbb{R}$. Montrer qu'il existe une unique famille $\left\{u_{i}^{n+1}, i=1, \ldots, N\right\}$ solution de 4.66.

Les trois questions suivantes donnent des estimations sur la solution approchée dont on vient de montrer l'existence et l'unicité.

5. (Estimation $L^{\infty}(] 0,1[\times] 0, T[)$ sur la solution approchée.)

On pose $A=\left\|u_{0}\right\|_{L^{\infty}(] 0,1[)}$ et $B=\|v\|_{L^{\infty}(] 0,1[\times] 0, T[)}$.

Montrer, par récurrence sur $n$, que $u_{i}^{n} \in[-A-n k B, A+n k B]$ pour tout $i=1, \ldots, N$ et tout $n=0, \ldots, M$. [On pourra, par exemple, considérer 4.66 avec $i$ t.q. $u_{i}^{n+1}=\min \left\{u_{j}^{n+1}, j=1, \ldots, N\right\}$.] 
6. (Estimation de la dérivée p.r. à $x$ de $\varphi(u)$.) Montrer qu'il existe $C_{1}$ (ne dépendant que de $T, \varphi, v$ et $u_{0}$ ) t.q., pour tout $n=0, \ldots, M-1$,

$$
\sum_{i=1}^{N-1}\left(\varphi\left(u_{i+1}^{n+1}\right)-\varphi\left(u_{i}^{n+1}\right)\right)^{2} \leq C_{1} \frac{h}{k} .
$$

[indication : multiplier 4.66 $\operatorname{par} u_{i}^{n+1}$ et sommer sur $i$.]

7. (Estimation de la dérivée p.r. à $t$ de $\varphi(u)$.) Montrer qu'il existe $C_{2}$ (ne dépendant que de $T, \varphi, v$ et $u_{0}$ ) t.q.

$$
\sum_{n=0}^{M-1} h \sum_{i=0}^{N+1}\left(\varphi\left(u_{i}^{n+1}\right)-\varphi\left(u_{i}^{n}\right)\right)^{2} \leq C_{2} k .
$$

[indication : multiplier 4.66 $\operatorname{par} \varphi\left(u_{i}^{n+1}\right)-\varphi\left(u_{i}^{n}\right)$ et sommer sur $i$ et $n$ ]

L'objectif est maintenant de passer à la limite sur les paramètres de discrétisation. Pour $M \in \mathbb{N}^{\star}$ donné, on prend $N=M^{2}$ (et donc $h$ et $k$ sont donnés et $k=T \sqrt{h}$ ), on définit, avec les $u_{i}^{n}$ trouvés dans les questions précédentes, une fonction, $u_{h}$, sur $[0,1] \times[0, T]$ en posant

$$
u_{h}(x, t)=\frac{t-n k}{k} u_{h}^{(n+1)}(x)+\frac{(n+1) k-t}{k} u_{h}^{(n)}(x), \text { si } t \in[n k,(n+1) k]
$$

et

$$
u_{h}^{(n)}(x)=u_{i}^{n}, \operatorname{si} x \in[(i-1) h, i h], i=1, \ldots, N, n=0, \ldots, M .
$$

8. Soient $A_{1}=\left\{u_{h}, M \in \mathbb{N}^{\star}\right\}$ et $A_{2}=\left\{\varphi\left(u_{h}\right), M \in \mathbb{N}^{\star}\right\}$.

Montrer que $A_{1}$ est relativement séquentiellement compacte dans $L^{\infty}(] 0,1[\times] 0, T[)$, pour la topologie faible- $\star$, et que $A_{2}$ est relativement (séquentiellement) compacte dans $C\left([0, T], L^{2}(] 0,1[)\right)$.

En déduire que l'on peut trouver une suite $\left(h_{n}\right)_{n \in \mathbb{N}}$ et $u \in L^{\infty}(] 0,1[\times] 0, T\left[\right.$ ) t.q., en posant $u_{n}=u_{h_{n}}$ (on rappelle que $k_{n}=T \sqrt{h}_{n}$ ), on ait, quand $n \rightarrow \infty$,

(a) $h_{n} \rightarrow 0$ et $k_{n} \rightarrow 0$

(b) $u_{n} \rightarrow u \star$-faiblement dans $L^{\infty}(] 0,1[\times] 0, T[)$,

(c) $\varphi\left(u_{n}\right) \rightarrow \varphi(u)$ dans $L^{p}(] 0,1[\times] 0, T[)$, pour tout $p \in[1, \infty[$.

9. Montrer que la fonction $u$ trouvée à la question précédente est solution de 4.63, (4.64).

Remarque. On peut aussi montrer l'unicité de la solution de 44.63, (4.64).

\section{Exercice 4.8 (Existence pour le problème de Stefan)}

Soient $\Omega$ un ouvert borné de $\mathbb{R}^{N}(N \geq 1), 0<T<+\infty, f \in L^{2}(] 0, T\left[,\left(L^{2}(\Omega)\right), u_{0} \in L^{2}(\Omega)\right.$ et $\varphi$ une fonction lipschitzienne de $\mathbb{R}$ de $\mathbb{R}$ croissante (mais non nécessairement strictement croissante, la fonction $\varphi$ peut être constante sur un intervalle de $\mathbb{R}$ de mesure strictement positive).

Dans cet exercice, on donne une preuve alternative à celle de [2] de l'existence d'une solution) (faible) à l'équation $\partial_{t} u-\Delta(\varphi(u))=f($ dans $\Omega \times] 0, T[)$ avec une condition aux limites de Dirichlet homogène et $u_{0}$ comme condition initiale. Plus précisément, la fonction $u$ sera solution au sens suivant : 


$$
\left\{\begin{array}{l}
\left.u \in L^{\infty}(] 0, T\left[, L^{2}(\Omega)\right), \partial_{t} u \in L^{2}(] 0, T\left[, H^{-1}(\Omega)\right), u \in C\left([0, T], H^{-1}(\Omega)\right)\right), \varphi(u) \in L^{2}(] 0, T\left[, H_{0}^{1}(\Omega)\right) \\
\int_{0}^{T}\left\langle\partial_{t} u, v\right\rangle_{H^{-1}, H_{0}^{1}} \mathrm{~d} t+\int_{0}^{T} \int_{\Omega} \nabla \varphi(u) \cdot \nabla v \mathrm{~d} x \mathrm{~d} t \\
=\int_{0}^{T} \int_{\Omega} f v \mathrm{~d} x \mathrm{~d} t=0, \forall v \in L^{2}(] 0, T\left[, H_{0}^{1}(\Omega)\right), \\
u(\cdot, 0)=u_{0} .
\end{array}\right.
$$

On rappelle que $L^{2}(\Omega)$ est, comme d'habitude, identifié à $L^{2}(\Omega)^{\prime}$.

Pour résoudre ce problème, on introduit pour $n>0$ la fonction $\varphi_{n}$ définie par $\varphi_{n}(s)=\varphi(s)+(1 / n) s$. On montre l'existence d'une solution au problème avec $\varphi_{n}$ au lieu de $\varphi$. Puis, on passe à la limite quand $n \rightarrow+\infty$.

1. Montrer que l'exercice 4.6 donne l'existence de $u_{n}$ solution de 4.67) avec $\varphi_{n}$ au lieu de $\varphi$, avec la régularité supplémentaire $u_{n} \in L^{2}(] 0, T\left[, H_{0}^{1}(\Omega)\right)$ (et donc $u_{n} \in C([0, T]), L^{2}(\Omega)$ ).

[On rappelle que le lemme 4.31 donne $\nabla \varphi(v)=\varphi^{\prime}(v) \nabla v$ p.p. si $v \in L^{2}(] 0, T\left[, H_{0}^{1}(\Omega)\right)$.]

Dans la suite, on étudie cette suite $\left(u_{n}\right)_{n \in \mathbb{N}^{*}}$

2. Montrer que la suite $\left(\varphi\left(u_{n}\right)\right)_{n \in \mathbb{N}^{\star}}$ est bornée dans $L^{2}(] 0, T\left[, H_{0}^{1}(\Omega)\right)$, la suite $\left(\partial_{t} u_{n}\right)_{n \in \mathbb{N}^{\star}}$ est bornée dans $L^{2}(] 0, T\left[, H^{-1}(\Omega)\right)$ et la suite $\left(u_{n}\right)_{n \in \mathbb{N}^{*}}$ est bornée dans $C(] 0, T\left[, L^{2}(\Omega)\right)$.

[Le lemme 4.31 est encore utile ici.]

Dans la suite, on montre que cette fonction $u$ est la solution recerchée.

3. Montrer qu'il existe $u$ et $\zeta$ tels que (quitte à extraire une sous suite, non renumérotée) $\varphi\left(u_{n}\right) \rightarrow \zeta$ faiblement dans $L^{2}(] 0, T\left[, H_{0}^{1}(\Omega)\right)$ et $u_{n} \rightarrow u$ dans $L^{2}(] 0, T\left[, H^{-1}(\Omega)\right)$.

[Pour la convergence de $u_{n}$, utiliser le théorème 4.42]]

4. Montrer que $\lim _{n \rightarrow+\infty} \int_{0}^{T} \int_{\Omega} \varphi\left(u_{n}\right) u_{n} \mathrm{~d} x \mathrm{~d} t=\int_{0}^{T} \int_{\Omega} \zeta u \mathrm{~d} x \mathrm{~d} t$.

5. Avec l'astuce de Minty (utilisée par exemple pour démontrer 3.25 ), montrer que $\varphi(u)=\zeta$ p.p.

6. Montrer que $u \in C\left([0, T], H^{-1}\right)$.

[Remarquer que la $\left(u_{n}\right)_{n \in \mathbb{N}^{\star}}$ est bornée dans $C\left([0, T], L^{2}(\Omega)\right)$ et raisonner alors comme dans l'étape 6 du théorème 4.28] ]

7. Montrer que $u$ est solution de 4.67).

Exercice 4.9 (Théorème de Kolmogorov, avec $\boldsymbol{B}=\mathbf{I R}$ ) Corrigé 4.6 Le but de cet exercice est de refaire la démonstration du théorème 4.40 dans le cas (plus simple) $B=\mathbb{R}$ et $p=1$.

Soit $T>0$. On note $L^{1}$ l'espace $L^{1}(] 0, T[, \mathbb{R})$. Soit $\left(u_{n}\right)_{n \in \mathbb{N}}$ une suite bornée de $L^{1}$ (on a donc $\sup _{n \in \mathbb{N}}\left\|u_{n}\right\|_{1}<$ $+\infty)$.

On suppose que pour tout $h \in] 0, T[$ et tout $n \in \mathbb{N}$ on a

$$
\int_{0}^{T-h}\left|u_{n}(t+h)-u_{n}(t)\right| d t \leq \eta(h),
$$

où $\eta$ est une fonction croissante de $] 0, T\left[\right.$ dans $\mathbb{R}_{+}$t.q. $\lim _{h \rightarrow 0^{+}} \eta(h)=0$.

L'objectif de l'exercice est de démontrer que la suite $\left(u_{n}\right)_{n \in \mathbb{N}}$ est relativement compacte dans $L^{1}$.

1. Soit $\delta, h \in] 0, T[$ t.q. $\delta+h \leq T$. Montrer que

$$
\int_{0}^{\delta}\left|u_{n}(t)\right| \mathrm{d} t \leq \int_{0}^{\delta}\left|u_{n}(t+h)\right| \mathrm{d} t+\int_{0}^{\delta}\left|u_{n}(t+h)-u_{n}(t)\right| \mathrm{d} t .
$$


2. Soit $\left.h_{0} \in\right] 0, T[$ et $\delta \in] 0, T-h_{0}[$, montrer que

$$
h_{0} \int_{0}^{\delta}\left|u_{n}(t)\right| \mathrm{d} t \leq \delta\left\|u_{n}\right\|_{1}+h_{0} \eta\left(h_{0}\right) .
$$

3. Montrer que $\int_{0}^{\delta}\left|u_{n}(t)\right| \mathrm{d} t \rightarrow 0$ quand $\delta \rightarrow 0^{+}$, uniformément par rapport à $n$.

4. Montrer que la suite $\left(u_{n}\right)_{n \in \mathbb{N}}$ est relativement compacte dans $L^{1}$.

[Appliquer le théorème de Kolmogorov, théorème 4.39, en utilisant le prolongement de $u_{n}$ par 0.]

\subsection{Corrigés d'exercices}

\section{Corrigé 4.1 (Solution classique en dimension 1)}

Soit $u_{0} \in L^{2}(] 0,1[)$. On s'intéresse ici au problème suivant :

$$
\left\{\begin{array}{l}
\left.\partial_{t} u(x, t)-\partial_{x}^{2} u(x, t)=0, x \in\right] 0,1[, t \in] 0,+\infty[ \\
u(0, t)=u(1, t)=0, t \in] 0,+\infty[ \\
u(x, 0)=u_{0}(x), x \in[0,1]
\end{array}\right.
$$

La notation $\partial_{t} u$ désigne la dérivée de $u$ par rapport à $t$ et $u_{x x}$ désigne la dérivée seconde de $u$ par rapport à $x$. On dit que $u$ est une solution classique de (4.70) si $u$ vérifie les trois conditions suivantes

(c1) $u$ est de classe $C^{2}$ sur $] 0,1[\times] 0,+\infty\left[\right.$ et vérifie au sens classique $\partial_{t} u-\partial_{x}^{2} u=0$ en tout point de $] 0,1[\times] 0,+\infty[$,

(c2) pour tout $t>0$, les fonctions $u, u_{x}$ et $u_{x x}$ sont continues sur $[0,1] \times[t,+\infty[$ et $u(0, t)=u(1, t)=0$,

(c3) $u(\cdot, t) \rightarrow u_{0}$ dans $L^{2}(] 0,1[)$ quand $t \rightarrow 0(t>0)$.

1. Montrer que le problème 4.70] admet au plus une solution classique. [On pourra reprendre la méthode développée à la section 4.1]

Corrigé - Si u et $\bar{u}$ sont deux solutions classiques de 4.70p. La fonction $(u-\bar{u})$ est alors une solution classique de 4.70 avec $u_{0}=0$ p.p. (sur ]0, 1[). Pour montrer que $u=\bar{u}$, il suffit donc de montrer que la fonction nulle est l'unique solution de 4.70 lorsque $u_{0}=0$ p.p.

On suppose donc que u est une solution classique de 4.70 avec $u_{0}=0$ p.p.. On va montrer que $u(x, t)=0$ pour tout $(x, t) \in[0,1] \times] 0,+\infty[$.

Soit $t>0$, les hypothèses $(c 1)$ et ( $c 2$ ) permettent de dire que

$$
\int_{0}^{1} \partial_{t} u(x, t) u(x, t) \mathrm{d} x=\frac{1}{2} \int_{0}^{1}\left(u^{2}\right)_{t}(x, t) \mathrm{d} x=\frac{1}{2} \frac{d}{d t}\left(\int_{0}^{1} u^{2}(x, t) \mathrm{d} x\right)
$$

et

$$
\int_{0}^{1} u_{x x}(x, t) u(x, t) \mathrm{d} x=-\int_{0}^{1} u_{x}(x, t)^{2} \mathrm{~d} x .
$$

Comme $\partial_{t} u u-u_{x x} u=0$ sur $] 0,1[\times] 0,+\infty$ (, on en déduit que

$$
\frac{1}{2} \frac{d}{d t}\left(\int_{0}^{1} u^{2}(x, t) \mathrm{d} x\right)+\int_{0}^{1} u_{x}(x, t)^{2} \mathrm{~d} x=0 .
$$

Soit $0<\varepsilon<T<+\infty$. En intégrant l'équation précédente entre $\varepsilon$ et $T$, on obtient

$$
\frac{1}{2} \int_{0}^{1} u^{2}(x, T) \mathrm{d} x+\int_{0}^{T} \int_{0}^{1} u_{x}^{2}(x, t) \mathrm{d} x \mathrm{~d} t=\frac{1}{2} \int_{0}^{1} u(x, \varepsilon) \mathrm{d} x,
$$

ce qui donne

$$
\int_{0}^{1} u^{2}(x, T) \mathrm{d} x \leq \int_{0}^{1} u^{2}(x, \varepsilon) \mathrm{d} x
$$


Quand $\varepsilon \rightarrow 0$, le membre de droite de cette inégalité tend vers 0 (par $(c 3))$, on a donc $\int_{0}^{1} u^{2}(x, T) \mathrm{d} x=0$, ce qui donne $u(\cdot, T)=0$ p.p. et donc $u(x, T)=0$ pour tout $x \in[0,1]($ car $u(\cdot, T)$ est supposée être continue sur $[0,1])$. Comme $T>0$ est arbitraire, on a bien montré que $u(x, t)=0$ pour tout $(x, t) \in[0,1] \times] 0,+\infty[$.

2. Montrer que le problème (4.1) admet une solution classique. [On pourra reprendre la méthode développée à la section 4.1 en explicitant une base hilbertienne convenable de $L^{2}(] 0,1[)$, voir l'exercice 2.2.]

Corrigé -

On utilise un résultat de l'exercice 2.2 Pour $n \in \mathbb{N}^{\star}$, on pose $c_{n}=2 \int_{0}^{1} u_{0}(t) \sin (n \pi t) d t$. L'exercice 2.2 donne que

$$
\left\|u_{0}-\sum_{p=1}^{n} c_{p} \sin (p \pi \cdot)\right\|_{2} \rightarrow 0, \text { quand } n \rightarrow \infty
$$

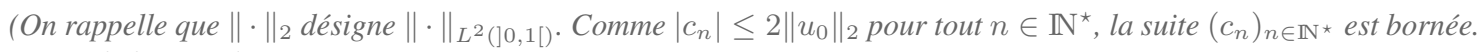
On en déduit que la série

$$
\sum_{n=1}^{+\infty} e^{-n^{2} \pi^{2} t} c_{n} \sin n \pi x
$$

est convergente (dans $\mathbb{R}$ ) pour tout $x \in \mathbb{R}$ et $t>0$. De plus, pour tout $\varepsilon>0$, les séries

$$
\sum_{n=1}^{+\infty} n e^{-n^{2} \pi^{2} \varepsilon}\left|c_{n}\right| \text { et } \sum_{n=1}^{+\infty} n^{2} e^{-n^{2} \pi^{2} \varepsilon}\left|c_{n}\right|
$$

sont convergentes. Ceci montre que la fonction

$$
x, t \mapsto \sum_{n=1}^{+\infty} e^{-n^{2} \pi^{2} t} c_{n} \sin n \pi x
$$

est, pour tout $\varepsilon>0$, de classe $C^{2}$ sur $\left.\mathbb{R} \times\right] \varepsilon,+\infty\left[\right.$ ( elle est même de classe $C^{\infty}$ ) et que la série peut être dérivée terme à terme, une fois en $t$ et deux fois en $x$. On pose donc, pour $x \in] 0,1[$ et $t>0$

$$
u(x, t)=\sum_{n=1}^{+\infty} e^{-n^{2} \pi^{2} t} c_{n} \sin n \pi x .
$$

La fonction u ainsi définie vérifie bien les conditions (c1) et (c2).

Il reste à montrer que u vérifie (c3). Soit $\varepsilon>0$. Pour tout $t>0$ et $n_{0}>0$, on a

$$
\left\|u(\cdot, t)-u_{0}\right\|_{2} \leq\left\|\sum_{n=1}^{n_{0}} c_{n}\left(1-e^{-n^{2} t^{2}}\right) \sin (n \pi \cdot)\right\|_{2}+2\left\|u_{0}-\sum_{n=1}^{n_{0}} c_{n} \sin (n \pi \cdot)\right\|_{2} .
$$

On commence par choisir $n_{0}$ pour que le deuxième terme du membre de droite de cette inégalité soit inférieur à $\varepsilon$. Puis, comme $n_{0}$ est maintenant fixé, on remarque qu'il existe $t_{0}>0$ t.q. le premier terme du membre de droite de cette inégalité est inférieur à $\varepsilon$ dès que $\left.t \in] 0, t_{0}\right]$. On en déduit bien que u vérifie (c3).

\section{Corrigé 4.2 (Dual de $\boldsymbol{L}_{E}^{p}$ )}

Soit $(X, T, m)$ un espace mesuré, $E$ un espace de Banach et $1<p<+\infty$. On pose $p^{\prime}=p /(p-1)$.

1. Soit $v \in L_{E^{\prime}}^{p^{\prime}}(X, T, m)$ et $u \in L_{E}^{p}(X, T, m)$. Montrer que l'application $x \mapsto\langle v(x), u(x)\rangle_{E^{\prime}, E}$ est $m$-mesurable de $X$ dans $\mathbb{R}$ puis que $\langle v, u\rangle_{E^{\prime}, E} \in L_{\mathbb{R}}^{1}(X, T, m)$ et

$$
\int\left|\langle v, u\rangle_{E^{\prime}, E}\right| d m \leq\|v\|_{L_{E^{\prime}}^{p^{\prime}}}\|u\|_{L_{E}^{p}}
$$


Corrigé - On choisit pour $u$ et $v$ des représentants, de sorte que $v \in \mathcal{L}_{E^{\prime}}^{p^{\prime}}(X, T, m)$ et $u \in \mathcal{L}_{E}^{p}(X, T, m)$. La $m$ mesurabilité de l'application $x \mapsto\langle u(x), v(x)\rangle_{E^{\prime}, E}$ (notée $\langle v, u\rangle_{E^{\prime}, E}$ ) est assez simple à montrer (et ne dépend pas des représentants choisis pour $u$ et $v$ ). Il suffit de remarquer qu'il existe deux suites de fonctions étagées $\left(v_{n}\right)_{n \in \mathbb{N}}$ et $\left(u_{n}\right)_{n \in \mathbb{N}}\left(v_{n}\right.$ est une fonction de $X$ dans $E^{\prime}$ et $u_{n}$ de $X$ dans $\left.E\right)$ t.q. $v_{n} \rightarrow$ v p.p. et $u_{n} \rightarrow$ u p.p. $(q u a n d n \rightarrow+\infty)$. Pour tout $n \in \mathbb{N}$, la fonction $\left\langle v_{n}, u_{n}\right\rangle_{E^{\prime}, E}$ est alors une fonction étagée de $X$ dans $\mathbb{R}$. Quand $n \rightarrow+\infty$, elle converge p.p. vers la fonction $\langle v, u\rangle_{E^{\prime}, E}$ qui est donc m-mesurable.

On remarque ensuite que $\left|\langle v(x), u(x)\rangle_{E^{\prime}, E}\right| \leq\|v(x)\|_{E^{\prime}}\|u(x)\|_{E}$ pour tout $x \in X$. En intégrant par rapport à la mesure $m$ (on utilise ici la monotonie de l'intégrale pour les fonctions $m$-mesurables à valeurs dans $\mathbb{R}$ et l'inégalité de Hölder), on obtient que $\langle v, u\rangle_{E^{\prime}, E} \in \mathcal{L}_{\mathbb{R}}^{1}(X, T, m)$ (et donc $\langle v, u\rangle_{E^{\prime}, E} \in L_{\mathbb{R}}^{1}(X, T, m)$ avec la confusion habituelle entre $\mathcal{L}^{1}$ et $L^{1}$ ) et

$$
\int\left|\langle v, u\rangle_{E^{\prime}, E}\right| d m \leq \in t\|v\|_{E^{\prime}}\|u\|_{E} d m \leq\|v\|_{L_{E^{\prime}}^{p^{\prime}}}\|u\|_{L_{E}^{p}}
$$

2. Soit $v \in L_{E^{\prime}}^{p^{\prime}}(X, T, m)$.

(a) Montrer que l'application $u \mapsto \int\langle v, u\rangle_{E^{\prime}, E} d m$ est bien définie, linéaire et continue de $L_{E}^{p}(X, T, m)$ dans $\mathbb{R}$.

Corrigé - La question précédente donne bien que $\langle v, u\rangle_{E^{\prime}, E} \in L_{E}^{1}(X, T, m)$ pour tout $u \in L_{E}^{p}(X, T, m)$. L'application $u \mapsto \int\langle v, u\rangle_{E^{\prime}, E} d m$ est donc bien définie de $L_{E}^{p}(X, T, m)$ dans $\mathbb{R}$. Elle est trivialement linéaire. Sa continuité est une conséquence du fait que

$$
\left|\int\langle v, u\rangle_{E^{\prime}, E} d m\right| \leq \in t\left|\langle v, u\rangle_{E^{\prime}, E}\right| d m \leq\|v\|_{L_{E^{\prime}}^{p^{\prime}}}\|u\|_{L_{E}^{p}}
$$

On note $T_{v}$ cette application (on a donc $T_{v} \in L_{E}^{p}(X, T, m)^{\prime}$.)

(b) Montrer que $\left\|T_{v}\right\|_{L_{E}^{p}(X, T, m)^{\prime}} \leq\|v\|_{L_{E^{\prime}}^{p^{\prime}}(X, T, m)}$.

Corrigé - Par définition de la norme dans le dual d'un espace de Banach, on a

$$
\left\|T_{v}\right\|_{\left(L_{E}^{p}\right)^{\prime}}=\sup \left\{\left|T_{v}(u)\right|, u \in L_{E}^{p} \text { t.q. }\|u\|_{L_{E}^{p}}=1\right\} .
$$

La question précédente donne que $\left|T_{v}(u)\right| \leq\|v\|_{L_{E^{\prime}}^{p^{\prime}}}$ si $\|u\|_{L_{E}^{p}}=1$, on a donc

$$
\left\|T_{v}\right\|_{L_{E}^{p}(X, T, m)^{\prime}} \leq\|v\|_{L_{E^{\prime}}^{p^{\prime}}(X, T, m)} .
$$

(c) (Question plus difficile) Montrer que $\left\|T_{v}\right\|_{L_{E}^{p}(X, T, m)^{\prime}}=\|v\|_{L_{E^{\prime}}^{p^{\prime}}(X, T, m)}$.

Corrigé - On pose $q=p^{\prime}$. On raisonne ici en deux étapes. On commence par montrer l'égalité demandée si v est une fonction étagée. Puis, on traite le cas général.

Etape 1

On suppose, dans cette étape, que v est une fonction étagée non nulle. Il existe donc $n \in \mathbb{N}^{\star}$, une famille $A_{1}, \ldots, A_{n}$ d'éléments de la tribu $T$ et une famille $b_{1}, \ldots, b_{n}$ d'éléments de $E^{\prime}$, non nuls, t.q.

$$
v=\sum_{i=1}^{n} b_{i} 1_{A_{i}} .
$$

Soit $\varepsilon>0$. De la définition de la norme dans $E^{\prime}$, on déduit que, pour tout $i \in\{1, \ldots, n\}$, il existe $\bar{a}_{i} \in E$ t.q.

$$
\left\|\bar{a}_{i}\right\|_{E}=1 \text { et }\left\langle b_{i}, \bar{a}_{i}\right\rangle_{E^{\prime}, E} \geq(1-\varepsilon)\left\|b_{i}\right\|_{E^{\prime}} .
$$

On pose $a_{i}=\left\|b_{i}\right\|_{E^{\prime}}^{q-1} \bar{a}_{i}$ pour tout $i \in\{1, \ldots, n\}$ et

$$
u=\sum_{i=1}^{n} a_{i} 1_{A_{i}} .
$$


La fonction u est une fonction étagée (de $X$ dans $E$ ) et on a

$$
\int\langle v, u\rangle_{E^{\prime}, E} d m=\sum_{i=1}^{n} m\left(A_{i}\right)\left\langle b_{i}, a_{i}\right\rangle_{E^{\prime}, E} \geq(1-\varepsilon) m\left(A_{i}\right)\left\|b_{i}\right\|_{E^{\prime}}^{q}=(1-\varepsilon)\|v\|_{L_{E^{\prime}}^{q}}^{q} .
$$

D'autre part, on a (comme $p(q-1)=q)$

$$
\|u\|_{L_{E}^{p}}^{p}=\sum_{i=1}^{n} m\left(A_{i}\right)\left\|b_{i}\right\|_{E^{\prime}}^{p(q-1)}=\sum_{i=1}^{n} m\left(A_{i}\right)\left\|b_{i}\right\|_{E^{\prime}}^{q}=\|v\|_{L_{E^{\prime}}^{q}}^{q} .
$$

On a donc

$$
\frac{1}{\|u\|_{L_{E}^{p}}} \int\langle v, u\rangle_{E^{\prime}, E} d m \geq(1-\varepsilon) \frac{\|v\|_{L_{E^{\prime}}^{q}}^{q}}{\|v\|_{L_{E^{\prime}}^{q}}^{q}}=(1-\varepsilon)\|v\|_{L_{E^{\prime}}^{q}}
$$

En posant $\bar{u}=\frac{u}{\|u\|_{L_{E}^{p}}}$, on a donc $\|\bar{u}\|_{L_{E}^{p}}=1$ et $\int\langle v, \bar{u}\rangle_{E^{\prime}, E} d m \geq(1-\varepsilon)\|v\|_{L_{E^{\prime}}^{q}}$. Ceci prouve que

$$
\left\|T_{v}\right\|_{\left(L_{E}^{p}\right)^{\prime}} \geq(1-\varepsilon)\|v\|_{L_{E^{\prime}}^{q}} .
$$

Comme $\varepsilon>0$ est arbitraire, on obtient donc, avec la question $2(b),\left\|T_{v}\right\|_{\left(L_{E}^{p}\right)^{\prime}}=\|v\|_{L_{E^{\prime}}^{q}}$.

\section{Etape 2}

On traite maintenant le cas général. Soit $v \in L_{E^{\prime}}^{q}$ non nulle ( $s i v=0$ p.p. l'égalité demandée est triviale). Il existe une suite $\left(v_{n}\right)_{n \in \mathbb{N}}$ de fonctions étagée (de $X$ dans $\left.E^{\prime}\right)$ t.q. $v_{n} \rightarrow v$ dans $L_{E^{\prime}}^{q}$ (cette suite peut se construire comme cela a été pour la définition de $L^{1}$, c'est-à-dire en construisant $v_{n} t . q .\left\|v_{n}(x)\right\|_{E^{\prime}} \leq 2\|v(x)\|_{E^{\prime}}$ pour presque tout $x \in E^{\prime}$ et pour tout $n \in \mathbb{N}$ ).

Soit $\varepsilon>0$, il existe donc $w$ fonction étagée t.q. $\|w-v\|_{L_{E^{\prime}}^{p}} \leq \varepsilon$. Par l'étape 1, il existe $u \in L_{E}^{p}$ t.q. $\|u\|_{L_{E}^{p}}=1$ et

On a donc (avec la question 1)

$$
\int\langle w, u\rangle_{E^{\prime}, E} d m \geq\|w\|_{L_{E^{\prime}}^{q}}-\varepsilon
$$

$$
\begin{gathered}
\int\langle v, u\rangle_{E^{\prime}, E} d m=\int\langle w, u\rangle_{E^{\prime}, E} d m+\int\langle v-w, u\rangle_{E^{\prime}, E} d m \geq\|w\|_{L_{E^{\prime}}^{q}}-\varepsilon-\|v-w\|_{L_{E^{\prime}}^{q}} . \\
\text { Comme }\|v-w\|_{L_{E^{\prime}}^{q}} \leq \varepsilon \text { et }\|w\|_{L_{E^{\prime}}^{q}} \geq\|v\|_{L_{E^{\prime}}^{q}}-\varepsilon, \text { on a donc } \\
\int\langle v, u\rangle_{E^{\prime}, E} d m \geq\|v\|_{L_{E^{\prime}}^{q}}-3 \varepsilon .
\end{gathered}
$$

Comme $\varepsilon>0$ est arbitraire, ceci permet d'affirmer que $\left\|T_{v}\right\|_{\left(L_{E}^{p}\right)^{\prime}} \geq\|v\|_{L_{E^{\prime}}^{q}}$. Finalement, avec la question $2(b)$, on a bien $\left\|T_{v}\right\|_{\left(L_{E}^{p}\right)^{\prime}}=\|v\|_{L_{E^{\prime}}^{q}}$.

\section{Corrigé 4.3 (Diffusion non homogène et non isotrope)}

On reprend les hypothèses du théorème 4.28 Soit donc $\Omega$ un ouvert borné de $\mathbb{R}^{N}, T>0$ et $u_{0} \in L^{2}(\Omega)$. On identifie $L^{2}(\Omega)$ avec son dual et on suppose que $f \in L^{2}(] 0, T\left[, H^{-1}(\Omega)\right)$.

On se donne aussi une application, notée $A$, de $\Omega$ dans $\mathcal{M}_{N}(\mathbb{R})$ (ensemble des matrices carrés à $N$ lignes et $N$ colonnes, à coefficients dans $\mathbb{R})$. On suppose que les coefficients de $A$ appartiennent à $L^{\infty}(\Omega)$ et qu'il existe $\alpha>0$ t.q.

$$
A \xi \cdot \xi \geq \alpha|\xi|^{2} \text { p.p., pour tout } \xi \in \mathbb{R}^{N} .
$$

En suivant la démonstration donnée dans ce chapitre, montrer qu'il existe un et un seul $u$ tel que

$$
\left\{\begin{array}{l}
u \in L^{2}(] 0, T\left[, H_{0}^{1}(\Omega)\right), \partial_{t} u \in L^{2}(] 0, T\left[, H^{-1}(\Omega)\right), \\
\in t_{0}^{T}<\partial_{t} u(s), v(s)>_{H^{-1}, H_{0}^{1}} d s+\int_{0}^{T}\left(\int_{\Omega} A \nabla u(s) \cdot \nabla v(s) \mathrm{d} x\right) d s= \\
\quad \int_{0}^{T}<f(s), v(s)>_{H^{-1}, H_{0}^{1}} d s \text { pour tout } v \in L^{2}(] 0, T\left[, H_{0}^{1}(\Omega)\right), \\
u(0)=u_{0} \text { p.p. }
\end{array}\right.
$$


Corrigé - Si A est symétrique, le plus simple consiste à utiliser une base hilbertienne de $L^{2}(\Omega)$ formée de fonctions propres de l'opérateur $u \mapsto-\operatorname{div}(A \nabla u)$ (avec condition de Dirichlet, c'est-à-dire $u=0$ sur $\partial \Omega$ ). On prend donc une famille $\left\{e_{n}, n \in \mathbb{N}^{\star}\right\}$ telle que $e_{n}$ est (pour tout $n$ ) une solution faible de

$$
\left\{\begin{array}{l}
-\operatorname{div} A \nabla e_{n}=\lambda_{n} e_{n} \\
e_{n}=0 \quad \text { sur } \partial \Omega
\end{array}\right.
$$

avec $\lambda_{n} \in \mathbb{R}$. (On a vu dans la section 2.2 qu'une telle base existait.) La démontration de l'existence (et de l'unicité) d'une solution de 4.71 est alors très voisine de celle donnée pour le cas où A est la matrice identité.

Le cas où A est non symétrique est plus difficile car on n'a pas nécessairement une base hilbertienne de $L^{2}(\Omega)$ formée de fonctions propres de l'opérateur $u \mapsto-\operatorname{div}(A \nabla u)$. Il faut alors modifier légèrement la démonstration. On va faire cette démonstration ici en prenant la base hilbertienne $\left\{e_{n}, n \in \mathbb{N}^{\star}\right\}$ associée au Laplacien (avec condition de Dirichlet). Pour $n \in \mathbb{N}^{\star}$, la fonction $e_{n}$ est donc solution faible (non nulle) de

$$
\begin{aligned}
& -\Delta e_{n}=\lambda_{n} e_{n} \text { dans } \Omega, \\
& e_{n}=0 \text { sur } \partial \Omega .
\end{aligned}
$$

On rappelle que $\left\|e_{n}\right\|_{L^{2}(\Omega)}=1, \lambda_{n}>0$ et $\lim _{n \rightarrow+\infty} \lambda_{n}=+\infty$. On a aussi $\left\|e_{n}\right\|_{H_{0}^{1}(\Omega)}=\sqrt{\lambda_{n}}$ et la famille $\left\{e_{n} / \sqrt{\lambda_{n}}, n \in \mathbb{N}^{\star}\right\}$ est une base hilbertienne de $H_{0}^{1}(\Omega)$. C'était l'étape 1 de la démonstration du théorème 4.28.

Remarque 4.56 Compte tenu de la manière dont $H_{0}^{1}(\Omega)$ s'injecte dans $H^{-1}(\Omega)$ (par l'identification de $L^{2}(\Omega)^{\prime}$ avec $L^{2}(\Omega)$ ) et de la définition du produit scalaire dans $H^{-1}(\Omega)$ (à partir du produit scalaire dans $H_{0}^{1}(\Omega)$ ), on peut aussi remarquer que

$$
\begin{aligned}
& \left(e_{n} / e_{m}\right)_{H^{-1}}=0 \text { si } n \neq m, \\
& \left(e_{n} / e_{m}\right)_{H^{-1}}=\frac{1}{\lambda_{n}} \text { si } n=m .
\end{aligned}
$$

La famille $\left\{\sqrt{\lambda_{n}} e_{n}, n \in \mathbb{N}^{\star}\right\}$ est donc une famille orthonormée de $H^{-1}(\Omega)$ (c'est même une base hilbertienne de $H^{-1}(\Omega)$ ). Pour montrer 4.72, on rappelle tout d'abord la définition du produit scalaire dans $H^{-1}(\Omega)$. Si $u \in H_{0}^{1}(\Omega)$, on définit $T_{u}$ dans $H^{-1}(\Omega)$ par

$$
\left\langle T_{u}, \varphi\right\rangle_{H^{-1}, H_{0}^{1}}=(u / \varphi)_{H_{0}^{1}}=\int_{\Omega} \nabla u \cdot \nabla \varphi \mathrm{d} x .
$$

L'application $T$ est bijective de $H_{0}^{1}(\Omega)$ dans $H^{-1}(\Omega)$. Pour $u, v \in H_{0}^{1}(\Omega)$, on définit alors le produit scalaire dans $H^{-1}(\Omega)$ par

$$
\left(T_{u} / T_{v}\right)_{H^{-1}(\Omega)}=(u / v)_{H_{0}^{1}(\Omega)}=\int_{\Omega} \nabla u \cdot \nabla v \mathrm{~d} x .
$$

Soit $n \in \mathbb{N}^{\star}$, compte tenu de la manière dont $H_{0}^{1}(\Omega)$ s'injecte dans $H^{-1}(\Omega)$ (par l'identification de $L^{2}(\Omega)^{\prime}$ avec $L^{2}(\Omega)$ ), on a, pour tout $\varphi \in H_{0}^{1}(\Omega)$,

$$
\lambda_{n}\left\langle e_{n}, \varphi\right\rangle_{H^{-1}, H_{0}^{1}}=\lambda_{n} \int_{\Omega} e_{n} \varphi \mathrm{d} x=\int_{\Omega} \nabla e_{n} \cdot \nabla \varphi \mathrm{d} x
$$

ce qui montre que $T_{e_{n}}=\lambda_{n} e_{n}$. On a donc, pour $n, m \in \mathbb{N}^{\star}$,

$$
\lambda_{n} \lambda_{m}\left(e_{n} / e_{m}\right)_{H^{-1}(\Omega)}=\left(T_{e_{n}} / T_{e_{m}}\right)_{H^{-1}(\Omega)}=\left(e_{n} / e_{m}\right)_{H_{0}^{1}(\Omega)}=\int_{\Omega} \nabla e_{n} \cdot \nabla e_{m} \mathrm{~d} x=\lambda_{n} \delta_{n, m}
$$

On en déduit bien 4.72 .

On construit maintenant une solution approchée (étape 2 du théorème 4.28 .

Soit $n \in \mathbb{N}^{\star}$. On pose $E_{n}=e v\left\{e_{p}, p=1, \ldots n\right\}$. On cherche une solution approchée $u_{n}$ sous la forme $u_{n}(t)=$ $\sum_{i=1}^{n} \alpha_{i}(t) e_{i}$ avec $\alpha_{i} \in C([0, T], \mathbb{R})$. Le calcul formel fait dans la démonstration du théorème 4.28 donne ici (en supposant que les $\alpha_{i}$ sont dérivables pour tout $\left.t\right)$, pour tout $\varphi \in H_{0}^{1}(\Omega)$ et presque tout $\left.t \in\right] 0, T$,

$$
\begin{aligned}
& \left\langle u_{n}^{\prime}(t)-\operatorname{div}\left(A \nabla u_{n}(t)\right)-f(t), \varphi\right\rangle_{H^{-1}(\Omega), H_{0}^{1}(\Omega)} \\
& =\sum_{i=1}^{n}\left(\alpha_{i}^{\prime}(t) \int_{\Omega} e_{i} \varphi \mathrm{d} x+\alpha_{i}(t) \int_{\Omega} A \nabla e_{i} \cdot \nabla \varphi \mathrm{d} x\right)-\langle f(t), \varphi\rangle_{H^{-1}(\Omega), H_{0}^{1}(\Omega)} .
\end{aligned}
$$


On souhaite alors choisir les fonctions $\alpha_{i}$ pour que $\left\langle u_{n}^{\prime}(t)-\operatorname{div}\left(A \nabla u_{n}\right)(t)-f(t), \varphi\right\rangle_{H^{-1}(\Omega), H_{0}^{1}(\Omega)}=0$ pour tout $\varphi \in E_{n}$.

On pose $f_{i}(t)=\left\langle f(t), e_{i}\right\rangle_{H^{-1}(\Omega), H_{0}^{1}(\Omega)}$ et on note $F(t)$ le vecteur donc les composantes sont les $f_{i}(t), i=1, \ldots, n$. On définit aussi la matrice $n \times n$ (à coefficients réels) $M$ en posant $M_{i, j}=\int_{\Omega} A \nabla e_{j} \cdot \nabla e_{i} \mathrm{~d} x\left(M_{i, j}\right.$ est le coefficient de $M$ en ligne $i$ et colonne $j$ ). en notant $\alpha(t)$ le vecteur donc les composantes sont les $\alpha_{i}(t), i=1, \ldots, n$, on souhaite donc avoir

$$
\alpha^{\prime}(t)+M \alpha(t)=F(t)
$$

En tenant compte de la condition initiale et en posant $\alpha_{i}^{(0)}=\left(u_{0} / e_{i}\right)_{2}$ et $\alpha^{(0)}$ le vecteur donc les composantes sont les $\alpha_{i}^{(0)}, i=1, \ldots, n$, ceci suggère donc de prendre

$$
\alpha(t)=e^{-M t} \alpha^{(0)}+\int_{0}^{t} e^{-M(t-s)} F(s) d s
$$

Les fonctions $\alpha_{i}$ ainsi définies appartiennent à $C([0, T], \mathbb{R})$ et on a donc $u_{n} \in C\left([0, T], E_{n}\right) \subset C\left([0, T], H_{0}^{1}(\Omega)\right)$ avec $u_{n}(t)=\sum_{i=1}^{n} \alpha_{i}(t) e_{i}$.

Nous avons ici raisonné à n fixé. La matrice $M$ et les fonctions $F$ et $\alpha$ dépendent donc de $n$.

Nous adaptons maintenant l'étape 3 du théorème 4.28 Soit $n \in \mathbb{N}^{\star}$ et $u_{n}$ la solution approchée donnée par l'étape précédente. On va préciser ici ce que vaut la dérivée (par transposition) de $u_{n}$. Cette dérivée est notée $\left(u_{n}\right)_{t}$. Par définition de la dérivation par transposition, $\left(u_{n}\right)_{t}$ est un élément de $\mathcal{D}_{E}^{\star}$ avec $E=H_{0}^{1}(\Omega)$. Soit $\varphi \in C_{c}^{\infty}(] 0, T[, \mathbb{R})$ on $a$

$$
\left\langle\left(u_{n}\right)_{t}, \varphi\right\rangle_{\mathcal{D}_{E}^{\star}, \mathcal{D}}=-\int_{0}^{T} u_{n}(t) \varphi^{\prime}(t) \mathrm{d} t \in E_{n} \subset H_{0}^{1}(\Omega) .
$$

Comme $u_{n}=\sum_{i=1}^{n} \alpha_{i} e_{i}$, on a donc

$$
\left\langle\left(u_{n}\right)_{t}, \varphi\right\rangle_{\mathcal{D}_{E}^{\star}, \mathcal{D}}=-\sum_{i=1}^{n} \int_{0}^{T} \alpha_{i}(t) e_{i} \varphi^{\prime}(t) \mathrm{d} t=-\sum_{i=1}^{n}\left(\int_{0}^{T} \alpha_{i}(t) \varphi^{\prime}(t) \mathrm{d} t\right) e_{i} .
$$

On utilise maintenant 4.73,

$$
\int_{0}^{T} \alpha_{i}(t) \varphi^{\prime} \mathrm{d} t=T_{i}+S_{i}
$$

avec

$$
\begin{gathered}
T_{i}=\int_{0}^{T}\left(e^{-M t} \alpha^{(0)}\right)_{i} \varphi^{\prime}(t) d t=\int_{0}^{T}\left(M e^{-M t} \alpha^{(0)}\right)_{i} \varphi(t) d t \\
S_{i}=\int_{0}^{T}\left(\int_{0}^{t} e^{-M(t-s)} F(s) d s\right)_{i} \varphi^{\prime}(t) \mathrm{d} t
\end{gathered}
$$

Pour transformer $S_{i}$ on utilise le théorème de Fubini et on obtient

$$
S_{i}=\int_{0}^{T}\left(\int_{0}^{t}\left(M e^{-M(t-s)} F(s)\right)_{i} d s\right) \varphi(t) d t-\int_{0}^{T} f_{i}(t) \varphi(t) d t .
$$

On en déduit que $T_{i}+S_{i}=\int_{0}^{T}(M \alpha(t))_{i} \varphi(t) \mathrm{d} t-\int_{0}^{T} f_{i}(t) \varphi(t) \mathrm{d} t$, et donc

$$
\left\langle\left(u_{n}\right)_{t}, \varphi\right\rangle_{\mathcal{D}^{\star}, \mathcal{D}}=-\sum_{i=1}^{n} \int_{0}^{T}(M \alpha(t))_{i} e_{i} \varphi(t) \mathrm{d} t+\sum_{i=1}^{n} \int_{0}^{T} f_{i}(t) e_{i} \varphi(t) d t .
$$

Comme $\varphi$ est arbitraire dans $C_{c}^{\infty}(] 0, T[, \mathbb{R})$, on a donc (p.p. en $\left.t\right)$

$$
\left(u_{n}\right)_{t}=-\sum_{i=1}^{n}(M \alpha)_{i} e_{i}+\sum_{i=1}^{n} f_{i} e_{i} \in L^{2}(] 0, T\left[, E_{n}\right) .
$$

Le premier terme du membre de droite de cette égalité est même continu de $[0, T]$ à valeurs dans $E_{n}$. En reprenant la définition de $M$, cette égalité donne (p.p. en $t$ )

$$
\left(u_{n}\right)_{t}=-\sum_{i=1}^{n}\left(\int_{\Omega} A \nabla u_{n} \cdot \nabla e_{i} \mathrm{~d} x\right) e_{i}+\sum_{i=1}^{n} f_{i} e_{i} .
$$


La situation est légérement différente de celle du théorème 4.28 Mais on a toujours

$$
\left(u_{n}\right)_{t} \in L^{2}(] 0, T\left[, E_{n}\right) \subset L^{2}(] 0, T\left[, H_{0}^{1}(\Omega)\right) \subset L^{2}(] 0, T\left[, H^{-1}(\Omega)\right) .
$$

Compte tenu de la manière dont $H_{0}^{1}(\Omega)$ s'injecte dans $H^{-1}(\Omega)$ (par l'identification de $L^{2}(\Omega)^{\prime}$ avec $L^{2}(\Omega)$ ) on a pour tout $v \in L^{2}(] 0, T\left[, H_{0}^{1}(\Omega)\right)$,

$$
\int_{0}^{T}\left\langle\left(u_{n}\right)_{t}(t), v(t)\right\rangle_{H^{-1}(\Omega), H_{0}^{1}(\Omega)} d t=-\int_{0}^{T} \int_{\Omega} \sum_{i=1}^{n}(M \alpha(t))_{i} e_{i} v \mathrm{~d} x \mathrm{~d} t+\sum_{i=1}^{n} \int_{0}^{T} \int_{\Omega} f_{i} e_{i} v \mathrm{~d} x \mathrm{~d} t .
$$

Ceci est intéressant surtout lorsque $v \in L^{2}(] 0, T\left[, E_{n}\right)$ car en reprenant la définition de $M$ on obtient

$$
\int_{0}^{T}\left\langle\left(u_{n}\right)_{t}(t), v(t)\right\rangle_{H^{-1}(\Omega), H_{0}^{1}(\Omega)} d t=-\int_{0}^{T} \int_{\Omega} A \nabla u_{n} \cdot \nabla v \mathrm{~d} x \mathrm{~d} t+\sum_{i=1}^{n} \int_{0}^{T} \int_{\Omega} f_{i} e_{i} v \mathrm{~d} x \mathrm{~d} t .
$$

Ceci donne, en revenant à la définition de $f_{i}$, toujours pour $v \in L^{2}(] 0, T\left[, E_{n}\right)$,

$$
\begin{aligned}
& \int_{0}^{T}\left\langle\left(u_{n}\right)_{t}(t), v(t)\right\rangle_{H^{-1}(\Omega), H_{0}^{1}(\Omega)} d t+\int_{0}^{T} \int_{\Omega} A \nabla u_{n} \cdot \nabla v \mathrm{~d} x \mathrm{~d} t \\
& =\sum_{i=1}^{n} \int_{0}^{T}\left\langle f(t), e_{i}\right\rangle_{H^{-1}(\Omega), H_{0}^{1}(\Omega)}\left(\int_{\Omega} e_{i} v \mathrm{~d} x\right) \mathrm{d} t=\int_{0}^{T}\langle f(t), v\rangle_{H^{-1}(\Omega), H_{0}^{1}(\Omega)} d t .
\end{aligned}
$$

On rapelle aussi que $u_{n} \in C\left([0, T], H_{0}^{1}(\Omega)\right)$ et $u_{n}(0)=P_{n} u_{0}$ où $P_{n}$ est l'opérateur de projection orthogonale dans $L^{2}(\Omega)$ sur le s.e.v. $E_{n}$.

On cherche maintenant (étape 4) des estimations sur la solution approchée.

Pour $n \in \mathbb{N}^{\star}$, on a

$$
u_{n} \in C\left([0, T], H_{0}^{1}(\Omega)\right) \subset L^{2}(] 0, T\left[, H_{0}^{1}(\Omega)\right) \text { et }\left(u_{n}\right)_{t} \in L^{2}(] 0, T\left[, E_{n}\right) \subset L^{2}(] 0, T\left[, H^{-1}(\Omega)\right) .
$$

D’après la section 4.2 on a donc

En prenant $v=u_{n}$ dans 4.75, on en déduit

$$
\frac{1}{2}\left\|u_{n}(T)\right\|_{2}^{2}-\frac{1}{2}\left\|u_{0}\right\|_{2}^{2}=\int_{0}^{T}\left\langle\left(u_{n}\right)_{t}, u_{n}\right\rangle_{H^{-1}, H_{0}^{1}} \mathrm{~d} t
$$

$$
\frac{1}{2}\left\|u_{n}(T)\right\|_{2}^{2}-\frac{1}{2}\left\|u_{0}\right\|_{2}^{2}+\int_{0}^{T} \int_{\Omega} A \nabla u_{n} \cdot \nabla u_{n} \mathrm{~d} x \mathrm{~d} t=\int_{0}^{T}\left\langle f, u_{n}\right\rangle_{H^{-1}, H_{0}^{1}} d t,
$$

et donc

$$
\alpha\left\|u_{n}\right\|_{L^{2}(] 0, T\left[, H_{0}^{1}(\Omega)\right)}^{2} \leq \int_{0}^{T} \int_{\Omega} A \nabla u_{n} \cdot \nabla u_{n} \mathrm{~d} x \mathrm{~d} t \leq \frac{1}{2}\left\|u_{0}\right\|_{2}^{2}+\int_{0}^{T}\left\langle f, u_{n}\right\rangle_{H^{-1}, H_{0}^{1}} d t .
$$

On en déduit

$$
\begin{aligned}
& \alpha\left\|u_{n}\right\|_{L^{2}(] 0, T\left[, H_{0}^{1}(\Omega)\right)}^{2} \leq \frac{1}{2}\left\|u_{0}\right\|_{2}^{2}+\int_{0}^{T}\left\langle f, u_{n}\right\rangle_{H^{-1}, H_{0}^{1}} d t \\
& \leq \frac{1}{2}\left\|u_{0}\right\|_{2}^{2}+\|f\|_{L^{2}(] 0, T\left[, H^{-1}(\Omega)\right)}\left\|u_{n}\right\|_{L^{2}(] 0, T\left[, H_{0}^{1}(\Omega)\right)} \\
& \leq \frac{1}{2}\left\|u_{0}\right\|_{2}^{2}+\frac{1}{2 \alpha}\|f\|_{L^{2}(] 0, T\left[, H^{-1}(\Omega)\right)}^{2}+\frac{\alpha}{2}\left\|u_{n}\right\|_{L^{2}(] 0, T\left[, H_{0}^{1}(\Omega)\right)}^{2} .
\end{aligned}
$$

On a donc

$$
\alpha\left\|u_{n}\right\|_{L^{2}(] 0, T\left[, H_{0}^{1}(\Omega)\right)}^{2} \leq\left\|u_{0}\right\|_{2}^{2}+\frac{1}{\alpha}\|f\|_{L^{2}(] 0, T\left[, H^{-1}(\Omega)\right)}^{2} .
$$

Ce qui donne une borne sur $u_{n}$ dans $L^{2}(] 0, T\left[, H_{0}^{1}(\Omega)\right)$.

Pour obtenir la borne sur $\left(u_{n}\right)_{t}$, on utilise 4.74. Pour presque tout t on a

$$
\left(u_{n}\right)_{t}(t)=-\sum_{i=1}^{n}\left(\int_{\Omega} A \nabla u_{n}(t) \cdot \nabla e_{i} \mathrm{~d} x\right) e_{i}+\sum_{i=1}^{n} f_{i}(t) e_{i} .
$$


Soit $v \in H_{0}^{1}(\Omega)$, on a $v=\sum_{i=1}^{\infty}\left(v / e_{i}\right)_{L^{2}} e_{i}$ et cette série est convergente dans $L^{2}(\Omega)$ et dans $H_{0}^{1}(\Omega)$. En posant $P_{n}(v)=\sum_{i=1}^{n}\left(v / e_{i}\right)_{L^{2}} e_{i}$ on a $\left\|P_{n} v\right\|_{H_{0}^{1}(\Omega)} \leq\|v\|_{H_{0}^{1}(\Omega)}$. Comme

$$
\begin{gathered}
\left\langle\left(u_{n}\right)_{t}(t), P_{n} v\right\rangle_{H^{-1}, H_{0}^{1}}=\left\langle\left(u_{n}\right)_{t}(t), v\right\rangle_{H^{-1}, H_{0}^{1}}=\int_{\Omega}\left(u_{n}\right)_{t}(t) v \mathrm{~d} x \\
=-\sum_{i=1}^{n}\left(\int_{\Omega} A \nabla u_{n}(t) \cdot \nabla e_{i} \mathrm{~d} x\right)\left(v / e_{i}\right)_{L^{2}}+\sum_{i=1}^{n} f_{i}(t)\left(v / e_{i}\right)_{L^{2}} .
\end{gathered}
$$

on en déduit que

$$
\left\|\left(u_{n}\right)_{t}(t)\right\|_{H^{-1}(\Omega)}=\sup \left\{\left\langle\left(u_{n}\right)_{t}(t), v\right\rangle_{H^{-1}, H_{0}^{1}}, v \in E_{n},\|v\|_{H_{0}^{1}(\Omega)} \leq 1\right\} .
$$

Or, pour $v \in E_{n}$, on obtient avec 4.76

$$
\left\langle\left(u_{n}\right)_{t}(t), v\right\rangle_{H^{-1}, H_{0}^{1}}=\int_{\Omega} A \nabla u_{n}(t) \cdot \nabla v \mathrm{~d} x+\langle f(t), v\rangle_{H^{-1}, H_{0}^{1}} .
$$

En utilisant le fait que les coefficients de A sont dans $L^{\infty}(\Omega)$, on en déduit l'existence de $\beta$, ne dépendant que de A, t.q. pour presque tout $t$,

$$
\left\|\left(u_{n}\right)_{t}(t)\right\|_{H^{-1}(\Omega)} \leq \beta\left\|u_{n}(t)\right\|_{H_{0}^{1}(\Omega)}+\|f(t)\|_{H^{-1}(\Omega)} .
$$

La suite $\left(u_{n}\right)_{n \in \mathbb{N}^{\star}}$ est bornée dans $L^{2}(] 0, T\left[, H_{0}^{1}(\Omega)\right)$, la suite $\left(\left(u_{n}\right)_{t}\right)_{n \in \mathbb{N}^{\star}}$ est donc bornée dans $L^{2}(] 0, T\left[, H^{-1}(\Omega)\right)$.

Il s'agit maintenant (étape 5) de passer à la limite quand $n \rightarrow+\infty$. Grâce aux estimations obtenues à l'étape précédente, on peut supposer, après extraction éventuelle d'une sous suite, que, quand $n \rightarrow+\infty$,

$$
\begin{gathered}
u_{n} \rightarrow u \text { faiblement dans } L^{2}(] 0, T\left[, H_{0}^{1}(\Omega)\right), \\
\left(u_{n}\right)_{t} \rightarrow w \text { faiblement dans } L^{2}(] 0, T\left[, H^{-1}(\Omega)\right) .
\end{gathered}
$$

Comme nous l'avons dans la démonstration du théorème 4.28 on a $w=\partial_{t} u$.

Soit $v \in L^{2}(] 0, T\left[, H_{0}^{1}(\Omega)\right)$. Soit $n \in \mathbb{N}^{\star}$. On pose $v_{n}(t)=P_{n}(v(t))$, de sorte que, par convergence dominée, $v_{n} \rightarrow v$ dans $L^{2}(] 0, T\left[, H_{0}^{1}(\Omega)\right)$. On utilise alors 4.75 avec $v_{n}$ au lieu de $v$. On obtient

$$
\int_{0}^{T}\left\langle\left(u_{n}\right)_{t}, v_{n}\right\rangle_{H^{-1}, H_{0}^{1}} d t+\int_{0}^{T} \int_{\Omega} A \nabla u_{n} \cdot \nabla v_{n} \mathrm{~d} x \mathrm{~d} x \mathrm{~d} t=\int_{0}^{T}\left\langle f, v_{n}\right\rangle_{H^{-1}, H_{0}^{1}} d t
$$

Les trois termes de cette égalité passent à limite quand $n \rightarrow+\infty$ grâce aux convergences de $u_{n}$, $v_{n}$ et $\left(u_{n}\right)_{t}$. On obtient ainsi

$$
\int_{0}^{T}\left\langle\partial_{t} u, v\right\rangle_{H^{-1}, H_{0}^{1}} d t+\int_{0}^{T} A \nabla u \cdot \nabla v \mathrm{~d} x \mathrm{~d} t=\int_{0}^{T}\langle f, v\rangle_{H^{-1}, H_{0}^{1}} d t
$$

Ce qui est bien ce qui était souhaité.

Comme $u \in L^{2}(] 0, T\left[, H_{0}^{1}(\Omega)\right)$ et $\partial_{t} u \in L^{2}(] 0, T\left[, H^{-1}(\Omega)\right)$, on sait que $u \in C\left([0, T], L^{2}(\Omega)\right)$. Pour terminer la démonstration du fait que u est solution de 4.71, il reste donc seulement à montrer que $u(0)=u_{0}$ p.p. (c'est-àdire $u(0)=u_{0}$ dans $L^{2}(\Omega)$ ). La démonstration de ce point est identique à celle donnée dans la démonstration du théorème 4.28 Cela termine la démonstration de l'existence d'une solution à 4.71.

On montre maintenant l'unicité de la solution de 4.71. La démonstration est très proche de celle donnée pour le théorème 4.28. Soit $u_{1}, u_{2}$ deux solutions de 4.71. On pose $u=u_{1}-u_{2}$. En faisant la différence des équations satisfaites par $u_{1}$ et $u_{2}$ et en prenant, pour $t \in[0, T], v=u 1_{0, t}$ comme fonction test, on obtient

$$
\int_{0}^{t}\left\langle\partial_{t} u(s), u(s)\right\rangle_{H^{-1}, H_{0}^{1}} d s+\int_{0}^{t} \int_{\Omega} A \nabla u(s) \cdot \nabla u(s) \mathrm{d} x d s=0 .
$$

Comme $u \in L^{2}(] 0, T\left[, H_{0}^{1}(\Omega)\right)$ et $\partial_{t} u \in L^{2}(] 0, T\left[, H^{-1}(\Omega)\right)$, on a, d'après la section 4.2

On en déduit, pour tout $t \in[0, T]$,

$$
\frac{1}{2}\left(\|u(t)\|_{2}^{2}-\left(\|u(0)\|_{2}^{2}\right)=\int_{0}^{t}\left\langle\partial_{t} u(s), u(s)\right\rangle_{H^{-1}, H_{0}^{1}} d s .\right.
$$

$$
\left(\|u(t)\|_{2}^{2}-\left(\|u(0)\|_{2}^{2}\right)+2 \int_{0}^{t} \int_{\Omega} A \nabla u(s) \cdot \nabla u(s) \mathrm{d} x \mathrm{~d} t=0 .\right.
$$


Enfin, comme $u(0)=0$ et $A \nabla u \cdot \nabla u \geq 0$ p.p., on obtient bien, finalement, $u(t)=0$ p.p. dans $\Omega$, pour tout $t \in[0, T]$. Ce qui termine la démonstration de l'unicité de la solution de 4.71.

\section{Corrigé 4.4 (Existence par le théorème de Schauder)}

Soit $\Omega$ un ouvert borné de $\mathbb{R}^{N}$ et $A: \mathbb{R} \rightarrow M_{N}(\mathbb{R})$ (où $M_{N}(\mathbb{R})$ désigne les matrices $N \times N$ à coefficients réels) t.q.

$$
\begin{array}{r}
\forall s \in \mathbb{R}, A(s)=\left(a_{i, j}(s)\right)_{i, j=1, \ldots, N} \text { où } a_{i, j} \in L^{\infty}(\mathbb{R}) \cap C(\mathbb{R}, \mathbb{R}), \\
\exists \alpha>0 ; A(s) \xi \cdot \xi \geq \alpha|\xi|^{2}, \forall \xi \in \mathbb{R}^{N}, \forall s \in \mathbb{R}, \\
f \in L^{2}(] 0, T\left[, H^{-1}(\Omega)\right) \text { et } u_{0} \in L^{2}(\Omega) .
\end{array}
$$

On identifie $L^{2}(\Omega)$ à $L^{2}(\Omega)^{\prime}$, comme d'habitude. On veut, dans cet exercice, montrer l'existence d'une solution au problème 4.32].

Soit $\bar{u} \in L^{2}(] 0, T\left[, L^{2}(\Omega)\right)$, on définit l'opérateur $T$ de $L^{2}(] 0, T\left[, L^{2}(\Omega)\right)$ dans $L^{2}(] 0, T\left[, L^{2}(\Omega)\right) \operatorname{par} T(\bar{u})=u$ où $u$ est la solution (donnée par l'exercice 4.5 ) du problème

$$
\left\{\begin{array}{l}
u \in L^{2}(] 0, T\left[, H_{0}^{1}(\Omega)\right), \partial_{t} u \in L^{2}(] 0, T\left[, H^{-1}(\Omega)\right), \\
\int_{0}^{T}\left\langle\partial_{t} u, v\right\rangle_{H^{-1}, H_{0}^{1}} \mathrm{~d} t+\int_{0}^{T} \int_{\Omega} A(\bar{u}) \nabla u \cdot \nabla v \mathrm{~d} x \mathrm{~d} t \\
\quad=\int_{0}^{T}\langle f, v\rangle_{H^{-1}, H_{0}^{1}} \mathrm{~d} t, \forall v \in L^{2}(] 0, T\left[, H_{0}^{1}(\Omega)\right), \\
u(\cdot, 0)=u_{0} .
\end{array}\right.
$$

1. Montrer que $T$ est continu de $L^{2}(] 0, T\left[, L^{2}(\Omega)\right)$ dans $L^{2}(] 0, T\left[, L^{2}(\Omega)\right)$.

Corrigé - Soit $\left(\bar{u}_{n}\right)_{n \in \mathbb{N}}$ une suite de $L^{2}(] 0, T\left[, L^{2}(\Omega)\right)$ et $\bar{u} \in L^{2}(] 0, T\left[, L^{2}(\Omega)\right)$ telle que $\bar{u}_{n} \rightarrow \bar{u}$ dans $L^{2}(] 0, T[$, $L^{2}(\Omega)$ ) quand $n \rightarrow+\infty$. On pose $u_{n}=T\left(\bar{u}_{n}\right)$ et $u=T(\bar{u})$. Pour montrer que $u_{n} \rightarrow$ u dans $L^{2}(] 0, T\left[, L^{2}(\Omega)\right)$ quand $n \rightarrow+\infty$, on raisonne par l'absurde. Si $u_{n} \nrightarrow \rightarrow$ u dans $L^{2}(] 0, T\left[, L^{2}(\Omega)\right.$ ), il existe $\varepsilon>0$ et une sous suite, encore notée $\left(u_{n}\right)_{n \in \mathbb{N}}$, t.q.

$$
\left\|u_{n}-u\right\|_{L^{2}(] 0, T\left[, L^{2}(\Omega)\right)} \geq \varepsilon \text { pour tout } n \in \mathbb{N} \text {. }
$$

Pour $n \in \mathbb{N}$, un est la solution de 4.80 avec $\bar{u}_{n}$ au lieu de $\bar{u}$. En prenant $v=u_{n}$ comme fonction test, on en déduit, grâce à 4.78 , que la suite $\left(u_{n}\right)_{n \in \mathbb{N}}$ est bornée dans $L^{2}(] 0, T\left[, H_{0}^{1}(\Omega)\right)$. Puis, comme

$$
\left(u_{n}\right)_{t}=\operatorname{div}\left(A\left(\bar{u}_{n}\right) \nabla u_{n}\right)+f
$$

dans $L^{2}(] 0, T\left[, H^{-1}(\Omega)\right)$, on en déduit que la suite $\left(\left(u_{n}\right)_{t}\right)_{n \in \mathbb{N}}$ est bornée dans $L^{2}(] 0, T\left[, H^{-1}(\Omega)\right)$.

Après extraction éventuelle de sous suite, on peut donc supposer qu'il existe $w \in L^{2}(] 0, T\left[, H_{0}^{1}(\Omega)\right)$ et $\zeta \in L^{2}(] 0, T[$, $H^{-1}(\Omega)$ t.q., quand $n \rightarrow+\infty$,

$$
\begin{aligned}
& u_{n} \rightarrow w \text { faiblement dans } L^{2}(] 0, T\left[, H_{0}^{1}(\Omega)\right), \\
& \left(u_{n}\right)_{t} \rightarrow \zeta \text { faiblement dans } L^{2}(] 0, T\left[, H^{-1}(\Omega)\right) .
\end{aligned}
$$

Par le théorème 4.42 on a aussi $u_{n} \rightarrow u$ dans $L^{2}(] 0, T\left[, L^{2}(\Omega)\right)$.

Comme cela a été montré dans la démonstration du théorème 4.28 on a nécessairement $\zeta=w_{t}$.

On peut également supposer, toujours après extraction de sous suite, que $\bar{u}_{n} \rightarrow \bar{u}$ p.p. de sorte que $A\left(\bar{u}_{n}\right) \rightarrow A(\bar{u})$ p.p. (grâce à la continuité des $\left.a_{i, j}\right)$.

Soit $v \in L^{2}(] 0, T\left[, H_{0}^{1}(\Omega)\right)$, on peut alors passer à la limite quand $n \rightarrow+\infty$ dans 4.80 écrit avec $\bar{u}_{n}$ et $u_{n}$ (au lieu de $\bar{u}$ et $u$ ). On obtient que $w$ est la solution de 4.80, c'est-à-dire que $w=u=T(\bar{u})$. On a donc $u_{n} \rightarrow u$ dans $L^{2}(] 0, T\left[, L^{2}(\Omega)\right)$, en contradiction avec 4.81 .

On a bien ainsi montré la continuité de $T$. 
2. Montrer que $T$ est compact de $L^{2}(] 0, T\left[, L^{2}(\Omega)\right)$ dans $L^{2}(] 0, T\left[, L^{2}(\Omega)\right)$.

Corrigé - Le début du raisonnement de la question précédente montre que $\operatorname{Im}(T)$ est bornée dans $L^{2}(] 0, T\left[, H_{0}^{1}(\Omega)\right)$ et que $\left\{\partial_{t} u, u \in \operatorname{Im}(T)\right\}$ est une partie bornée de $L^{2}(] 0, T\left[, H^{-1}(\Omega)\right)$. Le lemme de compacité 4.34 donne alors que $\operatorname{Im}(T)$ est relativement compacte dans $L^{2}(] 0, T\left[, L^{2}(\Omega)\right)$, ce qui donne la compacité de $T$.

3. Montrer qu'il existe $R>0$ t.q. $\|T(\bar{u})\|_{L^{2}(] 0, T\left[, L^{2}(\Omega)\right)} \leq R$ pour tout $u \in L^{2}(] 0, T\left[, L^{2}(\Omega)\right)$.

Corrigé - Ici encore, ceci découle du raisonnement fait à la première question. En effet, on sait que $\operatorname{Im}(T)$ est bornée dans $L^{2}(] 0, T\left[, H_{0}^{1}(\Omega)\right)$ et donc aussi dans $L^{2}(] 0, T\left[, L^{2}(\Omega)\right)$.

4. Montrer qu'il existe $u$ solution de 4.32 .

Corrigé - On note $B_{R}$ la boule de $L^{2}(] 0, T\left[, L^{2}(\Omega)\right)$ de centre 0 et de rayon $R$, avec $R$ donné à la question précédente. L'opérateur $T$ est continu et compact de $B_{R}$ dans $B_{R}$. Le théorème de Schauder donne alors l'existence de $u \in B_{R}\left(\right.$ et donc $\left.u \in L^{2}(] 0, T\left[, L^{2}(\Omega)\right)\right)$ t.q. $u=T(u)$, c'est-à-dire u solution de 4.32 .

5. On suppose maintenant de plus que $a_{i, j}$ est, pour tout $i, j$, une fonction lipschitzienne. Montrer que 4.32] admet une unique solution.

Corrigé - La démonstration est ici très proche de celle faite pour montrer l'unicité de la solution de 4.33.

Soit $u_{1}, u_{2}$ deux solutions de 4.32. On pose $u=u_{1}-u_{2}$ et on va montrer que $u=0$ p.p..

Pour $\varepsilon>0$ on définit la fonction $T_{\varepsilon}$ de $\mathbb{R}$ dans $\mathbb{R}$ par $T_{\varepsilon}(s)=\max \{-\varepsilon, \min \{s, \varepsilon\}\}$. On note aussi $\phi_{\varepsilon}$ la primitive de $T_{\varepsilon}$ s'annulant en 0 . En prenant $v=T_{\varepsilon}(u)$ dans les formulations faibles satisfaites par $u_{1}$ et $u_{2}$, on obtient

$$
\begin{array}{r}
\int_{0}^{T}\left\langle\partial_{t} u, T_{\varepsilon}(u)\right\rangle_{H^{-1}, H_{0}^{1}} \mathrm{~d} t+\int_{0}^{T} \int_{\Omega} A\left(u_{1}\right) \nabla u \cdot \nabla T_{\varepsilon}(u) \mathrm{d} x \mathrm{~d} t \\
=\int_{0}^{T} \int_{\Omega}\left(A\left(u_{2}\right)-A\left(u_{1}\right)\right) \nabla u_{2} \cdot \nabla T_{\varepsilon}(u) \mathrm{d} x \mathrm{~d} t .
\end{array}
$$

Comme $\nabla T_{\varepsilon}(u)=\nabla u 1_{0<|u|<\varepsilon}$ p.p., on en déduit que

$$
\begin{aligned}
\int_{\Omega} \phi_{\varepsilon}(u(x, T)) \mathrm{d} x- & \int_{\Omega} \phi_{\varepsilon}(u(x, 0)) \mathrm{d} x+\alpha \int_{0}^{T} \int_{\Omega} \nabla u \cdot \nabla u 1_{0<|u|<\varepsilon} d x \mathrm{~d} t \\
& \leq \int_{0}^{T} \int_{\Omega}\left|\left(A\left(u_{1}\right)-A\left(u_{2}\right)\right) \nabla u_{2}\right||\nabla u| 1_{0<|u|<\varepsilon} d x \mathrm{~d} t .
\end{aligned}
$$

Comme les $a_{i, j}$ sont des fonctions lipschitziennes, il existe $L$ t.q., pour tout $i, j \in\{1, \ldots, N\}$ et $s_{1}, s_{2} \in \mathbb{R}$,

$$
\left|a_{i, j}\left(s_{1}\right)-a_{i, j}\left(s_{2}\right)\right| \leq L\left|s_{1}-s_{2}\right|,
$$

On utilise alors le fait que $u_{0}=0$ p.p. et $\phi_{\varepsilon} \geq 0$ pour déduire de 4.82, avec $A_{\varepsilon}=\{0<|u|<\varepsilon\}$ et $y=(x, t)$,

$$
\alpha \int_{0}^{T} \int_{\Omega}\left|\nabla T_{\varepsilon}(u)\right|^{2} \mathrm{~d} x \mathrm{~d} t \leq N^{2} L \varepsilon\left(\int_{A_{\varepsilon}}\left|\nabla u_{2}\right|^{2} \mathrm{~d} y\right)^{\frac{1}{2}}\left(\int_{0}^{T} \int_{\Omega}\left|\nabla T_{\varepsilon}(u)\right|^{2} \mathrm{~d} x \mathrm{~d} t\right)^{\frac{1}{2}} .
$$

On a donc $\alpha\left\|\left|\nabla T_{\varepsilon}(u)\right|\right\|_{L^{2}(Q)} \leq a_{\varepsilon} \varepsilon$, avec $\left.Q=\right] 0, T[\times \Omega$ et

$$
a_{\varepsilon}=N^{2} L\left(\int_{A_{\varepsilon}}\left|\nabla u_{2}\right|^{2} \mathrm{~d} y\right)^{\frac{1}{2}} .
$$

Comme $\cap_{\varepsilon>0} A_{\varepsilon}=\emptyset$ la continuité décroissante d'une mesure donne que la mesure de Lebesgue $(N+1$ dimensionnelle $)$ de $A_{\varepsilon}$ tend vers 0 quand $\varepsilon \rightarrow 0$ et on a donc, comme $\nabla u_{2} \in L^{2}(Q)^{N}$, (noter que $L^{2}(Q)$ peut être identifié à $\left.L^{2}(] 0, T\left[, L^{2}(\Omega)\right)\right)$

$$
\lim _{\varepsilon \rightarrow 0} \int_{A_{\varepsilon}}\left|\nabla u_{2}\right|^{2} \mathrm{~d} y=0
$$

ce qui donne $\lim _{\varepsilon \rightarrow 0} a_{\varepsilon}=0$. Il nous reste maintenant à utiliser, par exemple, l'injection de $W_{0}^{1,1}(\Omega)$ dans $L^{1^{\star}}(\Omega)$. elle donne pour $t \in] 0, T[$,

$$
\left\|T_{\varepsilon}(u(t))\right\|_{L^{1^{\star}}(\Omega)} \leq\left\|\left|\nabla T_{\varepsilon}(u(t))\right|\right\|_{L^{1}(\Omega)} .
$$


On désigne par "mes" le mesure de le Lebesgue dans $\mathbb{R}^{N}$. On remarque maintenant que pour $\left.t \in\right] 0, T[$

On a donc, avec 4.83,

$$
\varepsilon m e s\{|u(t)| \geq \varepsilon\}^{\frac{1}{1^{\star}}} \leq\left(\int_{\Omega}\left|T_{\varepsilon}(u)\right|^{1^{\star}} \mathrm{d} x\right)^{\frac{1}{1^{\star}}} .
$$

$$
\varepsilon m e s\{|u(t)| \geq \varepsilon\}^{\frac{1}{1^{\star}}} \leq\left\|\left|\nabla T_{\varepsilon}(u(t))\right|\right\|_{L^{1}(\Omega)}=\int_{\Omega}\left|\nabla T_{\varepsilon}(u(x, t))\right| \mathrm{d} x,
$$

et, en intégrant par rapport à $t$, sachant que $1 / 1^{\star}=(N-1) / N$ et utilisant l'inégalité de Cauchy-Schwarz,

$$
\begin{array}{r}
\varepsilon \int_{0}^{T} \operatorname{mes}\{|u(t)| \geq \varepsilon\}^{\frac{N-1}{N}} \mathrm{~d} t \leq \int_{0}^{T} \int_{\Omega}\left|\nabla T_{\varepsilon}(u(x, t))\right| \mathrm{d} x \mathrm{~d} t \leq\left\|\left|\nabla T_{\varepsilon}(u)\right|\right\|_{L^{2}(Q)}(\operatorname{Tmes}(\Omega))^{\frac{1}{2}} \\
\leq \frac{(\operatorname{Tmes}(\Omega))^{\frac{1}{2}}}{\alpha} a_{\varepsilon} \varepsilon .
\end{array}
$$

On a donc

$$
\int_{0}^{T} \operatorname{mes}\{|u(t)| \geq \varepsilon\}^{\frac{N-1}{N}} \mathrm{~d} t \leq \frac{(\operatorname{Tmes}(\Omega))^{\frac{1}{2}}}{\alpha} a_{\varepsilon} .
$$

quand $\varepsilon \rightarrow 0$, par convergence dominée, on en déduit (comme $\lim _{\varepsilon \rightarrow 0} a_{\varepsilon}=0$ )

$$
\int_{0}^{T} \operatorname{mes}\{|u(t)|>0\}^{\frac{N-1}{N}} \mathrm{~d} t \leq 0 .
$$

Ce qui donne mes $\{|u(t)|>0\}=0$ p.p. en $t \in] 0, T[$ et donc $u=0$ p.p., ce qui termine cette preuve d'unicité.

\section{Corrigé 4.5 (Existence par schéma numérique)}

On se propose, dans cet exercice, de montrer l'existence d'une solution faible au problème 4.84-(4.86) en passant à la limite sur un solution approchée donnée par un schéma numérique.

$$
\begin{gathered}
\left.\frac{\partial u}{\partial t}(x, t)-\frac{\partial^{2} \varphi(u)}{\partial x^{2}}(x, t)=v(x, t), x \in\right] 0,1[, t \in] 0, T[ \\
\left.\frac{\partial \varphi(u)}{\partial x}(0, t)=\frac{\partial \varphi(u)}{\partial x}(1, t)=0, t \in\right] 0, T[ \\
\left.u(x, 0)=u_{0}(x), x \in\right] 0,1[
\end{gathered}
$$

où $\varphi, v, T, u_{0}$ sont donnés et sont t.q.

1. $T>0, v \in L^{\infty}(] 0,1[\times] 0, T[)$,

2. $\varphi$ croissante, lipschitzienne de $\mathbb{R}$ dans $\mathbb{R}$,

3. $u_{0} \in L^{\infty}(] 0,1[)$ et $\varphi\left(u_{0}\right)$ lipschitzienne de $[0,1]$ dans $\mathbb{R}$.

Un exemple important est donné $\operatorname{par} \varphi(s)=\alpha_{1} s$ si $s \leq 0, \varphi(s)=0$ si $0 \leq s \leq L$ et $\varphi(s)=\alpha_{2}(s-L)$ si $s \geq L$, avec $\alpha_{1}, \alpha_{2}$ et $L$ donnés dans $\mathbb{R}_{+}^{*}$. Noter pour cet exemple que $\varphi^{\prime}=0$ sur $] 0, L[$.

Les ensembles ]0,1[ et ]0,1[×]0,T[ sont munis de leur tribu borélienne et de la mesure de Lebesgue sur cette tribu. On appelle "solution faible" de 4.84, 4.85, 4.86 une solution de

$$
\begin{aligned}
u & \in L^{\infty}(] 0,1[\times] 0, T[), \\
\int_{0}^{T} \int_{0}^{1}\left(u(x, t) \frac{\partial \psi}{\partial t}(x, t)\right. & \left.+\varphi(u(x, t)) \frac{\partial^{2} \psi}{\partial x^{2}}(x, t)+v(x, t) \psi(x, t)\right) \mathrm{d} x \mathrm{~d} t \\
+ & \int_{0}^{1} u_{0}(x) \psi(x, 0) \mathrm{d} x=0, \quad \forall \psi \in C_{T}^{2,1}\left(\mathbb{R}^{2}\right),
\end{aligned}
$$

où $C_{T}^{2,1}\left(\mathbb{R}^{2}\right)=\left\{\psi: \mathbb{R}^{2} \rightarrow \mathbb{R}, 2\right.$ fois continûment dérivable par rapport à $x$ et une fois continûment dérivable par rapport à $t$, t.q. $\frac{\partial \psi}{\partial x}(0, t)=\frac{\partial \psi}{\partial x}(1, t)=0$ pour tout $t \in[0, T]$ et $\psi(x, T)=0$ pour tout $\left.x \in[0,1]\right\}$. 
1. (Question indépendante des questions suivantes.) On suppose, dans cette question seulement, que $\varphi$ est de classe $C^{2}, v$ est continue sur $[0,1] \times[0, T]$ et $u_{0}$ est continue sur $[0,1]$. Soit $w \in C^{2}\left(\mathbb{R}^{2}, \mathbb{R}\right)$ et $u$ la restriction de $w$ à $] 0,1[\times] 0, T[$. Montrer que $u$ est solution de (4.87), (4.88) si et seulement si $u$ vérifie (4.84), (4.85) et (4.86) au sens classique ( c'est-à-dire pour tout $(x, t) \in[0,1] \times[0, T]$ ).

2. (Un résulat de compacité.) Soit $A \subset C\left([0, T], L^{2}(] 0,1[)\right)$. On munit $C\left([0, T], L^{2}(] 0,1[)\right)$ de la norme usuelle, c'est-à-dire

$$
\|u\|=\sup \left\{\|u(t)\|_{\left.\left.L^{2}(] 0,1\right]\right)}, t \in[0, T]\right\} .
$$

On suppose que $A$ vérifie les 2 hypothèses suivantes

(a) Pour tout $t \in[0, T],\{u(t), u \in A\}$ est un borné de $L^{\infty}(] 0,1[)$ et il existe $C$ t.q.

$$
\int_{\mathbb{R}}|u(t)(x+\eta)-u(t)(x)|^{2} \mathrm{~d} x \leq C \eta,
$$

pour tout $\eta \in \mathbb{R}_{+}^{\star}$ et tout $u \in A$, avec $u(t)$ prolongée par 0 hors de $[0,1]$.

(b) Il existe $C \in \mathbb{R}$ t.q. $\|u(t)-u(s)\|_{\left.\left.L^{2}(] 0,1\right]\right)} \leq C|t-s|$, pour tout $t, s \in[0, T]$ et tout $u \in A$.

Montrer que $A$ est relativement compacte dans $C\left([0, T], L^{2}(] 0,1[)\right)$.

3. (Passage à la limite sur une non linéarité, cette question est indépendante de la question précédente.)

Soit $\left(u_{n}\right)_{n \in \mathbb{N}}$ une suite bornée de $L^{\infty}(] 0,1[\times] 0, T[)$. Soient $u \in L^{\infty}(] 0,1[\times] 0, T[)$ et $f \in L^{1}(] 0,1[\times] 0, T[)$.

On suppose que, quand $n \rightarrow \infty$,

(a) $u_{n} \rightarrow u \quad \star$-faiblement dans $L^{\infty}(] 0,1[\times] 0, T[)$,

(b) $\varphi\left(u_{n}\right) \rightarrow f$ dans $L^{1}(] 0,1[\times] 0, T[)$ et p.p..

Montrer que $\varphi(u)=f$ p.p. sur $] 0,1[\times] 0, T[$.

[Indication : Utiliser l'astuce de Minty...]

On cherche maintenant une solution approchée de 4.84, ,4.85, ,4.86).

Soient $N, M \in \mathbb{N}^{\star}$. On pose $h=\frac{1}{N}$ et $k=\frac{T}{M}$. On va construire une solution approchée de 4.84), (4.85), (4.86) à partir de la famille $\left\{u_{i}^{n}, i=1, \ldots, N, n=0, \ldots, M\right\}$ vérifiant les équations suivantes

$$
\begin{gathered}
u_{i}^{0}=\frac{1}{h} \int_{(i-1) h}^{i h} u_{0}(x) \mathrm{d} x, i=1, \ldots, N, \\
\frac{u_{i}^{n+1}-u_{i}^{n}}{k}-\frac{\varphi\left(u_{i-1}^{n+1}\right)-2 \varphi\left(u_{i}^{n+1}\right)+\varphi\left(u_{i+1}^{n+1}\right)}{h^{2}}=v_{i}^{n}, i=1, \ldots, N, n=0, \ldots, M-1,
\end{gathered}
$$

avec $u_{0}^{n+1}=u_{1}^{n+1}, u_{N+1}^{n+1}=u_{N}^{n+1}$, pour tout $n=0, \ldots, M-1$ et $v_{i}^{n}=\frac{1}{k h} \int_{n k}^{(n+1) k} \int_{(i-1) h}^{i h} v(x, t) \mathrm{d} x \mathrm{~d} t$, pour tout $i=1, \ldots, N$, pour tout $n=0, \ldots, M$.

4. (Existence et unicité de la solution approchée.)

Soit $n \in\{0, \ldots, M-1\}$. On suppose connu $\left\{u_{i}^{n}, i=1, \ldots, N\right\}$. 
(a) On suppose que $\varphi$ est bijective de $\mathbb{R}$ dans $\mathbb{R}$. Soit $\left\{u_{i}^{n+1}, i=1, \ldots, N\right.$. On pose $r_{i}=\varphi\left(u_{i}^{n+1}\right)$ pour tout $i=1, \ldots, N\}$. Montrer que $\left\{u_{i}^{n+1}, i=1, \ldots, N\right\}$ est solution de (4.90) si et seulement si $\left(r_{i}\right)_{i=1, \ldots, N}$ est un point fixe de l'application (bien définie...) qui à $w=\left(w_{i}\right)_{i=1, \ldots, N}$ associe $r=\left(r_{i}\right)_{i=1, \ldots, N}$ défini $\left(\right.$ avec $w_{0}=w_{1}$ et $\left.w_{N+1}=w_{N}\right)$ par

$$
\varphi^{-1}\left(r_{i}\right)+\frac{2 k}{h^{2}} r_{i}=\frac{k}{h^{2}}\left(w_{i-1}+w_{i+1}\right)+u_{i}^{n}+k v_{i}^{n}, i=1, \ldots, N .
$$

Montrer que cette application est strictement contractante (de $\mathbb{R}^{N}$ dans $\mathbb{R}^{N}$ ) pour la norme dite "du sup". En déduire qu'il existe une unique famille $\left\{u_{i}^{n+1}, i=1, \ldots, N\right\}$ solution de [4.90].

(b) (plus difficile) On ne suppose plus que $\varphi$ est bijective de $\mathbb{R}$ dans $\mathbb{R}$. Montrer qu'il existe une unique famille $\left\{u_{i}^{n+1}, i=1, \ldots, N\right\}$ solution de 4.90).

Les trois questions suivantes donnent des estimations sur la solution approchée dont on vient de montrer l'existence et l'unicité.

5. (Estimation $L^{\infty}(] 0,1[\times] 0, T[)$ sur la solution approchée.)

On pose $A=\left\|u_{0}\right\|_{L^{\infty}(] 0,1[)}$ et $B=\|v\|_{L^{\infty}(] 0,1[\times] 0, T[)}$.

Montrer, par récurrence sur $n$, que $u_{i}^{n} \in[-A-n k B, A+n k B]$ pour tout $i=1, \ldots, N$ et tout $n=0, \ldots, M$. [On pourra, par exemple, considérer 4.90) avec $i$ t.q. $u_{i}^{n+1}=\min \left\{u_{j}^{n+1}, j=1, \ldots, N\right\}$.]

6. (Estimation de la dérivée p.r. à $x$ de $\varphi(u)$.) Montrer qu'il existe $C_{1}$ (ne dépendant que de $T, \varphi, v$ et $u_{0}$ ) t.q., pour tout $n=0, \ldots, M-1$,

$$
\sum_{i=1}^{N-1}\left(\varphi\left(u_{i+1}^{n+1}\right)-\varphi\left(u_{i}^{n+1}\right)\right)^{2} \leq C_{1} \frac{h}{k}
$$

[indication : multiplier 4.90 par $u_{i}^{n+1}$ et sommer sur $i$.]

7. (Estimation de la dérivée p.r. à $t$ de $\varphi(u)$.) Montrer qu'il existe $C_{2}$ (ne dépendant que de $T, \varphi, v$ et $u_{0}$ ) t.q.

$$
\sum_{n=0}^{M-1} h \sum_{i=0}^{N+1}\left(\varphi\left(u_{i}^{n+1}\right)-\varphi\left(u_{i}^{n}\right)\right)^{2} \leq C_{2} k
$$

[indication : multiplier 4.90 $\operatorname{par} \varphi\left(u_{i}^{n+1}\right)-\varphi\left(u_{i}^{n}\right)$ et sommer sur $i$ et $n$ ]

L'objectif est maintenant de passer à la limite sur les paramètres de discrétisation. Pour $M \in \mathbb{N}^{\star}$ donné, on prend $N=M^{2}$ (et donc $h$ et $k$ sont donnés et $k=T \sqrt{h}$ ), on définit, avec les $u_{i}^{n}$ trouvés dans les questions précédentes, une fonction, $u_{h}$, sur $[0,1] \times[0, T]$ en posant

$$
u_{h}(x, t)=\frac{t-n k}{k} u_{h}^{(n+1)}(x)+\frac{(n+1) k-t}{k} u_{h}^{(n)}(x), \text { si } t \in[n k,(n+1) k]
$$

et

$$
u_{h}^{(n)}(x)=u_{i}^{n}, \operatorname{si} x \in[(i-1) h, i h], i=1, \ldots, N, n=0, \ldots, M
$$


8. Soient $A_{1}=\left\{u_{h}, M \in \mathbb{N}^{\star}\right\}$ et $A_{2}=\left\{\varphi\left(u_{h}\right), M \in \mathbb{N}^{\star}\right\}$.

Montrer que $A_{1}$ est relativement séquentiellement compacte dans $L^{\infty}(] 0,1[\times] 0, T[)$, pour la topologie faible- $\star$, et que $A_{2}$ est relativement (séquentiellement) compacte dans $C\left([0, T], L^{2}(] 0,1[)\right)$.

En déduire que l'on peut trouver une suite $\left(h_{n}\right)_{n \in \mathbb{N}}$ et $u \in L^{\infty}(] 0,1[\times] 0, T[)$ t.q., en posant $u_{n}=u_{h_{n}}$ (on rappelle que $k_{n}=T \sqrt{h}_{n}$ ), on ait, quand $n \rightarrow \infty$,

(a) $h_{n} \rightarrow 0$ et $k_{n} \rightarrow 0$,

(b) $u_{n} \rightarrow u \star$-faiblement dans $L^{\infty}(] 0,1[\times] 0, T[)$,

(c) $\varphi\left(u_{n}\right) \rightarrow \varphi(u)$ dans $L^{p}(] 0,1[\times] 0, T[)$, pour tout $p \in[1, \infty[$.

9. Montrer que la fonction $u$ trouvée à la question précédente est solution de 4.87, ,4.88).

Remarque. On peut aussi montrer l'unicité de la solution de 4.87), 4.88).

Corrigé 4.6 (Théorème de Kolmogorov, avec $B=\mathbb{R}$ ) Le but de cet exercice est de refaire la démonstration du théorème 4.40 dans le cas (plus simple) $B=\mathbb{R}$ et $p=1$.

Soit $T>0$. On note $L^{1}$ l'espace $L_{\mathbb{R}}^{1}(] 0, T[, \mathcal{B}(] 0, T[), \lambda)$. Soit $\left(u_{n}\right)_{n \in \mathbb{N}}$ une suite bornée de $L^{1}$ (on a donc $\left.\sup _{n \in \mathbb{N}}\left\|u_{n}\right\|_{1}<+\infty\right)$.

On suppose que pour tout $h \in] 0, T[$ et tout $n \in \mathbb{N}$ on a

$$
\int_{0}^{T-h}\left|u_{n}(t+h)-u_{n}(t)\right| d t \leq \eta(h),
$$

où $\eta$ est une fonction croissante de $] 0, T\left[\right.$ dans $\mathbb{R}_{+}$t.q. $\lim _{h \rightarrow 0^{+}} \eta(h)=0$.

L'objectif de l'exercice est de démontrer que la suite $\left(u_{n}\right)_{n \in \mathbb{N}}$ est relativement compacte dans $L^{1}$.

1. Soit $\delta, h \in] 0, T[$ t.q. $\delta+h \leq T$. Montrer que

$$
\int_{0}^{\delta}\left|u_{n}(t)\right| \mathrm{d} t \leq \int_{0}^{\delta}\left|u_{n}(t+h)\right| \mathrm{d} t+\int_{0}^{\delta}\left|u_{n}(t+h)-u_{n}(t)\right| \mathrm{d} t
$$

Corrigé - Pour tout $t \in] 0, \delta\left[\right.$ on a $\left|u_{n}(t)\right| \leq\left|u_{n}(t+h)\right|+\left|u_{n}(t+h)-u_{n}(t)\right|$. En intégrant cette inégalité entre 0 et $\delta$, on obtient bien 4.91 .

2. Soit $\left.h_{0} \in\right] 0, T[$ et $\delta \in] 0, T-h_{0}[$, montrer que

$$
h_{0} \int_{0}^{\delta}\left|u_{n}(t)\right| \mathrm{d} t \leq \delta\left\|u_{n}\right\|_{1}+h_{0} \eta\left(h_{0}\right) .
$$

Corrigé - Comme d'habitude, on choisit pour $u_{n}$ l'un de représentants, de sorte que $u_{n} \in \mathcal{L}_{\mathbb{R}}^{1}(] 0, T[, \mathcal{B}(] 0, T[), \lambda)$ (pour tout $n \in \mathbb{N}$ ).

L'inégalité 4.91] est vraie pour tout $h \in] 0, h_{0}\left[\right.$. En intégrant 4.91] entre 0 et $h_{0}$ et en remarquant que $\int_{0}^{\delta} \mid u_{n}(t+$ $h)-u_{n}(t) \mid \mathrm{d} t \leq \eta(h) \leq \eta\left(h_{0}\right)\left(\right.$ car $h \leq h_{0}$ et $\left.\delta \leq T-h_{0} \leq T-h\right)$ on obtient

$$
\begin{array}{r}
h_{0} \int_{0}^{\delta}\left|u_{n}(t)\right| \mathrm{d} t \leq \int_{0}^{h_{0}}\left(\int_{0}^{\delta}\left|u_{n}(t+h)\right| \mathrm{d} t\right) d h+\int_{0}^{h_{0}}\left(\int_{0}^{\delta}\left|u_{n}(t+h)-u_{n}(t)\right| \mathrm{d} t\right) d h \\
\leq \int_{0}^{h_{0}}\left(\int_{0}^{\delta}\left|u_{n}(t+h)\right| \mathrm{d} t\right) d h+h_{0} \eta\left(h_{0}\right) .
\end{array}
$$


La mesure de Lebesgue est $\sigma$-finie et l'application $(t, h) \mapsto u_{n}(t+h)$ est borélienne de $] 0, \delta[\times] 0, h_{0}[$ dans $\mathbb{R}$ (car c'est la composée de $(t, h) \mapsto t+h$ qui est continue donc borélienne et de $s \mapsto u_{n}(s)$ qui est borélienne). On peut donc appliquer le théorème de Fubini-Tonelli pour obtenir que

$$
\int_{0}^{h_{0}}\left(\int_{0}^{\delta}\left|u_{n}(t+h)\right| \mathrm{d} t\right) d h=\int_{0}^{\delta}\left(\int_{0}^{h_{0}}\left|u_{n}(t+h)\right| d h\right) \mathrm{d} t
$$

On en déduit 4.92.

$$
\leq \int_{0}^{\delta}\left(\int_{0}^{T}\left|u_{n}(s)\right| d s\right) \leq \delta\left\|u_{n}\right\|_{1}
$$

3. Montrer que $\int_{0}^{\delta}\left|u_{n}(t)\right| \mathrm{d} t \rightarrow 0$ quand $\delta \rightarrow 0^{+}$, uniformément par rapport à $n$.

Corrigé - Soit $\varepsilon>0$. On choisit d'abord $\left.h_{0} \in\right] 0, T\left[\right.$ t.q. $\eta\left(h_{0}\right) \leq \varepsilon$. Puis, avec $C=\sup _{n \in \mathbb{N}}\left\|u_{n}\right\|_{1}$, on pose $\bar{\delta}=\min \left\{T-h_{0}, \varepsilon h_{0} / C\right\}$. On obtient alors, pour tout $n \in \mathbb{N}$,

$$
0 \leq \delta \leq \bar{\delta} \Rightarrow \int_{0}^{\delta}\left|u_{n}(t)\right| \mathrm{d} t \leq 2 \varepsilon
$$

On a donc $\int_{0}^{\delta}\left|u_{n}(t)\right| \mathrm{d} t \rightarrow 0$ quand $\delta \rightarrow 0^{+}$, uniformément par rapport à $n$.

Une démonstration analogue donne aussi que $\int_{T-\delta}^{T}\left|u_{n}(t)\right| \mathrm{d} t \rightarrow 0$ quand $\delta \rightarrow 0^{+}$, uniformément par rapport à $n$ (il suffit de raisonner avec $v_{n}(t)=u_{n}(T-t)$ ).

4. Montrer que la suite $\left(u_{n}\right)_{n \in \mathbb{N}}$ est relativement compacte dans $L^{1}$.

[Appliquer le théorème de Kolmogorov, théorème 4.40, qui utilise le prolongement de $u_{n}$ par 0.]

Corrigé - On prolonge $u_{n}$ par 0 hors de ]0, T[ (et on note toujours $u_{n}$ la fonction prolongée). Pour appliquer le théorème 4.40, il suffit de montrer que

$$
\int_{\mathbb{R}}\left|u_{n}(t+h)-u_{n}(t)\right| \mathrm{d} t \rightarrow 0 \text { quand } h \rightarrow 0^{+}, \text {uniformément par rapport à } n \in \mathbb{N} .
$$

Pour cela, on remarque que pour $h>0$ et $n \in \mathbb{N}$,

$$
\begin{aligned}
\int_{\mathbb{R}} \mid u_{n}(t+h)- & u_{n}(t) \mid \mathrm{d} t \\
\leq \int_{-h}^{0}\left|u_{n}(t+h)\right| \mathrm{d} t & +\int_{0}^{T-h}\left|u_{n}(t+h)-u_{n}(t)\right| \mathrm{d} t+\int_{T-h}^{T}\left|u_{n}(t)\right| \mathrm{d} t \\
& =\int_{0}^{h}\left|u_{n}(t)\right| \mathrm{d} t+\int_{0}^{T-h}\left|u_{n}(t+h)-u_{n}(t)\right| \mathrm{d} t+\int_{T-h}^{T}\left|u_{n}(t)\right| d t .
\end{aligned}
$$

Soit $\varepsilon>0$. Il existe $h_{1}>0$ t.q. $\eta\left(h_{1}\right) \leq \varepsilon$. Puis, avec la question précédente, il existe $h_{2}>0$ t.q. (pour tout $\left.n \in \mathbb{N}\right)$

$$
0 \leq h \leq h_{2} \Rightarrow \int_{0}^{h}\left|u_{n}(t)\right| \mathrm{d} t \leq \varepsilon \text { et } \int_{T-h}^{T}\left|u_{n}(t)\right| d t \leq \varepsilon .
$$

Avec $h_{3}=\min \left\{h_{1}, h_{2}\right\}$, on a donc (pour tout $n \in \mathbb{N}$ )

Ceci termine la question.

$$
0 \leq h \leq h_{3} \Rightarrow \int_{\mathbb{R}}\left|u_{n}(t+h)-u_{n}(t)\right| \mathrm{d} t \leq 3 \varepsilon .
$$




\section{Chapitre 5}

\section{Problèmes hyperboliques}

Dans ce chapitre, nous allons nous intéresser tout d'abord aux équations hyperboliques scalaires, et nous allons démontrer le théorème d'existence et d'unicité de la solution entropique, dû à Kruzhkov ${ }^{1}$ Nous étudierons le cas unidimensionnel (section 5.1) puis multidimensionnel (section 5.2). Dans la section 5.3 nous donnerons quelques éléments pour l'étude des systèmes hyperboliques.

\subsection{Le cas unidimensionnel}

Les équations de type hyperbolique interviennent principalement en mécanique des fluides (aéronautique, écoulements diphasiques, modélisation de rupture de barrage et d'avalanches). Elles sont souvent obtenues en négligeant les phénomènes de diffusion (parce qu'ils sont faibles à l'échelle considérée) dans les équations de conservation de la mécanique. L'exemple le plus classique d'équation hyperbolique linéaire est l'équation de transport (ou d'advection).

$$
\partial_{t} u-c \partial_{x} u=0, t \in \mathbb{R}_{+}, x \in \mathbb{R},
$$

où $c$ est la vitesse de transport avec condition initiale :

$$
u(x, 0)=u_{0}(x) .
$$

Dans le cas où la condition initiale $u_{0}$ est suffisamment régulière, il est facile de voir que la fonction :

$$
u(x, t)=u_{0}(x+c t),
$$

est solution de (5.1)-(5.2). Si $u_{0}$ est non régulière (par exemple discontinue), nous verrons qu'il y a encore moyen de montrer que la fonction définie par (5.3) est solution en un sens que nous qualifierons de "faible".

Si l'équation est non linéaire, i.e.

$$
\partial_{t} u+\partial_{x}(f(u))=0, t \in \mathbb{R}_{+}, x \in \mathbb{R},
$$

avec par exemple $f(u)=u^{2}$, et condition initiale (5.2), on peut encore définir des solutions faibles, mais leur calcul est plus difficile.

Remarque 5.1 Sur le plan de la simulation numérique, les équations hyperboliques sont discrétisées de manière usuelle par la méthode des volumes finis. Les discrétisations par éléments finis mènent souvent à des schémas instables (en particulier, les solutions discrètes ne vérifient pas toujours certaines propriétés physiques souhaitables).

1. Stanislav Nikolaevich Kruzhkov, 1936-1997, mathématicien russe, spécialiste de l'analyse des EDP non linéaires. 
On se donne $f \in C^{1}(\mathbb{R}, \mathbb{R})$ et $u_{0} \in C^{1}(\mathbb{R})$ et on considère maintenant l'équation hyperbolique non linéaire :

$$
\begin{aligned}
& \partial_{t} u+\partial_{x}(f(u))=0, \quad(x, t) \in \mathbb{R} \times \mathbb{R}_{+}, \\
& u(x, 0)=u_{0}(x) .
\end{aligned}
$$

\subsubsection{Solutions classiques et courbes caractéristiques}

Commençons par donner la définition de solution classique de ce problème même si, comme nous le verrons après, le problème 5.5$]$ n'a pas, en général, de solution classique.

Notations. Soit $Q$ une partie de $\mathbb{R}^{p}(p \geq 1)$ et $k \in \mathbb{N}$. On dit que $u \in C^{k}(\bar{Q})$ si $u$ est la restriction à $Q$ d'une fonction de classe $C^{k}$ sur $\mathbb{R}^{p}$. Ceci est équivalent à la définition usuelle si $k=0$. On utilisera aussi la notation $u \in C_{c}^{k}(Q)$ qui signifie que $u \in C^{k}(\bar{Q})$ et qu'il existe $K \subset Q, K$ compact t.q. $u=0$ sur $K^{c}$. Cette notation sera utilisée par exemple pour $Q=\mathbb{R} \times \mathbb{R}_{+}$ou $Q=\mathbb{R} \times[0, T[$.

Définition 5.2 (Solution classique) On suppose que $u_{0} \in C^{1}(\mathbb{R})$ et $f \in C^{1}(\mathbb{R}, \mathbb{R})$. Alors u est solution classique de 5.5 si $u \in C^{1}\left(\mathbb{R} \times \mathbb{R}_{+}, \mathbb{R}\right)$ et u vérifie

$$
\left\{\begin{array}{l}
\partial_{t} u(x, t)+\partial_{x}(f(u))(x, t)=0, \quad \forall(x, t) \in \mathbb{R} \times \mathbb{R}_{+}^{\star}, \\
u(x, 0)=u_{0}(x), \quad \forall x \in \mathbb{R} .
\end{array}\right.
$$

Avant de donner un résultat de non existence d'une solution classique (proposition 5.5), nous rappelons le résultat classique d'existence et d'unicité locale de solutions pour une équation différentielle non linéaire (théorème de Cauchy-Lipschitz), avec une fonction $a$ localement lipschitzienne de $\mathbb{R} \times \mathbb{R}_{+}$dans $\mathbb{R}$ et $x_{0} \in \mathbb{R}$,

$$
\left\{\begin{array}{l}
x^{\prime}(t)=a(x(t), t), \quad t \in \mathbb{R}_{+}^{\star}, \\
x(0)=x_{0} .
\end{array}\right.
$$

Pour tout $T>0$, le problème 5.6 admet au plus une solution (classique) définie sur $\left[0, T\left[\right.\right.$. Il existe $T_{\max }>0$ (éventuellement égal à $+\infty$ ) et une fonction $x$ continue sur $\left[0, T_{\max }\left[\right.\right.$, de classe $C^{1}$ sur $] 0, T_{\max }[$, solution (classique) de 5.6. De plus, si $T_{\max }<+\infty$ alors $|x(t)| \rightarrow+\infty$ lorsque $t \rightarrow T_{\max }$.

Soit $u$ une solution classique de (5.5). On définit alors

$$
\begin{aligned}
& a \in C\left(\mathbb{R} \times \mathbb{R}_{+}, \mathbb{R}\right) \\
& (x, t) \mapsto a(x, t)=f^{\prime}(u(x, t)) .
\end{aligned}
$$

Il est clair que la fonction $u$ est alors une solution classique du problème suivant :

$$
\left\{\begin{array}{l}
\partial_{t} u(x, t)+a(x, t) \partial_{x} u(x, t)=0, \quad(x, t) \in \mathbb{R} \times \mathbb{R}_{+}^{\star}, \\
u(x, 0)=u_{0}(x) .
\end{array}\right.
$$

Nous donnons maintenant la définition des courbes caractéristiques pour l'équation (5.8), qui permet le lien entre les équations hyperboliques linéaires et les équations différentielles ordinaires.

Définition 5.3 (Courbe caractéristique) On suppose que la fonction a définie par (5.7) est localement lipschitzienne de $\mathbb{R} \times \mathbb{R}_{+}$dans $\mathbb{R}$. Soit $x_{0} \in \mathbb{R}$. On appelle courbe caractéristique du problème 5.8 issue de $x_{0} \in \mathbb{R}$, la courbe définie par le problème de Cauchy suivant :

$$
\left\{\begin{array}{l}
x^{\prime}(t)=a(x(t), t) \\
x(0)=x_{0}
\end{array}\right.
$$


Proposition 5.4 (Solutions classiques et courbes caractéristiques) Soit $f \in C^{2}(\mathbb{R}, \mathbb{R}), u_{0} \in C^{1}(\mathbb{R})$ et $u$ une solution classique de 5 5.5. Alors, pour tout $x_{0} \in \mathbb{R}$ et tout $t \in \mathbb{R}_{+}$, on a $u\left(x_{0}+f^{\prime}\left(u_{0}\left(x_{0}\right)\right) t, t\right)=u_{0}\left(x_{0}\right)$. Autrement dit, pour tout $x_{0} \in \mathbb{R}$, la fonction u est constante sur la droite $t \mapsto x(t)=x_{0}+f^{\prime}\left(u_{0}\left(x_{0}\right)\right) t$. (Cette droite est la courbe caractéristique du problème (5.8) issue de $x_{0} \in \mathbb{R}$ avec a $(x, t)=f^{\prime}(u(x, t))$.)

Démonstration On pose $a(x, t)=f^{\prime}(u(x, t))$. Comme $f \in C^{2}(\mathbb{R}, \mathbb{R})$ et que $u \in C^{1}\left(\mathbb{R} \times \mathbb{R}_{+}, \mathbb{R}\right)$, la fonction $a$ est bien localement lipschitzienne de $\mathbb{R} \times \mathbb{R}_{+}$dans $\mathbb{R}$. Le théorème de Cauchy-Lipschitz s'applique donc pour le problème $(5.8)$. Soit $x_{0} \in \mathbb{R}$, le problème $(5.8)$ admet alors une solution maximale $x(t)$ définie sur $\left[0, T_{\max }[\right.$, et $|x(t)|$ tend vers l'infini lorsque $t$ tend vers $T_{\max }$ si $T_{\max }<+\infty$. Les trois étapes de la démonstration sont les suivantes :

1. Comme $u$ est solution classique, on a $u(x(t), t)=u_{0}\left(x_{0}\right), \forall t \in\left[0, T_{\max }\right.$ [, et donc $u$ (solution de (5.5)) est constante sur la droite caractéristique issue de $x_{0}$. En effet, soit $\varphi$ définie par $\varphi(t)=u(x(t), t)$; en dérivant $\varphi$, on obtient : $\varphi^{\prime}(t)=\partial_{t} u(x(t), t)+\partial_{x} u(x(t), t) x^{\prime}(t)$. Comme la fonction $x$ vérifie [5.9), ceci entrâ̂ne : $\varphi^{\prime}(t)=\partial_{t} u(x(t), t)+f^{\prime}(u(x(t), t)) \partial_{x} u(x(t), t)$, et donc

$$
\varphi^{\prime}(t)=\partial_{t} u(x(t), t)+\partial_{x} u(f(u))(x(t), t)=0 .
$$

La fonction $\varphi$ est donc constante, et on a :

$$
u(x(t), t)=\varphi(t)=\varphi(0)=u(x(0), 0)=u\left(x_{0}, 0\right)=u_{0}\left(x_{0}\right), \forall t \in\left[0, T_{\max }[.\right.
$$

2. Les courbes caractéristiques sont des droites, car $u(x(t), t)=u_{0}\left(x_{0}\right), \forall t \in\left[0, T_{\max }\left[\right.\right.$, et donc $x^{\prime}(t)=$ $f^{\prime}\left(u_{0}\left(x_{0}\right)\right)$. En intégrant, on obtient que le système (5.9) décrit la droite d'équation :

$$
x(t)=f^{\prime}\left(u_{0}\left(x_{0}\right)\right) t+x_{0} .
$$

3. $T_{\max }=+\infty$ et donc $u(x, t)=u_{0}\left(x_{0}\right) \quad \forall t \in[0,+\infty[$.

En effet, puisque $x$ vérifie $(5.10)$, on a donc, si $T_{\max }<\infty$,

$$
\lim _{t \rightarrow T_{\max }}|x(t)|<+\infty \text {. On en déduit que } T_{\max }=+\infty .
$$

Proposition 5.5 (Non existence d'une solution classique) Soit $f \in C^{2}(\mathbb{R}, \mathbb{R})$, on suppose que $f^{\prime} n^{\prime}$ 'est pas constante, alors il existe $u_{0} \in C_{c}^{\infty}(\mathbb{R})$ telle que (5.5) n'admette pas de solution classique.

Démonstration Comme $f^{\prime}$ est non constante, il existe $v_{0}, v_{1}$ tel que $f^{\prime}\left(v_{0}\right)>f^{\prime}\left(v_{1}\right)$, et on peut construire $u_{0} \in$ $C_{c}^{\infty}(\mathbb{R}, \mathbb{R})$ telle que $u_{0}\left(x_{0}\right)=v_{0}$ et $u_{0}\left(x_{1}\right)=v_{1}$, où $x_{0}$ et $x_{1}$ sont donnés et $x_{0}<x_{1}$, voir figure 5.1. Supposons que $u$ soit solution classique avec cette donnée initiale. Alors, par la proposition 5.4:

$$
u\left(x_{0}+f^{\prime}\left(u_{0}\left(x_{0}\right)\right) t, t\right)=u_{0}\left(x_{0}\right)=v_{0} \text { et } u\left(x_{1}+f^{\prime}\left(u_{0}\left(x_{1}\right)\right) t, t\right)=u_{0}\left(x_{1}\right)=v_{1} .
$$

Soit $T$ tel que $x_{0}+f^{\prime}\left(v_{0}\right) T=x_{1}+f^{\prime}\left(v_{1}\right) T=\bar{x}$, c'est à dire

$$
T=\frac{x_{1}-x_{0}}{f^{\prime}\left(v_{0}\right)-f^{\prime}\left(v_{1}\right)} .
$$

On a alors :

$$
u(\bar{x}, T)=u_{0}\left(x_{0}\right)=v_{0}=u_{0}\left(x_{1}\right)=v_{1},
$$

ce qui est impossible. On en conclut que [5.5] n'admet pas de solution classique pour cette donnée initiale. 

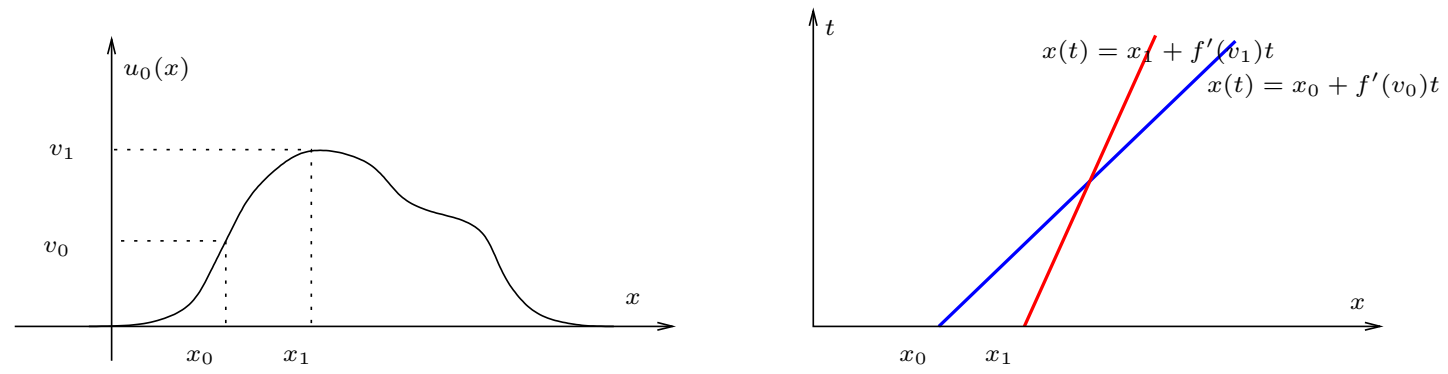

FIGURE 5.1 - Droites caractéristiques, cas non linéaire

\subsubsection{Solutions faibles}

Il n'existe donc pas toujours de solution au sens classique au problème (5.5). On va donc affaiblir le sens de solution, et définir des solutions dites faibles. On donne cette définition dans un cadre légèrement plus g'enéral consistant à supposer que $f$ est localement lipschitzienne (au lieu d'être de classe $C^{1}$ ). On note $\operatorname{Lip}_{\text {loc }}(\mathbb{R}, \mathbb{R})$ l'ensemble des fonctions localement lipschitziennes de $\mathbb{R}$ dans $\mathbb{R}$. On rappelle que si $f \in \operatorname{Lip}_{\text {loc }}(\mathbb{R}, \mathbb{R})$, la fonction $f$ est alors derivable p.p., sa dérivée est localement bornée et on a $f(c)-f(d)=\int_{c}^{d} f^{\prime}(t) d t$ pour tout $c, d \in \mathbb{R}$.

Définition 5.6 (Solution faible) Soit $u_{0} \in L^{\infty}(\mathbb{R})$ et $f \in \operatorname{Lip}_{\text {loc }}(\mathbb{R}, \mathbb{R})$, On appelle solution faible de 5.5 une fonction $u \in L^{\infty}\left(\mathbb{R} \times \mathbb{R}_{+}\right)$telle que

$$
\iint_{\mathbb{R} \times \mathbb{R}_{+}}\left[u(x, t) \varphi_{t}(x, t)+f(u(x, t)) \varphi_{x}(x, t)\right] d x \mathrm{~d} t+\int_{\mathbb{R}^{\prime}} u_{0}(x) \varphi(x, 0) \mathrm{d} x=0, \forall \varphi \in C_{c}^{1}\left(\mathbb{R} \times \mathbb{R}_{+}, \mathbb{R}\right) .
$$

Donnons maintenant les liens entre solution classique et solution faible.

Proposition 5.7 Soient $f \in C^{1}(\mathbb{R}, \mathbb{R})$ et $u_{0} \in L^{\infty}(\mathbb{R})$.

1. Si u est solution classique de (5.5) (et donc $u_{0} \in C^{1}(\mathbb{R}, \mathbb{R})$ ) alors u est solution faible de (5.5).

2. Si $u \in C^{1}\left(\mathbb{R} \times \mathbb{R}_{+}\right)$est solution faible de 5.5 ) alors $u_{0} \in C^{1}(\mathbb{R}, \mathbb{R}$ ) (au sens où la classe de fonctions $u_{0}$ admet un représentant de classe $C^{1}$ et est alors identifiée à ce représentant) et u est solution classique de (5.5).

3. Soit $\sigma \in \mathbb{R}, D_{1}=\left\{(x, t) \in \mathbb{R} \times \mathbb{R}_{+}^{\star} ; x<\sigma t\right\}$ et $D_{2}=\left\{(x, t) \in \mathbb{R} \times \mathbb{R}_{+}^{\star} ; x>\sigma t\right\}$.

(a) On suppose que $u \in C\left(\mathbb{R} \times \mathbb{R}_{+}^{\star}\right)$, que $u_{\mid D_{i}} \in C^{1}\left(\bar{D}_{i}, \mathbb{R}\right), i=1,2$, que l'équation (5.5a) est vérifiée pour tout $(x, t) \in D_{i}(i=1,2)$ et que la condition initiale $5.5 \mathrm{~b}$ ) est satisfaite p.p.. Alors u est solution faible de 5.5.

(b) Plus généralement, on suppose que $u_{\mid D_{i}} \in C^{1}\left(\bar{D}_{i}, \mathbb{R}\right)(i=1,2)$, que l'équation (5.5a) est vérifiée pour tout $(x, t) \in D_{i}(i=1,2)$ et que la condition initiale $5.5 \mathrm{~b}$ est satisfaite p.p.. Pour $t \in \mathbb{R}_{+}$, on pose

$$
\begin{gathered}
u_{+}(\sigma t, t)=\lim _{x \downarrow \sigma t} u(x, t) \text { et } u_{-}(\sigma t, t)=\lim _{x \uparrow \sigma t} u(x, t), \\
{[u](\sigma t, t)=u_{+}(\sigma t, t)-u_{-}(\sigma t, t),} \\
{[f(u)](\sigma t, t)=f\left(u_{+}(\sigma t, t)\right)-f\left(u_{-}(\sigma t, t)\right) .}
\end{gathered}
$$


Alors u est solution faible de [5.5] si et seulement si

$$
\sigma[u](\sigma t, t)=[f(u)](\sigma t, t) \text { pour tout } t \in \mathbb{R}_{+} .
$$

Cette condition s'appelle relation de Rankine ${ }^{2}-$ Hugoniot $t_{3}^{3}$

\section{Démonstration}

1. Supposons que $u$ est solution classique de $(5.5)$, c'est-à-dire de :

$$
\left\{\begin{array}{l}
\partial_{t} u+\partial_{x}(f(u))=0, \quad(x, t) \in \mathbb{R} \times \mathbb{R}_{+}^{\star}, \\
u(x, 0)=u_{0}(x), \quad x \in \mathbb{R} .
\end{array}\right.
$$

Soit $\varphi \in C_{c}^{1}\left(\mathbb{R} \times \mathbb{R}_{+}, \mathbb{R}\right)$. Multiplions (5.5) par $\varphi$ et intégrons sur $\mathbb{R} \times \mathbb{R}_{+}$. On obtient :

$$
\int_{\mathbb{R}} \int_{\mathbb{R}_{+}} \partial_{t} u(x, t) \varphi(x, t) \mathrm{d} t \mathrm{~d} x+\int_{\mathbb{R}} \int_{\mathbb{R}_{+}} \partial_{x}(f(u))(x, t) \varphi(x, t) \mathrm{d} t \mathrm{~d} x=0 .
$$

L'application du théorème de Fubini et une intégration par parties donnent alors :

$$
-\int_{\mathbb{R}} u(x, 0) \varphi(x, 0) \mathrm{d} x-\int_{\mathbb{R}} \int_{\mathbb{R}_{+}} u(x, t) \varphi_{t}(x, t) d t \mathrm{~d} x-\int_{\mathbb{R}_{+}} \int_{\mathbb{R}} f(u)(x, t) \varphi_{x}(x, t) \mathrm{d} x \mathrm{~d} t=0,
$$

(car le support de $\varphi$ est un compact de $\mathbb{R} \times \mathbb{R}_{+}$). On obtient donc bien la relation (5.11), grâce à la condition initiale $u(x, 0)=u_{0}(x)$.

2. Soit $u$ une solution faible de [5.5], qui vérifie de plus $u \in C^{1}(\mathbb{R} \times[0,+\infty[)$. On a donc suffisamment de régularité pour intégrer par parties dans 5.11 .

Commençons par prendre $\varphi$ à support compact dans $\mathbb{R} \times] 0,+\infty[$. On a donc $\varphi(x, 0)=0$, et une intégration par parties dans (5.11) donne :

$$
-\int_{\mathbb{R}} \int_{\mathbb{R}_{+}} \partial_{t} u(x, t) \varphi(x, t) \mathrm{d} t \mathrm{~d} x-\int_{\mathbb{R}_{+}} \int_{\mathbb{R}} \partial_{x}(f(u))(x, t) \varphi(x, t) \mathrm{d} x \mathrm{~d} t=0 .
$$

On a donc :

$$
\int_{\mathbb{R}^{R}} \int_{\mathbb{R}_{+}}\left(\partial_{t} u(x, t)+\partial_{x}(f(u))(x, t)\right) \varphi(x, t) \mathrm{d} t \mathrm{~d} x=0, \forall \varphi \in C_{c}^{1}(\mathbb{R} \times] 0,+\infty[) .
$$

Comme $\partial_{t} u+\partial_{x}(f(u))$ est continue sur $\mathbb{R} \times \mathbb{R}_{+}^{\star}$, on en déduit que $\partial_{t} u+\partial_{x}(f(u))=0$. En effet, on rappelle que si $h \in L_{\text {loc }}^{1}(\mathbb{R} \times] 0,+\infty[)$ et $\left.\int_{\mathbb{R}} \int_{\mathbb{R}_{+}} h(x, t)\right) \varphi(x, t) \mathrm{d} t \mathrm{~d} x=0$ pour toute fonction $\varphi$ appartenant à $C_{c}^{1}(\mathbb{R} \times] 0,+\infty[)$, alors $f=0$ p.p.; si de plus $h$ est continue sur $\left.\mathbb{R} \times\right] 0,+\infty[$, alors $f=0$ partout sur $\mathbb{R} \times] 0,+\infty[$.

On prend alors $\varphi \in C_{c}^{1}\left(\mathbb{R} \times \mathbb{R}_{+}\right)$. Dans ce cas, une intégration par parties dans (5.11) donne

$$
\int_{\mathbb{R}} u(x, 0) \varphi(x, 0) \mathrm{d} x-\int_{\mathbb{R}} \int_{\mathbb{R}_{+}}\left(\partial_{t} u(x, t)+\partial_{x}(f(u))(x, t)\right) \varphi(x, t) \mathrm{d} t \mathrm{~d} x-\int_{\mathbb{R}} u_{0}(x) \varphi(x, 0) \mathrm{d} x=0 .
$$

2. William John Macquorn Rankine (1820-1872), ingénieur écossais qui contribua aussi en physique et mathématiques.

3. Pierre-Henri Hugoniot (1851-1887), inventeur, mathématicien, and physicien français spécialiste de mécanique des fluide, en particulier des chocs. 
Mais on vient de montrer que $\partial_{t} u+\partial_{x}(f(u))=0$. On en déduit que

$$
\int_{\mathbb{R}}\left(u_{0}(x)-u(x, 0)\right) \varphi(x, 0) \mathrm{d} x=0, \forall \varphi \in C_{c}^{1}\left(\mathbb{R} \times R_{+}\right) .
$$

Ceci donne $u_{0}=u(\cdot, 0)$ p.p.. Comme $u$ est continue, on a donc $u_{0}$ continue (au sens où on identifie $u_{0}$ et $u(\cdot, 0))$ et $u$ est solution classique de 5.5 .

3. On montre directement l'item (b) (qui contient l'item (a)). On suppose que $u_{\mid D_{i}} \in C^{1}\left(\bar{D}_{i}, \mathbb{R}\right)(i=1,2)$, que la première équation de 5.5 ) est vérifiée pour tout $(x, t) \in D_{i}(i=1,2)$ et que la condition initiale (de (5.5) est satisfaite p.p. sur $\mathbb{R}$. Nous allons montrer que $u$ est solution faible de (5.5) si et seulement si (5.12) est vérifiée. Pour cela, on pose

$$
X=\int_{\mathbb{R}^{\mathbb{R}}} \int_{\mathbb{R}_{+}} u(x, t) \varphi_{t}(x, t) \mathrm{d} t \mathrm{~d} x+\int_{\mathbb{R}_{+}} \int_{\mathbb{R}^{R}} f(u)(x, t) \varphi_{x}(x, t) \mathrm{d} x \mathrm{~d} t .
$$

On a donc $X=X_{1}+X_{2}$, avec

$$
X_{1}=\int_{\mathbb{R}} \int_{\mathbb{R}_{+}} u(x, t) \varphi_{t}(x, t) \mathrm{d} t \mathrm{~d} x \text { et } X_{2}=\int_{\mathbb{R}_{+}} \int_{\mathbb{R}}(f(u))(x, t) \varphi_{x}(x, t) \mathrm{d} x \mathrm{~d} t .
$$

Calculons $X_{1}$. Comme $u$ n'est de classe $C^{1}$ que sur chacun des domaines $D_{i}$, on n'a pas le droit d'intégrer par parties sur $\mathbb{R} \times \mathbb{R}_{+}$entier. On va donc décomposer l'intégrale sur $D_{1}$ et $D_{2}$; supposons par exemple $\sigma<0$, voir figure 5.2 (Le cas $\sigma>0$ se traite de façon similaire et le cas $\sigma=0$ est plutôt plus simple). On a alors $D_{1}=\left\{(x, t) ; x \in \mathbb{R}_{-}\right.$et $\left.0<t<\frac{x}{\sigma}\right\}$ et $D_{2}=\mathbb{R}_{+} \times \mathbb{R}_{+} \cup\left\{(x, t) ; x \in \mathbb{R}_{-}\right.$et $\left.\frac{x}{\sigma}<t<+\infty\right\}$.

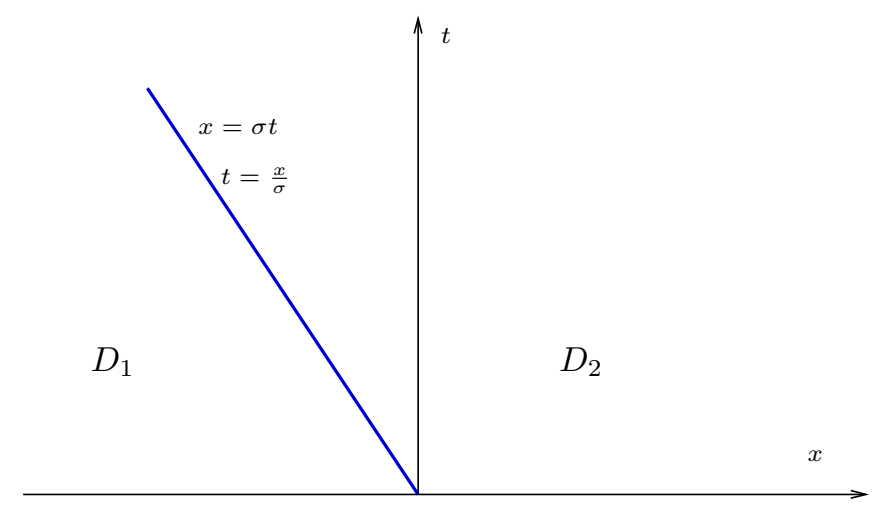

FIGURE $5.2-$ Les domaines $D_{1}$ et $D_{2}$

On a donc :

$X_{1}=\int_{\mathbb{R}_{-}} \int_{0}^{x / \sigma} u(x, t) \varphi_{t}(x, t) \mathrm{d} t \mathrm{~d} x+\int_{\mathbb{R}_{-}} \int_{\frac{x}{\sigma}}^{+\infty} u(x, t) \varphi_{t}(x, t) \mathrm{d} t \mathrm{~d} x+\int_{\mathbb{R}_{+}} \int_{\mathbb{R}_{+}} u(x, t) \varphi_{t}(x, t) \mathrm{d} t \mathrm{~d} x$. 
Comme $u$ est de classe $C^{1}$ sur chacun des domaines, on peut intégrer par parties, ce qui donne :

$$
\begin{array}{r}
X_{1}=\int_{\mathbb{R}_{-}} u_{-}\left(x, \frac{x}{\sigma}\right) \varphi\left(x, \frac{x}{\sigma}\right) \mathrm{d} x-\int_{\mathbb{R}_{-}} u(x, 0) \varphi(x, 0) \mathrm{d} x-\int_{\mathbb{R}_{-}} \int_{0}^{\frac{x}{\sigma}} \partial_{t} u(x, t) \varphi(x, t) \mathrm{d} t \mathrm{~d} x \\
-\int_{\mathbb{R}_{-}} u_{+}\left(x, \frac{x}{\sigma}\right) \varphi\left(x, \frac{x}{\sigma}\right) \mathrm{d} x-\int_{\mathbb{R}_{-}} \int_{\frac{x}{\sigma}}^{+\infty} \partial_{t} u(x, t) \varphi(x, t) \mathrm{d} t \mathrm{~d} x \\
\quad-\int_{\mathbb{R}_{+}} u(x, 0) \varphi(x, 0) \mathrm{d} x-\int_{\mathbb{R}_{+}} \int_{\mathbb{R}_{+}} \partial_{t} u(x, t) \varphi(x, t) \mathrm{d} t \mathrm{~d} x .
\end{array}
$$

En regroupant, il vient :

$$
\begin{array}{r}
X_{1}=-\int_{\mathbb{R}} u(x, 0) \varphi(x, 0) \mathrm{d} x-\iint_{D_{1}} \partial_{t} u(x, t) \varphi(x, t) \mathrm{d} t \mathrm{~d} x- \\
-\iint_{D_{2}} \partial_{t} u(x, t) \varphi(x, t) \mathrm{d} t \mathrm{~d} x \\
-\int_{\mathbb{R}_{-}}[u]\left(x, \frac{x}{\sigma}\right) \varphi\left(x, \frac{x}{\sigma}\right) \mathrm{d} x .
\end{array}
$$

Dans la dernière intégrale, on effectue le changement de varaible $t=x / \sigma$. On obtient

$$
\begin{aligned}
& X_{1}=-\int_{\mathbb{R}} u(x, 0) \varphi(x, 0) \mathrm{d} x-\iint_{D_{1}} \partial_{t} u(x, t) \varphi(x, t) \mathrm{d} t \mathrm{~d} x-\iint_{D_{2}} \partial_{t} u(x, t) \varphi(x, t) \mathrm{d} t \mathrm{~d} x \\
& +\sigma \int_{\mathbb{R}_{+}}[u](\sigma t, t) \varphi(\sigma t, t) \mathrm{d} t .
\end{aligned}
$$

On décompose de même $X_{2}$ sur $D_{1} \cup D_{2}$, en remarquant maintenant que $D_{1}=\left\{(x, t) \in \mathbb{R} \times \mathbb{R}_{+}^{\star} ; x<\sigma t\right\}$ et $D_{2}=\left\{(x, t) \in \mathbb{R} \times \mathbb{R}_{+}^{\star} ; x>\sigma t\right\}$ :

$$
X_{2}=\int_{\mathbb{R}_{+}} \int_{-\infty}^{\sigma t} f(u)(x, t) \varphi_{x}(x, t) \mathrm{d} x \mathrm{~d} t+\int_{\mathbb{R}_{+}} \int_{\sigma t}^{+\infty} f(u)(x, t) \varphi_{x}(x, t) \mathrm{d} x \mathrm{~d} t .
$$

La fonction $u$ est de classe $C^{1}$ sur chacun des domaines, on peut là encore intégrer par parties. Comme $\varphi$ est à support compact sur $\mathbb{R} \times \mathbb{R}_{+}$, on obtient :

$$
\begin{array}{r}
X_{2}=-\iint_{D_{1}}(f(u))_{x}(x, t) \varphi(x, t) \mathrm{d} x \mathrm{~d} t-\iint_{D_{2}}(f(u))_{x}(x, t) \varphi(x, t) \mathrm{d} x \mathrm{~d} t \\
-\int_{\mathbb{R}_{+}}[f(u)](\sigma t, t) \varphi(\sigma t, t) \mathrm{d} t .
\end{array}
$$

Comme $\partial_{t} u+\partial_{x}(f(u))=0$ sur $D_{1}$ et $D_{2}$, on a donc :

$$
X=X_{1}+X_{2}=-\int_{\mathbb{R}^{2}} u(x, 0) \varphi(x, 0) \mathrm{d} x+\int_{\mathbb{R}_{+}}(\sigma[u](\sigma t, t)-[f(u)](\sigma t, t)) \varphi(\sigma t, t) \mathrm{d} t .
$$

On en déduit bien que $u$ est solution faible de (5.5) si et seulement si (5.12) est vérifiée.

Notons qu'il existe souvent plusieurs solutions faibles. On a donc besoin d'une notion supplémentaire pour les distinguer. C'est la notion de solution entropique, qui nous permettra d'obtenir l'unicité. Donnons tout d'abord un 
exemple de non-unicité de la solution faible. Pour cela on va considérer une équation modèle, appelée équation de Burgers 4 qui s'écrit

$$
\partial_{t} u+\partial_{x}\left(u^{2}\right)=0
$$

et des données initiales particulières, sous la forme

$$
u_{0}(x)=\left\{\begin{array}{l}
u_{g} \text { si } x<0 \\
u_{d} \text { si } x>0
\end{array}\right.
$$

avec $u_{g}, u_{d} \in \mathbb{R}$. Ces données initiales définissent un problème de Cauchy particulier, qu'on appelle problème de Riemann 5

Nous considérons maintenant l'exemple simple obtenu avec $u_{g}=-1$ et $u_{d}=1$. Le problème considéré est donc le problème suivant, avec $f(u)=u^{2}, u_{g}=-1, u_{d}=1$ :

$$
\left\{\begin{array}{l}
\partial_{t} u+\partial_{x}(f(u))=0, \\
u_{0}(x)=\left\{\begin{array}{l}
u_{g} \text { si } x<0, \\
u_{d} \text { si } x>0 .
\end{array}\right.
\end{array}\right.
$$

On cherche tout d'abord une solution faible de la forme :

$$
u(x, t)=\left\{\begin{array}{l}
u_{g} \text { si } x<\sigma t \\
u_{d} \text { si } x>\sigma t .
\end{array}\right.
$$

Cette éventuelle solution est discontinue au travers de la droite d'équation $x=\sigma t$ dans le plan $(x, t)$. On remplace $u(x, t)$ par ces valeurs dans (5.11). D'après la proposition 5.7 on sait que $u$ est solution faible si la condition suivante (condition de Rankine et Hugoniot) est vérifiée :

$$
\sigma\left(u_{d}-u_{g}\right)=\left(f\left(u_{d}\right)-f\left(u_{g}\right)\right)
$$

ce qui avec la condition initiale particulière choisie ici, donne $2 \sigma=1^{2}-(-1)^{2}=0$.

Mais on peut trouver d'autres solutions faibles. Si $u$ est solution régulière, on sait que sur les courbes caractéristiques, qui ont pour équation $x(t)=x_{0}+f^{\prime}\left(u_{0}\left(x_{0}\right)\right) t$, la fonction $u$ est constante. Comme $f^{\prime}(u)=2 u$, les courbes caractéristiques sont donc des droites de pente -2 si $x_{0}<0$, et de pente 2 si $x_{0}>0$. Construisons ces caractéristiques sur la figure 5.3: Dans la zone du milieu, où l'on a représenté un point d'interrogation, on cherche $u$ sous la forme $u(x, t)=\varphi\left(\frac{x}{t}\right)$ et telle que $u$ soit continue sur $\mathbb{R} \times \mathbb{R}_{+}^{\star}$. La fonction $u$ suivante convient :

$$
u(x, t)=\left\{\begin{array}{l}
-1 \text { si } x<-2 t, \\
\frac{x}{2 t} \text { si }-2 t<x<2 t \\
1 \text { si } x>2 t .
\end{array}\right.
$$

\subsubsection{Solution entropique}

On vient de voir qu'il peut y avoir non unicité des solutions faibles. Comment choisir la "bonne" solution faible, entre (5.16) et 5.18) ? Comme les problèmes hyperboliques sont souvent obtenus en négligeant les termes de

4. Johannes Martinus Burgers (1895-1981) physicien néerlandais.

5. Georg Friedrich Bernhard Riemann (1826-1866), mathématicien allemand qui a apporté de nombreuses contributions importantes en particulier en topologie, analyse, et géométrie différentielle 

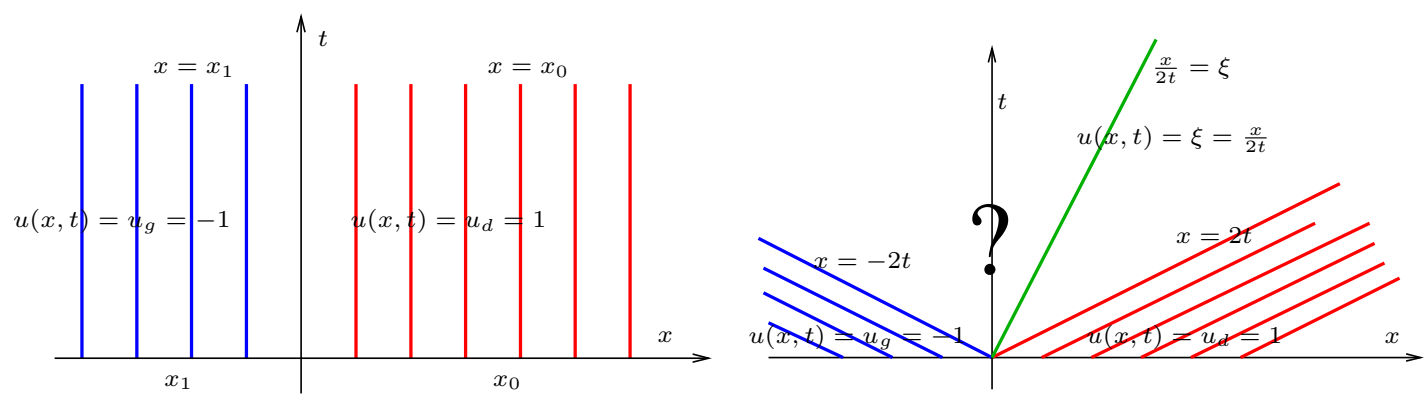

FIGURE 5.3 - Problème de Riemann pour l'équation de Burgers

diffusion dans des équations paraboliques, une technique pour choisir la solution est de chercher la limite du problème de diffusion associé qui s'écrit :

$$
\partial_{t} u+\partial_{x}(f(u))-\varepsilon \partial_{x x} u=0,
$$

lorsque le terme de diffusion devient négligeable, c'est-à-dire lorsque $\varepsilon$ tend vers 0 . Soit $u_{\varepsilon}$ la solution de (5.19) avec la condition initiale $u_{\varepsilon}(\cdot, 0)=u_{0}(\cdot)$ (on admet pour l'instant l'existence et l'unicité de $u_{\varepsilon}$ ). On peut montrer que $u_{\varepsilon}$ tend vers $u$ (en un sens convenable) lorsque $\varepsilon$ tend vers 0 , où $u$ est la "solution faible entropique" de (5.19), définie comme suit.

Définition 5.8 (Solution entropique) Soit $u_{0} \in L^{\infty}(\mathbb{R})$ et $f \in \operatorname{Lip}_{\text {loc }}(\mathbb{R}, \mathbb{R})$. Soit $u \in L^{\infty}\left(\mathbb{R} \times \mathbb{R}_{+}\right)$. On dit que u est solution faible entropique de (5.5) si pour toute fonction $\eta$ de $\mathbb{R}$ dans $\mathbb{R}$, convexe, appelée "entropie", et pour $\phi$ définie par $\phi(s)=\int_{0}^{s} f^{\prime}(\tau) \eta^{\prime}(\tau) d \tau$ (pour $s \in \mathbb{R}$ ), appelé "flux d'entropie", on a :

$$
\int_{\mathbb{R}_{+}} \int_{\mathbb{R}}\left(\eta(u) \partial_{t} \varphi+\phi(u) \partial_{x} \varphi\right)(x, t) \mathrm{d} x \mathrm{~d} t+\int_{\mathbb{R}} \eta\left(u_{0}(x)\right) \varphi(x, 0) \mathrm{d} x \geq 0, \forall \varphi \in C_{c}^{1}\left(\mathbb{R} \times \mathbb{R}_{+}, \mathbb{R}_{+}\right) .
$$

On rappelle que si la fonction $\eta$ (de $\mathbb{R}$ dans $\mathbb{R}$ ) est convexe, elle est localement lipschitzienne (ce qui permet de remarquer que $\phi$ est bien définie). Il est intéressant aussi de remarquer que dans la définition 5.8 on peut se limiter à des fonctions $\eta$ de classe $C^{2}$ (il suffit de régulariser $\eta$ avec une famille de noyaux régularisants pour s'en convaincre). Si $f$ et $\eta$ sont des fonctions de classe $C^{1}$, la fonction $\phi$ est simplement une fonction de classe $C^{1}$ telle que $\phi^{\prime}=\eta^{\prime} f^{\prime}$. Enfin, bien sûr, si $u$ est solution faible entropique alors $u$ est solution faible (proposition 5.11).

Remarque 5.9 (Condition initiale) Noter que dans la définition 5.8, on prend une fois de plus $\varphi \in C_{c}^{1}(\mathbb{R} \times$ $\mathbb{R}_{+}, \mathbb{R}_{+}$) de manière à bien prendre en compte la condition initiale, formulation introduite dans [14]; ceci n'est pas toujours fait de cette manière dans les travaux plus anciens sur le sujet, où la condition initiale était assurée par la condition supplémentaire, $u(t) \rightarrow u_{0}$ dans $L_{\text {loc }}^{1}$ quand $t \rightarrow 0$ (voir par exemple [10, Definition 5.11]). Si la condition initiale est prise en compte seulement dans la définition de solution faible (et n'est pas reprise dans la condition d'entropie), le choix de l'espace fonctionnel dans lequel on recherche la solution devient crucial pour ne pas perdre l'unicité de la solution entropique. Un exemple est donné dans l'exercice 5.11. On peut remarquer que si $u$ est solution entropique au sens de la définition 5.8, alors $u \in C\left(\left[0,+\infty\left[, L_{\text {loc }}^{1}(\mathbb{R})\right)\right.\right.$ et $u(t) \rightarrow u_{0}$ dans $L_{\text {loc }}^{1}$ quand $t \rightarrow 0$.

Nous démontrerons plus loin le théorème 5.24 dans le cadre multidimensionnel (mais avec la variable spatiale dans un domaine borné plutôt que dans tout l'espace). Ce théorème affirme que si $u_{0} \in L^{\infty}(\mathbb{R})$ et $f \in \operatorname{Lip}_{\text {loc }}(\mathbb{R}, \mathbb{R})$ alors il existe une unique solution entropique de (5.5) au sens de la définition 5.8. Voyons maintenant les liens entre solution classique, solution faible et solution entropique. 
Proposition 5.10 (Solution classique et solution faible entropique) Soit $f \in C^{1}(\mathbb{R}, \mathbb{R})$ et $u_{0} \in L^{\infty}(\mathbb{R}) \cap$ $C^{1}(\mathbb{R}, \mathbb{R})$. Si u est solution classique de (5.5), alors u est solution (faible) entropique.

Démonstration Soit $u$ une solution classique de 5.5 . Soit $\eta \in C^{1}(\mathbb{R})$ (la convexité de $\eta$ est inutile ici) et $\phi$ tel que $\phi^{\prime}=f^{\prime} \eta^{\prime}\left(\phi\right.$ est la fonction flux associée à $\eta$ ). Multiplions 5.5) par $\eta^{\prime}(u)$ :

$$
\eta^{\prime}(u) \partial_{t} u+f^{\prime}(u) \partial_{x} u \eta^{\prime}(u)=0
$$

Soit encore, puisque $\phi^{\prime}=f^{\prime} \eta^{\prime}$,

$$
(\eta(u))_{t}+\phi^{\prime}(u) u_{x}=0
$$

On a donc finalement :

$$
(\eta(u))_{t}+(\phi(u))_{x}=0
$$

De plus, comme $u(x, 0)=u_{0}(x)$, on a aussi : $\eta(u(x, 0))=\eta\left(u_{0}(x)\right)$. Soit $\varphi \in C_{c}^{1}\left(\mathbb{R} \times \mathbb{R}_{+}, \mathbb{R}\right)$, on multiplie (5.21) par $\varphi$, on intègre sur $\mathbb{R} \times \mathbb{R}_{+}$et on obtient 5.20) (avec égalité) en intégrant par parties. Dans le cas d'une solution classique, l'inégalité d'entropie est une égalité.

Une solution faible entropique est solution faible :

Proposition 5.11 Soit $f \in \operatorname{Lip}_{\text {loc }}(\mathbb{R}, \mathbb{R})$ et $u_{0} \in L^{\infty}(\mathbb{R})$. Si u est solution faible entropique de (5.5), alors u est solution faible de (5.5).

Démonstration Il suffit de prendre $\eta(u)=u$ et $\eta(u)=-u$ dans 5.20) pour se convaincre du résultat.

On déduit de la proposition 5.10 et du théorème 5.24 de Kruzhkov que si on a plusieurs solutions faibles au problème 5.5) et que l'une d'entre elles est régulière, alors cette dernière est forcément la solution entropique. La caractérisation suivante, que l'on admettra, est souvent utilisée en pratique :

Proposition 5.12 (Entropies de Kruzhkov) Soit $u_{0} \in L^{\infty}(\mathbb{R})$ et $f \in \operatorname{Lip}_{\text {loc }}(\mathbb{R}, \mathbb{R})$. Soit $u \in L^{\infty}\left(\mathbb{R} \times \mathbb{R}_{+}\right)$. La fonction u est solution entropique de (5.5) (au sens de la définition 5.8) si et seulement si pour tout $k \in \mathbb{R}$ (5.20) est vérifiée avec $\eta$ définie par $\eta(s)=|s-k|$, et $\phi$, flux d'entropie associé, défini par :

$$
\phi(u)=f(\max (u, k))-f(\min (u, k)) .
$$

Notons que la fonction $\eta$, dite “entropie de Kruzhkov”, n'est pas de classe $C^{1}$.

Nous examinons maintenant le cas particulier des solutions ayant une ligne de discontinuité, comme dans la dernière partie de la proposition 5.7

Proposition 5.13 Soient $f \in C^{1}(\mathbb{R}, \mathbb{R})$ et $u_{0} \in L^{\infty}(\mathbb{R})$. Soit $\sigma \in \mathbb{R}, D_{1}=\left\{(x, t) \in \mathbb{R} \times \mathbb{R}_{+}^{\star} ; x<\sigma t\right\}$ et $D_{2}=\left\{(x, t) \in \mathbb{R} \times \mathbb{R}_{+}^{\star} ; x>\sigma t\right\}$. On suppose que $u_{\mid D_{i}} \in C^{1}\left(\bar{D}_{i}, \mathbb{R}\right)(i=1,2)$, que la première équation de (5.5) est vérifiée pour tout $(x, t) \in D_{i}(i=1,2)$ et que la condition initiale (de (5.5) ) est satisfaite p.p.. Pour $t \in \mathbb{R}_{+}$, on pose

$$
\begin{gathered}
u_{+}(\sigma t, t)=\lim _{x \downarrow \sigma t} u(x, t) \text { et } u_{-}(\sigma t, t)=\lim _{x \uparrow \sigma t} u(x, t), \\
{[u](\sigma t, t)=u_{+}(\sigma t, t)-u_{-}(\sigma t, t),} \\
{[f(u)](\sigma t, t)=f\left(u_{+}(\sigma t, t)\right)-f\left(u_{-}(\sigma t, t)\right) .}
\end{gathered}
$$

Alors u est solution faible entropique de (5.5) si et seulement si

$$
\sigma[u](\sigma t, t)=[f(u)](\sigma t, t) \text { pour tout } t \in \mathbb{R}_{+}
$$

et, pour toute fonction $\eta \in C^{1}(\mathbb{R})$ convexe et $\phi \in C^{1}$ telle que $\phi^{\prime}=f^{\prime} \eta^{\prime}$,

$$
\sigma[\eta(u)](\sigma t, t) \geq[\phi(u)](\sigma t, t) \text { pour tout } t \in \mathbb{R}_{+} .
$$


Démonstration La proposition 5.7 montre que $u$ est solution faible si et seulement si (5.22) est satisfaite. En reprenant la démonstration de la proposition 5.7. on montre que $u$ est solution faible entropique si et seulement si (5.22) et (5.23) sont satisfaites. Ceci fait l'objet de la première question de l'exercice 5.12

Dans le cas où la fonction $f$ est strictement convexe, la proposition 5.13 peut être précisée. Ceci est fait dans la proposition 5.15 donnée ci après, dont la démonstration repose sur le petit lemme technique suivant.

Lemme 5.14 (Un résultat pour des fonctions convexes) Soit $f$ et $\eta$ deux fonctions convexes de $\mathbb{R}$ à $\mathbb{R}$. Soit $a, b \in \mathbb{R}, a<b$, et $\sigma=(f(b)-f(a)) /(b-a)$. Soit $\phi$ défini par $\phi(s)=\int_{0}^{s} \eta^{\prime}(t) f^{\prime}(t) d t$ pour $s \in \mathbb{R}$ (de sorte que $\phi^{\prime}=\eta^{\prime} f^{\prime}$ p.p. sur $\left.\mathbb{R}\right)$. Alors,

1. $\sigma(\eta(b)-\eta(a)) \leq(\phi(b)-\phi(a))$,

2. si $\eta$ est strictement convexe et $f$ est convexe et non affine entre a et b, alors $\sigma(\eta(b)-\eta(a))<(\phi(b)-\phi(a))$.

Démonstration Rappelons d'abord que si $\varphi$ est une fonction convexe de $\mathbb{R}$ à $\mathbb{R}$, alors c'est une fonction localement lipschitzienne. Elle est donc dérivable presque partout, sa dérivée est localement bornée et $\varphi(\alpha)-\varphi(\beta)=$ $\int_{\alpha}^{\beta} \varphi^{\prime}(t) d t$ pour tout $(\alpha, \beta) \in \mathbb{R}^{2}$. Pour tout $\gamma \in \mathbb{R}$,

$$
(\phi(b)-\phi(a))-\sigma(\eta(b)-\eta(a))=\int_{a}^{b} \eta^{\prime}(t)\left(f^{\prime}(t)-\sigma\right) d t=\int_{a}^{b}\left(\eta^{\prime}(t)-\gamma\right)\left(f^{\prime}(t)-\sigma\right) d t
$$

Puisque $f$ est convexe, la fonction $f^{\prime}$ est croissante. Puisque $\sigma$ est la valeur moyenne de $f^{\prime}$ sur $] a, b[$, il existe $c \in] a, b[$ tel que

$$
\left.f^{\prime}(t) \leq \sigma \text { for p.p. } t \in\right] a, c\left[, \quad f^{\prime}(t) \geq \sigma \text { for p.p. } t \in \in\right] c, b[.
$$

Soit maintenant $\gamma=\sup \left\{\eta^{\prime}(s), s \leq c\right\}$ dans (5.24) de sorte que $\eta^{\prime}(s) \leq \gamma$ si $s \leq c$ et $\eta^{\prime}(s) \geq \gamma$ si $s>c$. Bien sûr, si $\eta^{\prime}$ est continu, on a $\left.\gamma=\eta^{\prime}(c)\right)$. Comme $\left(\eta^{\prime}(t)-\gamma\right)\left(f^{\prime}(t)-\sigma\right) \geq 0$ for p.p. $\left.t \in\right] a, b[$, on obtient

$$
(\phi(b)-\phi(a))-\sigma(\eta(b)-\eta(a))=\int_{a}^{b}\left(\eta^{\prime}(t)-\gamma\right)\left(f^{\prime}(t)-\sigma\right) d t \geq 0,
$$

ce qui donne le premier item du lemme.

Pour le deuxième item, on remarque que $\sigma(\eta(b)-\eta(a))=(\phi(b)-\phi(a))$ donne $\left(\eta^{\prime}(t)-\gamma\right)\left(f^{\prime}(t)-\sigma\right)=0$ p.p. sur $] a, b\left[\right.$. Puisque $\eta$ est strictement convexe, on a $\left(\eta^{\prime}-\gamma\right) \neq 0$ p.p. sur $] a, b\left[\right.$. On a alors $f^{\prime}=\sigma$ p.p. sur $] a, b[$ et cela donne que $f$ est affine sur $] a, b[$, ce qui contredit l'hypothèse.

Proposition 5.15 Sous les hypothèses de la proposition 5.13 on suppose que u est solution faible de 5.5. On suppose de plus que f est strictement convexe, les trois conditions suivantes sont alors équivalentes :

1. u est solution faible entropique,

2. $u_{-}(\sigma t, t) \geq u_{+}(\sigma t, t)$ pour tout $t \in \mathbb{R}^{+}$,

3. il existe $\eta$ strictement convexe $($ de $\mathbb{R}$ dans $\mathbb{R})$ t.q. (5.23) est vérifiée (avec $\phi$ telle que $\left.\phi^{\prime}=f^{\prime} \eta^{\prime}\right)$.

Démonstration Prouvons d'abord l'équivalence entre les deux premiers items.

Si $u$ est une solution faible entropique, on a (5.23) pour tout $t$ et pour toute fonction $C^{1}$ convexe $\eta$. En prenant pour $\eta$ une fonction strictement convexe, le lemme 5.14 donne nécessairement, grâce au fait que $f$ est aussi strictement convexe, $u_{-}(\sigma t, t) \geq u_{+}(\sigma t, t)$ pour $t \in \mathbb{R}^{+}$.

Réciproquement, si $u$ satisfait $u_{-}(\sigma t, t) \geq u_{+}(\sigma t, t)$ pour $t \in \mathbb{R}^{+}$, alors le lemme 5.14) donne (5.23) pour tout $t$ et toute fonction $C^{1}$ convexe $\eta$ (et c'est également vrai si $f$ n'est qu' une fonction convexe). Ceci conclut l'équivalence entre les items 1 et 2 . 
Pour conclure la preuve de la proposition 5.15, on remarque que le premier item implique bien sûr le troisième. Réciproquement, si $u$ satisfait le troisième item, le lemme 5.14 donne nécessairement, grâce au fait que $f$ est également strictement convexe, que $u_{-}(\sigma t, t) \geq u_{+}(\sigma t, t)$ pour $t \in \mathbb{R}^{+}$, et $u$ est donc une solution faible entropique.

\section{Remarque 5.16 (Contre-exemple si $f$ n'est pas strictement convexe)}

L'équivalence entre les deux premiers items de la proposition 5.15 est fausse si l'on remplace l'hypothèse " $f$ strictement convexe" par " $f$ convexe". Bien sûr, cela est évident si $u_{0}$ prend ses valeurs dans un intervalle où $f$ est une fonction affine mais c'est aussi le cas pour les $u_{0}$ plus généraux. On commence par donner un exemple qui apparaît dans certains articles concernant la modélisation de la circulation routière. Ensuite, nous adaptons légèrement cet exemple afin d'être exactement dans les hypothèses de la proposition 5.15

Soit $\alpha>0, \beta<0$ et $a=-\beta /(\alpha-\beta)$ (noter que $a \in] 0,1[$ et $a \alpha=\beta(a-1)$ ). On définit $f$ par $f(s)=\alpha s$ pour $s \in[0, a], f(s)=\beta(s-1) s \in] a, 1\left[\right.$. Soit $\left.u_{g} \in\right] a, 1\left[\right.$ et $\left.u_{d} \in\right] 0, a\left[\right.$ et $u_{0}=u_{g}$ en $\mathbb{R}_{-}, u_{0}=u_{d}$ en $\mathbb{R}_{+}$. Dans ce cas, il est assez facile de prouver que la solution faible entropique de (5.5) est la fonction $u$ définie par

$$
u(x, t)=\left\{\begin{array}{l}
u_{g} \text { if } x<\beta t, \\
a \text { if } \beta t<x<\alpha t, \\
u_{d} \text { if } x>\alpha t .
\end{array}\right.
$$

Puisque $u_{g}>a$ (et aussi $a>u_{d}$ ) et puisque $f$ est concave, cette solution semble en contradiction avec la proposition 5.15

Dans cet exemple, la fonction $f$ est lipschitzienne et la solution comporte deux lignes de discontinuités. En moditiant légèrement cet exemple, on va obtenir une fonction $f \in C^{1}(\mathbb{R})$ et une seule discontinuité. On prend $a=1 / 2$ et $f(s)=\alpha s$ pour $s \in[0, a], f(s)=\beta s-\gamma s^{2}+\delta$ avec $\alpha=\gamma=4 / 3, \beta=8 / 3, \delta=-1 / 3$. La fonction $f$ est de la classe $C^{1}$, strictement concave sur $[a, 1]$, affine sur $[0, a]$. Comme précédemment, on prend $\left.u_{g} \in\right] a, 1[$ et $\left.u_{d} \in\right] 0, a\left[\right.$ et $u_{0}=u_{g}$ dans $\mathbb{R}_{-}, u_{0}=u_{d}$ dans $\mathbb{R}_{+}$. Alors la solution faible entropique de (5.5) est la fonction $u$ définie par (puisque $f^{\prime}(a)=\alpha$ )

$$
\begin{array}{r}
u(x, t)=u_{g} \text { if } x<f^{\prime}\left(u_{g}\right) t, \\
\left.u(x, t)=\xi \text { if } f^{\prime}\left(u_{g}\right) t<x<f^{\prime}(a) t \text { et } f^{\prime}(\xi)=\frac{x}{t}, \xi \in\right] a, u_{g}[, \\
u(x, t)=u_{d} \text { if } x>f^{\prime}(a) t=\alpha t .
\end{array}
$$

Ici aussi, puisque $a>u_{d}$ et $f$ est concave, cette solution semble en contradiction avec la proposition 5.15 (mais en fait non, puisque les hypothèses de la proposition ne sont pas respectées).

Les propositions 5.13 et 5.15 peuvent être généralisées aux cas de courbes de discontinuité.

Proposition 5.17 (Rankine-Hugoniot, cas courbe) Soient $f \in C^{1}(\mathbb{R}, \mathbb{R})$ et $u_{0} \in L^{\infty}(\mathbb{R})$. On suppose qu'il existe un nombre fini d'ouverts à frontière lipschitzienne, $D_{i}, i=1, \ldots, N$, tels que

1. $\mathbb{R} \times \mathbb{R}_{+}=\cup_{i=1}^{N} \bar{D}_{i}$.

2. Pour $i \neq j, \bar{D}_{i} \cap \bar{D}_{j}=\left\{\left(t, \sigma_{i, j}(t)\right), t \in I_{i, j}\right\}$ où $I_{i, j}$ est un intervalle de $\mathbb{R}_{+}$et $\sigma_{i, j}$ une fonction lipschitzienne de $I_{i, j}$ dans $\mathbb{R}$.

3. Pour tout $i, u_{\mid D_{i}}$ appartient à $C^{1}\left(\bar{D}_{i}, \mathbb{R}\right)$ et est solution (classique) de la première équation de (5.5) et satisfait (p.p.) la condition initiale (de (5.5)) lorsque $\bar{D}_{i}$ rencontre l'axe $t=0$. 
Pour $i, j \in\{1, \ldots, N\}$ et $t \in I_{i, j}$, on pose

$$
\begin{gathered}
u_{+}\left(\sigma_{i, j}(t), t\right)=\lim _{x \downarrow \sigma_{i, j}(t)} u(x, t) \text { et } u_{-}\left(\sigma_{i, j}(t), t\right)=\lim _{x \uparrow \sigma_{i, j}(t)} u(x, t), \\
{[u]\left(\sigma_{i, j}(t), t\right)=u_{+}\left(\sigma_{i, j}(t), t\right)-u_{-}\left(\sigma_{i, j}(t), t\right),} \\
{[f(u)]\left(\sigma_{i, j}(t), t\right)=f\left(u_{+}\left(\sigma_{i, j}(t), t\right)\right)-f\left(u_{-}\left(\sigma_{i, j}(t) t\right)\right) .}
\end{gathered}
$$

Alors u est solution faible entropique de 5.5 si et seulement si

$$
\sigma_{i, j}^{\prime}(t)[u]\left(\sigma_{i, j}(t), t\right)=[f(u)]\left(\sigma_{i, j}(t), t\right) \text { pour presque tout } t \in I_{i, j},
$$

et, pour toute fonction $\eta \in C^{1}(\mathbb{R})$ convexe et $\phi \in C^{1}$ telle que $\phi^{\prime}=f^{\prime} \eta^{\prime}$,

$$
\sigma_{i, j}^{\prime}(t)[\eta(u)]\left(\sigma_{i, j}(t) t, t\right) \geq[\phi(u)]\left(\sigma_{i, j}(t), t\right) \text { pour presque tout } t \in I_{i, j} .
$$

Démonstration La démonstration peut se faire en utilisant la formule de Stokes (espace temps) sur chaque $D_{i}$. En notant $u_{i}$ le prolongement par continuité de $u$ sur $\bar{D}_{i}$, la formule de Stokes fait apparaître pour tout couple $(i, j)$, $i \neq j$ (lorsque $I_{i, j}$ est non vide),

$$
\int_{I_{i, j}}\left[\begin{array}{c}
f\left(u_{i}(x, t)\right) \\
u_{i}(x, t)
\end{array}\right] \cdot \boldsymbol{n}_{i, j} d \gamma(x, t)
$$

où $\gamma$ désigne la mesure de Lebesgue 1-dimensionnelle sur $I_{i, j}$ et $\boldsymbol{n}_{i, j}$ est le vecteur normal à $I_{i, j}$ extérieur à $D_{i}$. Pour que $u$ soit solution faible, il faut et il suffit que le terme correspondant à $i$ sur $I_{i, j}$ se compense avec le terme correspondant à $j$. Comme $\boldsymbol{n}_{i, j}\left(\sigma_{i, j}(t), t\right)$ est colinéaire au vecteur $\left[\begin{array}{c}-1 \\ \sigma_{i, j}^{\prime}(t)\end{array}\right]$ (et que $\boldsymbol{n}_{i, j}=-\boldsymbol{n}_{j, i}$ ), on obtient la condition 5.25 .

Un raisonnement analogue avec le vecteur $\left[\begin{array}{l}\phi\left(u_{i}\right) \\ \eta\left(u_{i}\right)\end{array}\right]$ donne la condition 5.26).

Remarquons que les solutions d'une équation hyperbolique non linéaire respectent les bornes de la solution initiale.

Proposition 5.18 (Principe du maximum) Soit $u_{0} \in L^{\infty}(\mathbb{R})$ et soit $A$ et $B \in \mathbb{R}$ tels que $A \leq u_{0} \leq B$ p.p.. Soit $f \in \operatorname{Lip}_{\text {loc }}(\mathbb{R}, \mathbb{R})$, alors la solution entropique $u \in L^{\infty}\left(\mathbb{R} \times \mathbb{R}_{+}\right)$de 5.5 vérifie $: A \leq u(x) \leq B$ p.p. dans $\mathbb{R} \times \mathbb{R}_{+}$.

Cette propriété se démontre en passant à la limite soit sur les solutions de l'équation visqueuse associée, soit sur les solutions approchées par schéma numérique; il faut pour cela avoir pris soin de mettre au point un schéma qui respecte les bornes, mais ceci est de toutes façon souhaitable pour respecter les bornes physiques.

Remarque 5.19 (Domaine borné) Que faire si le domaine spatial est différent de $\mathbb{R}$, par exemple si le problème 5.5) est posé pour $x \in I$ où $I$ est un intervalle de $\mathbb{R}$. Si $f^{\prime}$ ne change pas de signe, il est assez facile de donner une bonne définition de solution entropique et de montrer un théorème d'existence et d'unicité de la solution entropique. Dans le cas où $f^{\prime}$ change de signe (et ce cas est très intéressant pour de nombreux problèmes), le problème est beaucoup plus difficile. Le premier résultat sur la question est celui de Bardos-Leroux-Nedelec (1979). Dans la thése de Otto (1996), il y a une très jolie formulation pour les conditions aux limites. Un intérêt considérable de cette formulation est qu'elle est très pratique pour montrer la convergence des schémas numériques. Dans le cas multidimensionnel de la section suivante, on s'intéressera à un problème similaire à (5.5) posé dans un domaine borné de $\mathbb{R}^{N}(N>1)$ mais sans aborder vraiment ce délicat problème des conditions aux limites (car dans le théorème 5.24 on considère un champ de vecteurs $b$ nul sur le bord du domaine considéré). 
On termine ce paragraphe en introduisant les notions de "discontinuité de contact", "onde de choc" et "onde de détente".

Si $f$ est linéaire (ou affine, ce qui revient au même car on peut supposer $f(0)=0$ ) et si $u_{0} \in L^{\infty}(\mathbb{R})$, la solution faible de (5.5) est unique (voir l'exercice 5.3), c'est donc la solution entropique. On peut aussi montrer dans ce cas que les inégalités d'entropie (5.20) sont des égalités. Si la solution $u$ a une courbe de discontinuité (nécessairement une demi droite en fait), on parle alors de "discontinuité de contact".

Si $f$ est strictement convexe et que la solution faible entropique $u$ de $(5.5)$ a une courbe de discontinuité, on parle d'un choc ou d'une onde de choc. On peut montrer dans ce cas que les inégalités d'entropie (5.20) sont strictes pour certains $\eta$ et $\varphi$.

Si $u_{0}$ a une discontinuité en un point mais que cette discontinuité ne se propage pas dans la solution faible entropique, on parle d'une détente" ou d'une onde de détente.

\subsubsection{Conditions limites}

On donne maintenant un résultat d'existence et d'unicité pour une équation hyperbolique avec conditions aux limites en utilisant la formulation due à Otto [24].

On s'intéresse donc au problème :

$$
\begin{aligned}
& \partial_{t} u+\partial_{x}(f(u))=0, \quad(x, t) \in \mathbb{R} \times \mathbb{R}_{+}, \\
& \left.u(x, 0)=u_{0}(x), \quad x \in\right] 0,1[, \\
& u(0, t)=\bar{u}(t), u(1, t)=\overline{\bar{u}}(t), \quad t \in \mathbb{R}_{+} .
\end{aligned}
$$

Mais, comme nous le verrons, les conditions aux limites ne sont que partiellement prises en compte dans la formulation faible entropique du problème.

Définition 5.20 (Solution entropique avec conditions aux limites, Otto)

Soient $u_{0} \in L^{\infty}([0,1]), \bar{u}, \overline{\bar{u}} \in L^{\infty}(] 0,+\infty[)$ et $f \in \operatorname{Lip}_{\text {loc }}(\mathbb{R}, \mathbb{R})$. Soit $u \in L^{\infty}\left(\mathbb{R} \times \mathbb{R}_{+}\right)$. Soit $A, B$ tels que $A \leq u_{0}, \bar{u}, \overline{\bar{u}} \leq B$ p.p.. On dit que $u$ est solution entropique de (5.27) si il existe $M \geq 0$ tel que

$$
\begin{array}{r}
\int_{0}^{+\infty} \int_{0}^{1} \eta(u(x, t)) \partial_{t} \varphi(x, t) \mathrm{d} x \mathrm{~d} t+\int_{0}^{1} \eta\left(u_{0}(x)\right) \varphi(x, 0) \mathrm{d} x \\
+\int_{0}^{+\infty} \int_{0}^{1} \Phi(u(x, t)) \partial_{x} \varphi(x, t) \mathrm{d} x+M \int_{\mathbb{R}_{+}} \varphi(0, t) \eta(\bar{u}(t)) \mathrm{d} t \\
+M \int_{0}^{+\infty} \varphi(1, t) \eta(\overline{\bar{u}}(t)) \mathrm{d} t \geq 0
\end{array}
$$

pour tout $\varphi \in C_{c}^{1}\left([0,1] \times \mathbb{R}_{+}, \mathbb{R}_{+}\right)$et pour toute fonction $\eta$ convexe positive telle qu'il existe $s_{0} \in[A, B]$ avec $\eta\left(s_{0}\right)=0$, et $\Phi$ telle que $\Phi(s)=\int_{s_{0}}^{s} \eta^{\prime}(t) f^{\prime}(t)$ t. (On rappelle qu' une fonction convexe de $\mathbb{R}$ dans $\mathbb{R}$ est localement lipschitzienne et donc dérivable p.p..)

Théorème 5.21 (Existence and unicité, avec conditions limites) Soient $u_{0} \in L^{\infty}([0,1]), \bar{u}, \overline{\bar{u}} \in L^{\infty}(] 0,+\infty[)$ et $f \in \operatorname{Lip}_{\text {loc }}(\mathbb{R}, \mathbb{R})$. Soit $A, B$ tels que $A \leq u_{0} \leq B$ p.p. et $A \leq \bar{u}, \overline{\bar{u}} \leq B$ p.p.. Soit $M \geq \max _{s \in[A, B]}\left|f^{\prime}(s)\right|$. Alors il existe une et une seule fonction $u \in L^{\infty}\left(\mathbb{R} \times \mathbb{R}_{+}\right)$solution entropique de (5.27). De plus,

$$
A \leq u(x, t) \leq B \text { p.p. }(x, t) \in] 0,1\left[\times \mathbb{R}_{+} .\right.
$$

La démonstration de l'existence de solution dans le théorème 5.21 s'obtient en prenant $M \geq \max _{s \in[A, B]}\left|f^{\prime}(s)\right|$ (la solution $u$ prend ses valeurs dans $[A, B]$ ). Un moyen assez simple de prouver cette existence est de passer 
à la limite sur des schémas numériques à flux monotone, voir la démonstration du théorème 5.30 dans le cas multidimensionnel. L'unicité est vraie pour toute valeur de $M$ (mais, évidemment, n'est intéressante que si il y a existence) et la solution (qui existe donc si $M \geq \max _{s \in[A, B]}\left|f^{\prime}(s)\right|$ ) ne dépend pas de $M$ ).

Si la solution est de classe $C^{1}$, cette solution est solution entropique sur ]0, 1 [, c'est-à-dire est solution de (5.28) pour tout $\varphi \in C_{c}^{1}(] 0,1\left[\times \mathbb{R}_{+}, \mathbb{R}_{+}\right)$et pour toute fonction $\eta$ convexe, et vérifie les conditions aux limites au sens donné par [5].

Ceci est encore vrai si la solution est encore de classe $B V$ (on dit aussi à variation bornée) en espace pour tout $t$, de sorte à ce que la solution ait, pour tout $t$, une limite en $x=0$ et $x=1)$. On rappelle que l'espace $B V([0,1])$ est défini par

Définition 5.22 (Fonction à variation bornée, espace $\boldsymbol{B} \boldsymbol{V}$ ) Soit $v:] 0,1[\rightarrow \mathbb{R}$, alors $v$ est a variation bornée, ou $v \in B V(] 0,1[)$, si $v \in L^{1}(] 0,1[)$ et si $|v|_{B V([0,1]}<+\infty$, avec

$$
|v|_{B V(\bar{\Omega})}=\sup \left\{\int_{\Omega} v \operatorname{div} \varphi \mathrm{d} x, \varphi \in C_{c}^{\infty}\left(\mathbb{R}^{N}, \mathbb{R}^{N}\right),\|\varphi\|_{L^{\infty}(\Omega)} \leq 1\right\} .
$$

Bien sûr, si $u \in L^{\infty}\left(\mathbb{R} \times \mathbb{R}_{+}\right)$est solution de $(5.28)$ comme dans la définition 5.20 , la fonction $u$ est aussi solution de (5.28) avec les entropies de Kruzhkov, c'est-à-dire $\eta$ définie (pour $k \in[A, B]$ ) par $\eta(s)=|s-k|$ (et donc $\phi$, flux d'entropie associé, définie par $\phi(s)=f(\max (s, k))-f(\min (s, k)))$. Par contre, le fait que $u \in L^{\infty}\left(\mathbb{R} \times \mathbb{R}_{+}\right)$ vérifie (5.28) pour les toutes les entropies de Kruzhkov n'est pas suffisant pour assurer l'unicité (alors que c'était suffisant dans le cas du problème posé sur tout $\mathbb{R}$, sans conditions aux limites). Un exemple de non unicité est donné dans la remarque 5.23. On obtient toutefois l'unicité si $u \in L^{\infty}\left(\mathbb{R} \times \mathbb{R}_{+}\right)$vérifie (5.28) pour toutes les "semi-entropies de Kruzhkov", c'est-à-dire les fonctions $\eta$ définies (pour $k \in[A, B]$ ) par $\eta(s)=(s-k)^{+}$(et donc $\phi(s)=f(s)-f(k)$ si $s \geq k$ et 0 sinon) et $\eta(s)=(s-k)^{-}$(et donc $\phi(s)=f(k)-f(s)$ si $s \leq k$ et 0 sinon).

\section{Remarque 5.23 (Contre exemple à l'unicité avec les entropies de Kruzhkov)}

Nous donnons dans cette remarque un exemple de non unicité si on se limite dans la definition 5.20 aux entropies de Kruzhkov, c'est-à-dire aux fonctions $\eta$ définies (pour $k \in[A, B]$ ) par $\eta(s)=|s-k|$ (et donc $\phi$, flux d'entropie associé, définie par $\phi(s)=f(\max (s, k))-f(\min (s, k)))$.

Pour cet exemple, $f(s)=s^{2}, u_{0}=1$ p.p. dans $] 0,1\left[, \bar{u}=-1\right.$ p.p. sur $\mathbb{R}_{+}$et $\overline{\bar{u}}=1$ p.p. sur $\mathbb{R}_{+}$. Il est donc possible de prendre $A=-1, B=1$ et $M=2$. Le théorème 5.21 nous donne une solution entropique de 5.27) avec ces valeurs de $A, B$ and $M$. On peut vérifier que cette solution est la fonction $u$ définie par

$$
u(x, t)=\frac{x}{2 t} \text { if } x<2 t, u(x, t)=1 \text {, if } x>2 t .
$$

Elle corespond à une onde de détente. Cette solution reste donc aussi solution de (5.28) en se limitant aux entropies de Kruzhkov.

On considère maintenant la fonction constante $u=1$ p.p. dans $] 0,1\left[\times \mathbb{R}_{+}\right.$. Cette fonction constante est aussi solution de (5.28) si on se limite dans cette définition aux entropies de Kruzhkov. Cette solution consiste en fait à propager une discontinuité non entropique au point $x=0$.

On a ainsi deux fonctions qui vérifient (5.28) si on se limite dans cette définition aux entropies de Kruzhkov.

\subsection{Cas multidimensionnel}

Soit $\Omega \subset \mathbb{R}^{N}, N=2,3, T>0, \boldsymbol{b} \in C^{1}(\bar{\Omega} \times[0, T])^{N}$ et $f \in C^{1}(\mathbb{R}, \mathbb{R})$ (mais on pourrait aussi considérer le cas $\left.f \in \operatorname{Lip}_{\text {loc }}(\mathbb{R}, \mathbb{R})\right)$. On étudie maintenant le problème suivant :

$$
\begin{array}{r}
\left.\partial_{t} u+\operatorname{div}(\boldsymbol{b} f(u))=0 \text { dans } \Omega \times\right] 0, T[, \\
u(x, 0)=u_{0}(x) \text { dans } \Omega .
\end{array}
$$


Plus précisément, nous allons démontrer, avec des hypothèses convenables sur les données, le théorème d'existence et d'unicité des solutions entropiques de ce problème, d'abord dans le cas sans conditions limites (Sect. 5.2.1), puis dans le cas d'un problème avec conditions limites (Sect. 5.2.2).

\subsubsection{Cas sans condition limite}

On suppose ici que $\boldsymbol{b}=0$ sur $\partial \Omega \times[0, T]$, et donc on n'a pas besoin de conditions aux limites sur $\partial \Omega$.

Théorème 5.24 (Kruzhkov,1955) Soit $\Omega$ un ouvert borné de $\mathbb{R}^{N}(N>1)$ à frontière lipschitzienne. Soit $T>0$ et $\boldsymbol{b} \in C^{1}(\bar{\Omega} \times[0, T])^{N}$ t.q. $\boldsymbol{b}=0$ sur $\partial \Omega \times[0, T]$ et $\operatorname{div} \boldsymbol{b}=0$ dans $\Omega \times[0, T]$. Soit $u_{0} \in L^{\infty}(\Omega)$ et $f \in C^{1}(\mathbb{R}, \mathbb{R})$. Alors il existe une unique solution entropique de [5.29], c'est-à-dire solution de

$$
\begin{aligned}
& u \in L^{\infty}(\Omega \times] 0, T[), \\
& \int_{0}^{T} \int_{\Omega}\left(\eta(u) \varphi_{t}+\Phi(u) \boldsymbol{b} \cdot \nabla \varphi\right) \mathrm{d} x \mathrm{~d} t+\int_{\Omega} \eta\left(u_{0}(x)\right) \varphi(x, 0) \mathrm{d} x \geq 0, \\
& \forall \varphi \in C_{c}^{\infty}\left(\Omega \times\left[0, T\left[, \mathbb{R}_{+}\right), \forall \eta \in C^{2}(\mathbb{R}, \mathbb{R}) \text { convexe et } \Phi \text { tel que } \Phi^{\prime}=\eta^{\prime} f^{\prime}\right.\right.
\end{aligned}
$$

De plus, si $A \leq 0$ et $B \geq 0$ sont t.q. $A \leq u_{0} \leq B$ p.p. sur $\Omega$, on a alors $A \leq u \leq B$ p.p. sur $\left.\Omega \times\right] 0, T[$.

\section{Démonstration}

Etape 1 Construction d'une solution approchée. Soient $n \in \mathbb{N}^{\star}$ et $u^{(n)}$ solution de

$$
\begin{aligned}
& u^{(n)} \in L^{2}(] 0, T\left[, H_{0}^{1}(\Omega)\right), \\
& \quad-\int_{0}^{T} \int_{\Omega} u^{(n)} \varphi_{t} \mathrm{~d} x \mathrm{~d} t-\int_{0}^{T} \int_{\Omega} \boldsymbol{b} f\left(u^{(n)}\right) \cdot \nabla \varphi \mathrm{d} x \mathrm{~d} t+\frac{1}{n} \int_{0}^{T} \int_{\Omega} \nabla u^{(n)} \cdot \nabla \varphi \mathrm{d} x \mathrm{~d} t \\
& \quad-\int_{\Omega} u_{0}(x) \varphi(x, 0) \mathrm{d} x=0, \forall \varphi \in C_{c}^{\infty}(\Omega \times[0,+\infty[) .
\end{aligned}
$$

On connaît l'existence et l'unicité de $u^{(n)}$ par l'étude de l'quation de convection-diffusion faite au chapitre 4 . (Le fait d'avoir $1 / n$ au lieu de 1 ne pose aucune difficulté dans cette étude.)

On a vu aussi au chapitre 4 que cette formulation est équivalente au problème suivant :

$$
\begin{aligned}
& u^{(n)} \in L^{2}(] 0, T\left[, H_{0}^{1}(\Omega)\right), \partial_{t} u^{(n)} \in L^{2}(] 0, T\left[, H^{-1}(\Omega)\right), u^{(n)}(0)=u_{0}, \\
& \int_{0}^{T}\left\langle\partial_{t} u^{(n)}, v\right\rangle_{H^{-1}, H_{0}^{1}} \mathrm{~d} t-\int_{0}^{T} \int_{\Omega} \boldsymbol{b} f\left(u^{(n)}\right) \cdot \nabla v \mathrm{~d} x \mathrm{~d} t+\frac{1}{n} \int_{0}^{T} \int_{\Omega} \nabla u^{(n)} \cdot \nabla v \mathrm{~d} x \mathrm{~d} t=0, \\
& \forall v \in L^{2}(] 0, T\left[, H_{0}^{1}(\Omega)\right) .
\end{aligned}
$$

On va se servir fortement de cette équivalence.

\section{Etape 2 Estimations sur la solution approchée.}

Soit $u_{0} \in L^{\infty}(\Omega)$ il existe $A$ et $B \in \mathbb{R}, A \leq 0 \leq B$, tels que $A \leq u_{0} \leq B$ p.p. ( $A$ et $B$ sont donc indépendants de $n$ ). On en déduit (remarque 4.36 du chapitre 4 que pour tout $t \in[0, T], A \leq u^{(n)} \leq B$ p.p.. La suite $\left(u^{(n)}\right)_{n \in \mathbb{N}}$ est donc bornée dans $L^{\infty}(\Omega \times] 0, T[)$. On prend maintenant $v=u^{(n)}$ dans $\left[5.32\right.$, on obtient, en utilisant $\int_{\Omega} b f\left(u^{(n)}\right)$. $\nabla u^{(n)} \mathrm{d} x=0$ p.p. (grâce à $\operatorname{div} \boldsymbol{b}=0$, voir 3.12 ) :

$$
\frac{1}{2}\left(\left\|u^{(n)}(T)\right\|_{L^{2}(\Omega)}^{2}-\left\|u_{0}\right\|_{L^{2}(\Omega)}^{2}\right)+\frac{1}{n} \int_{0}^{T} \int_{\Omega}\left|\nabla u^{(n)}\right|^{2} \mathrm{~d} x \mathrm{~d} t=0 .
$$


On en déduit que

$$
\frac{1}{n} \int_{0}^{T} \int_{\Omega}\left|\nabla u^{(n)}\right|^{2} \mathrm{~d} x \mathrm{~d} t \leq \frac{1}{2}\left\|u_{0}\right\|_{L^{2}(\Omega)}^{2}<+\infty
$$

ce qui donne une estimation $L^{2}(\Omega \times] 0, T[)^{d} \operatorname{sur}\left(\frac{1}{\sqrt{n}} \nabla u^{(n)}\right)_{n \in \mathbb{N}}$. Cette estimation ne donne rien pour la compacité, mais elle est utile pour passer à la limite (quand $n \rightarrow+\infty$ ).

Grâce à la première estimation (l'estimation de $u_{n}$ dans $L^{\infty}(\Omega \times] 0, T[)$ ), après extraction d'une sous suite, on peut supposer que $u^{(n)} \rightarrow u \star$-faiblement dans $L^{\infty}(\Omega \times] 0, T[)$.

Si $f(u)=u$, on montre assez facilement que $u$ est solution faible de 5.29) ( c'est-à-dire solution de 5.30 avec seulement $\eta(s)=s$ mais avec tout $\varphi$ dans $C_{c}^{\infty}(\Omega \times[0, T[, \mathbb{R})$ et avec $=$ au lieu de $\geq)$. Puis on peut montrer (un peu moins facilement) que $u$ est solution de (5.30) et cela termine la partie "existence" du théorème 5.24. Si la fonction $f^{\prime}$ est non constante, la situation est beaucoup plus difficile, même pour montrer seulement que $u$ est solution faible de 5.29), car la convergence de $u^{(n)}$ vers $u$ n'est que faible et donc on ne sait pas si $f\left(u^{(n)}\right)$ tend vers $f(u)$ (et, plus généralement, on ne sait pas si $\eta\left(u^{(n)}\right)$ tend vers $\eta(u)$ et $\phi\left(u^{(n)}\right)$ tend vers $\phi(u)$ ). Pour résoudre cette difficulté, deux méthodes ont été développées.

Une première méthode, due à Kruzhkov, suppose, dans un premier temps, que la donnée initiale $u_{0}$ appartient à l'espace $B V(\bar{\Omega})$ (on dit aussi que $u_{0}$ est "à variation bornée"), c'est-à-dire que $u_{0} \in L^{1}(\Omega)$ et $\left|u_{0}\right|_{B V(\bar{\Omega})}<+\infty$, avec, par définition,

$$
\left|u_{0}\right|_{B V(\bar{\Omega})}=\sup \left\{\int_{\Omega} u_{0} \operatorname{div} \varphi \mathrm{d} x, \varphi \in C_{c}^{\infty}\left(\mathbb{R}^{N}, \mathbb{R}^{N}\right),\|\varphi\|_{L^{\infty}(\Omega)} \leq 1\right\}
$$

On démontre alors que la suite $\left(u^{(n)}\right)_{n \in \mathbb{N}}$ est bornée dans $B V(\bar{\Omega} \times[0, T])$. L'idée, pour montrer cette borne sur $u^{(n)}$ est de dériver la première équation de (5.29) par rapport à $x_{i}$ et de multiplier par $\operatorname{sign}\left(\partial_{i} u\right)$. Grâce à cette estimation $\operatorname{sur} u^{(n)}$, on peut appliquer ensuite le théorème de Helly 6 donné ci après.

Théorème 5.25 (Helly) Soit $d \geq 1$ et $\left(u_{n}\right)_{n \in \mathbb{N}}$ une suite bornée dans $L^{1}(Q)$ et bornée dans $B V(Q)$ où $Q$ est un compact de $\mathbb{R}^{d}$ alors $\left(u_{n}\right)_{n \in \mathbb{N}}$ est relativement compact dans $L^{1}(Q)$.

On applique donc le théorème de Helly avec $Q=\bar{\Omega} \times[0, T]($ et $d=N+1)$. Puisque $u^{(n)} \rightarrow u$ dans $L^{1}(Q)$, à une sous suite près, on a $u^{(n)} \rightarrow u$ dans $L^{p}(Q)$ pour tout $p<+\infty$ et on peut aussi supposer (toujours après extraction éventuelle d'une sous suite) que $u^{(n)} \rightarrow u$ p.p.. On peut alors montrer que $u$ est solution de (5.30) (ce qu'on fera a l'étape 3 plus loin), ce qui donne l'existence d'une solution à $\left(5.30\right.$ ) si $u_{0} \in B V(\bar{\Omega})$. Si $u_{0}$ n'est que dans $L^{\infty}(\Omega)$ (et c'est probablement ici l'apport majeur de Kruzhkov), on peut approcher $u_{0}$ par une suite d'éléments de $L^{\infty}(\Omega) \cap B V(\bar{\Omega})$ et montrer que la suite des solutions entropiques associées converge (en un sens convenable, après extraction d'une sous suite) vers une solution entropique associée à $u_{0}$. On peut également montrer l'unicité de la solution de (5.30) (étape 4 ci après). L'inconvénient majeur de cette méthode est qu'elle ne semble pas marcher pour montrer la convergence des schémas numériques car même si la condition initiale est supposée être dans $B V(\bar{\Omega})$, la solution approchée obtenue par un schéma numérique n'est pas bornée dans $B V(\bar{\Omega} \times[0, T])$ indépendamment des paramètres de discrétisation (sauf dans le cas des maillages cartésiens).

C'est pour cela qu'on peut lui préférer la deuxième méthode, qui ne passe pas par l'estimation $B V$. On prend uniquement $u_{0}$ dans $L^{\infty}(\Omega)$, et on ne cherche pas à montrer directement une compacité forte de la suite $u^{(n)}$ dans $L^{1}(\Omega \times] 0, T[)$. Grâce à l'estimation de $u^{(n)}$ dans $L^{\infty}(\Omega \times] 0, T[)$, on montre que (après extraction d'une sous suite) $u^{(n)} \rightarrow \tilde{u}$, en un sens convenable, où $\tilde{u}$ dépend d'une variable supplémentaire. (Il s'agit donc d'un théorème de

6. Eduard Helly, 1884-1943, mathématicien autrichien, prisonnier en Sibérie pendant et après la première guerre mondiale, il n'obtient pas de poste universitaire en raison de sa judéité et il s'exile aux USA après l'Anschluss en 1938. * Il a donné de nombreuses contributions en Analyse fonctionnelle. 
compacité un peu inhabituel donnant une convergence que nous appelons "convergence non linéaire faible- $\star$ "). Puis, on montre que $\tilde{u}$ est une solution du problème en un sens plus général que [5.30), que nous appelons "sens processus". Cette preuve est très voisine de celle de l'étape 3 ci après. On démontre ensuite l'unicité de la solution au sens processus et que la solution processus est solution entropique ( c'est-à-dire solution de $(5.30)$ ). Cette preuve d'unicité est très voisine de celle donnée dans l'étape 4 ci après. Ceci termine la démonstration de l'existence d'une solution à $(5.30)$ (directement avec $u_{0}$ dans $L^{\infty}(\Omega)$ ). (L'unicité est toujours donnée par l'étape 4.) Un sous produit de cette démonstration est la convergence (forte) de $u^{(n)}$ vers $u$ dans tout les espaces $L^{p}(\Omega \times] 0, T[), p<+\infty$, y compris si $f$ est linéaire (ou est linéaire sur des intervalles de $\mathbb{R}$ ). L'idée essentielle a donc été de remplacer le théorème de compacité (forte) de Helly par un théorème de compacité plus faible combiné avec un résulat d'unicité de la solution "au sens processus" de (5.29).

Etape 3. On reprend ici la méthode 1, et on va effectuer le passage à la limite, quand $n \rightarrow+\infty$, en supposant que $u_{0} \in B V$ et donc que $u^{(n)} \rightarrow u$ p.p. (pour une sous suite). On admet donc la partie "estimation $B V$ de $u^{(n)}$ ".

1. Montrons que $u$ est solution faible. Soit $\varphi \in C_{c}^{\infty}(\Omega \times[0,+\infty[)$, on a

$$
\int_{0}^{T} \int_{\Omega}\left(u^{(n)} \varphi_{t}+\boldsymbol{b} f\left(u^{(n)}\right) \cdot \nabla \varphi\right) \mathrm{d} x \mathrm{~d} t+\int_{\Omega} u_{0}(x) \varphi(x, 0) \mathrm{d} x-\frac{1}{n} \int_{0}^{T} \int_{\Omega} \nabla u^{(n)} \cdot \nabla \varphi \mathrm{d} x \mathrm{~d} t=0
$$

On remarque tout d'abord que le dernier terme du membre de gauche tend vers 0 , grâce à l'estimation (5.33) et à l'inégalité de Cauchy-Schwarz, en effet

$$
\left|\frac{1}{n} \int_{0}^{T} \int_{\Omega} \nabla u^{(n)} \cdot \nabla \varphi \mathrm{d} x \mathrm{~d} t\right| \leq \frac{1}{\sqrt{n}}\left\|\frac{1}{\sqrt{n}}\left|\nabla u^{(n)}\right|\right\|_{\left.\left.L^{2}(\Omega \times] 0, T\right]\right)}\||\nabla \varphi|\|_{L^{2}(\Omega \times] 0, T[)}
$$

et $\left\|\frac{1}{\sqrt{n}}\left|\nabla u^{(n)}\right|\right\|_{L^{2}(\Omega \times] 0, T[)} \leq(1 / \sqrt{2})\left\|u_{0}\right\|_{L^{2}(\Omega)}$ par (5.33) et donc $\frac{1}{n} \iint \nabla u^{(n)} \cdot \nabla \varphi \rightarrow 0$ lorsque $n \rightarrow 0$. Les autres termes convergent par convergence dominée, et donc en passant à la limite, on obtient

$$
\int_{0}^{T} \int_{\Omega}\left(u \varphi_{t}+\boldsymbol{b} f(u) \cdot \nabla \varphi\right) \mathrm{d} x \mathrm{~d} t+\int_{\Omega} u_{0}(x) \varphi(x, 0) \mathrm{d} x=0 .
$$

2. Montrons que $u$ est solution entropique. Comme $u^{(n)}$ est solution faible de

$$
u_{t}^{(n)}+\operatorname{div}\left(\boldsymbol{b} f\left(u^{(n)}\right)\right)-\frac{1}{n} \Delta u^{(n)}=0,
$$

on peut montrer (on l'admettra) que $u^{(n)} \in C^{2}(\Omega \times] 0, T[)$ (c'est ce que l'on appelle l'effet régularisant pour une équation parabolique). La fonction $u^{(n)}$ est donc solution classique de (5.36). On peut alors mutiplier cette équation par $\eta^{\prime}\left(u^{(n)}\right)$ avec $\eta \in C^{2}(\mathbb{R}, \mathbb{R})$ convexe. On obtient, sur $\left.\Omega \times\right] 0, T$,

$$
\left(\eta\left(u^{(n)}\right)\right)_{t}+\boldsymbol{b} f^{\prime}\left(u^{(n)}\right) \eta^{\prime}\left(u^{(n)}\right) \cdot \nabla u_{n}-\frac{1}{n} \eta^{\prime}\left(u^{(n)}\right) \Delta u^{(n)}=0 .
$$

On en déduit :

$$
\left(\eta\left(u^{(n)}\right)\right)_{t}+\boldsymbol{b} \cdot \nabla\left(\Phi\left(u^{(n)}\right)-\frac{1}{n} \operatorname{div}\left(\eta^{\prime}\left(u^{(n)}\right) \nabla u^{(n)}\right)+\frac{1}{n} \eta^{\prime \prime}\left(u^{(n)}\right)\left|\nabla u^{(n)}\right|^{2}=0 .\right.
$$

Mais $\frac{1}{n} \eta^{\prime \prime}\left(u^{(n)}\right)\left|\nabla u^{(n)}\right|^{2} \geq 0$, on a donc

$$
\left(\eta\left(u^{(n)}\right)\right)_{t}+\boldsymbol{b} \cdot \nabla\left(\Phi\left(u^{(n)}\right)-\frac{1}{n} \operatorname{div}\left(\eta^{\prime}\left(u^{(n)}\right) \nabla u^{(n)}\right)\right) \leq 0 .
$$


En multipliant cette équation par $\varphi$, avec $\varphi \in C_{c}^{\infty}\left(\Omega \times\left[0, T\left[, \mathbb{R}_{+}\right)\right.\right.$, on obtient, toujours sur $\left.\Omega \times\right] 0, T$,

$$
\varphi\left(\eta\left(u^{(n)}\right)\right)_{t}+\varphi \boldsymbol{b} \cdot \nabla\left(\Phi\left(u^{(n)}\right)-\frac{1}{n} \varphi \operatorname{div}\left(\eta^{\prime}\left(u^{(n)}\right) \nabla u^{(n)}\right)\right) \leq 0
$$

On intègre sur $[\varepsilon, T[\times \Omega$ avec $\varepsilon>0$ et, après intégration par parties, on obtient :

$$
-\int_{\varepsilon}^{T} \int_{\Omega}\left(\eta\left(u^{(n)}\right)\right) \varphi_{t}-\int_{\Omega} \eta\left(u^{(n)}(\varepsilon)\right) \varphi(x, \epsilon) \mathrm{d} x-\int_{\varepsilon}^{T} \int_{\Omega}\left(\boldsymbol{b} \Phi\left(u^{(n)}\right) \cdot \nabla \varphi+\frac{1}{n} \eta^{\prime}\left(u^{(n)}\right) \nabla u^{(n)} \cdot \nabla \varphi\right) \mathrm{d} x \mathrm{~d} t \leq 0 .
$$

Mais on a, quand $\varepsilon \rightarrow 0, u^{(n)}(\varepsilon) \rightarrow u^{(n)}(0)=u_{0}$ dans $L^{2}(\Omega)$ et $\eta\left(u^{(n)}(\varepsilon)\right) \rightarrow \eta\left(u_{0}\right)$ dans $L^{2}(\Omega)$ et donc

$$
-\int_{0}^{T} \int_{\Omega}\left(\eta\left(u^{(n)}\right)\right) \varphi_{t}-\int_{\Omega} \eta\left(u_{0}\right) \varphi(x, 0) \mathrm{d} x-\int_{0}^{T} \int_{\Omega}\left(\boldsymbol{b} \Phi\left(u^{(n)}\right) \cdot \nabla \varphi+\frac{1}{n} \eta^{\prime}\left(u^{(n)}\right) \nabla u^{(n)} \cdot \nabla \varphi\right) \mathrm{d} x \mathrm{~d} t \leq 0 .
$$

Lorsque $n \rightarrow \infty$, on a $\eta\left(u^{(n)}\right) \rightarrow \eta(u)$ et $\Phi\left(u^{(n)}\right) \rightarrow \Phi(u)$ dans $L^{2}(\Omega \times] 0, T[)$ et, avec $C_{\eta, A, B}=$ $\max \left\{\left|\eta^{\prime}(s)\right|, A \leq s \leq B\right\}$,

$$
\left.\left|\frac{1}{n} \int_{0}^{T} \int_{\Omega} \eta^{\prime}\left(u^{(n)}\right) \nabla u^{(n)} \cdot \nabla \varphi \mathrm{d} x \mathrm{~d} t\right| \leq C_{\eta, A, B} \frac{1}{\sqrt{n}}\left\|\frac{1}{\sqrt{n}}\left|\nabla u^{(n)}\right|\right\|_{L^{2}(\Omega \times] 0, T[}\right)\||\nabla \varphi|\|_{L^{2}(\Omega \times] 0, T[)} \rightarrow 0
$$

lorsque $n \rightarrow+\infty$.

On obtient ainsi, finalement,

$$
\int_{0}^{T} \int_{\Omega}(\eta(u)) \varphi_{t}+\boldsymbol{b} \cdot \Phi\left(u^{(n)}\right) \nabla \varphi \mathrm{d} x \mathrm{~d} t+\int_{\Omega} \eta\left(u_{0}\right) \varphi(x, 0) \mathrm{d} x \geq 0
$$

pour tout $\varphi \in C_{c}^{\infty}\left(\Omega \times\left[0, T\left[, \mathbb{R}_{+}\right)\right.\right.$, ce qui termine la preuve de l'existence.

\section{Etape 4. Unicité}

Soit $u$ une solution de 5.30 . On montre tout d'abord que l'on peut prendre $\varphi \in C_{c}^{\infty}\left(\mathbb{R}^{N} \times\left[0, T\left[, \mathbb{R}_{+}\right)\right.\right.$dans [5.30). C'est ici que l'hypothèse $\boldsymbol{b}=0$ sur le bord de $\Omega$ est utile.

On constuit une suite $\left(\varphi_{n}\right)_{n \in \mathbb{N}}$ appartenant à $C_{c}^{\infty}(\Omega)$ et telle que $\varphi_{n}=1$ sur $K_{n}=\left\{x \in \Omega ; d(x, \partial \Omega) \geq \frac{1}{n}\right\}$, $0 \leq \varphi_{n} \leq 1$ et $\left|\nabla \varphi_{n}\right| \leq C_{\Omega} n$, où $C_{\Omega}$ ne dépend que $\Omega$ (la régularité lipschitzienne de $\Omega$ est importante ici). Soit $\varphi \in C_{c}^{\infty}\left(\mathbb{R}^{N} \times\left[0, T\left[, \mathbb{R}_{+}\right)\right.\right.$, On prend alors $\varphi(x, t) \varphi_{n}(x)$ comme fonction test dans [5.30], on obtient

$$
\int_{0}^{T} \int_{\Omega}\left(\varphi_{n} \eta(u) \varphi_{t}+\varphi_{n} \Phi(u) \boldsymbol{b} \cdot \nabla \varphi\right) \mathrm{d} x \mathrm{~d} t+\int_{\Omega} \eta\left(u_{0}(x)\right) \varphi_{n}(x) \varphi(x, 0) \mathrm{d} x+\int_{0}^{T} \int_{\Omega} \boldsymbol{b} \Phi(u) \varphi \cdot \nabla \varphi_{n} \mathrm{~d} x \mathrm{~d} t \geq 0 .
$$

Les premiers termes convergent par convergence dominée. Appelons $R_{n}$ le dernier terme. On va montrer sa convergence assez facilement grâce au fait qu'on a supposé $\boldsymbol{b}$ nul sur le bord.

$$
R_{n}=\int_{0}^{T} \int_{\Omega} \boldsymbol{b} \Phi(u) \varphi \cdot \nabla \varphi_{n} \mathrm{~d} x \mathrm{~d} t=\int_{0}^{T} \int_{C_{n}} \boldsymbol{b} \Phi(u) \varphi \cdot \nabla \varphi_{n},
$$

où $C_{n}=\Omega \backslash K_{n}$. On a donc

$$
\left|R_{n}\right| \leq T\|\boldsymbol{b}\|_{L^{\infty}\left(C_{n}\right)} C_{u, \phi}\|\varphi\|_{\infty} C_{\Omega} n \operatorname{mes}\left(C_{n}\right),
$$


où $C_{u, \phi}=\max \{|\phi(s)|, s \in[-\gamma, \gamma]\}$, avec $\gamma=\|u\|_{L^{\infty}(\Omega \times] 0, T[)}$. Comme $\boldsymbol{b}=0$ sur $\partial \Omega \times[0, T]$ (et $\boldsymbol{b}$ continue), on a $\|\boldsymbol{b}\|_{L^{\infty}\left(C_{n}\right)} \rightarrow 0$ quand $n \rightarrow \infty$. Enfin, la suite $\left(n \operatorname{mes}\left(C_{n}\right)\right)_{n \in \mathbb{N}}$ est bornée. On a donc $\lim _{n \rightarrow+\infty} R_{n}=0$ et on obtient donc

$$
\int_{0}^{T} \int_{\Omega} \eta(u) \varphi_{t}+\boldsymbol{b} \Phi(u) \cdot \nabla \varphi \mathrm{d} x \mathrm{~d} t+\int_{\Omega} \eta\left(u_{0}\right) \varphi(x, 0) \mathrm{d} x \geq 0 \forall \varphi \in C_{c}^{\infty}\left(\mathbb{R}^{N} \times\left[0, T\left[, \mathbb{R}_{+}\right),\right.\right.
$$

pour tout $\eta \in C^{2}(\mathbb{R}, \mathbb{R}), \eta$ convexe.

Par un procédé de régularisation, il est alors assez facile de montrer que l'hypothèse de régularité sur $\eta$ ( c'est-àdire $\eta$ de classe $C^{2}$ ) peut être remplacée par l'hypothèse plus faible " $\eta$ localement lipschitzienne", ce qui a l'intérêt de pouvoir utiliser les entropies de Kruzhkov.

On peut maintenant montrer l'unicité de la solution de (5.30). Soient $u$ et $v$ deux solutions de (5.30). On va utiliser (5.30) en prenant pour $\eta$ une entropie de Kruzhkov et des fonctions $\varphi \in C_{c}^{\infty}\left(\mathbb{R}^{N} \times\left[0, T\left[, \mathbb{R}_{+}\right)\right.\right.$(on vient de montrer que cela est possible). On reprend ici une idée de Kruzhkov, dite de dédoublement de variables. Elle consiste tout d'abord à choisir, dans $5.30, k=v(y, s)$ et à prendre $\varphi(x, t)=\psi(t) \rho_{n}(x-y) \bar{\rho}_{n}(t-s)$ avec $\psi \in C_{c}^{\infty}\left(\left[0, T\left[, \mathbb{R}_{+}\right), \rho_{n}(x)=n^{N} \rho(n x)\right.\right.$ et $\bar{\rho}_{n}(t)=n \bar{\rho}(n t)$, où $\rho$ et $\bar{\rho}$ sont des noyaux régularisants, et à intégrer par rapport à $y$ et $s$. La fonction $\rho$ est à valeurs positives, elle est de classe $C^{\infty} \operatorname{sur} \mathbb{R}^{N}$, elle a son support dans la boule de rayon 1 et son intégrale sur $\mathbb{R}^{N}$ vaut 1 . De même, la fonction $\bar{\rho}$ est à valeurs positives, elle est de classe $C^{\infty}$ sur $\mathbb{R}$, elle a son support dans la boule de rayon 1 et son intégrale sur $\mathbb{R}$ vaut 1 . De plus, on choisit $\bar{\rho}$ de manière à ce que son support soit dans $\mathbb{R}_{-}$. Avec ce choix de fonction test (et $n$ assez grand pour que la fonction test soit admissible dans (5.30) $)$ écrit avec des éléments de $\varphi \in C_{c}^{\infty}\left(\mathbb{R}^{N} \times[0, T[, \mathbb{R})\right.$, on obtient :

$$
A_{1, n}+A_{2, n}+A_{3, n}+A_{4, n} \geq 0
$$

avec

$$
\begin{aligned}
& A_{1, n}=\int_{0}^{T} \int_{\Omega} \int_{0}^{T} \int_{\Omega}|u(x, t)-v(y, s)| \psi^{\prime}(t) \rho_{n}(x-y) \bar{\rho}_{n}(t-s) \mathrm{d} x \mathrm{~d} t \mathrm{~d} y \mathrm{~d} s, \\
& A_{2, n}=\int_{0}^{T} \int_{\Omega} \int_{0}^{T} \int_{\Omega}|u(x, t)-v(y, s)| \psi(t) \rho_{n}(x-y) \bar{\rho}_{n}^{\prime}(t-s) \mathrm{d} x \mathrm{~d} t \mathrm{~d} y \mathrm{~d} s, \\
& A_{3, n}=\int_{0}^{T} \int_{\Omega} \int_{0}^{T} \int_{\Omega}\left(f(u(x, t))-f(v(y, s))\left(\operatorname{sign}(u(x, t)-v(y, s)) \psi(t) \boldsymbol{b} \cdot \nabla \rho_{n}(x-y) \bar{\rho}_{n}(t-s) \mathrm{d} x \mathrm{~d} t \mathrm{~d} y \mathrm{~d} s,\right.\right. \\
& A_{4, n}=\int_{0}^{T} \int_{\Omega} \int_{\Omega}\left|u_{0}(x)-v(y, s)\right| \psi(0) \rho_{n}(x-y) \bar{\rho}_{n}(-s) \mathrm{d} x \mathrm{~d} y d s .
\end{aligned}
$$

On passe maintenant à limite quand $n \rightarrow+\infty$ dans (5.37). Il n'est pas difficile de montrer que

$$
\left.\lim _{n \rightarrow+\infty} A_{1, n}=\int_{0}^{T} \int_{\Omega} \mid u(x, t)-v(x, t)\right) \mid \psi^{\prime}(t) \mathrm{d} x \mathrm{~d} t .
$$

On montre ensuite que $A_{2, n}+A_{3, n} \leq 0$. Pour cela, on considère la formulation entropique pour $v$, écrite avec $y$ et $s$ comme variables. On choisit l'entropie de Kruzhkov associée à $k=u(x, t)$ et $\varphi(y, s)=\psi(t) \rho_{n}(x-y) \bar{\rho}_{n}(t-s)$. Enfin, en intégrant par rapport à $x \in \Omega$ et $t \in \mathbb{R}_{+}$, on obtient

$$
\begin{gathered}
-\int_{0}^{T} \int_{\Omega} \int_{0}^{T} \int_{\Omega}|v(y, s)-u(x, t)| \psi(t) \rho_{n}(x-y) \bar{\rho}_{n}^{\prime}(t-s) \mathrm{d} y \mathrm{~d} s \mathrm{~d} x \mathrm{~d} t \\
-\int_{0}^{T} \int_{\Omega} \int_{0}^{T} \int_{\Omega}\left(f(v(y, s))-f(u(x, t))\left(\operatorname{sign}(v(y, s)-u(x, t)) \psi(t) \boldsymbol{b} \cdot \nabla \rho_{n}(x-y) \bar{\rho}_{n}(t-s) \mathrm{d} y \mathrm{~d} s \mathrm{~d} x \mathrm{~d} t \geq 0 .\right.\right.
\end{gathered}
$$


Ce qui donne $A_{2, n}+A_{3, n} \leq 0$. On notera que le terme associé à la condition initiale est nul car $\bar{\rho}_{n}(t)=0$ si $t \geq 0$. Il suffit maintenant de montrer que $\lim _{n \rightarrow+\infty} A_{4, n}=0$ pour conclure en passant à limite dans (5.37) que

$$
\int_{0}^{T} \int_{\Omega}|u(x, t)-v(x, t)| \psi^{\prime}(t) \mathrm{d} x \mathrm{~d} t \geq 0
$$

Pour montrer que $\lim _{n \rightarrow+\infty} A_{4, n}=0$, on reprend la formulation entropique pour $v$ écrite avec $y$ et $s$ comme variables. On choisit l'entropie de Kruzhkov associée à $k=u_{0}(x)$ et $\varphi(y, s)=\psi(0) \rho_{n}(x-y) \int_{s}^{\infty} \bar{\rho}_{n}(-\tau) d \tau$ (avec $n$ assez grand pour cette fonction test $\varphi$ soit admissible). Enfin, on intègre par rapport à $x \in \Omega$. On obtient

$$
\begin{array}{r}
-A_{4, n}-\int_{0}^{T} \int_{\Omega} \int_{\Omega}\left(f(v(y, s))-f\left(u_{0}(x)\right)\left(\operatorname{sign}\left(v(y, s)-u_{0}(x)\right) \psi(0) \boldsymbol{b} \cdot \nabla \rho_{n}(x-y)\left(\int_{s}^{\infty} \bar{\rho}_{n}(-\tau) d \tau\right) \mathrm{d} y \mathrm{~d} s \mathrm{~d} x\right.\right. \\
+\int_{\Omega} \int_{\Omega}\left|u_{0}(y)-u_{0}(x)\right| \psi(0) \rho_{n}(x-y) \mathrm{d} x \mathrm{~d} y \geq 0 .
\end{array}
$$

On a donc

$$
0 \leq A_{4, n} \leq A_{5, n}+A_{6, n}
$$

avec

$A_{5, n}=-\int_{0}^{T} \int_{\Omega} \int_{\Omega}\left(f(v(y, s))-f\left(u_{0}(x)\right)\left(\operatorname{sign}\left(v(y, s)-u_{0}(x)\right) \psi(0) \boldsymbol{b} \cdot \nabla \rho_{n}(x-y)\left(\int_{s}^{\infty} \bar{\rho}_{n}(-\tau) d \tau\right) \mathrm{d} y \mathrm{~d} s \mathrm{~d} x\right.\right.$,

$A_{6, n}=\int_{\Omega} \int_{\Omega}\left|u_{0}(y)-u_{0}(x)\right| \psi(0) \rho_{n}(x-y) \mathrm{d} x \mathrm{~d} y$.

Il n'est pas difficile de montrer que $\lim _{n \rightarrow+\infty} A_{5, n}=\lim _{n \rightarrow+\infty} A_{6, n}=0$. On en déduit que $\lim _{n \rightarrow+\infty} A_{4, n}=0$ et, finalement, on obtient 5.38.

On peut maintenant conclure. Soit $0<\varepsilon<T$, on choisit $\psi \in C_{c}^{\infty}\left(\left[0, T\left[, \mathbb{R}_{+}\right)\right.\right.$t.q. $\psi^{\prime}<0$ sur $\left.] 0, T-\varepsilon\right]$. L'inégalité [5.38 donne alors $u=v$ p.p. sur $\Omega \times] 0, T-\varepsilon[$. Comme $\varepsilon$ est arbitrairement petit, on en conclut que $u=v$ p.p. sur $\Omega \times] 0, T[$, ce qui termine la preuve de l'unicité.

Remarque 5.26 (Pour le cas où $\Omega$ est non borné) Dans la partie “unicité" (Etape 4) de la démonstration précédente, il aurait été possible de prendre une fonction $\psi$ dépendant aussi de $x$. On aurait alors obtenu

$$
\int_{0}^{T} \int_{\Omega}|u-v| \psi_{t} \mathrm{~d} x \mathrm{~d} t+\int_{0}^{T} \int_{\Omega} \boldsymbol{b}\left(f(u)-f(v) \operatorname{sign}(u-v) \cdot \nabla \psi \mathrm{d} x \mathrm{~d} t \geq 0 \forall \psi \in C_{c}^{\infty}\left(\mathbb{R}^{N} \times\left[0, T\left[, \mathbb{R}_{+}\right) .\right.\right.\right.
$$

Ceci est intéressant pour montrer alors l'unicité dans le cas où l'ouvert $\Omega$ est non borné (par exemple, $\Omega=\mathbb{R}^{N}$ ) en profitant de la propriété de "propagation à vitesse finie" pour les problèmes hyperboliques. Plus précisément, on prend dans 5.39)

$$
\psi(x, t)=r(t) \varphi_{a}(|x|+\omega t) \operatorname{avec} \omega=L_{f}\|\boldsymbol{b}\|_{\infty},
$$

où $L_{f}$ est un majorant de $\left|f^{\prime}\right|$ sur l'intervalle $[-\gamma, \gamma]$, avec

$$
\begin{aligned}
& \gamma=\max \left\{\|u\|_{\infty},\|v\|_{\infty}\right\}, r(t)=(1 / T)(T-t)^{+} \\
& \varphi_{a} \in C_{c}^{\infty}\left(\left[0, \infty\left[, \mathbb{R}_{+}\right), \text {avec } \varphi_{a}=1 \text { sur }[0, a] \text { avec } a>0 \text { donné et } \varphi_{a}\right.\right. \text { décroissante }
\end{aligned}
$$


on peut remarquer qu' un argument simple de régularisation autorise à prendre une telle fonction $\psi$ dans (5.39). On obtient alors

$$
\begin{array}{rl}
-\frac{1}{T} \int_{0}^{T} \int_{\mathbb{R}^{N}}|u-v| \varphi_{a}(|x|+\omega t) \mathrm{d} & x \mathrm{~d} t+\int_{0}^{T} \int_{\mathbb{R}^{N}}|u-v| r(t) \varphi_{a}^{\prime}(|x|+\omega t) \omega \mathrm{d} x \mathrm{~d} t \\
& +\int_{0}^{T} \int_{\mathbb{R}^{N}} \boldsymbol{b}(f(u)-f(v)) \operatorname{sign}(u-v) r(t) \varphi_{a}^{\prime}(|x|+\omega t) \frac{x}{|x|} d x \mathrm{~d} t \geq 0 .
\end{array}
$$

Mais

$$
\begin{aligned}
\int_{0}^{T} \int_{\mathbb{R}^{N}} \boldsymbol{b}\left(f(u)-f(v) \operatorname{sign}(u-v) r(t) \varphi_{a}^{\prime}(|x|+\omega t) \frac{x}{|x|} \leq\right. & -\int_{0}^{T} \int_{\mathbb{R}^{N}}\|\boldsymbol{b}\| L_{f}|u-v| r(t) \varphi_{a}^{\prime}(|x|+\omega t) \mathrm{d} x \mathrm{~d} t \\
& \leq-\int_{0}^{T} \int_{\mathbb{R}^{N}}|u-v| r(t) \varphi_{a}^{\prime}(|x|+\omega t) \omega d x d t,
\end{aligned}
$$

$\operatorname{car} \omega=L_{f}\|\boldsymbol{b}\|$. On a donc

$$
-\frac{1}{T} \int_{0}^{T} \int_{\mathbb{R}^{N}}|u-v| \varphi_{a}(|x|+\omega t) \mathrm{d} x \mathrm{~d} t \geq 0 .
$$

On en déduit, avec $B_{a, t}=\{x$ t.q. $|x|+\omega t \leq a\}$ que $\int_{0}^{T}\left(\int_{B_{a, t}}|u-v| \mathrm{d} x\right) d t=0$. On fait tendre maintenant $a$ vers $+\infty$. On obtient, par convergence monotone, $\int_{0}^{T} \int_{\Omega}|u-v| \mathrm{d} x \mathrm{~d} t=0$ et donc $u=v$ p.p. $\left.\operatorname{sur} \Omega \times\right] 0, T[$.

\section{Remarque 5.27 (hypothèses sur $b$ )}

1. On a utilisé la régularité $C^{1}$ de $\boldsymbol{b}$ pour obtenir l'estimation $B V$ sur les solutions approchées. Si on n'utilise pas l'estimation $B V$, on utilise quand même la régularité $C^{1}$ de $\boldsymbol{b}$ pour l'unicité. En fait, on peut remarquer que les démonstrations de l'estimation $B V$ et de l'unicité restent justes dés que $\boldsymbol{b}$ est localement lipschitzienne.

2. On a supposé $\operatorname{div} \boldsymbol{b}=0$. On pourrait remplacer cette hypothèse par $\operatorname{div} \boldsymbol{b} \in L^{\infty}$ à condition de supposer que $f$ soit lipschitzienne. On a aussi supposé que $\boldsymbol{b}=0$ sur $\partial \Omega$ (pour ne pas traiter le cas, difficile, des conditions aux limites) mais on pourrait remplacer cette condition par $\boldsymbol{b} \cdot \boldsymbol{n}=0$ sans grande difficulté supplémentaire. Le problème des conditions aux limites interviendrait si $\boldsymbol{b} \cdot \boldsymbol{n} \neq 0$.

\subsubsection{Cas des conditions aux limites}

Cette section est consacrée à une généralisation du théorème 5.21 dans le cas scalaire multidimensionnel ainsi qu'à une esquisse de preuve. Nous considérons le problème (5.29) et ne supposons plus que $\boldsymbol{b}=0$ sur la frontière.

Définition 5.28 (Solution faible entropique, avec conditions limites, [24]) Soit $\Omega$ un sous-ensemble ouvert borné de $\mathbb{R}^{N}(N \geq 1)$ avec une frontière de Lipschitz. Soit $T>0, f \in C^{1}(\mathbb{R}, \mathbb{R})$ (ou $f: \mathbb{R} \rightarrow \mathbb{R}$ lipschitzienne) et $\boldsymbol{b} \in C^{1}(\bar{\Omega} \times[0, T])^{N}$. Soit $u_{0} \in L^{\infty}(\Omega)$ et $\bar{u} \in L^{\infty}(\partial \Omega \times(0, T))$. Soient $A, B \in \mathbb{R}$ tels que $A \leq u_{0} \leq B$ a.e. sur $\Omega$ et $A \leq \bar{u} \leq B$ p.p. sur $\partial \Omega \times(0, T)$.

Une fonction $u: \Omega \times(0, T) \rightarrow \mathbb{R}$ est une solution entropique faible de (5.29) satisfaisant (faiblement) la condition limite $\bar{u}$ si

$$
\begin{aligned}
& u \in L^{\infty}(\Omega \times(0, T)) \text { and } \forall \kappa \in[A, B], \forall \varphi \in C_{c}^{1}\left(\bar{\Omega} \times[0, T), \mathbb{R}_{+}\right), \\
& \int_{0}^{T} \int_{\Omega}\left[(u-\kappa)^{ \pm} \varphi_{t}+\operatorname{sign}_{ \pm}(u-\kappa)(f(u)-f(\kappa)) \boldsymbol{b} \cdot \operatorname{grad} \varphi\right] \mathrm{d} x \mathrm{~d} t \\
& +M \int_{0}^{T} \int_{\partial \Omega}(\bar{u}(t)-\kappa)^{ \pm} \varphi(x, t) d \gamma(x) \mathrm{d} t+\int_{\Omega}\left(u_{0}-\kappa\right)^{ \pm} \varphi(x, 0) \mathrm{d} x \geq 0,
\end{aligned}
$$


où $d \gamma(x)$ représente l'intégration par rapport à la mesure de Lebesgue $(N-1)$-dimensionnelle sur la frontière de $\Omega$ et $M$ est tel que $\|\boldsymbol{b}\|_{\infty}\left|f\left(s_{1}\right)-f\left(s_{2}\right)\right| \leq M\left|s_{1}-s_{2}\right|$ pour tous $s_{1}, s_{2} \in[A, B]$, où $\|\boldsymbol{b}\|_{\infty}=$ $\sup _{(x, t) \in \Omega \times[0, T]}|\boldsymbol{b}(x, t)|\left(\right.$ et $|\cdot|$ désigne ici la norme euclidienne dans $\mathbb{R}^{2}$ ).

\section{Remarque 5.29}

1. Si u satisfait la famille d'inégalités (5.40), on peut prouver que u est une solution d'une forme faible de 5.29 et qu'elle satisfait certaines inégalités d'entropie dans $\Omega \times(0, T)$, à savoir

$$
|u-\kappa|_{t}+\operatorname{div}(\boldsymbol{b}(f(\max (u, \kappa))-f(\min (u, \kappa)))) \leq 0 \text { pour tout } \kappa \text { dans } \mathbb{R},
$$

mais aussi sur la frontière $\partial \Omega$ et au temps $t=0$. La solution faible entropique u satisfait la condition initiale $\left(u(\cdot, 0)=u_{0}\right)$ et satisfait partiellement la condition aux limites. Par exemple, si $f^{\prime}>0$ et que $u$ et $\Omega$ sont suffisamment réguliers, alors $u(x, t)=\bar{u}(x, t)$ si $x \in \partial \Omega, t \in(0, T)$ et $\boldsymbol{b}(x, t) \cdot \boldsymbol{n}(x, t)<0$, où $\boldsymbol{n}$ est le vecteur normal extérieur à $\partial \Omega$.

2. Soit $\bar{M} \geq 1$. Il est intéressant de remarquer que u est une solution de (5.40) si et seulement si u est solution de [5.40] où le terme $\int_{\Omega}\left(u_{0}-\kappa\right)^{ \pm} \varphi(x, 0) \mathrm{d} x$ est remplacé par $\bar{M} \int_{\Omega}\left(u_{0}-\kappa\right)^{ \pm} \varphi(x, 0) \mathrm{d} x$.

Théorème 5.30 (Existence et unicité, Otto, 1996) Sous les hypothèses de la définition 5.28. supposons que divb = 0 dans $\Omega \times[0, T]$; alors il existe une solution entropique unique $u \in L^{\infty}\left(\mathbb{R} \times \mathbb{R}_{+}\right)$satisfaisant $[5.40$. De plus,

$$
A \leq u(x, t) \leq B \text { p.p. }(x, t) \in] 0,1\left[\times \mathbb{R}_{+} .\right.
$$

Démonstration Nous ne donnons ici qu'une esquisse de la preuve dans le cas $\Omega$ un sous-ensemble ouvert borné polygonal (ou polyédrique) de $\mathbb{R}^{N}$; cette preuve est basée sur la convergence des approximations numériques [31].

ÉTAPE 1 : SOLUTIONS APPROCHÉES. En considérant un maillage assez général de $\Omega$ (avec des triangles, par exemple dans le cas bidimensionnel), noté $\mathcal{T}$, et un pas de temps $k$, une solution approchée $u_{\mathcal{T}, k}$ du problème 5.29 peut être définie en utilisant des flux numériques à deux points (sur les bords des mailles) construits avec une fonction de flux numérique $g$ telle que

- $g$ est croissante par rapport à son premier argument et décroissante par rapport à son second argument,

- $g(s, s)=f(s)$, pour tout $s \in[A, B]$,

- $g$ est localement lipschitzienne (ou localement "Lip-diag", voir [16, Définition 3.1]).

Sous une condition dite de CFL, du type $k \leq(1-\zeta) \frac{h}{L}$ avec $\zeta>0$, il est facile de prouver que

$$
A \leq u_{\mathcal{T}, k} \leq B \text { a.e. on } \Omega \times(0, T) .
$$

Malheureusement, il ne semble pas facile d'obtenir directement un résultat de compacité forte sur la famille des solutions approximatives (bien que ce résultat de compacité forte soit vrai, comme nous le verrons plus loin).

ÉTAPE 2 : COMPACITÉ FAIBLE. En utilisant seulement cette borne $L^{\infty}$ sur $u_{\mathcal{T}, k}$, on peut supposer (à une soussuite près) que $u_{\mathcal{T}, k} \rightarrow u$, lorsque le pas du maillage tend vers 0 (avec la condition CFL), dans un "sens non linéaire faible" (similaire à la convergence vers une mesure de Young, voir [13, Section 30] par exemple), c'est-àdire $u \in L^{\infty}(\Omega \times(0, T) \times(0,1))$ et

$$
\begin{array}{r}
\int_{0}^{T} \int_{\Omega} \phi\left(u_{\mathcal{T}, k}(x, t)\right) \varphi(x, t) \mathrm{d} x \mathrm{~d} t \rightarrow \int_{0}^{1} \int_{0}^{T} \int_{\Omega} \phi(u(x, t, \alpha)) \varphi(x, t) \mathrm{d} x \mathrm{~d} t \mathrm{~d} \alpha, \\
\text { for all } \varphi \in L^{1}(\Omega \times(0, T)) \text { and all } \phi \in C(\mathbb{R}, \mathbb{R}) .
\end{array}
$$


ÉTAPE 3 : PASSAGE À LA LIMITE. En utilisant la monotonie des flux numériques, les solutions approchées satisfont certaines inégalités d'entropie discrète. En passant à la limite sur ces inégalités, on obtient que $u$ (définie à l'étape 2) satisfait certaines inégalités très similaires à (5.40), à savoir :

$$
\begin{array}{r}
u \in L^{\infty}(\Omega \times(0, T) \times(0,1)), \\
\int_{0}^{1} \int_{0}^{T} \int_{\Omega}\left[(u-\kappa)^{ \pm} \varphi_{t}+\operatorname{sign}_{ \pm}(u-\kappa)(f(u)-f(\kappa)) \boldsymbol{b} \cdot \operatorname{grad} \varphi\right] \mathrm{d} x \mathrm{~d} t \mathrm{~d} \alpha \\
+M \int_{0}^{T} \int_{\partial \Omega}(\bar{u}(t)-\kappa)^{ \pm} \varphi(x, t) d \gamma(x) \mathrm{d} t \\
\quad+\int_{\Omega}\left(u_{0}-\kappa\right)^{ \pm} \varphi(x, 0) \mathrm{d} x \geq 0, \\
\forall \kappa \in[A, B], \forall \varphi \in C_{c}^{1}\left(\bar{\Omega} \times[0, T), \mathbb{R}_{+}\right),
\end{array}
$$

Nous choisissons ici $M$ non seulement plus grand que la constante de Lipschitz de $\|\boldsymbol{b}\|_{\infty} f$ sur $[A, B]$, mais aussi plus grand que la constante de Lipschitz (sur $[A, B]^{2}$ ) des flux numériques associés aux bords des mailles. Ce choix de $M$ est possible car la solution unique de $(5.40)$ ne dépend pas de $M$ à condition que $M$ soit supérieur à la constante de Lipschitz de $\|\boldsymbol{b}\|_{\infty} f$ sur $[A, B]$ et car la fonction de flux numérique peut être choisie avec une constante de Lipschitz bornée par la constante de Lipschitz de $\|\boldsymbol{b}\|_{\infty} f$ (le flux de Godunov par exemple). Cette méthode conduit à un résultat d'existence avec $M$ seulement supérieur à la constante de Lipschitz de $\|\boldsymbol{b}\|_{\infty} f$ sur $s \in[A, B]$, en passant à la limite sur les solutions approchées données avec ces flux numériques.

ÉTAPE 4 : Unicité DE LA SOlution De (5.41). Dans cette étape, la méthode de "dédoublement de variables" de Krushkov est utilisée pour prouver l'unicité de la solution de (5.41). En effet, si $u$ et $w$ sont deux solutions de (5.41), la méthode de dédoublement de variables conduit à :

$$
\begin{aligned}
& \int_{0}^{1} \int_{0}^{1} \int_{0}^{T} \int_{\Omega}|u(x, t, \alpha)-w(x, t, \beta)| \varphi_{t} \mathrm{~d} x \mathrm{~d} t \mathrm{~d} \alpha \mathrm{d} \beta \\
& +\int_{0}^{1} \int_{0}^{1} \int_{0}^{T^{2}} \int_{\Omega}(f(\max (u, w))-f(\min (u, w))) \boldsymbol{b} \cdot \operatorname{grad} \varphi \mathrm{d} x \mathrm{~d} t \mathrm{~d} \alpha \mathrm{d} \beta \geq 0 \\
& \forall \varphi \in C_{c}^{1}\left(\bar{\Omega} \times[0, T), \mathbb{R}_{+}\right),
\end{aligned}
$$

En prenant $\varphi(x, t)=(T-t)^{+}$dans 5.42 (ce qui est en effet possible), on obtient que $u$ ne dépend pas de $\alpha, w$ ne dépend pas de $\beta$ et $u=w$ a.e. sur $\Omega \times(0, T)$. Par conséquent, $u$ est également la solution unique de 5.40).

ÉTAPE 5 : CONCLUSION. L'étape 4 donne, en particulier, l'unicité de la solution de 5.40 . Elle donne également que la limite faible- $\star$ non linéaire des suites de solutions approchées est solution de (5.40) et, par conséquent, l'existence de la solution de $(5.40)$.

De plus, puisque la limite faible non linéaire des suites de solutions approchées ne dépend pas de $\alpha$, il est assez facile de déduire que cette limite est "forte" dans $L^{p}(\Omega \times(0, T))$ pour tout $p \in[1, \infty)$ (voir [13], par exemple); grâce à l'unicité de la limite, la convergence a lieu sans extraction de sous-suite.

\subsection{Systèmes hyperboliques}

\subsubsection{Définitions}

On s'intéresse dans ce paragraphe aux systèmes hyperboliques dans le cas unidimensionnel, c'est-à-dire que la variable dite "d'espace", généralement notée $x$, appartient à $\mathbb{R}$ (et la variable dite "de temps", notée $t$, appartient à $\mathbb{R}_{+}$) et référons à [7] pour l'étude des systèmes multidimensionnels.

Soit $p \in \mathbb{N}$ le nombre d'équations (scalaires) aux dérivées partielles du système considéré ( $p=1$ dans le cas des équations scalaires considérées dans les paragraphes précédents) et $D$ le domaine de $\mathbb{R}^{p}$ dans lequel l'inconnue 
vectorielle de ce système de $p$ équations prend ses valeurs ( $D$ est dit "domaine des valeurs admissibles"). Soit $F \in C^{1}\left(D, \mathbb{R}^{p}\right)$ et $U_{0} \in\left(L^{\infty}(\mathbb{R})\right)^{p}$, à valeurs dans $D$, ce que noterons parfois $L^{\infty}(\mathbb{R} ; D)$; on cherche une fonction vectorielle $U: \mathbb{R} \times \mathbb{R}_{+} \rightarrow D$ solution, en un sens à définir, du système

$$
\begin{aligned}
& \partial_{t} U+\partial_{x}(F(U))=0, x \in \mathbb{R}, t \in \mathbb{R}_{+}, \\
& U(x, 0)=U_{0}(x), x \in \mathbb{R} .
\end{aligned}
$$

Par exemple, dans le cas des des équations d'Euler pour un écoulement compressible isentropique, on a $p=2$, $U=\left[\begin{array}{c}\rho \\ \rho u\end{array}\right]$ où $\rho$ est la masse volumique $(\rho>0)$ et $u$ la vitesse ( $\rho u$ est donc la quantité de mouvement) et donc le domaine des valeurs admissibles est $D=\mathbb{R}_{+} \times \mathbb{R} \subset \mathbb{R}^{2}$. La fonction $F$ est donnée par $F(U)=\left[\begin{array}{c}\rho u \\ \left(\rho u^{2}+p\right)\end{array}\right]$, avec $p=\rho^{\gamma}$, où $\gamma>1$ est un nombre réel donné.

Le système (5.43) n'est pas toujours bien posé, et sa nature hyperbolique dépend des valeurs propres de la matrice jacobienne de $F$, ce que l'on précise dans la définition qui suit.

Définition 5.31 (Système hyperbolique et strictement hyperbolique) Soient $p>1, D$ un domaine de $\mathbb{R}^{p}$ et $F \in C^{1}\left(D, \mathbb{R}^{p}\right)$. Le système (5.43a) est dit :

- "hyperbolique" (plus précisément "hyperbolique dans $D$ ") si, pour tout $U \in D$, la matrice jacobienne $J_{F}(U) \in \mathcal{M}_{p}(\mathbb{R})$ de l'application $F$ au point $U$ est diagonalisable dans $\mathbb{R}$ (rappelons que les coefficients de la matrice jacobienne de $F$ sont $\left.\left(J_{F}(U)\right)_{i, j}=\partial_{j} F_{i}(U)\right)$;

- "strictement hyperbolique" si, pour tout $U \in D$, la matrice jacobienne $J_{F}(U)$ admet p valeurs propres réelles distinctes.

L'exemple le plus simple de système est le cas linéaire : $F(U)=A U$ où $A \in \mathcal{M}_{p}(\mathbb{R})$ et $D=\mathbb{R}^{p}$. D'après la définition 5.31, le système (5.43a) est alors hyperbolique si $A$ est diagonalisable dans $\mathbb{R}$, c'est-à-dire s'il existe une base $\left(\varphi_{1}, \ldots, \varphi_{p}\right)$ de $\mathbb{R}^{p}$ et une famille $\left(\lambda_{1}, \ldots, \lambda_{p}\right) \in \mathbb{R}^{p}$ telles que $A \varphi_{i}=\lambda_{i} \varphi_{i}$ pour tout $i=1, \ldots, p$. Dans ce cas, on peut décomposer la condition initiale $U_{0}$ sur la base $\left(\varphi_{1}, \ldots, \varphi_{p}\right)$; en notant $\alpha_{1}, \ldots, \alpha_{p}$ les composantes de $U_{0}$ dans cette base, on a donc :

$$
U_{0}(x)=\sum_{i=1}^{p} \alpha_{i}(x) \varphi_{i}
$$

et l'unique solution faible du système (5.43) sécrit alors

$$
U(x, t)=\sum_{i=1}^{p} \alpha_{i}\left(x-\lambda_{i} t\right) \varphi_{i}
$$

voir à ce sujet l'exercice 5.2 .

\subsubsection{Solutions faibles, solutions entropiques}

On se place dans ce paragraphe dans le cadre de la definition 5.31. Comme dans le cas scalaire (qui en est de fait un exemple particulier), le problème (5.43) n'admet en général pas de solution classique (c'est-à-dire une fonction régulière qui satisfait la condition initiale (5.43b) et le système (5.43a). On définit donc la notion de solution faible, qui revient, comme dans le cas scalaire (voir définition [5.6, à porter les dérivées sur les fonctions test. 
Définition 5.32 (Solution faible d'un système hyperbolique) Soient $p \geq 1, U_{0} \in\left(L^{\infty}(\mathbb{R})\right)^{p}$ une fonction (vectorielle) de $\mathbb{R}$ à valeurs dans le domaine $D$ de $\mathbb{R}^{p}$ et $F \in C^{1}\left(D, \mathbb{R}^{p}\right)$. On dit que $U$ est solution faible du système (5.43) si $U \in\left(L^{\infty}\left(\mathbb{R} \times \mathbb{R}_{+} ; D\right)\right)$ et vérifie

$$
\begin{aligned}
\iint_{\mathbb{R} \times \mathbb{R}_{+}} U(x, t) \partial_{t} \varphi(x, t) \mathrm{d} x \mathrm{~d} t+\iint_{\mathbb{R} \times \mathbb{R}_{+}} F(U(x, t)) \partial_{x} \varphi(x, t) \mathrm{d} x \mathrm{~d} t \\
\quad+\int_{\mathbb{R}} U_{0}(x) \varphi(x, 0) \mathrm{d} x=0, \forall \varphi \in C_{c}^{1}\left(\mathbb{R} \times \mathbb{R}_{+} ; \mathbb{R}\right) .
\end{aligned}
$$

Considérons par exemple le cas d'un problème de Riemann, c'est-à-dire le système 5.43 avec comme condition initiale

$$
U_{0}=\left\{\begin{array}{l}
U_{g} \text { pour } x<0, \\
U_{d} \text { pour } x>0,
\end{array}\right.
$$

avec $U_{g}, U_{d} \in D$. Comme dans le cas scalaire ( $p=1$ ), on peut montrer qu'une fonction de la forme, avec $\sigma \in \mathbb{R}$,

$$
U(x, t)=\left\{\begin{array}{l}
U_{g} \text { pour } x<\sigma t, \\
U_{d} \text { pour } x>\sigma t,
\end{array}\right.
$$

est solution faible si et seulement si la relation de Rankine et Hugoniot est vérifée, c'est-à-dire si et seulement si

$$
\sigma[U]=[F(U)]
$$

(on rappelle que $[U]=U_{d}-U_{g}$ désigne le saut de $U$ ). La démonstration de cette équivalence est ici identique à cette faite pour $p=1$ car elle se fait en raisonnant avec chacune des $p$ équations scalaires du système (indépendamment des autres équations).

Voyons maintenant le cas du problème de Riemann pour un système hyperbolique linéaire, c'est-à-dire pour le système $(5.43)$ avec $F(U)=A U$, où $A$ est une matrice diagonalisable dans $\mathbb{R}$, et avec une condition initiale de la forme (5.44). Soient $\lambda_{1}, \ldots, \lambda_{p}$ les valeurs propres (réelles) de $A$ et $\varphi_{1}, \ldots, \varphi_{p}$ une base (de $\mathbb{R}^{p}$ ) de vecteurs propres associés. On décompose $U_{0}$ sur la base des vecteurs propres :

$$
U_{0}=\sum_{i=1}^{p} \alpha_{i} \varphi_{i} \text { avec } \alpha_{i}=\left\{\begin{array}{l}
\alpha_{g, i} \text { pour } x<0, \\
\alpha_{d, i} \text { pour } x>0 .
\end{array}\right.
$$

On peut alors montrer (voir exercice 5.2 que la fonction $U$ définie par

$$
U(x, t)=\sum_{i=1}^{p} \alpha_{i}\left(x-\lambda_{i} t\right) \varphi_{i}
$$

est l'unique solution faible. Examinons un peu la structure de cette solution : elle est formée de $p$ états constants, et on change d'état à chaque fois que l'on traverse une droite $x=\lambda_{i} t$, puisque

$$
\alpha_{i}\left(x-\lambda_{i} t\right)=\left\{\begin{array}{l}
\alpha_{g, i} \text { pour } x<\lambda_{i} t, \\
\alpha_{d, i} \text { pour } x>\lambda_{i} t .
\end{array}\right.
$$

Revenons au cas général du système (5.43). Comme dans le cas scalaire, pour un système non linéaire, on n'a pas unicité des solutions faibles. On imite le cas scalaire et on introduit la notion de solution entropique. Pour cela, on commence par définir la notion d'entropie pour un système hyperbolique. 
Définition 5.33 (Entropie et flux d'entropie) Soient $p>1, D$ un domaine de $\mathbb{R}^{p}$ et $F \in C^{1}\left(D, \mathbb{R}^{p}\right)$. Une fonction $\eta$ de $D$ dans $\mathbb{R}$ est une entropie pour le système (5.43) si

1. $\eta \in C^{1}(D, \mathbb{R})$ et $\eta$ est convexe;

2. il existe une fonction $\Phi \in C^{1}(D, \mathbb{R})$, appelée flux d'entropie, telle que, pour tout $U \in D$,

$$
\nabla \Phi(U)=J_{F}(U)^{t} \nabla \eta(U),
$$

ce qui s'écrit encore, composante par composante :

$$
\partial_{i} \Phi(U)=\sum_{j=1}^{p} \partial_{i} F_{j}(U) \partial_{j} \eta(U) .
$$

Existe-t-il des entropies? La réponse est évidemment oui, il suffit de prendre $\eta$ et $\Phi$ constantes (ce qui ne donne pas beaucoup d'information sur les solutions), ou encore $\eta(U)=U_{i}$ et $\Phi(U)=F_{i}(U)$ (ce qui nous ramène aux solutions faibles). Ce sont des entropies dites "triviales". En existe-t-il des non triviales? La réponse est différente selon la valeur de $p$ :

$-p=1$ (cas scalaire). Toute fonction $\eta$ convexe est une entropie, et le flux associé est une primitive de $\eta^{\prime} F^{\prime}$.

$-p=2$. Des entropies non triviales existent (voir, par exemple, [26]).

$-p \geq 3$. Pour un système hyperbolique quelconque, il n'existe pas en général pas d'entropie (autre que les entropies triviales). Toutefois, de nombreux systèmes modélisant des phénomènes physiques possèdent une entropie, bien connue de la physicienne (et celle ci l'indique généreusement à la mathématicienne).

Définition 5.34 (Solution entropique d'un système hyperbolique) Soit $U_{0} \in\left(L^{\infty}(\mathbb{R})\right)^{p}$ une fonction (vectorielle) de $\mathbb{R}$ à valeurs dans $D \subset \mathbb{R}^{p}$. On dit que $U$ est solution entropique du système (5.43) si $U \in L^{\infty}(\mathbb{R} \times$ $\left.\mathbb{R}_{+} ; D\right)$ et vérifie

$$
\begin{aligned}
& \iint_{\mathbb{R}_{\times} \mathbb{R}_{+}} \eta(U(x, t)) \partial_{t} \varphi(x, t) \mathrm{d} x \mathrm{~d} t+\iint_{\mathbb{R} \times \mathbb{R}_{+}} \Phi(U(x, t)) \partial_{x} \varphi(x, t) \mathrm{d} x \mathrm{~d} t \\
& +\int_{\mathbb{R}} \eta\left(U_{0}(x)\right) \varphi(x, 0) \mathrm{d} x \geq 0, \forall \varphi \in C_{c}^{1}\left(\mathbb{R} \times \mathbb{R}_{+} ; \mathbb{R}_{+}\right), \text {pour }(\eta, \Phi) \text { entropie et flux d'entropie associé. }
\end{aligned}
$$

Notons qu'une solution entropique est forcément une solution faible. Il suffit pour s'en convaincre de prendre $\eta$ linéaire.

Comme dans le cas $p=1$, on peut montrer qu'une solution de la forme, avec $U_{g}, U_{d} \in D, \sigma \in \mathbb{R}$,

$$
U(x, t)=\left\{\begin{array}{l}
U_{g} \text { pour } x<\sigma t, \\
U_{d} \text { pour } x>\sigma t .
\end{array}\right.
$$

est solution entropique si et seulement si, pour toute entropie $\eta$ de flux associé $\Phi$, les conditions de RankineHugoniot

$$
\begin{array}{ll}
\text { (i) } & \sigma[U]=[F(U)], \\
\text { (ii) } & \sigma[\eta(U)] \geq[\Phi(U)],
\end{array}
$$

sont vérifiées (on rappelle que $[V]=V_{d}-V_{g}$ ). Notons que $(i i) \Rightarrow(i)$. La démonstration de cette équivalence est ici aussi identique à cette faite pour $p=1$ car $(i i)$ est une équation scalaire (et $(i)$ est équivalent à dire que $U$ est solution faible). 
Y a-til existence et unicité de la solution entropique? On a vu le théorème de Kruzhkov qui nous permet de répondre par l'affirmative dans le cas $p=1$. Dans le cas $p>1$ la situation est, bien sûr, plus complexe parce qu'un système strictement hyperbolique n'admet pas toujours une entropie (non triviale). On utilise alors, en particulier lors de l'étude du problème de Riemann (section 5.3.3), une condition introduite par P. D. Lax dite "condition d'entropie de Lax" ou "condition de Lax". Dans le cas $p=1$ et d'une équation strictement hyperbolique, c'està-dire que $F$ est strictement convexe ou strictement concave, il est facile de voir que cette condition de Lax est équivalente à la condition d'entropie. Cette équivalence est encore vraie pour certains cas avec $p>1$. Ceci est démontré dans l'exercice 5.18 pour les équations de Saint-Venant? La démonstration est faite dans le cas d'une ligne de discontinuité de la solution autosimilaire du problème de Riemann mais se généralise au cas d'une courbe régulière de discontinuité.

\subsubsection{Résolution du problème de Riemann}

La résolution du problème de Riemann pour certains problèmes issus de la physique est intéressante d'une part parce qu'elle permet de comprendre la structure des solutions entropiques, et d'autre part parce que certains schémas numériques d'approximation de ces systèmes sont fondés sur cette résolution. Dans ce paragraphe, on se place dans le cadre des systèmes hyperboliques décrits dans définition 5.31

\section{Définitions}

On rappelle que le problème de Riemann s'écrit

$$
\begin{aligned}
& \partial_{t} U+\partial_{x}(F(U))=0, x \in \mathbb{R}, t \in \mathbb{R}_{+}, \\
& U(x, 0)=\left\{\begin{array}{l}
U_{g} \text { pour } x<0, \\
U_{d} \text { pour } x>0,
\end{array}\right.
\end{aligned}
$$

avec $F \in C^{1}\left(D, \mathbb{R}^{p}\right)$ et $U_{g}, U_{d} \in D$. La solution en est connue lorsque $F(U)=A U$ (et $D=\mathbb{R}^{p}$, voir le paragraphe précédent). On cherche une solution "autosimilaire" c'est-à-dire une solution de la forme $U(x, t)=$ $V\left(\frac{x}{t}\right)$. On rappelle qu'une telle solution définie par zone est solution faible si et seulement si

1. elle est solution classique sur chaque zone

2. elle satisfait les conditions de Rankine et Hugoniot au passage de chaque discontinuité $x=\sigma t$ entre deux zones : $\sigma[U]=[F(U)]$.

A cette notion de solution faible, il faut ajouter une condition d'entropie. Si le système contient une entropie (ou des entropies) non triviale(s) naturelle(s), la solution faible est entropique si et seulement si elle vérifie $\sigma[\eta(U)] \geq$ $[\phi(U)]$ pour toute entropie et flux associé au passage de chaque discontinuité $x=\sigma t$. En pratique, on utilise aussi la condition d'entropie de Lax, condition (5.41) (qui ne demande pas l'existence d'une entropie). Le lien entre ces deux conditions d'entropie est étudié dans l'exercice 5.18 pour les équations de Saint-Venant.

On suppose dans toute la suite que $F \in C^{2}\left(D, \mathbb{R}^{p}\right)$ et que le problème (5.45) est strictement hyperbolique, c'està-dire que la matrice jacobienne de $F$ est toujours diagonalisable dans $\mathbb{R}$ et que ses valeurs propres sont distinctes; pour tout $U \in D$, il existe donc $\lambda_{1}(U)<\ldots<\lambda_{p}(U)$ valeurs propres de $J_{F}(U)$. On note $\left(\varphi_{1}(U), \ldots, \varphi_{p}(U)\right)$ une base de $\mathbb{R}^{p}$ formée de vecteurs propres associés :

$$
J_{F}(U) \varphi_{i}(U)=\lambda_{i}(U) \varphi_{i}(U), \forall U \in D, i=1, \ldots, p .
$$

On admettra que $\lambda_{i}(U) \in C^{1}(D, \mathbb{R})$ et qu'il est possible de choisir la fonction $\varphi_{i}$ pour que $\varphi_{i} \in C^{1}\left(D, \mathbb{R}^{p}\right)$. On appelle " $i$-ème champ" l'application de $D$ dans $\mathbb{R}$ qui à $U \in D$ associe $\lambda_{i} \in \mathbb{R}$.

\footnotetext{
7. Adhémar Jean Claude Barré de Saint-Venant (1797-1886) ingénieur et mathematicien spécialiste de mécanique des fluides.
} 


\section{Définition 5.35 (Champ vraiment non linéaire, champ linéairement dégénéré)}

En utilisant les définitions ci dessus de $\lambda_{i}(U)$ et $\varphi_{i}(U)$, on dit que le $i$-ème champ associé à la fonction non linéaire F est

— "vraiment non linéaire" (VNL) si

$$
\nabla \lambda_{i}(U) \cdot \varphi_{i}(U) \neq 0, \forall U \in D,
$$

et on normalise alors $\varphi_{i}(U)$ de manière à ce que $\nabla \lambda_{i}(U) \cdot \varphi_{i}(U)=1$,

- "linéairement dégénéré" $(L D)$ si

$$
\nabla \lambda_{i}(U) \cdot \varphi_{i}(U)=0, \forall U \in D .
$$

(On admet ici aussi qu'il est possible de choisir $\varphi_{i} \in C^{1}\left(D, \mathbb{R}^{p}\right)$.)

Dans le cas scalaire $p=1$, il n'y a qu' une seule valeur propre $\lambda_{1}(U)=F^{\prime}(U)$, on peut choisir $\varphi_{1}(U)=1$, et

- le cas VNL correspond au cas où $F^{\prime \prime}$ ne s'annule jamais, auquel cas $F$ est strictement convexe ou concave,

- le cas LD correspond au cas $F^{\prime \prime}(u)=0$ pour tout $u$, auquel cas la fonction $F$ est linéaire.

Ce ne sont bien sûr pas les seuls cas possibles, voir par exemple l'équation de Buckley-Leverett (exercice 5.8.

Dans le cas d'un système linéaire, les valeurs propres $\lambda_{i}$ ne dépendent pas de $U$ et tous les champs sont linéairement dégénérés.

Un exemple intéressant est le système des équations d'Euler pour l'écoulement des fluides compressibles. Pour le système complet (conservation de la masse, de la quantité de mouvement et de l'énergie) avec la loi d'état des gaz parfaits, on peut montrer qu'on a deux champs VNL et un champ LD. Le cas des équations d'Euler barotrope (la pression ne dépend que de $\rho$ ) est un peu plus simple. Pour ce système, on a $D=\mathbb{R}_{+}^{\star} \times \mathbb{R}$ et le système s'écrit :

$$
\begin{aligned}
& \partial_{t} \rho+\partial_{x}(\rho u)=0, \\
& \partial_{t}(\rho u)+\partial_{x}\left(\rho u^{2}+p\right)=0,
\end{aligned}
$$

où $p$ est relié à $\rho$ par une fonction donnée $\mathcal{P}$ strictement croissante convexe dérivable de $\mathbb{R}_{+}^{\star}$ dans $\mathbb{R}_{+}^{\star}$, c'est-à-dire $p=\mathcal{P}(\rho)$. Notons que les équations de Saint-Venant pour les écoulements en eau peu profonde rentrent dans ce cadre, avec $\rho$ la hauteur d'eau, et $p=\alpha \rho^{2}$, avec $\alpha>0$ (voir exercice (5.15)).

Posons $q=\rho u$ (la quantité de mouvement); le système (5.46) s'écrit alors :

$$
\partial_{t} U+\partial_{x}(F(U))=0, \text { avec } U=\left[\begin{array}{l}
\rho \\
q
\end{array}\right], F(U)=\left[\begin{array}{c}
q \\
\frac{q^{2}}{\rho}+\mathcal{P}(\rho)
\end{array}\right] .
$$

On pose $c=\sqrt{\mathcal{P}^{\prime}(\rho)}$. La matricienne jacobienne de $F$ est alors :

$$
J_{F}(U)=\left[\begin{array}{cc}
0 & 1 \\
-\frac{q^{2}}{\rho^{2}}+\mathcal{P}^{\prime}(\rho) & \frac{2 q}{\rho}
\end{array}\right]=\left[\begin{array}{cc}
0 & 1 \\
-u^{2}+c^{2} & 2 u
\end{array}\right]
$$

Le polynôme caractéristique de $J_{F}(U)$ sécrit donc

$$
\begin{aligned}
P_{J_{F}(U)}(X) & =X^{2}-2 \frac{q}{\rho} X+\frac{q^{2}}{\rho^{2}}-\mathcal{P}^{\prime}(\rho) \\
& =X^{2}-2 u X+u^{2}-c^{2}
\end{aligned}
$$


Les valeurs propres et des vecteurs propres associés (non normalisés) sont donc

$$
\lambda_{1}(U)=u-c, \lambda_{2}(U)=u+c, \varphi_{1}(U)=\left[\begin{array}{c}
1 \\
u-c
\end{array}\right], \varphi_{2}(U)=\left[\begin{array}{c}
1 \\
u+c
\end{array}\right] .
$$

On a donc, pour tout $U \in D=\left\{(\rho, q)^{t} ; \rho>0\right\}$,

$$
\nabla \lambda_{1}(U) \cdot \varphi_{1}(U)=-\frac{c}{\rho}-\frac{\mathcal{P}^{\prime \prime}(\rho)}{2 c}<0, \nabla \lambda_{2}(U) \cdot \varphi_{2}(U)=\frac{c}{\rho}+\frac{\mathcal{P}^{\prime \prime}(\rho)}{2 c}>0,
$$

Car $\mathcal{P}^{\prime}>0$ et $\mathcal{P}^{\prime \prime} \geq 0$. Les deux champs sont donc vraiment non linéaires.

\section{Etude d'un système découplé}

L'étude d'un système découplé dont tous les champs sont soit LD soit VNL est facile et instructif, donc nous n'allons pas nous en priver... Un système découplé s'écrit sous la forme

$$
\begin{aligned}
& \partial_{t} u_{i}+\partial_{x}\left(f_{i}\left(u_{i}(x, t)\right)\right)=0, x \in \mathbb{R}, t \in \mathbb{R}_{+}, i=1, \ldots, p \\
& u_{i}(x, 0)=u_{i}(0), x \in \mathbb{R}, i=1, \ldots, p,
\end{aligned}
$$

où chaque composante $f_{i}$ de la fonction $F$ ne dépend donc que de la composante $u_{i}$ de l'inconnue. Le domaine $D$ dans lequel l'inconnue vectorielle $U$ (dont les composantes sont $u_{1}, \ldots, u_{p}$ ) est ici égal à $\mathbb{R}^{p}$. Comme tous les champs sont soit VNL soit LD, chaque fonction $f_{i}$ est soit strictement convexe, soit strictement concave, soit linéaire.

Prenons par exemple le cas $p=2$ et $f_{1}$ et $f_{2}$ strictement convexes. Considérons le problème de Riemann associé, c'est-à-dire

$$
U(x, 0)=\left\{\begin{array} { l } 
{ U _ { g } \text { pour } x < 0 , } \\
{ U _ { d } \text { pour } x > 0 . }
\end{array} , \text { avec } \left\{\begin{array}{l}
u_{g, 1}<u_{d, 1} \\
u_{g, 2}<u_{d, 2} .
\end{array}\right.\right.
$$

Nous avons donc pour chaque composante de $U$ un problème de Riemann scalaire. Ces deux problèmes de Riemann correspondent à des détentes. En notant $u_{1}$ et $u_{2}$ les composantes d'un vecteur $U$ de $\mathbb{R}^{2}$, les valeurs propres de la matrice jacobienne du flux de ce système sont $f_{1}^{\prime}\left(u_{1}\right)$ et $f_{2}^{\prime}\left(u_{2}\right)$. La stricte hyperbolicité du système nous permet alors d'affirmer (quitte à changer l'ordre des équations) que

$$
f_{1}^{\prime}\left(u_{1}\right)=\lambda_{1}(U)<\lambda_{2}(U)=f_{2}^{\prime}\left(u_{2}\right), \text { pour tout } u_{1}, u_{2} \in \mathbb{R} .
$$

En particulier $f_{1}^{\prime}\left(u_{d, 1}\right)<f_{2}^{\prime}\left(u_{g, 2}\right)$, et donc les zones de détentes (dans le plan $\mathbb{R} \times \mathbb{R}_{+}^{\star}$ ) pour les inconnues $u_{1}$ et $u_{2}$ sont complètement séparées, la solution du problème de Riemann, dans le plan $\mathbb{R} \times \mathbb{R}_{+}^{\star}$, apparaît comme la superposition des solutions des deux problèmes de Riemann sur $u_{1}$ et $u_{2}$.

On peut noter que sans la condition de stricte hyperbolicité, les ondes de détente ne sont plus séparées. (On peut toutefois construire la solution du problème de Riemann en profitant du fait que les deux équations du système sont découplées, ceci sera moins facile pour les systèmes où les équations sont couplées.)

Les autres cas des positions relatives entre $u_{g, 1}, u_{d, 1}$ et $u_{g, 2}, u_{d, 2}$ se traitent de manière similaire. Par exemple, si $u_{g, 1}>u_{d, 1}$ et $u_{g, 2}<u_{d, 2}$, la solution est formée (dans le plan $\mathbb{R} \times \mathbb{R}_{+}^{\star}$ ) d'un choc, que l'on appellera "1choc", situé sur la droite $x=\sigma t$, avec $\sigma=\left(f_{1}\left(u_{g, 1}\right)-f_{1}\left(u_{d, 1}\right) /\left(u_{g, 1}\right)-u_{d, 1}\right)$ (correspondant à la première équation), et d'une détente, que l'on appellera "2-détente", située entre les droites $x=f_{2}^{\prime}\left(u_{g, 2}\right) t$ et $x=f_{2}^{\prime}\left(u_{d, 2}\right) t$ (correspondant à la deuxième équation). Le 1-choc n'est pas dans la zone de 2-détente car $\sigma \in] f_{1}^{\prime}\left(u_{d, 1}\right), f_{1}^{\prime}\left(u_{g, 1}\right)[$ et, grâce à (5.48), $f_{1}^{\prime}\left(u_{g, 1}\right)<f_{2}^{\prime}\left(u_{g, 2}\right)$. 
Dans le cas d'un système non découplé, on se sert des relations de Rankine-Hugoniot pour construire les solutions discontinues, appelées "ondes de choc", correspondant aux champs VNL, comme dans le cas scalaire. Pour déterminer les solutions continues correspondant aux champs VNL, qui sont les "ondes de détente" (on dit aussi "ondes de raréfaction"), on va se servir des invariants de Riemann qui sont définis ci-après. Enfin pour déterminer les "ondes de contact", qui correspondent aux champs LD et sont discontinues, on se servira soit des relations de Rankine-Hugoniot soit des invariants de Riemann (car une onde de contact peut être vue comme le cas limite d'une onde de détente ou d'une onde de choc).

\section{Invariants de Riemann et ondes de détente}

On rappelle que l'on se place toujours dans le cadre des systèmes hyperboliques (definition 5.31).

Définition 5.36 (Invariant de Riemann) Soit $1 \leq i \leq p$, on appelle $i$-invariant de Riemann pour le système 5.43a une application $r \in C^{1}(D, \mathbb{R})$ non constante telle que $\nabla r(U) \cdot \varphi_{i}(U)=0$ pour tout $U \in D$.

La notion d'invariant de Riemann n'a d'intérêt que pour $p \geq 2$. En effet pour $p=1, \varphi_{1}(U)$ est n'importe quel réel non nul. Il n'y a pas d'invariant de Riemann.

Dans le cas du système découplé vu précédemment, on a $\lambda_{i}(U)=f_{i}^{\prime}\left(u_{i}\right)$ et $\varphi_{i}$ est colinéaire au $i$-ème vecteur de la base canonique. Donc toutes les applications $U \mapsto u_{j}, j \neq i$, sont des $i$-invariants de Riemann. On a ainsi pour chaque champ $(p-1)$ invariants de Riemann indépendants (au sens que ces $(p-1)$ applications sont linéairement indépendantes). On peut montrer que cette situation se généralise de la manière suivante (voir, par exemple, [26]) : Pour tout $U_{0} \in D$, il existe un voisinage $\mathcal{V}$ de $U_{0}$ et $(p-1) i$-invariants de Riemann définis sur $\mathcal{V}$ et linéairement indépendants, ce qui est équivalent à dire que leurs gradients sont indépendants.

Calculons les invariants de Riemann pour les équations d'Euler barotrope (5.46). On rappelle que les valeurs propres et des vecteurs propres (non normalisés) de la jacobienne $J_{F}(U)$ sont

$$
\lambda_{1}(U)=u-c, \lambda_{2}(U)=u+c, \varphi_{1}(U)=\left[\begin{array}{c}
1 \\
u-c
\end{array}\right], \varphi_{2}(U)=\left[\begin{array}{c}
1 \\
u+c
\end{array}\right] .
$$

Cherchons un 1-invariant de Riemann sous la forme $r(U)=\frac{q}{\rho}+h(\rho)$. On a alors

$$
\nabla r(U)=\left[\begin{array}{c}
-\frac{q}{\rho^{2}}+h^{\prime}(\rho) \\
\frac{1}{\rho}
\end{array}\right]
$$

et donc

$$
\nabla r(U) \cdot \varphi_{1}(U)=-\frac{q}{\rho^{2}}+h^{\prime}(\rho)+\frac{1}{\rho}\left(\frac{q}{\rho}-c\right)=h^{\prime}(\rho)-\frac{c}{\rho} .
$$

La fonction $r$ est donc un 1-invariant de Riemann si (et seulement si) $h^{\prime}(\rho)=\frac{c}{\rho}$.

Il suffit donc de prendre pour $h$ une primitive de la fonction $\rho \mapsto \sqrt{\mathcal{P}^{\prime}(\rho)} / \rho$. Dans le cas du système des équations de Saint-Venant (pour lequel $\mathcal{P}(\rho)=\alpha \rho^{2}, \alpha>0$ ), on a $c=\sqrt{2 \alpha \rho}$, et un 1-invariant de Riemann est $r_{1}(U)=$ $\frac{q}{\rho}+2 c=u+2 c$, voir aussi l'exercice 5.15 .

De la même manière, on calcule un 2-invariant de Riemann : $r_{2}(U)=\frac{q}{\rho}-h(\rho)$, avec pour $h$ une primitive de la fonction $\rho \mapsto \sqrt{\mathcal{P}^{\prime}(\rho)} / \rho$. Dans le cas des équations de Saint-Venant, ce 2 -invariant de Riemann s'écrit : $r_{2}(U)=u-2 c$.

Sous des hypothèses de régularité, pour un système de $p$ équations, un invariant de Riemann commun à $p-1$ ondes satisfait une équation particulièrement simple, comme le montre la proposition suivante (voir aussi, par exemple, [26]). 
Proposition 5.37 (Equation d'évolution pour un invariant de Riemann) Soit $p>1$; on considère un système strictement hyperbolique (définition 5.31) de la forme (5.43). On note $\lambda_{i}(U), i=1, \ldots, p$, les $p$ valeurs propres réelles distinctes de la matrice jacobienne de $F$ au point $U \in D$. Soit $i \in\{1, \ldots, p\}$; on suppose qu'il existe une application $r \in C^{1}(D, \mathbb{R})$ qui soit un $j$-invariant de Riemann pour tout $j \neq i$. Alors, pour toute fonction $U \in C^{1}\left(\mathbb{R} \times R_{+}, D\right)$ satisfaisant 5.43a), on a

$$
\partial_{t}(r(U))+\lambda_{i}(U) \partial_{x}(r(U))=0 .
$$

Démonstration : Pour simplifier les notations, on note $A=J_{F}$. Pour tout $V \in D$, on note $\left\{\varphi_{1}(V), \ldots, \varphi_{p}(V)\right\}$ une base de $\mathbb{R}^{p}$ telle que $A(V) \varphi_{j}(V)=\lambda_{j} \varphi_{j}(V)$ pour tout $j \in\{1, \ldots, n\}$. Comme les valeurs propres de $A(V)^{t}$ sont les mêmes que celles de $A(V)$, il existe aussi $\left\{\psi_{1}(V), \ldots, \psi_{p}(V)\right\}$ base de $\mathbb{R}^{p}$ telle que $A(V)^{t} \psi_{j}(V)=$ $\lambda_{j} \psi_{j}(V)$ pour tout $j \in\{1, \ldots, n\}$.

Soit $k \neq j$, comme $\lambda_{k}(V) \neq \lambda_{j}(V)$, on a

$$
\lambda_{j}(V) \psi_{j}(V) \cdot \varphi_{k}(V)=A(V)^{t} \psi_{j}(V) \cdot \varphi_{k}(V)=\psi_{j}(V) \cdot A(V) \varphi_{k}(V)=\lambda_{k}(V) \psi_{j}(V) \cdot \varphi_{k}(V),
$$

et donc $\left(\lambda_{j}(V)-\lambda_{k}(V)\right) \psi_{j}(V) \cdot \varphi_{k}(V)=0$. Ceci entrâne que $\psi_{j}(V) \cdot \varphi_{k}(V)=0$.

On en déduit aussi, comme $\left\{\varphi_{1}(V), \ldots, \varphi_{p}(V)\right\}$ et $\left\{\psi_{1}(V), \ldots, \psi_{p}(V)\right\}$ sont des bases de $\mathbb{R}^{n}$, que $\psi_{j}(V)$. $\varphi_{j}(V) \neq 0$ pour tout $j \in\{1, \ldots, n\}$.

On utilise maintenant la régularité de $r$, et on décompose $\nabla r(V)$ dans la base $\left\{\psi_{1}(V), \ldots, \psi_{p}(V)\right\}, \nabla r(V)=$ $\sum_{k=1}^{n} \alpha_{k}(V) \psi_{k}(V)$. Comme $r$ est un $j$-invariant de Riemann pour $j \neq i$, on a, pour tout $j \neq i$,

$$
0=\nabla r(V) \cdot \varphi_{j}(V)=\sum_{k=1}^{n} \alpha_{k}(V) \psi_{k}(V) \cdot \varphi_{j}(V)=\alpha_{j}(V) \psi_{j}(V) \cdot \varphi_{j}(V),
$$

et donc $\alpha_{j}(V)=0$, ce qui prouve que $\nabla r(V)=\alpha_{i}(V) \psi_{i}(V)$.

On utilise enfin la régularité de la fonction $U$,

$$
\begin{aligned}
\partial_{t} r(U)= & \nabla r(U) \cdot \partial_{t} U=\alpha_{i}(U) \psi_{i}(U)^{t} \partial_{t} U \\
=-\alpha_{i}(U) \psi_{i}(U)^{t} A(U) \partial_{x} U=-\alpha_{i}(U)\left(\partial_{x} U\right)^{t} A(U)^{t} \psi_{i}(U)=-\alpha_{i}(U) \lambda_{i}(U)\left(\partial_{x} U\right)^{t} \psi_{i}(U) & \quad=-\lambda_{i}(U)\left(\partial_{x} U\right)^{t}\left(\alpha_{i}(U) \psi_{i}(U)\right)=-\lambda_{i}(U) \nabla r(U) \cdot \partial_{x} U=-\lambda_{i}(U) \partial_{x} r(U) .
\end{aligned}
$$

La proposition 5.37 permet en quelque sorte d'obtenir un système diagonal avec les fonctions $r_{i}(U)$ comme inconnues (où $r_{i}$ est un $j$-invariant de Riemann pour tout $j \neq i$ ).

Supposons par exemple $p=2$ dans la proposition 5.37, l'application $r_{1}$ est un 1-invariant de Riemann et l'application $r_{2}$ est un 2-invariant de Riemann. La proposition 5.37]donne, si $U$ est régulière,

$$
\begin{aligned}
& \partial_{t}\left(r_{1}(U)\right)+\lambda_{2}(U) \partial_{x}\left(r_{1}(U)\right)=0, \\
& \partial_{t}\left(r_{2}(U)\right)+\lambda_{1}(U) \partial_{x}\left(r_{2}(U)\right)=0 .
\end{aligned}
$$

Si l'application $U \mapsto R(U)=\left[\begin{array}{l}r_{1}(U) \\ r_{2}(U)\end{array}\right]$ est un difféomorphisme (de $D$ dans son image), on note $s$ son application réciproque, de sorte que $U=s(R)$. Les fonctions $(x, t) \mapsto r_{1}(U(x, t))$ et $(x, t) \mapsto r_{2}(U(x, t))$, que l'on note $\bar{r}_{1}$ et $\bar{r}_{2}$ ci après, sont alors solution du problème de transport suivant :

$$
\begin{aligned}
& \partial_{t}\left(\bar{r}_{1}\right)+\lambda_{2}(s(\bar{R})) \partial_{x}\left(\bar{r}_{1}\right)=0, \\
& \partial_{t}\left(\bar{r}_{2}\right)+\lambda_{1}(s(\bar{R})) \partial_{x}\left(\bar{r}_{2}\right)=0,
\end{aligned}
$$


où $\bar{R}$ est la fonction vectorielle dont les composantes sont $\bar{r}_{1}$ et $\bar{r}_{2}$.

Ceci permet par exemple d'obtenir des estimations sur les invariants de Riemann en les considérant comme solution de ce problème de transport.

Les invariants de Riemann vont nous permettre de construire les ondes de détente, qui sont des solutions continues sur $\mathbb{R} \times \mathbb{R}_{+}^{\star}$ et autosimilaires du problème de Riemann, c'est-à-dire qu'il existe $-\infty<a<b<+\infty$ tels que $U=U_{g}$ sur $D_{1}=\{(x, t), x \leq a t\}, U(x, t)=V\left(\frac{x}{t}\right)$ sur $D_{2}=\{(x, t), a t<x<b t\}, U(x, t)=U_{d}$ sur $D_{3}=\{(x, t), x \geq b t\}$ et $U$ solution classique dans $D_{2}$. La continuité de $U$ impose donc que $V(a)=U_{g}$ et $V(b)=U_{d}$.

On fixe $a$ et $b(a<b)$. On cherche donc $V \in C([a, b]) \cap C^{1}(] a, b[)$ tels que $V(a)=U_{g}, V(b)=U_{d}$, et $U$ solution classique de (5.45a) dans $D_{2}$ avec $U(x, t)=V\left(\frac{x}{t}\right)$. On a, dans $D_{2}$,

$$
\partial_{t} U(x, t)=-\frac{x}{t^{2}} V^{\prime}\left(\frac{x}{t}\right) \text { et } \partial_{x}(F(U(x, t)))=J_{F}(U(x, t)) \partial_{x}(U(x, t))=\frac{1}{t} J_{F}\left(V\left(\frac{x}{t}\right)\right) V^{\prime}\left(\frac{x}{t}\right) .
$$

Donc, dans $D_{2}$,

$$
\partial_{t} U(x, t)+\partial_{x}(F(U(x, t)))=\frac{1}{t}\left(J_{F}\left(V\left(\frac{x}{t}\right)\right)-\frac{x}{t}\right) V^{\prime}\left(\frac{x}{t}\right) .
$$

Si on se limite à chercher une solution avec $V^{\prime} \neq 0$ sur $] a, b\left[\right.$, pour que $\partial_{t} U+\partial_{x}(F(U))=0$ dans $D_{2}$, il faut donc que, pour tout $\left.\frac{x}{t} \in\right] a, b[$,

$$
\frac{x}{t} \text { soit valeur propre de } J_{F}\left(V\left(\frac{x}{t}\right)\right) \text {, et } V^{\prime}\left(\frac{x}{t}\right) \text { soit vecteur propre associé non nul. }
$$

Il existe donc $i \in \llbracket 1, p \rrbracket$ tel que $\frac{x}{t}=\lambda_{i}\left(V\left(\frac{x}{t}\right)\right)$. On est sûr que $i$ ne dépend pas de $\frac{x}{t}$, parce qu'on a supposé $V$ continue et que le système est strictement hyperbolique.

Noter que l'existence et l'unicité de $i$ est encore valable si $V^{\prime}$ s'annule en un point entre ] $a, b$ [ (toujours grâce au fait que le système est strictement hyperbolique). Par contre si $V^{\prime}$ est nul sur tout un intervalle $[c, d], a<c<d<b, i$ peut être différent sur $] a, c[$ et sur $] d, b[$. Cette situation apparaitra lorsque le problème de Riemann considéré aura une solution formée de deux détentes (chaque détente correspondant à une valeur de $i$ ).

Finalement, sous cette condition $V^{\prime} \neq 0$ (ou si $V^{\prime}$ ne s'annule qu'en des points isolés), pour que la fonction $V$ recherchée convienne, il faut et il suffit qu'il existe $i \in \llbracket 1, p \rrbracket$ tel que

$$
\begin{aligned}
& \forall s \in] a, b\left[, \lambda_{i}(V(s))=s \text { et } V^{\prime}(s)=\alpha(s) \varphi_{i}(V(s)), \text { avec } \alpha(s) \in \mathbb{R},\right. \\
& V(a)=U_{g}, V(b)=U_{d} .
\end{aligned}
$$

On rappelle que l'on cherche $V \in C([a, b]) \cap C^{1}(] a, b[)$. Noter que la condition 5.50) et le fait que $\lambda_{i}(V(s))=s$ (pour $s \in] a, b\left[\right.$ et donc aussi $s \in[a, b]$ ) donne $\lambda_{i}\left(U_{g}\right)=a$ et $\lambda_{i}\left(U_{d}\right)=b$.

La condition (5.49) implique que le champ $i$ est VNL. En effet, comme l'application $U \mapsto \lambda_{i}(U)$ est dérivable et que l'on cherche $V$ de classe $C^{1}$ sur $] a, b[$, les égalités précédentes donnent que

$$
\left.1=\nabla \lambda_{i}(V(s)) \cdot V^{\prime}(s)=\alpha(s) \nabla \lambda_{i}(V(s)) \cdot \varphi_{i}(V(s)), \forall s \in\right] a, b[.
$$

Ceci prouve que $\nabla \lambda_{i}(V(s)) \cdot \varphi_{i}(V(s)) \neq 0$ (et aussi $\alpha(s) \neq 0$ ) et donc que le champ $i$ est VNL. Enfin, en normalisant $\varphi_{i}(U)$ par la condition $\nabla \lambda_{i}(U) \cdot \varphi_{i}(U)=1$, l'égalité (5.51) donne $\alpha(s)=1$ et $V^{\prime}(s)=\varphi_{i}(V(s))$ pour tout $s \in] a, b[$.

La condition nécessaire et suffisante sur $V$ est donc que $V$ soit solution de l'équation différentielle (avec condition initiale et finale) suivante :

$$
\begin{aligned}
& V \in C([a, b], D) \cap C^{1}(] a, b[, D), \\
& \left.V^{\prime}(s)=\varphi_{i}(V(s)), \text { pour tout } s \in\right] a, b[, \\
& V(a)=U_{g}, \lambda_{i}\left(U_{g}\right)=a, V(b)=U_{d} .
\end{aligned}
$$


En effet, on vient de montrer que cette condition est bien nécessaire. Pour voir qu'elle est suffisante, il suffit de remarquer que si $V$ est solution de cette équation différentielle, on a bien $\lambda_{i}(V(s))=s$ pour tout $\left.s \in\right] a, b$ [ car $\lambda_{i}(V(a))=\lambda_{i}\left(U_{g}\right)=a$ et $\lambda_{i}(V(s))^{\prime}=\nabla \lambda_{i}(V(s)) \cdot V^{\prime}(s)=\nabla \lambda_{i}(V(s)) \cdot \varphi_{i}(V(s))=1$ pour tout $\left.s \in\right] a, b[$. En particulier, on rappelle que ceci donne $\lambda_{i}\left(U_{d}\right)=\lambda_{i}(V(b))=b$.

Définition 5.38 (Onde de détente) Une $i$-onde de détente du système (5.45) est une solution de (5.45) telle que

1. $U(x, t)=U_{g}$ si $x \leq \lambda_{i}\left(U_{g}\right) t$,

2. $U(x, t)=U_{d}$ si $x \geq \lambda_{i}\left(U_{d}\right)$ t (on a donc $\lambda_{i}\left(U_{d}\right)>\lambda_{i}\left(U_{g}\right)$ ),

3. $U(x, t)=V\left(\frac{x}{t}\right)$ si $\lambda_{i}\left(U_{g}\right) t<x<\lambda_{i}\left(U_{d}\right) t$, avec $\frac{x}{t}=\lambda_{i}\left(V\left(\frac{x}{t}\right)\right)$,

4. $V \in C\left(\left[\lambda_{i}\left(U_{g}\right), \lambda_{i}\left(U_{d}\right)\right], \mathbb{R}\right), V\left(\lambda_{i}\left(U_{g}\right)\right)=U_{g}$ et $V\left(\lambda_{i}\left(U_{d}\right)\right)=U_{d}$.

5. $V \in C^{1}(] \lambda_{i}\left(U_{g}\right), \lambda_{i}\left(U_{d}\right)[, D), V^{\prime}\left(\frac{x}{t}\right)=\varphi_{i}\left(\left(V\left(\frac{x}{t}\right)\right)\right.$ pour $\lambda_{i}\left(U_{g}\right) t<x<\lambda_{i}\left(U_{d}\right) t$.

On dit alors que $U_{d}$ est reliable a $U_{g}$ par une i-détente (noter que cette relation est non symétrique car $\lambda_{i}\left(U_{g}\right)<$ $\left.\lambda_{i}\left(U_{d}\right)\right)$.

(On rappelle que $\varphi_{i}(U)$ est normalisé par la condition $\nabla \lambda_{i}(U) \cdot \varphi_{i}(U)=1$.)

Le calcul précédent montre donc que $U_{d}$ est reliable à $U_{g}$ par une $i$-détente si et seulement si $U_{d} \in \Gamma_{i}\left(U_{g}\right)=\left\{V(s), s>a, V\right.$ solution de [5.55)-(5.56), avec $\left.a=\lambda_{i}\left(U_{g}\right)\right\}$,

$$
\begin{aligned}
& V^{\prime}(s)=\varphi_{i}(V(s)), s>a, \\
& V(a)=U_{g} .
\end{aligned}
$$

En effet, si $U_{d}$ est reliable à $U_{g}$ par une $i$-détente, la fonction $V$ donnée par la définition 5.38 est solution de (5.55)-(5.56) sur l'intervalle $[a, b]$ avec $b=\lambda_{i}\left(U_{d}\right)$ et $U_{d}=V\left(\lambda_{i}\left(U_{d}\right)\right)=V(b) \in \Gamma_{i}\left(U_{g}\right)$. Réciproquement si $U_{d} \in \Gamma_{i}\left(U_{g}\right)$, il existe $b$ t.q $U_{d}=V(b)$ avec $V$ solution de (5.55)-(5.56) sur l'intervalle $[a, b]$ et donc $V$ est solution (5.52)-5.54), ce qui donne bien que $U_{d}$ est reliable à $U_{g}$ par une $i$-détente.

Comme la fonction $\varphi_{i}$ est de classe $C^{1}$, l'équation différentielle (5.55) admet avec la condition initiale (5.56) une unique solution locale. L'ensemble $\Gamma_{i}\left(U_{g}\right)$ est donc la trajectoire (dans $\left.\mathbb{R}^{p}\right)$ de la solution de cette équation différentielle. Le vecteur $\varphi_{i}\left(U_{g}\right)$ est un vecteur tangent à cette trajectoire au point $U_{g}$. Ceci est résumé dans le théorème 5.39 .

Théorème 5.39 (Courbe de détente) Soit $i$ un champ VNL et $U_{g} \in D$,. alors $\Gamma_{i}\left(U_{g}\right)$ est une courbe (dans l'espace $\mathbb{R}^{p}$ ) partant de $U_{g}$ et le vecteur $\varphi_{i}\left(U_{g}\right)$ est un vecteur tangent à cette trajectoire au point $U_{g}$.

Lorqu'on veut résoudre le problème de Riemann, on veut déterminer s'il existe des ondes de détente, et si un état $U_{d}$ à droite est reliable à un état $U_{g}$ à gauche par une onde de détente, c'est-à-dire si $U_{d} \in \Gamma_{i}\left(U_{g}\right)$. Pour cela, un moyen assez simple est de faire intervenir les invariants de Riemann. Supposons que $U$ soit une $i$-onde de détente. En reprenant les notations de la définition 5.38. $U(x, t)=V\left(\frac{x}{t}\right)$ pour $a=\lambda_{i}\left(U_{g}\right)<x / t<\lambda_{i}\left(U_{d}\right)=b$, $V \in C^{1}(] a, b[) \cap C([a, b])$. Soit $r$ un $i$-invariant de Riemann, alors $r\left(U_{g}\right)=r\left(U_{d}\right)$, puisque $r(U)$ est constant dans la zone de détente : en effet, pour tout $\xi \in] a, b[$,

$$
r(V(\xi))^{\prime}=\nabla r(V(\xi)) \cdot V^{\prime}(\xi)=0 \operatorname{car} V^{\prime}(\xi)=\varphi_{i}(V(\xi)) .
$$

Ceci montre que

$$
\Gamma_{i}\left(U_{g}\right) \subset \bar{\Gamma}_{i}\left(U_{g}\right)=\left\{U_{d} \in D: r\left(U_{d}\right)=r\left(U_{g}\right), \text { pour tout } r \text { invariant de Riemann }\right\} .
$$

Comme on a en général $p-1 i$-invariants de Riemann indépendants, $\bar{\Gamma}_{i}\left(U_{g}\right)$ est une courbe de $\mathbb{R}^{p}$ passant par $U_{d}$ et $U_{g}$. 
D'autre part si $U_{d} \in \Gamma_{i}\left(U_{g}\right)$, on a nécessairement $\lambda_{i}\left(U_{g}\right)<\lambda_{i}\left(U_{d}\right)$. L'ensemble $\Gamma_{i}\left(U_{g}\right)$ correspond donc à "la moitié" de la courbe $\bar{\Gamma}_{i}\left(U_{g}\right)$. Plus précisément,

$$
\Gamma_{i}\left(U_{g}\right) \subset\left\{U_{d} \in \bar{\Gamma}_{i}\left(U_{g}\right) \text { tel que } \lambda_{i}\left(U_{g}\right)<\lambda_{i}\left(U_{d}\right)\right\}=\tilde{\Gamma}_{i}\left(U_{g}\right) .
$$

On a en général $\Gamma_{i}\left(U_{g}\right)=\tilde{\Gamma}_{i}\left(U_{g}\right)$ (si il y a bien $p-1 i$-invariants de Riemann indépendants).

Résoudre un problème de Riemann passe donc par la construction des courbes $\Gamma_{i}\left(U_{g}\right)$. En pratique, on construit l'ensemble $\bar{\Gamma}_{i}\left(U_{g}\right)$, et on ne conserve que la partie telle que $\lambda_{i}\left(U_{g}\right)<\lambda_{i}\left(U_{d}\right)$.

Commençons par l'exemple simple d'un système découplé, avec $p=2$. Dans ce cas on a vu qu'un 1-invariant de Riemann est $u_{2}$, et donc l'ensemble $\bar{\Gamma}_{i}\left(U_{g}\right)$ est la droite $u_{2}=u_{g, 2}$. Si $f_{1}$ est strictement convexe, la condition $\lambda_{1}\left(U_{g}\right)<\lambda_{1}\left(U_{d}\right)$ donne que $u_{g, 1}<u_{d, 1}$, ce qui donne une demi droite. L'ensemble des points $U_{d}$ reliables à $U_{g}$ sont les points t.q. $u_{d, 2}=u_{g, 2}$ et $u_{d, 1}>u_{g, 1}$. La solution $U$ est alors une fonction dont la seconde composante est constant (et égale à $u_{g, 2}$ ) et dont la première composante correspond à une détente pour une équation scalaire.

Considérons maintenant le cas du système des équations d'Euler barotrope (5.46). On a vu qu'un 1-invariant

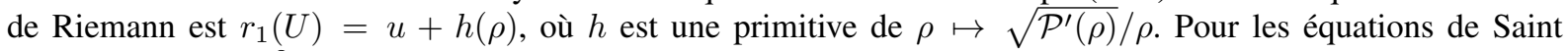
Venant $\mathcal{P}(\rho)=\alpha \rho^{2}, \alpha>0$, on a donc $r_{1}(U)=u+2 c$ (on rappelle que $c=\sqrt{2 \alpha \rho}$ ); on peut tracer la courbe $\bar{\Gamma}_{1}\left(U_{g}\right)=\left\{U_{d} \in D: r\left(U_{d}\right)=r\left(U_{g}\right)\right.$ pour tout $r$ 1-invariant de Riemann $\}$; dans les variables $\rho$, $u$, en posant $\beta_{g}=u_{g}+2 \sqrt{2 \alpha \rho_{g}}, r_{1}\left(U_{d}\right)=r_{2}\left(U_{g}\right)$ revient à écrire que $u+2 \sqrt{2 \alpha \rho}=\beta_{g}$ soit encore

$$
u=\beta_{g}-2 \sqrt{2 \alpha} \sqrt{\rho} .
$$

On obtient alors la demi courbe $\tilde{\Gamma}_{1}\left(U_{g}\right)$ en imposant que $\lambda_{1}\left(U_{g}\right)<\lambda_{1}\left(U_{d}\right)$, c'est-à-dire $u_{g}-c_{g}<u-c$ ou encore $\frac{q_{g}}{\rho_{g}}-\sqrt{2 \alpha \rho_{g}}<\frac{q}{\rho}-\sqrt{2 \alpha \rho}$.

\section{Ondes de choc, discontinuités de contact}

On s'intéresse toujours au problème de Riemann 5.45. Comme pour l'étude des ondes de détentes, on fixe $U_{g}$ et on étudie la possibilité qu'il y ait une solution autosimilaire reliant $U_{g}$ et $U_{d}$ avec une discontinuité, c'est-àdire qu'il existe $\sigma \in \mathbb{R}$ tel que la fonction $U$ définie par $U(x, t)=U_{g}$ si $x<\sigma t$ et $U(x, t)=U_{d}$ si $x>\sigma t$ soit solution de (5.45). On a déjà vu que pour qu'une telle fonction $U$ soit solution faible de (5.45) il faut et il suffit que

$$
\sigma[U]=\sigma\left(U_{d}-U_{g}\right)=\left(F\left(U_{d}\right)-F\left(U_{g}\right)\right)=[F(U)] .
$$

La relation 5.57] nous donne de nombreux couples $\left(U_{g}, U_{d}\right)$ possibles, et en particulier des couples pour lesquels $\lambda_{i}\left(U_{g}\right)<\lambda_{i}\left(U_{d}\right)$ avec un certain $i$ correspondant à un champ VNL et pour lesquels il y a une solution sous forme de détente. Dans ce cas, il n'y a pas unicité de la solution faible. Le problème est le même que dans le cas scalaire, il faut ajouter une condition supplémentaire pour tenter d'assurer l'unicité. Dans le cas général des systèmes étudié ici, il n'y a pas forcément d'entropie au sens de la définition 5.45 . On va plutôt utiliser la condition d'entropie appelée "condition d'entropie de Lax". Cette condition consiste à demander qu'il existe $i$ t.q.

$$
\begin{aligned}
& \lambda_{i}\left(U_{g}\right)>\sigma>\lambda_{i}\left(U_{d}\right) \text { si le champ } i \text { est VNL, } \\
& \lambda_{i}\left(U_{g}\right)=\sigma=\lambda_{i}\left(U_{d}\right) \text { si le champ } i \text { est LD. }
\end{aligned}
$$

Pour un système comme celui de Saint-Venant, cette condition de Lax est équivalente la condition d'entropie de la definition 5.34). Ceci est démontré dans l'exercice 5.18 .

En éliminant $\sigma$ par l'une des équations (5.57) et en le remplaçant dans les autres, on obtient $(p-1)$ équations sur $U_{d}$. Les solutions de ces $p-1$ équations donnent en général, au moins dans un voisinage de $U_{g}, p$ courbes dans 
le plan $\mathbb{R}^{d}$. (Ceci n'est pas démontré dans ce cours mais un exemple est donné dans l'exercice 5.15.) On peut alors déterminer comment ces courbes se comportent au point $U_{g}$. Soit $V:\left[0, \bar{\varepsilon}\left[\rightarrow\left(\mathbb{R}^{d}\right)^{\star}\right.\right.$ une application telle que $\lim _{s \rightarrow 0} V(s)=0$ et telle que, pour tout $s \in\left[0, \bar{\varepsilon}\left[, U_{d}=U_{g}+V(s)\right.\right.$ est reliable à $U_{g}$ par une discontinuité, c'est-à-dire que (5.57) est vérifiée pour un certain $\sigma=\sigma(s)$.

On considère une suite $\left(s_{n}\right)_{n \in \mathbb{N}}$ de $] 0, \bar{\varepsilon}\left[\right.$ telle que $\lim _{n \rightarrow+\infty} s_{n}=0$. Quitte à extraire une sous-suite, on peut supposer que

$$
\lim _{n \rightarrow+\infty} \frac{V\left(s_{n}\right)}{\left\|V\left(s_{n}\right)\right\|}=\varphi
$$

avec $\varphi \in \mathbb{R}^{d}$ (et $\|\varphi\|=1$ ). On écrit la relation de Rankine Hugoniot (5.57) avec $U_{d}=U_{g}+V\left(s_{n}\right)$; en utilisant le caractère $C^{1}$ de $F$, on obtient donc

$$
\left.\sigma\left(s_{n}\right) V\left(s_{n}\right)=F\left(U_{g}+V\left(s_{n}\right)\right)-F\left(U_{g}\right)\right)=D F\left(U_{g}\right)\left(V\left(s_{n}\right)\right)+\left\|V\left(s_{n}\right)\right\| \varepsilon_{n},
$$

avec $\lim _{n \rightarrow+\infty} \varepsilon_{n}=0$, et donc

$$
\sigma\left(s_{n}\right) \frac{V\left(s_{n}\right)}{\left\|V\left(s_{n}\right)\right\|}=D F\left(U_{g}\right) \frac{V\left(s_{n}\right)}{\left\|V\left(s_{n}\right)\right\|}+\varepsilon_{n} .
$$

Ceci montre que la suite $\left(\sigma\left(s_{n}\right)\right)_{n \in \mathbb{N}}$ a une limite, que l'on note $\lambda$ et que $\lambda \varphi=D F\left(U_{g}\right) \varphi$. Comme $\varphi \neq 0$, il existe donc $i \in\{1, \ldots, p\}$ tel que $\lambda=\lambda_{i}\left(U_{g}\right)$ et $\varphi$ est colinéaire à $\varphi_{i}\left(U_{g}\right)$ (et non nul). Les vecteurs $\varphi_{1}\left(U_{g}\right), \ldots, \varphi_{p}\left(U_{g}\right)$ sont donc des vecteurs tangents (au point $U_{g}$ ) aux $p$ courbes de vecteurs $U_{d}$ reliables à $U_{g}$ par une discontinuité.

On note $\Gamma_{i}\left(U_{g}\right)$ la courbe associée au champ $i$ ( c'est-à-dire que le vecteur $\varphi_{i}\left(U_{g}\right)$ est tangent à cette courbe au point $\left.U_{g}\right)$ et on suppose que ce champ est VNL. La condition de Lax (5.58) nous donne alors $\lambda_{i}\left(U_{g}\right)>\sigma\left(s_{n}\right)>$ $\lambda_{i}\left(U_{d}\right)$ au moins pour $n$ assez grand et pour $\left\|U_{g}-U_{d}\right\|$ assez petit (pour assurer, grâce à la condition de stricte hyperbolicité et au fait que $\sigma\left(s_{n}\right) \rightarrow \lambda_{i}\left(U_{g}\right)$, que cette condition de Lax ne puisse pas être vérifiée pour une autre valeur de $i$ ).

En utilisant le caractère $C^{1}$ de $\lambda_{i}$, cette condition de Lax donne alors

$$
\lambda_{i}\left(U_{d}\right)=\lambda_{i}\left(U_{g}\right)+\nabla \lambda_{i}\left(U_{g}\right) \cdot V\left(s_{n}\right)+\left\|V\left(s_{n}\right)\right\| \varepsilon_{n}<\sigma\left(s_{n}\right)<\lambda_{i}\left(U_{g}\right),
$$

avec $\lim _{n \rightarrow+\infty} \varepsilon_{n}=0$, et donc

$$
\nabla \lambda_{i}\left(U_{g}\right) \cdot \frac{V\left(s_{n}\right)}{\left\|V\left(s_{n}\right)\right\|}+\varepsilon_{n}<0 .
$$

Quand $n \rightarrow+\infty$ on en déduit $\nabla \lambda_{i}\left(U_{g}\right) \cdot \varphi \leq 0$, ce qui prouve que $\varphi=\alpha \varphi_{i}\left(U_{g}\right)$ avec $\alpha<0$ (car $\varphi_{i}\left(U_{g}\right)$ est normalisé $\operatorname{par} \nabla \lambda_{i}\left(U_{g}\right) \cdot \varphi_{i}\left(U_{g}\right)=1$ et $\varphi$ est non nul colinéaire à $\left.\varphi_{i}\left(U_{g}\right)\right)$.

La courbe $\Gamma_{i}\left(U_{g}\right)$ a donc la même tangente au point $U_{g}$ que la courbe $\Gamma_{i}\left(U_{g}\right)$ vue dans le cas des détentes mais ces deux courbes partent de $U_{g}$ dans des directions opposées.

Dans le cas des équations de Saint-Venant, $\mathcal{P}(\rho)=\alpha \rho^{2}$ avec $\alpha=1 / 2$, l'exercice 5.15 donne l'ensemble des états $U$ reliable à $U_{g}$ par un 1-choc (resp. 2-choc) Avec les inconnues $(h, u)$, c'est l'ensemble des couples $(h, u)$ tels que $h>h_{g}\left(\right.$ resp. $\left.h<h_{g}\right)$ et $u=u_{g}-S, S=\sqrt{\left(h-h_{g}\right)\left(h^{2}-h_{g}^{2}\right) /\left(2 h_{g} h\right)}$. Etant donnés deux états $U_{g}$ et $U_{d}$, on peut alors constuire, pour les équations de Saint-Venant, tous les états reliables à $U_{g}$ par un 1-choc ou une 1-détente et tous les états reliables à $U_{d}$ par un 2-choc ou une 2-détente. On obtient ainsi deux courbes (l'une passant par $U_{g}$ et l'autre par $U_{d}$ ). L'intersection de ces deux courbes donne un état, appelé état intermédiaire, qui permet de construire la solution du problème de Riemann. Celle ci est formée d'une 1-onde reliant $U_{g}$ à l'état intermédiaire suivie par une 2-onde reliant l'état intermédiaire à $U_{d}$. La figure 5.4 donnent ces courbes avec un choix particulier de $U_{g}$ et $U_{d}$.

A partir de cette étude (ondes de choc, ondes de détentes et discontinuités de contact), il est possible de démontrer le théorème 5.40, dû à P. D. Lax, voir [19, Theorem 5.4] pour l'énoncé initial et la démonstration. 


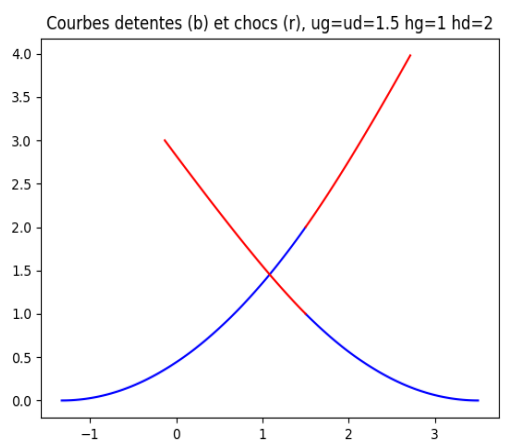

FIgURE 5.4 - La vitesse (u) est en abscisse et la hauteur (h) en ordonnée. L'état intermédiaire est donné par l'intersection de la courbe rouge partant de $U_{g}$ et de la courbe bleue partant de $U_{d}$

Théorème 5.40 (Solution du problème de Riemann, Lax) On considère un système strictement hyperbolique, avec des champs VNL ou LD, et $U_{g} \in D$. Alors il existe $\varepsilon>0$ tel que si $\left|U_{g}-U_{d}\right|<\varepsilon$, il existe une solution au problème de Riemann 5.45, formée d'au plus $p+1$ états constants reliés par p ondes (détentes, discontinuités de contact ou chocs) et vérifiant la condition de Lax. 


\subsection{Exercices}

Exercice 5.1 $\left(\boldsymbol{L}^{\mathbf{1}}(\mathbb{R}) \cap \boldsymbol{B} \boldsymbol{V}(\mathbb{R}) \subset \boldsymbol{L}^{\infty}(\mathbb{R})\right)$ Corrigé en page 243

Pour $u \in L_{\text {loc }}^{1}(\mathbb{R})$ on pose

$$
|u|_{B V}=\sup \left\{\int_{\Omega} u \operatorname{div} \varphi \mathrm{d} x, \varphi \in C_{c}^{\infty}(\mathbb{R}, \mathbb{R}),\|\varphi\|_{L^{\infty}(\Omega)} \leq 1\right\},
$$

et on rappelle que $u \in B V(\mathbb{R})$ si $|u|_{B V}<+\infty$.

Soit $u \in L^{1}(\mathbb{R}) \cap B V(\mathbb{R})$. Montrer que $u \in L^{\infty}(\mathbb{R})$ et

$$
\|u\|_{\infty} \leq|u|_{B V} .
$$

Exercice 5.2 (Système hyperbolique linéaire) Corrigé en page 244 Soit $A \in \mathcal{M}_{n}(\mathbb{R})$ et $u_{0} \in L^{\infty}(\mathbb{R})^{n}$. On suppose $A$ diagonalisable dans $\mathbb{R}$ et on cherche $u \in L^{\infty}\left(\mathbb{R} \times \mathbb{R}_{+}\right)^{n}$ solution faible du problème suivant :

$$
\begin{aligned}
& \left.\partial_{t} u(x, t)+A \partial_{x} u(x, t)=0, x \in \mathbb{R}, t \in\right] 0,+\infty[, \\
& u(x, 0)=u_{0}(x), x \in \mathbb{R} .
\end{aligned}
$$

Soit $\left\{v_{i}, i \in\{1, \ldots, n\}\right.$ une base de $\mathbb{R}^{n}$ formée de vecteurs propres de $A$. On a donc $A v_{i}=\lambda_{i} v_{i}$ pour tout $i \in\{i, \ldots, n\}$ avec $\lambda_{i} \in \mathbb{R}$. Pour $x \in \mathbb{R}$, on décompose $u_{0}(x)$ sur la base $\left\{v_{i}, i \in\{i, \ldots, n\}\right\}$. On a donc $u_{0}=\sum_{i=1}^{n} a_{i} v_{i}$ p.p. avec $a_{i} \in L^{\infty}(\mathbb{R})$ Montrer que $u$ défini presque partout par $u(x, t)=\sum_{i=1}^{n} a_{i}\left(x-\lambda_{i} t\right) v_{i}$ est solution faible de (5.60).

Exercice 5.3 (Unicité de la solution faible du problème linéaire par dualité) Soit $c \in \mathbb{R}$ et $u_{0} \in L^{\infty}(\mathbb{R})$. L'objet de cet exercice est de prouver l'unicité de la solution faible $u$ (dans $L^{\infty}\left(\mathbb{R} \times \mathbb{R}_{+}\right)$) du problème de transport suivant :

$$
\begin{aligned}
& \left.\partial_{t} u(x, t)+c \partial_{x} u(x, t)=0, x \in \mathbb{R}, t \in\right] 0,+\infty[ \\
& u(x, 0)=u_{0}(x), x \in \mathbb{R} .
\end{aligned}
$$

1. Montrer qu'il suffit de prouver l'unicité de la solution pour $u_{0}=0$ p.p..

On suppose dès lors que $u$ est une solution faible de (5.61) avec $u_{0}=0$ p.p..

2. Soit $\psi \in C_{c}(\mathbb{R} \times \mathbb{R})$. Pour $x \in \mathbb{R}$ et $t \in \mathbb{R}_{+}$on pose $\varphi(x, t)=-\int_{t}^{+\infty} \psi(x-c(t-s), s) d s$.

(a) Montrer que $\varphi \in C_{c}^{1}\left(\mathbb{R} \times\left[0,+\infty[)\right.\right.$ et que $\varphi_{t}+c \varphi_{x}=\psi$ in $\mathbb{R} \times[0,+\infty[)$.

(b) Montrer que $\int_{0}^{+\infty} \int_{\mathbb{R}} u(x, t) \psi(x, t) \mathrm{d} x \mathrm{~d} t=0$.

3. Montrer que $u=0$ p.p..

4. En déduire que la solution faible du problème (5.61) est unique, ainsi que que celle du système (5.60) de l'exercice 5.2 .

Exercice 5.4 (Construction d'une solution faible entropique, I) Corrigé en page 245

On considère dans cet exercice l'équation de Burgers avec une condition initiale :

$$
\left\{\begin{array}{l}
\partial_{t} u+\partial_{x}\left(u^{2}\right)=0 \text { dans } \mathbb{R} \times \mathbb{R}_{+}^{\star}, \\
u(\cdot, 0)=u_{0} \text { dans } \mathbb{R} .
\end{array}\right.
$$

Construire une solution faible de (5.62) pour $u_{0}$ définie par :

$$
u_{0}(x)= \begin{cases}1 & \text { si } x<0 \\ 1-x & \text { si } 0<x<1 \\ 0 & \text { si } x>1\end{cases}
$$




\section{Exercice 5.5 (Non unicité des solutions faibles)}

On considère l'équation

$$
\left\{\begin{array}{l}
\partial_{t} u+\partial_{x}\left(u^{2}\right)=0 \\
u(0, x)= \begin{cases}u_{g} & \text { si } x<0 \\
u_{d} & \text { si } x>0\end{cases}
\end{array}\right.
$$

avec $u_{g}<u_{d}$.

1. Montrer qu'il existe $\sigma \in \mathbb{R}$ tel que si $\left\{\begin{array}{ll}u(t, x)=u_{g} & \text { si } x<\sigma t \\ u(t, x)=u_{d} & \text { si } x>\sigma t\end{array}\right.$ alors $u$ est solution faible de (5.64). Vérifier que $u$ n'est pas solution entropique de (5.64).

2. Montrer que $u$ définie par :

$$
\begin{cases}u(t, x)=u_{g} & \text { si } x<2 u_{g} t \\ u(t, x)=\frac{x}{2 t} & \text { si } 2 u_{g} t \leq x \leq 2 u_{d} t \\ u(t, x)=u_{d} & \text { si } x>2 u_{d} t\end{cases}
$$

alors $u$ est solution faible entropique de 5.64).

Exercice 5.6 (Construction d'une solution faible entropique, II) Corrigé en page 246

On considère dans cet exercice l'équation de Burgers avec une condition initiale :

$$
\left\{\begin{array}{l}
\partial_{t} u+\partial_{x}\left(u^{2}\right)=0 \text { dans } \mathbb{R} \times \mathbb{R}_{+}^{\star} \\
u(\cdot, 0)=u_{0} \text { dans } \mathbb{R}
\end{array}\right.
$$

Construire la solution entropique de (5.66) pour $u_{0}$ définie par :

$$
u_{0}(x)= \begin{cases}0 & \text { si } x<0 \\ 1-x & \text { si } 0<x<1 \\ 1 & \text { si } x>1\end{cases}
$$

Exercice 5.7 (Problème de Riemann (1)) Soit $u_{d}$ et $u_{g}$ des nombres réels et $f$ une fonction de $\mathbb{R}$ dans $\mathbb{R}$ de classe $C^{1}$.

1. On suppose que $f$ est strictement convexe. Calculer la solution entropique du problème de Riemann (5.15) avec données $u_{d}$ et $u_{g}$. Expliciter la solution dans le cas $f(s)=s^{4}$.

2. On suppose que $f$ est strictement concave. Calculer la solution entropique du problème de Riemann (5.15) avec données $u_{d}$ et $u_{g}$.

\section{Exercice 5.8 (Equation de Buckley-Leverett) Corrigé en page 247}

On s'intéresse au problème de Riemann

$$
\begin{aligned}
& \partial_{t} u+\partial_{x} f(u)=0 \text { sur } \mathbb{R} \times \mathbb{R}_{+}, \\
& u(x, 0)=u_{g} \text { pour } x<0, u(x, 0)=u_{d} \text { pour } x>0 .
\end{aligned}
$$

On suppose ici que la fonction $f$ de $\mathbb{R}$ dans $\mathbb{R}$ est convexe puis concave : plus précisément, on considère $f \in$ $C^{2}(\mathbb{R}, \mathbb{R})$ avec

(i) $f(0)=0, f^{\prime}(0)=f^{\prime}(1)=0$

(ii) $\exists a \in] 0,1[$, tel que $f$ est strictement convexe sur $] 0, a[$, $f$ est strictement concave sur $] a, 1[$.

On suppose de plus que $u_{g}=1, u_{d}=0$. 
1. Montrer qu'il existe un unique point $b$ de l'intervalle $] a, 1\left[\right.$ tel que $\frac{f(b)}{b}=f^{\prime}(b)$. Puis, montrer qu'il existe un unique point $c$ de $] 0, b\left[\right.$ tel que $f^{\prime}(c)=f^{\prime}(b)$.

On conserve dans la suite cette notation des points $b$ et $c$.

2. On définit (p.p.) la fonction $u$ de $\mathbb{R} \times \mathbb{R}_{+}$dans $\mathbb{R}$ par :

$$
\begin{cases}u(x, t)=1 & \text { si } x \leq 0 \\ u(x, t)=\xi & \text { si } x=f^{\prime}(\xi) t, b<\xi<1 \\ u(x, t)=0 & \text { si } x>f^{\prime}(b) t\end{cases}
$$

Montrer que $u$ est la solution faible entropique de 5.15.

[Pour montrer que la condition d'entropie, on pourra commencer par remarquer que pour toute fonction $\eta$ $($ de $\mathbb{R}$ dans $\mathbb{R})$ de classe $C^{1}$ et convexe, on a $\int_{0}^{b}\left(f^{\prime}(b)-f^{\prime}(x)\right)\left(\eta^{\prime}(x)-\eta^{\prime}(c)\right) \mathrm{d} x \leq 0$.]

On peut ainsi construire la solution entropique du problème de Riemann dans le cas intéressant pour l'ingénierie pétrolière, de l'équation de Buckley-Leverett, c'est-à-dire pour

$$
f(u)=\frac{u^{2}}{u^{2}+\frac{(1-u)^{2}}{4}} \text { et } u_{g}, u_{d} \in[0,1]
$$

Pour cela, on distingue les cas où la fonction $f$ est convexe-concave ou convexe ou concave entre $u_{g}$ et $u_{d}$, selon les valeurs de $u_{g}$ et $u_{d}$.

Exercice 5.9 (Construction d'une solution faible entropique, III) Corrigé de l'exercice en page 248

Construire la solution entropique du problème

$$
\left\{\begin{array}{l}
\partial_{t} u+\partial_{x}\left(u^{2}\right)=0 \\
u(x, 0)=u_{0}(x)= \begin{cases}0 & \text { si } x<0 \\
x & \text { si } x \in[0,1] \\
0 & \text { si }>1\end{cases}
\end{array}\right.
$$

Vérifier que pour tout $t>0$ on a bien $\int_{\mathbb{R}} u(x, t) \mathrm{d} x=\int_{\mathbb{R}} u_{0}(x) \mathrm{d} x$.

Exercice 5.10 (Construction d'une solution faible entropique, IV) Corrigé en page 249

Construire la solution entropique du problème

$$
\left\{\begin{array}{l}
\partial_{t} u+\partial_{x}\left(u^{2}\right)=0 \\
u(x, 0)=u_{0}(x)= \begin{cases}1 & \text { si } x<-1 \\
0 & \text { si }-1<x<0, \\
2 & \text { si } 0<x<1 \\
0 & \text { si } x>1 .\end{cases}
\end{array}\right.
$$

Exercice 5.11 (Solution non entropique) Corrigé en page 251

On s'intéresse à l'équation de Burgers.

$$
\begin{gathered}
\partial_{t} u(x, t)+\partial_{x}\left(u^{2}\right)(x, t)=0, x \in \mathbb{R}, t \in \mathbb{R}_{+}, \\
u(x, 0)=0, x \in \mathbb{R} .
\end{gathered}
$$

1. (Question de cours...) Donner le sens de " $u$ solution faible de 5.69)-5.70" et " $u$ solution entropique de 5.69-5.70". 
On définit $u$ de $\mathbb{R} \times \mathbb{R}_{+}$dans $\mathbb{R}$ par :

$$
u(x, t)=\left\{\begin{array}{l}
0 \text { pour } x \in \mathbb{R}, t \in \mathbb{R}_{+}, x<-\sqrt{t}, \\
\frac{x}{2 t} \text { pour } x \in \mathbb{R}, t \in \mathbb{R}_{+}^{\star},-\sqrt{t}<x<\sqrt{t}, \\
0 \text { pour } x \in \mathbb{R}, t \in \mathbb{R}_{+}, x>\sqrt{t} .
\end{array}\right.
$$

2. Montrer que $u^{2} \in L_{\text {loc }}^{1}\left(\mathbb{R} \times \mathbb{R}_{+}\right)$et $u \in L_{\text {loc }}^{1}\left(\mathbb{R} \times \mathbb{R}_{+}\right)$. (On rappelle qu'une fonction $v$ de $\mathbb{R} \times \mathbb{R}_{+}$ dans $\mathbb{R}$ appartient à $L_{\text {loc }}^{1}\left(\mathbb{R} \times \mathbb{R}_{+}\right)$si $v \mathbf{1}_{K} \in L^{1}\left(\mathbb{R} \times \mathbb{R}_{+}\right)$pour tout $K \subset \mathbb{R} \times \mathbb{R}_{+}=\mathbb{R} \times[0, \infty[, \mathbf{K}$ compact.)

3. (Solution faible (1)) Montrer que $u$ vérifie :

$$
\int_{\mathbb{R}_{+}} \int_{\mathbb{R}^{R}}\left(u(x, t) \partial_{t} \varphi(x, t)+u^{2}(x, t) \partial_{x} \varphi(x, t)\right) \mathrm{d} x \mathrm{~d} t=0, \forall \varphi \in C_{c}^{1}\left(\mathbb{R}_{+}^{\star} \times \mathbb{R}, \mathbb{R}\right) .
$$

4. (Solution faible (2)) Montrer que $u$ est solution faible de (5.69)-(5.70), c'est-à-dire que $u$ vérifie :

$$
\int_{\mathbb{R}_{+}} \int_{\mathbb{R}^{R}}\left(u(x, t) \partial_{t}(x, t)+u^{2}(x, t) \partial_{x} \varphi(x, t)\right) \mathrm{d} x \mathrm{~d} t=0, \forall \varphi \in C_{c}^{1}\left(\mathbb{R}_{+} \times \mathbb{R}, \mathbb{R}\right) .
$$

5. (Solution entropique?) Soit $\eta$ une fonction convexe de $\mathbb{R}$ dans $\mathbb{R}$, de classe $C^{1}$. On définit $\phi$ de $\mathbb{R}$ dans $\mathbb{R}$ par : $\phi(s)=\int_{0}^{s} \eta^{\prime}(\xi) 2 \xi d \xi$, pour tout $s \in \mathbb{R}$. Montrer que $u$ vérifie :

$$
\int_{\mathbb{R}_{+}} \int_{\mathbb{R}}\left(\eta(u)(x, t) \partial_{t} \varphi(x, t)+\phi(u)(x, t) \partial_{x} \varphi(x, t)\right) \mathrm{d} x \mathrm{~d} t \geq 0, \forall \varphi \in C_{c}^{1}\left(\mathbb{R} \times \mathbb{R}_{+}^{\star}, \mathbb{R}_{+}\right) .
$$

[On pourra commencer par étudier, grâce à la convexité de $\eta$, le signe de $\phi(s)-s(\eta(s)-\eta(0))$.]

6. Montrer que la fonction $u$ n'est pas la solution entropique de (5.69)-5.70).

N.B : La question 5 montre que $\partial_{t} \eta(u)+\partial_{x} \phi(u) \leq 0$ au sens des dérivées par transposition dans $\mathbb{R} \times \mathbb{R}_{+}^{\star}$. Mais on ne peut pas montrer 5.73 (en ajoutant $\int_{\mathbb{R}} \eta(0) \varphi(x, 0) \mathrm{d} x$ si $\eta(0) \neq 0$ ) pour tout $\varphi \in C_{c}^{1}\left(\mathbb{R} \times \mathbb{R}_{+}, \mathbb{R}_{+}\right.$), même en se limitant à considérer les entropies de Krushkov. Ceci est dû au fait que $u(\cdot, t) \not \rightarrow 0$ dans $L_{\text {loc }}^{1}(\mathbb{R})$ quand $t \rightarrow 0$ (cette convergence dans $L_{\mathrm{loc}}^{1}(\mathbb{R})$ serait d'ailleurs vraie si $u$ était solution entropique de $(5.69)-(5.70)$ ). On a seulement $u(\cdot, t) \rightarrow 0$ dans $\mathcal{D}^{\star}(\mathbb{R})$ et c'est ce qui a été utilisé dans la question 4 pour démontrer que $u$ est solution faible.

Exercice 5.12 (Flux strictement convexe et entropie) Soit $f \in C^{1}(\mathbb{R}, \mathbb{R})$ et $u_{0} \in L^{\infty}(\mathbb{R})$. On s'intéresse ici à la solution entropique du problème suivant :

$$
\left\{\begin{array}{l}
\partial_{t} u+\partial_{x}(f(u))=0, \quad(x, t) \in \mathbb{R} \times \mathbb{R}_{+}, \\
u(x, 0)=u_{0}(x)
\end{array}\right.
$$

Soit $\sigma \in \mathbb{R}, D_{1}=\left\{(x, t) \in \mathbb{R} \times \mathbb{R}_{+}^{\star} ; x<\sigma t\right\}$ et $D_{2}=\left\{(x, t) \in \mathbb{R} \times \mathbb{R}_{+}^{\star} ; x>\sigma t\right\}$. On suppose que $u_{\mid D_{i}} \in C^{1}\left(\bar{D}_{i}, \mathbb{R}\right)(i=1,2)$ et que $u$ est solution faible de (5.74). En particulier, on a donc (voir la proposition 5.7)

$$
\sigma[u](\sigma t, t)=[f(u)](\sigma t, t) \text { pour tout } t \in \mathbb{R}_{+} .
$$

1. Montrer que $u$ est solution entropique de (5.74) si et seulement si

$$
\sigma[\eta(u)](\sigma t, t) \geq[\phi(u)](\sigma t, t) \text { pour tout } t \in \mathbb{R}_{+},
$$

pour toute fonction $\eta \in C^{1}(\mathbb{R}, \mathbb{R})$ convexe et $\phi \in C^{1}(\mathbb{R}, \mathbb{R})$ telle que $\phi^{\prime}=f^{\prime} \eta^{\prime}$. 
2. Si $f$ est strictement convexe et $u$ est solution entropique de (5.74), montrer que $u_{-}(\sigma t, t) \geq u_{+}(\sigma t, t)$ pour tout $t \in \mathbb{R}^{+}$. [On pourra choisir $\eta=f$ dans 5.76 .]

On rappelle que $u_{+}(\sigma t, t)=\lim _{x \downarrow \sigma t} u(x, t), u_{-}(\sigma t, t)=\lim _{x \uparrow \sigma t} u(x, t),[u](\sigma t, t)=u_{+}(\sigma t, t)-u_{-}(\sigma t, t)$ et $[g(u)](\sigma t, t)=g\left(u_{+}(\sigma t, t)\right)-g\left(u_{-}(\sigma t, t)\right)$ pour $g=f, \eta$ ou $\phi$.

\section{Exercice 5.13 (Effet Landau 8 ) Corrigé en page 252}

Soit $f$ une fonction borélienne bornée et périodique de $\mathbb{R}$ dans $\mathbb{R}$ (pour simplifier, on peut supposer que $f$ est continue périodique de $\mathbb{R}$ dans $\mathbb{R}$ ). On s'intéresse dans cet exercice à la limite quand $t \rightarrow+\infty$ de la solution (faible) du problème suivant :

$$
\begin{aligned}
& \frac{\partial u}{\partial t}(x, y, t)+y \frac{\partial u}{\partial x}(x, y, t)=0, x, y \in \mathbb{R}, t \in \mathbb{R}_{+}^{\star}, \\
& u(x, y, 0)=f(x), x, y \in \mathbb{R} .
\end{aligned}
$$

1. Donner explicitement en fonction de $f$ l'unique solution faible de 5.77).

Dans la suite, on note $u$ cette solution faible.

On remarquera que $u$ est continue de $\mathbb{R}_{+}$dans $L_{l o c}^{p}\left(\mathbb{R}^{2}\right)$ pour tout $p<\infty$.

On note aussi $m$ la moyenne de $f$ sur une période. Enfin, pour tout $y \in \mathbb{R}$ et $r>0$, on pose

$$
F(y, r)=\frac{1}{2 r} \int_{y-r}^{y+r} f(z) d z .
$$

2. (Question liminaire) Montrer que $\lim _{r \rightarrow+\infty} F(y, r)=m$, uniformément par rapport à $y \in \mathbb{R}$.

3. Soit $a, b \in \mathbb{R}$ et $\delta>0$. Montrer que

$$
\lim _{t \rightarrow+\infty} \int_{b-\delta}^{b+\delta} \int_{a-\delta}^{a+\delta} u(x, y, t) \mathrm{d} x \mathrm{~d} y=4 \delta^{2} m .
$$

[On pourra remarquer que $\int_{b-\delta}^{b+\delta} f(x-y t) \mathrm{d} y=2 \delta F(x-b t, \delta t)$.]

4. Montrer que $u(\cdot, \cdot, t) \rightarrow m \star$-faiblement dans $L^{\infty}\left(\mathbb{R}^{2}\right)$, quand $t \rightarrow+\infty$.

5. Montrer que le résultat de la question 4 reste vrai si on remplace dans (5.77) y $\frac{\partial u}{\partial x}$ par $a(y) \frac{\partial u}{\partial x}$ où $a \in$ $C^{1}(\mathbb{R}, \mathbb{R})$ et $a^{\prime}(y) \neq 0$ pour tout $y \in \mathbb{R}$. (Plus généralement, ce résultat reste vrai sous l'hypothèse plus faible demandant que l'ensemble des points ou $a^{\prime}$ s'annule soit de mesure nulle.)

Exercice 5.14 (Principe du maximum et positivité) Soit $v$ une fonction lipschitzienne et de classe $C^{1}$ de $\mathbb{R}$ dans $\mathbb{R}$. Soit $u_{0} \in C_{b}(\mathbb{R}, \mathbb{R})$. On s'intéresse aux deux problèmes suivants :

$$
\begin{array}{r}
\frac{\partial u}{\partial t}(x, t)+v \frac{\partial u}{\partial x}(x, t)=0, x \in \mathbb{R}, t \in \mathbb{R}_{+}^{\star}, \\
u(x, 0)=u_{0}(x), x \in \mathbb{R} . \\
\frac{\partial u}{\partial t}(x, t)+\frac{\partial v u}{\partial x}(x, t)=0, x \in \mathbb{R}, t \in \mathbb{R}_{+}^{\star}, \\
u(x, 0)=u_{0}(x), x \in \mathbb{R} .
\end{array}
$$


1. Soit $A, B \in \mathbb{R}$ t.q. $A \leq u_{0}(x) \leq B$ pour tout $x \in \mathbb{R}$. Soit $u$ la solution (continue) de (5.78), montrer que $A \leq u(x, t) \leq B$ pour tout $x \in \mathbb{R}$ et $t \in \mathbb{R}_{+}$. Montrer (en donnant un exemple) que cette propriété peut être fausse si $u$ est solution de 5.79 .

2. On suppose que $u_{0}(x) \geq 0$ pour tout $x \in \mathbb{R}$. Soit $u$ la solution (continue) de (5.79], montrer que $u(x, t) \geq 0$ pour tout $x \in \mathbb{R}$ et $t \in \mathbb{R}_{+}$.

\section{Exercice 5.15 (Equations de Saint-Venant) Corrigé en page 255}

On considère, dans cet exercice, le système des équations de Saint-Venant à une dimension d'espace, c'est-à-dire le système suivant :

$$
\begin{aligned}
& \partial_{t} h(x, t)+\partial_{x}(h u)(x, t)=0, x \in \mathbb{R}, t \in \mathbb{R}_{+}, \\
& \partial_{t}(h u)(x, t)+\partial_{x}\left(h u^{2}+\frac{g}{2} h^{2}\right)(x, t)=0, x \in \mathbb{R}, t \in \mathbb{R}_{+},
\end{aligned}
$$

où $g$ est un nombre réel donné, $g>0$. Le système (5.82) est une modélisation simple du problème de l'écoulement d'un fluide en eau peu profonde. L'inconnue $h(x, t)$ est la hauteur de la colonne d'eau située au point $x$ à l'instant $t$. L'inconnue $u(x, t)$ est la vitesse de cette colonne d'eau (située au point $x$ à l'instant $t$ ). Le nombre $g$ correspond à l'intensité de la gravité.

La fonction $h$ prend ses valeurs dans $\mathbb{R}_{+}^{\star}$ et la fonction $u$ prend ses valeurs dans $\mathbb{R}$.

On introduit deux nouvelles inconnues :

- la quantité de mouvement, $q: \mathbb{R} \times \mathbb{R}_{+} \rightarrow \mathbb{R}$ définie par $q=h u$,

- la célérité des ondes, $c: \mathbb{R} \times \mathbb{R}_{+} \rightarrow \mathbb{R}_{+}^{\star}$, définie par $c=\sqrt{g h}$.

On note également

$$
U=\left[\begin{array}{l}
h \\
q
\end{array}\right], V=\left[\begin{array}{c}
u \\
2 c
\end{array}\right], p=\frac{g h^{2}}{2} \text { et } D=\left\{\left[\begin{array}{l}
h \\
q
\end{array}\right] \in \mathbb{R}^{2}, h>0\right\} .
$$

1. (Forme équivalente) En définissant $F: D \rightarrow \mathbb{R}^{2}$ convenablement, montrer que le système 5.80-5.81) s'écrit aussi

$$
\partial_{t} U(x, t)+\partial_{x}(F(U))(x, t)=0, x \in \mathbb{R}, t \in \mathbb{R}_{+} .
$$

Dans toute la suite de l'exercice, $F$ est la fonction définie dans cette première question.

2. (Hyperbolicité) Pour tout $U \in D$, calculer les valeurs propres de la matrice jacobienne de $F$ au point $U$ et donner une base de $\mathbb{R}^{2}$ formée de vecteurs propres de cette matrice jacobienne. Montrer que le système (5.82) est strictement hyperbolique dans le domaine $D$.

Dans la suite, on note $\lambda_{1}(U)$ et $\lambda_{2}(U)$ (avec $\lambda_{1}(U)<\lambda_{2}(U)$ ) les deux valeurs propres de la matrice jacobienne de $F$ au point $U$.

3. (Nature des champs) Montrer que les deux champs du système 5.82 ) sont VNL (c'est-à-dire vraiment non linéaires, au sens de la definition 5.35) dans tout le domaine $D$.

4. (Invariants) Calculer des invariants de Riemann (non triviaux) associés à chacun des deux champs du système (5.82) (voir définition 5.36. On pourra les chercher de la forme $u+\psi(h)$.

5. (Entropie) Pour tout $U=\left[\begin{array}{l}h \\ q\end{array}\right] \in D$, on pose $\eta(U)=\frac{1}{2} h u^{2}+p$ (on rappelle que $q=h u$ et $p=g \frac{h^{2}}{2}$ ). Montrer que $\eta(U)$ est une entropie du système, c'est-à-dire que $\eta$ est convexe et qu'il existe une fonction $\Phi$ telle que $\partial_{t} \eta(U)+\partial_{x}(\Phi(U))=0$ pour toute solution régulière de (5.80)-5.81) (et donc de (5.82).

N.B. On peut remarquer que pour le système de Saint-Venant (5.82), la quantité $\eta(U(x, t))$ est l'énergie totale de cette colonne d'eau située au point $x$ à l'instant $t$, c'est-à-dire la somme de l'énergie cinétique et de l'énergie potentielle. 
6. (Limite de solutions visqueuses.) On ajoute des termes de régularisation dans le système $(5.80)-(5.81)$, plus précisément $-\varepsilon \partial_{x}^{2} h$ dans la première équation et $-\varepsilon \partial_{x}^{2} q$ dans la deuxième équation (avec $\epsilon>0$ ). On note $h_{\varepsilon}$ et $u_{\varepsilon}$ (et donc $q_{\varepsilon}=h_{\varepsilon} u_{\varepsilon}$ ) les solutions de ce nouveau système. On suppose que ce sont des fonctions régulières, bornées dans $L^{\infty}\left(\mathbb{R} \times \mathbb{R}_{+}\right)$indépendamment de $\varepsilon$, et qu'elles convergent dans $L_{\text {loc }}^{1}\left(\mathbb{R} \times \mathbb{R}_{+}\right)$, quand $\varepsilon \rightarrow 0$ vers des fonctions $h$ et $u$ respectivement (avec $h>0$ ). On suppose aussi que $\sqrt{\varepsilon} \partial_{x} h$ et $\sqrt{\varepsilon} \partial_{x} q$ sont bornées dans $L_{l o c}^{2}\left(\mathbb{R} \times \mathbb{R}_{+}\right)$. Montrer que le couple $(h, u)$ est solution de (5.80)-(5.81) et vérifie, au sens des distributions,

$$
\partial_{t} \eta(U)+\partial_{x} \Phi(U) \leq 0
$$

7. (Forme équivalente de 5.80)-5.81) pour des solutions régulières). Soit $(h, u)$ une solution régulière de 5.80)5.81. Montrer que $V=\left[\begin{array}{c}u \\ 2 c\end{array}\right]$ est solution du système

$$
\partial_{t} V(x, t)+B(V) \partial_{x} V(x, t)=0, x \in \mathbb{R}, t \in \mathbb{R}_{+}, \text {avec } B(V)=\left[\begin{array}{ll}
u & c \\
c & u
\end{array}\right] .
$$

On s'intéresse maintenant au problème de Riemann, c'est-à-dire au système [5.80)-(5.81) (équivalent à 5.82) avec la condition initiale

$$
\begin{aligned}
& h(x, 0)=h_{g}, u(x, 0)=u_{g}, x<0, \\
& h(x, 0)=h_{d}, u(x, 0)=u_{d}, x>0,
\end{aligned}
$$

où $h_{g}, h_{d} \in \mathbb{R}_{+}^{\star}$ et $u_{d}, u_{g} \in \mathbb{R}$ sont donnés. On pose $q_{g}=h_{g} u_{g}, q_{d}=h_{d} u_{d}, c_{g}=\sqrt{g h_{g}}, c_{d}=\sqrt{g h_{d}}$.

On suppose que $u_{d}-u_{g}<2\left(c_{g}+c_{d}\right)$ (cette condition est nécessaire pour que le problème [5.82), (5.84)-(5.85) ait une solution prenant ses valeurs dans $D$, c'est la condition de "non apparition du vide").

8. (Deux détentes) On suppose, dans cette question, que $2\left|c_{g}-c_{d}\right| \leq u_{d}-u_{g}$. Construire la solution du problème de Riemann (5.82), 5.84)-(5.85). [Cette solution est formée de 2 détentes reliées par un état dit intermédiaire, noté $\left(h_{\star}, u_{\star}\right)$, avec $h_{\star}>0$. On pourra chercher la solution dans les zones de détente avec $u$ et $c$ sous la forme de fonctions affines de $x / t$.]

9. (Détente-choc, choc-détente et choc-choc) On suppose, dans cette question, que $2\left|c_{g}-c_{d}\right|>u_{d}-u_{g}$ et on pose

$$
S=\sqrt{\frac{g\left(h_{g}-h_{d}\right)\left(h_{g}^{2}-h_{d}^{2}\right)}{2 h_{g} h_{d}} .}
$$

(a) (Calcul d'un choc) On suppose dans cette question qu'il existe $\sigma \in \mathbb{R}$, tel que

$$
U(x, t)=U_{g} \text { si } x<\sigma t, U(x, t)=U_{d} \text { si } x>\sigma t .
$$

i. Montrer que $U$ est solution faible de (5.82), 5.84 - (5.85) si et seulement si $u_{d}=u_{g} \pm S$ et $\sigma\left(h_{d}-h_{g}\right)=$ $\left(q_{d}-q_{g}\right)$.

Définition 5.41 (Condition de Lax) Cette solution faible est un 1-choc si $\lambda_{1}\left(U_{g}\right)>\sigma>\lambda_{1}\left(U_{d}\right)$ et c'est un 2-choc si $\lambda_{2}\left(U_{g}\right)>\sigma>\lambda_{2}\left(U_{d}\right)$.

Montrer que $U$ est un 1-choc si et seulement si $u_{d}=u_{g}-S$ et $h_{g}<h_{d}$. Montrer que $U$ est un 2-choc si et seulement si $u_{d}=u_{g}-S$ et $h_{g}>h_{d}$.

[On pourra commencer par montrer que la condition de Lax et la valeur de $\sigma$ imposent $u_{d}<u_{g}$.]

Pour $x \geq 1$, on pose $\varphi(x)=\sqrt{\frac{(x-1)\left(x^{2}-1\right)}{x}}$. 
ii. Montrer que $\varphi$ est strictement croissante.

Montrer que pour tout $u_{g}, h_{g}$ et $h_{d}$, avec $h_{d}>h_{g}$, il existe un seul $u_{d}$ tel que $U$ soit un 1-choc.

(b) (Détente-choc) On suppose dans cette question que $u_{g}-u_{d}<S$ et $h_{d}<h_{g}$. Montrer qu'on peut construire une solution de 5.82, 5.84-5.85) formée d'une 1-détente et d'un 2-choc reliés par un état dit intermédiaire, noté $\left(h_{\star}, u_{\star}\right)$.

[Remarquer qu'on cherche $\left(h_{\star}, u_{\star}\right)$ tel que $u_{\star}+2 c_{\star}=u_{g}+2 c_{g}, u_{\star}=u_{d}+\sqrt{g h_{d} / 2} \varphi\left(\frac{h_{\star}}{h_{d}}\right), h_{\star}>h_{d}$ et qu'il faut $u_{g}-c_{g}<u_{\star}-c_{\star}<\sigma$ où $\sigma$ est la vitesse du 2-choc.]

(c) (Choc-détente) On suppose dans cette question que $u_{g}-u_{d}<S$ et $h_{d}>h_{g}$. Montrer qu'on peut construire une solution de 5.82, 5.84)-5.85) formée d'un 1-choc et d'une 2-détente.

(d) (Choc-choc) On suppose dans cette question que $u_{g}-u_{d}>S$. Montrer qu'on peut construire une solution de 5.82, 5.84-5.85) formée d'un 1-choc et d'un 2-choc.

10. (Problème de Riemann linéarisé) On pose $\bar{V}=\left[\begin{array}{c}\bar{u} \\ 2 \bar{c}\end{array}\right], \bar{u}=\left(u_{g}+u_{d}\right) / 2$ et $\bar{c}=\left(c_{g}+c_{d}\right) / 2$.

On remplace dans le problème de Riemann (5.82, 5.84)- 5.85, l'équation 5.82 par l'équation suivante :

$$
\partial_{t} V(x, t)+B(\bar{V}) \partial_{x} V(x, t)=0, x \in \mathbb{R}, t \in \mathbb{R}_{+} .
$$

où $B$ est définie par [5.83). Construire la solution du problème de Riemann linéarisé [5.86, 5.84)- 5.85).

N.B. : On remarque que, grâce à la condition $u_{d}-u_{g}<2\left(c_{g}+c_{d}\right)$, ce nouveau problème de Riemann admet une solution avec un état intermédiaire $\left(u_{\star}, 2 c_{\star}\right)$ t.q. $c_{\star}>0$, et donc $c_{\star}=\sqrt{g h_{\star}}$ avec un $h_{\star}>0$. (Ceci n'est pas le cas si $u_{d}-u_{g} \geq 2\left(c_{g}+c_{d}\right)$.) Le fait que $h_{\star}>0$ est important lorsque que l'on remplace dans le schéma de Godunov la résolution du problème de Riemann par la résolution de ce problème de Riemann linéarisé.

Exercice 5.16 (Entropie pour les équations de Saint-Venant avec gradient de fond) Corrigé page 263

On s'intéresse dans cet exercice au système d'équations (à une dimension d'espace) modélisant un écoulement d'eau sur un fond non plat. On note $z$ la fonction régulière de $\mathbb{R}$ dans $\mathbb{R}_{+}$donnant la cote du fond et $g$ l'intensité de la gravité. Le système considéré sécrit alors

$$
\begin{aligned}
& \partial_{t} h(x, t)+\partial_{x}(h u)(x, t)=0, x \in \mathbb{R}, t \in \mathbb{R}_{+}, \\
& \partial_{t}(h u)(x, t)+\partial_{x}\left(h u^{2}+g \frac{h^{2}}{2}\right)(x, t)+g h z^{\prime}(x)=0, x \in \mathbb{R}, t \in \mathbb{R}_{+},
\end{aligned}
$$

On reprend ici les questions 5 et 6 de l'exercice 5.15

1. (Entropie) Pour tout $U=\left[\begin{array}{l}h \\ q\end{array}\right] \in D$, on pose $\eta(U)=\frac{1}{2} h u^{2}+p+g h z$ (avec $q=h u$ et $p=g h^{2} / 2$ ). Montrer que $\eta(U)$ est une entropie du système, c'est-à-dire que $\eta$ est convexe et qu'il existe une fonction $\Phi$ telle que $\partial_{t} \eta(U)+\partial_{x}(\Phi(U))=0$ pour toute solution régulière de 5.87)-(5.88).

N.B. Comme dans l'exercice 5.15, question 5, la quantité $\eta(U(x, t))$ est l'énergie totale de la colonne d'eau située au point $x$ à l'instant $t$ (c'est-à-dire la somme de l'énergie cinétique et de l'énergie potentielle).

2. (Limite de solutions visqueuses) On ajoute des termes de régularisation dans le système 5.87)-(5.88), c'est-àdire $-\varepsilon \partial_{x}^{2} h$ pour la première équation et $-\varepsilon \partial_{x}^{2} q$ pour la deuxième équation (avec $\varepsilon>0$ ). On note $h_{\varepsilon}$ et $u_{\varepsilon}$ (et donc $q_{\varepsilon}=h_{\varepsilon} u_{\varepsilon}$ ) les solutions de ce nouveau système. On suppose que ce sont des fonctions régulières bornées dans $L^{\infty}\left(\mathbb{R} \times \mathbb{R}_{+}\right)$indépendamment de $\varepsilon$, et qu'elles convergent dans $L_{\text {loc }}^{1}\left(\mathbb{R} \times \mathbb{R}_{+}\right)$, quand $\varepsilon \rightarrow 0$, vers des fonctions $h$ et $u$ (avec $h>0$ ). On suppose aussi que $\varepsilon \partial_{x} h$ et $\varepsilon \partial_{x} q$ sont bornées dans $L_{l o c}^{2}\left(\mathbb{R} \times \mathbb{R}_{+}\right)$. Montrer que le couple $(h, u)$ est solution de 5.87)-(5.88) et vérifie, au sens des distributions,

$$
\partial_{t} \eta(U)+\partial_{x} \Phi(U) \leq 0
$$


Exercice 5.17 (Solutions stationnaires régulières pour les équations de Saint-Venant) Corrigé en page 263 On cherche à construire, dans cet exercice, des solutions stationnaires régulières au système d'équations (à une dimension d'espace) modélisant un écoulement d'eau sur un fond non plat. On note $z$ la fonction régulière de $\mathbb{R}$ dans $\mathbb{R}_{+}$donnant la cote du fond et $g$ l'intensité de la gravité. Le système considéré sécrit alors

$$
\begin{aligned}
& \partial_{t} h(x, t)+\partial_{x}(h u)(x, t)=0, x \in \mathbb{R}, t \in \mathbb{R}_{+}, \\
& \partial_{t}(h u)(x, t)+\partial_{x}\left(h u^{2}+g \frac{h^{2}}{2}\right)(x, t)+g h z^{\prime}(x)=0, x \in \mathbb{R}, t \in \mathbb{R}_{+},
\end{aligned}
$$

Dans la suite de cet exercice, on note $q$ la fonction $h u$ et $\psi$ la fonction $\frac{u^{2}}{2}+g h+g z$.

Dans la suite, on appelle "solution stationnaire régulière" un couple de fonctions de classe $C^{1}$, notée $(h, u)$, de $\mathbb{R}$ dans $\mathbb{R}_{+}^{\star} \times \mathbb{R}$, solution stationnaire de (5.89)-(5.90) (noter que $h(x)>0$ pour tout $x \in \mathbb{R}$ ). On suppose aussi que $z$ est de classe $C^{1}$.

1. Montrer que le couple $(h, u)\left(\right.$ de $\mathbb{R}$ dans $\mathbb{R}_{+}^{\star} \times \mathbb{R}$ ) est une solution stationnaire régulière de 5.89 - 5.90 si et seulement si les fonctions $q$ et $\psi$ sont des fonctions constantes.

On se donne donc deux nombres positifs $\alpha$ et $\beta$ et on cherche un couple de fonctions $(h, u)$ tel que $q(x)=\alpha$ et $\psi(x)=\beta$ pour tout $x \in \mathbb{R}$.

On note $z_{m}$ le maximum de la fonction $z$, et on suppose (bien sûr) que $z_{m}<+\infty$.

2. (Lac au repos) On suppose, dans cette question, que $\alpha=0$. Montrer qu'il n'y a pas de solution stationnaire régulière si $\beta \leq g z_{m}$ (on rappelle que la fonction $h$ doit être à valeurs strictement positives) et que la seule solution stationnaire régulière est donnée par $h(x)=\beta / g-z(x)$ (pour tout $x \in \mathbb{R}$ ) si $\beta>g z_{m}$.

Dans la suite de l'exercice, on suppose $\alpha>0$ et on pose on pose $\beta_{m}=g z_{m}+(3 / 2)(\alpha g)^{2 / 3}$.

3. Montrer que :

(a) $\mathrm{Si} \beta<\beta_{m}$, il n'y pas de solution stationnaire régulière associée au couple $(\alpha, \beta)$,

(b) $\mathrm{Si} \beta>\beta_{m}$, il y a (exactement) deux solutions stationnaires régulières associées au couple $(\alpha, \beta)$,

4. On suppose, dans cette question, que $\beta=\beta_{m}$. Montrer que :

(a) si $z$ est une fonction constante, il y a (exactement) une solution stationnaire régulière associée au couple $(\alpha, \beta)$,

(b) si $z(x) \neq z_{m}$ pour tout $x$ (et donc $z$ est non constante), il y a (exactement) deux solutions stationnaires régulières associées au couple $(\alpha, \beta)$.

Dans la suite de l'exercice, on fixe $\alpha$ et $\beta$ avec $\alpha>0$ et $\beta>\beta_{m}$. On note $\left(h_{i}, u_{i}\right), i=1,2$, les deux couples de solutions stationnaires régulières.

5. Montrer que $h_{1}-h_{2}$ a un signe constant. On peut donc supposer $h_{1}(x)<h_{2}(x)$ pour tout $x$. Montrer qu'il existe des fonctions régulières $\varphi_{1}$ et $\varphi_{2}$ telle que $h_{i}(x)=\varphi_{i}(z(x))$ pour tout $x$.

Montrer que $h_{2}+z$ est décroissante quand $z$ est croissante (et croissante quand $z$ est décroissante)

Montrer que $h_{1}+z$ est croissante quand $z$ est décroissante (et croissante quand $z$ est décroissante)

6. Déterminer la nature des écoulements trouvés (on rappelle que $\alpha>0$ et $\beta>\beta_{m}$ ), c'est-à-dire a-t-on $u>\sqrt{g h}$ ou $u<\sqrt{g h}$ ?

Exercice 5.18 (Equations de Saint-Venant, entropie au sens de Lax) 
On considère une nouvelle fois le système des équations de Saint-Venant à une dimension d'espace, c'est-à-dire le système suivant :

$$
\begin{aligned}
& \partial_{t} h(x, t)+\partial_{x}(h u)(x, t)=0, x \in \mathbb{R}, t \in \mathbb{R}_{+}, \\
& \partial_{t}(h u)(x, t)+\partial_{x}\left(h u^{2}+\frac{g}{2} h^{2}\right)(x, t)=0, x \in \mathbb{R}, t \in \mathbb{R}_{+},
\end{aligned}
$$

où $g$ est un nombre réel donné, $g>0$. On pose $q=h u$.

Soit $u_{g}, u_{d} \in \mathbb{R}, h_{g}, h_{d} \in \mathbb{R}^{\star}$ et $\sigma \in \mathbb{R}$. On pose $q_{g}=h_{g} u_{g}, q_{d}=h_{d} u_{d}$. On définit la fonction $U=\left[\begin{array}{c}h \\ q\end{array}\right]$ de $\mathbb{R} \times \mathbb{R}_{+}$dans $\mathbb{R}_{+}^{\star} \times \mathbb{R}$ par

$$
\begin{aligned}
& U(x, t)=\left[\begin{array}{l}
h_{g} \\
q_{g}
\end{array}\right] \text { si } x<\sigma t, \\
& U(x, t)=\left[\begin{array}{l}
h_{d} \\
q_{d}
\end{array}\right] \text { si } x>\sigma t .
\end{aligned}
$$

On suppose que $U$ est solution faible discontinue de (5.91)-5.92) avec la condition initiale $U_{0}=\left[\begin{array}{c}h_{g} \\ q_{g}\end{array}\right]$ si $x<0$, $U_{0}=\left[\begin{array}{l}h_{d} \\ q_{d}\end{array}\right]$ si $x>0$.

1. Montrer que $U$ est solution entropique au sens de la définition 5.34 avec l'entropie $\eta(U)=h u^{2} / 2+g h^{2} / 2$ si et seulement si $u_{d}<u_{g}$.

Corrigé - La fonction U est solution faible si et seulement les conditions de Rankine-Hugoniot sont satisfaites sur la droite $x=\sigma t$, c'est-à-dire, avec les notations du cours, $\sigma[h]=[h u]$ et $\sigma[h u]=\left[h u^{2}+p\right]$. On remarque tout d'abord que $h_{d} \neq h_{g}$, sinon $\sigma[h]=[h u]$ donne $u_{g}=u_{d}$ en contradiction avec le fait que $U$ est discontinue. On a alors aussi $u_{g} \neq u_{d}$ car $u_{g}=u_{d}$ donne $\sigma=u_{g}$ et $\sigma[h u]=\left[h u^{2}+p\right]$ donne alors $[p]=0$ et donc $h_{g}=h_{d}$, en contradiction avec le fait que $U$ est discontinue.

Pour le calcul ci dessous, on suppose, par simple souci de lisibilité, que $g=1$ et on pose $v_{g}=\bar{v}, v_{d}=v$ pour $v=h$, u et $p\left(p=h^{2} / 2\right)$. Les conditions de Rankine-Hugoniot s'écrivent

$$
\begin{array}{r}
\sigma(h-\bar{h})=(h u-\bar{h} \bar{u}) \\
\sigma(h u-\bar{h} \bar{u})=\left(h u^{2}+p-\bar{h} \bar{u}^{2}-\bar{p}\right) .
\end{array}
$$

On multiplie la lere équation par $(h+\bar{h}) / 2-(u+\bar{u})^{2} / 8$, la 2eme par $(u+\bar{u}) / 2$ et on additionne, on obtient

$$
\sigma\left[p+\frac{1}{2} h u^{2}\right]-\sigma \frac{[h]}{8}(u-\bar{u})^{2}=\left[\frac{1}{2} h u^{3}+2 p u\right]-\frac{[h u]}{8}(u-\bar{u})^{2}-\frac{[u]}{4}[h]^{2},
$$

et donc, comme $\sigma[h]=[h u]$, avec $\eta(U)=\frac{1}{2} h u^{2}+p$ et $\phi(U)=\frac{1}{2} h u^{3}+2 p u$,

$$
\sigma[\eta(U)]=[\phi(U)]-\frac{[u]}{4}[h]^{2} .
$$

Ceci prouve que est solution entropique au sens de la définition 5.34 avec l'entropie $\eta(U)=h u^{2} / 2+g h^{2} / 2$ si et seulement si $[u] \leq 0$, c'est-à-dire $u_{d}<u_{g}$ (car on sait déjà que $\left.u_{g} \neq u_{d}\right)$.

2. En utilisant l'exercice 5.15 question 9a montrer que $U$ est solution entropique au sens de la définition 5.34 avec l'entropie $\eta(U)=h u^{2} / 2+g h^{2} / 2$ si et seulement si $U$ vérifie la condition de Lax 5.41 .

Corrigé - Si U est solution entropique au sens de la définition 5.34 avec l'entropie $\eta(U)=h u^{2} / 2+g h^{2} / 2$, la question précedente nous montre que $u_{d}<u_{g}$, c'est-à-dire, avec les notations de l'exercice 5.15 question 9a que 
$u_{d}=u_{g}-S$ (on a $S>0$ ). La question $9 a$ de l'exercice 5.15 donne alors que $U$ est 1 -choc si $h_{g}<h_{d}$ et un 2 -choc si $h_{d}<h_{g}$. La fonction $U$ vérifie donc la condition de Lax.

Réciproquement, si U est une solution faible qui vérifie la condition de Lax, on a nécessairement $u_{d}<u_{g}$, c'est la première étape de la démonstration de la question 9 a de l'exercice 5.15 (et on montre ensuite dans cette exercice que c'est un 1-choc si $h_{g}<h_{d}$ et un 2 -choc si $h_{g}>h_{d}$ ). La question précédente nous donne alors

$$
\sigma[\eta(U)]=[\phi(U)]-\frac{[u]}{4}[h]^{2}>[\phi(U)]
$$

ce qui prouve que $U$ est solution entropique au sens de la définition 5.34 avec l'entropie $\eta(U)=h u^{2} / 2+g h^{2} / 2$.

Exercice 5.19 (Equation linéaire avec terme source singulier) Corrigé en page 266

Soient $a, b, c, u_{g}, u_{d} \in \mathbb{R}$. On définit la mesure $\mu$ sur $\mathbb{R} \times \mathbb{R}_{+}$par :

$$
\int_{\mathbb{R} \mathbb{R}_{+}} \varphi d \mu=b \int_{0}^{+\infty} \varphi(c t, t) d t \text { pour tout } \varphi \in C_{c}^{\infty}\left(\mathbb{R} \times \mathbb{R}_{+}, \mathbb{R}\right) .
$$

La mesure $\mu$ est donc une mesure portée par la demi-droite $\Gamma_{c}=\left\{(c t, t), t \in \mathbb{R}_{+}\right\}$. On définit $u_{0}$ par $u_{0}(x)=u_{g}$ si $x<0$ et $u_{0}(x)=u_{d}$ si $x>0$. On s'intéresse au problème

$$
\begin{array}{r}
\partial_{t} u+a \partial_{x} u=\mu \text { dans } \mathbb{R} \times \mathbb{R}_{+}, \\
u(\cdot, 0)=u_{0} \text { dans } \mathbb{R} .
\end{array}
$$

Une solution faible de (5.96)-5.97) est une fonction $u \in L^{\infty}\left(\mathbb{R} \times \mathbb{R}_{+}\right)$telle que

$$
\begin{aligned}
& \int_{\mathbb{R} \times \mathbb{R}_{+}} u\left(\partial_{t} \varphi+a \partial_{x} \varphi\right) d(x, t)+\int_{\mathbb{R}} u_{0}(x) \varphi(x, 0) \mathrm{d} x=-b \int_{0}^{+\infty} \varphi(c t, t) d t \\
& \quad \text { pour tout } \varphi \in C_{c}^{\infty}\left(\mathbb{R} \times \mathbb{R}_{+}, \mathbb{R}\right) .
\end{aligned}
$$

1. Unicité Montrer que 5.96-5.97) a au plus une solution faible. [On pourra se ramener à l'exercice 5.3.]

2. Existence On suppose dans cette question que $c \neq a$. Montrer que (5.96-5.97) a une (unique) solution faible et la construire.

3. Non existence On suppose dans cette question que $c=a$ et $b \neq 0$. Montrer que (5.96)-(5.97) n'a pas de solution faible.

\subsection{Corrigés d'exercices}

\section{Exercice 5.1 page $233\left(L^{1}(\mathbb{R}) \cap B V(\mathbb{R}) \subset L^{\infty}(\mathbb{R})\right)$}

Soit $x, y$ des points de Lebesgue de $u, x<y$. Pour $n$ tel que $2 / n<y-x$, on choisit $\varphi$ continue et telle que

$$
\left\{\begin{array}{l}
\varphi(z)=0 \text { si } z \leq x-1 / 2 n \text { ou } z \leq x-1 / 2 n \\
\varphi(z)=1 \text { si } z \leq x+1 / 2 n \text { ou } z \leq x-1 / 2 n \\
\varphi \text { affine sur }[x-1 / 2 n, x+1 / 2 n] \text { et }[y-1 / 2 n, y+1 / 2 n 1 / 2 n] .
\end{array}\right.
$$

On a $\left|\int u(z) \varphi^{\prime}(z) d z\right| \leq|u|_{B V}$. 
En faisant tendre $n \rightarrow+\infty$, on obtient $|u(x)-u(y)| \leq|u|_{B V}$ et donc

$$
|u(x)| \leq|u|_{B V}+|u(y)|,
$$

pour tous $x, y$ points de Lebesgue.

Mais comme $u \in L^{1}(\mathbb{R})$, pour tout $\varepsilon>0$, il existe $y$ point de Lebesgue tel que $|u(y)| \leq \varepsilon\left(\operatorname{sinon} \int|u(y)| d y \geq\right.$ $\varepsilon(+\infty)=+\infty)$. On en déduit que $|u(x)| \leq|u|_{B V}$ pour tout $x$ point de Lebesgue et donc

$$
\|u\|_{\infty} \leq|u|_{B V} .
$$

\section{Exercice 5.2 page 233 (Système hyperbolique linéaire)}

Soit $u \in L^{\infty}\left(\mathbb{R} \times \mathbb{R}_{+}\right)^{n}$; décomposons $u$ sur la base des vecteurs propres $\left\{v_{i}, i \in\{1, \ldots, n\}\right.$ :

$$
u(x, t)=\sum_{i=1}^{n} u_{i}(x, t) v_{i}, \text { et donc } \partial_{t} u(x, t)=\sum_{i=1}^{n} \partial_{t} u_{i}(x, t) v_{i} \text { et } A \partial_{x} u(x, t)=\sum_{i=1}^{n} \lambda_{i} \partial_{x} u_{i}(x, t) v_{i} .
$$

On en déduit que $u=\sum_{i=1}^{n} u_{i}(x, t) v_{i}$ est solution faible de (5.60) si et seulement si pour tout $i=1, \ldots, n$,

$$
\begin{aligned}
& \left.\partial_{t} u_{i}(x, t)+\lambda_{i} \partial_{x} u_{i}(x, t)=0, x \in \mathbb{R}, t \in\right] 0,+\infty[, \\
& u_{i}(x, 0)=a_{i}(x), x \in \mathbb{R} .
\end{aligned}
$$

Montrons que la fonction $u_{i} \in L^{\infty}\left(\mathbb{R} \times \mathbb{R}_{+}\right)$définie par $u_{i}(x, t)=a_{i}(x-\lambda t)$ est une solution faible de (5.99). Remarquons d'abord que si $a_{i}$ est une fonction régulière, alors $u_{i}$ ainsi définie est solution classique, donc faible et on a terminé. Maintenant si $a_{i}$ est seulement $L^{\infty}$, on a bien $u_{i} \in L^{\infty}\left(\mathbb{R} \times \mathbb{R}_{+}\right)$et il nous reste à montrer que pour toute fonction $\varphi \in C_{c}^{1}\left(\mathbb{R} \times \mathbb{R}_{+}, \mathbb{R}\right)$, la fonction $u_{i}$ satisfait :

$$
\iint_{\mathbb{R} \times \mathbb{R}_{+}}\left[u_{i}(x, t) \partial_{t} \varphi(x, t)+\lambda_{i} u_{i}(x, t) \partial_{x} \varphi(x, t)\right] \mathrm{d} x \mathrm{~d} t+\int_{\mathbb{R}} a_{i}(x) \varphi(x, 0) \mathrm{d} x=0 .
$$

Posons

$$
X=\iint_{\mathbb{R} \times \mathbb{R}_{+}}\left[u_{i}(x, t) \partial_{t} \varphi(x, t)+\lambda_{i} u_{i}(x, t) \partial_{x} \varphi(x, t)\right] \mathrm{d} x \mathrm{~d} t .
$$

Puisque $u_{i}(x, t)=a_{i}(x-c t)$, on a donc :

$$
X=\iint_{{\mathbb{R} \times \mathbb{R}_{+}}}\left[u_{0}(x-c t) \partial_{t} \varphi(x, t)+c u_{0}(x-c t) \partial_{x} \varphi(x, t)\right] \mathrm{d} x \mathrm{~d} t .
$$

En appliquant le changement de variable $y=x-\lambda_{i} t$ et en utilisant le théorème de Fubini, on obtient :

$$
X=\int_{\mathbb{R}^{R}} a_{i}(y) \int_{\mathbb{R}_{+}}\left[\partial_{t} \varphi\left(y+\lambda_{i} t, t\right)+\lambda_{i} \partial_{x} \varphi\left(y+\lambda_{i} t, t\right)\right] \mathrm{d} t \mathrm{~d} y .
$$

Posons alors

$$
\psi_{y}(t)=\varphi\left(y+\lambda_{i} t, t\right)
$$

On a donc :

$$
X=\int_{\mathbb{R}}\left(a_{i}(y) \int_{0}^{+\infty} \psi_{y}^{\prime}(t) \mathrm{d} t\right) \mathrm{d} y,
$$


et comme $\psi$ est à support compact sur $[0,+\infty[$, on a

$$
X=-\int_{\mathbb{R}} a_{i}(y) \psi_{y}(0) \mathrm{d} y=-\int_{\mathbb{R}} a_{i}(y) \varphi(y, 0) \mathrm{d} y .
$$

On a ainsi démontré que la fonction $u$ définie par $u(x, t)=a_{i}\left(x-\lambda_{i} t\right)$ est solution faible de l'équation (5.99). On en déduit que la fonction $u:(x, t) \mapsto \sum_{i=1}^{n} a_{i}\left(x-\lambda_{i} t\right)$ est solution faible du système [5.60).

\section{Exercice 5.3 page 233 (Unicité de la solution faible du problème linéaire par dualité)}

1. Soient $u_{1}$ et $u_{2}$ des solutions faibles de (5.61), alors $u_{1}-u_{2}$ est solution faible de (5.61) avec $u_{0}=0$; on en déduit qu'il est équivalent de montrer qu'il existe une unique solution à (5.61) que de montrer que la fonction nulle est l'unique solution faible de (5.61) avec $u_{0}=0$.

2. (a) Montrer que $\varphi \in C_{c}^{1}\left(\mathbb{R} \times\left[0,+\infty[)\right.\right.$ et que $\varphi_{t}+c \varphi_{x}=\psi$ in $\mathbb{R} \times[0,+\infty[)$.

\section{Exercice 5.4 (Construction d'une solution faible entropique, I)}

On commence par construire, pour chaque $x_{0}$, la courbe caractéristique issue de $x_{0}$.

- Pour $x_{0}<0$, la courbe caractéristique est la demi-droite $\left\{\left(x_{0}+2 t, t\right), t \geq 0\right\}$.

- Pour $0 \leq x_{0} \leq 1$, la courbe caractéristique est la demi-droite $\left\{\left(x_{0}+2\left(1-x_{0}\right) t, t\right), t \geq 0\right\}$, car $f^{\prime}\left(u_{0}\left(x_{0}\right)\right)=$ $2\left(1-x_{0}\right)$.

- Pour $x_{0}>1$, la courbe caractéristique est la demi-droite $\left\{\left(x_{0}, t\right), t \geq 0\right\}$.

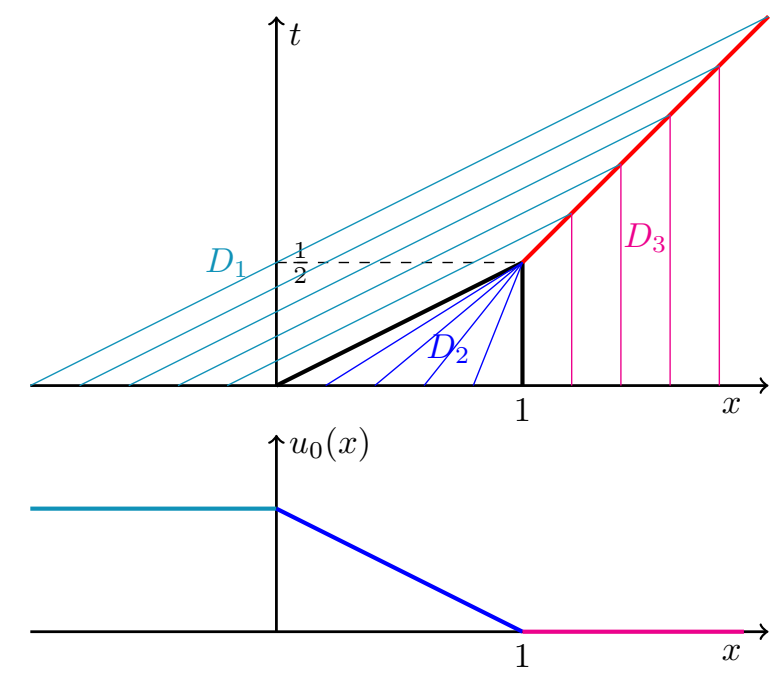

FIGURE 5.5 - Exercice 5.4 page 233 En haut : Droites caractéristiques pour l'équation de Burgers avec condition initiale 5.63 En rouge la ligne de choc, en noir les lignes de discontinuité $C^{1}$. - En bas : allure de la condition initiale $u_{0}$.

Pour $0<t<1 / 2$, les courbes caractéristiques ne se rencontrent pas, comme indiqué sur la figure 5.5 . La solution est donc continue pour $0<t<1 / 2$ et elle est constante sur chaque courbe caractéristique. 
Par exemple, si $0<t<1 / 2$ et $x=x_{0}+2\left(1-x_{0}\right) t$ avec $x_{0} \in[0,1]$, on a $u(x, t)=u_{0}\left(x_{0}\right)=1-x_{0}=$ $(1-x) /(1-2 t) \operatorname{car} x_{0}(1-2 t)=x-2 t$.

En $t=1 / 2$,les courbes caractéristiques issues des points $x_{0}$ de l'intervalle $[0,1]$ se rencontrent (au point $x=1$ ); une discontinuité apparait et se propage à une vitesse conforme à la relation de Rankine Hugoniot. Ceci nous permet de construire la solution $u(x, t)$ pour tout $(x, t) \in \mathbb{R} \times R_{+}$de la manière suivante. On pose :

$$
\begin{array}{ll}
u(x, t)=1 & \text { si }(x, t) \in D_{1}=\left\{(x, t), 0<t \leq \frac{1}{2}, x<2 t\right\} \cup\left\{(x, t), t>\frac{1}{2}, x<t+\frac{1}{2}\right\}, \\
u(x, t)=\frac{1-x}{1-2 t} & \text { si }(x, t) \in D_{2}=\left\{(x, t), 0<t<\frac{1}{2}, 2 t<x<1\right\}, \\
u(x, t)=0 & \text { si }(x, t) \in D_{3}=\left\{(x, t), 0<t \leq \frac{1}{2}, 1<x\right\} \cup\left\{(x, t), t>\frac{1}{2}, t+\frac{1}{2}<x\right\} .
\end{array}
$$

La fonction $u$ est bien solution faible de 5.62 . Cette solution est même entropique (voir la proposition 5.15.

\section{Exercice 5.6 (Construction d'une solution faible entropique, II)}

Comme dans le corrigé de l'exercice 5.4, on commence par construire, pour tout $x_{0} \in \mathbb{R}$, la courbe caractéristique issue de $x_{0}$.

- Pour $x_{0}<0$, la courbe caractéristique est la demi-droite $\left\{\left(x_{0}, t\right), t \geq 0\right\}$.

- Pour $0 \leq x_{0} \leq 1$, la courbe caractéristique est la demi-droite $\left\{\left(x_{0}+2\left(1-x_{0}\right) t, t\right), t \geq 0\right\}$, car $f^{\prime}\left(u_{0}\left(x_{0}\right)\right)=$ $2\left(1-x_{0}\right)$.

- Pour $x_{0}>1$, la courbe caractéristique est la demi-droite $\left\{\left(x_{0}+2 t, t\right), t \geq 0\right\}$.

Pour $0<t<1 / 2$, les courbes caractéristiques ne se rencontrent pas, comme indiqué sur la figure 5.6 La solution est donc continue pour $0<t<1 / 2$ et elle est constante sur chaque courbe caractéristique.

Comme dans le corrigé de l'exercice 5.4. on a, par exemple, si $0<t<1 / 2$ et $x=x_{0}+2\left(1-x_{0}\right) t$ avec $x_{0} \in[0,1]$, $u(x, t)=u_{0}\left(x_{0}\right)=1-x_{0}=(1-x) /(1-2 t) \operatorname{car} x_{0}(1-2 t)=x-2 t$.

La différence avec l'exercice 5.4 est que la solution comporte maintenant deux détentes prenant leur origine aux points 0 et 1 .

Dans la zone de détente issue du point 0 , on a $u(x, t)=\xi$ pour $x=2 \xi t$ et $\xi \in[0,1]$ (et donc $u(x, t)=x /(2 t)$ ). Dans la zone de détente issue du point 1 , on a $u(x, t)=\xi$ pour $x=2 \xi t+1$ et $\xi \in[0,1]$ (et donc $u(x, t)=$ $(x-1) /(2 t))$.

Puis, en $t=1 / 2$, un choc apparaît. En utilisant la relation de Rankine-Hugoniot, on montre (avec le calcul de la solution sur les caractéristiques de l'équation, voir ci après) que ce choc se propage à vitesse 1 . La solution est bien entropique (grâce à la proposition 5.15.

En résumé, ceci donne la solution suivante :

$$
\begin{array}{ll}
u(x, t)=0 & \text { sur } D_{1}=\{(x, t), 0<t, x<0\}, \\
u(x, t)=\frac{x}{2 t} & \text { sur } D_{2}=\left\{(x, t), 0<t \leq \frac{1}{2}, 0<x<2 t\right\} \cup\left\{(x, t), t>\frac{1}{2}, 0<x<t+\frac{1}{2}\right\}, \\
u(x, t)=\frac{1-x}{1-2 t} & \text { sur } D_{3}=\left\{(x, t), 0<t<\frac{1}{2}, 2 t<x<1\right\}, \\
u(x, t)=\frac{x-1}{2 t} & \text { sur } D_{4}=\left\{(x, t), 0<t \leq \frac{1}{2}, 1<x<1+2 t\right\} \cup\left\{(x, t), t>\frac{1}{2}, t+\frac{1}{2}<x<1+2 t\right\}, \\
u(x, t)=1 & \text { sur } D_{5}=\{(x, t), 0<t, 1+2 t<x\} .
\end{array}
$$




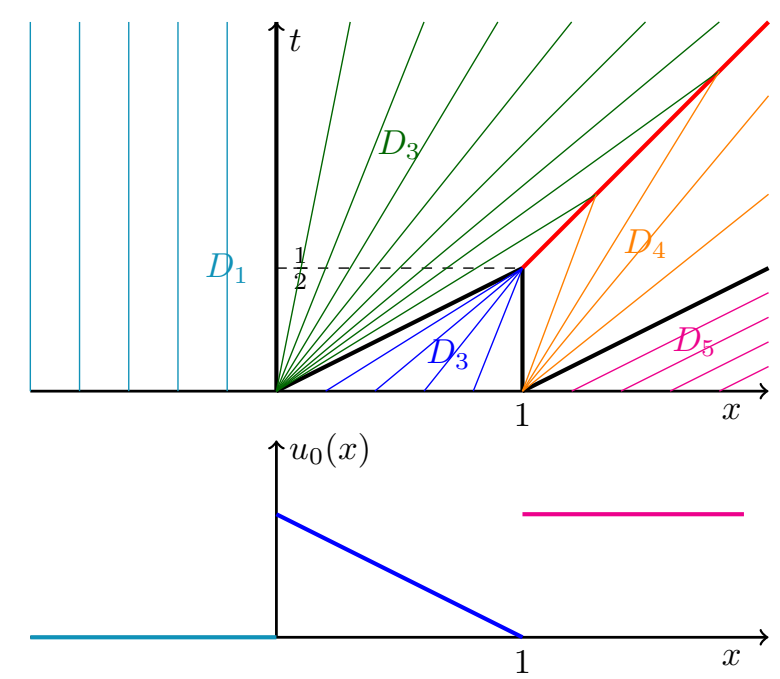

FiguRE 5.6 -5.6- En haut : Droites caractéristiques pour l'équation de Burgers avec condition initiale $u_{0}$; en rouge la ligne de choc, en noir les lignes de discontinuité $C^{1}-$ En bas : allure de la condition initiale $u_{0}$.

La fonction $u$ est discontinue sur l'ensemble $\{(x, t), t>1 / 2, x=t+1 / 2\}$ (ligne de choc, en rouge sur la figure). On vérifie que la relation de Rankine-Hugoniot est satisfaite en tout point de cet ensemble. En effet, soit $t>1 / 2$ et $x=t+1 / 2$. Avec les notations de la proposition 5.15 , on a

$$
\begin{gathered}
u_{-}(x, t)=\frac{x}{2 t}=\frac{t+1 / 2}{2 t}=\frac{1}{2}+\frac{1}{4 t}\left(\text { on utilise ici } D_{2}\right), \\
u_{+}(x, t)=\frac{x-1}{2 t}=\frac{t-1 / 2}{2 t}=\frac{1}{2}-\frac{1}{4 t}\left(\text { on utilise ici } D_{4}\right) .
\end{gathered}
$$

Ceci donne $u_{-}(x, t)+u_{+}(x, t)=1$ et la relation de Rankine-Hugoniot est bien vérifiée. D'autre part, la solution contruite est bien entropique car $u_{-}>u_{+}$.

\section{Exercice 5.8 (Equation de Buckley-Leverett)}

1. On remarque tout d'abord que $f^{\prime}$ est strictement croissante sur $[0, a]$ puis strictement décroissante sur $[a, 1]$. On a donc $f^{\prime}(x)>0$ et $f(x)>0$ pour tout $\left.\left.x \in\right] 0,1\right]$.

Comme la fonction $f$ est strictement convexe sur $] 0, a\left[\right.$ (et de classe $C^{1}$ sur $\mathbb{R}$ ),

$$
\left.\left.0>f(x)+(0-x) f^{\prime}(x), \text { pour tout } x \in\right] 0, a\right],
$$

et donc $f^{\prime}(x)>f(x) / x$ pour tout $\left.\left.x \in\right] 0, a\right]$. En particulier $f^{\prime}(a)>f(a) / a$.

Pour $x \in[a, 1]$, on pose $h(x)=f(x)-x f^{\prime}(x)$, de sorte que $h(a)<0$ et $h(1)=f(1)>0$. Puis, comme $h^{\prime}(x)=-x f^{\prime \prime}(x)$, la fonction $h$ est strictement croissante sur $[a, 1]$. Il existe donc un et un seul point $b \in] a, 1\left[\right.$ tel que $h(b)=0$, c'est-à-dire $f^{\prime}(b)=f(b) / b$.

Enfin comme la fonction $f^{\prime}$ est strictement croissante de 0 à $\left.f^{\prime} a\right)$ sur $[0, a]$ puis strictement décroissante de $f^{\prime}(a)$ à 0 sur $[a, 1]$, il existe un unique point $\left.c \in\right] 0, a\left[\right.$ tel que $f^{\prime}(c)=f^{\prime}(b)($ car $b \in] a, 1[$ ). 
2. On décompose le demi-plan $\mathbb{R} \times \mathbb{R}_{+}$en 3 zones,

$$
\begin{aligned}
& D_{1}=\{(x, t), t \geq 0, x<0\}, \\
& D_{2}=\left\{(x, t), t \geq 0, x=f^{\prime}(\xi) t, b<\xi<1\right\}, \\
& D_{3}=\left\{(x, t), t \geq 0, x>f^{\prime}(b) t\right\} .
\end{aligned}
$$

Dans chacune de ces trois zones, la fonction $u$ est une solution classique de (5.67) (et vérifie bien (5.68)). La fonction $u$ est continue à la frontière entre $D_{1}$ et $D_{2}$ (elle vaut 1 , on rappelle que $f^{\prime}(1)=0$ ). La fonction $u$ est discontinue à la frontière entre $D_{2}$ et $D_{3}$. Du coté de $D_{2}$, elle vaut $b$ et elle vaut 0 dans $D_{3}$. La frontière entre $D_{2}$ et $D_{3}$ est la demi-droite d'équation $x=f^{\prime}(b) t$. Comme $f^{\prime}(b)=f(b) / b=(f(b)-f(0)) /(b-0)$, la relation de Rankine-Hugoniot est bien vérifiée à la frontière entre $D_{2}$ et $D_{3}$. Ceci montre que $u$ est solution faible de 5.67)-5.68.

Il reste à vérifier la condition d'entropie à la frontière entre $D_{2}$ et $D_{3}$.

Soit $\eta$ une fonction de $\mathbb{R}$ dans $\mathbb{R}$ de classe $C^{1}$ et convexe. Comme $\left(f^{\prime}(b)-f^{\prime}(x)\right)\left(\eta^{\prime}(x)-\eta^{\prime}(c)\right) \leq 0$ pour presque tout $x \in[0, b]$ (pour le voir, il suffit de distinguer les cas $x<c$ et $x>c$ et d'utiliser $f^{\prime}(b)=f^{\prime}(c)$ ), on a bien $\int_{0}^{b}\left(f^{\prime}(b)-f^{\prime}(x)\right)\left(\eta^{\prime}(x)-\eta^{\prime}(c)\right) \mathrm{d} x \leq 0$.

En notant $\phi$ la primitive de $\eta^{\prime} f^{\prime}$, ceci donne

$$
f^{\prime}(b)(\eta(b)-\eta(0))-f^{\prime}(b) \eta^{\prime}(c) b-(\phi(b)-\phi(0))+f(b) \eta^{\prime}(c) \leq 0 .
$$

Comme $b f^{\prime}(b)=f(b)$, on en déduit $(\phi(0)-\phi(b)) \leq f^{\prime}(b)(\eta(0)-\eta(b))$, ce qui est bien la condition d'entropie à la frontière entre $D_{2}$ et $D_{3}$.

\section{Exercice 5.9 (Construction d'une solution faible entropique, III)}

$\mathrm{Au}$ vu de la condition initiale, on peut se douter que la solution entropique contient une discontinuité issue du point $x=1$. On cherche donc la solution sous la forme d'une fonction continue à gauche et à droite d'une ligne de discontinuité notée $L$ (en rouge sur la figure 5.7), définie par $L=\{(x, t), t>0, x=\sigma(t)\}$, où $\sigma$ est une fonction de classe $C^{1}$ croissante telle que $\sigma(0)=1$ et $\sigma^{\prime}(t)<2$ pour tout $t>0$.

On pose (voir figure 5.7) :

$$
\begin{aligned}
& D_{1}=\{(x, t), 0<t, x<0\}, \\
& D_{2}=\{(x, t), 0<t, 0<x<\sigma(t)\}, \\
& D_{3}=\{(x, t), 0<t, \sigma(t)<x\} .
\end{aligned}
$$

On prend $u(x, t)=0$ si $(x, t) \in D_{1}$. Dans $D_{2}, u$ est construite en utilisant les caractéristiques, ce qui donne $u(x, t)=x /(1+2 t)$ si $(x, t) \in D_{2}$. Enfin, on pose $u(x, t)=0$ si $(x, t) \in D_{3}$.

Pour que $u$ soit solution faible du problème considéré, il suffit de vérifer la relation de Rankine-Hugoniot sur $L$, c'est-à-dire (avec les notations de la proposition 5.15 que

$$
\sigma^{\prime}(t)=u_{-}(x, t)+u_{+}(x, t) \text { pour tout }(x, t) \in L .
$$

Soit $t>0$ et $x=\sigma(t)$, on a $u_{-}(x, t)+u_{+}(x, t)=\sigma(t) /(1+2 t)$. Il suffit donc que

$$
\begin{aligned}
& \sigma^{\prime}(t)=\sigma(t) /(1+2 t) \text { pour tout } t>0 \\
& \sigma(0)=1 .
\end{aligned}
$$




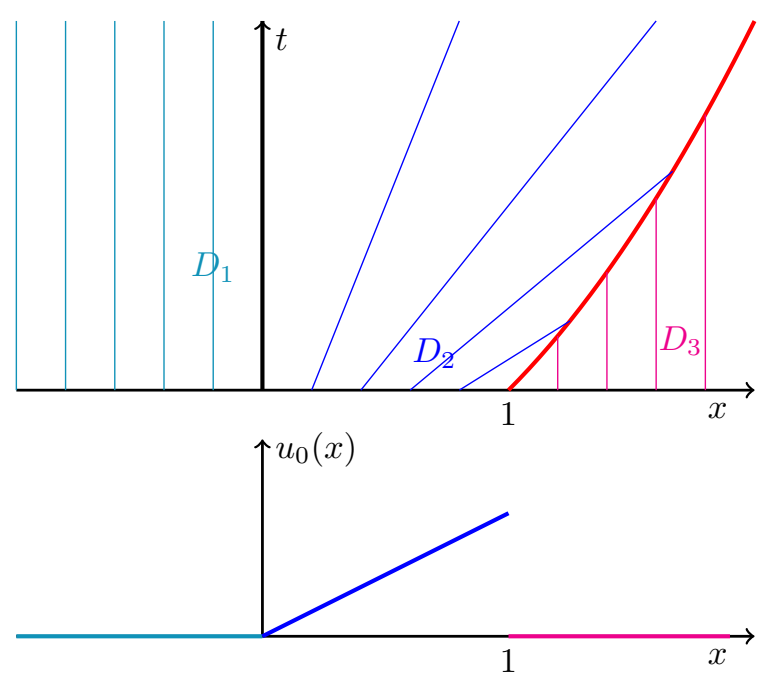

FIGURE 5.7 - En haut : Droites caractéristiques pour l'équation de Burgers avec condition initiale $u_{0}$; en rouge la ligne de choc, en noir les lignes de discontinuité $C^{1}-$ En bas : allure de la condition initiale $u_{0}$.

La solution de cette équation différentielle est $\sigma(t)=\sqrt{1+2 t}$ (pour tout $t>0$ ). Avec ce choix de la fonction $\sigma$, la fonction $u$ ainsi construite est solution faible du problème considéré. Cette fonction est même solution entropique car $u_{-}>u_{+}$sur $L$ (voir la proposition 5.15).

Pour $t>0, \int_{\mathbb{R}} u(x, t) \mathrm{d} x=\int_{0}^{\sqrt{1+2 t}} \frac{x}{1+2 t} \mathrm{~d} t=\frac{1}{2}=\int_{\mathbb{R}} u_{0}(x) \mathrm{d} x$.

\section{Exercice 5.10 (Construction d'une solution faible entropique, IV)}

L'allure de la solution entropique est donnée sur la figure 5.8. La discontinuité de $u_{0}$ en $x=-1$, commence par se propager à la vitesse 1 (c'est-à-dire sur la droite $x=-1+t$ ), c'est une onde de choc; elle sépare les régions $D_{1}$ et $D_{2}$ sur la figure. Noter que, conformément à la théorie, les caractéristiques "rentrent" dans la ligne de choc. La discontinuité de $u_{0}$ en $x=1$, commence par se propager à la vitesse 2 (c'est-à-dire sur la droite $x=1+2 t$ ), c'est aussi une onde de choc. La discontinuité de $u_{0}$ en $x=0$ disparait, elle donne une onde de détente (région $\left.D_{3}\right)$. Dans cette onde de détente, on a $u(x, t)=x /(2 t)$.

Puis, en $t=1 / 2$, la "tête" de l'onde détente rattrape l'onde de choc de droite (au point $x=2$ ) qui alors "ralentit" et continue sur une courbe que nous notons $L_{1}$, avec $L_{1}=\left\{(x, t), t>1 / 2, x=\sigma_{1}(t)\right\}$. En $t=1$, l'onde de choc de gauche rattrape le "pied" de l'onde de détente (au point $x=0$ ). L'onde de choc "ralentit" et continue sur une courbe que notons $L_{2}$, avec $L_{2}=\left\{(x, t), t>1, x=\sigma_{1}(t)\right\}$. Les fonctions $\sigma_{1}$ et $\sigma_{2}$ se calculent grâce aux relations de Rankine et Hugoniot.

1. Calcul de $\sigma_{1}$. Soit $(x, t) \in L_{1}$. L'ensemble $L_{1}$ sépare l'onde de détente $D_{2}$ de la zone $D_{5}$ dans laquelle $u=0$. On a donc, avec la relation de Rankine-Hugoniot,

$$
\sigma_{1}^{\prime}(t)=u_{-}(x, t)+u_{+}(x, t)=\frac{x}{2 t}=\frac{\sigma_{1}(t)}{2 t} .
$$

Comme $\sigma_{1}(1 / 2)=2$, la résolution de cette équation différentielle donne $\sigma_{1}(t)=2 \sqrt{2 t}$ pour tout $t>1 / 2$. 


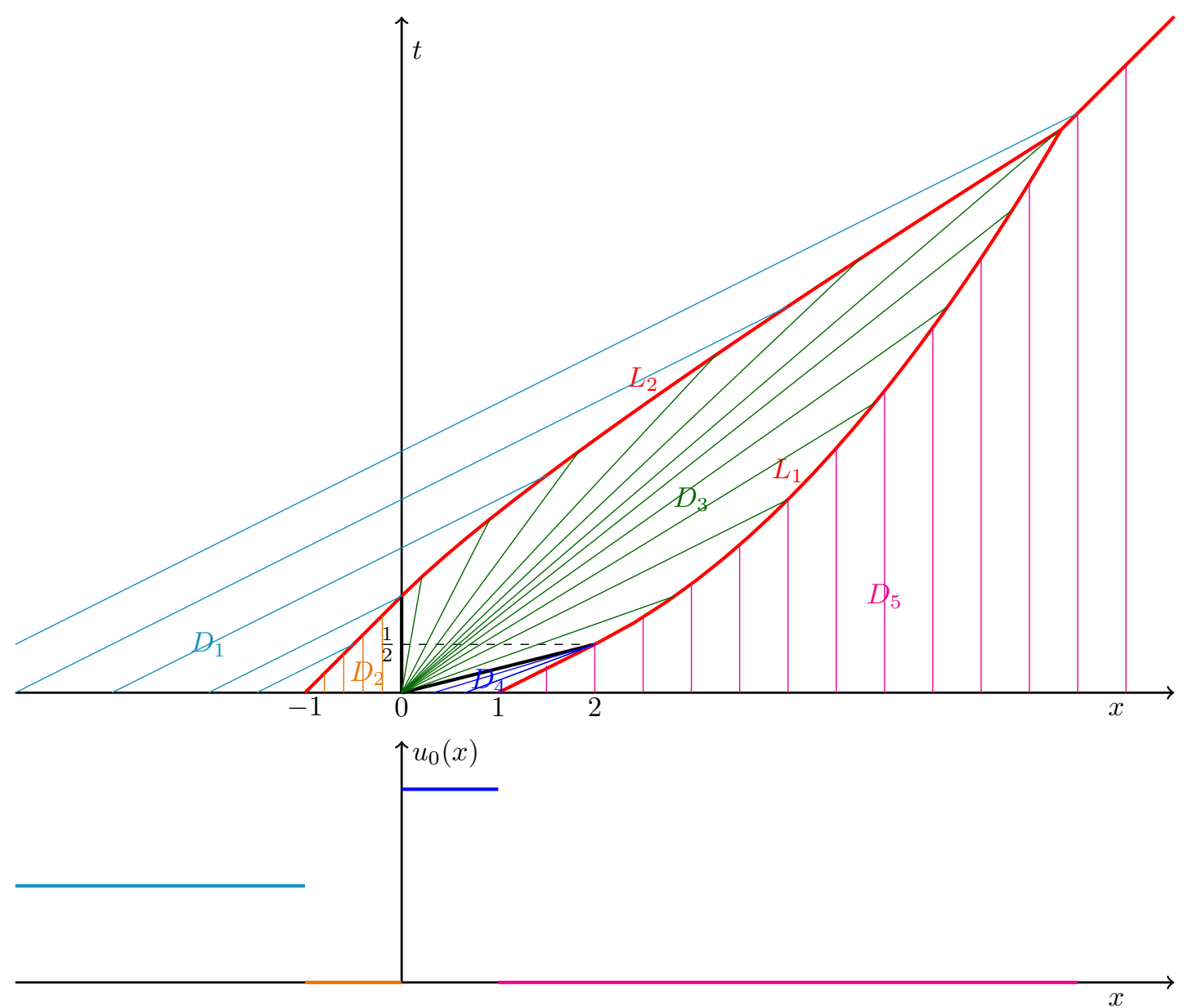

FIGURE 5.8 - En haut : Droites caractéristiques pour l'équation de Burgers avec condition initiale $u_{0}$; en rouge la ligne de choc, en noir les lignes de discontinuité $C^{1}-$ En bas : allure de la condition initiale $u_{0}$.

2. Calcul de $\sigma_{2}$. Soit $(x, t) \in L_{2}$. L'ensemble $L_{2}$ sépare la zone $D_{1}$ dans laquelle $u=1$ de l'onde de détente $D_{2}$. On a donc, avec la relation de Rankine-Hugoniot,

$$
\sigma_{2}^{\prime}(t)=u_{-}(x, t)+u_{+}(x, t)=1+\frac{x}{2 t}=1+\frac{\sigma_{2}(t)}{2 t}
$$

Comme $\sigma_{2}(1)=0$, la résolution de cette équation différentielle donne $\sigma_{2}(t)=2(t-\sqrt{t})$ pour tout $t>1$.

Les courbes $L_{1}$ et $L_{2}$ se rencontrent pour en $t$ tel que $1+\frac{\sigma_{2}(t)}{2 t}=\frac{\sigma_{1}(t)}{2 t}$, c.à.d. $t=3+2 \sqrt{2}$, pour donner naissance à une seule discontinuité qui se propage à la vitesse 1 , car cette discontinuité sépare la zone $D_{1}$ dans laquelle $u=1$ de la zone $D_{5}$ dans laquelle $u=0$.

La solution ainsi construite est bien entropique car sur chaque courbe de discontinuité on a $u_{g}>u_{d}$, or la fonction flux $s \mapsto s^{2}$ de l'équation de Burgers est bien convexe. 


\section{Exercice 5.11 (Solution non entropique)}

1. voir cours.

2. Comme $L_{\text {loc }}^{1} \subset L_{\text {loc }}^{2}$, il suffit de montrer que $u^{2} \in L_{\text {loc }}^{1}\left(\mathbb{R} \times \mathbb{R}_{+}\right)$.

Soit $T>0$. Pour $0<t<T$,

$$
\int_{\mathbb{R}} u^{2}(x, t) \mathrm{d} x=\int_{-\sqrt{t}}^{\sqrt{t}} \frac{x^{2}}{4 t^{2}} d x=\frac{1}{6 t^{\frac{1}{2}}}
$$

et donc

$$
\int_{0}^{T}\left(\int_{\mathbb{R}} u^{2}(x, t) \mathrm{d} x\right) \mathrm{d} t=\int_{0}^{T} \frac{1}{6 t^{\frac{1}{2}}} \mathrm{~d} t<+\infty .
$$

Ce qui prouve que $u^{2} \in L_{\text {loc }}^{1}\left(\mathbb{R} \times \mathbb{R}_{+}\right)$.

3. Une démonstration (rapide) de cette question consiste à utiliser la proposition 5.17 avec, pour tout $\varepsilon>0$, $\mathbb{R} \times\left[\varepsilon,+\infty\left[\right.\right.$ au lieu de $\mathbb{R} \times \mathbb{R}_{+}$. Nous donnons ici une autre démonstration, plus proche de celle donné dans la proposition 5.13 .

Soit $\varphi \in C_{c}^{1}\left(\mathbb{R} \times \mathbb{R}_{+}^{\star}, \mathbb{R}\right)$. En utilisant une intégration par parties, on obtient, pour tout $x \in \mathbb{R}$,

$$
\int_{0}^{\infty} u(x, t)(x, t) \partial_{t} \varphi(x, t) \mathrm{d} t=\int_{x^{2}}^{+\infty} \frac{x}{2 t^{2}} \varphi(x, t) \mathrm{d} t-\frac{1}{2 x} \varphi\left(x, x^{2}\right) .
$$

En notant que les deux termes de droite sont intégrables $(\operatorname{car} \varphi(x, t)=0$ pour $t$ proche de 0$)$,

$$
\int_{\mathbb{R}} \int_{0}^{\infty} u(x, t)(x, t) \partial_{t} \varphi(x, t) \mathrm{d} t=\int_{\mathbb{R}} \int_{x^{2}}^{+\infty} \frac{x}{2 t^{2}} \varphi(x, t) \mathrm{d} t \mathrm{~d} x-\int_{\mathbb{R}} \frac{1}{2 x} \varphi\left(x, x^{2}\right) \mathrm{d} x .
$$

De même, avec une intégration par parties, on obtient, pour tout $t \in \mathbb{R}_{+}^{\star}$,

$$
\int_{\mathbb{R}} u^{2}(x, t) \partial_{x} \varphi(x, t) \mathrm{d} x=-\int_{-\sqrt{t}}^{\sqrt{t}} \frac{x}{2 t^{2}} \mathrm{~d} x+\frac{1}{4 t} \varphi(\sqrt{t}, t)-\frac{1}{4 t} \varphi(-\sqrt{t}, t) .
$$

Ici encore les termes de droite sont intégrables $(\operatorname{car} \varphi(x, t)=0$ pour $t$ proche de 0$)$,

$$
\int_{0}^{+\infty} \int_{\mathbb{R}} u^{2}(x, t) \partial_{x} \varphi(x, t) \mathrm{d} x=-\int_{0}^{+\infty} \int_{-\sqrt{t}}^{\sqrt{t}} \frac{x}{2 t^{2}} \mathrm{~d} x \mathrm{~d} t+\int_{0}^{+\infty} \frac{1}{4 t}(\varphi(\sqrt{t}, t)-\varphi(-\sqrt{t}, t)) d t
$$

Le théorème de Fubini nous donne $\int_{\mathbb{R}} \int_{x^{2}}^{+\infty} \frac{x}{2 t^{2}} \varphi(x, t) \mathrm{d} t \mathrm{~d} x=\int_{0}^{+\infty} \int_{-\sqrt{t}}^{\sqrt{t}} \frac{x}{2 t^{2}} \mathrm{~d} x \mathrm{~d} t$. Puis le changement de variable $t=x^{2}$ donne

$$
\begin{array}{r}
\int_{0}^{+\infty} \frac{1}{4 t} \varphi(\sqrt{t}, t) d t=\int_{0}^{+\infty} \frac{1}{4 x^{2}} \varphi\left(x, x^{2}\right) 2 x \mathrm{~d} x=\int_{0}^{+\infty} \frac{1}{2 x} \varphi\left(x, x^{2}\right) \mathrm{d} x \\
\int_{0}^{+\infty} \frac{1}{4 t} \varphi(-\sqrt{t}, t) d t=-\int_{-\infty}^{0} \frac{1}{4 x^{2}} \varphi\left(x, x^{2}\right) 2 x \mathrm{~d} x=-\int_{-\infty}^{0} \frac{1}{2 x} \varphi\left(x, x^{2}\right) \mathrm{d} x .
\end{array}
$$

En additionnant 5.103 et 5.104 on obtient 5.71).

4. On se donne une fonction $\psi \in C^{\infty}(\mathbb{R}, \mathbb{R})$ telle que $\psi(t)=0$ pour $\left.\left.t \in\right]-\infty, 1\right]$ et $\psi(t)=1$ pour $t \in\left[2,+\infty\left[\right.\right.$. Puis, pour $n \in \mathbb{N}^{\star}$, on pose $\psi_{n}(t)=\psi(n t)$. 
Soit $\varphi \in C_{c}^{1}\left(\mathbb{R} \times \mathbb{R}_{+}, \mathbb{R}\right)$. Comme la fonction $(x, t) \mapsto \varphi(x, t) \psi_{n}(t)$ est un élément de $C_{c}^{\infty}\left(\mathbb{R} \times \mathbb{R}_{+}^{\star}, \mathbb{R}\right)$, la question précédente donne

$$
\int_{\mathbb{R}_{+}} \int_{\mathbb{R}^{R}}\left(u(x, t) \partial_{t}\left(\varphi \psi_{n}\right)(x, t)+u^{2}(x, t) \partial_{x}\left(\varphi \psi_{n}\right)(x, t)\right) \mathrm{d} x \mathrm{~d} t=0,
$$

et donc

$$
\begin{aligned}
\int_{\mathbb{R}_{+}} \int_{\mathbb{R}} u(x, t) \partial_{t} \varphi(x, t) \psi_{n}(x, t) \mathrm{d} x \mathrm{~d} t+n & \int_{1 / n}^{2 / n} \int_{\mathbb{R}} u(x, t) \varphi(x, t) \psi^{\prime}(n t) \mathrm{d} x \mathrm{~d} t \\
& +\int_{\mathbb{R}_{+}} \int_{\mathbb{R}^{R}} u^{2}(x, t) \partial_{x} \varphi(x, t) \psi_{n}(x, t) \mathrm{d} x \mathrm{~d} t=0 .
\end{aligned}
$$

La question 2 donne $u \partial_{t} \varphi, u^{2} \partial_{x} \varphi \in L^{1}\left(\mathbb{R} \times \mathbb{R}_{+}\right)$. On en déduit, par le théorème de convergence dominée,

$$
\begin{array}{r}
\lim _{n \rightarrow+\infty} \int_{\mathbb{R}_{+}} \int_{\mathbb{R}} u(x, t) \partial_{t} \varphi(x, t) \psi_{n}(x, t) \mathrm{d} x \mathrm{~d} t=\int_{\mathbb{R}_{+}} \int_{\mathbb{R}^{R}} u(x, t) \partial_{t} \varphi(x, t) \mathrm{d} x \mathrm{~d} t, \\
\lim _{n \rightarrow+\infty} \int_{\mathbb{R}_{+}} \int_{\mathbb{R}} u^{2}(x, t) \partial_{x} \varphi(x, t) \psi_{n}(x, t) \mathrm{d} x \mathrm{~d} t=\int_{\mathbb{R}_{+}} \int_{\mathbb{R}^{R}} u^{2}(x, t) \partial_{x} \varphi(x, t) \mathrm{d} x \mathrm{~d} t .
\end{array}
$$

Puis,

$$
n \int_{1 / n}^{2 / n} \int_{\mathbb{R}} u(x, t) \varphi(x, t) \psi^{\prime}(n t) \mathrm{d} x \mathrm{~d} t=n \int_{1 / n}^{2 / n} \int_{0}^{\sqrt{t}} \frac{x}{2 t}(\varphi(x, t)-\varphi(-x, t)) \psi^{\prime}(n t) \mathrm{d} x \mathrm{~d} t .
$$

En notant par $M$ un majorant de $\psi^{\prime} \partial_{x} \varphi$, on obtient

$$
\left|n \int_{1 / n}^{2 / n} \int_{\mathbb{R}} u(x, t) \varphi(x, t) \psi^{\prime}(n t) \mathrm{d} x \mathrm{~d} t\right| \leq n M \int_{1 / n}^{2 / n} \int_{0}^{\sqrt{t}} \frac{x^{2}}{t} d x \mathrm{~d} t \leq n M \int_{1 / n}^{2 / n} \sqrt{t} \mathrm{~d} t \leq M \sqrt{2 / n} .
$$

En passant à limite quand $n \rightarrow+\infty$ dans 5.105, on en déduit 5.72.

5. On peut supposer $\eta(0)=0$, cela ne change pas les termes de (5.73). On pose $\psi(s)=\phi(s)-s \eta(s)$ de sorte que $\psi^{\prime}(s)=s \eta^{\prime}(s)-\eta(s)$. La convexité de $\eta$ donne alors $\psi^{\prime}(s) \geq 0$ pour $s \geq 0$ et donc $\psi(s) \geq 0$ pour $s \geq 0$.

Pour démontrer 5.73, on peut alors utiliser la proposition 5.17 avec, pour tout $\varepsilon>0, \mathbb{R} \times[\varepsilon,+\infty[$ au lieu de $\mathbb{R} \times \mathbb{R}_{+}$ou reprendre la démontration de la question 3 en remplaçant $u$ par $\eta(u)$ et $u^{2}$ par $\phi(u)$.

6. La solution entropique de 5.69)-(5.70) est la fonction identiquement nulle sur $\mathbb{R} \times \mathbb{R}_{+}$. La fonction $u$ n'est pas la solution entropique de (5.69)-(5.70).

\section{Exercice 5.13 (Effet "Landau")}

1. La première équation de 5.77) peut s'écrire $\frac{\partial u}{\partial t}(x, y, t)+\frac{\partial y u}{\partial x}(x, y, t)=0$. La notion de solution faible pour cette équation est donc parfaitement définie.

\section{Etape 1, construction d'une solution}

Le problème (5.77) correspond à une équation de transport dans la direction $x$, la vitesse du transport dépendant de la variable $y$ (que l'on peut voir ici comme un paramètre). Le début du chapitre 5 nous suggère alors la forme de la solution faible. Pour $(x, y, t) \in \mathbb{R} \times \mathbb{R} \times \mathbb{R}_{+}$, on pose

$$
u(x, y, t)=f(x-y t) \text {. }
$$


La fonction $u$ ainsi définie appartient bien à $L^{\infty}\left(\mathbb{R} \times \mathbb{R} \times \mathbb{R}_{+}\right)$. On montre maintenant que $u$ est solution faible de (5.77).

Soit $\varphi \in C_{c}^{1}\left(\mathbb{R}^{2} \times \mathbb{R}_{+}, \mathbb{R}\right)$. On va montrer que

$$
\int_{\mathbb{R}_{+}} \int_{\mathbb{R}} \int_{\mathbb{R}} u(x, y, t)\left(\varphi_{t}(x, y, t)+y \varphi_{x}(x, y, t)\right) \mathrm{d} x \mathrm{~d} y \mathrm{~d} t=-\int_{\mathbb{R}} f(x) \varphi(x, y, 0) \mathrm{d} x \mathrm{~d} y .
$$

Ceci montrera bien que $u$ est solution faible de 5.77$)$. On considère de terme de gauche de $(5.106)$ en remplaçant $u(x, y, t)$ par $f(x-y t)$ et on utilise dans l'intégrale par rapport à $x$ le changement de variable $x-y t=z$ (pour $y$ et $t$ fixés, on profite aussi ici du théorème de Fubini). On obtient

$$
\begin{aligned}
& \int_{\mathbb{R}_{+}} \int_{\mathbb{R}} \int_{\mathbb{R}} f(x-y t)\left(\varphi_{t}(x, y, t)+y \varphi_{x}(x, y, t)\right) \mathrm{d} x \mathrm{~d} y \mathrm{~d} t \\
& =\int_{\mathbb{R}_{+}} \int_{\mathbb{R}} \int_{\mathbb{R}} f(z)\left(\varphi_{t}(z+y t, y, t)+y \varphi_{x}(z+y t, y, t)\right) \mathrm{d} z \mathrm{~d} y \mathrm{~d} t .
\end{aligned}
$$

(Noter que $\varphi_{x}$ désigne toujours la dérivée de $\varphi$ par rapport à sa première variable et $\varphi_{t}$ désigne toujours la dérivée de $\varphi$ par rapport à sa troisième variable.)

Pour $z, y \in \mathbb{R}$ et $t \in \mathbb{R}_{+}$, on pose $\psi(z, y, t)=\varphi(z+y t, y, t)$, de sorte que $\psi_{t}(z, y, t)=y \varphi_{x}(z+y t, y, t)+$ $\varphi_{t}(z+y t, y, t)$. On obtient ainsi

$$
\int_{\mathbb{R}_{+}} \int_{\mathbb{R}} \int_{\mathbb{R}} f(x-y t)\left(\varphi_{t}(x, y, t)+y \varphi_{x}(x, y, t)\right) \mathrm{d} x \mathrm{~d} y \mathrm{~d} t=\int_{\mathbb{R}_{+}} \int_{\mathbb{R}} \int_{\mathbb{R}} f(z) \psi_{t}(z, y, t) \mathrm{d} z \mathrm{~d} y \mathrm{~d} t .
$$

On peut maintenant intégrer le terme de droite d'abord par rapport à $t$ (grâce au théorème de Fubini), on obtient

$$
\int_{\mathbb{R}_{+}} \int_{\mathbb{R}} \int_{\mathbb{R}} f(x-y t)\left(\varphi_{t}(x, y, t)+y \varphi_{x}(x, y, t)\right) \mathrm{d} x \mathrm{~d} y \mathrm{~d} t=-\int_{\mathbb{R}} \int_{\mathbb{R}} f(z) \psi(z, y, 0) \mathrm{d} z \mathrm{~d} y .
$$

Ce qui donne

$$
\int_{\mathbb{R}_{+}} \int_{\mathbb{R}} \int_{\mathbb{R}} f(x-y t)\left(\varphi_{t}(x, y, t)+y \varphi_{x}(x, y, t)\right) \mathrm{d} x \mathrm{~d} y \mathrm{~d} t=-\int_{\mathbb{R}} \int_{\mathbb{R}} f(z) \varphi(z, y, 0) \mathrm{d} z \mathrm{~d} y .
$$

On a bien montré 5.106. La fonction $u$ est donc bien une solution faible de 5.77).

\section{Unicité de la solution faible de (5.77)}

Grâce à la linéarité de la première équation de (5.77), il suffit de montrer que si $u$ est solution de (5.106) (pour tout $\varphi \in C_{c}^{1}\left(\mathbb{R}^{2} \times \mathbb{R}_{+}, \mathbb{R}\right)$ ) avec $f=0$ p.p., alors $u=0$ p.p.. On suppose donc que $u$ appartient à $L^{\infty}\left(\mathbb{R}^{2} \times \mathbb{R}_{+}\right)$et vérifie

$$
\int_{\mathbb{R}_{+}} \int_{\mathbb{R}} \int_{\mathbb{R}} u(x, y, t)\left(\varphi_{t}(x, y, t)+y \varphi_{x}(x, y, t)\right) \mathrm{d} x \mathrm{~d} y \mathrm{~d} t=0 \text { pour tout } \varphi \in C_{c}^{1}\left(\mathbb{R}^{2} \times \mathbb{R}_{+}, \mathbb{R}\right) .
$$

On va montrer que $u=0$ p.p..

Soit $\psi \in C_{c}^{1}\left(\mathbb{R}^{2} \times \mathbb{R}_{+}, \mathbb{R}\right)$. Pour $x, y \in \mathbb{R}$ et $t \in \mathbb{R}_{+}$, on pose

$$
\varphi(x, y, t)=-\int_{t}^{+\infty} \psi(x-y(t-s), y, s) \mathrm{d} s .
$$


On a aussi $\varphi \in C_{c}^{1}\left(\mathbb{R}^{2} \times \mathbb{R}_{+}, \mathbb{R}\right)$ et on remarque que pour tout $x, y \in \mathbb{R}$ et tout $t>0$ on a

$$
\begin{array}{r}
\varphi_{t}(x, y, t)+y \varphi_{x}(x, y, t)=\psi(x, y, t)+y \int_{t}^{+\infty} \psi_{x}(x-y(t-s), y, s) \mathrm{d} s \\
-y \int_{t}^{+\infty} \psi_{x}(x-y(t-s), y, s) \mathrm{d} s
\end{array}
$$

et donc

$$
\varphi_{t}(x, y, t)+y \varphi_{x}(x, y, t)=\psi(x, y, t) .
$$

En prenant cette fonction $\varphi$ dans (5.107) on obtient

$$
\int_{\mathbb{R}_{+}} \int_{\mathbb{R}} \int_{\mathbb{R}} u(x, y, t) \psi(x, y, t) \mathrm{d} x \mathrm{~d} y \mathrm{~d} t=0 \text { pour tout } \psi \in C_{c}^{1}\left(\mathbb{R}^{2} \times \mathbb{R}_{+}, \mathbb{R}\right) .
$$

On en déduit que $u=0$ p.p..

N.B. La méthode que nous venons d'utiliser est une méthode classique pour obtenir l'unicité d'un problème par la résolution du problème adjoint (qui est ici $\varphi_{t}+y \varphi_{x}=\psi$ avec $\varphi=0$ comme donnée "finale".)

2. Soit $T>0$ t.q. $f(z+T)=f(z)$ pour tout $z \in \mathbb{R}$ (la fonction $f$ est donc de période $T$ ).

Soit $y \in \mathbb{R}$ et $r>T$. Il existe $p, q \in \mathbb{Z}$ t.q. $(p-1) T<y-r \leq p T<q T \leq y+r<(q+1) T$. On a alors

$$
2 r F(y, r)=\int_{y-r}^{p T} f(z) d z+\int_{p T}^{q T} f(z) d z+\int_{q T}^{y+r} f(z) d z=\int_{y-r}^{p T} f(z) d z+(q-p) m T+\int_{q T}^{y+r} f(z) d z .
$$

Ceci donne

$$
F(y, r)=m+\left(\frac{(q-p) T}{2 r}-1\right) m+\frac{1}{2 r} \int_{y-r}^{p T} f(z) d z+\frac{1}{2 r} \int_{q T}^{y+r} f(z) d z .
$$

Comme $0 \leq 2 r-(q-p) T \leq 2 T$ et que, avec $M=\max \{|f(z)|, z \in \mathbb{R}\}$,

$$
\left|\int_{y-r}^{p T} f(z) d z\right| \leq \int_{y-r}^{p T}|f(z)| d z \leq M T, \quad\left|\int_{q T}^{y+r} f(z) d z\right| \leq \int_{q T}^{y+r}|f(z)| d z \leq M T,
$$

on a donc

$$
|F(y, r)-m| \leq \frac{(|m|+M) T}{r} .
$$

ce qui prouve bien que $\lim _{r \rightarrow+\infty} F(y, r)=m$, uniformément par rapport à $y \in \mathbb{R}$.

3. Soit $x \in \mathbb{R}$ et $t>0$. En utilisant le changement de variable $z=x-y t$, c'est-à-dire $y=(x-z) / t$, on obtient

$$
\int_{b-\delta}^{b+\delta} f(x-y t) \mathrm{d} y=\int_{x-b t-\delta t}^{x-b t+\delta t} \frac{f(z)}{t} d z=2 \delta F(x-b t, \delta t) .
$$

Soit maintenant $t>0$. On sait que $u(x, y, t)=f(x-y t)$, on a donc

$$
\int_{b-\delta}^{b+\delta} \int_{a-\delta}^{a+\delta} u(x, y, t) \mathrm{d} x \mathrm{~d} y=\int_{b-\delta}^{b+\delta} \int_{a-\delta}^{a+\delta} f(x-y t) \mathrm{d} x \mathrm{~d} y .
$$

Avec le théorème de Fubini, on a donc

$$
\int_{b-\delta}^{b+\delta} \int_{a-\delta}^{a+\delta} u(x, y, t) \mathrm{d} x \mathrm{~d} y=\int_{a-\delta}^{a+\delta}\left(\int_{b-\delta}^{b+\delta} f(x-y t) \mathrm{d} y\right) \mathrm{d} x=2 \delta \int_{a-\delta}^{a+\delta} F(x-b t, \delta t) \mathrm{d} x .
$$


La deuxième question donne $\lim _{t \rightarrow \infty} F(x-b t, \delta t)=m$, uniformément par rapport à $x$, on a donc bien

$$
\lim _{t \rightarrow+\infty} \int_{b-\delta}^{b+\delta} \int_{a-\delta}^{a+\delta} u(x, y, t) \mathrm{d} x \mathrm{~d} y=4 \delta^{2} m .
$$

4. On remarque d'abord que (avec $M=\max \{|f(z)|, z \in \mathbb{R}\}$ )

$$
\|u(\cdot, \cdot, t)\|_{L^{\infty}\left(\mathbb{R}^{2}\right)} \leq M \text { pour tout } t>0 .
$$

On pose

$$
\mathcal{C}=\left\{1_{] a-\delta, a+\delta[\times] b-\delta, d+\delta[}, a, b \in \mathbb{R}, \delta>0\right\} .
$$

La question précédente montre que pour tout $\varphi \in \mathcal{C}$ on a

$$
\lim _{t \rightarrow \infty} \int_{\mathbb{R}} \int_{\mathbb{R}} u(x, y, t) \varphi(x, y) \mathrm{d} x \mathrm{~d} y=m \int_{\mathbb{R}} \int_{\mathbb{R}} \varphi(x, y) \mathrm{d} x \mathrm{~d} y .
$$

On note maintenant $E$ l'espace vectoriel engendré par $\mathcal{C}$. Par linéarité de l'intégrale, on a alors 5.108$)$ pour tout $\varphi \in E$.

L'espace vectoriel $E$ est dense dans $L^{1}\left(\mathbb{R}^{2}\right)$ (pour la mesure de Lebesgue). Pour montrer ceci, il suffit, par exemple, d'utiliser la densité de $C_{c}\left(\mathbb{R}^{2}, \mathbb{R}\right)$ dans $L^{1}\left(\mathbb{R}^{2}\right)$ puis de remarquer que tout élément de $C_{c}\left(\mathbb{R}^{2}, \mathbb{R}\right)$ peut être approché d'aussi près que l'on veut pour la norme de $L^{1}\left(\mathbb{R}^{2}\right)$ par un élément de $E$. On en déduit bien que $E$ est dense dans $L^{1}\left(\mathbb{R}^{2}\right)$. Grâce à cette densité et à la borne $L^{\infty}\left(\mathbb{R}^{2}\right)$ sur $u(\cdot, \cdot, t)$, on conclut facilement que (5.108) est vrai pour tout $u \in L^{1}\left(\mathbb{R}^{2}\right)$. Ceci donne bien que $u(., ., t) \rightarrow m \star$-faiblement dans $L^{\infty}\left(\mathbb{R}^{2}\right)$, quand $t \rightarrow+\infty$.

\section{Exercice 5.15 (Equations de Saint-Venant)}

1. La fonction $F$ est définie (sur $D$ ) par $F(U)=\left[\begin{array}{c}q \\ \frac{q^{2}}{h}+\frac{g}{2} h^{2}\end{array}\right]$.

2. La matrice jacobienne de $F$ au point $U$ est

$$
D F(U)=\left[\begin{array}{cc}
0 & 1 \\
-u^{2}+g h & 2 u
\end{array}\right]
$$

Le polynôme caractéristique de cette matrice est $P_{U}(\lambda)=\lambda^{2}-2 u \lambda+u^{2}-g h=(u-\lambda)^{2}-g h$. Les valeurs propres de $D F(U)$ sont donc $\lambda_{1}(U)=u-c$ et $\lambda_{2}(U)=u+c$. Elles sont réelles et distinctes, et le système est donc strictement hyperbolique. Une base de $\mathbb{R}^{2}$ formée de vecteurs propres de cette matrice jacobienne est alors $\left\{\varphi_{1}(U), \varphi_{2}(U)\right\}$ avec

$$
\varphi_{1}(U)=\left[\begin{array}{c}
1 \\
u-c
\end{array}\right] \text { et } \varphi_{2}(U)=\left[\begin{array}{c}
1 \\
u+c
\end{array}\right]
$$

3. Soit $U \in D$. Par la question précédente, on a $\lambda_{1}(U)=u-c=q / h-\sqrt{g h}$ et donc

$$
\nabla \lambda_{1}(U)=\left[\begin{array}{c}
-\frac{q}{h^{2}}+\frac{g}{2 \sqrt{g h}} \\
\frac{1}{h}
\end{array}\right]=\left[\begin{array}{c}
-\frac{u}{h}+\frac{g}{2 c} \\
\frac{1}{h}
\end{array}\right],
$$


ce qui donne, avec les notations précédentes :

$$
\nabla \lambda_{1}(U) \cdot \varphi_{1}(U)=-\frac{u}{h}+\frac{g}{2 c}+\frac{u-c}{h}=-\frac{g}{2 c} \neq 0 .
$$

Comme $\lambda_{2}(U)=u+c$, on a

$$
\nabla \lambda_{2}(U)=\left[\begin{array}{c}
-\frac{u}{h}-\frac{g}{2 c} \\
\frac{1}{h}
\end{array}\right] \text { et } \nabla \lambda_{2}(U) \cdot \varphi_{1}(U)=-\frac{u}{h}-\frac{g}{2 c}+\frac{u+c}{h}=\frac{g}{2 c} \neq 0 .
$$

Les deux champs sont donc VNL.

4. Un 1-invariant de Riemann est une fonction $r_{1}$ de $D$ dans $\mathbb{R}$ de classe $C^{1}$ telle que $\nabla r_{1}(U) \cdot \varphi_{1}(U)=0$ pour tout $U$ dans $D$. Si on cherche $r_{1}$ sous la forme $r_{1}(U)=u+\psi(h)$ (parce que ça marche!), la condition sur $r_{1}$ devient

$$
\left[\begin{array}{c}
-\frac{q}{h^{2}}+\psi^{\prime}(h) \\
\frac{1}{h}
\end{array}\right] \cdot\left[\begin{array}{c}
1 \\
u-c
\end{array}\right]=-\frac{u}{h}+\psi^{\prime}(h)+\frac{u}{h}-\frac{c}{h}=\psi^{\prime}(h)-\frac{c}{h}=0,
$$

Une solution consiste à prendre $\psi(h)=2 c$, ceci donne bien $\psi^{\prime}(h)=\frac{g}{c}=\frac{c}{h}$. On choisit donc $r_{1}(U)=u+2 c$. De manière analogue, un 2-invariant de Riemann est $r_{2}(U)=u-2 c$.

5. On considère une solution régulière de (5.80)-5.81). On multiplie (5.80) par $g h$ et (5.81) par $u$, on obtient

$$
\begin{aligned}
& \partial_{t}\left(\frac{g h^{2}}{2}\right)+g h^{2} \partial_{x} u+u \partial_{x}\left(\frac{g h^{2}}{2}\right)=0 \\
& h \partial_{t}\left(\frac{u^{2}}{2}\right)+u^{2} \partial_{t} h+u^{2} \partial_{x}(h u)+h u^{2} \partial_{x} u+u \partial_{x} p=0 .
\end{aligned}
$$

On additionne ces deux équations. En utilisant (5.80) et $p=g h^{2} / 2$, on obtient

$$
\partial_{t} p+h \partial_{t}\left(\frac{u^{2}}{2}\right)+h u^{2} \partial_{x} u+2 p \partial_{x} u+2 u \partial_{x} p=0
$$

En utilisant encore (5.80), $h \partial_{t}\left(\frac{u^{2}}{2}\right)=\partial_{t}\left(h \frac{u^{2}}{2}\right)-\frac{u^{2}}{2} \partial_{t} h=\partial_{t}\left(h \frac{u^{2}}{2}\right)+\frac{u^{2}}{2} \partial_{x}(h u)$ et l'égalité précédente donne

$$
\partial_{t} \eta(U)+\frac{u^{2}}{2} \partial_{x}(h u)+h u^{2} \partial_{x} u+\partial_{x}(2 p u)=0 .
$$

En posant $\phi(U)=(1 / 2) h u^{3}+2 p u$, l'égalité précédente s'écrit

$$
\partial_{t} \eta(U)+\partial_{x} \phi(U)=0 .
$$

Un autre moyen d'obtenir ce résultat (et nous prendrons cette méthode plus générale dans la question suivante) est de remarquer que

$$
\eta(U)=\frac{1}{2} h u^{2}+p=\frac{q^{2}}{2 h}+\frac{g h^{2}}{2}
$$

de sorte que

$$
\nabla \eta(U)=\left[\begin{array}{c}
-\frac{u^{2}}{2}+g h \\
u
\end{array}\right]
$$


On multiplie alors (5.80) par $\partial_{h} \eta(U)=-\frac{u^{2}}{2}+g h$ et (5.81) par $\partial_{q} \eta(U)=u$ et par des manipulations semblables aux précédentes on obtient aussi 5.109).

Noter que $\partial_{h} \eta$ et $\partial_{q} \eta$ désignent les dérivées partielles de la fonction $\eta$, c'est-à-dire de la fonction $U \mapsto \eta(U)$ (de $D$ dans $\mathbb{R}$ ) et donc que $\partial_{h} \eta(U)$ et $\partial_{q} \eta(U)$ sont ces dérivées partielles prises au point $U$ (appartenant à $D$ ), alors que $\partial_{t} \eta(U)$ et $\partial_{x} \eta(U)$ désignent les dérivées partielles de la fonction $\eta \circ U$, c'est-à-dire de la fonction $(x, t) \mapsto \eta(U(x, t))\left(\right.$ de $\mathbb{R} \times \mathbb{R}_{+}^{\star}$ dans $\left.\mathbb{R}\right)$.

Il reste à vérifier que $\eta$ est une fonction convexe de $D$ dans $\mathbb{R}$, ce qui est équivalent à montrer que sa matrice hessienne $H(U)$ est positive pour tout $U \in D$, ou encore que $\xi^{t} H(U) \xi \geq 0$ pour tout $U \in D$ et $\xi \in \mathbb{R}^{2}$.

Comme $\nabla \eta(U)=\left[\begin{array}{c}-\frac{q^{2}}{2 h^{2}}+g h \\ \frac{q}{h}\end{array}\right]$, on a $H(U)=\left[\begin{array}{cc}\frac{u^{2}}{h}+g & -\frac{u}{h} \\ -\frac{u}{h} & \frac{1}{h}\end{array}\right]$, et donc $\operatorname{tr} H(U)=\frac{u^{2}}{h}+g+\frac{1}{h}>0$ et $\operatorname{det} H(U)=\frac{u^{2}}{h^{2}}+\frac{g}{h}-\frac{u^{2}}{h^{2}}=\frac{g}{h}>0$. Ceci montre que $H(U)$ est bien positive pour tout $U \in D$.

6. Comme $h_{\varepsilon}$ et $u_{\varepsilon}$ convergent vers $h$ et $u$ dans $L_{\text {loc }}^{1}\left(\mathbb{R} \times \mathbb{R}_{+}\right)$, les dérivées $\partial_{x}^{2} h_{\varepsilon}$ et $\partial_{x}^{2} u_{\varepsilon}$ convergent vers $\partial_{x}^{2} h$ et $\partial_{x}^{2} u$ au moins au sens des distributions et donc $\varepsilon \partial_{x}^{2} h_{\varepsilon}$ et $\varepsilon \partial_{x}^{2} u_{\varepsilon}$ convergent vers 0 au sens des distributions. Ceci prouve que $(h, u)$ est solution faible de 5.80-5.81.

On note $U_{\varepsilon}=\left[\begin{array}{l}h_{\varepsilon} \\ q_{\varepsilon}\end{array}\right]$. On remarque tout d'abord que $\eta\left(U_{\varepsilon}\right)$ et $\phi\left(U_{\varepsilon}\right)$ convergent dans $L_{\text {loc }}^{1}\left(\mathbb{R} \times \mathbb{R}_{+}\right)$, quand $\varepsilon \rightarrow 0$, vers $\eta(U)$ et $\phi(U)$ respectivement.

En reprenant la méthode de la question précédente, c'est-à-dire multiplication de (5.80) par $\partial_{h} \eta\left(U_{\varepsilon}\right)$ et (5.81) $\operatorname{par} \partial_{q} \eta\left(U_{\varepsilon}\right)$, on obtient

$$
\partial_{t} \eta\left(U_{\varepsilon}\right)+\partial_{x} \phi\left(U_{\varepsilon}\right)+R_{\varepsilon}=0
$$

avec $R_{\varepsilon}=-\varepsilon \partial_{h} \eta\left(U_{\varepsilon}\right) \partial_{x}^{2} h_{\varepsilon}-\varepsilon \partial_{q} \eta\left(U_{\varepsilon}\right) \partial_{x}^{2} q_{\varepsilon}=S_{\varepsilon}+T_{\varepsilon}$, et

$$
\begin{aligned}
& S_{\varepsilon}=-\sqrt{\varepsilon} \partial_{x}\left(\partial_{h} \eta\left(U_{\varepsilon}\right) \sqrt{\varepsilon} \partial_{x} h_{\varepsilon}\right)-\sqrt{\varepsilon} \partial_{x}\left(\partial_{q} \eta\left(U_{\varepsilon}\right) \sqrt{\varepsilon} \partial_{x} q_{\varepsilon}\right), \\
& T_{\varepsilon}=\varepsilon \partial_{x}\left(\partial_{h} \eta\left(U_{\varepsilon}\right)\right) \partial_{x} h_{\varepsilon}+\varepsilon \partial_{x}\left(\partial_{q} \eta\left(U_{\varepsilon}\right)\right) \partial_{x} q_{\varepsilon} .
\end{aligned}
$$

D'une part, $S_{\varepsilon} \rightarrow 0$ au sens des distributions quand $\varepsilon \rightarrow 0$ car $\left.\partial_{h} \eta\left(U_{\varepsilon}\right)\right)$ et $\left.\partial_{q} \eta\left(U_{\varepsilon}\right)\right)$ sont bornées dans $L^{\infty}\left(\mathbb{R} \times \mathbb{R}_{+}\right)$et $\sqrt{\varepsilon} \partial_{x} h$ et $\sqrt{\varepsilon} \partial_{x} q$ sont bornées dans $L_{\text {loc }}^{1}\left(\mathbb{R} \times \mathbb{R}_{+}\right)$.

D'autre part $T_{\varepsilon}=\left(\partial_{x} U_{\varepsilon}\right)^{t} H\left(U_{\varepsilon}\right) \partial_{x} U_{\varepsilon}$, où $H\left(U_{\varepsilon}\right)$ désigne la matrice hessienne de $\eta$ au point $U_{\varepsilon}$. Or, on a montré à la question précédente que cette matrice hessienne est positive, on a donc $T_{\varepsilon} \geq 0$ et donc, en passant à la limite dans 5.110 quand $\varepsilon \rightarrow 0$, on obtient qu'au sens des distributions, $U$ vérifie,

$$
\partial_{t} \eta(U)+\partial_{x} \phi(U) \leq 0
$$

7. L'équation 5.81 donne

$$
h \partial_{t} u+u \partial_{t} h+u \partial_{x}(h u)+h u \partial_{x} u+g h \partial_{x} h=0 .
$$

En utilisant 5.80 et en divisant par $h$ (on rappelle que $h>0$ ),

$$
\partial_{t} u+u \partial_{x} u+g \partial_{x} h=0 .
$$

Comme $\partial_{x}(2 c)=\frac{c}{h} \partial_{x} h$ on a $g \partial_{x} h=\frac{g h}{c} \partial_{x}(2 c)=c \partial_{x}(2 c)$ et donc

$$
\partial_{t} u+u \partial_{x} u+c \partial_{x}(2 c)=0 .
$$


L'équation (5.80) donne

$$
g \partial_{t} h+g\left(\partial_{x} h\right) u+g h \partial_{x} u=0 .
$$

On a déjà vu que $g \partial_{x} h=c \partial_{x}(2 c)$. De même $g \partial_{t} h=c \partial_{t}(2 c)$. On en déduit

$$
c \partial_{t}(2 c)+c u \partial_{x}(2 c)+c^{2} \partial_{x} u=0 .
$$

Ce qui donne, en divisant par $c$,

$$
\partial_{t}(2 c)+u \partial_{x}(2 c)+c \partial_{x} u=0
$$

et donc 5.83 .

8. On cherche une solution sous la forme d'une 1-détente et d'une 2-détente séparées par un état intermédiaire noté $\left(h_{\star}, u_{\star}\right)$. On note $U_{g}, U_{d}$ et $U_{\star}$ les états constants de cette solution.

On sait qu'un $i$-invariant de Riemann est constant dans une $i$-détente. On a donc $u_{g}+2 c_{g}=u_{\star}+2 c_{\star}$ et $u_{d}-2 c_{d}=u_{\star}-2 c_{\star}$. Ceci permet de calculer $h_{\star}$ et $c_{\star}$,

$$
\begin{aligned}
& u_{\star}=\frac{u_{g}+u_{d}}{2}+c_{g}-c_{d}, \\
& c_{\star}=\frac{u_{g}-u_{d}}{4}+\frac{c_{g}+c_{d}}{2} .
\end{aligned}
$$

On a bien $c_{\star}>0$ car $u_{d}-u_{g}<2\left(c_{g}+c_{d}\right)$ (qui est justement la condition de non apparition du vide).

Pour construire la solution, on sait que la 1-détente correspond à la zone $\left\{\lambda_{1}\left(U_{g}\right) t \leq x \leq \lambda_{1}\left(U_{\star}\right) t\right\}$ et que la 2-détente correspond à la zone $\left\{\lambda_{2}\left(U_{\star}\right) t \leq x \leq \lambda_{2}\left(U_{d}\right)\right\}$. La construction de cette solution formée de deux détentes est donc possible si et seulement si

$$
\begin{aligned}
& \lambda_{1}\left(U_{g}\right)=u_{g}-c_{g} \leq u_{\star}-c_{\star}=\lambda_{1}\left(U_{\star}\right) \\
& \lambda_{2}\left(U_{\star}\right)=u_{\star}+c_{\star} \leq u_{d}+c_{d}=\lambda_{2}\left(U_{d}\right)
\end{aligned}
$$

La condition 5.111) est équivalente à $u_{g}-u_{d} \leq 2\left(c_{g}-c_{d}\right)$ et la condition 5.112 est équivalente à $u_{g}-u_{d} \leq$ $2\left(c_{d}-c_{g}\right)$. Ces deux conditions sont satisfaites grâce à la condition $2\left|c_{g}-c_{d}\right| \leq u_{d}-u_{g}$.

La solution du problème de Riemann (5.82), (5.84)-(5.85) est donc bien formée de deux détentes. On la construit en distinguant cinq zones $D_{i}, i=1, \ldots, 5$; avec

$$
\begin{aligned}
& D_{1}=\left\{x \leq\left(u_{g}-c_{g}\right) t\right\}, \\
& D_{2}=\left\{\left(u_{g}-c_{g}\right) t<x<\left(u_{\star}-c_{\star}\right) t\right\}, \\
& D_{3}=\left\{\left(u_{\star}-c_{\star}\right) t<x<\left(u_{\star}+c_{\star}\right) t\right\}, \\
& D_{4}=\left\{\left(u_{\star}+c_{\star}\right) t<x<\left(u_{d}+c_{d}\right) t\right\}, \\
& D_{5}=\left\{\left(u_{d}+c_{d}\right) t<x\right\} .
\end{aligned}
$$

— Sur $D_{1}$, on a $U=U_{g}$.

- Sur $D_{3}$, on a $U=U_{\star}$.

- Sur $D_{5}$, on a $U=U_{d}$.

- Sur $D_{2}$, on a affaire à une 1-détente. On cherche la solution sous la forme $U(x, t)=V\left(\frac{x}{t}\right)$. Donc sur la droite $x=\alpha t$ avec $\left(u_{g}-c_{g}\right)<\alpha<\left(u_{\star}-c_{\star}\right)$, la solution $U(x, t)$ est donnée par $\alpha=\frac{x}{t}=\lambda_{1}(V(\alpha))=$ 
$u-c$. En écrivant de plus l'invariance du 1-invariant de Riemann, on obtient la solution en résolvant le système

$$
\begin{aligned}
& u-c=\alpha, \\
& u+2 c=u_{g}+2 c_{g},
\end{aligned}
$$

ce qui donne $3 u=2 \alpha+u_{g}+2 c_{g}, 3 c=u_{g}+2 c_{g}-\alpha$.

— Enfin sur $D_{4}$, on a affaire à une 2-détente. Donc pour $x=\alpha t$ avec $\left(u_{\star}+c_{\star}\right)<\alpha<\left(u_{d}+c_{d}\right)$, la solution $U(x, t)$ est donnée par $\alpha=\frac{x}{t}=\lambda_{2}(V(\alpha))$, et par invariance du 2-invariant de Riemann, on obtient donc la solution en résolvant le système

$$
\begin{aligned}
& u+c=\alpha, \\
& u-2 c=u_{d}-2 c_{d},
\end{aligned}
$$

ce qui donne $3 u=2 \alpha+u_{d}-2 c_{d}, 3 c=-u_{d}+2 c_{d}+\alpha$.

9. (Détente-choc, choc-détente et choc-choc) Dans cette question on suppose que $2\left|c_{g}-c_{d}\right|>u_{d}-u_{g}$. Si $U$ est solution faible on a donc $h_{g} \neq h_{d}$ (en effet, si $h_{g}=h_{d}$, la relation de Rankine-Hugoniot sur la droite $x=\sigma t$ donne, avec les notations du cours, $\sigma[h]=[h u]$ et donc $u_{g}=u_{d}$ en contradiction avec $2\left|c_{g}-c_{d}\right|>u_{d}-u_{g}$ ).

(a) (Calcul d'un choc)

i. La fonction $U$ est solution faible si et seulement si les conditions de Rankine-Hugoniot sont satisfaites sur la droite $x=\sigma t$, c'est-à-dire, avec les notations du cours, $\sigma[h]=[h u]$ et $\sigma[h u]=\left[h u^{2}+p\right]$, ce qui est equivalent à $[h u]^{2}=[h]\left[h u^{2}+p\right]$ et $\sigma[h]=[h u]$.

Comme

$$
\begin{aligned}
& {[h u]^{2}=\left(h_{d} u_{d}-h_{g} u_{g}\right)^{2}=h_{d}^{2} u_{d}^{2}+h_{g}^{2} u_{g}^{2}-2 h_{g} h_{d} u_{g} u_{d} \text { et }} \\
& {[h]\left[h u^{2}+p\right]=h_{d}^{2} u_{d}^{2}+h_{g}^{2} u_{g}^{2}-h_{d} h_{g}\left(u_{g}^{2}+u_{d}^{2}\right)+[h][p],}
\end{aligned}
$$

la fonction $U$ est solution faible si et seulement si $h_{d} h_{g}\left(u_{g}-u_{d}\right)^{2}=[h][p]$ et $\sigma[h]=[h u]$. Ceci correspond bien à $\left(u_{g}-u_{d}\right)^{2}=S$ et $\sigma[h]=[h u]$.

On montre maintenant que $U$ est un 1-choc si et seulement si $u_{d}=u_{g}-S$ et $h_{g}<h_{d}$.

Condition nécessaire. On suppose que $U$ est un 1-choc. La condition de Lax donne alors, en utilisant $\sigma[h]=[h u]$,

$$
u_{g}-c_{g}>\sigma=u_{g}+\frac{h_{d}\left(u_{g}-u_{d}\right)}{h_{g}-h_{d}}=u_{d}+\frac{h_{g}\left(u_{g}-u_{d}\right)}{h_{g}-h_{d}}>u_{d}-c_{d} .
$$

La première inégalité donne que $u_{g}-u_{d}$ et $h_{g}-h_{d}$ sont non nuls et de signe contraire (car $c_{g}>0$ ). Comme $u_{g}-c_{g}>u_{d}-c_{d}$, on a aussi $u_{g}-u_{d}>c_{g}-c_{d}$. Comme $c_{g}-c_{d}$ a le même signe que $h_{g}-h_{d}$ et donc le signe contraire de celui de $u_{g}-u_{d}$; on en déduit $u_{g}-u_{d}>0>h_{g}-h_{d}$. Ceci donne bien $u_{d}=u_{g}-S$ et $h_{g}<h_{d}$.

Condition suffisante. On suppose maintenant que $u_{d}=u_{g}-S$ et $h_{g}<h_{d}$ et on veut montrer que $U$ est un 1-choc, c'est-à-dire que 5.113 est vérifiée. La première inégalité est vraie si

$$
g h_{g}<\frac{h_{d}^{2}\left(u_{g}-u_{d}\right)^{2}}{\left(h_{g}-h_{d}\right)^{2}}=\frac{h_{d}^{2}}{\left(h_{g}-h_{d}\right)^{2}} S^{2}=\frac{h_{d}^{2}}{\left(h_{g}-h_{d}\right)^{2}} \frac{g\left(h_{g}-h_{d}\right)\left(h_{g}^{2}-h_{d}^{2}\right)}{2 h_{g} h_{d}}=g \frac{h_{d}}{h_{g}} \frac{h_{g}+h_{d}}{2} .
$$

Ceci est vrai car $h_{g}<h_{d}$. 
La deuxième inégalité est vraie si

$$
g h_{d}>\frac{h_{g}^{2}\left(u_{g}-u_{d}\right)^{2}}{\left(h_{g}-h_{d}\right)^{2}}=\frac{h_{g}^{2}}{\left(h_{g}-h_{d}\right)^{2}} S^{2}=\frac{h_{g}^{2}}{\left(h_{g}-h_{d}\right)^{2}} \frac{g\left(h_{g}-h_{d}\right)\left(h_{g}^{2}-h_{d}^{2}\right)}{2 h_{g} h_{d}}=g \frac{h_{g}}{h_{d}} \frac{h_{g}+h_{d}}{2} .
$$

Ceci est vrai car $h_{d}>h_{g}$.

De manière analogue on montre que $U$ est un 2-choc si et seulement si $u_{d}=u_{g}-S$ et $h_{g}>h_{d}$. La condition de Lax (qui est (5.113) pour les 1-choc) devient

$$
u_{g}+c_{g}>\sigma=u_{g}+\frac{h_{d}\left(u_{g}-u_{d}\right)}{h_{g}-h_{d}}=u_{d}+\frac{h_{g}\left(u_{g}-u_{d}\right)}{h_{g}-h_{d}}>u_{d}+c_{d} .
$$

La deuxième inégalité donne que $u_{g}-u_{d}$ et $h_{g}-h_{d}$ sont non nuls et de même signe (car $c_{d}>0$ ). Comme $u_{g}+c_{g}>u_{d}+c_{d}$, on a aussi $u_{g}-u_{d}>c_{d}-c_{g}$. Comme $c_{d}-c_{g}$ a le même signe que $h_{d}-h_{g}$ et donc le signe contraire de celui de $u_{g}-u_{d}$, on en déduit $u_{g}-u_{d}>0>h_{d}-h_{g}$. Ceci donne bien $u_{d}=u_{g}-S$ et $h_{g}>h_{d}$. Ceci montre que la condition $u_{d}=u_{g}-S$ et $h_{g}>h_{d}$ est nécessaire pour avoir un 2-choc.

On montre maintenant que cette condition est suffisante. On suppose donc que $u_{d}=u_{g}-S$ et $h_{g}>h_{d}$ et on veut montrer 5.114.

La première inégalité est équivalente à montrer que

$$
g h_{g}>\frac{h_{d}^{2}\left(u_{g}-u_{d}\right)^{2}}{\left(h_{g}-h_{d}\right)^{2}}=\frac{h_{d}^{2}}{\left(h_{g}-h_{d}\right)^{2}} S^{2}=\frac{h_{d}^{2}}{\left(h_{g}-h_{d}\right)^{2}} \frac{g\left(h_{g}-h_{d}\right)\left(h_{g}^{2}-h_{d}^{2}\right)}{2 h_{g} h_{d}}=g \frac{h_{d}}{h_{g}} \frac{h_{g}+h_{d}}{2} .
$$

Ceci est vrai car $h_{g}>h_{d}$.

La deuxième inégalité est équivalente à montrer que

$$
g h_{d}<\frac{h_{g}^{2}\left(u_{g}-u_{d}\right)^{2}}{\left(h_{g}-h_{d}\right)^{2}}=\frac{h_{g}^{2}}{\left(h_{g}-h_{d}\right)^{2}} S^{2}=\frac{h_{g}^{2}}{\left(h_{g}-h_{d}\right)^{2}} \frac{g\left(h_{g}-h_{d}\right)\left(h_{g}^{2}-h_{d}^{2}\right)}{2 h_{g} h_{d}}=g \frac{h_{g}}{h_{d}} \frac{h_{g}+h_{d}}{2} .
$$

Ceci est vrai car $h_{d}<h_{g}$.

ii. La fonction $x \mapsto(1-1 / x)\left(x^{2}-1\right)$ est strictement croissante sur $[1,+\infty[$ (comme produit de fonctions strictement croissantes positives); la fonction $x \mapsto \sqrt{x}$ étant strictement croissante sur $R_{+}$, on en déduit que que $\varphi$ est strictement croissante.

Soient $u_{g} \in \mathbb{R}, h_{g}, h_{d} \in \mathbb{R}_{+}^{\star}$ avec $h_{d}>h_{g}$. Pour que $U$ soit un 1-choc, il faut et il suffit que $u_{d}=u_{g}-S$ (et $\sigma=[h u] /[h]$ ), c'est-à-dire

$$
u_{d}=u_{g}-\sqrt{\frac{g\left(h_{g}-h_{d}\right)\left(h_{g}^{2}-h_{d}^{2}\right)}{2 h_{g} h_{d}}}=u_{g}-\sqrt{\frac{g h_{g}}{2}} \varphi\left(\frac{h_{d}}{h_{g}}\right) .
$$

Bien sûr le raisonnement est complètement semblable pour un 2-choc. Soient $u_{g} \in \mathbb{R}, h_{g}, h_{d} \in \mathbb{R}_{+}^{\star}$ avec $h_{d}<h_{g}$. Pour que $U$ soit un 2-choc, il faut et il suffit que $u_{d}=u_{g}-S$ (et $\sigma=[h u] /[h]$ ), c'est-à-dire

$$
u_{d}=u_{g}-\sqrt{\frac{g\left(h_{g}-h_{d}\right)\left(h_{g}^{2}-h_{d}^{2}\right)}{2 h_{g} h_{d}}}=u_{g}-\sqrt{\frac{g h_{d}}{2}} \varphi\left(\frac{h_{g}}{h_{d}}\right) .
$$

(b) (Détente-choc) On note $U$ la solution recherchée. Dans la zone $D_{1}=\left\{x \leq\left(u_{g}-c_{g}\right) t\right\}$, on a $U=U_{g}$. La zone $D_{2}=\left\{\left(u_{g}-c_{g}\right) t<x<\left(u_{\star}-c_{\star}\right) t\right\}$ correspond à la 1-détente, la solution peut être calculée comme cela été fait dans la question 8 . L'invariance des 1-invariants de Riemann dans cette zone donne $u_{g}+2 c_{g}=u_{\star}+2 c_{\star}$. 
Dans la zone $D_{3}=\left\{\left(u_{\star}-c_{\star}\right) t \leq x<\sigma t\right\}$, la solution est $U=U_{\star}$. Dans la zone $D_{4}=\{x>\sigma t\}$, la solution est $U=U_{d}$ et la question 9 a montre que la condition nécessaire et suivante pour qu'il s'agisse bien d'un 2-choc est que $h_{\star}>h_{d}, u_{d}=u_{\star}-\sqrt{\frac{g h_{d}}{2}} \varphi\left(\frac{h_{\star}}{h_{d}}\right)$ et $\sigma=\left(h_{d} u_{d}-h_{\star} u_{\star}\right) /\left(h_{d}-h_{\star}\right)$.

En résumé, la construction d'une solution formée d'une 1-détente et d'un 2-choc reliés par un état intermédiaire $\left(h_{\star}, u_{\star}\right)$, est possible si et seulement si

$$
\begin{aligned}
& u_{g}-c_{g}<u_{\star}-c_{\star}, \\
& u_{g}+2 c_{g}=u_{\star}+2 c_{\star}, \\
& h_{\star}>h_{d}, \\
& u_{\star}=u_{d}+\sqrt{\frac{g h_{d}}{2}} \varphi\left(\frac{h_{\star}}{h_{d}}\right), \\
& u_{\star}-c_{\star}<\sigma=\frac{h_{d} u_{d}-h_{\star} u_{\star}}{h_{d}-h_{\star}} .
\end{aligned}
$$

Pour $h \in\left[h_{d}, h_{g}\right]$, on pose $F(h)=u_{d}+\sqrt{\frac{g h_{d}}{2}} \varphi\left(\frac{h}{h_{d}}\right)+2 \sqrt{g h}$. La fonction $F$ est continue, strictement croissante, $F\left(h_{d}\right)=u_{d}+2 c_{d}$ et $F\left(h_{g}\right)=u_{d}+S+2 c_{g}>u_{g}+2 c_{g}$. Comme $u_{g}+2 c_{g}>u_{d}+2 c_{d}$ (car $\left.2\left(c_{g}-c_{d}\right)=2\left|c_{g}-c_{d}\right|>u_{d}-u_{g}\right)$, il existe donc un (unique) $\left.h_{\star} \in\right] h_{d}, h_{g}\left[\right.$ tel que $F\left(h_{\star}\right)=u_{g}+2 c_{g}$. On pose alors $u_{\star}=u_{d}+\sqrt{\frac{g h_{d}}{2}} \varphi\left(\frac{h_{\star}}{h_{d}}\right)$ et les équations (5.116), 5.117) et 5.118) sont bien vérifiées. Compte tenu de 5.116, l'inégalité 5.115) est équivalente à $c_{\star}<c_{g}$, ce qui est bien vrai car $h_{\star}<h_{g}$.

Il reste à montrer (5.119). Comme

$$
\sigma=\frac{h_{\star} u_{\star}-h_{d} u_{d}}{h_{\star}-h_{d}}=u_{\star}+\frac{h_{d}}{h_{\star}-h_{d}}\left(u_{\star}-u_{d}\right),
$$

la condition 5.119) est vérifiée car $c_{\star}>0, h_{\star}>h_{d}$ et $u_{\star}>u_{d}$ (par 5.118).

(c) (Choc-détente) Le raisonnement est ici très voisin du précédent. On note $U$ la solution recherchée et $\left(h_{\star}, u_{\star}\right)$ l'état intermédiaire.

Dans la zone $D_{1}=\{x \leq \sigma t\}$, on a $U=U_{g}$ et la question 9a montre que la condition nécessaire et suivante pour qu'il s'agisse bien d'un 1 -choc est que $h_{g}<h_{\star}, u_{\star}=u_{g}-\sqrt{\frac{g h_{g}}{2}} \varphi\left(\frac{h_{\star}}{h_{g}}\right)$ et $\sigma=\left(h_{g} u_{g}-h_{\star} u_{\star}\right) /\left(h_{g}-h_{\star}\right)$. Dans la zone $D_{2}=\left\{\sigma t<x<\left(u_{\star}+c_{\star}\right) t\right\}$, la solution est $U=U_{\star}$. La zone $D_{3}=\left\{\left(u_{\star}+c_{\star}\right) t<x<\right.$ $\left.\left(u_{d}+c_{d}\right) t\right\}$ correspond à la 2-détente, la solution peut être calculée comme cela été fait dans la question 8 (zone $D_{4}$ de la question 8). L'invariance des 2-invariants de Riemann dans cette zone donne $u_{d}-2 c_{d}=u_{\star}-2 c_{\star}$. Dans la zone $D_{4}=\left\{\left(u_{d}+c_{d}\right) t \leq x\right\}$, la solution est $U=U_{d}$.

En résumé, la construction d'une solution formée d'une 1-choc et d'un 2-détente reliés par un état intermédiaire noté $\left(h_{\star}, u_{\star}\right)$, est possible si et seulement si

$$
\begin{aligned}
& h_{\star}>h_{g}, \\
& u_{\star}=u_{g}-\sqrt{\frac{g h_{g}}{2}} \varphi\left(\frac{h_{\star}}{h_{g}}\right), \\
& \sigma=\frac{h_{g} u_{g}-h_{\star} u_{\star}}{h_{g}-h_{\star}}<u_{\star}+c_{\star}, \\
& u_{\star}+c_{\star}<u_{d}+c_{d}, \\
& u_{d}-2 c_{d}=u_{\star}-2 c_{\star},
\end{aligned}
$$


Pour $h \in\left[h_{g}, h_{d}\right]$, on pose $F(h)=u_{g}-\sqrt{\frac{g h_{g}}{2}} \varphi\left(\frac{h}{h_{g}}\right)-2 \sqrt{g h}$. La fonction $F$ est continue, strictement décroissante, $F\left(h_{g}\right)=u_{g}-2 c_{g}$ et $F\left(h_{d}\right)=u_{g}-S-2 c_{d}<u_{d}-2 c_{d}$. Comme $u_{d}-2 c_{d}<u_{g}-2 c_{g}$ (car $2\left(c_{d}-c_{g}\right)=2\left|c_{g}-c_{d}\right|>u_{d}-u_{g}$ ), il existe un (unique) $\left.h_{\star} \in\right] h_{g}, h_{d}\left[\right.$ tel que $F\left(h_{\star}\right)=u_{d}-2 c_{d}$. On pose alors $u_{\star}=u_{g}-\sqrt{\frac{g h_{g}}{2}} \varphi\left(\frac{h_{\star}}{h_{g}}\right)$ et les équations 5.124, 5.120 et 5.121) sont bien vérifiées. Compte tenu de 5.124), l'inégalité (5.123) est équivalente à $c_{\star}<c_{d}$, ce qui est bien vrai car $h_{\star}<h_{d}$.

Il reste à montrer 5.122). Comme

$$
\sigma=\frac{h_{\star} u_{\star}-h_{g} u_{g}}{h_{\star}-h_{g}}=u_{\star}+\frac{h_{g}}{h_{\star}-h_{g}}\left(u_{\star}-u_{g}\right),
$$

la condition 5.122) est vérifiée car $c_{\star}>0, h_{\star}>h_{g}$ et $u_{\star}<u_{g}$ (par 5.121) ).

(d) (Choc-choc) Pour que la solution $U$ soit formée de deux chocs séparés par un état intermédiaire noté $\left(h_{\star}, u_{\star}\right)$, il faut et il suffit, d'après la question $9 \mathrm{a}$, d'avoir les conditions suivantes

$$
\begin{aligned}
& u_{\star}=u_{g}-\sqrt{\frac{g h_{g}}{2}} \varphi\left(\frac{h_{\star}}{h_{g}}\right), h_{g}<h_{\star}, \\
& u_{\star}=u_{d}+\sqrt{\frac{g h_{d}}{2}} \varphi\left(\frac{h_{\star}}{h_{d}}\right), h_{d}<h_{\star}, \\
& \frac{h_{g} u_{g}-h_{\star} u_{\star}}{h_{g}-h_{\star}}<\frac{h_{d} u_{d}-h_{\star} u_{\star}}{h_{d}-h_{\star}} .
\end{aligned}
$$

La condition (5.127) indiquant que la vitesse du 1-choc est inférieure à vitesse du 2-choc.

On note $h_{m}=\min \left(h_{d}, h_{g}\right)$. Pour $h \geq h_{m}$, on pose $G(h)=\sqrt{\frac{g h_{d}}{2}} \varphi\left(\frac{h}{h_{d}}\right)+\sqrt{\frac{g h_{g}}{2}} \varphi\left(\frac{h}{h_{g}}\right)$. Les conditions (5.125)-5.126 donnent

$$
u_{g}-u_{d}=G\left(h_{\star}\right) .
$$

La fonction $G$ est continue et strictement croissante sur l'intervalle $\left[h_{m},+\infty\left[\right.\right.$. Comme $G\left(h_{m}\right)=S, u_{g}-$ $u_{d}>S$ et que $\lim _{h \rightarrow \infty} G(h)=+\infty$, il existe un (unique) $\left.h_{\star} \in\right] h_{m},+\infty\left[\right.$ tel que $G\left(h_{\star}\right)=u_{g}-u_{d}$. On définit alors $u_{\star}$ avec les conditions [5.125]-5.126) (qui sont identiques avec ce choix de $h_{\star}$ ). Il reste à vérifier 5.127. Ceci découle de $h_{\star}>\max \left(h_{g}, h_{d}\right), u_{g}-u_{\star}>0$ et $u_{d}-u_{\star}<0$ car

$$
\frac{h_{g} u_{g}-h_{\star} u_{\star}}{h_{g}-h_{\star}}=u_{\star}+h_{g} \frac{u_{g}-u_{\star}}{h_{g}-h_{\star}}<u_{\star}+h_{d} \frac{u_{d}-u_{\star}}{h_{d}-h_{\star}}=\frac{h_{d} u_{d}-h_{\star} u_{\star}}{h_{d}-h_{\star}} .
$$

NB. Si $u_{g}-u_{d}=S$, on trouve une solution de 5.82, 5.84-5.85 contenant seulement un 1-choc dans le cas $h_{g}<h_{d}$ et contenant seulement un 2-choc dans le cas $h_{g}>h_{d}$.

10. (Problème de Riemann linéarisé) Les valeurs propres de la matrice $B(\bar{V})$ sont $\lambda_{1}=\bar{u}-\bar{c}$ et $\lambda_{2}=\bar{u}+\bar{c}$. Une base de $\mathbb{R}^{2}$ de vecteurs propres associés est $\varphi_{1}=\left[\begin{array}{c}1 \\ -1\end{array}\right], \varphi_{2}=\left[\begin{array}{l}1 \\ 1\end{array}\right]$. La décomposition du vecteur $V=\left[\begin{array}{c}u \\ 2 c\end{array}\right]$ dans la base $\left\{\varphi_{1}, \varphi_{2}\right\}$ est

$$
V=\frac{u-2 c}{2} \varphi_{1}+\frac{u+2 c}{2} \varphi_{2}
$$

La solution de ce nouveau problème de Riemann est donc $(u, c)=\left(u_{g}, c_{g}\right)$ pour $x<(\bar{u}-\bar{c}) t,(u, c)=\left(u_{d}, c_{d}\right)$ pour $x>(\bar{u}+\bar{c}) t$ et $(u, c)=\left(u_{\star}, c_{\star}\right)$ pour $(\bar{u}-\bar{c}) t<x<(\bar{u}+\bar{c}) t$ avec

$$
\begin{aligned}
& u_{\star}+2 c_{\star}=u_{g}+2 c_{g}, \\
& u_{\star}-2 c_{\star}=u_{d}-2 c_{d},
\end{aligned}
$$

c'est-à-dire $u_{\star}=\frac{u_{g}+u_{d}}{2}+\left(c_{g}-c_{d}\right), c_{\star}=\frac{u_{g}-u_{d}}{4}+\frac{c_{g}+c_{d}}{2}$. 


\section{Exercice 5.16 (Entropie pour les équations de Saint-Venant avec gradient de fond)}

1. (Entropie)

Comme $\eta(U)=\frac{1}{2} h u^{2}+p+g h z=\frac{q^{2}}{2 h}+g \frac{h^{2}}{2}+g h z$, on a

$$
\nabla \eta(U)=\left[\begin{array}{c}
-\frac{u^{2}}{2}+g h+g z \\
u
\end{array}\right] .
$$

On multiplie (5.87) par $-\frac{u^{2}}{2}+g h+g z$ et 5.88) par $u$. On obtient

$$
-\left(\frac{u^{2}}{2}\right) \partial_{t} h+\partial_{t}\left(\frac{g h^{2}}{2}\right)+g z \partial_{t} h-\left(\frac{u^{3}}{2}\right) \partial_{x} h-h \frac{u^{2}}{2} \partial_{x} u+g h^{2} \partial_{x} u+u \partial_{x}\left(\frac{g h^{2}}{2}\right)+g z \partial_{x}(h u)=0
$$

et

$$
h \partial_{t}\left(\frac{u^{2}}{2}\right)+u^{2} \partial_{t} h+u^{3} \partial_{x} h+u^{2} h \partial_{x} u+h u^{2} \partial_{x} u+u \partial_{x}\left(\frac{g h^{2}}{2}\right)+g h u z^{\prime}=0 .
$$

En additionnant ces deux équations, on obtient

$$
\partial_{t} \eta(U)+\partial_{x} \phi(U)=0
$$

$\operatorname{avec} \Phi(U)=\frac{1}{2} h u^{3}+g u h^{2}+g h u z=u\left(\frac{1}{2} h u^{2}+2 p+g h z\right)$.

La démonstration du fait que $\eta$ est une fonction convexe de $D$ dans $\mathbb{R}$ est identique à celle de l'exercice 5.15 . question 5, car la fonction $\eta$ définie ici est la même que celle de l'exercice 5.15 au terme $g h z$ près, qui, étant linéaire, est lui même convexe.

2. Les termes de diffusion ajoutés sont les mêmes que ceux de la question 6 de l'exercice 5.15. Cette question se fait donc de manière identique à la question 6 de l'exercice 5.15 .

\section{Exercice 5.17(Solutions stationnaires régulières pour les équations de Saint-Venant)}

1. Soit $(h, u)$ un couple de fonctions de classe $C^{1}$ de $\mathbb{R}$ dans $\mathbb{R}_{+}^{\star} \times \mathbb{R}$. Le couple $(h, u)$ est une solution stationnaire régulière si et seulement si $q$ est une fonction constante et

$$
u(x) q^{\prime}(x)+h(x) u(x) u^{\prime}(x)+g h(x) h^{\prime}(x)+g h(x) z^{\prime}(x)=0, \text { pour tout } x \in \mathbb{R} .
$$

Comme $q$ est une fonction constante et $h(x)>0$ pour tout $x$, cette dernière équation est équivalente à $\psi^{\prime}(x)=0$ pour tout $x$, c'est-à-dire à $\psi$ constante.

2. Soit $(h, u)$ une solution stationnaire régulière avec $\alpha=0$. On a donc $u(x)=0$ pour tout $x(\operatorname{car} h(x)>0)$ et donc $g h(x)=\beta-g z(x)$ pour tout $x$. Ceci n'est possible que si $\beta>g z_{m}$ et la solution stationnaire correspondante est alors donnée par $u(x)=0$ et $h(x)=\beta / g-z(x)$ pour tout $x$.

N.B. Si on autorise $h$ à prendre la valeur 0 et à être éventuellement une fonction non régulière, on peut construire, dans le cas ou $z$ est une fonction non constante, une infinité d'autres solutions stationnaires avec $u=0$.

3. Soit $(h, u)$ une solution stationnaire régulière associée au couple $(\alpha, \beta)$. On a donc $u(x)=\alpha / h(x)$ pour tout $x$ (on rappelle que $h(x)>0$ ). Comme $\psi(x)=\beta$ pour tout $x$, on a donc $\alpha^{2} /\left(2 h^{2}\right)+g h+g z=\beta$, c'est-à-dire

$$
g h^{3}(x)+h^{2}(x)(g z(x)-\beta)+\alpha^{2} / 2=0, \text { pour tout } x \in \mathbb{R} .
$$


Comme $h(x)>0$, l'équation (5.128) est impossible si $\beta \leq g z(x)$. Une première condition nécessaire (pour avoir une solution stationnaire régulière) est donc $\beta>g z_{m}$ (ce qui donne $\beta>g z(x)$ pour tout $x$ ).

Pour $g a<\beta$, on définit le polynôme $P_{a}$ par $P_{a}(y)=g y^{3}+y^{2}(g a-\beta)+\alpha^{2} / 2$ de sorte que (5.128) sécrit $P_{z(x)}(h(x))=0$.

Comme $P_{a}^{\prime}(y)=3 g y^{2}+2 y(g a-\beta)$, le polynôme $P_{a}$ a un maximum local (strictement positif) en 0 et un minimum local au point $y_{a}$ donné par $y_{a}=(2 /(3 g))(\beta-g a)$. La valeur de ce minimum local est

$$
P_{a}\left(y_{a}\right)=g\left(\frac{2}{3 g}\right)^{3}(\beta-g a)^{3}-\left(\frac{2}{3 g}\right)^{2}(\beta-g a)^{3}+\alpha^{2} / 2=-\left(\frac{4}{27 g^{2}}\right)(\beta-g a)^{3}+\alpha^{2} / 2 .
$$

On a donc $P_{a}\left(y_{a}\right)>0$ si $0<(\beta-g a)<(3 / 2)(\alpha g)^{2 / 3}$ et $P_{a}\left(y_{a}\right)<0$ si $(\beta-g a)>(3 / 2)(\alpha g)^{2 / 3}$. Ceci explique l'introduction de $\beta_{m}=g z_{m}+(3 / 2)(\alpha g)^{2 / 3}$.

(a) On suppose $\beta<\beta_{m}$. Dans ce cas, il existe des points $x$ de $\mathbb{R}$ pour lesquels $\beta-g z(x)<(3 / 2)(\alpha g)^{2 / 3}$. Pour tous ces points, $P_{z(x)}(y)>0$ pour $y>0$. On ne peut donc pas voir $P_{z(x)}(h(x))=0$. Ceci prouve qu'il n'y a pas de solution stationnaire régulière associée au couple $(\alpha, \beta)$.

(b) On suppose $\beta>\beta_{m}$. Dans ce cas, pour tout $a \leq z_{m}$, l'équation $P_{a}(y)=0$ a deux solutions strictement positives notées $\varphi_{1}(a)$ et $\varphi_{2}(a)$ avec $\varphi_{1}(a)<y_{a}=(2 /(3 g))(\beta-g a)<\varphi_{2}(a)$. Pour tout $x \in \mathbb{R}$, on a $h(x) \in\left\{\varphi_{1}(z(x)), \varphi_{2}(z(x))\right\}$. Comme $h$ est continue, on doit donc avoir $h(x)=\varphi_{1}(z(x))$ pour tout $x \in \mathbb{R}$ ou $h(x)=\varphi_{2}(z(x))$ pour tout $x \in \mathbb{R}$.

Ceci suggère deux solutions stationnaires $\left(h_{1}, u_{1}\right)$ et $\left(h_{2}, u_{2}\right)$ avec, pour $i=1,2, h_{i}(x)=\varphi_{i}(z(x))$ et $u_{i}(x)=\alpha / h_{i}(x)$. Pour montrer que ces deux solutions sont bien des solutions stationnaires régulières, il reste à vérifier que $h_{1}$ et $h_{2}$ sont des fonctions de classe $C^{1}$. Ceci est une conséquence du fait que $\varphi_{1}$ et $\varphi_{2}$ sont des fonctions de classes $C^{1}$ au voisinage du point $a$ pour $a$ tel que $(\beta-g a)>(3 / 2)(\alpha g)^{2 / 3}$. En effet, on pose $F(a, y)=P_{a}(y)$ et on remarque que $\partial_{y} F(a, y)=3 g y^{2}+2 y(g a-\beta) \neq 0$ pour $y=\varphi_{i}(a)(i=1$ ou $\left.2,(\beta-g a)>(3 / 2)(\alpha g)^{2 / 3}\right)$. Le théorème des fonctions implicites appliqué à l'équation $F(a, y)=0$ au voisinage des points $\left(a, \varphi_{i}(a)\right)$ donne alors le caractère $C^{1}$ des fonctions $\varphi_{i}$.

Comme on a supposé que $z$ était de classe $C^{1}$, on en déduit bien que les fonctions $h_{i}$ sont de classe $C^{1}$ (et donc aussi les fonctions $u_{i}$ ).

Enfin, comme on a raisonné par condition nécessaire, il y a bien seulement deux solutions stationnaires régulières. La figure 5.9 donne un exemple de solutions stationnaires régulières.

4. Comme dans la question 3 et avec les mêmes notations, pour tout $a<z_{m}$, l'équation $P_{a}(y)$ a deux solutions strictement positives notées $\varphi_{1}(a)$ et $\varphi_{2}(a)$ avec $\varphi_{1}(a)<y_{a}=(2 /(3 g))(\beta-g a)<\varphi_{2}(a)$. Mais pour $a=z_{m}$, on a $\varphi_{1}(a)=y_{a}=(2 /(3 g))(\beta-g a)=\varphi_{2}(a)$. Les deux solutions données dans la question 3 semblent exister encore ici et sont d'ailleurs confondues si $z$ est une fonction constante. la seule question restante est sur la régularité des fonctions $h_{i}$.

(a) $\mathrm{Si} z$ est une fonction constante (donc $\left.z=z_{m}\right)$, il y a une solution unique qui est $h(x)=y_{z_{m}}=(2 /(3 g))\left(\beta_{m}-\right.$ $g z_{m}$ ) pour tout $x$.

(b) $\mathrm{Si} z(x) \neq z_{m}$ pour tout $x$, il y a exactement deux solutions stationnaires régulières associées au couple $(\alpha, \beta)$. Ce sont celles calculées pour le cas $\beta>\beta_{m}$ (et la preuve est identique).

Si $z$ est une fonction non constante et qu'il existe $x$ tel que $z(x)=z_{m}$ (et toujours $\beta=\beta_{m}$ ) la situation est un peu plus complexe. Le problème est dû ici au fait que les fonctions $\varphi_{i}$ ne sont pas dérivables au point $z_{m}$. Plus précisément $\lim _{z \rightarrow z_{m}, z<z_{m}}\left|\varphi_{i}^{\prime}(z)\right|=+\infty$. Toutefois, si le maximum de $z$ est atteint en un point unique noté $x_{m}$ et que $z^{\prime \prime}\left(x_{m}\right)<0$, on peut montrer qu'il y a (exactement) deux solutions stationnaires régulières associées au couple $(\alpha, \beta)$, obtenues en prenant $i$ différent selon que $x<x_{m}$ et $x>x_{m}$. Ce cas n'est pas détaillé ici. 


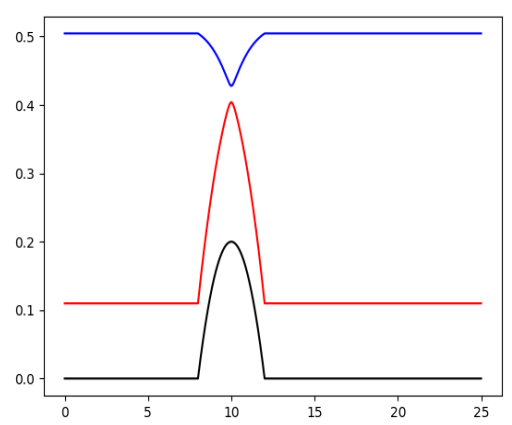

FIGURE 5.9 - Solutions stationnaires pour $\beta=\beta_{m}+0.001$ et $\alpha=0.1$. Solution surcritique $(u>c)$ en rouge et souscritique $(u<c)$ en bleu. Le fond est en noir

5. La première partie de cette question a été résolue à la question $3 \mathrm{~b}$ Avec les notations de la question $3 \mathrm{~b}$, on a bien, pour $i=1,2, h_{i}(x)=\varphi_{i}(z(x))$ pour tout $x \in \mathbb{R}$ et les fonctions $\varphi_{i}$ sont régulières. Enfin, on a bien $h_{1}<h_{2}$.

Pour la seconde partie de la question on remarque que $h_{i}(x)+z(x)=\psi_{i}(z(x))$ avec $\psi_{i}(a)=\varphi_{i}(a)+a$ (noter que $\psi_{i}(a)$ est définie, comme $\varphi_{i}(a)$, pour tout $a$ tel que $\left.g a<\beta-(3 / 2)(\alpha g)^{2 / 3}\right)$.

Comme $h_{i}^{\prime}(x)+z^{\prime}(x)=\psi_{i}^{\prime}(z(x)) z^{\prime}(x)$, la seconde partie de la question est une conséquence du fait que $\psi_{2}^{\prime}(a)<0$ et $\psi_{1}^{\prime}(a)>0$ pour tout $a \leq z_{m}$. Pour montrer ce fait, soit $a \leq z_{m}$. On a $\left.g a<\beta-(3 / 2)(\alpha g)^{2 / 3}\right\}$ et

$$
g \varphi_{i}^{3}(a)+\varphi_{i}^{2}(a)(g a-\beta)+\alpha^{2} / 2=0 .
$$

Cette première égalité donne en particulier $g \varphi_{i}(a)+(g a-\beta)<0$. D'autre part, en dérivant cette équation par rapport à $a$, on obtient

$$
\varphi_{i}^{\prime}(a) \varphi_{i}(a)\left(3 g \varphi_{i}(a)+2(g a-\beta)\right)=-g \varphi_{i}^{2}(a) .
$$

Pour $i=2$, on utilise $g \varphi_{2}(a)+g a-\beta<0$ et $y_{a}=(2 /(3 g))(\beta-g a)<g \varphi_{2}(a)$, cela donne $0<\left(3 g \varphi_{2}(a)+\right.$ $2(g a-\beta))<g \varphi_{2}(a)$. On en déduit, avec 5.129), $\varphi_{2}^{\prime}(a)<-1$ et donc $\psi_{2}^{\prime}(a)<0$.

Pour $i=1$, on utilise $y_{a}=(2 /(3 g))(\beta-g a)>\varphi_{1}(a)$, cela donne $\left(3 g \varphi_{1}(a)+2(g a-\beta)\right)<0$. On en déduit, avec 5.129, $\varphi_{1}^{\prime}(a)>0$ et donc $\psi_{1}^{\prime}(a)>1>0$.

6. On reprend les notations des corrigés des questions précédentes. Pour $i=1$ ou $2, u_{i}^{2}=\alpha^{2} / h_{i}^{2}$, ce qui donne avec 5.128

$$
2 g h_{i}(x)+2(g z(x)-\beta)+u_{i}^{2}(x)=0, \text { pour tout } x \in \mathbb{R},
$$

et donc

$$
3 g h_{i}(x)+2(g z(x)-\beta)=g h_{i}(x)-u_{i}^{2}(x), \text { pour tout } x \in \mathbb{R} .
$$

On rappelle que le choix de $\varphi_{1}$ et $\varphi_{2}$ est tel que $\varphi_{1}(a)<(2 /(3 g))(\beta-g a)<\varphi_{2}(a)$ pour tout $a \leq z_{m}$.

Pour $i=2$, ceci donne, avec $a=z(x), u_{2}^{2}(x)<g h_{2}(x)$ et donc $0<u_{2}(x)<\sqrt{g h_{2}(x)}$ pour tout $x \in \mathbb{R}$. L'écoulement est ici subsonique (on rappelle que $\sqrt{g h}$ est, pour ce système, la "vitesse du son").

Pour $i=1$, ceci donne, avec $a=z(x), u_{1}^{2}(x)>g h_{1}(x)$ et donc $u_{1}(x)>\sqrt{g h_{1}(x)}$ pour tout $x \in \mathbb{R}$. L'écoulement est ici supersonique. 


\section{Exercice 5.19 (Equation linéaire avec terme source singulier)}

1. Soient $u, v$ deux solutions faibles de (5.96)-5.97). On pose $w=u-v$. La fonction $w$ est alors solution faible de (5.96)-5.97) avec $b=0$ et $u_{0}=0$. L'exercice 5.3 donne alors $w=0$ p.p.. Ceci prouve l'unicié de la solution faible 5.96-5.97.

2. On suppose $c>a$ (le cas $c<a$ est similaire). On pose $D_{1}=\{(x, t), t>0, x<a t\}, D_{2}=\{(x, t), t>0$, at $<x<c t\}$ et $D_{3}=\{(x, t), t>0, x>c t\}$. On va chercher la solution faible $u$ de (5.96)-(5.97) sous la forme :

$$
u=u_{g} \text { dans } D_{1}, u=\bar{u} \text { dans } D_{2}, u=u_{d} \text { dans } D_{3},
$$

avec $\bar{u} \in \mathbb{R}$. On cherche donc $\bar{u}$ pour que $u$ défini par 5.131) vérifie (5.98).

Soit $\varphi \in C_{c}^{\infty}\left(\mathbb{R} \times \mathbb{R}_{+}, \mathbb{R}\right)$. On pose

$$
E(\varphi)=\int_{\mathbb{R} \times \mathbb{R}_{+}} u\left(\partial_{t} \varphi+a \partial_{x} \varphi\right) d(x, t)+\int_{\mathbb{R}} u_{0}(x) \varphi(x, 0) \mathrm{d} x+b \int_{0}^{+\infty} \varphi(c t, t) d t .
$$

On cherche donc $\bar{u}$ (indépendant de $\varphi$ ) pour que $E(\varphi)=0$.

On pose $v=\left[\begin{array}{l}a \\ 1\end{array}\right]$. On note $n_{i}$ le vecteur normal extérieur au domaine $D_{i}$, div l'opérateur divergence dans le plan $\mathbb{R} \times \mathbb{R}_{+}$et $y$ le point $(x, t)$; on a alors

$$
\int_{\mathbb{R} \times \mathbb{R}_{+}} u\left(\partial_{t} \varphi+a \partial_{x} \varphi\right) d(x, t)=\int_{\mathbb{R} \times \mathbb{R}_{+}} u \operatorname{div}(v \varphi) d(x, t)=\sum_{i=1}^{3} \int_{\partial D_{i}} u(y) \varphi(y) v \cdot n_{i}(y) d \gamma(y),
$$

où $\gamma$ désigne la mesure de Lebesgue 1-dimensionnelle sur $\partial D_{i}$ (qui est le bord de $D_{i}$ ) et la valeur de $u$ dans l'intégrale sur $\partial D_{i}$ est prise du coté de $D_{i}$. En posant $\Gamma_{a}=\left\{(a t, t), t \in \mathbb{R}_{+}\right\}$, on en déduit que

$$
\begin{array}{r}
\int_{\mathbb{R} \times \mathbb{R}_{+}} u\left(\partial_{t} \varphi+a \partial_{x} \varphi\right) d(x, t)+\int_{\mathbb{R}_{0}} u_{0}(x) \varphi(x, 0) \mathrm{d} x=\int_{\Gamma_{a}}\left(u_{g}-\bar{u}\right) \varphi(y) v \cdot n_{1}(y) d \gamma(y) \\
+\int_{\Gamma_{c}}\left(\bar{u}-u_{d}\right) \varphi(y) v \cdot n_{2}(y) d \gamma(y)
\end{array}
$$

On remarque maintenant que $n_{1}=\left(1 / \sqrt{1+a^{2}}\right)\left[\begin{array}{c}1 \\ -a\end{array}\right]$ et $n_{2}=\left(1 / \sqrt{1+c^{2}}\right)\left[\begin{array}{c}1 \\ -c\end{array}\right]$. On a donc

$$
E(\varphi)=\int_{\Gamma_{c}}\left(\bar{u}-u_{d}\right) \frac{1}{\sqrt{1+c^{2}}} \varphi(c t, t)(a-c) d \gamma(y)+b \int_{0}^{+\infty} \varphi(c t, t) d t .
$$

En paramétrant $\Gamma_{c}$ par $t$ (ce qui correspond à un changement de variable), l'élément d'intégration $d \gamma(y)$ devient $\sqrt{1+c^{2}} d t$. On obtient donc

$$
E(\varphi)=\int_{0}^{+\infty}\left(\bar{u}-u_{d}\right)(a-c) \varphi(c t, t) d t+b \int_{0}^{+\infty} \varphi(c t, t) d t .
$$

On en déduit que $E(\varphi)=0$ en prenant $\bar{u}$ tel que $\bar{u}=u_{d}+\frac{b}{c-a}$.

3. Si $c=a$, on a, avec les notations de la question précédente, $D_{2}=\emptyset$. Le raisonnement d'unicité de l'exercice 5.3 permet alors de montrer que $u=u_{g}$ sur $D_{1}$ et $u=u_{d}$ sur $D_{3}$. Le raisonnement de la question précédente donne alors, pour tout $\varphi \in C_{c}^{\infty}\left(\mathbb{R} \times \mathbb{R}_{+}, \mathbb{R}\right)$,

$$
E(\varphi)=b \int_{0}^{+\infty} \varphi(c t, t) d t
$$


Si $b \neq 0$, on en déduit qu'il existe $\varphi \in C_{c}^{\infty}\left(\mathbb{R} \times \mathbb{R}_{+}, \mathbb{R}\right)$ tel que $E(\varphi) \neq 0$. Le problème [5.96)-[5.97] n'a donc pas de solution faible. 


\section{Bibliographie}

[1] R. A. Adams and J. J. F. Fournier. Sobolev spaces, volume 140 of Pure and Applied Mathematics (Amsterdam). Elsevier/Academic Press, Amsterdam, second edition, 2003.

[2] H. W. Alt and S. Luckhaus. Quasilinear elliptic-parabolic differential equations. Math. Z., 183(3) :311-341, 1983.

[3] M. Artola. Sur une classe de problèmes paraboliques quasi-linéaires. Boll. Un. Mat. Ital. B (6), 5(1) :51-70, 1986.

[4] J.-P. Aubin. Analyse mathematique-un theoreme de compacite. COMPTES RENDUS HEBDOMADAIRES DES SEANCES DE L ACADEMIE DES SCIENCES, 256(24) :5042-5044, 1963.

[5] C. Bardos, A. LeRoux, and J. Nédélec. First order quasilinear equations with boundary conditions. Comm. Partial Differential Equations, 9 :1017-1034, 1979.

[6] P. Bénilan, L. Boccardo, T. Gallouët, R. Gariepy, M. Pierre, and J. L. Vázquez. An $L^{1}$-theory of existence and uniqueness of solutions of nonlinear elliptic equations. Ann. Scuola Norm. Sup. Pisa Cl. Sci. (4), 22(2) :241273, 1995.

[7] S. Benzoni-Gavage and D. Serre. Multidimensional hyperbolic partial differential equations. Oxford Mathematical Monographs. The Clarendon Press, Oxford University Press, Oxford, 2007. First-order systems and applications.

[8] H. Brezis. Functional analysis, Sobolev spaces and partial differential equations. Universitext. Springer, New York, 2011.

[9] L. E. J. Brouwer. Über Abbildung von Mannigfaltigkeiten. Math. Ann., 71(4) :598, 1912.

[10] G. A. Chechkin and A. Y. Goritsky. S.N. Kruzhkov's lectures on first-order quasilinear PDEs. In E. Emmrich and P. Wittbold, editors, De Gruyter Proceedings in Mathematics, Analytical and Numerical Aspects of Partial Differential Equations, pages pp. 1-68. De Gruyter, July 2009. traduit du russe par B. Andreianov.

[11] K. Deimling. Nonlinear functional analysis. Springer-Verlag, Berlin, 1985.

[12] J. Droniou. Quelques Résultats sur les Espaces de Sobolev. working paper or preprint, Apr. 2001.

[13] R. Eymard, T. Gallouët, and R. Herbin. Finite volume methods. In Handbook of numerical analysis, Vol. VII, Handb. Numer. Anal., VII, pages 713-1020. North-Holland, Amsterdam, 2000.

[14] R. Eymard, T. Gallouët, and R. Herbin. Existence and uniqueness of the entropy solution to a nonlinear hyperbolic equation. Chin. Ann. of Math., 16B(1) :1-14, 1995.

[15] T. Gallouët and R. Herbin. Mesure, intégration, probabilités. Ellipses, 2013.

[16] T. Gallouët, R. Herbin, and J.-C. Latché. Lax-wendroff consistency of finite volume schemes for systems of non linear conservation laws : extension to staggered schemes. SeMA, 2021. 
[17] P. Grisvard. Elliptic problems in nonsmooth domains, volume 69 of Classics in Applied Mathematics. Society for Industrial and Applied Mathematics (SIAM), Philadelphia, PA, 2011.

[18] O. Guibé, A. Mokrane, Y. Tahraoui, and G. Vallet. Lewy-Stampacchia's inequality for a pseudomonotone parabolic problem. Adv. Nonlinear Anal., 9(1) :591-612, 2020.

[19] P. D. Lax. 1. Hyperbolic Systems of Conservation Laws and the Mathematical Theory of Shock Waves, pages $1-48$.

[20] J. Leray. Sur le mouvement d'un liquide visqueux emplissant l'espace. Acta Math., 63(1) :193-248, 1934.

[21] J. Leray and L. Jacques-Louis. Quelques résultats de višik sur les problèmes elliptiques non linéaires par les méthodes de minty-browder. Bulletin de la S. M. F., 93 :97-107, 1965.

[22] J. Leray and J. Schauder. Topologie et équations fonctionnelles. Ann. Sci. École Norm. Sup. (3), 51 :45-78, 1934.

[23] N. G. Meyers. An $L^{p}$ e-estimate for the gradient of solutions of second order elliptic divergence equations. Ann. Scuola Norm. Sup. Pisa Cl. Sci. (3), 17 :189-206, 1963.

[24] F. Otto. Initial-boundary value problem for a scalar conservation law. C. R. Acad. Sci. Paris Sér. I Math., $8: 729-734,1996$.

[25] A. Prignet. Remarks on existence and uniqueness of solutions of elliptic problems with right-hand side measures. Rend. Mat. Appl. (7), 15(3):321-337, 1995.

[26] D. Serre. Systems of Conservation Laws 1 : Hyperbolicity, Entropies, Shock Waves. Cambridge University Press, 1999.

[27] J. Serrin. Pathological solutions of elliptic differential equations. Ann. Scuola Norm. Sup. Pisa Cl. Sci. (3), $18: 385-387,1964$.

[28] J. Simon. Compact sets in the space $L^{p}(0, T ; B)$. Ann. Mat. Pura Appl. (4), 146 :65-96, 1987.

[29] J. Smoller. Shock waves and reaction-diffusion equations., volume 258 of Grundlehren der Mathematischen Wissenschaften [Fundamental Principles of Mathematical Sciences]. Springer-Verlag, New York, 1994.

[30] G. Teschl. Topics in Linear and Nonlinear Functional Analysis, volume to appear of Graduate Studies in Mathematics. American Mathematical Society (AMS), Providence, Rhode Island, 2021,https ://www.mat.univie.ac.at/ gerald/ftp/book-fa/fa.pdf.

[31] J. Vovelle. Convergence of finite volume monotone schemes for scalar conservation laws on bounded domains. Numer. Math., 90(3) :563-596, 2002. 


\section{Index}

adjoint, 37

alternative de Fredholm, 87

Aubin-Simon, 163,167

autoadjoint, 37

autosimilaire, 223

Banach, 5

barotrope, 224, 226

base hibertienne, 185

base hilbertienne, 9, 37, 39, 48, 49, 126, 141, 182

bidual, 9

Brouwer, 82, 83

Buckley-Leverett, 234

Burgers, 203, 233,235

$\mathrm{BV}, 210,212$

Carathéodory, 85

Cauchy-Schwarz (inégalité de), 19

choc, 209,226

coercive, 34

compacité, 31

compacité faible, 10

compacité faible- $\star, 10$

compacité, 12, 82, 84

compacité, 12, 13, 29, 46,83

condition d'entropie de Lax, 230

condition de Lax, 223, 232

conditions limites, hyperbolique, 209

contact, 56

convection, 86

convergence faible, 10, 27, 97, 121

convergence faible- $\star, 10,130$

courbe caractéristique, 197

degré topologique, 82,84

densité, 10

dérivée au sens des distributions, 6

dérivée faible, 5,165

dérivée par transposition, 6 détente, 209. 226

diffusion, 86

Dirichlet, 36, 38, 40, 45, 57

discontinuité de contact, 209

donnée $L^{1}, 129$

dual algébrique, 6

dual topologique, 9

effet Landau, 237

éléments finis, 103

entropie, 241

Euler, 132, 220, 224

Faedo-Galerkin, 131, 141

Fourier, 37, 48, 49, 57, 125

gradient de fond, 240

Hahn-Banach, 9

harmonique, 80

Hausdorff, 12

Heaviside, 6

Helly, 212

Hilbert, 8

Hille-Yosida, 128

Hodge, 37

injection canonique, 38

intégration par parties, 12

invariant de Riemann, 226, 238

Kolmogorov, 12

Kruzkhov, 211

Landau, 237

Laplacien, 128

Lax-Milgram, 33, 35, 36, 55, 64, 129,130

Lebesgue, 5

Leray, 5

Leray-Lions, 96, 102, 121,123 
Leray-Schauder, 84

LES (Large Eddy Simulation), 96

linéairement dégénéré, 224

Lions, 154

lipchitzien(ne) (ouvert, frontière), $10,13,21,22$

lipchitzienne (fonction), 21

localement intégrable, 5

$m$-accrétif (opérateur), 127

maximal monotone (opérateur), 128

mesure de Hausdorff, 12

Meyers, 131

mild, 129

Minty, 102, 121

monotonie, 82, 95, 96, 102

Neumann, 37, 53

Nirenberg, 24, 41

noyau régularisant, 159

onde de choc, voir choc

onde de détente, voir détente

opérateur coercif, 98

opérateur transposé, 29

$p$-Laplacien, 96

Poincaré, 34

point fixe, 83,84

problème de Riemann, 203, 221, 223, 232, 234

prolongement, 9

Rankine-Hugoniot, 200, 221,223, 226

réflexif, 9, 10

Rellich, 12

Riemann, 203

Riesz, 34, 54, 136, 138

Robin, 37

réaction, 86

Saint-Venant, 223, 224, 226, 230, 231, 238, 240, 241.

$$
\text { 255, } 263
$$

Schauder, 84

Schrödinger, 57

semi-groupe, 128

séparable, 9

Smagorinsky, 96

Sobolev, 5, 7, 24

solution classique, 197 solution faible, 199

Stokes, 61, 62, 208

suite $B$-limite-incluse, 171

suite compacte-continue, 167

suite compactement incluse, 166

trace, $11,12,27,45,46,57,59,71,77,78,174$

transformée de Fourier, 124

troncature, 131

Trundiger-Möser, 60

variation bornée, voir $\mathrm{BV}$

variationnelle (formulation), 33

vraiment non linéaire, 224, 238

Wentzel, 61 